\title{
Copyright
}

by

Patricia LaVern Vahle

2004 
The Dissertation Committee for Patricia LaVern Vahle certifies that this is the approved version of the following dissertation:

\section{Electromagnetic Interactions in the MINOS Detectors}

Committee:

Karol Lang, Supervisor

Sacha Kopp

Roy Schwitters

Duane Dicus

David Lambert 


\title{
Electromagnetic Interactions in the MINOS Detectors
}

by

Patricia LaVern Vahle, B.S.

\author{
Dissertation \\ Presented to the Faculty of the Graduate School of \\ The University of Texas at Austin \\ in Partial Fulfillment \\ of the Requirements \\ for the Degree of \\ Doctor of Philosophy
}

The University of Texas at Austin

August 2004 


\section{Acknowledgments}

There are a number of people who helped to make the work described here both possible and enjoyable. Many thanks to Karol, who was a genuinely helpful and attentive advisor, and to Jenny, who kept me motivated. Thanks to the entire CalDet crew, in particular Gordon who kept me well fed and always had a room for me in London. To Madame and Monsieur D'Oro, and the Baron de Lestac, thanks for the relaxation provided after a long day in the test beam. Thanks to Marek for the technical know-how and the drawings, and to the rest of the Texas gang for making these six years memorable. To my Mom, Dad, Kayleen, and Aaron who were unwaivering in their support, and to Mike, who was right there with me through it all, thanks for the encouragement, understanding, and confidence.

Patricia LaVern VAhle

The University of Texas at Austin

August 2004 


\title{
Electromagnetic Interactions in the MINOS Detectors
}

\author{
Publication No. \\ Patricia LaVern Vahle, Ph.D. \\ The University of Texas at Austin, 2004
}

Supervisor: Karol Lang

MINOS is a long-baseline neutrino experiment designed to observe the oscillation of neutrinos traveling between two detectors, a Near Detector at Fermi National Accelerator Laboratory and a Far Detector at the Soudan Underground Laboratory in northern Minnesota. Precision measurement of the oscillation parameters requires a better than $5 \%$ absolute energy calibration which is derived using a dedicated calibration detector, called CalDet. A smaller version of the MINOS detectors, the CalDet was exposed to particle beams in the CERN PS East Area test beams in 2001-2003. This document describes the conditions under which the CalDet beam data were taken, establishes selection criteria to identify a sample of electrons, and discusses the characteristics of electromagnetic interactions in the CalDet. Showers initiated by electrons at a range of incident momenta are compared to Monte Carlo modeling and contrasted against hadronic showers. Finally, the detector electromagnetic response and energy resolution are parameterized as a function of incident momentum, and showers induced by single electrons are compared to showers induced by simulated neutrino interactions. 


\section{Contents}

Acknowledgments iv

$\begin{array}{ll}\text { Abstract } & \text { v }\end{array}$

Chapter 1 The Mysterious Neutrino 1

1.1 "A Desperate Remedy" . . . . . . . . . . . . . . . . . . . 2

1.2 Neutrino Oscillations . . . . . . . . . . . . . . . . 3

1.2.1 The Theory of Neutrino Oscillation . . . . . . . . . . . . 3

1.2.2 Evidence for Oscillations . . . . . . . . . . . . . . . 5

1.3 The Future of Neutrino Oscillation Experiments . . . . . . . . . . . 10

$\begin{array}{lll}\text { Chapter } 2 & \text { MINOS } & 13\end{array}$

2.1 MINOS Capabilities . . . . . . . . . . . . . . . . 14

2.2 The Beam . . . . . . . . . . . . . . . . . . 16

2.3 The Detectors . . . . . . . . . . . . . . . . . 21

2.3 .1 The Far Detector . . . . . . . . . . . . . . . 22

2.3.2 The Near Detector . . . . . . . . . . . . . . . 24

2.3.3 The Calibration Detector . . . . . . . . . . . 26

$\begin{array}{lll}\text { Chapter } 3 & \text { The CalDet Beam Runs } & 30\end{array}$

3.1 The Beam Lines . . . . . . . . . . . . . . . . . . . . . . . 30 
3.1.1 The T11 Beam Line . . . . . . . . . . . . . . . 31

3.1.2 The T7 Beam Line . . . . . . . . . . . . . . . . . . . 33

3.2 Particle Identification . . . . . . . . . . . . . . . . . . 34

3.2.1 Čerenkov Detectors. . . . . . . . . . . . . . 35

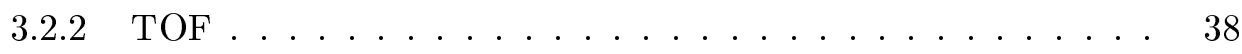

3.3 MINOS in a Beam . . . . . . . . . . . . . . . . 41

3.4 Run Configurations . . . . . . . . . . . . . . . . . . 43

$\begin{array}{lll}\text { Chapter } 4 & \text { The MINOS Calibration Scheme } & 47\end{array}$

4.1 Charge Injection . . . . . . . . . . . . . . . . . . . 48

4.2 Light Injection . . . . . . . . . . . . . . . . . . . . . . . 49

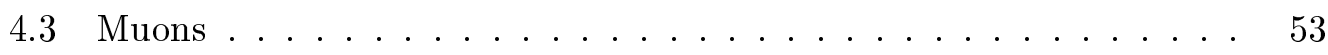

$\begin{array}{lll}\text { Chapter } 5 & \text { Electron Selection Criteria } & 62\end{array}$

5.1 Pre-selection Criteria . . . . . . . . . . . . . . . . . . 62

5.2 Electron Event Selection Cuts . . . . . . . . . . . . . 63

$\begin{array}{llr}\text { Chapter } 6 & \text { Beam Line Systematics } & 87\end{array}$

6.1 The External TOF Enable . . . . . . . . . . . . . . 87

6.2 Beam Intensity . . . . . . . . . . . . . . . . . . . 88

6.3 Differences between Spills . . . . . . . . . . . . . . . . . . . 90

6.4 Response Measurement Repeatability . . . . . . . . . . . . . . . . 92

6.5 Simulating Energy Losses in Upstream Material . . . . . . . . . . . . 98

$\begin{array}{llr}\text { Chapter } 7 & \text { Electron Characteristics } & 115\end{array}$

7.1 Electromagnetic Interactions in Matter . . . . . . . . . . . . 115

7.2 Comparison with Monte Carlo _ . . . . . . . . . . . . . . 117

7.3 Contrasting Electrons and Hadrons . . . . . . . . . . . . . . . . . . 119 
Chapter 8 Response and Resolution

8.1 Deriving the Response . . . . . . . . . . . . . . . . . . 136

8.2 Correcting for Upstream Energy Loss . . . . . . . . . . . . . . . . . . 142

8.3 Response and Resolution Versus Momentum . . . . . . . . . . . . . . 144

8.4 Comparison of Near FEE Response . . . . . . . . . . . . . . . . . 151

$\begin{array}{lll}\text { Chapter } 9 & \text { Looking Toward Neutrino Interactions } & 158\end{array}$

9.1 Electrons at Angled Incidence . . . . . . . . . . . . . . . . . . 159

9.2 First Plane Effects . . . . . . . . . . . . . . . . . . 165

9.3 Neutrinos in CalDet . . . . . . . . . . . . . . . . . . 172

$\begin{array}{lr}\text { Chapter } 10 \text { Conclusions } & 184\end{array}$

$\begin{array}{lr}\text { Appendix A Event Displays } & 187\end{array}$

$\begin{array}{lll}\text { Appendix B Electron Selection Cuts } & 206\end{array}$

B.1 The Cut Distributions . . . . . . . . . . . . . 206

B.2 The Number of Planes Hit Distributions . . . . . . . . . . . . . 212

$\begin{array}{lll}\text { Appendix C Beam Line Simulation Plots } & \mathbf{2 1 5}\end{array}$

C.1 Momentum, Vertex Position, and Beam Spot . . . . . . . . . . 215

C.2 Particle Multiplicities and Energy . . . . . . . . . . . . . . 223

$\begin{array}{ll}\text { Appendix D Comparisons with Monte Carlo } & 231\end{array}$

Appendix E CalDetDST Offline Analysis Package $\quad \mathbf{2 6 7}$

E.1 Introduction . . . . . . . . . . . . . . . . . 267

E.2 CalDetSI . . . . . . . . . . . . . . . . . . . . 268

E.3 CalDetPID . . . . . . . . . . . . . . . . . 270

E.4 CalDetDST . . . . . . . . . . . . . . . . . . 272 
$\begin{array}{lll}\text { Appendix F } & \text { CalDetDBUtils Package } & \mathbf{2 7 9}\end{array}$

F.1 DCSEnvCal . . . . . . . . . . . . . . . . . . . . . 279

F.2 DCSMagCal . . . . . . . . . . . . . . . . . . . 279

F.3 CalDetSuperCycle . . . . . . . . . . . . . . . . . . . . 282

F.4 SpillErrors . . . . . . . . . . . . . . . . . . . . 282

F.5 MomSetErrors . . . . . . . . . . . . . . . . . . 282

F.6 BeamLoss . . . . . . . . . . . . . . . . . . 283

$\begin{array}{ll}\text { Bibliography } & 285\end{array}$

$\begin{array}{lr}\text { Vita } & \mathbf{2 9 0}\end{array}$ 


\section{Chapter 1}

\section{The Mysterious Neutrino}

In recent years experimental physicists have exerted widespread effort to determine whether or not neutrinos experience oscillations, a phenomenon in which a neutrino generated as one flavor is later detected as a neutrino of a different flavor. To undergo oscillations, the neutrino must have mass, undermining one of the basic tenets of the Standard Model of particle interactions. Understandably, this hint of physics beyond the scope of one of the most successful physics theories of the 20th century has inspired a host of experiments. Among the latest, Super Kamiokande studies the properties of neutrinos produced by the interaction of cosmic rays in the atmosphere and as well as the properties of neutrinos produced in the sun, the Sudbury Neutrino Observatory studies neutrinos produced in the sun, while KamLAND observes neutrinos produced in nuclear reactors. While these experiments provide consistent evidence that neutrinos undergo oscillations, another experiment, LSND, suggests that more exotic phenomena may be at work in neutrino interactions. The Main Injector Neutrino Oscillation Search, or MINOS, will remove any doubt concerning the occurrence of oscillations by providing a precision measurement of the parameters involved in the oscillation of this elusive lepton. 


\section{1 "A Desperate Remedy"}

The history of the neutrino begins in 1930. In a letter addressed to "Dear Radioactive Ladies and Gentlemen", W. Pauli postulated the existence of a new particle [1]. His famous letter proposed this formerly outlandish idea in order to avoid the even more unsavory conclusion that energy was not conserved in $\beta$ decay. Pauli's particle had to be light, with a mass "of the same order of magnitude as the electron mass", and had to be weakly interacting in order to have escaped detection. In 1953, the first experimental evidence indicating the existence of this particle, which had come to be known as the neutrino, was obtained by Cowan and Reines [2]. Their original experiment investigated the reaction $\bar{\nu}_{e}+p \rightarrow n+e^{+}$. Using neutrinos produced in the Hanford nuclear power reactor incident on a liquid scintillator detector, Cowan and Reines looked for a signal from the annihilation of the positron followed by signal coming from neutron capture $5 \mu \mathrm{s}$ later. This technique of looking for the delayed-coincidence of two interactions allowed for a demonstration of the existence of this extremely weakly interacting particle. The cross section for the process studied in the Cowan and Reines experiment is $6 \times 10^{-20}$ barn. Neutrinos are likely to pass through a light-year long block of lead without interacting, explaining the 23 years it took to confirm the existence of Pauli's lepton. Since the confirmation of the existence of the first neutrino, now called the electron neutrino, two more flavors of neutrinos, the muon neutrino and the tau neutrino, have been discovered [3], [4]. Furthermore, it has been established that there are precisely three weakly interacting neutrinos [5].

Despite the difficulties involved in detecting neutrinos, these particles have played a fundamental role in probing the interactions of other particles. The Standard Model calls for two types of weak interactions. The first, called the charged current (CC) interaction, proceeds via the exchange of a $W$ charged boson, with a charged lepton in the final state. The second, called the neutral current (NC) 
interaction, proceeds via the exchange of the $Z^{0}$ neutral boson, with an electrically neutral lepton in the final state. Neutrinos were discovered via the charged current interaction, but before the Standard Model, neutral current interactions had not been observed. However, using a beam of neutrinos to induce reactions of the type $\nu_{\mu}+N \rightarrow \nu_{\mu}+$ hadrons, neutral current interactions were discovered, and the observation of such interactions established the Standard Model of particle interactions as a viable theory [6]. Ironically, neutrinos now provide the impetus for extensions to the Standard Model. The Standard Model assumes the neutrino is massless, and for many years, this assumption has not been challenged experimentally. However, 50 years since it's discovery, evidence is emerging that indicates the neutrino mass is non-zero.

\subsection{Neutrino Oscillations}

In the years shortly after the confirmation of the existence of the neutrino, an experiment was conducted that attempted to use the neutrino as a probe of the fusion processes that fuel the sun. The Homestake experiment was designed to count the number of electron neutrinos coming from sun; however, it was soon discovered that there was a deficiency in the number of neutrinos below that predicted by the Standard Solar Model [7]. Eventually a new "desperate remedy", that of neutrino oscillation, emerged as the leading explanation of the discrepancy between the calculation and the experimental data.

\subsubsection{The Theory of Neutrino Oscillation}

The theory of neutrino oscillation hinges on the assumption that neutrinos have mass, and that the mass eigenstates are not the same as the flavor eigenstates. If this is the case, the flavor eigenstates can be represented as a linear superposition 
of the mass eigenstates:

$$
\left|\nu_{f}>=\sum_{m=1}^{3} U_{f m}\right| \nu_{m}>
$$

where $\mid \nu_{f}>$ represents a flavor eigenstate, $\nu_{e}, \nu_{\mu}$, or $\nu_{\tau}, \mid \nu_{m}>$ represents a mass eigenstate, and $U_{f m}$ is the $f, m$ element of a unitary matrix giving the amplitude of the mixing among the different states.

The equations governing oscillations are simplified by considering the case in which only two flavors are involved in oscillation. Moreover, most observations can be understood, at least to first order, in terms of the case of two flavor and two mass states. Taking the flavor states $\nu_{\mu}$ and $\nu_{\tau}$, and the mass states $\nu_{2}$ and $\nu_{3}$, Equation 1.1 can then be written as

$$
\begin{aligned}
& \left|\nu_{\mu}>=\cos \theta\right| \nu_{2}>+\sin \theta \mid \nu_{3}> \\
& \left|\nu_{\tau}>=-\sin \theta\right| \nu_{2}>+\cos \theta \mid \nu_{3}>
\end{aligned}
$$

As mass eigenstates, $\mid \nu_{2}>$ and $\mid \nu_{3}>$ are also eigenstates of the free particle Hamiltonian with energies $E_{2}=\sqrt{p^{2}+m_{2}^{2}}$ and $E_{3}=\sqrt{p^{2}+m_{3}^{2}}$ respectively. Applying the time evolution operator, $e^{-i H t}$, to the $\nu_{\mu}$ flavor eigenstate dictates how that state propagates with time:

$$
\left|\nu_{\mu}(t)>=\cos \theta e^{-i E_{2} t}\right| \nu_{2}>+\sin \theta e^{-i E_{3} t} \mid \nu_{3}>
$$

in units where $\hbar=c=1$. Then, the probability that a neutrino originally of the $\nu_{\mu}$ flavor will at a time $t$ be detected as a $\nu_{\tau}$ is

$$
\begin{aligned}
P\left(\nu_{\mu} \rightarrow \nu_{\tau}\right) & =\mid\left\langle\nu_{\tau}\left|\nu_{\mu}(t)>\right|^{2}\right. \\
& =\sin ^{2} \theta \cos ^{2} \theta\left|-e^{-i E_{2} t}+e^{-i E_{3} t}\right|^{2} \\
& =\sin ^{2}(2 \theta) \sin ^{2}\left(\frac{\left(E_{3}-E_{2}\right) t}{2}\right) .
\end{aligned}
$$

For relativistic particles created with the same momentum,

$$
E_{3}-E_{2}=\sqrt{p^{2}+m_{3}^{2}}-\sqrt{p^{2}+m_{2}^{2}}
$$




$$
\begin{aligned}
& \approx \frac{m_{3}^{2}-m_{2}^{2}}{2 E} \\
& =\frac{\Delta m^{2}}{2 E}
\end{aligned}
$$

where $\Delta m^{2} \equiv m_{3}^{2}-m_{2}^{2}$. If $L$ is the distance the particle travels in time $t$, Equation 1.4 becomes

$$
P\left(\nu_{\mu} \rightarrow \nu_{\tau}\right)=\sin ^{2}(2 \theta) \sin ^{2}\left(\frac{\Delta m^{2} L}{4 E}\right),
$$

and the survival probability, or the probability that a $\nu_{\mu}$ will be detected as a $\nu_{\mu}$ after traversing a distance $L$ is

$$
\begin{aligned}
P\left(\nu_{\mu} \rightarrow \nu_{\mu}\right) & =1-P\left(\nu_{\mu} \rightarrow \nu_{\tau}\right) \\
& =1-\sin ^{2}(2 \theta) \sin ^{2}\left(\frac{1.27 \Delta m^{2} L}{E}\right)
\end{aligned}
$$

where $\Delta m^{2}$ is measured in eV, $L$ is measured in $\mathrm{km}, E$ is measured in $\mathrm{GeV}$, and the factor of 1.27 arises from the unit conversion. Figure 1.1 illustrates the behavior of Equation 1.7 with respect to neutrino energy for different values of $\Delta m^{2}$, with fixed values of $\sin ^{2}(2 \theta)=1$ and $L=735 \mathrm{~km}$. The curves are characterized by an oscillatory behavior. The survival probability depends on two unknown parameters, the mixing angle and the difference of the squares of the masses between the two neutrinos involved in the mixing. The amplitude of the oscillation is modulated by the value of $\sin ^{2}(2 \theta)$, and for a given length, the energy of the first minimum in the survival probability decreases with a smaller $\Delta m^{2}$. At energies below the first few minima, small changes in the neutrino energy result in large changes in the survival probability. With limited experimental resolution, a measurement of survival probability would yield an average of $\frac{1}{2}$.

\subsubsection{Evidence for Oscillations}

Since the Homestake experiment, many other experiments have added convincing evidence supporting the existence of neutrino oscillations. Three of these exper- 


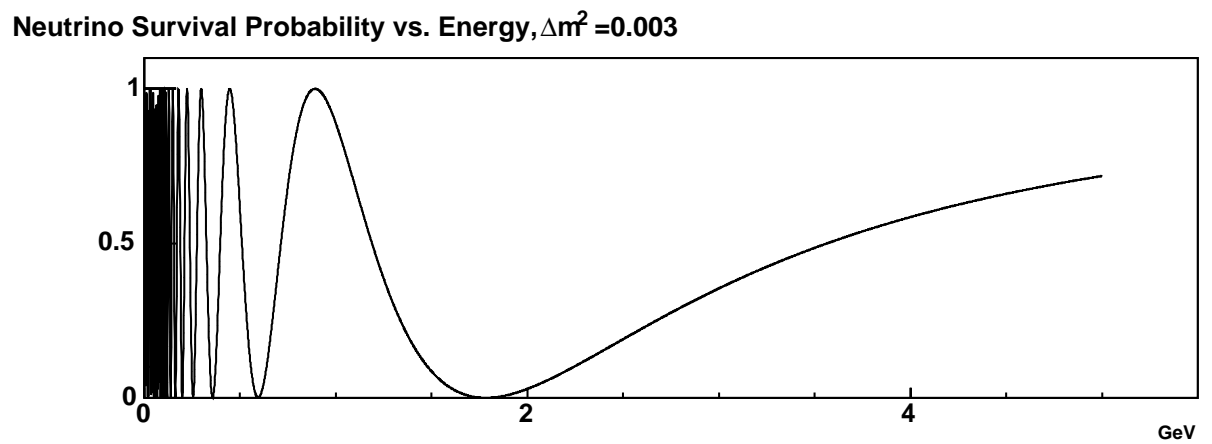

Neutrino Survival Probability vs. Energy, $\Delta \mathrm{m}^{2}=0.002$

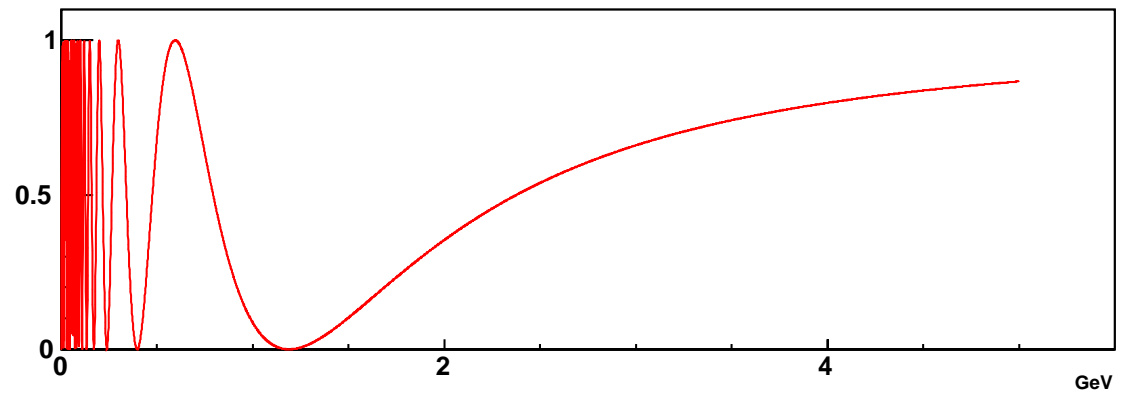

Neutrino Survival Probability vs. Energy, $\Delta \mathrm{m}^{2}=0.001$

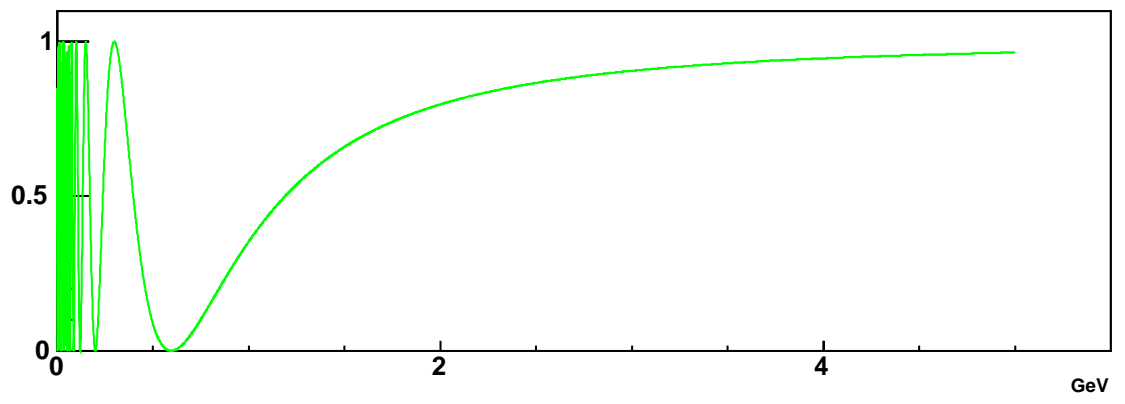

Figure 1.1: Neutrino survival probability versus energy for $\sin ^{2}(2 \theta)=1$ and $L=$ $735 \mathrm{~km}$. Top plot, $\Delta m^{2}=.003 \mathrm{eV}^{2}$; middle plot, $\Delta m^{2}=.002 \mathrm{eV}^{2}$; bottom plot $\Delta m^{2}=.001 \mathrm{eV}^{2}$. 
iments, Super Kamiokande, which investigates the interactions of both solar and atmospheric neutrinos, SNO, which investigates the interactions of solar neutrinos, and KamLAND, which investigates the interactions of reactor neutrinos, are discussed further here.

\section{Super Kamiokande}

Super Kamiokande uses a water Čerenkov detector to detect neutrinos produced by cosmic ray interactions in the atmosphere [8]. Atmospheric neutrino production is dominated by the process $\pi^{ \pm} \rightarrow \mu^{ \pm}+\nu_{\mu}\left(\bar{\nu}_{\mu}\right)$. The $\mu^{ \pm}$then decays via the reaction $\mu^{ \pm} \rightarrow e^{ \pm}+\nu_{e}\left(\bar{\nu}_{e}\right)$. Both the $\nu_{\mu}$ and the $\nu_{e}$ are detected in the Cerenkov, and the number of each neutrino type observed is compared to expectation. Super Kamiokande reports their results as a double ratio, $R$, defined as the measured ratio of $\mu_{\text {like }}$ to $e_{\text {like }}$ events divided by the same ratio as predicted by Monte Carlo simulation. They find a value of $R$ of $0.638_{-0.017}^{+0.017} \pm 0.050$ for sub-GeV events, and $0.675_{-0.032}^{+0.034} \pm 0.080$ for multi-GeV events [9]. In the absence of oscillations, one would expect $R=1$, thus the Super Kamiokande results provide a more than 5 sigma deviation from expectation in the case of no oscillations. Moreover, since the production of neutrinos in the atmosphere should be roughly isotropic, the dependence of the $\nu_{\mu}$ and $\nu_{e}$ fluxes on zenith angle, hence distance traveled, can be used to extract measurements of the mixing parameters. Figure 1.2 shows the results of 1289 days of data collection at the Super Kamiokande experiment. The left column of plots shows the number of $\nu_{e}$ events recorded versus the zenith angle while the right column of plots shows the number of $\nu_{\mu}$ events versus the zenith angle. The distributions of $\nu_{e}$ events agree well with the expectation from no oscillations, while the $\nu_{\mu}$ distributions do not, indicating that $\nu_{\mu}$ oscillate into $\nu_{\tau}$ with $\Delta m^{2}=$ $2.5 \times 10^{-3} \mathrm{eV}^{2}$ and $\sin ^{2}(2 \theta)=1.00$.

The Super Kamiokande detector is instrumental in another measurement 
of oscillations done by the KEK to Kamioka (K2K) neutrino oscillation experiment [10]. This experiment probes the neutrino oscillation parameters by studying accelerator neutrinos produced using $12 \mathrm{GeV}$ protons from the KEK proton synchrotron incident on an aluminum target. The K2K collaboration measures a reduction in $\nu_{\mu}$ flux, 56 observed events compared to 80.1 $1_{-5.4}^{+6.2}$ expected events, coupled with a distortion of the energy distribution of interactions in the SuperK detector relative to that measured in two smaller detectors $250 \mathrm{~km}$ upstream of the large water Cerenkov. The oscillation parameters derived from these observations are consistent with the measurement conducted with atmospheric neutrinos.

\section{SNO}

While the Super Kamiokande experiment also studies the interactions of solar neutrinos, the latest measurement using solar neutrinos comes from the Sudbury Neutrino Observatory, or SNO. SNO also uses a water Čerenkov detector, with heavy water as the active medium. SNO detects neutrino interactions via 3 reactions. The first, the charged current reaction, $\nu_{e}+d \rightarrow p+p+e^{-}$is initiated only by electron flavored neutrinos. On the other hand, the neutral current reaction, $\nu_{x}+d \rightarrow p+n+\nu_{x}$ and the elastic scattering reaction, $\nu_{x}+e^{-} \rightarrow \nu_{x}+e^{-}$can be induced by any neutrino flavor. Fusion processes in the sun only produce electron flavored neutrinos. These $\nu_{e}$ 's travel 150 million $\mathrm{km}$ from the sun before interacting in the SNO detector. If neutrinos do not oscillate, then the measured flux of non-electron type neutrinos from the sun should be zero. SNO measures a total neutrino flux consistent with the Standard Solar Model. It finds the component of the flux due to electron neutrinos to be $1.76_{-0.05}^{+0.05}$ (stat.) ${ }_{-0.09}^{+0.09}($ syst. $) \times 10^{6} \mathrm{~cm}^{-2} \mathrm{~s}^{-1}$ and measures the component of the

flux due to non-electron neutrinos to be $3.41_{-0.45}^{+0.45}($ stat. $){ }_{-0.45}^{+0.48}($ syst. $) \times 10^{6} \mathrm{~cm}^{-2} \mathrm{~s}^{-1}$, a result $5.3 \sigma$ greater than the expectation of zero [11]. SNO clearly demonstrates 

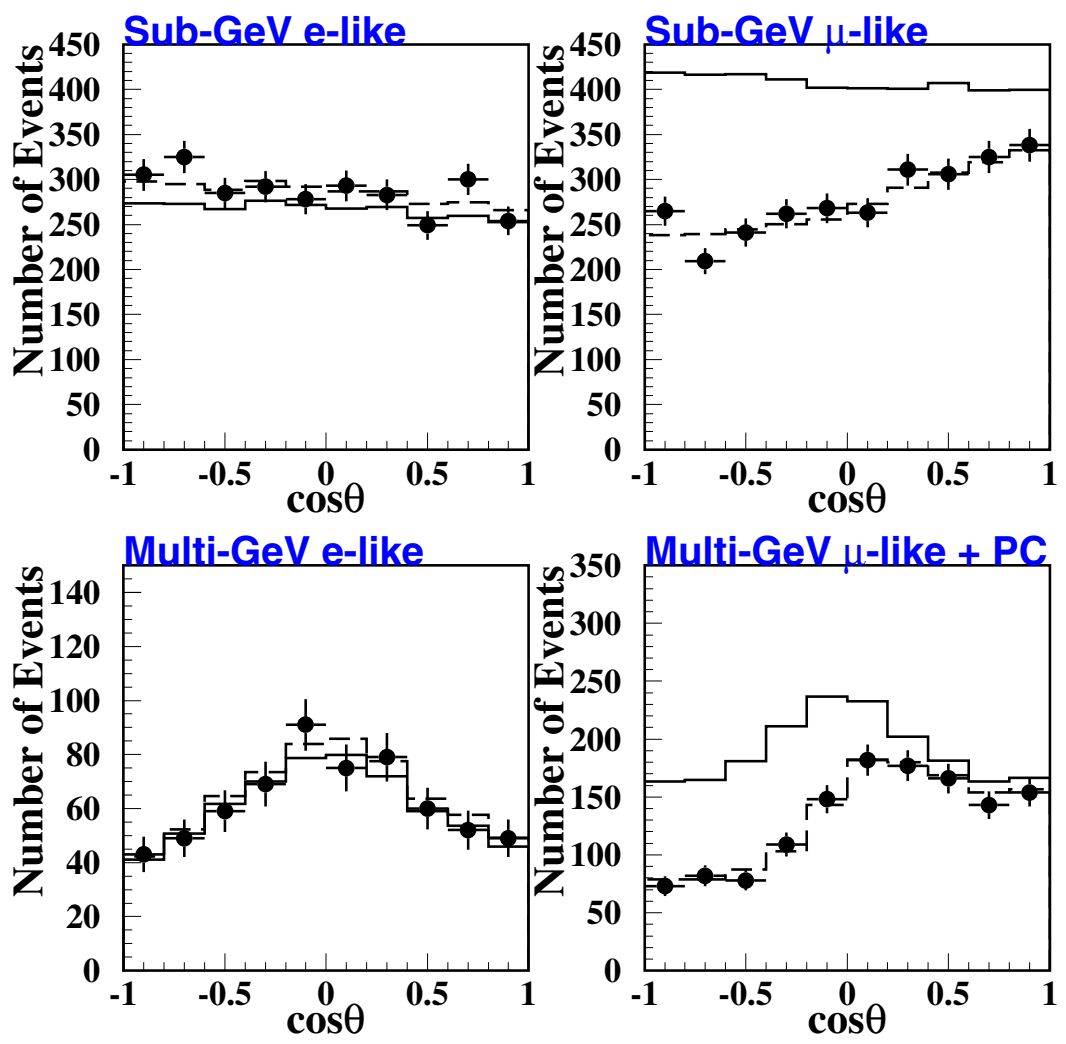

Figure 1.2: Results of 1289 days of data collection from the Super Kamiokande experiment. The left column of plots shows the number of $\nu_{e}$ events recorded versus the zenith angle $(\cos \theta=1$ corresponds to downward going neutrinos) while the right column of plots shows the number of $\nu_{\mu}$ events versus the zenith angle. The top row of plots corresponds to neutrinos with less than $1.3 \mathrm{GeV}$ incident energy, while the bottom row corresponds to higher energy neutrinos. The dots correspond to the data, the solid line to Monte Carlo predictions without oscillations, and the dashed line to Monte Carlo predictions with oscillations assuming $\nu_{\mu} \rightarrow \nu_{\tau}$ with $\Delta m^{2}=2.5 \times 10^{-3} \mathrm{eV}^{2}$ and $\sin ^{2}(2 \theta)=1.00[9]$. 
that the flavor of solar neutrinos changes during the journey from the sun to the earth.

\section{KamLAND}

Both Super Kamiokande and SNO rely on neutrinos from natural sources. Another experiment, the Kamioka Liquid scintillator Anti-Neutrino Detector (KamLAND), studies the behavior of man made neutrinos, namely those produced in the fission reactions that power nuclear power plants. Using a liquid scintillator detector, KamLAND measures the $\nu_{e}$ flux from many nuclear reactors at an average distance of $\approx 180 \mathrm{~km}$. KamLAND observes 258 events with an expected $365 \pm 24$ events in the case of no oscillations [12]. Moreover, as shown in Figure 1.3, the deficit of measured neutrino events compared to expectation depends on the energy of the incident neutrino. A fit to the spectral shape gives values of the mixing parameters of $\sin ^{2}(2 \theta)=0.83$ and $\Delta m^{2}=8.3 \times 10^{-5} \mathrm{eV}^{2}$, results consistent with the SNO measurement of $\nu_{e}$ oscillations.

\subsection{The Future of Neutrino Oscillation Experiments}

With three neutrino flavors, there are 2 independent mass squared differences. The experiments described above provide consistent measurements of the two possible values of $\Delta m^{2}$. However, another experiment, the Liquid Scintillator Neutrino Detector (LSND), also provides evidence of neutrino oscillations, but with a value of $\Delta m^{2}$ much larger that that seen in other experiments. LSND uses a beam of accelerator produced $\bar{\nu}_{\mu}$ and searches for the appearance of $\bar{\nu}_{e}$ in the liquid scintillator detector $30 \mathrm{~m}$ downstream of the neutrino source. LSND reports the appearance of $51.8_{-16.9}^{+18.7} \pm 8.0$ excess $\bar{\nu}_{e}$ events above that expected from background [13]. In order to explain the LSND results in terms of oscillations, $\Delta m^{2}$ must be on the order of $1 \mathrm{eV}^{2}$, a requirement not consistent with the measurement of $\Delta m^{2}$ on the order of 


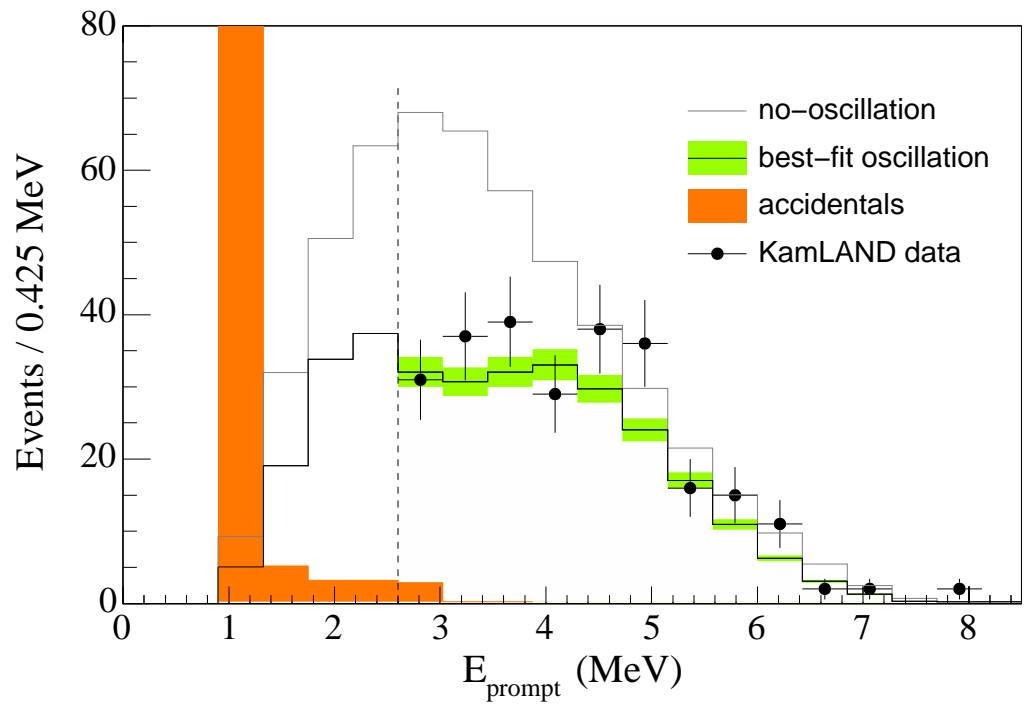

Figure 1.3: Prompt event energy spectrum of the KamLAND $\bar{\nu}_{e}$ candidate events [12].

$10^{-3} \mathrm{eV}^{2}$ of the atmospheric experiments, nor with $\Delta m^{2}$ on the order of $10^{-5} \mathrm{eV}^{2}$ of the solar experiments. Proposed solutions to resolve the conflict range from adding a fourth, non-interacting, or sterile, neutrino to the lepton family to allowing the violation of the Charge-Parity-Time symmetry of the Standard Model [14]. Another experiment, MiniBoone, is currently taking data to confirm or refute the measurement [15]. If the results are confirmed, the story of neutrino interactions will change dramatically.

Beyond exploring the questions raised by the LSND result, future neutrino experiments aim at studying effects of flavor transitions implied by considering the full, 3-flavor oscillation analysis. Both the newly named NuMI Off-Axis $\nu_{e}$ Appearance Experiment $(\mathrm{NO} \nu \mathrm{A})$ and the Japanese project JHF-Kamioka propose to measure the sub-dominant mixing of $\nu_{\mu} \rightarrow \nu_{e}[16]$, [17]. Such a measurement can shed light on the neutrino mass hierarchy and also probe Charge-Parity violation. 
Other studies comparing the oscillation among anti-neutrinos to that among neutrinos provide tests sensitive to the violation of the Charge-Parity-Time symmetry thought to be strictly obeyed by nature.

Strong evidence indicates that something happens to neutrinos as they move away from their source. Neutrino oscillation is the most likely candidate to explain this behavior, but a precision measurement of the parameters dictating the mixing has yet to be made. The Main Injector Neutrino Oscillation Search, or MINOS, is poised to make this measurement and unambiguously demonstrate that neutrinos oscillate. 


\section{Chapter 2}

\section{MINOS}

MINOS is a long-baseline neutrino oscillation experiment designed to provide a precision measurement of the oscillation parameters governing the mixture of neutrinos in the parameter space suggested by atmospheric neutrino studies. The MINOS experiment uses an intense beam of $\nu_{\mu}$ produced at the Neutrinos at the Main Injector (NuMI) beam line at Fermi National Accelerator Laboratory. In order to minimize systematic uncertainties of the beam flux and composition, MINOS employs two similar detectors to observe neutrino interactions. The first detector, or Near Detector, is located on-site at Fermilab, $1000 \mathrm{~m}$ downstream of the neutrino production target. The second detector, or Far Detector, is located $735 \mathrm{~km}$ away in the Soudan Underground Laboratory in northern Minnesota. A third, smaller detector, or Calibration Detector, is used to calibrate the response of the MINOS detectors. Figure 2.1 shows a schematic of the locations of the large MINOS detectors. 


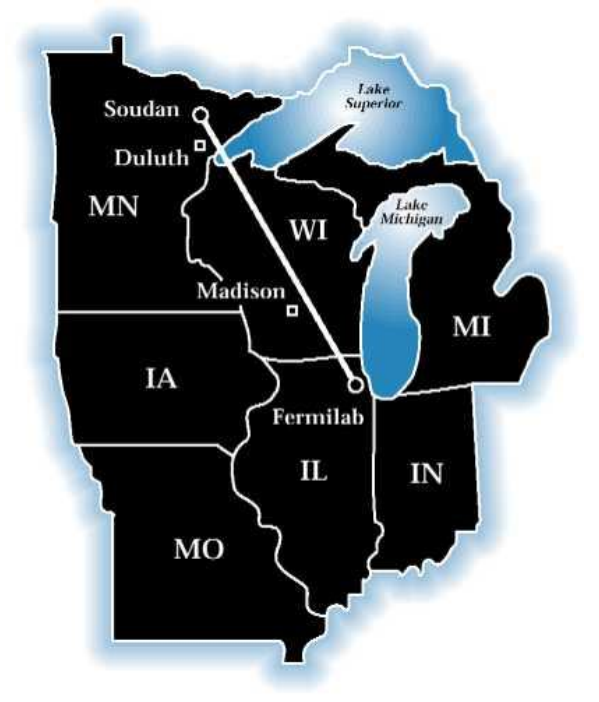

Figure 2.1: MINOS detector locations

\subsection{MINOS Capabilities}

MINOS will verify the oscillatory nature of neutrinos by measuring a statistically significant difference in the nature of the neutrino beam at the Far Detector as compared to that predicted by the Near Detector. The primary MINOS measurement involves comparing the $\nu_{\mu}$ charged current energy spectrum at the Far Detector with the extrapolated Near Detector spectrum. In this way MINOS will obtain the total survival probability of $\nu_{\mu}$ as a function of neutrino energy. With values of the oscillation parameters as suggested by Super Kamiokande, MINOS will be able to observe a dip in the survival probability around $1.5 \mathrm{GeV}$ incident neutrino energy, then a rise in the spectrum toward lower energies. Such an observation will be a powerful tool in discriminating among alternate mechanisms, such as neutrino decay or neutrino decoherence, that could cause the disappearance of neutrinos and will unambiguously indicate that the disappearance of neutrinos is caused by oscillations [18], [19]. Figure 2.2 shows the expected ratio of the number of charged 

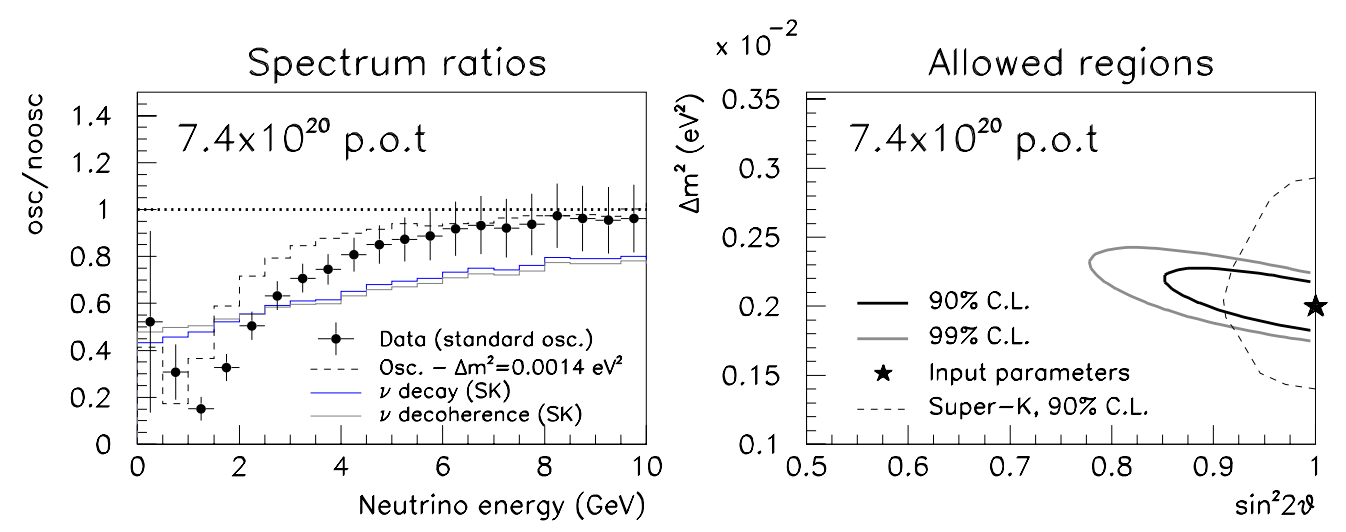

Figure 2.2: To the left, the ratio of $\nu_{\mu}$ charged current events expected in the Far Detector with oscillations to the predicted number of events with no oscillations as a function of neutrino energy. To the right, the confidence level regions from the MINOS measurement [20].

current events in the Far Detector with oscillations to the number of charged current events in the case of no oscillations. The dependence of the survival probability on neutrino energy is obvious, as is the first minimum and the rise at lower energies. If oscillations are observed, the energy dependence will provide precision measurements of $\Delta m^{2}$ and $\sin ^{2}(2 \theta)$. MINOS has particularly good sensitivity to the value of $\Delta m^{2}$. For $\sin ^{2}(2 \theta)$ near 1 , and with enough neutrinos to minimize statistical errors, MINOS will constrain $\Delta m^{2}$ to better than $10 \%$ [20].

An independent confirmation of the oscillatory nature of neutrinos can be derived by comparing the ratio of charged current events to neutral current events in each of the detectors. A test of this nature has power to distinguish between the case of oscillations versus that of no oscillations, and it is sensitive to the mode of the mixing. In particular, the shape of the neutral current energy spectrum can be used to discriminate between $\nu_{\mu} \rightarrow \nu_{\tau}$ and $\nu_{\mu} \rightarrow \nu_{\text {sterile }}$ oscillations [21]. Since sterile neutrinos will not interact at all, any component of oscillation into sterile 
neutrinos will suppress the number of neutral current interactions recorded at the Far Detector. Figure 2.3 shows the energy spectrum of neutral current events for different components of oscillation into sterile neutrinos along with the unoscillated spectrum.

Another important goal of the MINOS experiment is to search for a small component of $\nu_{\mu} \rightarrow \nu_{e}$ oscillation by identifying an excess of $\nu_{e}$ charged current interactions in the MINOS Far Detector. Study of the sub-dominant neutrino oscillation modes provides the impetus behind many of the next generation neutrino experiments. Figure 2.4 shows the parameter space for which MINOS could observe $\nu_{e}$ appearance [20]. MINOS has the capability to extend the search for sub-dominant mixing between $\nu_{\mu}$ and $\nu_{e}$ beyond the region excluded by the CHOOZ experiment, which currently provides the best limits on this mode of mixing [22].

Finally, MINOS is the first large underground detector with a magnetic field. Using the magnetic field to charge separate the particles that result from atmospheric neutrino interactions, MINOS will be able to compare the oscillation properties of neutrinos and anti-neutrinos.

\subsection{The Beam}

The NuMI neutrino beam is a tertiary beam resulting from the decays of pions and kaons produced by $120 \mathrm{GeV}$ protons from the Main Injector interacting in a graphite target [23]. The charged hadrons produced in the target are focused into a beam using two magnetic horns. Once focused, the secondary particles travel down a $675 \mathrm{~m}$ long, $2 \mathrm{~m}$ diameter pipe, where they decay into the neutrinos used for the MINOS experiment. At the end of the decay pipe, hadron and muon absorbers allow for the ranging out of any hadrons that did not decay and the muons created in the decays. Monitors are stationed at the end of the decay pipe to measure the 

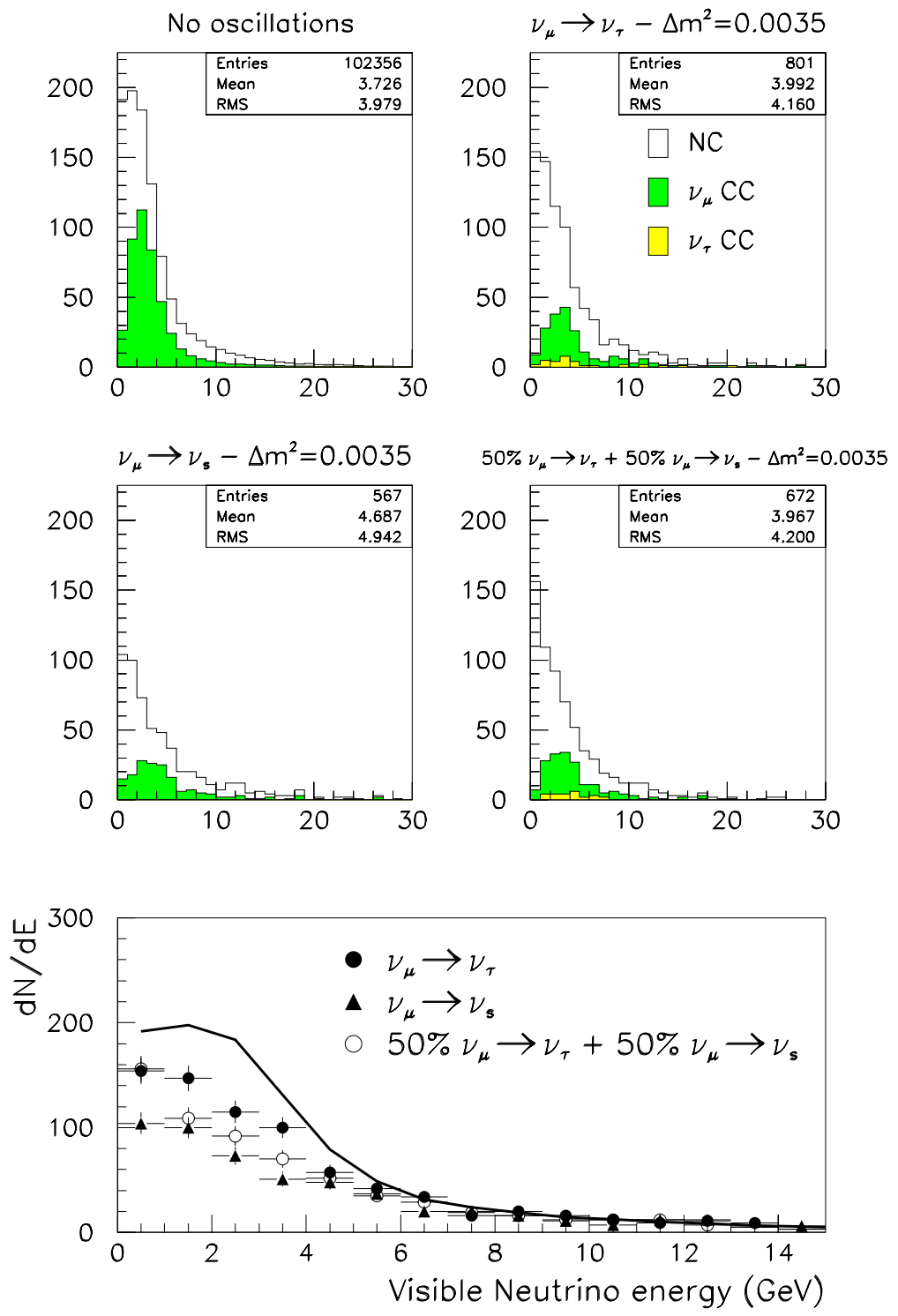

Figure 2.3: Energy distribution of neutral current candidates. Top left plot shows the energy distribution in the Far Detector with no oscillations. Top right shows the neutral current energy distribution in the Far Detector if $\nu_{\mu}$ oscillate to $\nu_{\tau}$ with $\Delta m^{2}=0.0035 \mathrm{eV}^{2}$. Middle left shows the distribution if $\nu_{\mu}$ oscillate to sterile neutrinos with $\Delta m^{2}=0.0035 \mathrm{eV}^{2}$. Middle right shows the distribution if $\nu_{\mu}$ oscillate to an equal admixture of $\nu_{\tau}$ and sterile. The bottom plot shows a comparison of the energy distribution for each of the scenarios [21]. 
$3 \sigma$ Contours

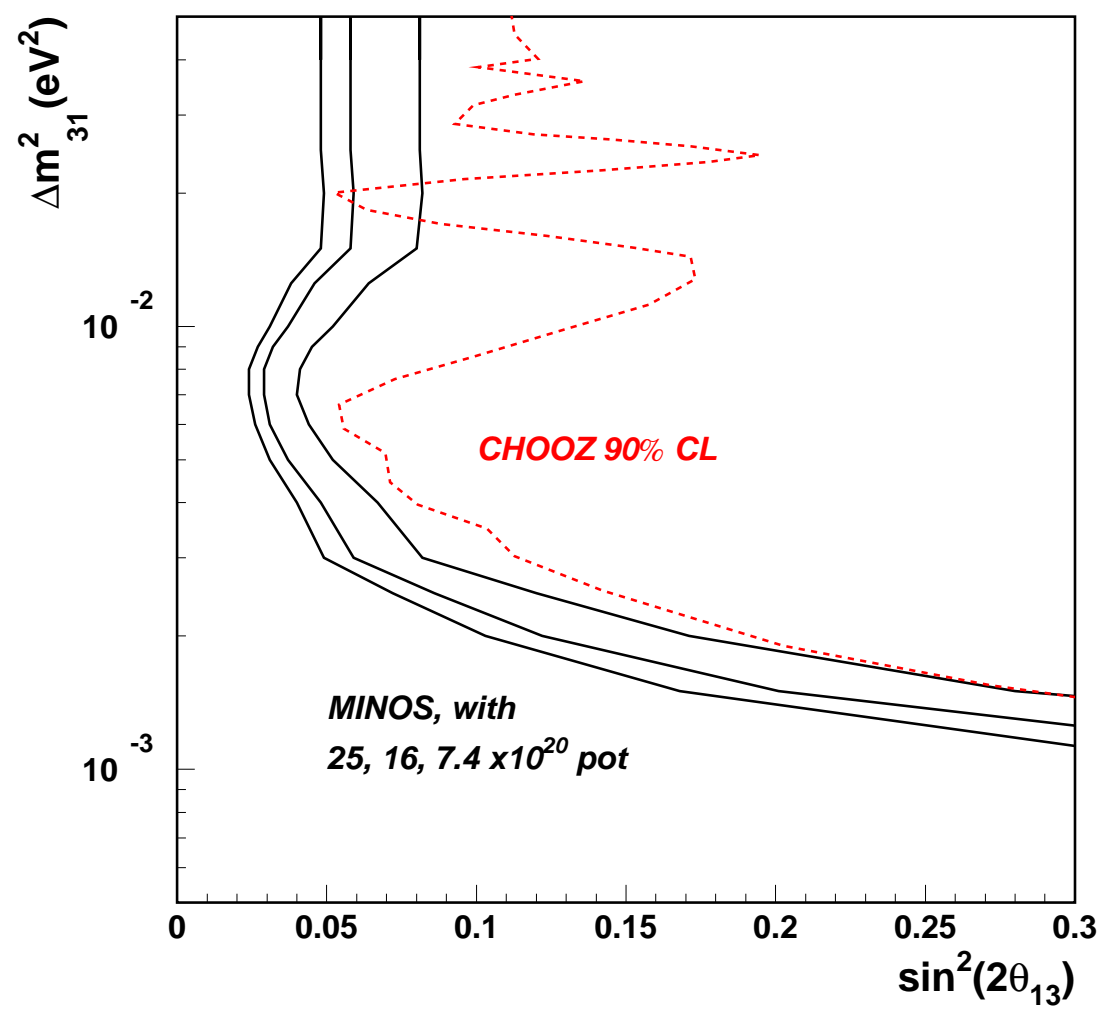

Figure 2.4: The parameter limits for which MINOS can make a $3 \sigma$ discovery of $\nu_{\mu} \rightarrow$ $\nu_{e}$, assuming $\Delta m^{2}$ is known from $\nu_{\mu}$ disappearance and that systematic uncertainty on background subtraction is $5 \%$. The dotted red curve outlines the area excluded by CHOOZ, the most sensitive measurement to date [20]. 
properties of the hadron and muon components of the beam [24]. Figure 2.5 shows a schematic of the NuMI beam line.

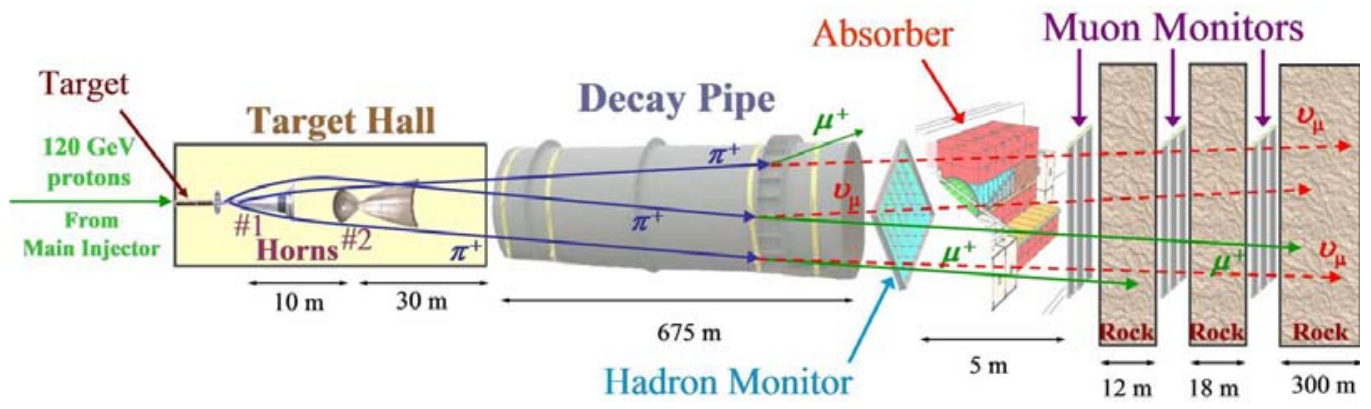

Figure 2.5: A schematic of the NuMI beam line. Courtesy of B. Zwaska

The optimally focused neutrino momentum depends on the relative placement of the target and two horns; by changing the relative placement, the peak momentum of the resultant neutrino beam can be changed. This situation allows for the possibility of three different beams, termed high energy, medium energy and low energy, and provides MINOS with the flexibility to optimize the measurement of the survival probability for different $\Delta m^{2}$. Figure 2.6 shows the energy spectrum of $\nu_{\mu}$ interactions to be observed at the Far Detector in the absence of oscillations for each of the beam configurations. The low energy beam peaks at about $3 \mathrm{GeV}$, the medium energy beam peaks around $6-8 \mathrm{GeV}$, while the high energy beam has a wide peak ranging from about $10-20 \mathrm{GeV}$. The current accepted values for neutrino oscillations in the MINOS regime dictate that the best sensitivity will be achieved most quickly using the low energy beam. In addition to being an intense $\nu_{\mu}$ source, the NuMI beam is very pure. Figure 2.7 shows the composition of the Low Energy Beam. The beam consists mainly of $\nu_{\mu}$. The contribution of $\nu_{e}$ and $\bar{\nu}_{e}$ in the peak energy range is less than $1 \%$. 


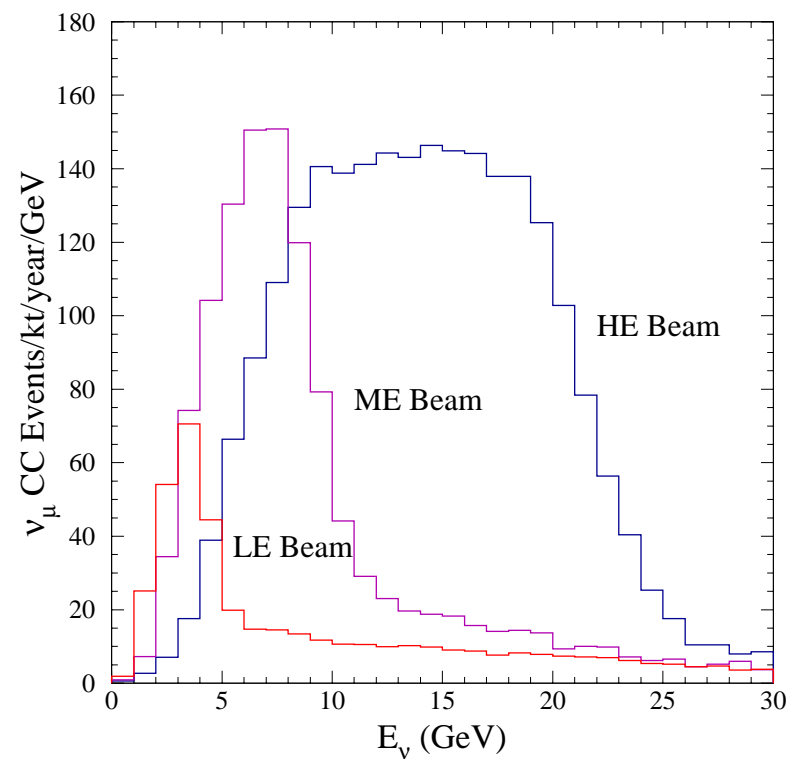

Figure 2.6: The predicted, unoscillated beam energy spectra at the Far Detector for each of the beam line configurations [20].

FLUKA

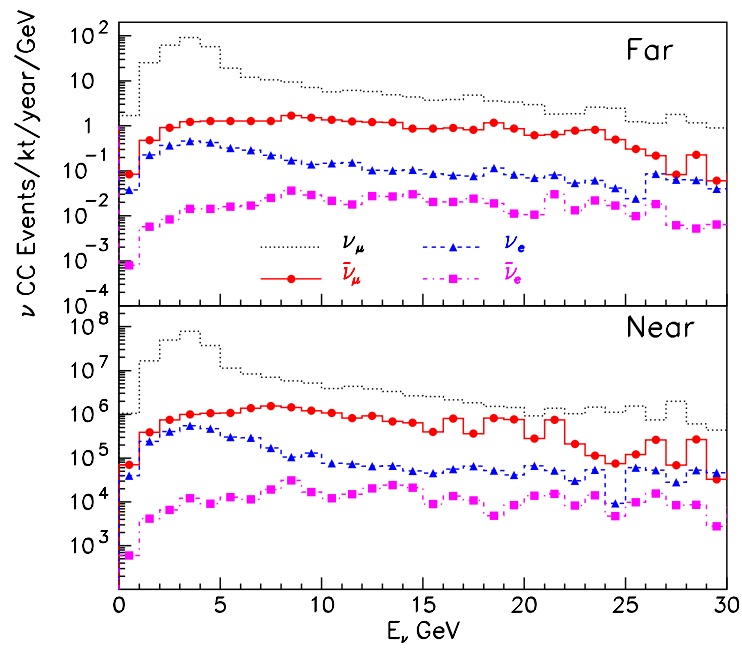

Figure 2.7: Predicted components of the low energy neutrino beam at each detector. Black shows the $\nu_{\mu}$ spectrum; red, $\bar{\nu}_{\mu}$; blue, $\nu_{e}$; magenta, $\bar{\nu}_{e}$. Courtesy of S. Kopp. 


\subsection{The Detectors}

The MINOS detectors are tracking, sampling calorimeters. The active medium in each of the detectors is $4.1 \mathrm{~cm}$ wide, $1 \mathrm{~cm}$ thick, plastic scintillator strips, coextruded with a $\mathrm{TiO}_{2}$ reflective sheath. The scintillator consists of polystyrene doped with $1 \%$ PPO and $0.030 \%$ POPOP fluors [25]. Arrayed side by side, the strips are grouped into planes and then backed by a $2.54 \mathrm{~cm}$ thick iron sheet that comprises the absorber of the calorimeter. Planes of scintillator and steel are hung vertically at a pitch of $5.94 \mathrm{~cm}$ to make up the depth of the detector. Consecutive planes of scintillator strips are rotated $90^{\circ}$ with respect to the previous plane to allow for tracking in both the horizontal and vertical directions.

The neutrinos interact primarily in the steel. Daughter particles created in the interaction that escape the steel and traverse the scintillator ionize the base plastic. The short wavelength radiation emitted by the excited molecules of the base plastic excites the primary fluor, which in turn emits ultra-violet radiation that excites the secondary fluor. The secondary fluor then emits longer wavelength blue light. Each scintillator strip has a wavelength-shifting fiber glued into a groove along the center of the strip. This fiber absorbs the scintillator light, shifts the wavelength from blue to green, then carries the light to a manifold at the edge of the detector. From there the light is transported via clear fiber cables to multi-anode photomultiplier tubes (PMTs). Light is converted into charge, which is measured by front end electronics (FEE). The amount of charge measured is proportional to the energy deposited in the scintillator by the daughter particles. Figure 2.8 shows a schematic diagram of the detector components.

All of the MINOS detectors have identical segmentation and are constructed from identical materials to reduce systematic effects arising from differences in detector operation; however, the environment in which each detector operates, along with the need to minimize cost, dictates some differences among the detectors. 


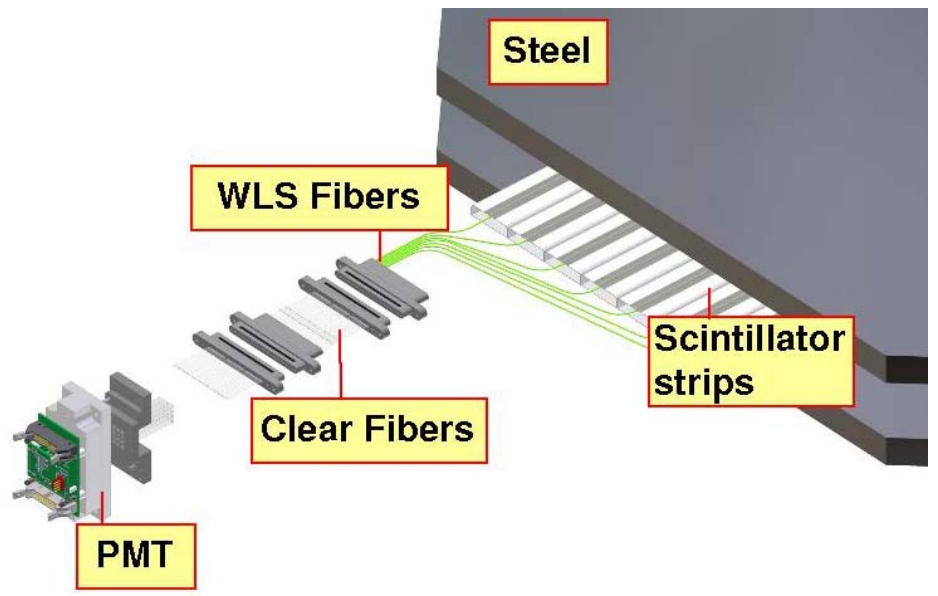

Figure 2.8: A schematic of the components of the MINOS detectors. Courtesy of M. Proga

\subsubsection{The Far Detector}

The MINOS Far detector is located 2,341 ft under ground, on the 27th level of the historic Soudan Iron Mine. At a distance of $735 \mathrm{~km}$ from the neutrino source, the Far Detector is designed to measure the energy spectrum of the neutrino beam after oscillations have taken place. The calorimeter is octagonally shaped and stands $8 \mathrm{~m}$ tall. It consists of 485 layers of iron and scintillator planes, with each plane consisting of 192 scintillator strips. The total mass of the Far Detector is $5.4 \mathrm{kt}$. The steel is magnetized with a field strength of $1.3 \mathrm{~T}$ at a radius of $2 \mathrm{~m}$ from the center of the detector [26]. A picture of the Far Detector is shown in Figure 2.9.

\section{Far Detector Front End Electronics}

In the absence of oscillations, the number of events expected in the Far Detector is only a few thousand per year, and with oscillations, that number is significantly reduced. Moreover, the detector depth limits the cosmic ray trigger rate to $0.4 \mathrm{~Hz}$ [27]. 


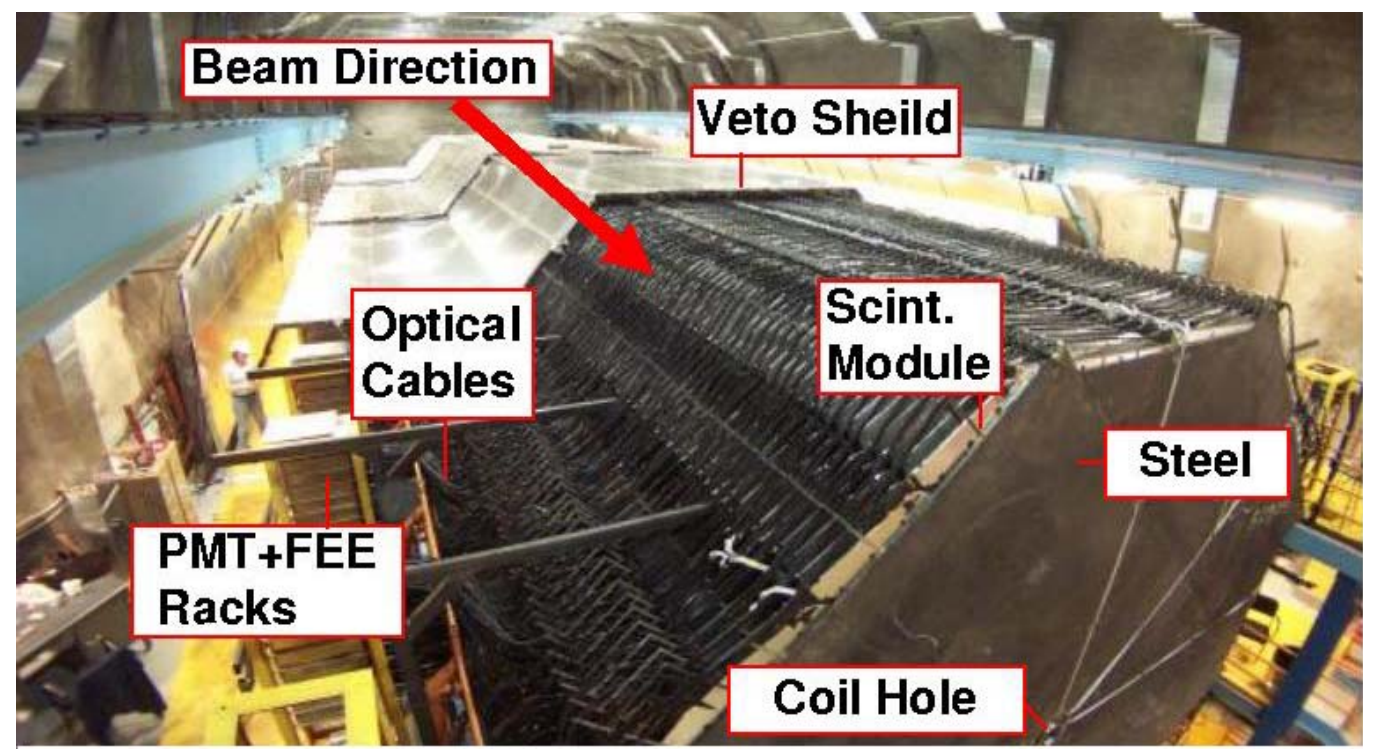

Figure 2.9: MINOS Far Detector. At the time of the photo, detector construction was complete, but the final modules of the Cosmic Ray Shield were not yet in place, allowing for clear viewing of the layers of the detector. Photo courtesy of J. Meyer

Therefore, the Far Detector FEE system was designed to take advantage of the low event rates expected at the Far Detector. The light produced in the far detector is measured at each end of the scintillator strip by Hamamatsu R5900-00-M16 photomultiplier tubes [28]. This phototube features 16 anode channels, or pixels, with a common dynode signal, in a compact glass enclosure. The PMT anodes are read out using the Viking VA chip, manufactured by IDE AS of Hovik, Norway. The VA chip features a charge sensitive preamplifier, a shaper, a sample and hold circuit for each of the anode channels, and a analog output multiplexer [29]. Three VA chips are housed on a single VA Front-end Board (VFB), which also contains a discriminator chip that acts as the hardware trigger. Analog signals are sent from the VFB to the VARC Mezzanine Modules (VMM), where they are digitized. Six VMMs are housed on a VA Readout Control board (VARC), which provides data timestamps 
and control of the VA devices. Read out of the front end electronics is activated by the PMT dynode signal. When the dynode signal from any of the three PMTs on a VFB goes above a tunable threshold, generally set at $\frac{1}{3}$ photoelectrons $(\mathrm{PE})$, the signals from all of the 16 anodes on that tube are sent to the VARC and digitized. Only the signals from anodes that are above a sparsification threshold of 4 times the pedestal width are forwarded to the output data stream. The Far Detector FEE uses a 14 bit analog to digital converter (ADC), giving a charge resolution of $2 \mathrm{fC} /$ count. In addition, the Far Detector FEE provides a 1.5625 ns resolution time stamp.

Since the average transverse size of neutrino events in the MINOS detector is expected to be less than $1 \mathrm{~m}$, the light from 8 strips separated by more than $1 \mathrm{~m}$ is "multiplexed"; the light from those 8 strips is read out by the same PMT pixel and FEE channel. Since the light is read out from each end of the scintillator strip and the multiplexing pattern is different on each end of the detector, this cost saving measure still permits unambiguous mapping of the signal to a given strip in the detector.

\subsubsection{The Near Detector}

The Near Detector is located a short distance from the neutrino source, and thus measures the unoscillated energy spectrum of the neutrino beam. The beam conditions at the Near Detector site allow for a smaller detector than that further away. Shaped like a "squashed" octagon, the Near Detector stands $3.8 \mathrm{~m}$ high, $4.8 \mathrm{~m}$ wide and has a mass of $0.98 \mathrm{kt}$. It consists of 282 planes. The Near Detector is also magnetized with a field strength of $1.2 \mathrm{~T}$ at the location of the neutrino beam incidence. Figure 2.10 shows the Near Detector during installation. 


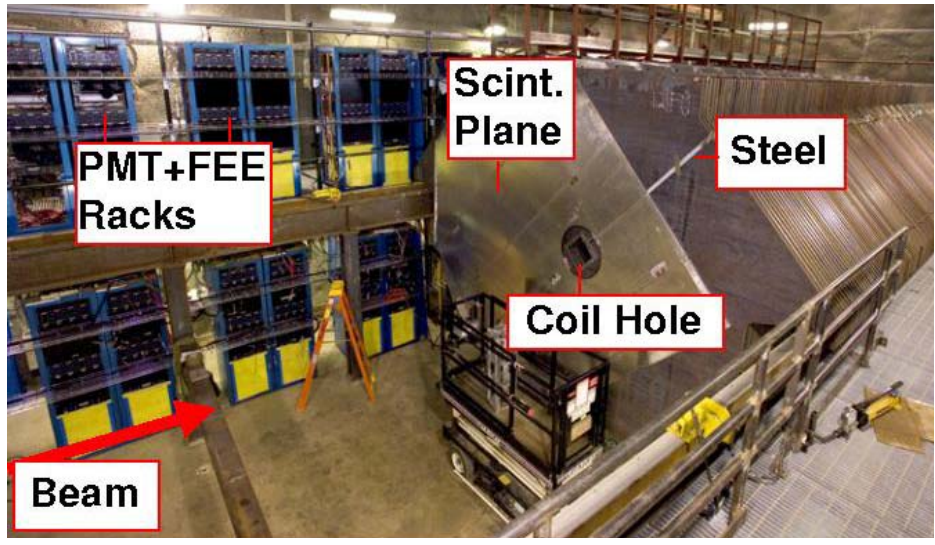

Figure 2.10: MINOS Near Detector during the construction phase. Photo courtesy of Fermilab Visual Media Services

\section{Near Detector Front End Electronics}

At only $100 \mathrm{~m}$ underground, the cosmic ray rate at the Near Detector is expected to be $270 \mathrm{~Hz}$ [26]. Moreover, when using the high energy beam hundreds of events per $8 \mu$ s spill are expected in the Near Detector [30]. These conditions demand electronics capable of operating at higher rates than the Far Detector electronics. Furthermore, the event rate will not allow for the use of the multiplexing technique of the Far Detector, hence the scintillation light is read out using the Hamamatsu M64 photomultipliers. These PMTs are operationally quite similar to the M16s but have 4 times as many pixels [31]. Each PMT anode is read out via a Charge to Current Encoder (QIE) chip. The QIE chip is capable of continuous analog processing at $53 \mathrm{MHz}$ without dead time incurred from reading out the chip [32]. The QIE operates by splitting an incoming charge signal into 8 binary-weighted fractions. Each of the charge fractions are integrated on separate integrating capacitors. At the end of the integration period, only one of the integrating capacitors will have a voltage in the digitization range of the ADC. To achieve the continuous operation that is necessary at the Near Detector, each QIE is equipped with four charge 
integrating capacitor circuits so that one circuit is always available to record a new event while the others are being digitized or reset. A single QIE chip is housed on a small board, called the MENU Module, that also contains the ADC and a FIFO for storing the data. Sixteen MENU modules are held on a MINDER module, which applies the 19 ns resolution time stamp to the data, provides power, control and interface to the QIEs, and controls multiplexing of the data from the MENU boards to a VME readout board, or MASTER Module. The data that arrive at the MASTER Module consist of 13 bits, 2 bits to encode the integrating capacitor circuit number (capid), 3 bits to encode the range of the signal, and a final 8 bits to encode the ADC value. A look-up-table is stored in the MASTER with an entry for each capid and range that provides a conversion to linearize the ADC value. The MASTER module then performs zero suppression, associates the linearized ADC with the correct time stamp and channel ID, then stores the data until it can be forwarded to the output data stream.

Because of its smaller size, the light in the Near Detector is only read out at one end of a scintillator strip. The other end of the strip is coated with a reflective paint to increase the light yield.

\subsubsection{The Calibration Detector}

With a mass of only 12 metric tons, the Calibration Detector (CalDet) is the smallest of the MINOS detectors. The CalDet is composed of sixty, $2.5 \mathrm{~cm}^{1}$ steel planes, each with a scintillator module attached to its front. The scintillator modules are $1 \mathrm{~m} \times 1 \mathrm{~m}$ and contain 24 strips. Although the CalDet is considerably smaller than the other MINOS detectors, it plays a fundamental role in the MINOS experiment. The MINOS measurements hinge on the fact that the oscillation probability depends

\footnotetext{
${ }^{1}$ Purchased in Europe, The CalDet steel measured $2.5 \mathrm{~cm}$ in thickness instead of 1 in.
} 
upon the energy of the neutrino; therefore, an accurate determination of the neutrino energy is paramount to the success of the MINOS experiment.

Since the Far and Near MINOS detectors are large and built in-situ, it is impossible to expose these detectors to a calibration beam. Therefore, to obtain an absolute energy scale of the particles interacting in the MINOS detectors, a dedicated calibration module was built and exposed to a beam of known particles with known momenta. Pictured in Figure 2.11, the CalDet is large enough to contain single particle electromagnetic and hadronic interactions up to $10 \mathrm{GeV}$ incident energy, but at the same time small enough to be readily moved into a beam line. Using the CalDet, the response of the detector can be determined and characterized in terms of its response to muons. This characterization will allow the calibration to be carried over to the larger detectors, where a better than $5 \%$ absolute calibration is required to determine the neutrino oscillation parameters to the desired precision. Moreover, the CalDet can be equipped with both the Far FEE and the Near FEE simultaneoulsy, providing a direct comparison of the response of both systems.

The CalDet data will also be used to provide more accurate physics input to the MINOS event reconstruction and classification. Event classification will be determined by event topology. Qualitatively, a $\nu_{\mu}$ charged current interaction is characterized by the presence of a long track indicating the production of a muon. Neutral current events are characterized by the absence of a track, and a diffuse energy deposition. A $\nu_{e}$ charged current interaction is characterized by a relatively short event with a small, dense deposition of energy indicating the production of an electron. Figure 2.12 shows examples of simulated events in each category. In order to make precision measurements, a quantitative definition that allows efficient identification of each event class is necessary. To aid in the event classification, the CalDet data, in conjunction with Monte Carlo, will be used to study the topology of events with the goal of fine tuning pattern recognition algorithms. Additionally, 


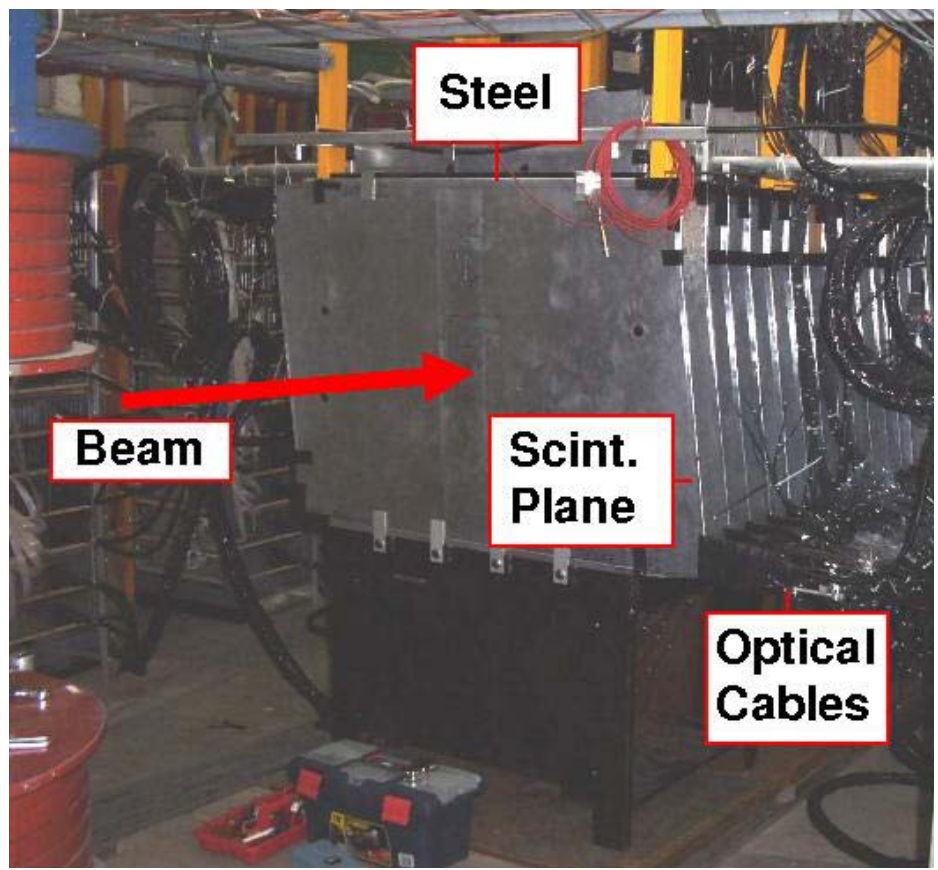

Figure 2.11: MINOS Calibration Detector

the measurements made at the CalDet allow the study of the hadronic response of sampling calorimeters at energies in the few $\mathrm{GeV}$ region. These interactions are poorly modeled in simulations making such measurements interesting to the particle physics community at large, but are particularly important for MINOS since the signature of oscillations will be most apparent at these lower energies. 


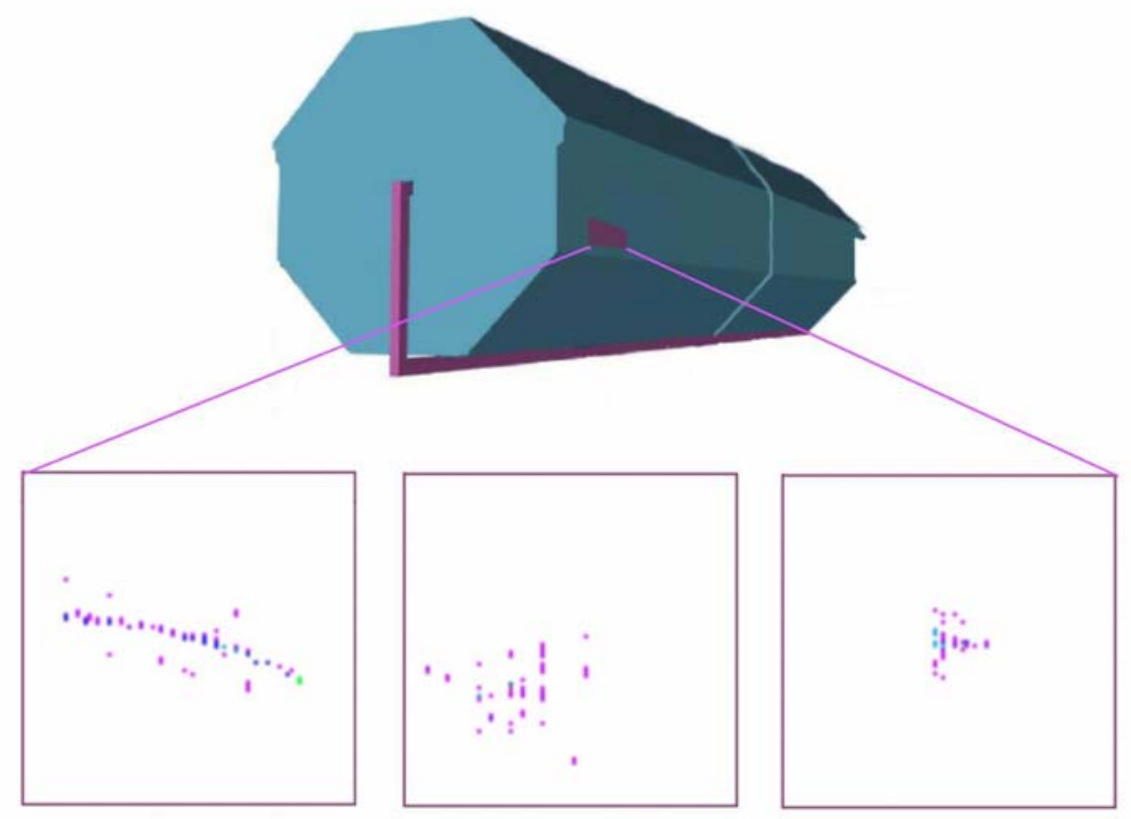

Figure 2.12: Three simulated events in the MINOS detector. To the left, a $2.6 \mathrm{GeV}$ $\nu_{\mu}$ charged current interaction. In the middle, a $4.1 \mathrm{GeV} \nu_{\mu}$ neutral current interaction. To the right, a $3.9 \mathrm{GeV} \nu_{e}$ charged current interaction. 


\section{Chapter 3}

\section{The CalDet Beam Runs}

In 2001, 2002, and 2003, the CalDet was exposed to particle beams in the CERN PS East Area complex. Over the 5 integrated months of beam data taking, the CalDet recorded the characteristics of over 30 million particles interacting within the detector. The run program consisted of data taking at momenta ranging from $200 \mathrm{MeV}$ to $10 \mathrm{GeV}$. Two different beam lines were used to span the momentum range, namely the T11 beam line for the lower end of the momentum spectrum, and the T7 beam line for the higher end. Between the beam running periods, the CalDet was operated in an enclosure in the PS East hall, where it took cosmic muon data. Each time the detector was moved from the enclosure to the beam area or back again, the entire detector had to be completely de-cabled, hoisted into position, then rebuilt. In all, the CalDet was dismantled and rebuilt 16 times.

\subsection{The Beam Lines}

The beams of the CERN PS East area complex are secondary beams produced from the interaction of a primary beam of protons accelerated to $24 \mathrm{GeV}$. On average $4 \times 10^{11}$ protons hit each secondary beam target during a spill roughly $500 \mathrm{~ms}$ 


\begin{tabular}{|r|r|r|}
\hline Start Date & End Date & Super cycle Period(sec) \\
\hline Sept. 4, 2002 & Sept. 18, 2002 & 16.8 \\
Sept. 18, 2002 & Sept. 27, 2002 & 21.6 \\
Sept. 27, 2002 & Oct. 17, 2002 & 19.2 \\
Sept. 3, 2003 & Sept. 8, 2003 & 16.8 \\
Sept. 8, 2003 & Sept. 9, 2003 & 21.6 \\
Sept. 9, 2003 & Sept. 17, 2003 & 16.8 \\
Sept. 17, 2003 & Sept. 22, 2003 & 12.0 \\
Sept. 22, 2003 & Oct. 8, 2003 & 19.2 \\
Oct. 8, 2003 & Oct. 9, 2003 \\
Oct. 9, 2003 & Oct. 22, 2003 & 21.6 \\
\hline
\end{tabular}

Table 3.1: CERN PS super cycle period over the dates spanned by the beam runs.

long [33]. The time between spills and the number of spills received varies with the mode of operation and the period of the primary Proton Synchrotron (PS), called the super cycle. Table 3.1 lists the PS super cycle period during the beam runs.

Each of the secondary beam areas is provided with a momentum selected beam with a resolution on the order of $1 \%$. Momentum selection is achieved via quadrupoles, bending magnets, and variable-aperture horizontal collimators. A number of other quadrupoles provide beam focusing, while vertical collimators are provided to tune the beam intensity. Figure 3.1 shows a schematic of the PS East hall and details the position of the beam elements [33]. The secondary beams can be charge selected and are composed primarily of electrons, pions, protons, muons, and kaons.

\subsubsection{The T11 Beam Line}

The T11 beam line delivers particles with momentum ranging from $0.2-3.6 \mathrm{GeV}$. In order to produce a low energy beam, the T11 beam area is positioned at an angle of $148 \mathrm{mrad}$ in the horizontal direction and $16 \mathrm{mrad}$ in the vertical direction relative to the incidence of the primary beam on the target. The distance from the 


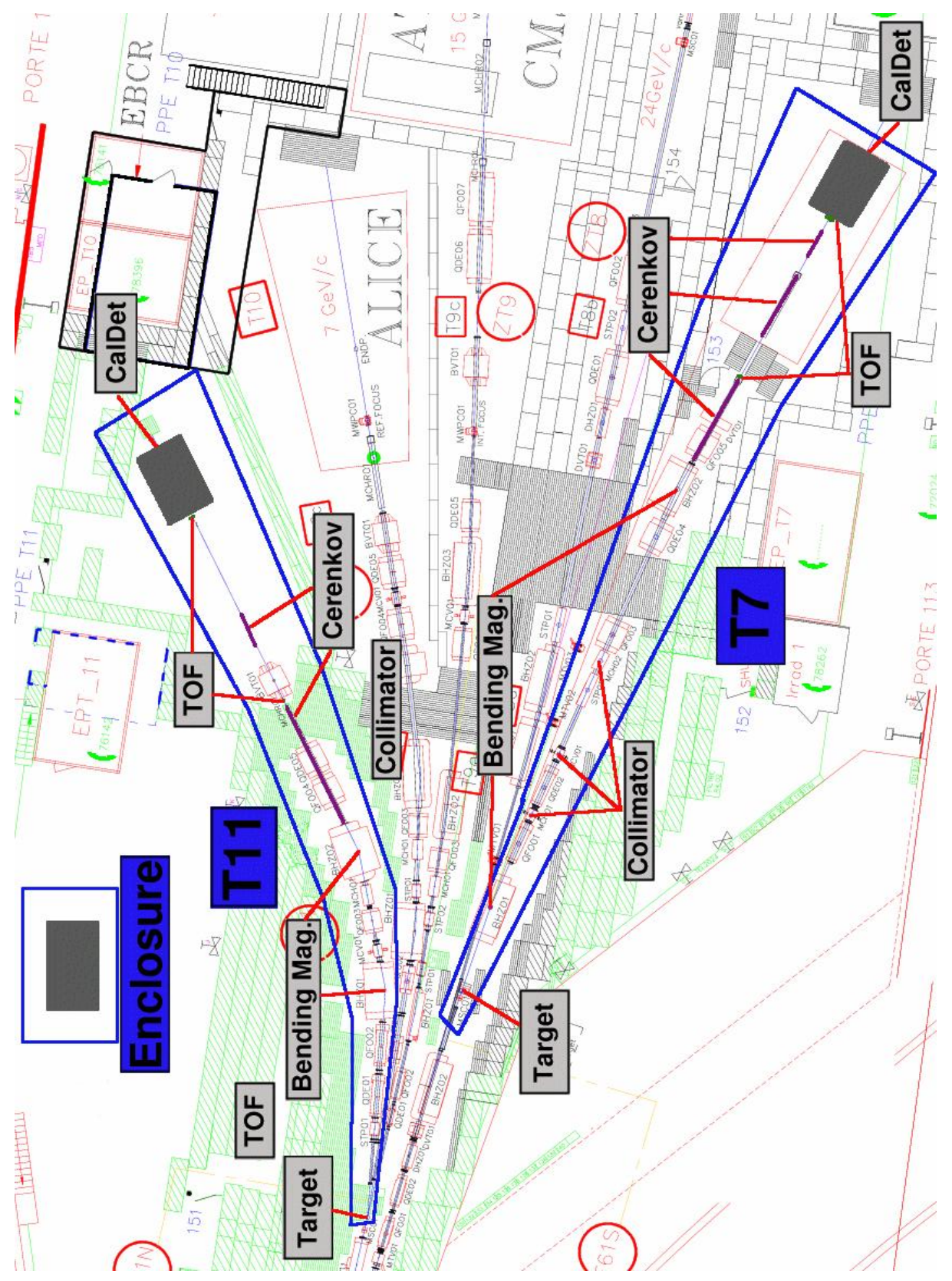

Figure 3.1: Schematic of PS East Area Hall Secondary beam lines. Courtesy of CERN, PS division [33]. 


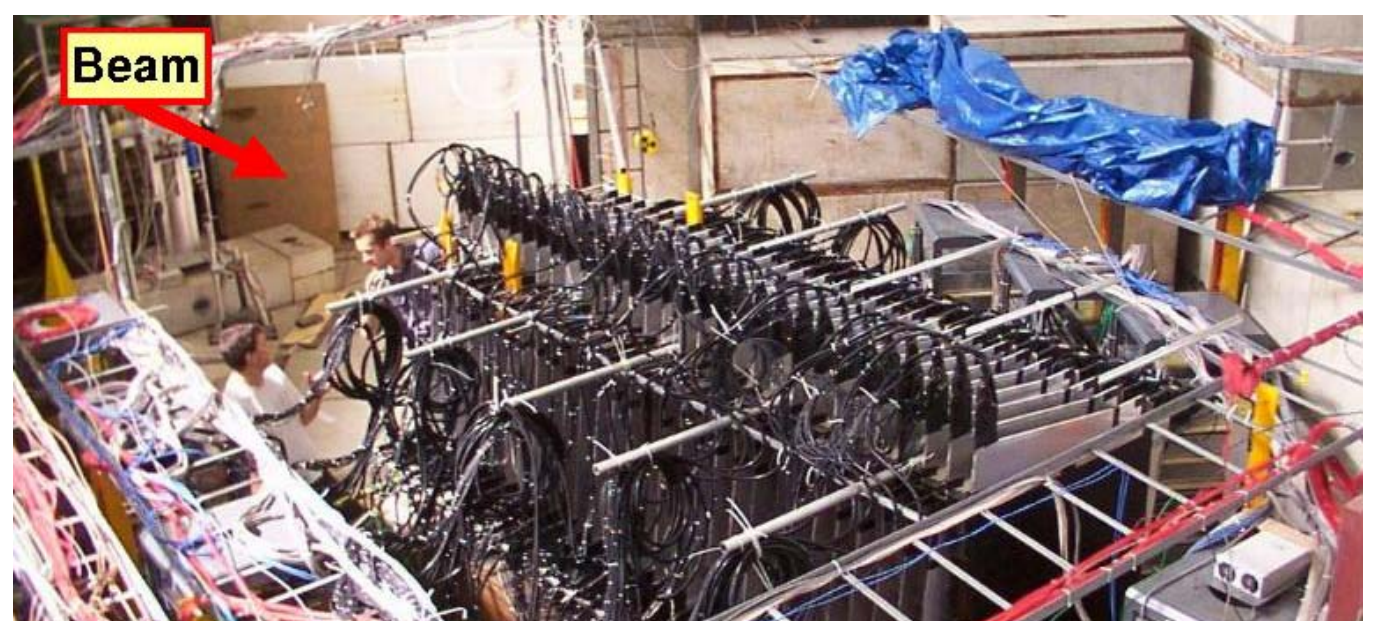

Figure 3.2: CalDet in the T11 beam area

target to the focusing point is $28 \mathrm{~m}$. The target supplying this beam line is shared with two other secondary beam lines and was typically an aluminum target $5 \mathrm{~mm}$ in diameter, and $250 \mathrm{~mm}$ long. For some runs a tungsten enriched target was used, which increased the electron yield. Figure 3.2 shows CalDet in the T11 beam area.

\subsubsection{The T7 Beam Line}

The T7 beam line delivers particles with momenta up to $10.0 \mathrm{GeV}$. The secondary particles of the $\mathrm{T} 7$ beam are produced along the direction of the incident beam, and the target is $45 \mathrm{~m}$ upstream of the reference focus. The T7 target supplied one beam line, and could be changed frequently. Generally, an aluminum target similar to the T11 target was used, though a tungsten enriched target was also used to increase electron yield, and a shorter aluminum target was occasionally used to lower the intensity. Figure 3.3 shows CalDet in the T7 beam area. 


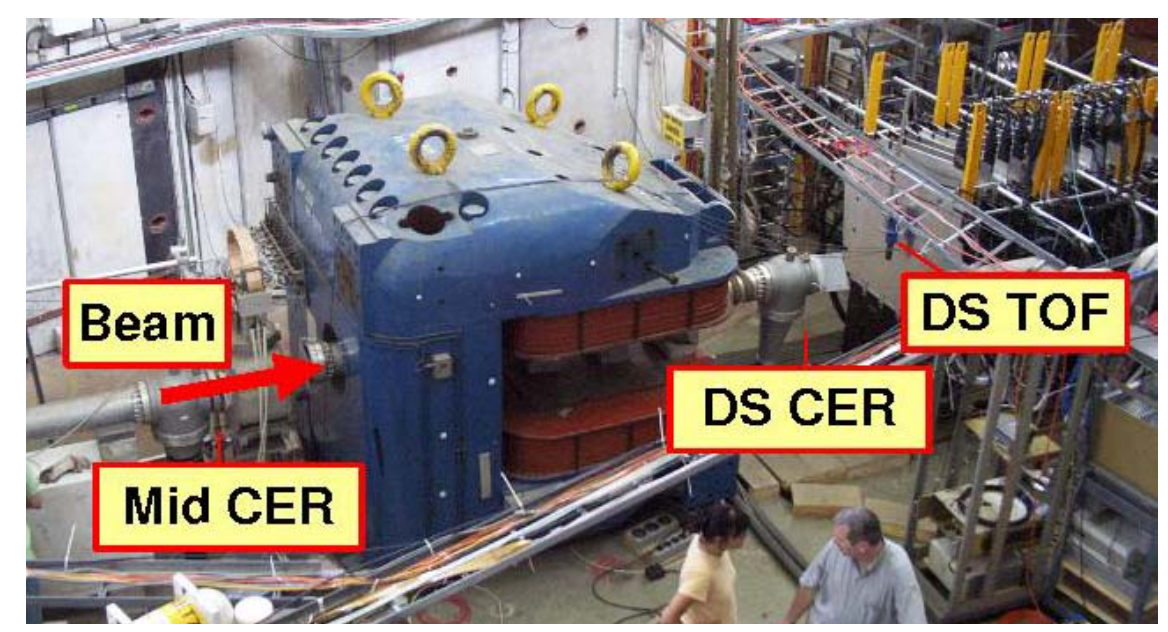

Figure 3.3: CalDet in the T7 beam area

\begin{tabular}{|r|r|r|r|r|r|r|}
\hline Detector & Crate & VARC & VMM & VAADC & VACHIP & VACHAN \\
\hline U.S. CER & 0 & 1 & 5 & 1 & 1 & 16 \\
Middle CER & 0 & 2 & 5 & 1 & 2 & 16 \\
D.S CER & 0 & 1 & 5 & 1 & 2 & 16 \\
Trigger PMT & 0 & 2 & 5 & 0 & 1 & 4 \\
VARC Trig. & 0 & 2 & 6 & 0 & 0 & 2 \\
\hline
\end{tabular}

Table 3.2: Electronics channel information for Particle ID readout

\section{$3.2 \quad$ Particle Identification}

Particle identification was accomplished using Čerenkov counters and a Time of Flight system (TOF). The read out of these external detectors was accomplished via auxiliary MINOS VA electronics. Table 3.2 lists the electronic channel information for each of the external signals. 


\subsection{1 Čerenkov Detectors}

The Čerenkov detectors used in the CERN test beam runs were threshold Čerenkov Detectors. These devices consist of a aluminum tube $15 \mathrm{~cm}$ in diameter, enclosing a volume of carbon dioxide gas, optics to focus light, and a PMT to detect the light produced. Figure 3.4 shows one of the Čerenkov used in the T7 beam line.

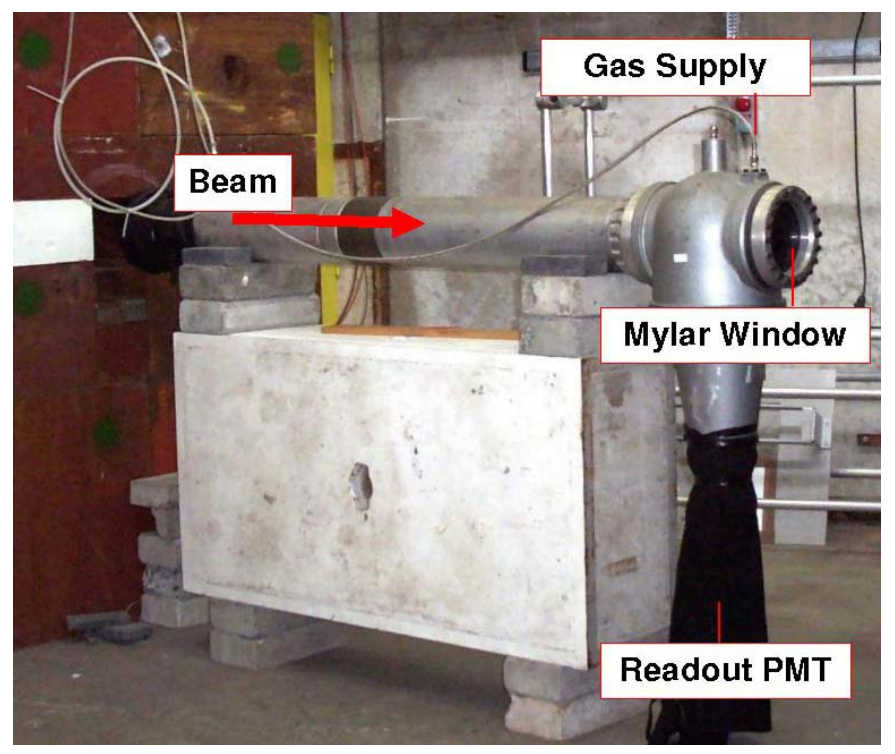

Figure 3.4: One of the Čerenkov detectors used in the $\mathrm{T} 7$ beam line.

Čerenkov detectors take advantage of the radiation emitted when a charged particle traverses a medium, traveling with a speed greater than the speed of light in that medium. By varying the index of refraction of that medium, one can discriminate on the velocity of particles in the beam. Since the beam of particles all have the same momentum, determining whether a particle that transversed the detector has a velocity above the speed of light in the medium identifies an upper limit on the mass of the particle. The speed of light in a gas varies with the pressure of the gas; therefore, at a given momentum, varying the pressure of the gas in the Cerenkov 
Pressure Threshold vs. Momentum, Electrons

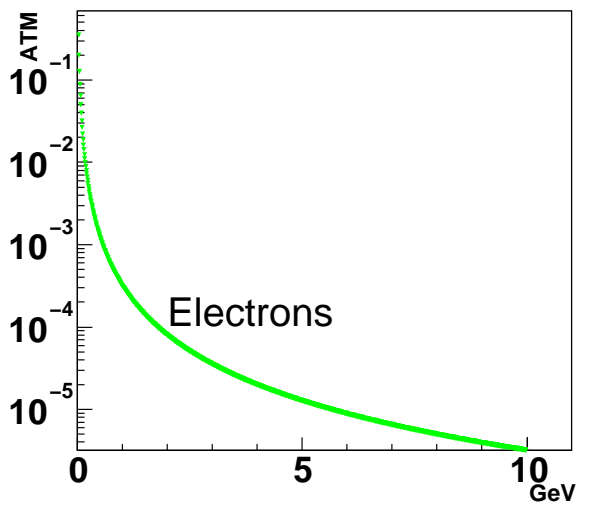

Pressure Threshold vs. Momentum

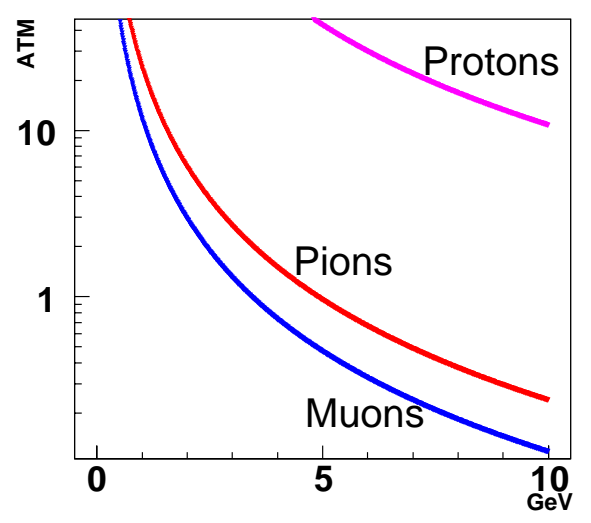

Figure 3.5: Minimum pressure in $\mathrm{CO}_{2}$ needed to detect each particle species as a function of momentum.

allows one to change which particles that Čerenkov will detect. The formula determining the minimum pressure of $\mathrm{CO}_{2}$ needed to detect a particle of mass $m$ at a momentum $p$ is given by

$$
\begin{aligned}
P & =\frac{1}{\eta}\left(\sqrt{\frac{m^{2}}{p^{2}}+1}-1\right) \\
\eta & =n-1 \\
& =4.10 \times 10^{-4}
\end{aligned}
$$

where $n$ is the index of refraction of $\mathrm{CO}_{2}$. Figure 3.5 illustrates the minimum pressure of $\mathrm{CO}_{2}$ needed to detect each particle species as a function of momentum.

The number of Čerenkovs used varied among the running periods. In the 2002 T11 beam run, one Čerenkov detector, 5 m long, was available. In T7 2002, three Čerenkovs were available. The upstream Čerenkov measured $4.4 \mathrm{~m}$ in length, while the middle and downstream Čerenkovs were shorter, $2 \mathrm{~m}$ and $3 \mathrm{~m}$ respectively. The middle and downstream counters shared a common gas system and thus were always run at the same pressure. In both the T11 and T7 runs of 2003, two Čerenkovs were 


\begin{tabular}{|l|l|l|l|l|}
\hline $\mathrm{P}(\mathrm{GeV})$ & Beam Line & Year & US CER (ATM) & DS(Mid) CER (ATM) \\
\hline $0.2-3.6$ & T11 & 2002 & 1.05 & - \\
$1.0-3.0$ & T7 & 2002 & 1.0 & 1.0 \\
$4.0-6.0$ & T7 & 2002 & 0.3 & 3.3 \\
$5.0-6.0$ & T7 & 2002 & 0.3 & 3.3 \\
7.0 & T7 & 2002 & 0.2 & 3.3 \\
$8.0-9.0$ & T7 & 2002 & 0.1 & 3.3 \\
$4.0-6.0$ (e-trig) & T7 & 2002 & 0.3 & 0.3 \\
7.0 (e-trig) & T7 & 2002 & 0.2 & 0.2 \\
$8.0-9.0$ (e-trig) & T7 & 2002 & 0.1 & 0.1 \\
\hline
\end{tabular}

Table 3.3: Čerenkov pressure setting versus beam momentum

used. The upstream counter in T11 measured $5 \mathrm{~m}$ while the upstream counter in T7 measured $4.4 \mathrm{~m}$. The downstream counters in both beam lines measured $3 \mathrm{~m}$. In general, at energies below $4 \mathrm{GeV}$, all Čerenkov counters were used to tag electrons in the beam. At higher energies, the upstream counter would be set to tag electrons, while the downstream counters would be set to trigger on electrons, muons and pions. Kaons and protons were below the Čerenkov threshold. Also at the higher energies, special runs were taken with all Čerenkovs set to tag only electrons. To increase the efficiency of electron event collection, the Čerenkov signals were incorporated into the external trigger, which is described later in this chapter. Table 3.3 lists the pressure settings used for production runs in each of the 2002 beam runs. During the 2003 run period, the pressures in the Čerenkov counters were recorded using digital pressure transducers. The pressures for a given run can be retrieved from the MINOS offline database table "DCS_ENV_CAL". See Appendix F for further details. 


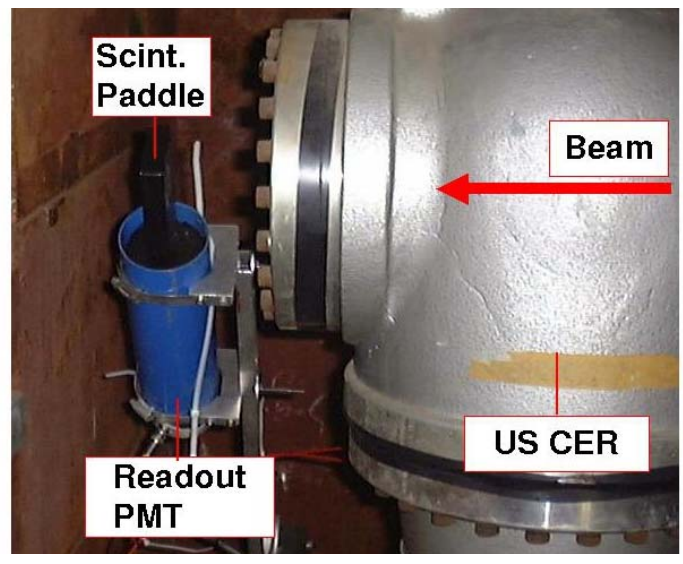

Figure 3.6: The upstream TOF paddle used in T7.

\subsubsection{TOF}

Threshold Čerenkov detectors only determine whether a particle's velocity is above a threshold, the Time of Flight (TOF) system, on the other hand, actually measures the velocity of that particle. The TOF system consists of two or more scintillator paddles, read out by PMTs, separated by some known distance. Figure 3.6 shows the upstream TOF paddle used in the T7 beam line. A beam particle produces a signal in each scintillator paddle it crosses. When signals from multiple TOF

paddles are detected within a given time window, a VME TDC module, with a timing resolution of 35 ps per TDC count, measures the difference in time between signals in the paddles, thus measuring how long it takes a particle to travel from one paddle to the next. The difference in time of flight for two different particles of mass $m_{1}$ and $m_{2}$ at a given beam momentum is given by

$$
\Delta t=\frac{L}{c}\left[\sqrt{1+\frac{m_{1}^{2} c^{2}}{p^{2}}}-\sqrt{1+\frac{m_{2}^{2} c^{2}}{p^{2}}}\right]
$$




\begin{tabular}{|l|l|}
\hline Configuration & Length $(\mathrm{m})$ \\
\hline T11, 2002 & 11.8 \\
T7, 2002 & 9.2 \\
T7, 2003 & 9.1 \\
T11, 2003 & 7.3 \\
\hline
\end{tabular}

Table 3.4: TOF base line lengths

Figure 3.7 shows the time of flight difference between different particle species as a function of beam momentum at a baseline length of $9.1 \mathrm{~m}$.

The separation between the TOF paddles varied with the space available in the beam area. Table 3.4 lists the distance between the TOF paddles in each of the beam lines. With these baselines and the intrinsic resolution of the system, the TOF was most useful in distinguishing protons from the other particle species. Figure 3.8 shows a typical time of flight distribution from a $1 \mathrm{GeV}$ run with paddle spacing of $11.8 \mathrm{~m}$. The proton peak is clearly separated from the peak containing electrons, muons and pions. Clear separation of the pion and protons peaks could be achieved up to $4.0 \mathrm{GeV}$. In 2003 the addition of extra TOF paddles and upgrades to the NIM circuitry used in the trigger improved the resolution of the system. In 2003, pion-proton separation could be achieved even at $5 \mathrm{GeV}$. Figure 3.9 shows the time of flight distribution for a $4 \mathrm{GeV}$ run from the $\mathrm{T} 72003$ run along with cuts on the Čerenkov detectors. The conjunction of the TOF and Čerenkov systems allowed for the identification of samples of electrons, pions and muons, and protons and kaons over the range of energies studied. Figure 3.10 shows the total number of each particle type collected at each beam momentum summed over all beam runs. 
Time of Flight Difference vs. P, $9.1 \mathrm{~m}$

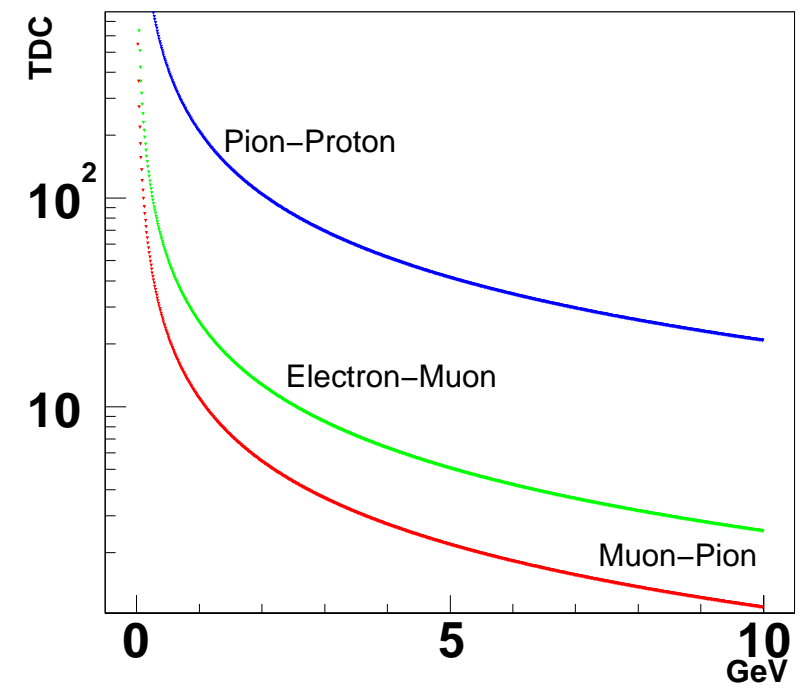

Figure 3.7: Difference in Time of Flight for different particle species versus beam momentum

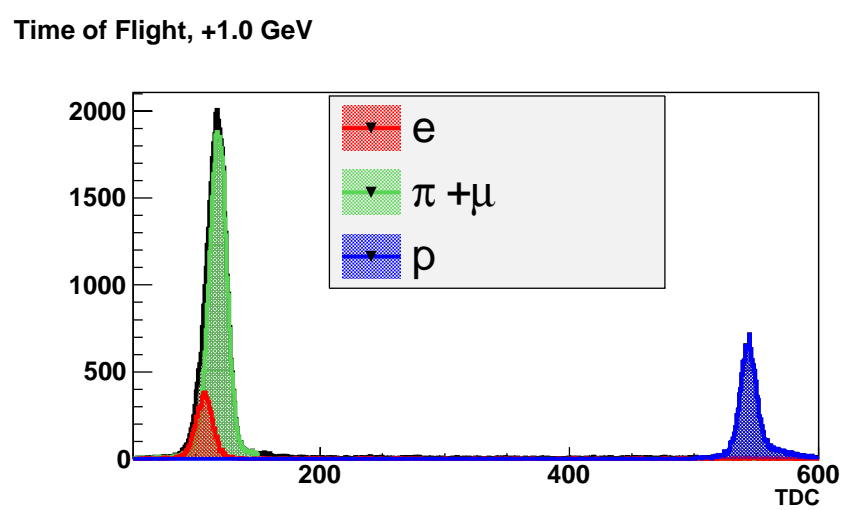

Figure 3.8: Time of Flight distribution at $1 \mathrm{GeV}$, with paddle spacing of $11.8 \mathrm{~m}$. 


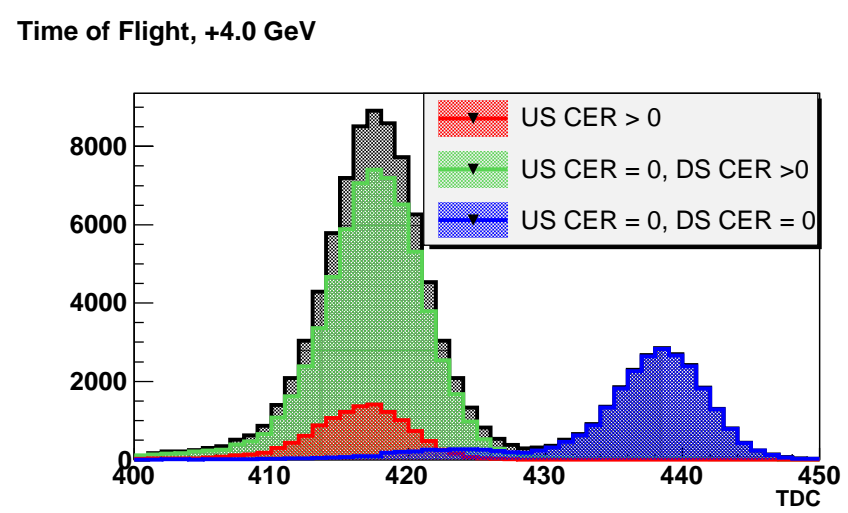

Figure 3.9: Time of Flight distribution at $4 \mathrm{GeV}$, with paddle spacing of $9.1 \mathrm{~m}$ is shown in black. Electrons selected by the requirement of two Cerenkov signals are shown in red, while pions and muons selected by the requirement of an signal in the downstream Črenkov and no signal in the upstream Cerenkov are shown in green. Protons and kaons are selected by the requirement of no Čerenkov signal and are shown in blue.

\subsection{MINOS in a Beam}

CalDet was intended to collect data in the same manner as that used at the larger MINOS detectors; however, high rate related electronic effects and beam line conditions made readout triggered solely by the PMT dynode signal not feasible. Instead, through a combination of VARC firmware upgrades and NIM logic, an external enable was added to the electronics triggering scheme. Instead of reading out a PMT whenever the dynode signal from that PMT went above threshold, a further trigger requirement allowed the detector to be read out only when a particle that passed through the TOF paddles interacted in the detector. The test beams were a noisy environment and not all particles inducing signal in the CalDet were associated with the beam. Requiring the particle to go through a TOF paddle ensured the particles accepted were associated with the beam. Another problem arising from running 


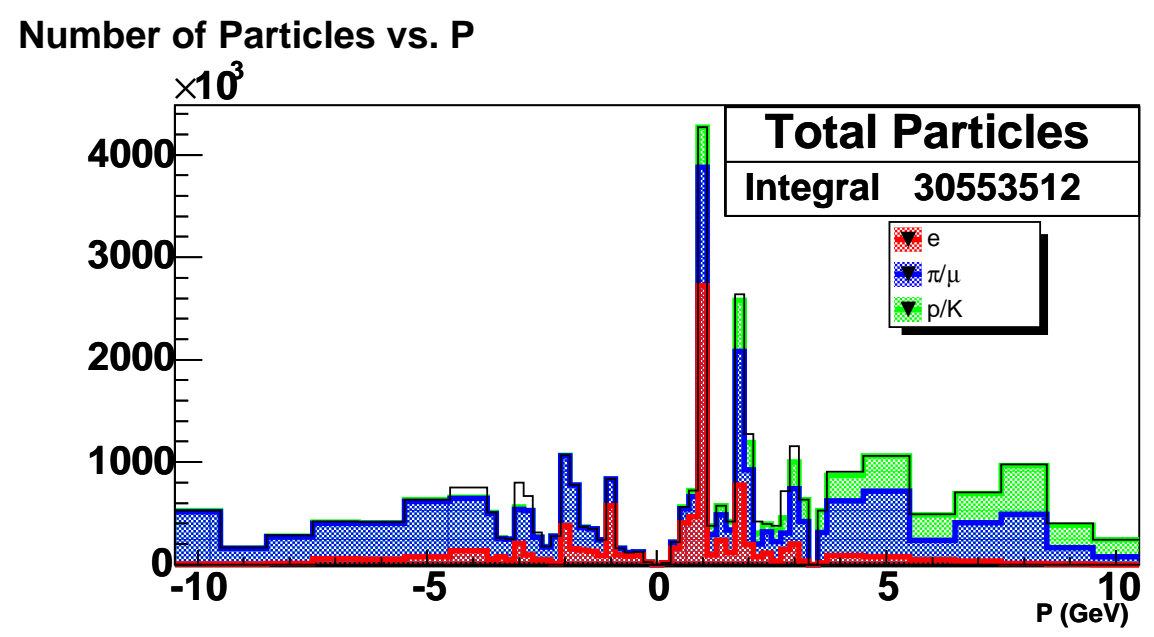

Figure 3.10: Total number of particles collected as a function of beam momentum. Portion of the bar in red shows the fraction of electrons, blue shows the fraction of pions and muons, while green shows the fraction of protons and kaons.

without a beam trigger resulted from using the Far Detector FEE, which was designed for a low rate environment. Reading out and digitizing the signals from a single VA chip takes approximately $5 \mu \mathrm{s}$. Since the 6 chips serviced by a single ADC are read out sequentially, a given chip can be unavailable for nearly $30 \mu \mathrm{s}$. During that read out time, the chip is dead and will not read out new signals. As a result, regions of the detector are dead asynchronously depending on previous activity, and the measure of the energy deposited is dramatically skewed. With the external enable, and using external NIM veto circuitry, readouts that came within $50 \mu \mathrm{s}(70 \mu \mathrm{s}$ in 2003) of a previous particle going through the detector were vetoed. Using the TOF enable and veto mitigated the effects of asynchronous dead time. 


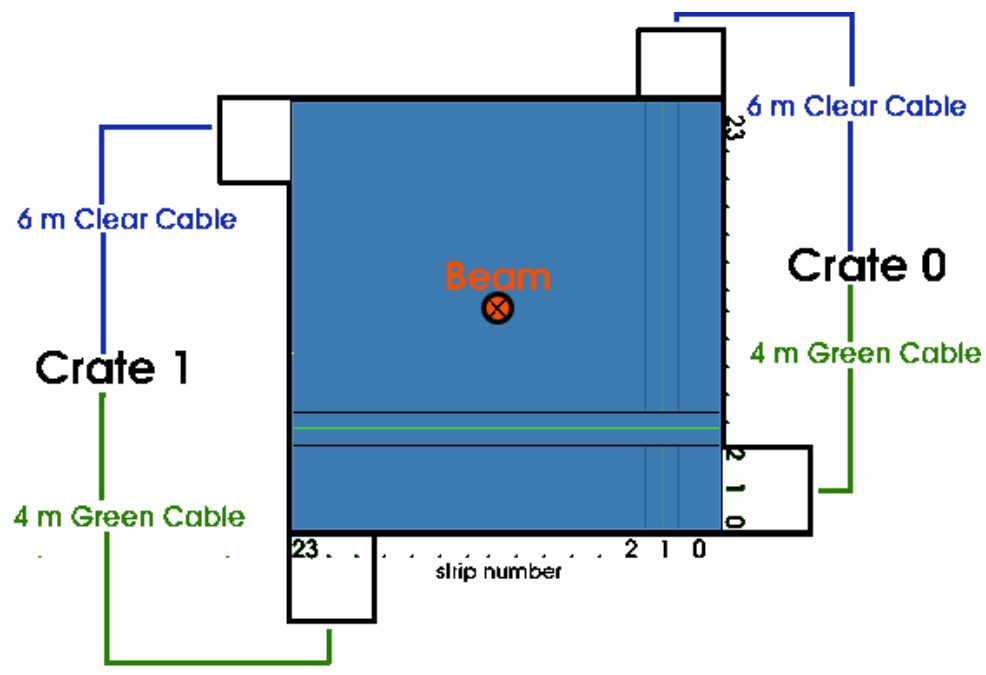

Figure 3.11: Schematic of the CalDet in the Far detector configuration

\subsection{Run Configurations}

During three years of operation, the CalDet was run in several different configurations. When operated in the Far Detector mode, 180 Hamamatsu M16 PMTs read out the light generated in the 1440 scintillator strips of the CalDet. The PMT signals were read out using the Far Detector FEE and two crates of VARC modules. The strips were read out from both ends. Light from one end of the scintillator modules was carried to the PMTs via $6 \mathrm{~m}$ clear fiber optics. To mimic the attenuation in the larger Far Detector, the other end of each module was read out through $4 \mathrm{~m}$ wavelength shifting green cables. Figure 3.11 illustrates the configuration of the CalDet in the Far Detector mode.

In the Near Detector mode, the read out was accomplished using thirty, M64 PMTs plus the Near Detector FEE. The green cables were replaced first with $3 \mathrm{~m}$, then with $1 \mathrm{~m}$, green cables with a mylar reflector at the free end of the cable. 


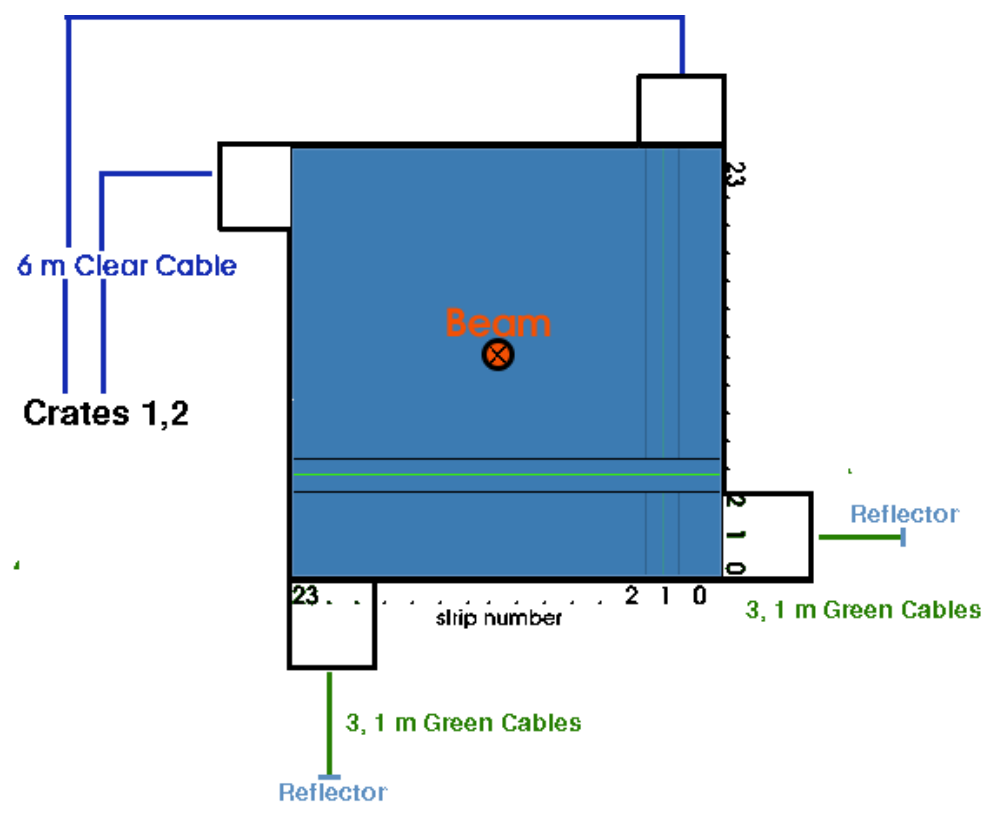

Figure 3.12: Schematic of the CalDet in the Near detector configuration

This configuration is illustrated in Figure 3.12. The CalDet was also operated in a Near/Far mode in which each side of the scintillator modules was read out with a different FEE. Figure 3.13 illustrates the mixed mode configuration.

In 2001, the CalDet was operated in Far mode in the T11 beam line. Then in June, 2002, the CalDet was run in Far mode in the T7 beam line. At this point, the external TOF enable was not in place. The data from these runs were used mainly as a basis to understand the operation of the detector and the characteristics of the beam line. The experience gained in 2001 was vital to the successful beam runs over the next two years.

In 2002 the CalDet returned to the T11 beam line. For the first two weeks, the CalDet was operated in a Near/Far mode, where 7 planes were read out on one end with Near Detector FEE and the other end with Far Detector FEE. The remaining 


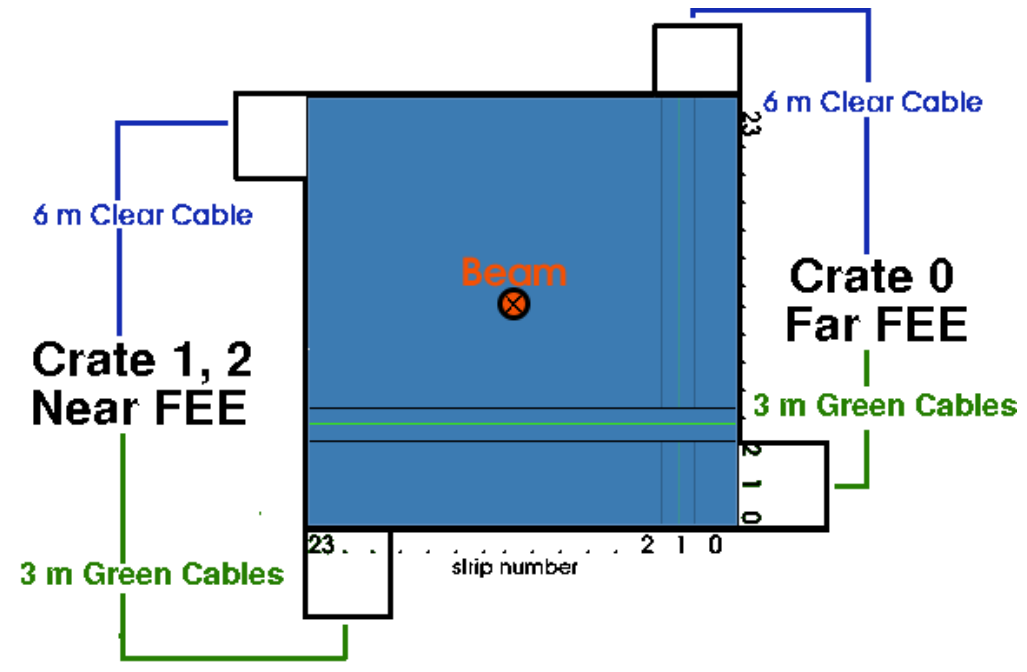

Figure 3.13: Schematic of the CalDet in the mixed Far/Near configuration

planes were instrumented on both sides with the Far Detector front end. After 2 weeks in the Near/Far mode, the Near Detector instrumentation was removed and replaced with Far Detector instrumentation, and the CalDet was operated for another 4 weeks in Far/Far mode. At the end of the 2002 T11 run, the detector was run in a configuration in which the planes were oriented at an angle of $30^{\circ}$ to the beam. After the T11 run, the CalDet was moved to the T7 beam line, where Far/Far data were collected for another two weeks.

In 2003, the CalDet was operated in a full Near/Far configuration for four weeks in the $\mathrm{T} 7$ beam line. In this run every plane was read out on one end using Near Detector electronics and the other end with Far Detector electronics. At the end of that run cycle, the Far Detector electronics system was removed, and reflector connectors were added at the end of the green cables. For one week the detector ran in T7 with single ended, Near readout with $3 \mathrm{~m}$ green cables, then for one more week with $1 \mathrm{~m}$ green cables. Finally in 2003, the detector was moved back to the 
T11 line and read out using only Near Detector electronics. Data were taken at normal incidence as well as at a series of angles. Table 3.5 lists run configurations, dates of the run period, and the run numbers of the data sets taken.

\begin{tabular}{|c|c|c|c|c|c|}
\hline Beam & Electronics & Dates & Angle & First Run & Last Run \\
\hline $\mathrm{T} 11$ & $\begin{array}{l}\text { Far } \\
\text { No Ext. Trig. }\end{array}$ & Sept. 2001 & $0^{\circ}$ & 10000 & \\
\hline $\mathrm{T} 7$ & $\begin{array}{l}\text { Far } \\
\text { No Ext. Trig. }\end{array}$ & June. 2002 & $0^{\circ}$ & 20000 & \\
\hline $\mathrm{T} 11$ & $\begin{array}{l}\text { Far/Near } \\
7 \text { Planes }\end{array}$ & Sept. 8-15, 2002 & $0^{\circ}$ & 31122 & 31573 \\
\hline $\mathrm{T} 11$ & Far & $\begin{array}{l}\text { Sept. 16- } \\
\text { Oct. } 2,2002\end{array}$ & $0^{\circ}$ & 40615 & 40962 \\
\hline $\mathrm{T} 11$ & Far & Oct. 3,2002 & $30^{\circ}$ & 41008 & 41017 \\
\hline $\mathrm{T} 7$ & Far & Oct. $4-16,2002$ & $0^{\circ}$ & 50001 & 50702 \\
\hline $\mathrm{T} 7$ & Far/Near & Sept. 4-25, 2003 & $0^{\circ}$ & 70001 & 71587 \\
\hline $\mathrm{T} 7$ & $\begin{array}{l}\text { Near } \\
3 \mathrm{~m} \text { cables }\end{array}$ & $\begin{array}{l}\text { Sept. 26- } \\
\text { Oct. } 1,2003\end{array}$ & $0^{\circ}$ & 80001 & 80319 \\
\hline $\mathrm{T} 7$ & $\begin{array}{l}\text { Near } \\
1 \mathrm{~m} \text { cables }\end{array}$ & Oct. 1-6, 2003 & $0^{\circ}$ & 90015 & 90332 \\
\hline $\mathrm{T} 11$ & $\begin{array}{l}\text { Near } \\
1 \mathrm{~m} \text { cables }\end{array}$ & Oct. $10-13,2003$ & $0^{\circ}$ & 100078 & 100344 \\
\hline $\mathrm{T} 11$ & $\begin{array}{l}\text { Near } \\
1 \mathrm{~m} \text { cables }\end{array}$ & Oct. $13-15,2003$ & $45^{\circ}$ & 101012 & 101142 \\
\hline $\mathrm{T} 11$ & $\begin{array}{l}\text { Near } \\
1 \mathrm{~m} \text { cables }\end{array}$ & Oct. $15-17,2003$ & $30^{\circ}$ & 101143 & 101263 \\
\hline $\mathrm{T} 11$ & $\begin{array}{l}\text { Near } \\
1 \mathrm{~m} \text { cables }\end{array}$ & Oct. $17-20,2003$ & $15^{\circ}$ & 102010 & 102264 \\
\hline
\end{tabular}

Table 3.5: Run Configurations 


\section{Chapter 4}

\section{The MINOS Calibration}

\section{Scheme}

The CalDet data provide the conversion factor to convert the charge in phototubes responding to a particle interacting in a MINOS detector to the energy of that incident particle. However, before the detector response can be interpreted as an indicator of the interacting particle's energy, the response of each detector cell must be normalized.

There are three systems involved in the intra-calibration of a MINOS detector. First, using electronic charge injection, a known value of charge is injected into the read out chain. By varying the input, a relation between charge and ADC value is determined. Next, light from a light-emitting diode (LED), monitored by independent PIN diodes, is injected into the optical path. This allows for the determination of channel gain, for the tracking of gain variations over time, and for the measurement of non-linearity in PMT+electronics response. Finally, cosmic ray muons are used to determine the light output of each scintillator strip relative to the average strip light output. After each detector cell has been intra-calibrated, muons of a known energy are studied to determine the response of the normalized detector, 


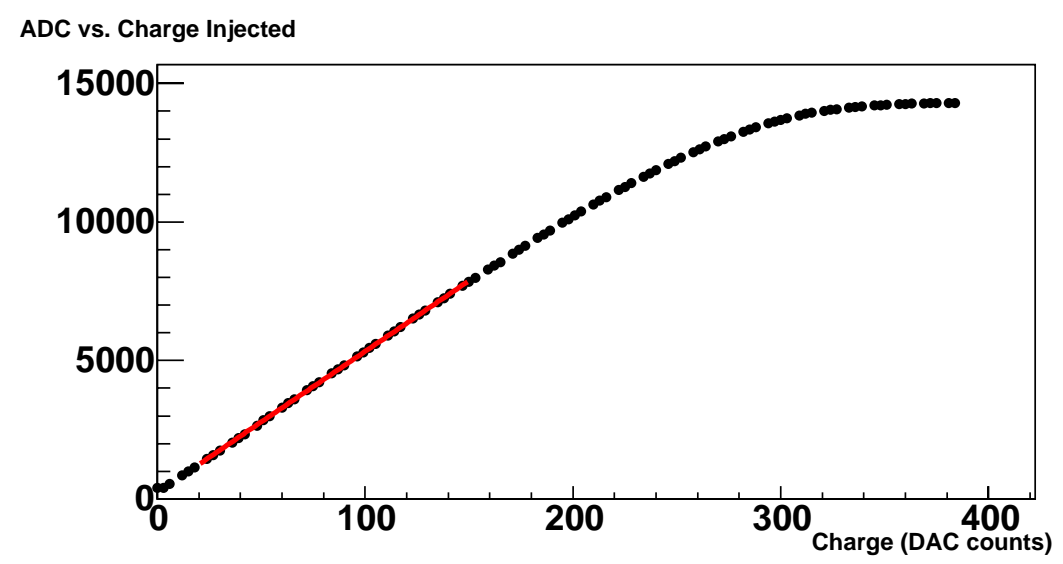

Figure 4.1: ADC versus charge injected for a typical Far Detector channel.

and the detector response of other particle types are compared to the response of muons. The following sections describe the calibration chain, illustrate principles, and demonstrate the capabilities of the various steps of the calibration.

\subsection{Charge Injection}

Both the Far and Near Detector FEE are equipped with the ability to inject a known amount of charge into the electronic read out chain with the purpose of determining the output ADC of the electronic channel as a function of the input charge. Scanning over the input charge, then interpolating between input values, allows for a relation between input charge and output ADC to be obtained. Figure 4.1 shows this relation for a typical Far Detector channel. The relationship is linear to about 8000 ADC counts, at which point the electronics begin to saturate. A minimum ionizing particle typically induces a response of a few hundred ADC counts, a level well within the linear region of the electronics. In Far Detector operation, the data from a charge 
injection run is used only to correct the non-linearity of the PIN diode readout, which is used in the light injection calibration. Charge injection in the Near Detector, on the other hand, is used to generate the look-up-table which converts the capid, range, and ADC information output from the QIE chip into a linearized ADC.

\subsection{Light Injection}

MINOS uses a Light Injection (LI) system to measure and compensate for the individual gains of each of the optical readout channels, to track drifts in response on a channel by channel basis, and to linearize the response of the PMT and electronics [34]. The LI system injects light into the standard optical path, and then compares the result to an independent measure of the light injected. Specifically, the injected light originates from blue LEDs. Pulsed LED light is carried from the LEDs via clear fiber optics to a manifold at the end of each of the MINOS scintillator modules. In this manifold, the LED light illuminates a set of wavelength shifting fibers from the scintillator strips. The light is absorbed in the WLS fibers, then conveyed to the PMT and read out in the same way that light from particle interactions in the scintillator is read out. The intensity of the LED light is also monitored by a PIN photodiode for an independent measurement of the injected light level. Figure 4.2 provides a schematic diagram of the components of the LI system.

The gain of the optical readout channels is determined by injecting a moderate amount of light into the optical readout chain. Photon statistics is then used to relate the number of photoelectrons (PE) produced by the PMT cathode to the histogram mean $(\mu)$ and RMS $(\sigma)$. Once the light level in PE is known, the gain can be computed. The equations for $\mathrm{PE}$ and gain are given by

$$
P E=\frac{\mu^{2}}{\sigma^{2}}
$$




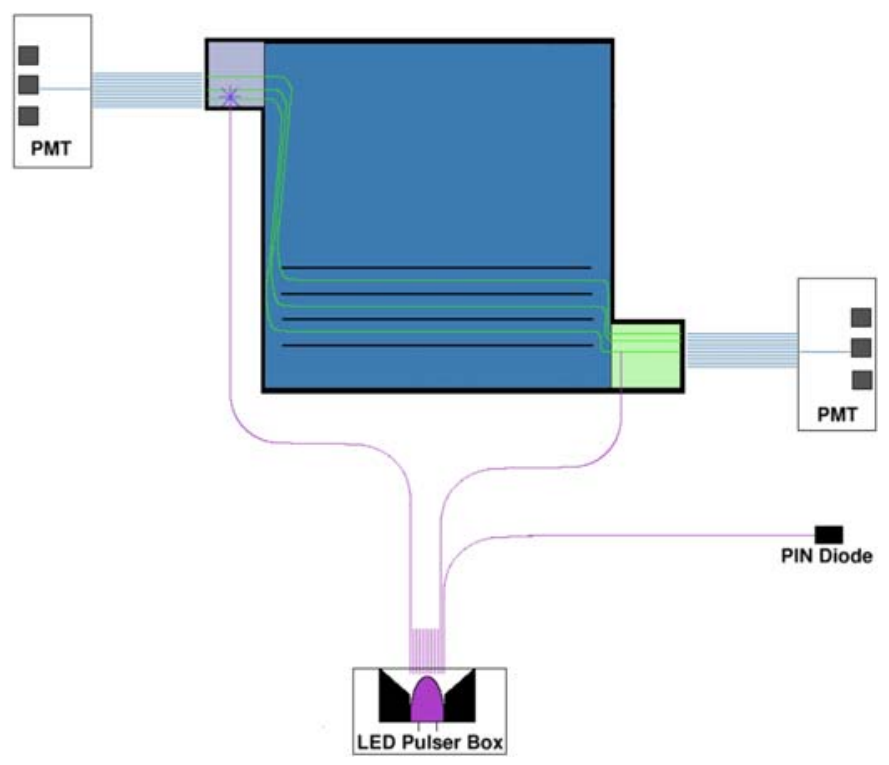

Figure 4.2: Schematic of the LI system.

$$
\begin{aligned}
G & =\frac{\mu}{P E} \times s \\
G & =\frac{\sigma^{2}}{\mu} \times s
\end{aligned}
$$

where $\mathrm{s} \approx 1.2$ is a correction factor that depends on the secondary emission of the first PMT dynode and is determined empirically [35]. Figure 4.3 shows the gains of all CalDet channels at a given point in time. The position of the $1 \mathrm{PE}$ peak is 76.8 ADC, corresponding to a gain of roughly $10^{6}$, with a spread of about $24 \%$.

After the gain of each channel is determined at a reference time, special LI runs are performed at 20 minute intervals during the data taking to compare the gain at that time to the gain at the reference time and to account for any drift. Correction for gain drifts is accomplished via the relation

$$
C=\frac{\left(\mu_{P M T} / \mu_{P I N}\right)_{t}}{\left(\mu_{P M T} / \mu_{P I N}\right)_{t=t_{r e f}}}
$$

where $\mu_{P M T}$ is the mean signal of the phototube, $\mu_{P I N}$ is the mean signal from 


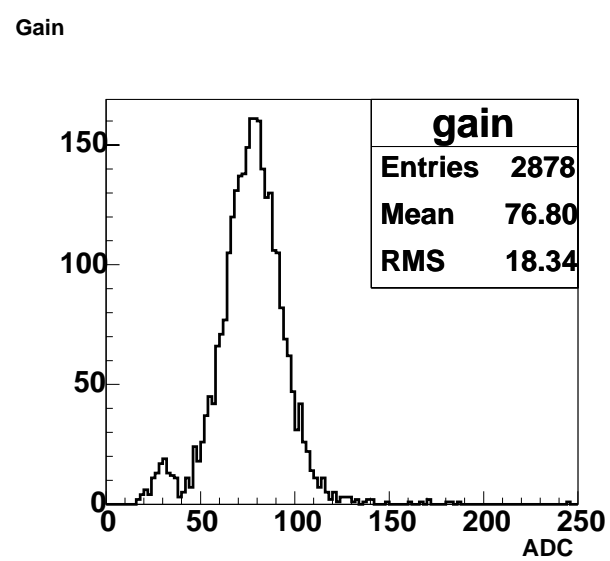

Figure 4.3: The gains of all CalDet channels at a reference time.

the PIN diode, and $t=t_{r e f}$ is the time of the reference gain measurement. The comparison to the PIN response is performed in order to eliminate any drift in the light output of the LED with time. Figure 4.4 shows the magnitude of gain drift at the CalDet. The left plot shows the drift in the gain of a typical channel over the period of several days. The variations in the gain correspond to variations in the ambient temperature between day and night. The right plot shows a histogram of the ratio of the gain to the gain at the reference time of every channel over a 6 day period. While a few channels showed a more dramatic gain shift, roughly $90 \%$ of channels drifted less than $5 \%$ over the 6 day period.

The final purpose of the LI system is to correct for non-linearities in the PMT and electronics readout chain. The MINOS Far Detector PMTs are known to have a linear relation between input light level and output charge up to $\sim 100 \mathrm{PE}$, however, above $100 \mathrm{PE}$, the PMT begins to saturate and the relation between the incident light level and the resulting charge flattens [28]. Moreover, as was shown above, the VA readout channels saturate above 8000 ADC counts, adding to the effect of the non linearity. In order to correct for this effect, the LEDs are pulsed 


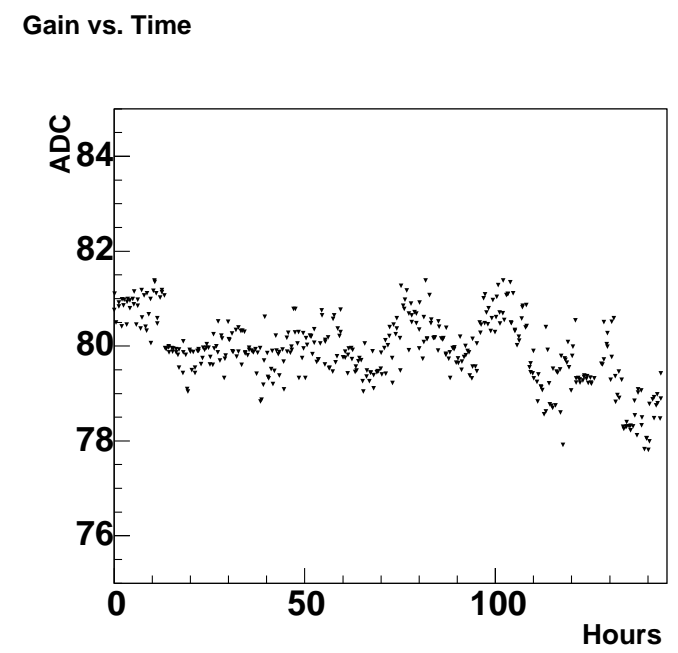

Ratio of Gain to Reference Gain

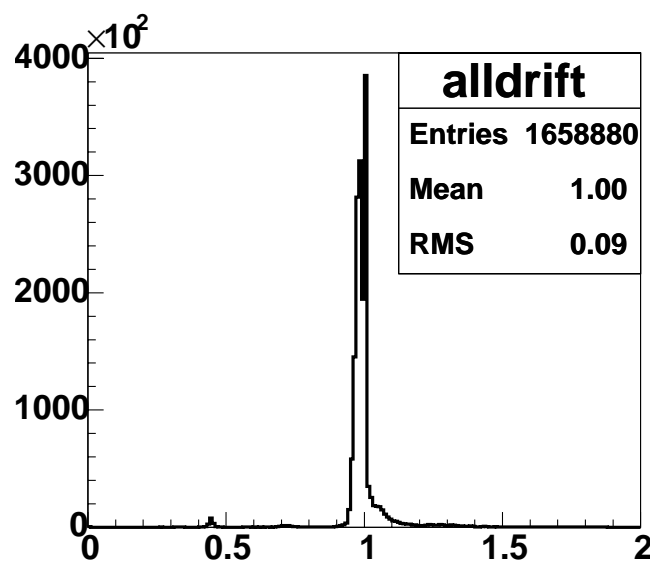

Figure 4.4: The left shows the gain of a specific channel versus time. The right shows the ratio of the gain to the gain at the reference time in each CalDet channel over a roughly six day period.

at different light levels, from $1-200 \mathrm{PE}$, and the PMT response is compared to the PIN response. While the PINs themselves prove to be very linear up to high incident light levels, non-linearities of the VA electronics induce a non-linearity in the PIN readout that must be removed using charge injection [35]. In principle, any remaining non-linearity in the PMT versus PIN relation is an effect of the PMT and electronics. However, it was discovered that the wavelength of the emitted light from the blue LEDs originally used in the LI system depended on the voltage used to drive the LED. This effect, convoluted with the absorption spectrum of the wavelength shifting fiber, induced a non linearity in the light level incident on the PMT face relative to the light level measured at the PIN. In 2003, the LEDs were replaced with UV LEDs that did not display the same wavelength dependence [36]. Figure 4.5 compares the linearity of the old LEDs to that of the new, UV LEDs. The top left plot shows an example of the PMT versus PIN response for the non-linear 
LEDs. The region between the green dotted lines is fitted to a straight line. In the bottom plot left plot, the fractional residual relative to the fit is plotted. At low light levels, a deviation from linearity of up to $10 \%$ can be seen. The top right plot shows the PMT versus PIN curve for the new LEDs, while the lower right plot shows the residuals for the new LEDs. No non-linearities at low light levels are observed.

To use the LI calibration data collected before the LED change, a bootstrapping method had to be employed to obtain a linearity correction. This method relied on the fact that when the LI system pulsed a given strip end, the PMT on the far end of the strip also received light, and due to the attenuation in the green fiber, the light measured on the far end was lower than at the pulsed end. Thus, the pulsed strip end could be used as a reference against which the far end could be linearized. Figure 4.6 shows the effect of the linearity correction for several channels. The linearity correction in general has no effect below 6000 ADC, but can have as large an effect as a few tens of percent at the highest ADC values.

\subsection{Muons}

Whether they be from cosmic sources or induced by neutrino interactions, muons are abundant at each of the detector sites. Moreover, muons are highly penetrating and the energy deposited in a detector cell is only weakly dependent on momentum. Together, these facts make muons an ideal tool to perform the final steps in the energy calibration. Charge and light injection only correct for variations in the response of the PMTs and electronic channels. Further non-uniformities arise from the variations in scintillator strip light yield, defects in the wavelength shifting fibers, and differences in the interfaces among optical elements [37], [38]. These variations are calibrated by comparing the average light yield from each strip when the detector is illuminated by cosmic ray muons. Figure 4.7 shows the response of several different strips due to cosmic muons. Normalizing the mean of such distributions for each 

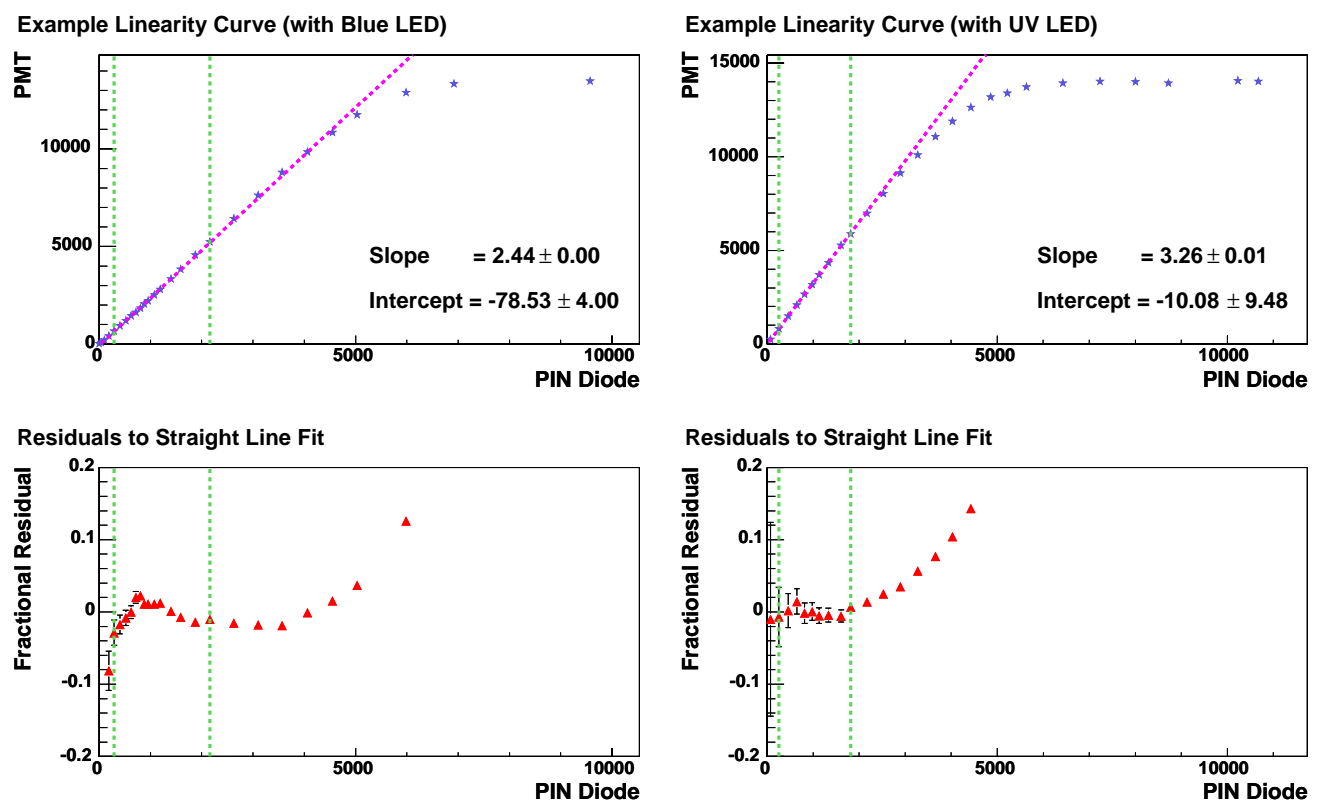

Figure 4.5: Top left plot shows the PMT response in ADC counts versus the PIN diode response for varying light levels. The bottom left plot shows the fractional residual from a linear fit over the range indicated by the dotted lines. The nonlinearity at higher light levels is expected as an effect of PMT and readout electronics saturation. The non-linearity at low light levels is induced by the wavelength shift of the LED light with driving voltage convoluted with the absorption spectrum of the wavelength shifting fiber. This effect can be as much as $10 \%$ at the lower light levels. Top right plot shows the PMT response in ADC counts versus the PIN diode response for varying light levels for the UV LEDs. The bottom right plot shows the fractional residual from a fit over the range indicated by the dotted lines. The non-linearity at the lower light levels is no longer present [35]. 


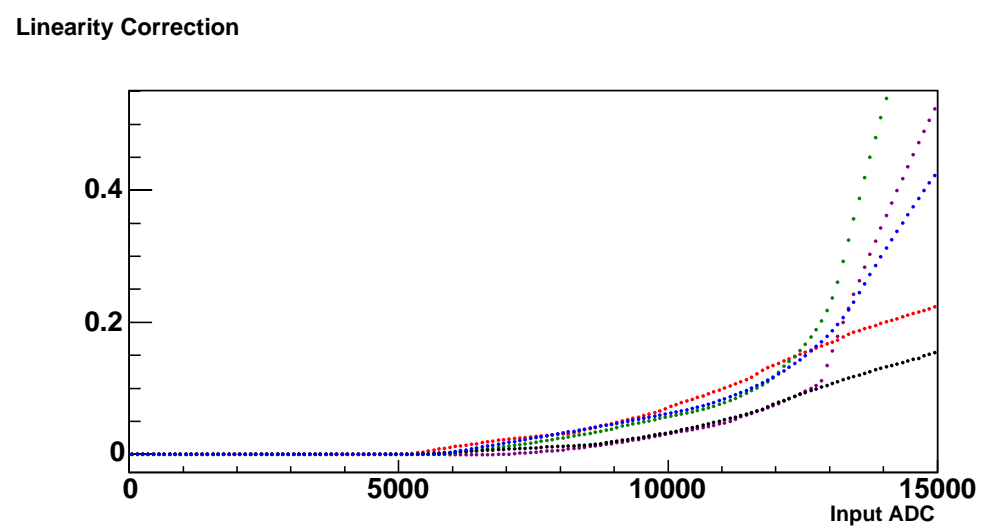

Figure 4.6: The relative effect of applying the linearity calibration versus ADC for a few central strips.

channel gives a correction factor that can be applied to the data to eliminate nonuniformities in scintillator strip light output. Figure 4.8 shows the average response of each cell in ADC when that strip is traversed by cosmic muons. Figure 4.9 shows the response in a plane in these normalized strip units versus plane number for $1.8 \mathrm{GeV}$ muons. The variation in the plane response of $2.5 \%$ gives a measure of the quality of the strip-to-strip calibration.

The results describing the detector response in this document are given relative to the mean response read out from a single strip end when that strip is traversed by cosmic muons. While the cosmic muons used to derive this mean response are not precisely minimum ionizing particles, this unit is called a MIP and allows for the direct comparison of all CalDet data. To use the CalDet results to infer the energy of an incident particle at the large detectors, one must define a unit of energy that has the same meaning for each of the detectors. Since each of the detectors is at a different depth and has a different overburden, the cosmic ray muon spectrum will be different at each detector location and thus can not be used as the standard calibration "candle". Instead, MINOS will define an energy unit, 

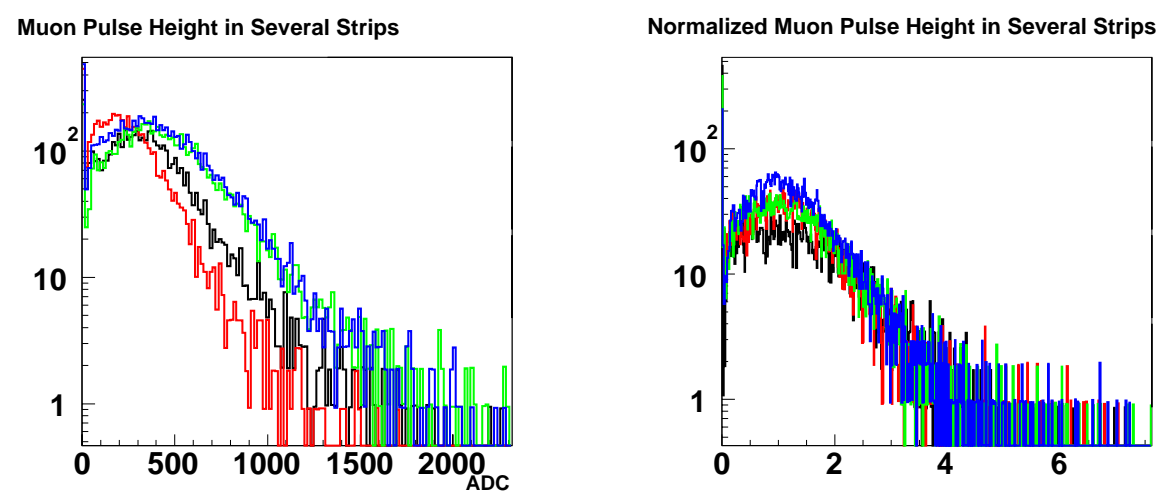

Figure 4.7: Pulse Height distribution from cosmic muons. Left plot shows the muon distributions before the strip-to-strip correction, while the right plot shows the distributions after applying the strip-to-strip correction.

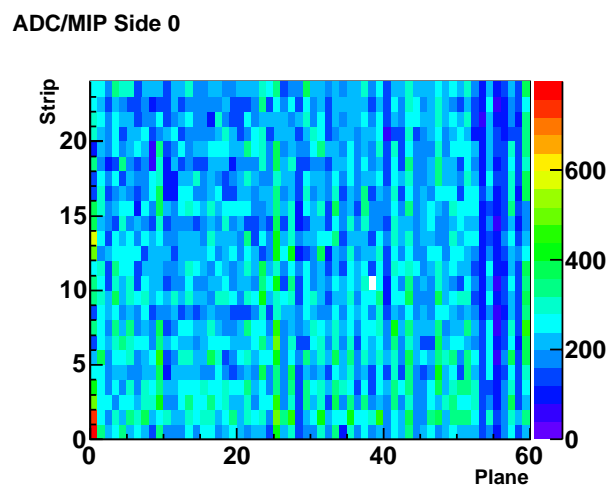

ADC/MIP Side 1

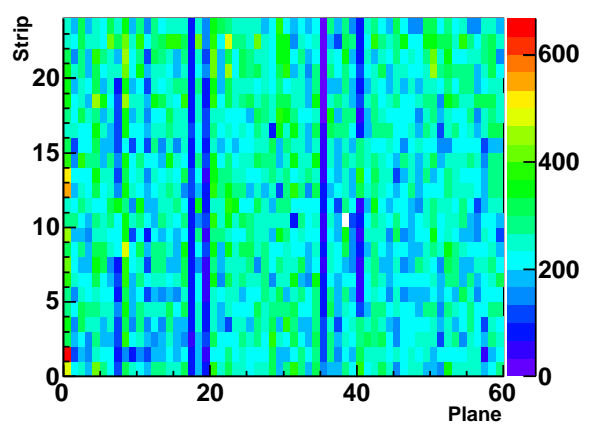

Figure 4.8: The average ADC produced in each strip end when that strip is traversed by cosmic muons. Left plot shows the crate 0 strip ends, while the right plot shows the crate 1 strip ends. 
Muon Response versus Plane

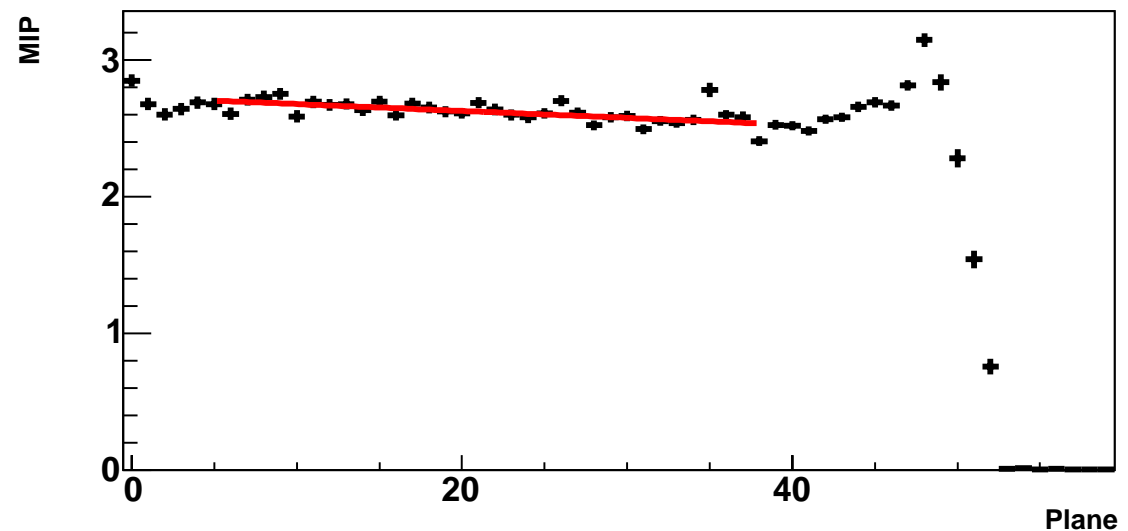

Relative Residual vs. Plane

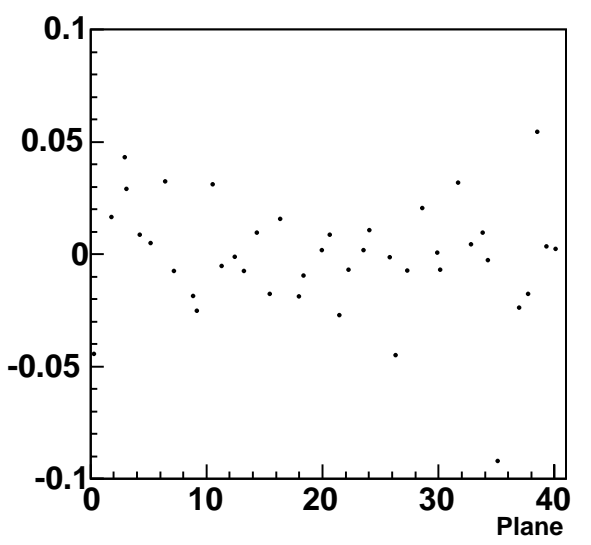

Relative Residual

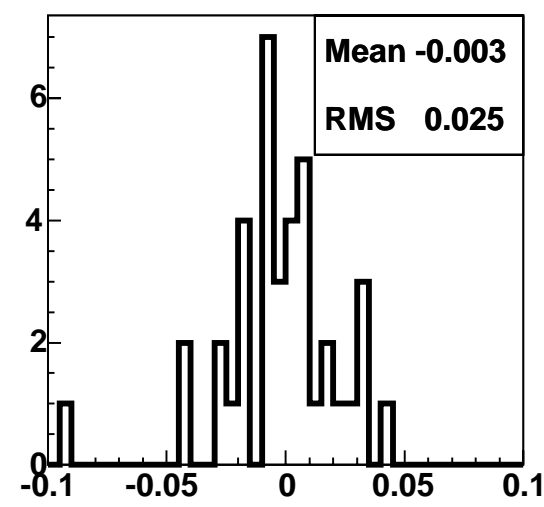

Figure 4.9: The top plot shows the average response versus plane for $1.8 \mathrm{GeV}$ stopping muons. The bottom left plot shows the residual of the data relative to a straight line fit over the range from plane 5 to plane 40 . The bottom right shows the y-projection of the relative residuals. The spread in the residuals of $2.5 \%$ is a measure of the quality of the strip-to-strip calibration. 
to be called the Muon Energy Unit (MEU), by studying the response of stopping muons over a range of planes near the end of the track [39]. By looking at the response of stopping muons in planes over a fixed range from the end of the track, one ensures that the signal is initiated by muons of the same energy. While the details of selection criteria and the range of planes have yet to be fixed for the sample of muons that will provide the definition of the MEU, an outline of the procedure is provided here. A sample of muons of varying incident momenta are selected by requiring that the event penetrates deeper than 25 planes. The stopping plane for each event is computed by selecting the most downstream plane on the track that meets the criterion that at least one out of four consecutive planes has a signal greater than 0.5 MIP in it. Then for muons with a stopping plane between planes 25 and 58 , the response versus plane relative to the stopping plane is histogrammed, then the average response is computed over a window spanning 15-25 planes before the end of the track. The average response in these planes is defined to be 1 MEU. Figure 4.10 illustrates the steps in this procedure. The top plot shows the response in a plane versus the distance from the end of the track. The second plot shows the profile histogram. The average response in a plane falls from 3.15 MIP in the stopping plane to a minimum of about 2.65 MIP around the tenth plane before the end of the track, then rises slightly with the distance from the end of the track. The bottom left plot shows the response versus plane for planes in the window of 15-25 planes before the end of the muon track, while the bottom right plot shows the y-projection. The mean indicates that $1 \mathrm{MEU}$ is equivalent to $2.67 \mathrm{MIP}$. The response given in this document can be converted to MEU by dividing by 2.67

Figure 4.11 demonstrates the efficacy of the calibration chain. The top row of plots shows the summed ADC distribution produced in the detector in response to $1 \mathrm{GeV}$ electrons. The left column shows the response as measured by the Far Detector front end, while the right column shows the response as measured by the 
MIP vs. Planes from Last Plane

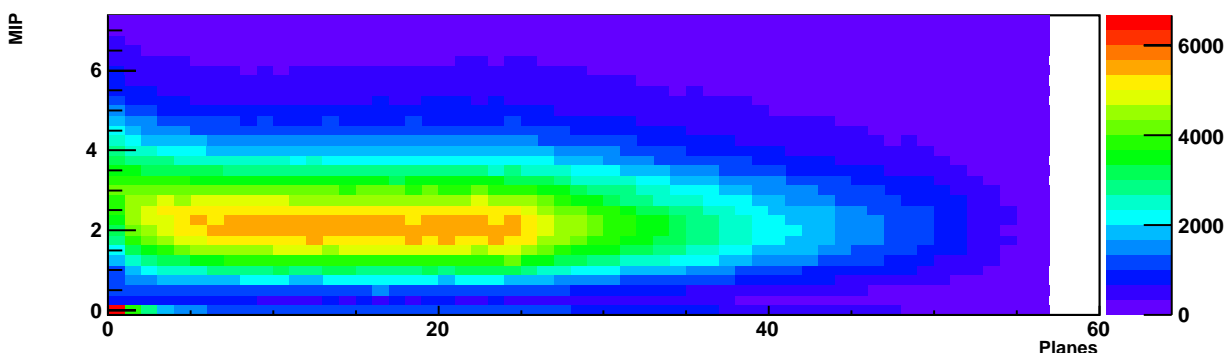

MIP vs. Planes from Last Plane

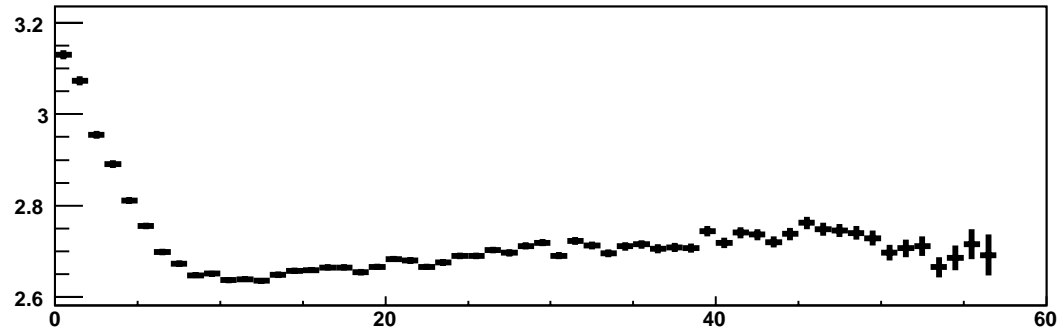

MIP vs. Planes from Last Plane (zoomed)

$\frac{a}{\Sigma}$

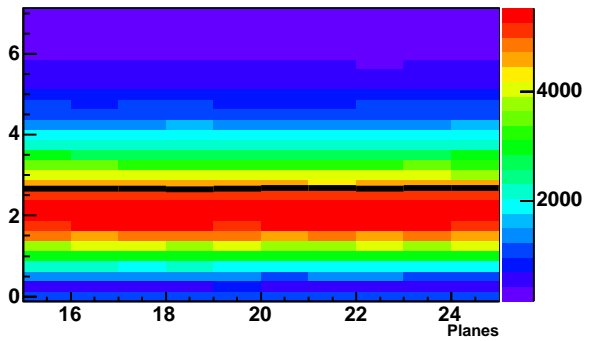

MIP vs. Planes from Last Plane (zoomed)

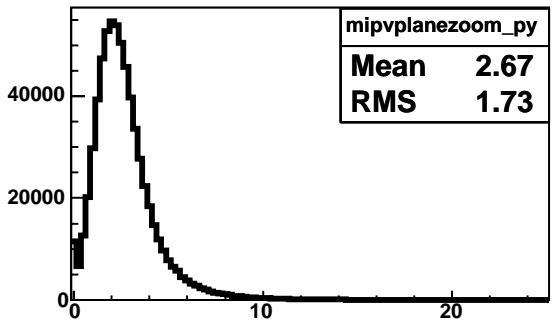

Figure 4.10: The top plot shows the response in a plane versus the distance to the end of the track (in planes). The second plot shows the profile histogram. The bottom left plot shows the response versus plane for planes in the window of $15-25$ planes before the end of the muon track, while the bottom right plot shows the y-projection. The mean indicates that $1 \mathrm{MEU}$ is equivalent to 2.67 MIP. 
Near Detector front end. The black distribution comes from a run in which the PMT high voltages were set to give an average gain of $10^{6}$. The red distribution comes from a run in which all the PMT high voltages were lowered by $25 \mathrm{~V}$. Such a change produced a $30-40 \%$ change in detector response. The middle row of plots shows the same distributions after applying the charge and light injection corrections. The bottom row of plots show the detector response after applying the strip to strip calibration. After calibration, the response of the detector differs by less than $0.5 \%$ between the two runs. The calibration removes the detector non-uniformities and drifts with respect to time, allowing for the comparison of the response of samples of $1 \mathrm{GeV}$ electrons despite significant changes in detector conditions.

Armed with the knowledge that the calibration chain works, the rest of this document describes the procedure used to measure electron interactions in the CalDet and determine the response induced by electrons versus momentum. 

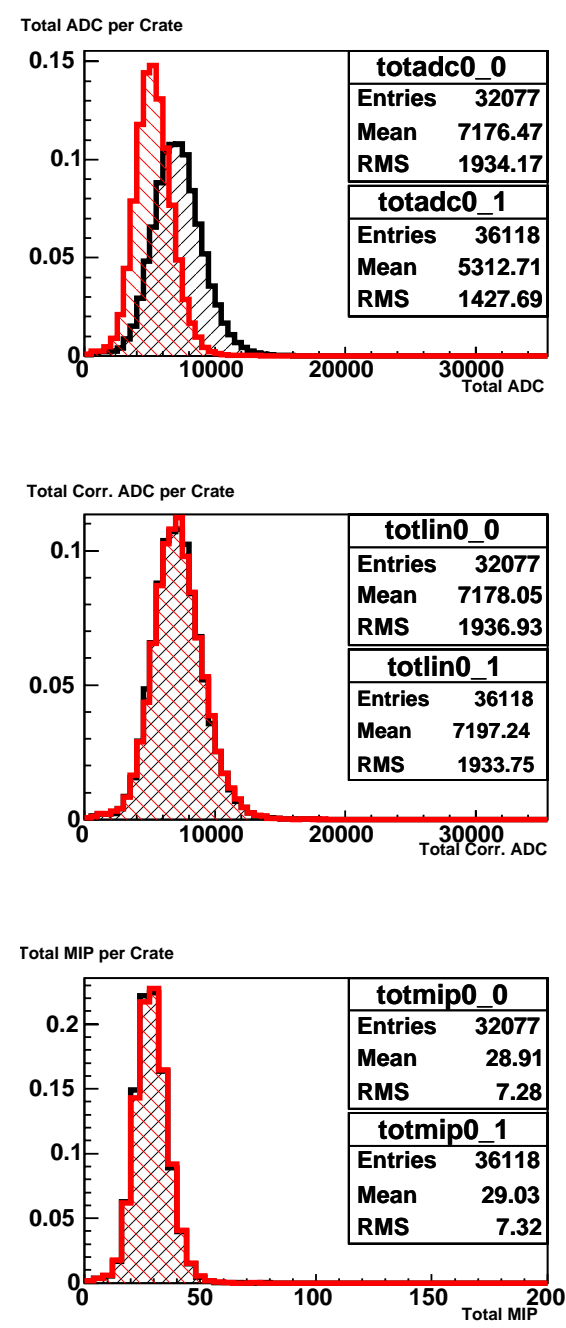
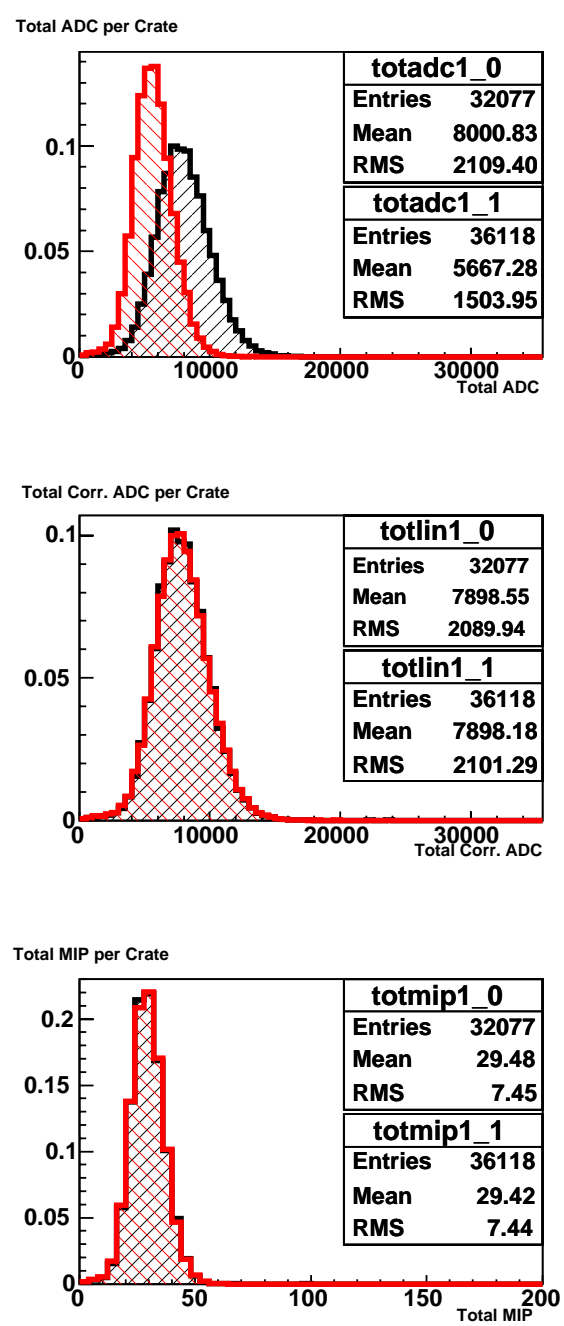

Figure 4.11: The effect of applying the calibration chain. The left column of plots shows quantities measured with the Far FEE, while the right column shows quantities measured with the Near FEE. Top row of plots shows the detector response in summed ADC values. The red histogram is taken from a run in which the high voltage on all PMTs was reduced by $25 \mathrm{~V}$ relative to the run histogrammed in black. The second row of plots shows the detector response after correcting for the gain change induced by the change in high voltage. The final row of plots shows the response in MIP. After the calibration chain is applied, the mean response of the two runs agrees within $0.5 \%$ 


\section{Chapter 5}

\section{Electron Selection Criteria}

Electrons events are initially tagged using a combination of the TOF and the Čerenkov detectors, but further cuts are applied to extract a sample of clean electron events. This chapter describes the cuts and explains why they are necessary.

\subsection{Pre-selection Criteria}

The CalDet beam data sets were collected using the data acquisition mode in which every hit that passed the external enable, the dynode threshold trigger, and the sparsification threshold was written to disk (NULL DAQ trigger). The collection of hits were then broken up into events using an offline, gap trigger [40]. This offline trigger sorts the hits by time, then looks for time gaps greater than $150 \mathrm{~ns}$. Such gaps form the boundaries of events. Figure 5.1 shows a schematic illustrating the process of event forming.

The events formed by the offline trigger were then scanned for electronics errors and dead chips in planes upstream of plane 15. Events without dead chips or electronics errors were further analyzed and subjected to a series of cuts designed to 


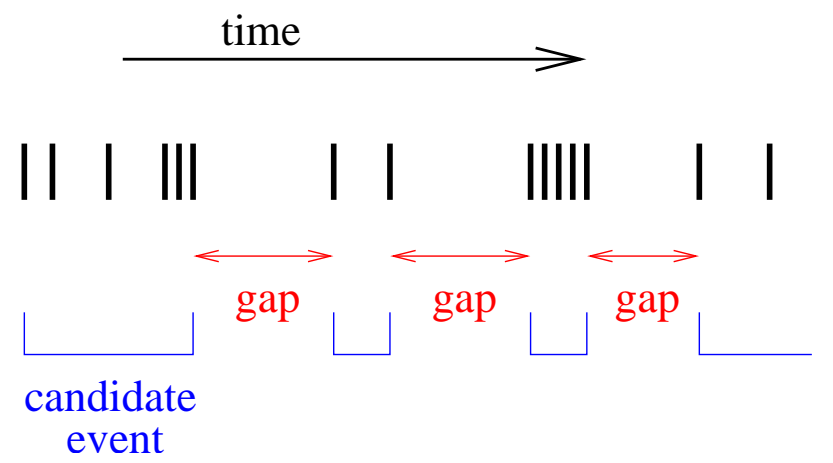

Figure 5.1: Schematic illustrating the event making algorithm of the offline trigger [40].

cull a clean electron sample. The runs from the 2002 beam runs used in the electron analysis are listed in Tables 5.1 and 5.2.

\subsection{Electron Event Selection Cuts}

The events that qualified for the analysis stage were analyzed using an offline software package called CalDetDST. Documentation of this package and further details are given in Appendix E. The cuts designed to isolate a clean sample of electron events are as follows:

- Require the ADC in the Čerenkov set for electron identification to be between $\pm 2.5 \sigma$ around the peak of the Cerenkov ADC distribution. If a second Cerenkov was set to trigger on electrons, a further requirement of signal in the other Čerenkov was imposed.

- Require the time of the VARC enable signal minus the time of any Čerenkov signal to come within $25 \mathrm{~ns}$ of the peak of that distribution. This cut minimizes accidentals arising from noise in the Čerenkov system. 


\begin{tabular}{|l|l|l|l|}
\hline $\mathrm{P}(\mathrm{GeV})$ & Run Numbers & $\mathrm{P}(\mathrm{GeV})$ & Run Numbers \\
\hline 0.2 & $40767-40769$ & -0.2 & $40771-40773$ \\
0.4 & $40777-40779$ & -0.4 & 40775 \\
0.6 & 40781 & -0.6 & 40783 \\
0.8 & $40813-40815$, & -0.8 & 40821 \\
& 40628 & & \\
1.0 & $40622-40626$ & -1.0 & 40758 \\
1.2 & $40862-40864$ & -1.2 & $40874-40876$ \\
1.4 & $40709-40711$ & -1.4 & 40760 \\
1.6 & 40817 & -1.6 & $40878-40880$ \\
1.8 & $40616-40620$ & -1.8 & $40763-40765$ \\
2.0 & $40715-40719$ & -2.0 & $40722-40724$ \\
2.2 & $40785-40789$ & -2.2 & $40805-40811$ \\
2.4 & $40910-40912$ & -2.4 & $40914-40918$ \\
2.6 & $40728-40734$ & -2.6 & $40748-40756$ \\
2.8 & 40922 & -2.8 & \\
3.0 & 40924 & -3.0 & \\
3.2 & $40844-40860$ & -3.2 & $40894-40900$ \\
3.6 & $40736-40746$, & -3.6 & $40791-40803$ \\
& $40926-40932$ & & \\
\hline
\end{tabular}

Table 5.1: T11, 2002 Run Numbers used in the CalDet Electron Analysis 


\begin{tabular}{|l|l|l|l|}
\hline $\mathrm{P}(\mathrm{GeV})$ & Run Numbers & $\mathrm{P}(\mathrm{GeV})$ & Run Numbers \\
\hline 1.0 & $50505-50507$ & -1.0 & $50456-50460$, \\
& & & $50643-50645$ \\
2.0 & $50491-50493$ & -2.0 & 50454 \\
2.4 & $50483-50485$ & -2.4 & 50462 \\
2.8 & $50487-50489$ & -2.8 & 50481 \\
3.0 & $50495-50497$ & -3.0 & $50262-50264$, \\
& & & 50456 \\
4.0 & 50531 & -4.0 & 50553 \\
5.0 & $50533-50535$ & -5.0 & $50555-50559$ \\
6.0 & $50537-50539$ & -6.0 & $50588-50592$ \\
7.0 & $50601-50605$, & -7.0 & $50509-50517$ \\
& $50662-50664$ & & $50656-50660$ \\
8.0 & $50611-50627$ & -8.0 & $50594-50596$ \\
9.0 & $50667-50673$ & -9.0 & $50700-50702$ \\
\hline
\end{tabular}

Table 5.2: T7, 2002 Run Numbers used in the CalDet Electron Analysis

- Require the TDC measurement provided by the TOF system to be within $\pm 2.5 \sigma$ around the peak of the electron time of flight distribution.

- Require the strip of maximum energy deposition in plane 0 to be either strip 11 or 12 . This cut minimizes the occasions when the particle initiating the external enable traveled through the body of the TOF system instead of the scintillator paddle. This cut implicitly requires energy to be deposited in plane 0 .

- Require the hit times of each digitized channel in an event to be consistent with the external enable time. This cut removes events in which two particles were responsible for the signal but recorded as one event. The cut is made using a test statistic called $\chi_{o l}^{2}$ and is described in detail later in this chapter.

A sample of electron events at $1 \mathrm{GeV}$ that pass the above cuts are shown in Figures 5.2 through 5.4. These events are characterized by a dense energy deposition 
limited to the front of the detector. The low pulse height hits at the edges of the detector are due to PMT cross talk, a phenomenon in which charge is recorded in a pixel other than the illuminated pixel. More electron events at other momenta, as well as muon and hadronic events are included in Appendix A.

Figures 5.5 and 5.6 show the distribution of the cut quantities for $1 \mathrm{GeV}$ positrons from the T11 and T7 2002 beam runs. Each histogram shows the cut quantity for events with a hit in the upstream Cerenkov, the cut quantity when all the cuts except the one shown are applied, and finally the cut quantity when all cuts are applied. Figure 5.7 shows the number of planes hit in the event, each color showing the distribution when all but one cut is applied. The blue histogram in these plots show the result after all cuts have been applied. In both the cases shown, the number of hit planes distribution is a tight distribution with few outliers, indicating the selected electron sample is relatively free from contamination by other particle species. Cut and planes hit histograms for other runs used in this analysis are included in Appendix B.

Either a noise hit in the Čerenkov system or a real signal caused by the production of a $\delta$-ray above the Cerenkov threshold in the Cerenkov windows or gas volume could lead to the inclusion of a pion or muon in the electron sample. The probability of either a muon or pion producing a $\delta$ in a Čerenkov window is on the order of $10^{-4}$. At low to intermediate energies, the number of pions and muons is of the same magnitude as the number of electrons, and the contamination of the sample is small. At higher energies, where the number of pions is many orders of magnitude greater than the number of electrons, the contamination due to $\delta$ - ray production could be important. Above $1 \mathrm{GeV}$, most muons and many pions penetrate deeper than plane 25, whereas electrons should not. Assuming the events that pass the electron cuts but travel further than 25 planes are either pions or muons, then comparing the number of such events to the number of pions and 


\begin{tabular}{c} 
Run: 40622 \\
Snarl: 26 \\
PID: Electron \\
Momentum: $1.0 \mathrm{GeV}$ \\
Total MIP: 68.11 , Even: 40.79 , Odd: 27.31 \\
\hline
\end{tabular}
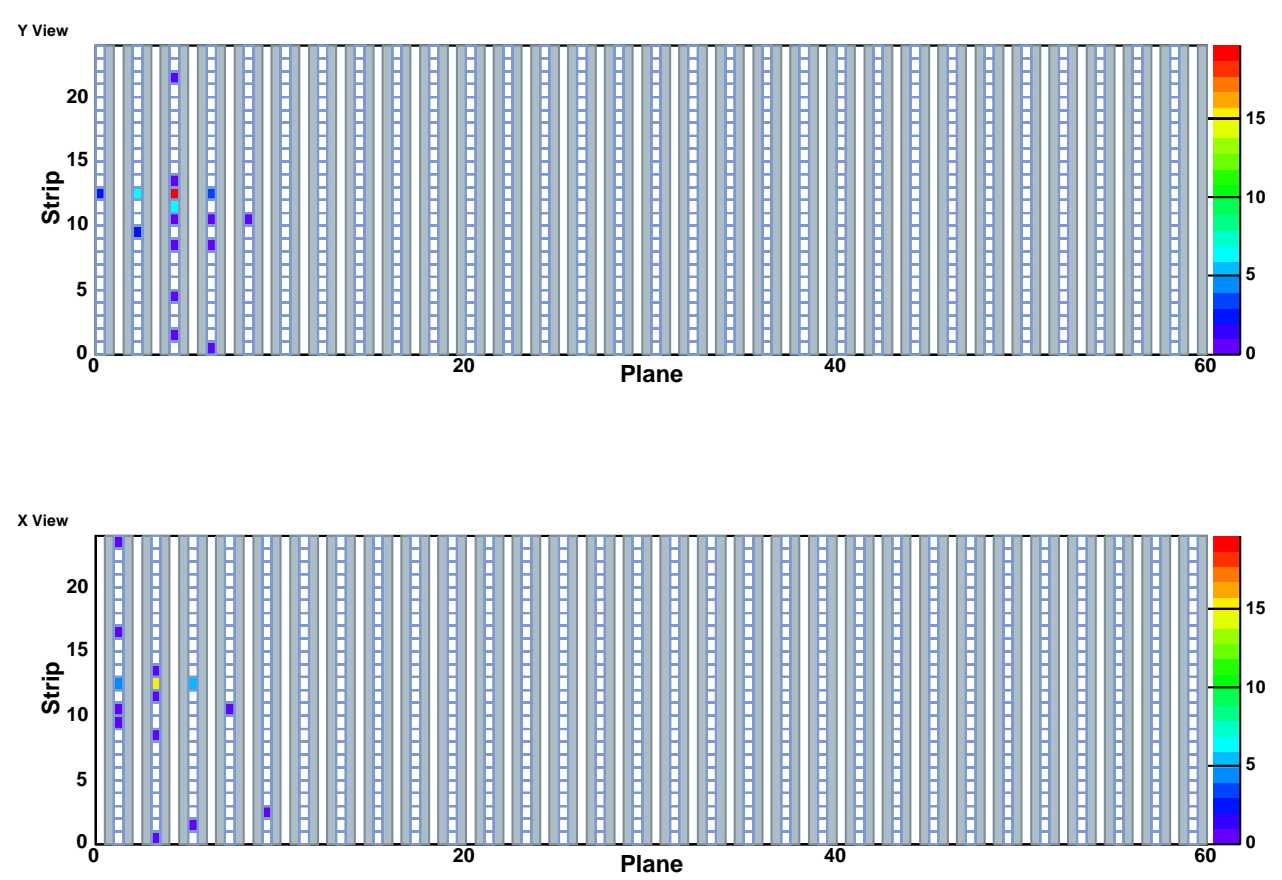

Figure 5.2: A $1 \mathrm{GeV}$ electron event. The top plot shows the hit strips in the Y-view, or even planes, while the bottom plot shows the hit strips in the $\mathrm{X}$-view, or odd planes. The pulse height, in MIP units, is given by the color scale. 


\begin{tabular}{c} 
Run: 40622 \\
Snarl: 63 \\
PID: Electron \\
Momentum: $1.0 \mathrm{GeV}$ \\
Total MIP: 36.09 , Even: 22.29 , Odd: 13.80 \\
\hline
\end{tabular}
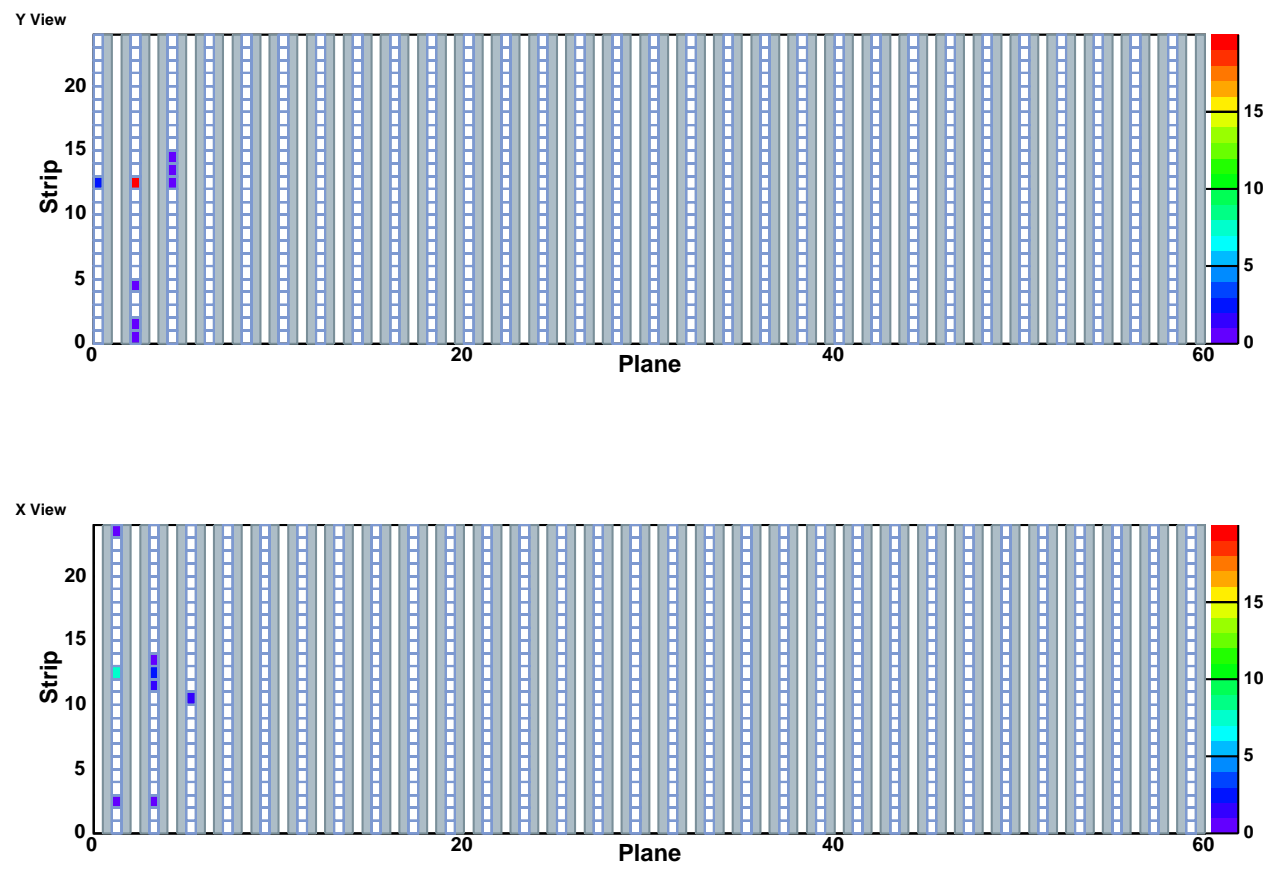

Figure 5.3: A $1 \mathrm{GeV}$ electron event. 


\begin{tabular}{c} 
Run: 40622 \\
Snarl: 67 \\
PID: Electron \\
Momentum: $1.0 \mathrm{GeV}$ \\
Total MIP: 91.52 , Even: 44.00 , Odd: 47.52 \\
\hline
\end{tabular}
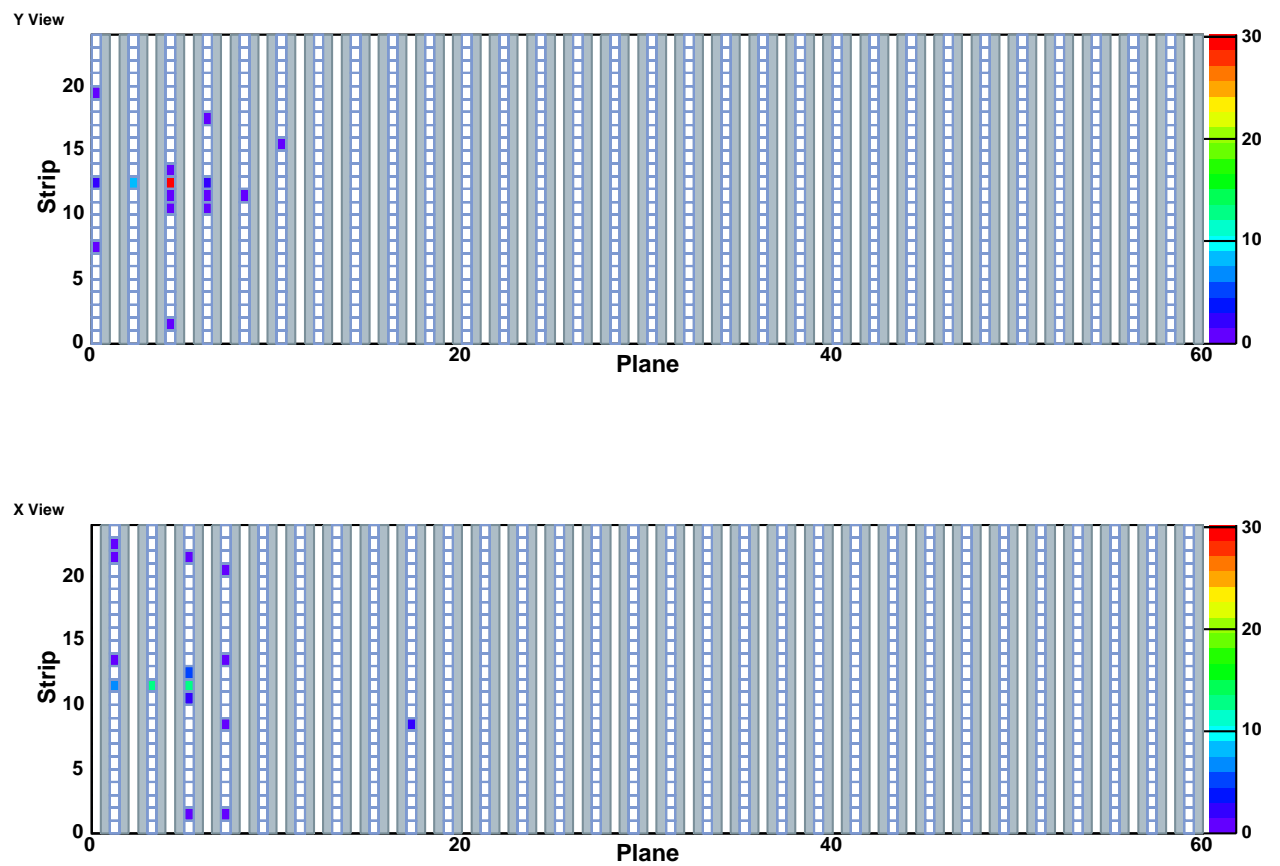

Figure 5.4: A $1 \mathrm{GeV}$ electron event. 


\section{Cerenkov ADC}

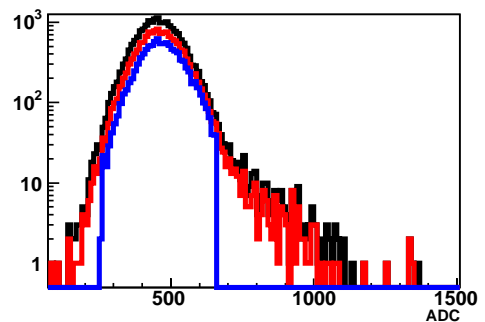

Cerenkov Time

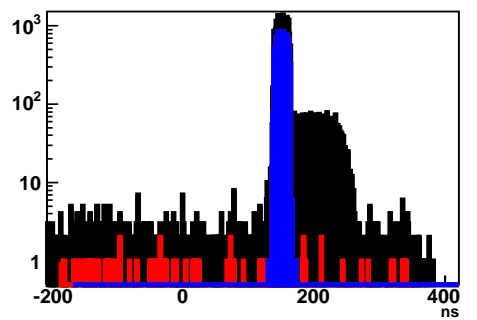

Time of Flight

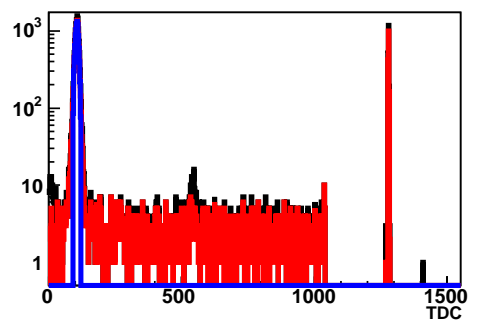

Overlapping Events

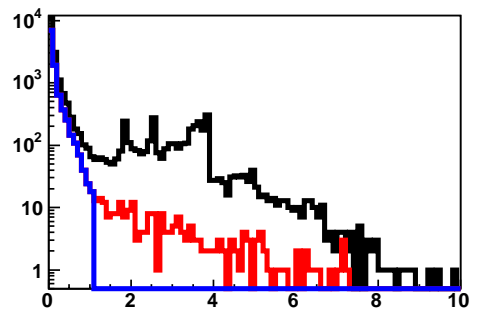

Plane 0, Strip of Max Signal

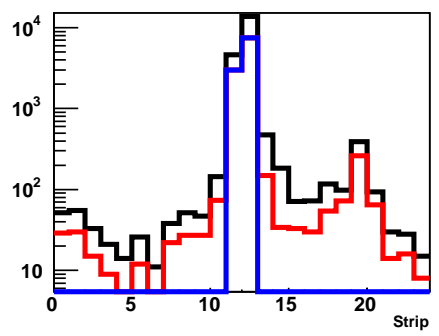

Figure 5.5: Distributions showing the electron selection cuts from a $+1 \mathrm{GeV}$ run from the T11 2002 data sample. Top left shows the Cerenkov ADC distribution. Top right shows the time of flight distribution. Middle left shows the distribution of Cerenkov hit time minus the trigger time. Middle right shows the distribution of the $\chi_{o l}^{2}$. Bottom shows the strip of maximum signal in plane 0 . In each of the plots, the black histogram shows the distribution for all events with a signal in the upstream Cerenkov, blue shows the distribution when all cuts but the one shown have been made, and red shows the distribution after all cuts have been made. The Čerenkov ADC and TOF cuts are applied simultaneously in the analysis. Their combined effect is shown in the top two plots. 

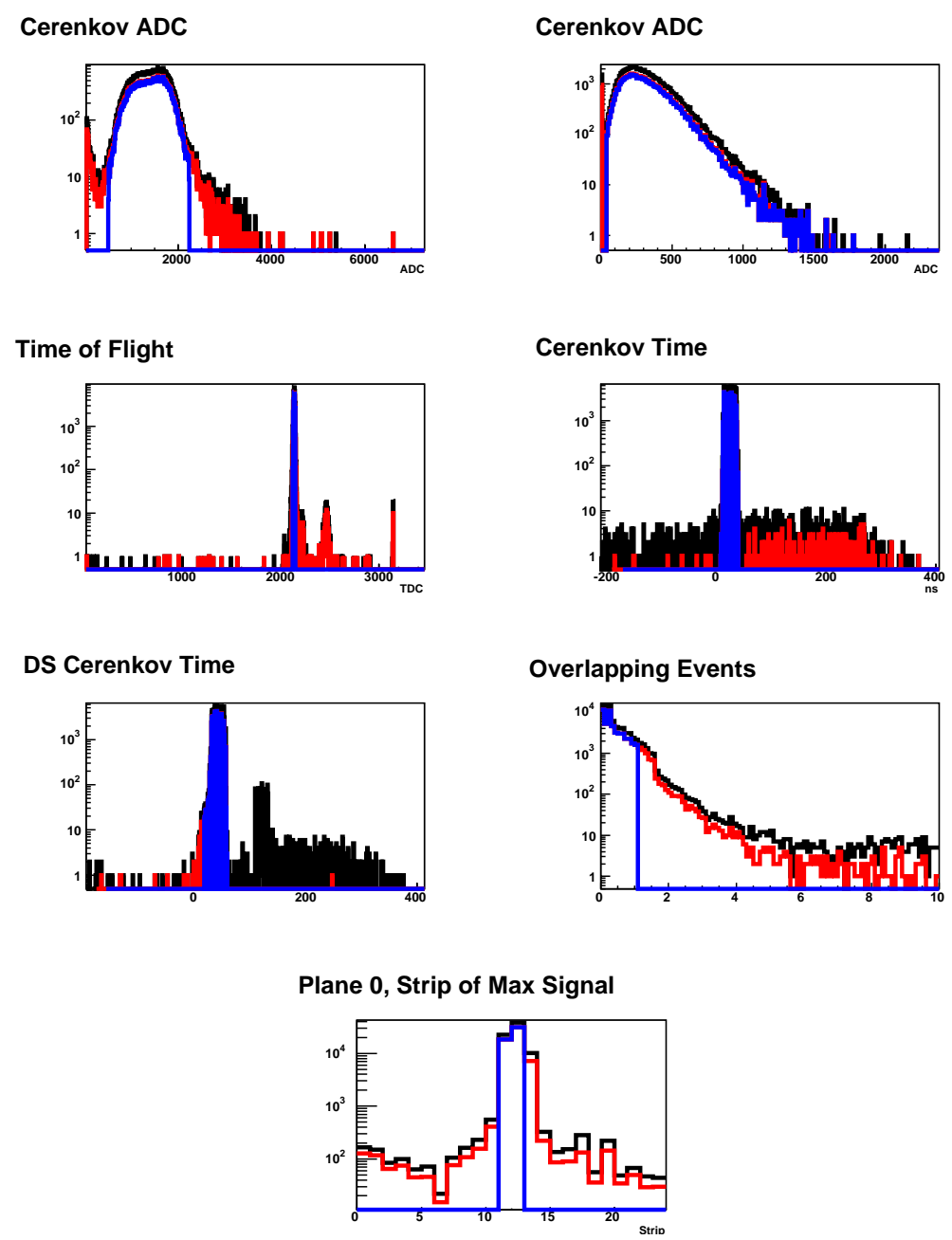

Figure 5.6: Distributions showing the electron selection cuts from a $+1 \mathrm{GeV}$ run from the T7 2002 data sample. Top plots show the upstream and downstream Čerenkov ADC distributions. Second row, left shows the time of flight distribution. Second row, right and third row left show the distribution of upstream and downstream Cerenkov hit time minus the trigger time. Third row, right shows the distribution of the $\chi_{o l}^{2}$. Bottom shows the strip of maximum signal in plane 0 . In each of the plots, the black histogram shows the distribution for all events with a signal in the upstream Cerenkov, blue shows the distribution when all cuts but the one shown have been made, and red shows the distribution after all cuts have been made. The Cerenkov ADC and TOF cuts are applied simultaneously in the analysis. Their combined effect is shown in the top two plots. 

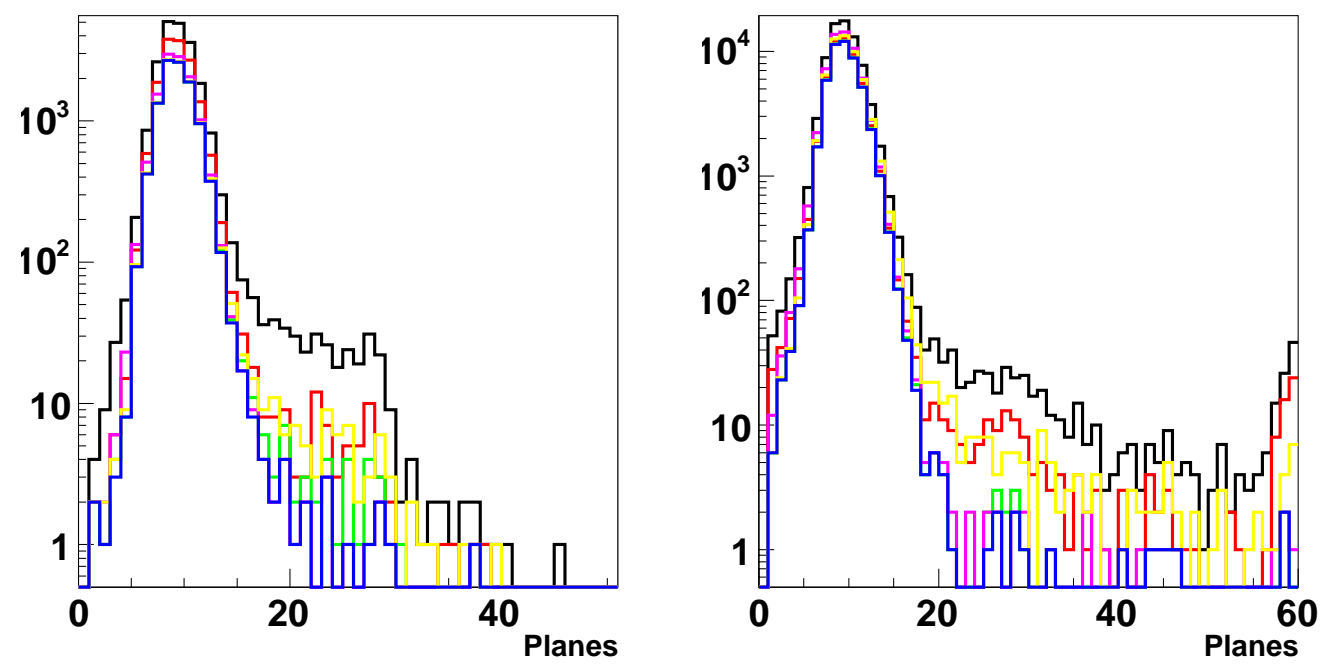

Figure 5.7: The distribution of number of hit planes from $1 \mathrm{GeV}$ positron events. Data from T11 2002 is shown to the left, data from T7 2002 is shown to the right. Black shows the number of hit planes for events with signal in the upstream Cerenkov; red, after applying all cuts but the pid cut; green, after applying all cuts but the Cerenkov timing cut; yellow, after applying all cuts but the $\chi_{o l}^{2}$ cut; purple, after applying all cuts but the plane 0 strip of maximum signal cut. Blue shows the effect of applying all cuts. The final distribution is a narrow distribution with few outliers, indicating a relatively pure sample of electron events.

muons gives an estimate of the level of pion or muon contamination. The runs used in the electron analysis all show much less than $1 \%$ pion and muon contamination. Since even a $1 \%$ contamination of pions or muons in the event sample would give rise to a tenth of a percent deviation in the measurement of the electromagnetic detector response, the contamination is considered negligible. 


\section{Overlapping Events}

The final cut listed above was designed to address the contamination of the event sample by a class of events dubbed "overlapping". Figure 5.8 and 5.10 show examples of typical overlapping events. In each display, an electron-like shower is seen, accompanied by a long muon track. In Figures 5.9 and 5.11, the hit times of each hit in the event are shown relative to the external enable time. In both these events, there are two distinct peaks in the timing distribution, indicating that two particles were responsible for the energy deposition in the detector. It is not sufficient to further divide this class of events into two sub-events since the signal from the channels where the events overlap spatially is summed in the FEE. Events of this nature are removed from the event sample.

Removal of overlapping events is done by comparing the hit time distributions of each event to a template timing histogram. The template histogram comes from a data run with a low rate, hence a low population of overlapping events. To create the template, first, any gross timing offsets between readout channels are corrected, then the hit time minus the external enable time is histogrammed for each detector side. The histograms are then normalized so that the area is equal to 1. Figure 5.12 shows the timing template histograms used for the T11 2002 runs. Once the template histograms are created, the hit time minus the external enable time is histogrammed for each event. Figure 5.13 shows an example of event time histograms.

Next, for each event, a test statistic is computed that compares the event timing characteristics to the template. If $T_{i}^{s}$ is the bin content of the $i^{\text {th }}$ bin of the template histogram from side $s, E_{i}^{s}$ is the bin content of the $i^{\text {th }}$ bin of the event timing histogram from side $s$, and $N^{s}$ is the number of digitizations from side $s$, 


Run: 50505
Snarl: 3271
PID: Electron
Momentum: $1.0 \mathrm{GeV}$
Total MIP: 147.51 , Even: 68.67 , Odd: 78.84
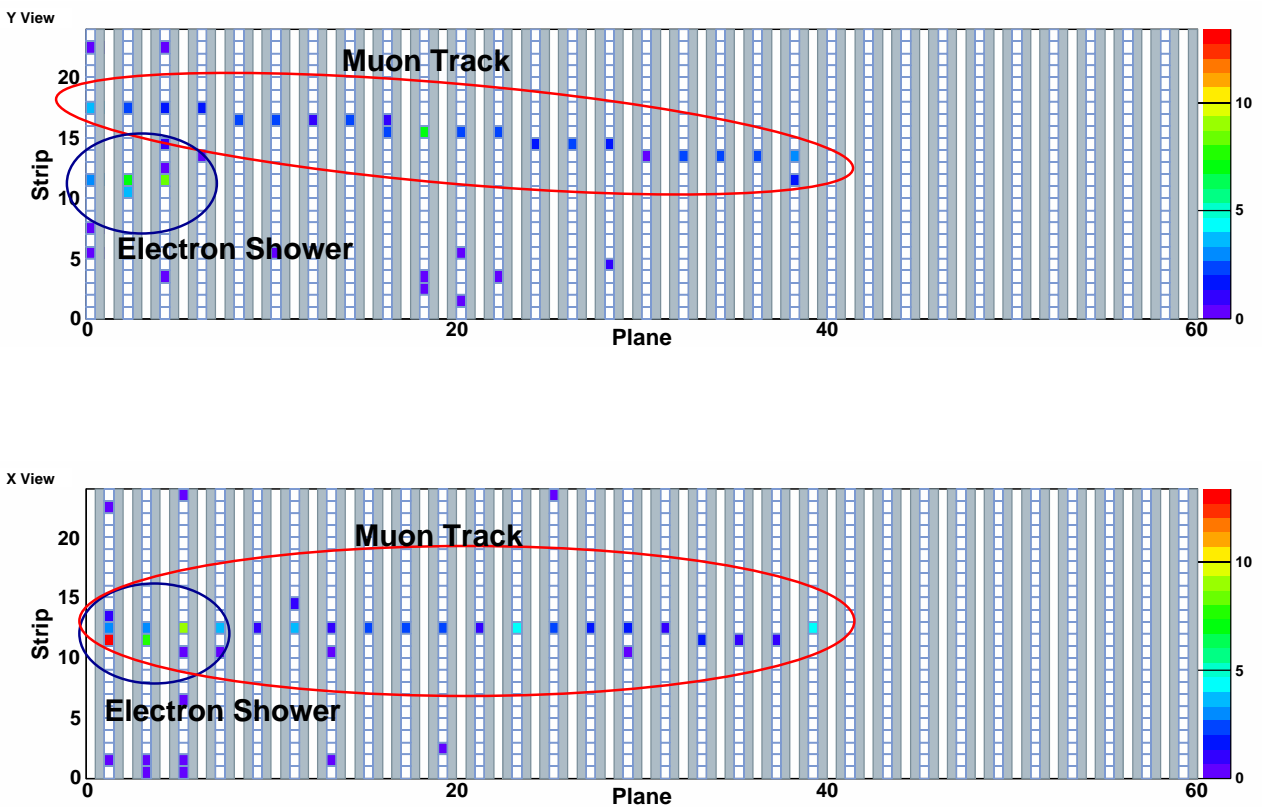

Figure 5.8: An example of an overlapping event. 


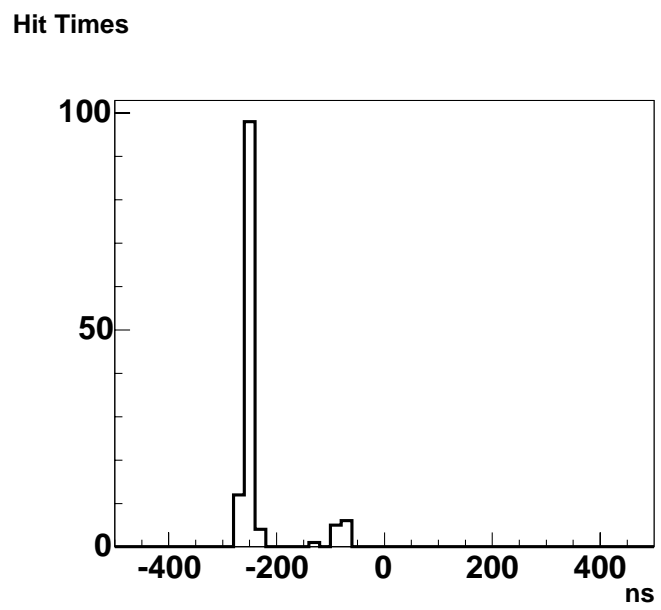

Figure 5.9: The hit times relative to the external enable for the overlapping event shown in Figure 5.8 .

then define

$$
P=\prod_{s} \prod_{i \in b i n s} \frac{P_{i}\left(E_{i}^{s}, T_{i}^{s} N^{s}\right)}{P_{i}\left(E_{i}^{s}, E_{i}^{s}\right)}
$$

where

$$
P_{i}\left(E_{i}^{s}, T_{i}^{s} N^{s}\right)=\frac{\left(T_{i}^{s} N^{s}\right)^{E_{i}^{s}} e^{-T_{i}^{s} N}}{\Gamma\left(E_{i}^{s}\right)}
$$

is the probability of getting $E_{i}^{s}$ hits from a Poisson distribution with mean $T_{i}^{s} N^{s}$, and

$$
P_{i}\left(E_{i}^{s}, E_{i}^{s}\right)=\frac{\left(E_{i}^{s}\right)^{E_{i}^{s}} e^{-E_{i}^{s}}}{\Gamma\left(E_{i}^{s}\right)}
$$

is a normalization factor. Taking the natural log of both sides of Equation 5.1 gives

$$
\begin{aligned}
L & =\ln P \\
& =\sum_{s} \sum_{\text {bins }} \ln \left(\frac{P_{i}\left(E_{i}^{s}, T_{i}^{s} N^{s}\right)}{P_{i}\left(E_{i}^{s}, E_{i}^{s}\right)}\right) \\
& =\sum_{s} \sum_{\text {bins }}\left(\ln P_{i}\left(E_{i}^{s}, T_{i}^{s} N^{s}\right)-\ln P_{i}\left(E_{i}^{s}, E_{i}^{s}\right)\right) \\
& =\sum_{s} \sum_{\text {bins }}\left[\left(E_{i}^{s}\right) \ln \left(T_{i}^{s} N^{s}\right)-T_{i}^{s} N-\left(\left(E_{i}^{s}\right) \ln \left(E_{i}^{s}\right)-E_{i}^{s}\right)\right] .
\end{aligned}
$$


Run: 50505

Snarl: 21626

PID: Electron

Momentum: $1.0 \mathrm{GeV}$

Total MIP: 143.42, Even: 61.71 , Odd: 81.71
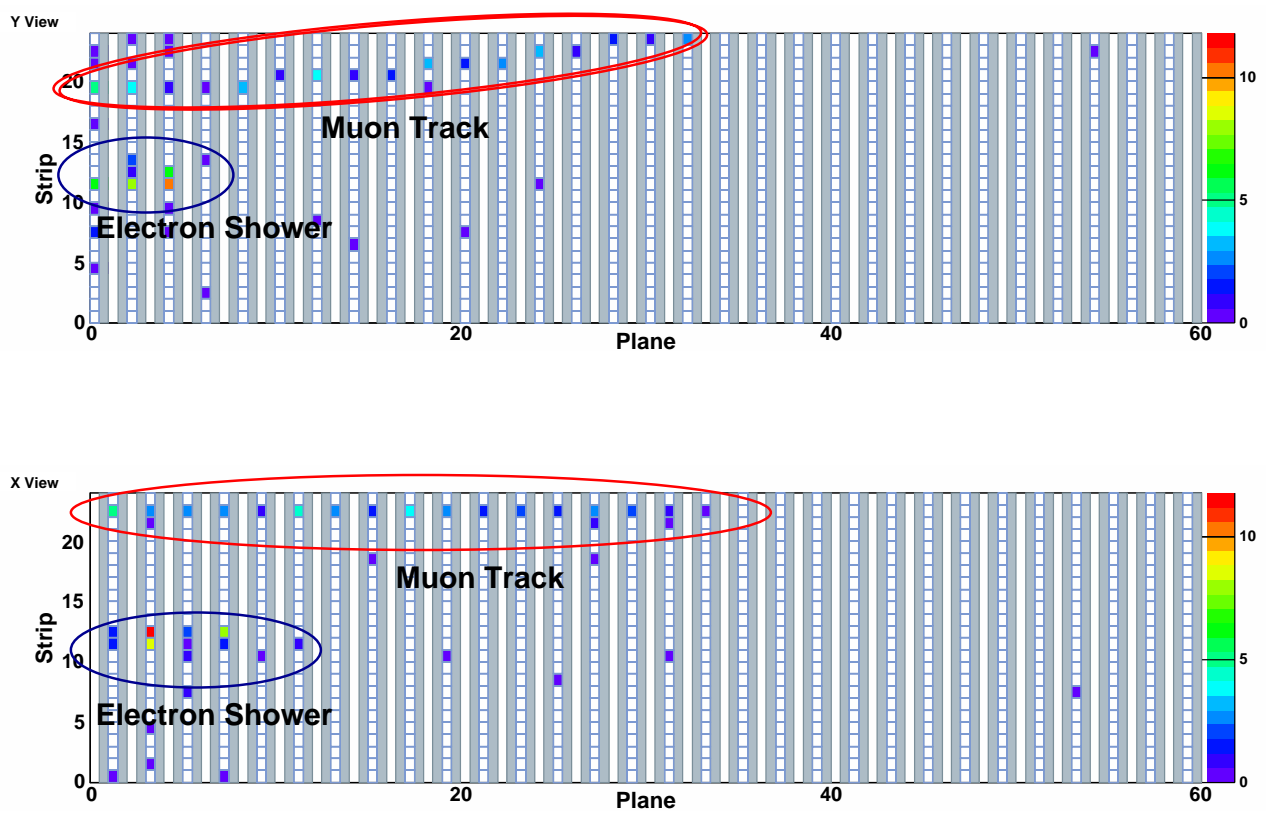

Figure 5.10: An example of an overlapping event. 


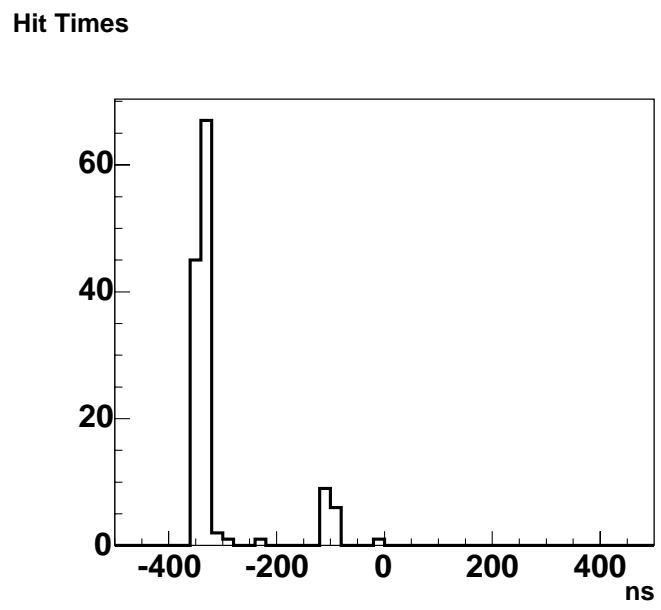

Figure 5.11: The hit times relative to the external enable for the overlapping event shown in Figure 5.10.
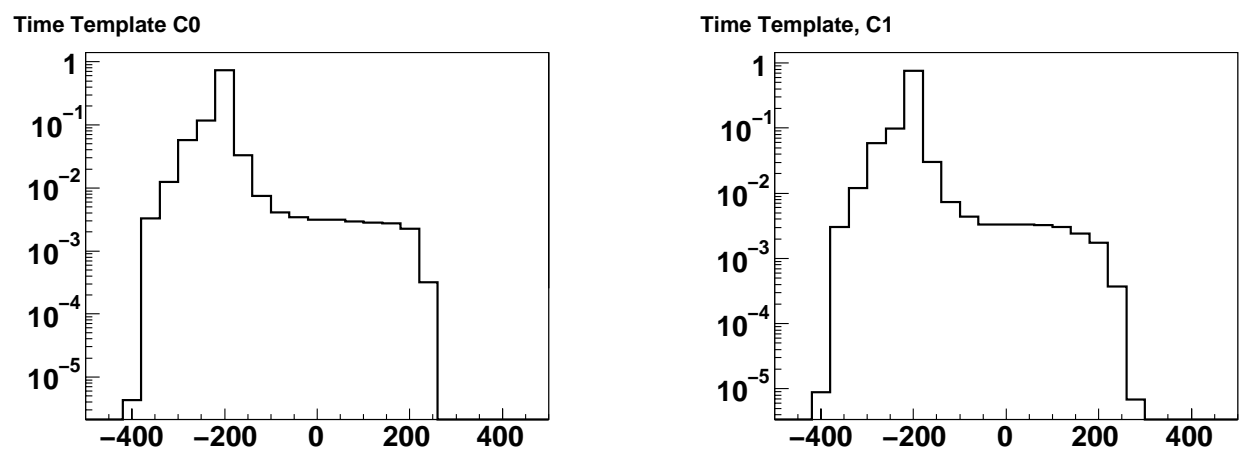

Figure 5.12: The template timing histograms for the T11 2002 runs. To the left, the hit time minus trigger time for all hits on the negative strip ends in a low rate, $1 \mathrm{GeV}$ run, normalized so that the area is 1 . The right plot shows the same for the positive ends. 

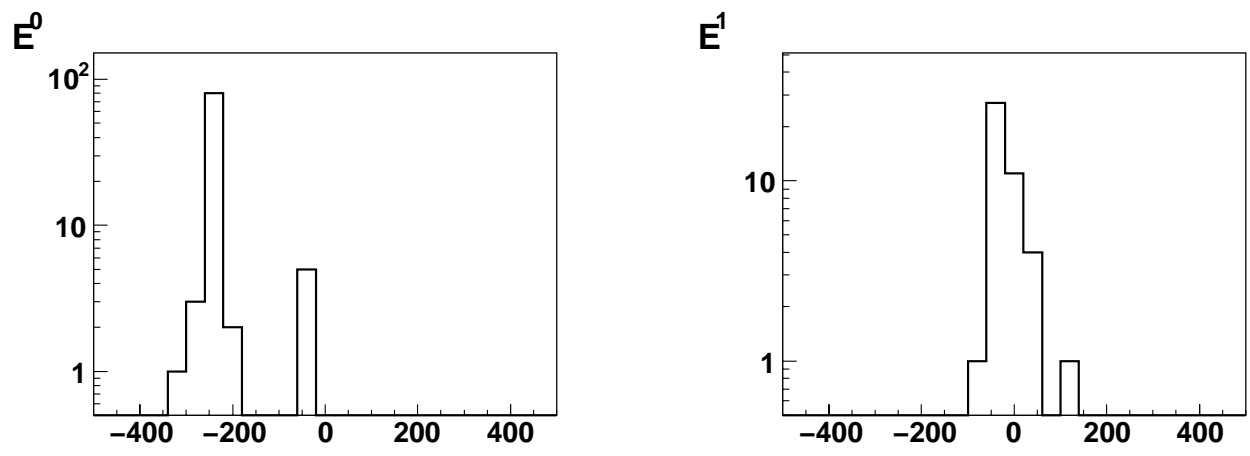

Figure 5.13: The timing histograms for one event. Left shows the hit times minus trigger time for negative strip ends, right shows the same for positive strip ends.

Finally, define the test statistic, $\chi_{o l}^{2}$, as

$$
\chi_{o l}^{2}=\frac{-2 L}{N^{0}+N^{1}}
$$

Then $\chi_{o l}^{2}$ provides for the separation of overlapping events. Events with $\chi_{o l}^{2} \geq 1.1$ are removed from the data sample. Figure 5.14 shows the percentage of overlapping events in a run as a function of the average rate in the upstream TOF paddle. As expected, the number of overlapping events increases with beam intensity.

To further illustrate the success of this method at identifying overlapping events, and to estimate the efficiency of this cut, $\chi_{o l}^{2}$ was calculated over a sample of events which were artificially overlapped. Events with a value of $\chi_{o l}^{2}<1.1$ were selected from a run, then all the hit times were collectively shifted. The size of the shift was randomly selected from a uniform distribution spanning the electronics readout window. Any hits that fell outside the readout window after shifting were discarded. Then, the shifted event was combined with another, non-shifted event, and the value of $\chi_{o l}^{2}$ was computed for the compound event. Figure 5.15 shows 


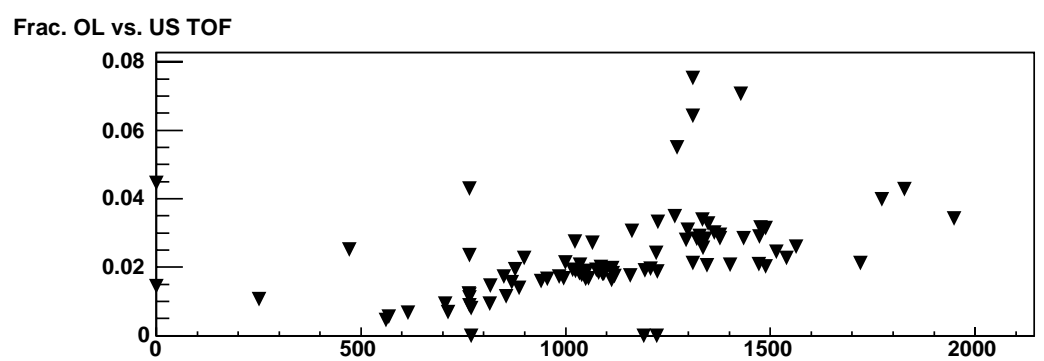

Figure 5.14: The fraction of events with $\chi_{o l}^{2} \geq 1.1$ as a function of the rate in the upstream TOF counter. Each point corresponds to a run. The rate in the upstream TOF counter is averaged over each spill in the run.

the resulting distribution of $\chi_{o l}^{2}$. The artificially overlapped events in general have a value of $\chi_{o l}^{2}$ greater than 1.1. The overlapped events with $\chi_{o l}^{2}<1.1$ arise from events in which the shift time was small, meaning that two events interact within the detector very close in time. By scaling the artificially overlapped distribution of $\chi_{o l}^{2}$ so that the tail matches the number of overlapping events in the tail of the data distribution, and looking at the number of artificially overlapped events with $\chi_{o l}^{2}<1.1$, one can approximately determine the level of overlapping events remaining in the electron sample after the cut on $\chi_{o l}^{2}$. Most runs used in the electron analysis have fewer than $1 \%$ overlapping events remaining after the cut on $\chi_{o l}^{2}$, though a few runs have contamination at the 3-5\% level. Overlapping events are expected to have much larger energy depositions than the average electron events, hence these events populate the tails of the response distribution. When the response is derived, a fit is performed over a limited range of the response distribution, minimizing the effect that overlapping events can have on the measurement. A complete determination of the effect of overlapping events requires a detailed simulation of the electronics timing and the ability to overlay two events temporally, features not yet available in the CalDet Monte Carlo. 
Chi2

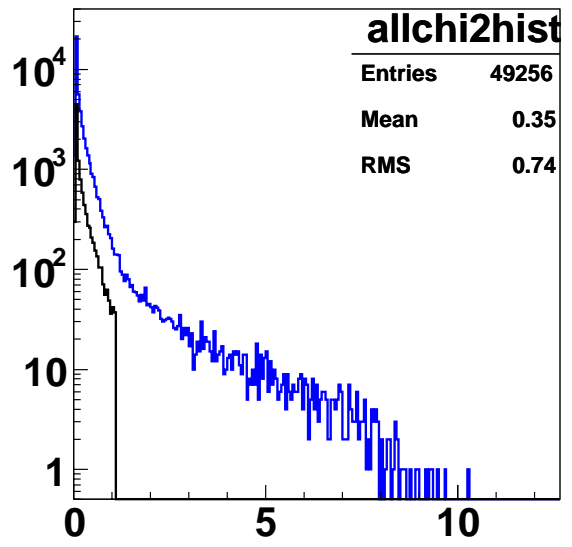

Shifted Chi2

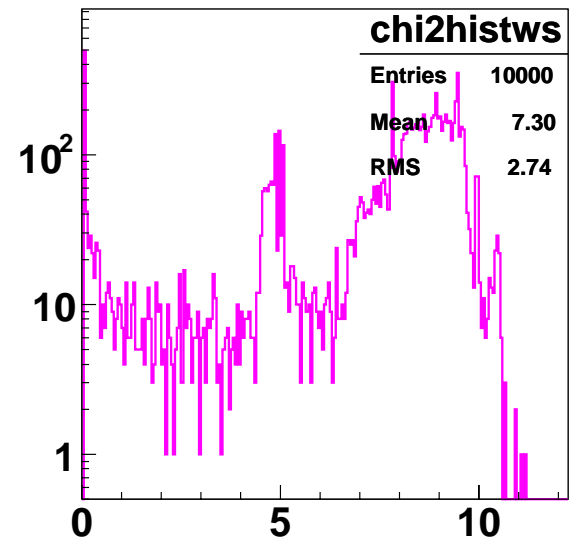

Compound Chi2

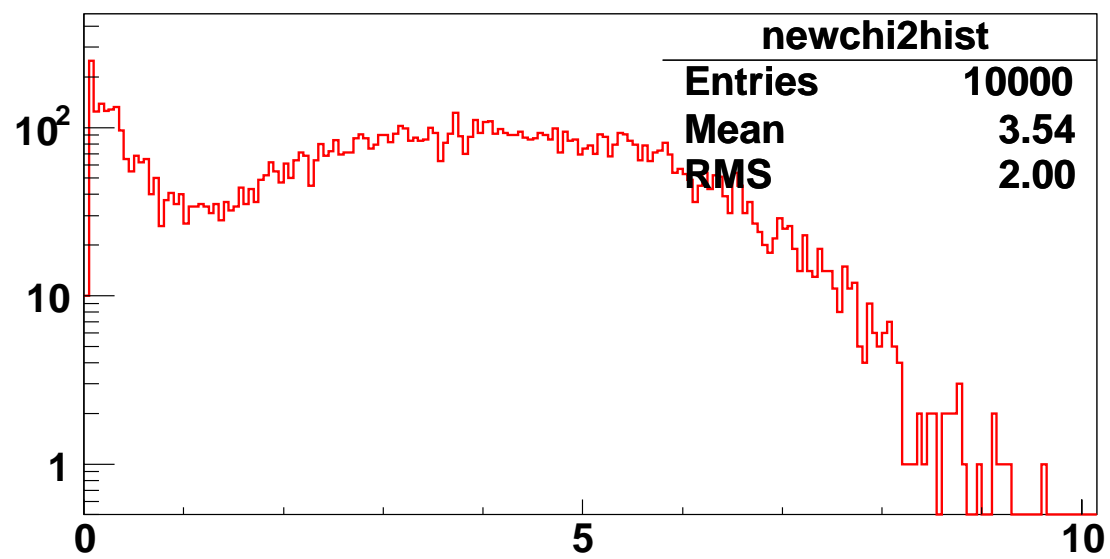

Figure 5.15: Top left shows the distribution of $\chi_{o l}^{2}$ for all electron events in blue, and for events with $\chi_{o l}^{2}<1.1$ selected for shifting in black. Top right shows the $\chi_{o l}^{2}$ distribution for events that have been uniformly shifted by a random offset. The bottom plot shows the $\chi_{o l}^{2}$ distribution for events that have been artificially overlapped using the method described in the text. The majority of overlapped events have a $\chi_{o l}^{2}$ greater than 1.1. The number of events with $\chi_{o l}^{2}<1.1$ in the artificially overlapped sample gives an estimate of the contamination of overlapping events remaining in the electron sample after the cut on $\chi_{o l}^{2}$ is applied. 


\section{Application of A Plane Trigger}

Initially, the MINOS data acquisition system relied upon a plane trigger requirement to reduce the data rate. Events had to pass a $n$ out of $n+1$ sequential hit planes requirement, where typically $\mathrm{n}=4$, in order to be written to disk. At CalDet, the external enable reduced data rates enough to take data without the plane trigger. Figures 5.16 through 5.18 show the response distributions induced by electrons, pions, and protons at sub-GeV beam momenta with progressively more restrictive plane trigger cuts. Not only does the efficiency for selecting the lowest energy particles decrease for more restrictive plane cuts, but the mean response is biased toward larger values. Figure 5.19 shows the efficiency of the different plane cuts as a function of beam momentum. Figure 5.20 shows the shift in the mean response for different plane trigger cuts as a function of beam momentum. In order not to bias the data sample and maintain particle detection efficiency at the lowest energies, steps are being taken to disable the plane trigger requirement at the large MINOS detectors during the NuMI beam spill. No plane trigger requirement is applied to the events used in this analysis. 


\section{Total MIP, $0.4 \mathrm{e}^{+}$}

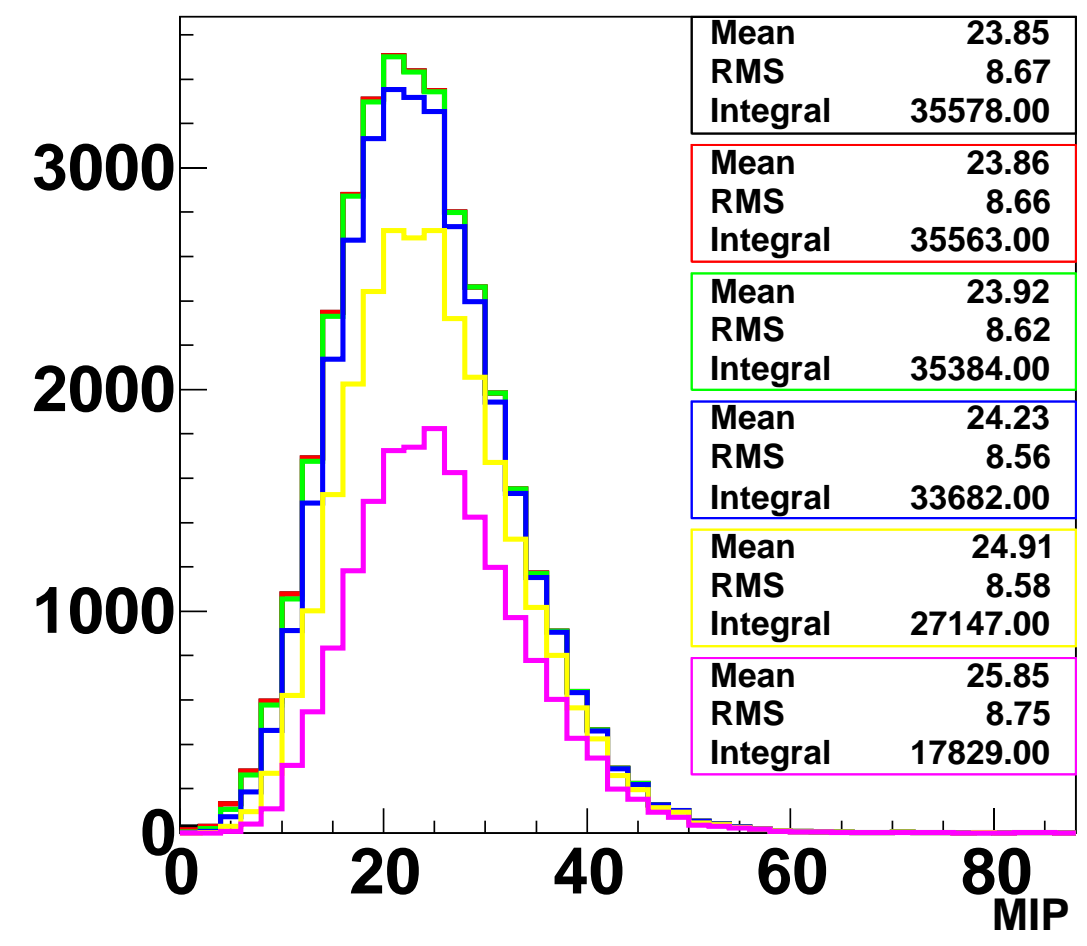

Figure 5.16: The response distributions arising from $0.4 \mathrm{GeV}$ electron interactions. In black, all electrons; red, electrons that pass a $2 / 3$ plane trigger; green, $3 / 4$; blue, 4/5; yellow, 5/6; pink, 6/7. 


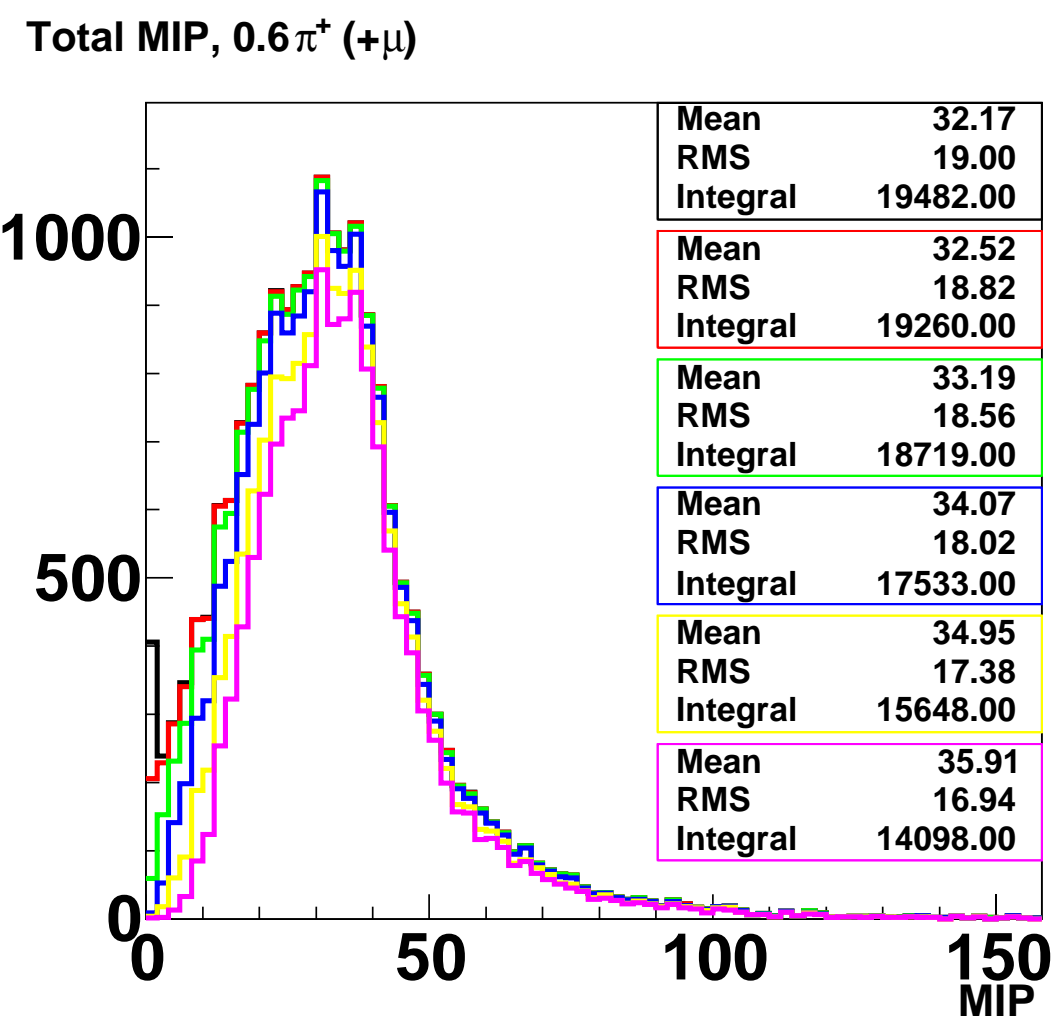

Figure 5.17: The response distributions arising from $0.6 \mathrm{GeV}$ pion interactions. In black, all pions; red, pions that pass a $2 / 3$ plane trigger; green, $3 / 4$; blue, $4 / 5$; yellow, $5 / 6$; pink, $6 / 7$. 


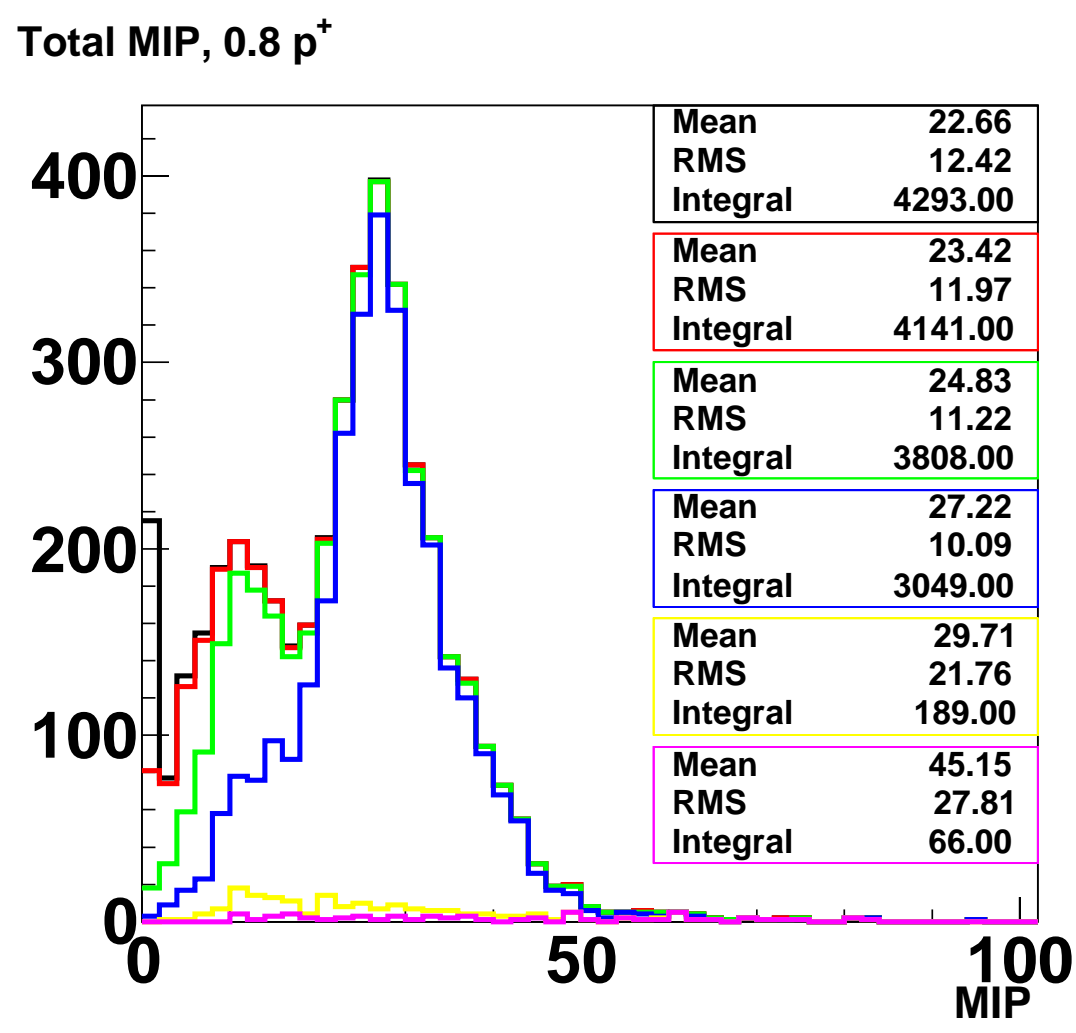

Figure 5.18: The response distributions arising from $0.8 \mathrm{GeV}$ proton interactions. In black, all protons; red protons that pass a $2 / 3$ plane trigger; green, $3 / 4$; blue, 4/5; yellow, $5 / 6$; pink, $6 / 7$. For low energy protons, the energy deposited is strongly correlated with the range in the detector. The lower peak in the distribution arises from protons that range out earlier than the events in the second peak. 

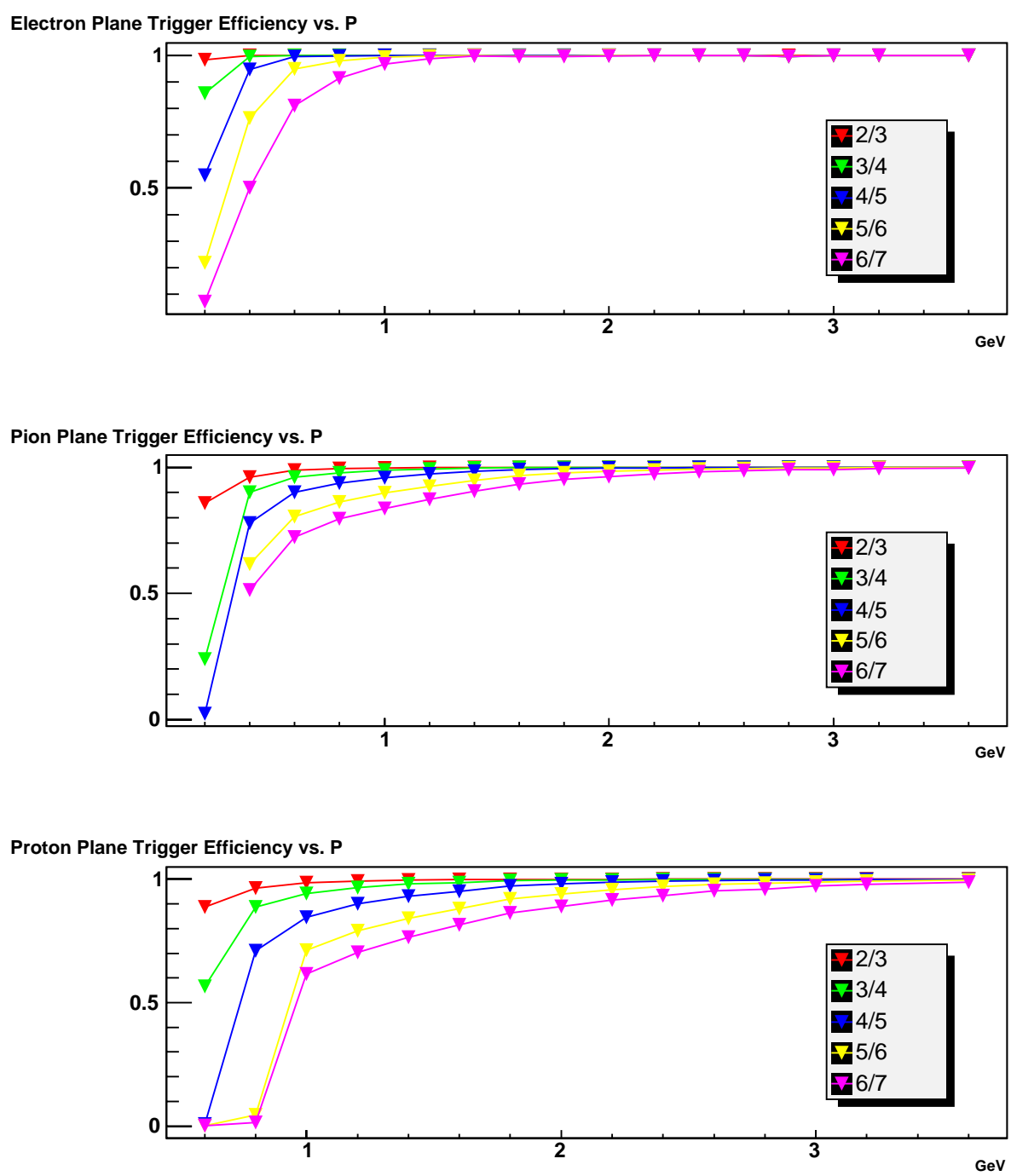

Figure 5.19: Top plot shows the fraction of electron events that pass a given plane trigger versus beam momentum, middle shows the same for pions, bottom shows the same from protons. 

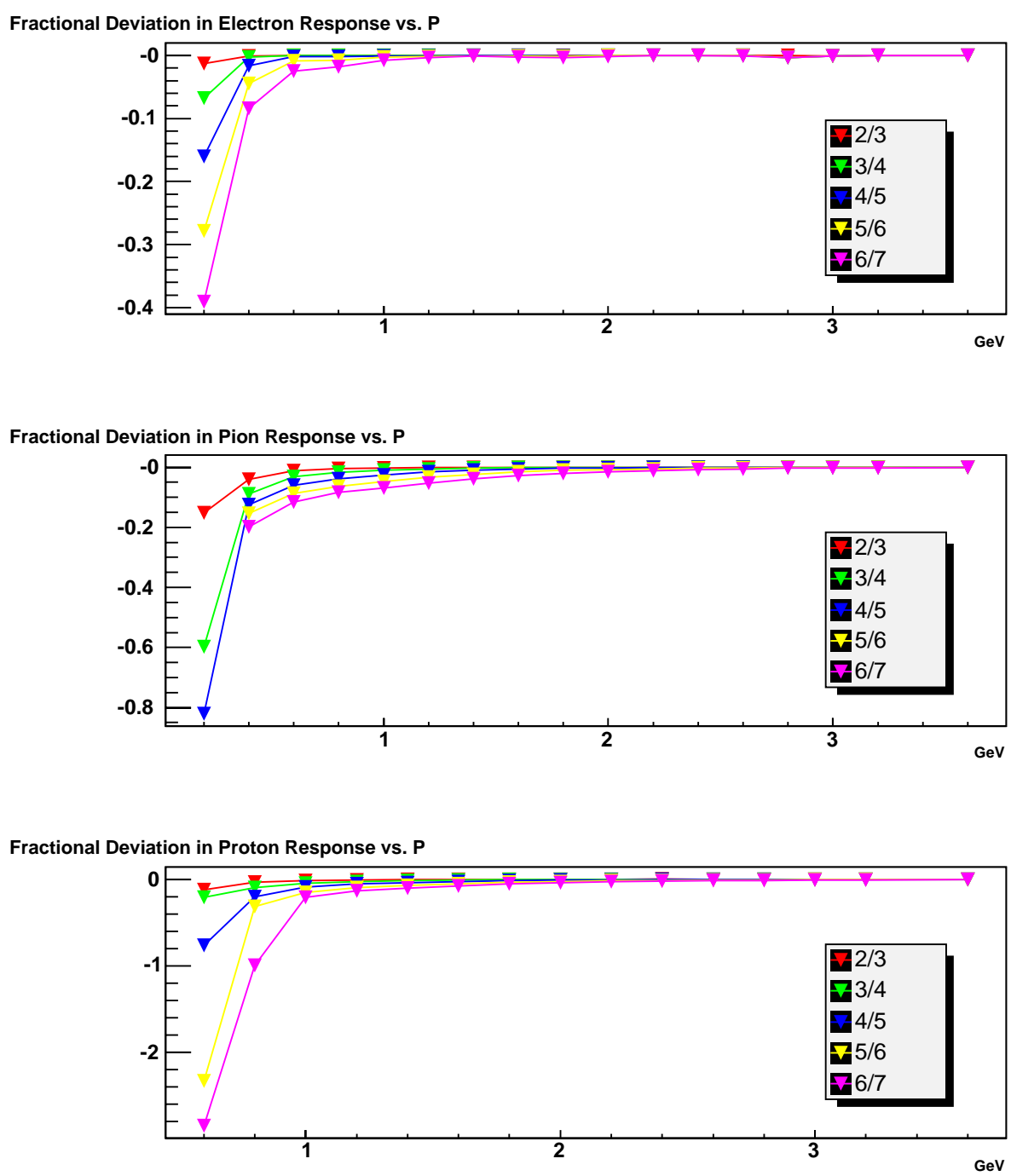

Figure 5.20: Top plot shows the relative shift in the electron mean response caused by applying a plane trigger versus beam momentum. Middle plot shows the same for pions, while bottom plot shows the effect for protons. 


\section{Chapter 6}

\section{Beam Line Systematics}

Several systematic effects of running the CalDet in the CERN beam lines could affect the measurement of the response of the detector to electromagnetic showers. In this chapter, five of these effects are considered. In particular, the effect of the external TOF enable, the effect of beam intensity, the differences between spills, the repeatability of the response measurement, and the energy loss of particles traversing the upstream beam elements are discussed.

\subsection{The External TOF Enable}

As discussed in section 3.3, the strict dynode triggering scheme originally envisioned for MINOS running prevented the collection of bias free beam data samples. Instead, at CalDet, the trigger algorithm was modified to include an external enable based on the coincidence of signals from the combination of either two or three TOF paddles. To understand any differences arising from the two trigger configurations, special runs were taken, and the detector response with the external enable was compared to the response with pure dynode triggering. Figure 6.1 compares the energy deposition distribution of $1.0 \mathrm{GeV}$ positrons interacting in the CalDet with 


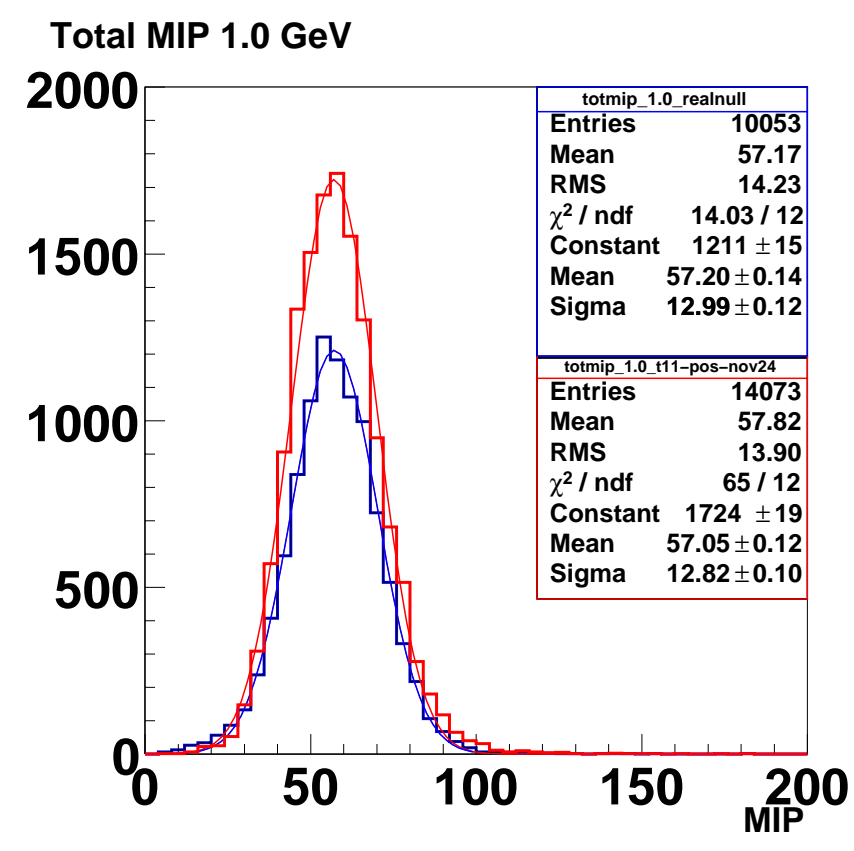

Figure 6.1: Comparison of observed energy deposited by $1.0 \mathrm{GeV}$ positrons with external enable on and off. The red histogram comes from a run with the external enable on, while the blue histogram comes from the run with the external enable off (Data shown use an older set of MIP calibration constants. Both histograms use the same calibration, but the scale is different from the results presented later in this document).

the external enable operating to that taken with the external enable not operating. The distributions agree within a few tenths of a percent, which in view of other systematic effects, was considered negligible. Using the external enable allowed for improved performance of the CalDet without introducing biasing effects.

\subsection{Beam Intensity}

The beam intensity was chosen to maximize data taking efficiency. In general, the beam intensity was adjusted to maintain about 250-600 TOF coincidences per spill. 


\section{Total MIP $+1.8 \mathrm{GeV}$}

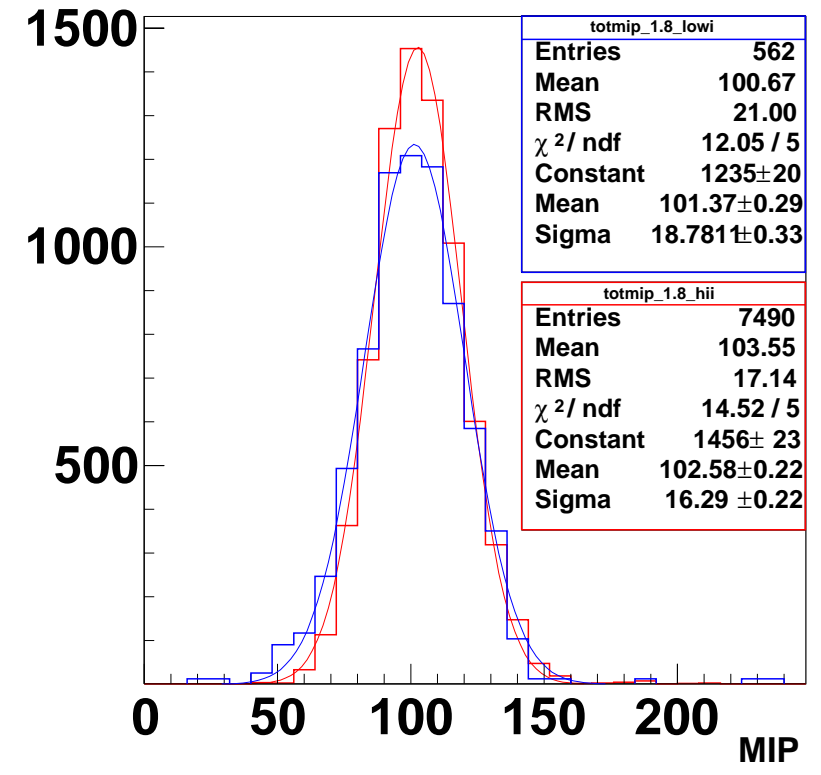

Figure 6.2: Observed energy deposition of $1.8 \mathrm{GeV}$ positrons. Red histogram comes from a run at the nominal intensity of about 250 particles/spill. Blue histogram comes from a run at a lower intensity of about 50 particles/spill. The means from Gaussian fits agree at the 1\% level (Data shown use an older set of MIP calibration constants. Both histograms use the same calibration, but the scale is different from the results presented later in this document).

More than 600 TOF coincidences per spill would cause the TDC buffers to fill before a readout cycle, and moreover increase the rate of overlapping events. Figure 6.2 compares the observed energy deposited in the CalDet for $1.8 \mathrm{GeV}$ positrons at two different intensities. The means of the distributions agree at the $1 \%$ level, indicating no intensity related effects. The tails of the distributions differ somewhat, which is most likely an effect of the beam defocussing done to achieve the lower intensity. 


\subsection{Differences between Spills}

The upstream portion of the T7 channel is shared with the T8 secondary beam line. In order to operate both beam lines at the same time, the current in the first momentum selection magnet (BHZ01) in the $\mathrm{T} 7$ beam line had to be ramped to zero during the T8 extraction, then ramped back up to the nominal value for the T7 spill. Depending on the PS super-cycle and the specifics of the distribution of spills to each of the secondary beam lines, the current in BHZ01 sometimes could not return to the nominal value in time for the $\mathrm{T} 7$ spill. Figure 6.3 shows the current in this magnet versus time at three different beam momenta. The two bands of currents correspond to different spills. Depending on the spill order within the super cycle, the current in BHZ01 during the first spill in the super cycle could be below the nominal value by a few percent. In the T11 beam line, no magnet ramping occurred, and this systematic difference in magnet currents was not observed.

Figure 6.4 shows the effect of the differences in spills on the electron response for two different runs in the T7 beam line. The top left plot shows the spill structure for a $0.6 \mathrm{GeV}$ run. The first spill corresponds to the spill before BHZ01 reached its nominal value. That spill contained about half the number of particles relative to the spill where BHZ01 attained its nominal setting. The top right plot shows the detector response to electrons for each of the spills. The response obtained when both spills are considered together is shown in black, the response to particles in the first spill is shown in red, while the response to particles in the second spill is shown in blue. The mean difference in response found by including events from all spills compared to using only events from the second spill is $1.6 \%$. However, the second row of plots in Figure 6.4 shows that the effect of the magnet ramping depends on the specifics of the run conditions. The lower left plot shows the spill structure of a specific $1.0 \mathrm{GeV}$ run. During this run, the T7 beam line was receiving 4 spills per supercycle. The bottom right plot shows the response of the detector 


\section{Current in BHZ01 vs. Time}

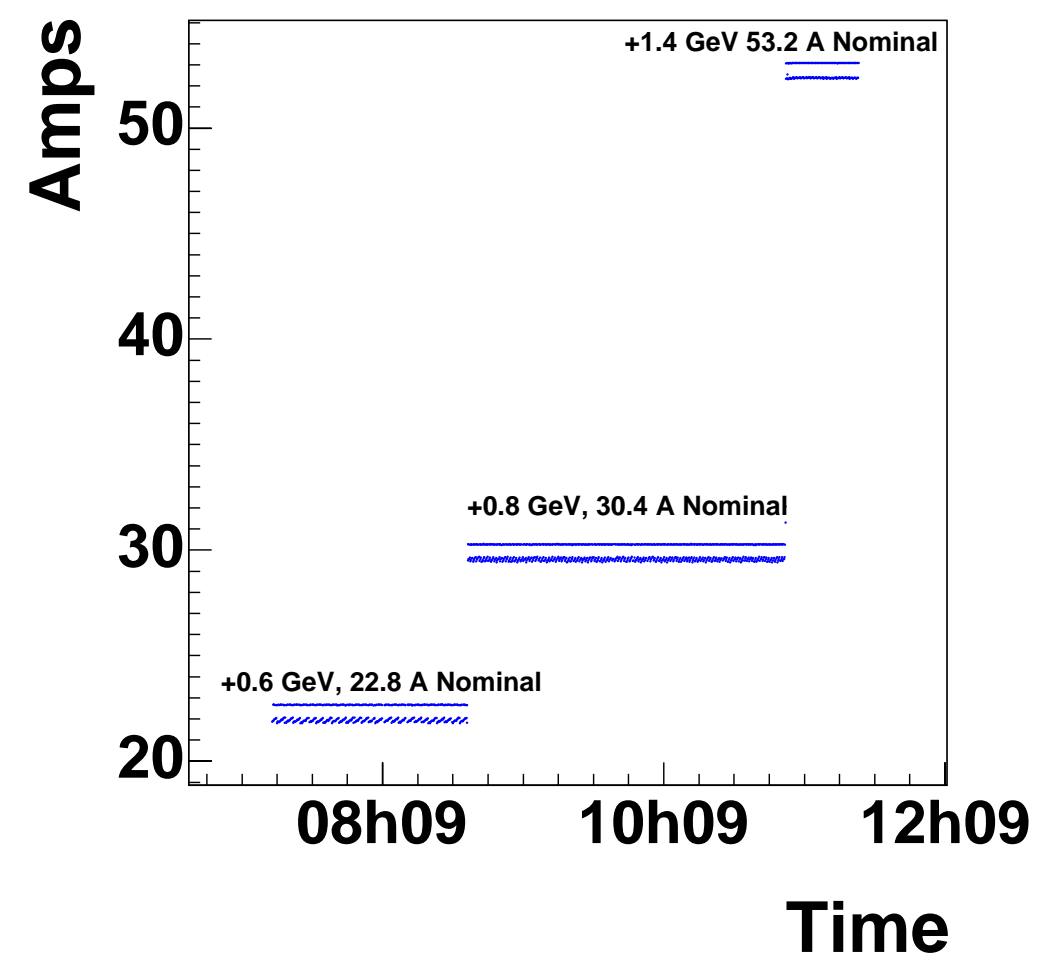

Figure 6.3: The current in the T7 upstream bending magnet (BHZ01) versus time. 
to particles in each spill. For this run, the difference in the mean response among spills is negligible.

Figure 6.5 shows the differences between the mean response of electrons from the spill with the most events and the mean response averaged over all spills for 56 different runs at varying beam momenta. The left plot shows the relative difference between the response averaged over all spills relative to the response obtained using only the spill with the most particles versus beam momentum. The right plot shows the projection of the relative differences. On average, the differences in the response among spills is small, on the order of $0.3 \%$, but can be larger than $1 \%$.

In principle, one could use the value of the magnet current to correct the events in the spill with the below-nominal current settings. Unfortunately the magnet monitoring software was not introduced until the 2003 beam runs; no magnet current data is available for the 2002 runs. Even without the magnet current data, an analysis could be done using only the events from the spills with the maximum number of particles at the expense of lower statistics. In order to facilitate a future analysis incorporating the effects of the spill to spill differences, the difference in response between the maximum spill and the average over all spills is calculated for each run, then the values of the error arising from spill differences is stored in the database table "CalDetSpillErrors". See Appendix F for further details.

\subsection{Response Measurement Repeatability}

In both the T11 and T7 beam lines, it was observed that repeated measurement of the detector response at a given momentum showed fluctuations larger than that expected from statistical errors. Figure 6.6 shows this effect for several $1 \mathrm{GeV}$ runs taken in 2003 in the T7 beam line. Some portion of this fluctuation may derive from uncorrected detector drift. For instance, the scintillator light output is known to decrease by about $0.4 \%$ per ${ }^{\circ} \mathrm{C}$ increase in temperature and is an effect not 

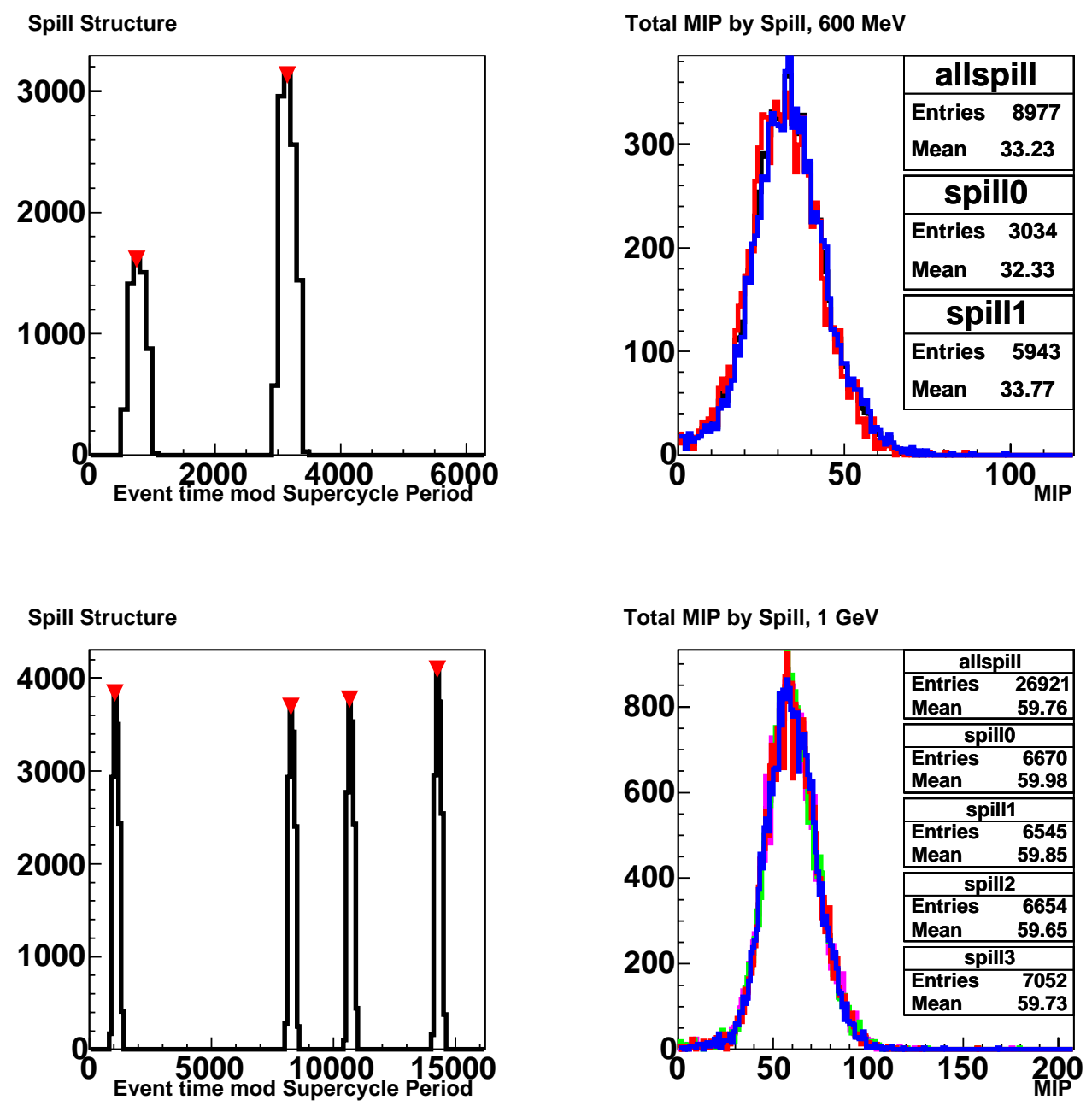

Figure 6.4: Spill structure of two different runs in T7. The top left plot shows the spill structure during run 70561, a $600 \mathrm{MeV}$ run. Two spills were being delivered to the $\mathrm{T} 7$ beam line during this run. The top right plot shows the detector response to positrons from all spills together in black, the first spill in red, and the second spill in blue. The difference in means between the histogram with all spills, and the histogram with only the second spill is about $1 \%$. The lower left plot shows the spill structure from run 70771 , a $1 \mathrm{GeV}$ run. During this run, 4 spills were directed to the $\mathrm{T} 7$ beam line. The lower right plot shows the response distributions for electrons in each of the spills. The difference in means for this run is negligible. 

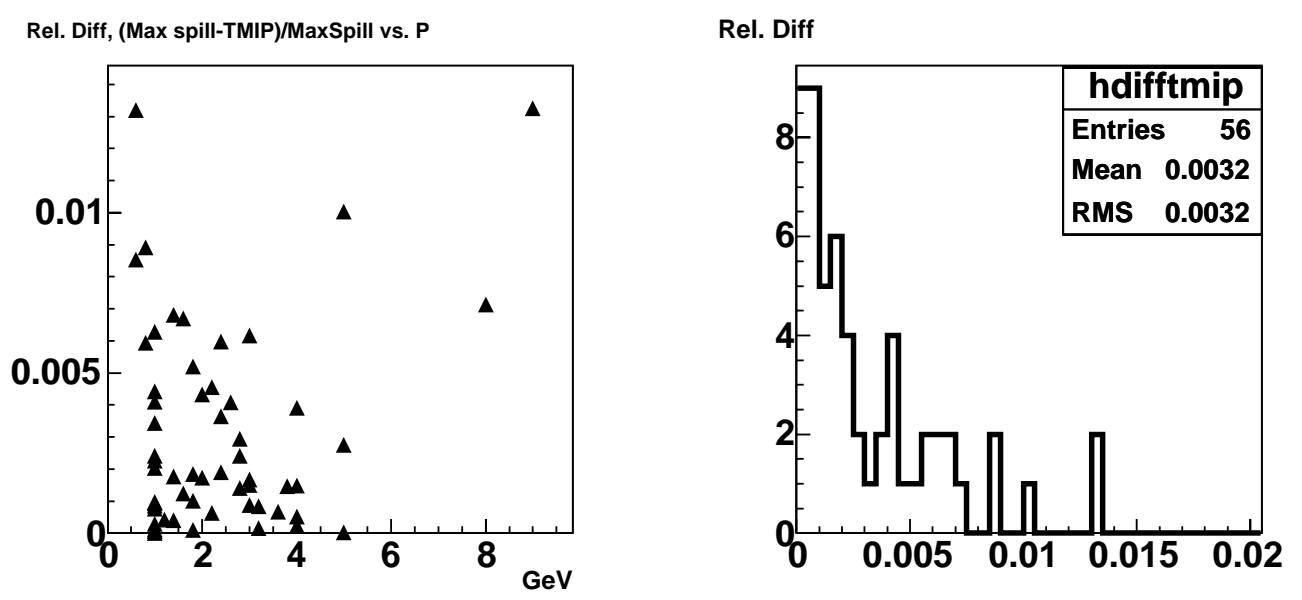

Figure 6.5: Spill differences for many runs. The left plot shows the relative difference between the response averaged over all spills relative to the response from the spill with the most events versus beam momentum. The right plot shows the projection of the relative differences. On average, the differences in the response among spills is about $0.3 \%$, but can be larger than $1 \%$ for some runs.
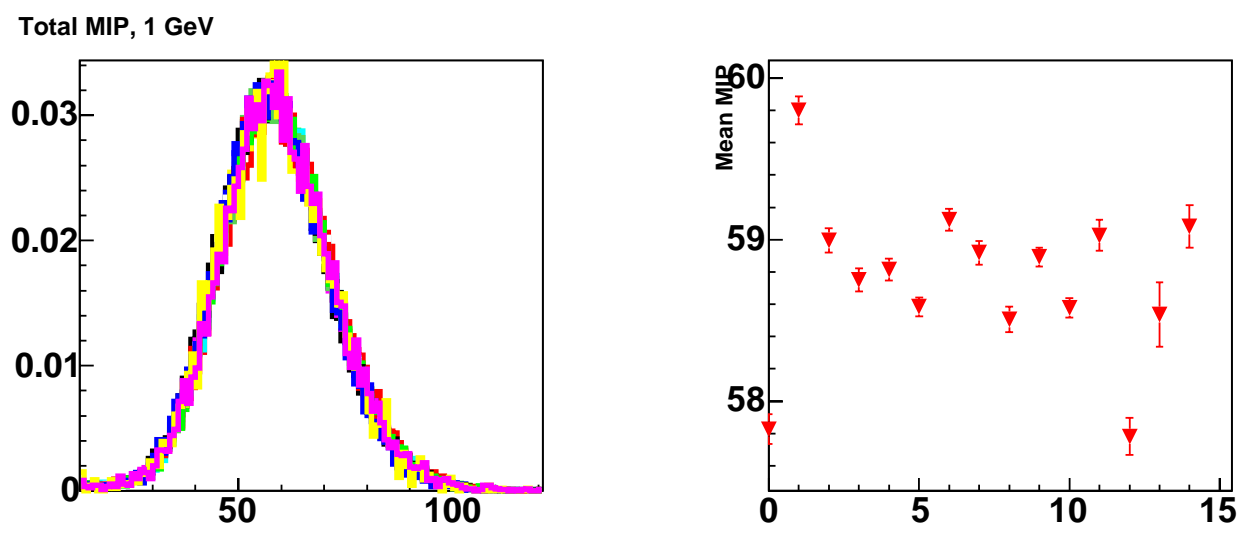

Figure 6.6: To the left, the response distributions of positrons from many $1 \mathrm{GeV}$, T7 2003 runs. To the right, the mean response of each of the runs. The dependence of scintillator light output on temperature is not accounted for in this plot. 


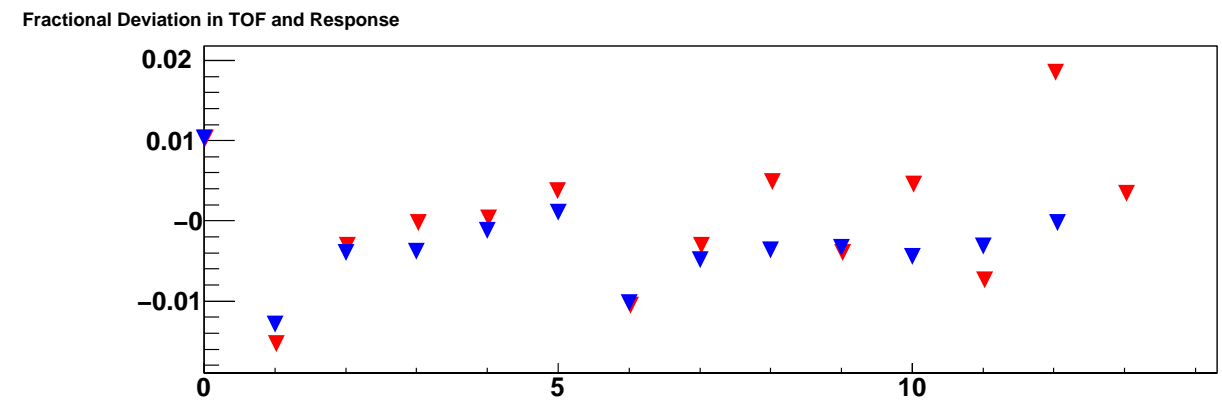

Figure 6.7: Fractional Deviation of detector response versus run is shown in red. Fractional shift in momentum as measured by the difference in time of flight between electrons and protons is shown in blue.

accounted for in Figure 6.6 [38]. However, Figure 6.7 shows the fractional change in momentum as calculated from the separation of the electron and proton peaks in the time of flight distribution. Superimposed on this graph is the fractional deviation in the detector response during the same run. The deviation in the detector response follows the deviation in the time of flight measurement, suggesting that a major component in the response measurement fluctuation is intrinsic in the setting of the beam momentum.

Figure 6.8 shows the deviation of response for several energies. The top plot shows the response over beam momentum from events in the maximum spill of each run versus the beam momentum. The lower left plot shows the RMS over the mean of each of the runs taken at a given momentum versus that momentum. The lower right plot shows the histogram of the fractional deviation for all momenta together. The mean of $0.8 \%$ is included as an additional error in the response, added in quadrature with the statistical error. Figure 6.9 shows the same quantities for the T11 beam line. The additional error to be included in the T11 response measurement was found to be $0.5 \%$. 

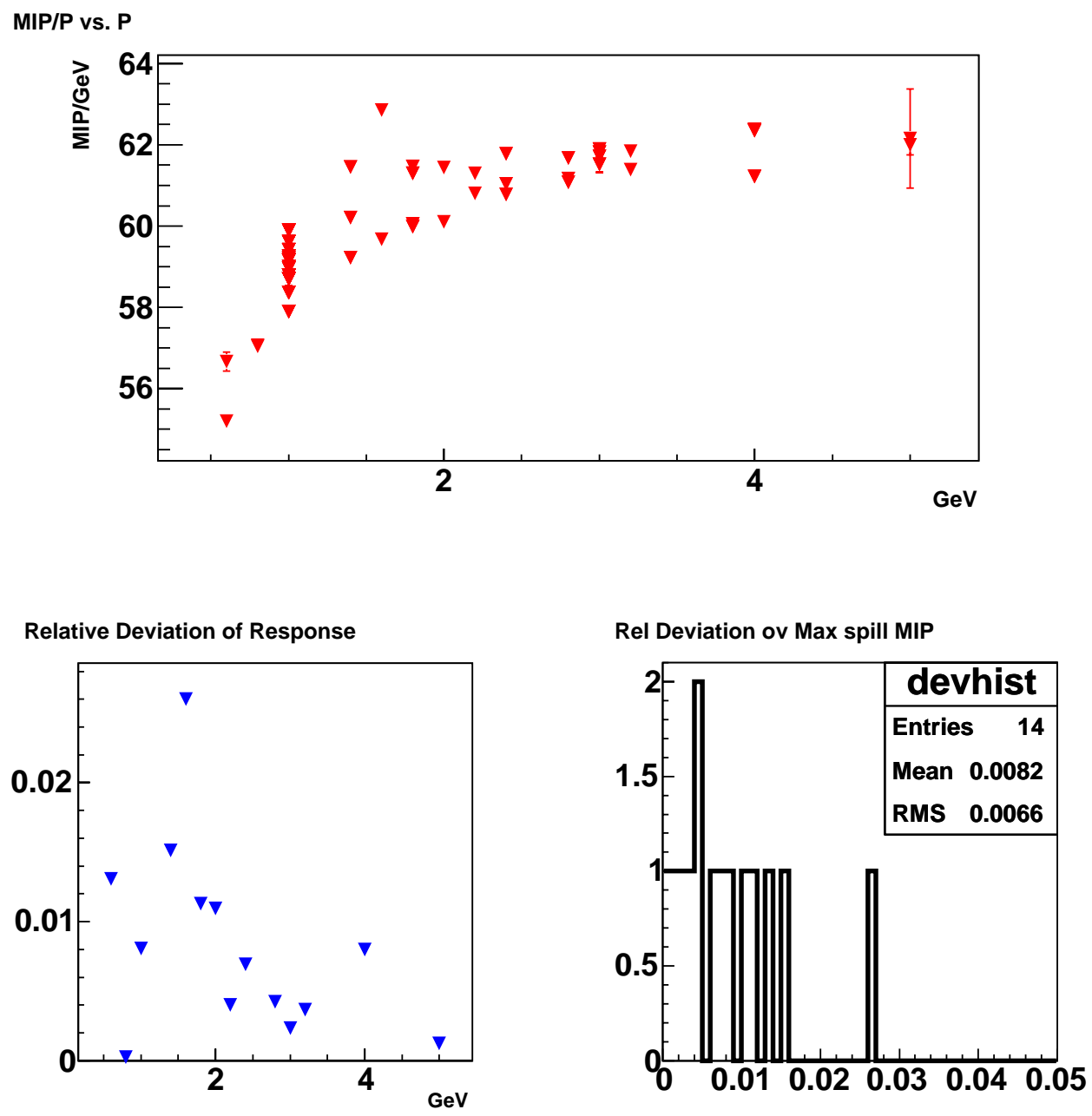

Figure 6.8: The deviation in detector response at many energies from runs in the T7 beam line. The top plot shows the response divided by momentum versus momentum for many repeated runs. The bottom left plot shows the fractional deviation in the response versus beam momentum. The bottom right shows the deviation of all momenta together. The average variation in the response measurement between runs is $0.8 \%$. 

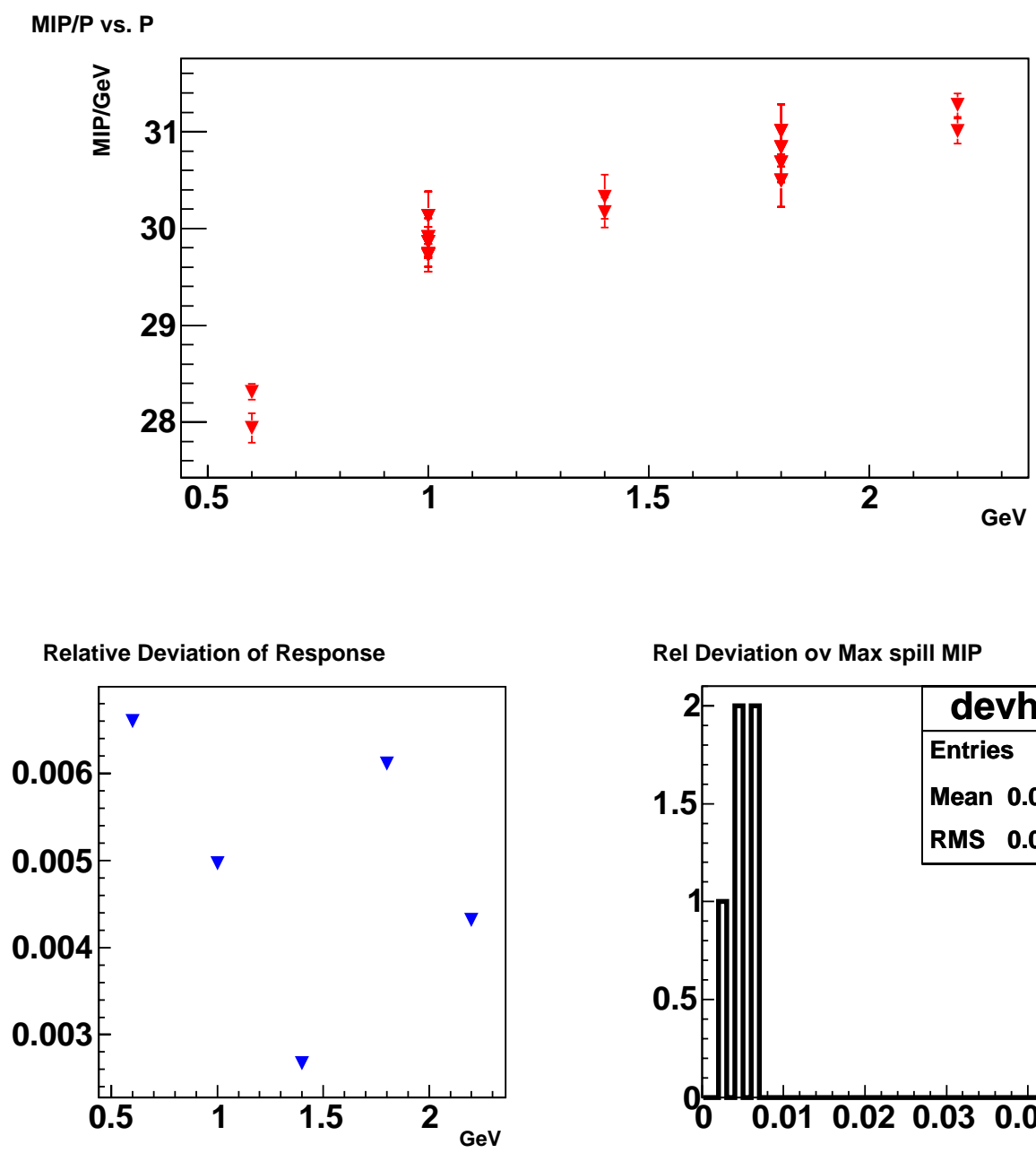

Rel Deviation ov Max spill MIP

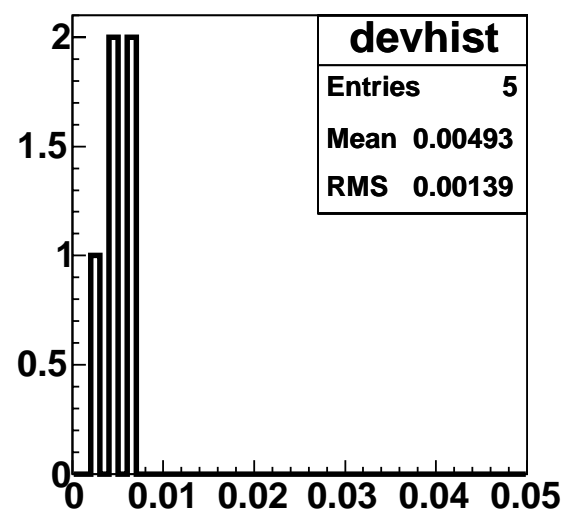

Figure 6.9: The deviation in detector response at many energies from runs in the T11 beam line. The top plot shows the response divided by momentum versus momentum for many repeated runs. These runs were taken with single ended readout, making the response approximately half the response shown previously. The bottom left plot shows the fractional deviation in the response versus beam momentum. The bottom right shows the deviation of all momenta together. The average variation in the response measurement from run to run is $0.5 \%$. 


\subsection{Simulating Energy Losses in Upstream Material}

On their way from the target to the detector, the beam particles lose some amount of their initial energy due to interactions in the particle identification detectors and other upstream beam elements. In order to study the extent of this energy loss, the material upstream of the detector in each of the beam lines has been modeled. The beam line simulation consists of three steps. First, particles are tracked through the magnetic channels of the beam lines using Decay Turtle [41]. The momentum spectrum and the positions of the particles are recorded at the position of the last vacuum window, then input into a GEANT3 simulation describing the material upstream of the CalDet [42]. At the first CalDet plane, the position and momentum of a particle and any daughter particles are recorded. This collection of particles is then used as input into the standard MINOS Monte Carlo to simulate the CalDet response. By comparing the simulated response of the CalDet to particles that travel through the upstream material to the simulated response of particles that do not, one can extract the effect of any energy loss upstream of the detector. The description of the beam line simulation and its output are discussed further here. Deriving and applying the correction for upstream energy loss is described in Chapter 8.

The amount of material upstream of the detector was different for each of the beam lines, and moreover, was different from year to year. Thus, four standard geometries were defined and used to study the effect of upstream energy loss. Figure 6.10 shows a schematic of the geometry used to simulate energy losses in the T11 beam line for the 2002 run, while Figure 6.11 shows the geometry used to simulate energy losses in the T7 beam line in 2002. Figure 6.12 shows the geometry used for T7 2003 runs, and Figure 6.13 shows the geometry of the T11 2003 runs.

In each beam lines, the upstream end of the upstream Cerenkov was was positioned inside the last beam quadrupole magnet. In the simulation, the resulting field was incorporated as an ideal quadrupole field limited to the volume of the Čerenkov that 
T11 2002
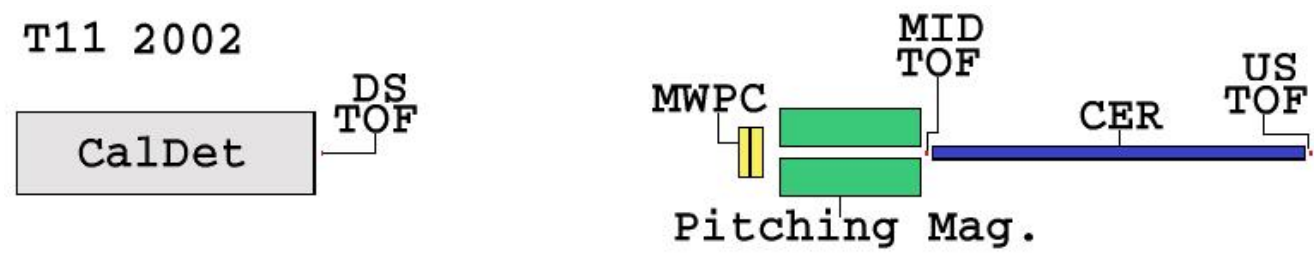

Figure 6.10: The upstream beam elements of the T11, 2002 energy loss simulation. US, MID, and DS refer to the upstream, middle, and downstream TOF paddles. MWPC refers to the (unused) multi-wire proportional chamber available in the beam line.
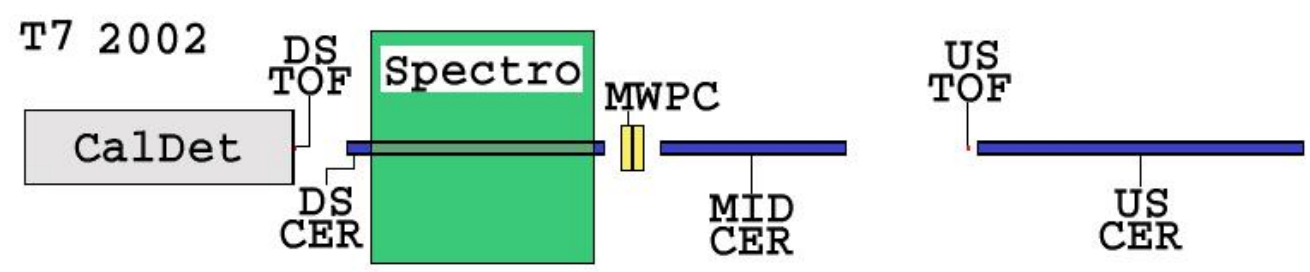

Figure 6.11: The upstream beam elements of the T7, 2002 energy loss simulation. Spectro refers to a large, but unenergized spectrometer magnet located in the T7 beam line.

overlapped with the magnet. Table 6.1 lists the dimensions and other parameters of each of the elements included in the geometries.

Figures 6.14 and 6.15 show 15 simulated $1 \mathrm{GeV}$ positron events passing through the beam elements of the T11, 2002 and T7, 2002 beam line geometries. The red, solid paths represent electrons or positrons, while the blue, dotted paths represent photons. Most electrons and positrons passing through the upstream material experience bremsstrahlung, a process in which the initial particle, decelerated by the material it traverses, emits a photon. This photon, and any any additional particles that could be produced along the way, may or may not eventually deposit energy in the CalDet along with the parent particle. It is the acceptance of and the detector response to these extra particles that makes the detailed simulation of 


\begin{tabular}{|c|c|c|c|c|c|}
\hline Element & Shape & Material & Dimension & Length & Comment \\
\hline US TOF & BOX & Scint. & $5 \times 12 \mathrm{~cm}^{2}$ & $1.5 \mathrm{~cm}$ & \\
\hline MID TOF & BOX & Scint. & $5 \times 12 \mathrm{~cm}^{2}$ & $1.5 \mathrm{~cm}$ & T11 2002 \\
\hline DS TOF & BOX & Scint. & $5 \times 12 \mathrm{~cm}^{2}$ & $1.5 \mathrm{~cm}$ & \\
\hline US TOF 2 & BOX & Scint. & $5 \times 5 \mathrm{~cm}^{2}$ & $1.5 \mathrm{~cm}$ & 2003 only \\
\hline DS TOF 2 & BOX & Scint. & $5 \times 5 \mathrm{~cm}^{2}$ & $1.5 \mathrm{~cm}$ & 2003 only \\
\hline CER Window & TUBE & Mylar & $r=7.5 \mathrm{~cm}$ & $0.06 \mathrm{~cm}$ & \\
\hline T11 US CER & TUBE & $\mathrm{CO}_{2}$ & $r=7.5 \mathrm{~cm}$ & $5.0 \mathrm{~m}$ & $\begin{array}{l}\text { Gas vol. in } 1 \mathrm{~cm} \\
\text { thick Al Shell } \\
\text { Pres. varies } \\
\text { Quad. B field }\end{array}$ \\
\hline T7 US CER & TUBE & $\mathrm{CO}_{2}$ & $r=7.5 \mathrm{~cm}$ & $4.4 \mathrm{~m}$ & $\begin{array}{l}\text { Gas vol. in } 1 \mathrm{~cm} \\
\text { thick Al Shell } \\
\text { Pres. varies } \\
\text { Quad. B field }\end{array}$ \\
\hline MID CER & TUBE & $\mathrm{CO}_{2}$ & $r=7.5 \mathrm{~cm}$ & $2.5 \mathrm{~m}$ & $\begin{array}{l}\text { Gas vol. in } 1 \mathrm{~cm} \\
\text { thick Al Shell } \\
\text { Pres. same } \\
\text { as DS CER }\end{array}$ \\
\hline DS CER & TUBE & $\mathrm{CO}_{2}$ & $r=7.5 \mathrm{~cm}$ & $3.47 \mathrm{~m}$ & $\begin{array}{l}\text { Gas vol. in } 1 \mathrm{~cm} \\
\text { thick Al Shell } \\
\text { Pres. varies }\end{array}$ \\
\hline MWPC & BOX & $\mathrm{Al}$ & $60 \times 60 \mathrm{~cm}^{2}$ & $0.003 \mathrm{~cm}$ & $\begin{array}{l}3 \text { sheets } \\
1 \mathrm{~cm} \text { apart } \\
2002\end{array}$ \\
\hline Pitching Mag. & $\mathrm{BOX}$ & $\mathrm{Fe}$ & $0.45 \times 3.5 \mathrm{~m}^{2}$ & $170 \mathrm{~cm}$ & No B field \\
\hline Spectro & TUBE & $\mathrm{Fe}$ & $\begin{array}{l}r_{1}=1.57 \mathrm{~m} \\
r_{2}=10 \mathrm{~cm}\end{array}$ & $3 \mathrm{~m}$ & No B field \\
\hline
\end{tabular}

Table 6.1: Elements of the beam line simulation 
T7 2003
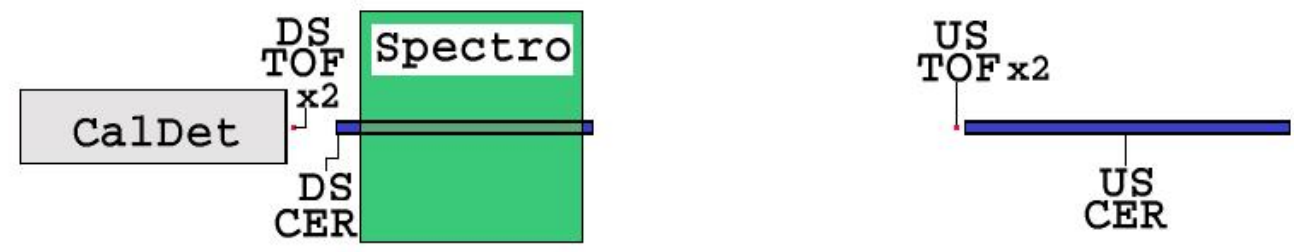

Figure 6.12: The upstream beam elements of the T7, 2003 energy loss simulation.

T11 2003

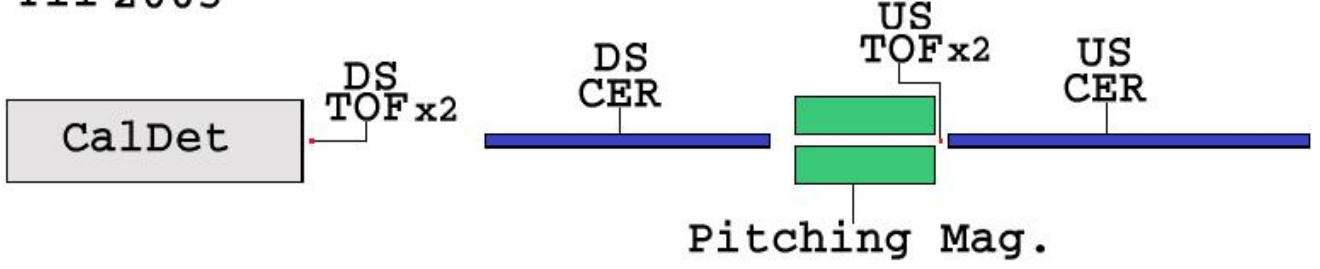

Figure 6.13: The upstream beam elements of the T11, 2003 energy loss simulation.

the beam line and detector essential to the study of the effect of energy losses by electrons traveling through the upstream material.

In order to more closely simulate the data taking conditions at the CalDet, only events that pass a trigger requirement are input into the detector Monte Carlo. To pass the trigger requirement, a particle must deposit $400 \mathrm{keV}$ of energy in the TOF paddles corresponding to the paddles used to form the coincidence for the external enable. Table 6.2 lists the trigger conditions for each configuration. Figures 6.16 and 6.17 show the input momentum derived from Decay Turtle, the vertex position of daughter particles produced in the beam line simulation, and the beam spot at the position of CalDet plane 0 for both triggered and untriggered events for $1 \mathrm{GeV}$ positrons from both beam lines. Appendix $\mathrm{C}$ shows the same quantities at other momenta.

Figures 6.18 and 6.19 show characteristics of events output by the beam line 


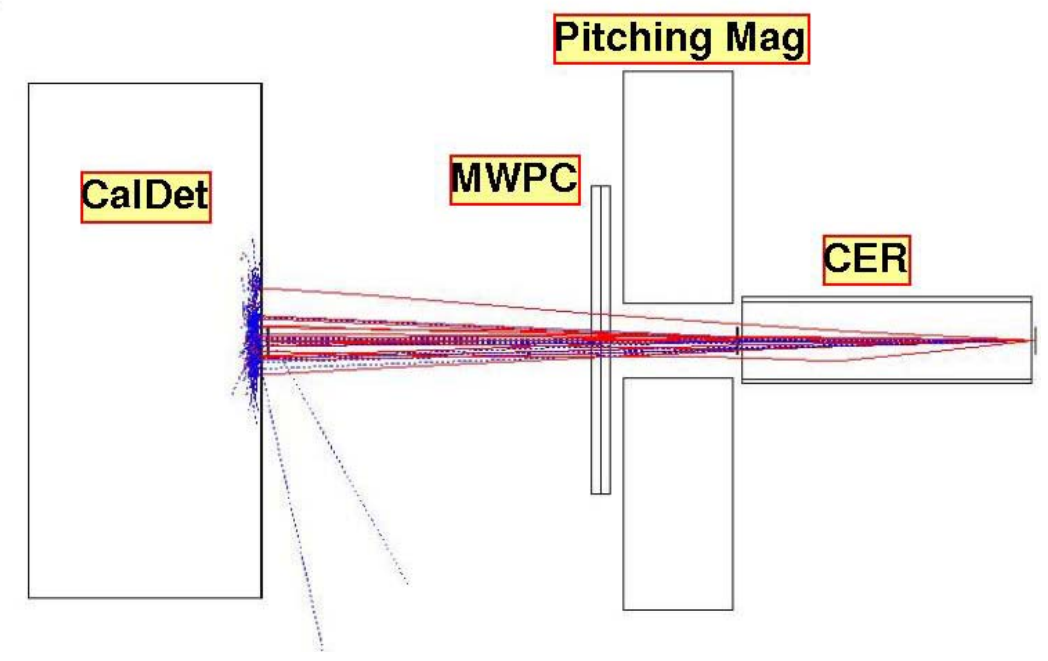

Figure 6.14: 15 simulated $1 \mathrm{GeV}$ positron events passing through the T11 beam elements, The horizontal dimension is scaled by a factor of 2 to show detail.

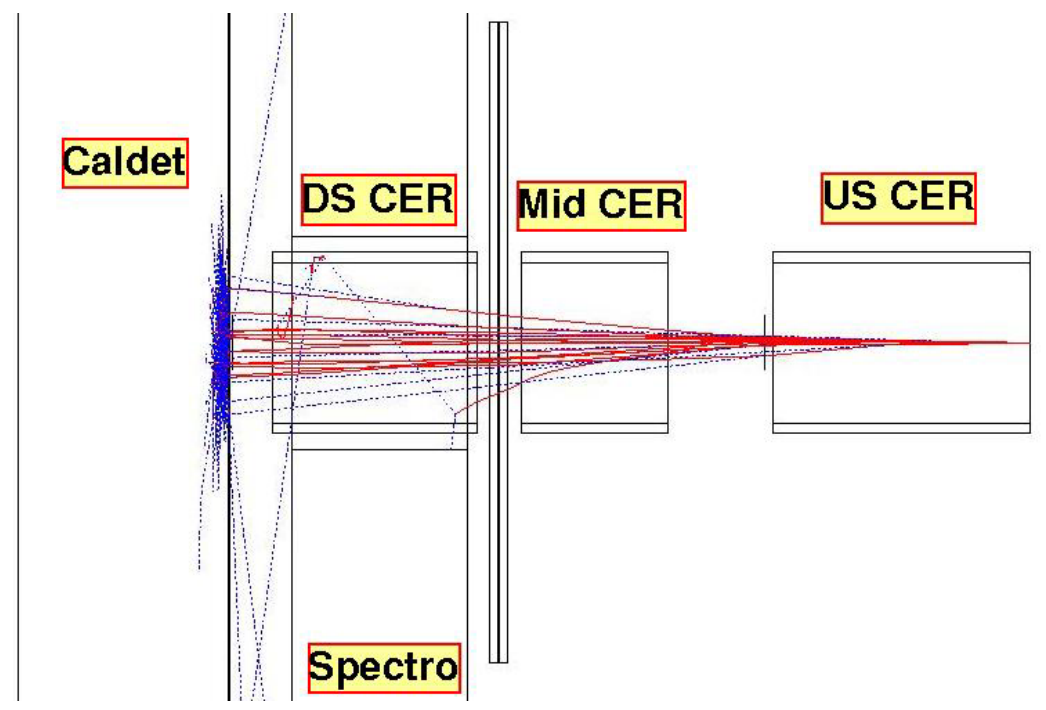

Figure 6.15: 15 simulated $1 \mathrm{GeV}$ positron events passing through the $\mathrm{T} 7$ beam elements. The horizontal dimension is scaled by a factor of 2 to show detail. 


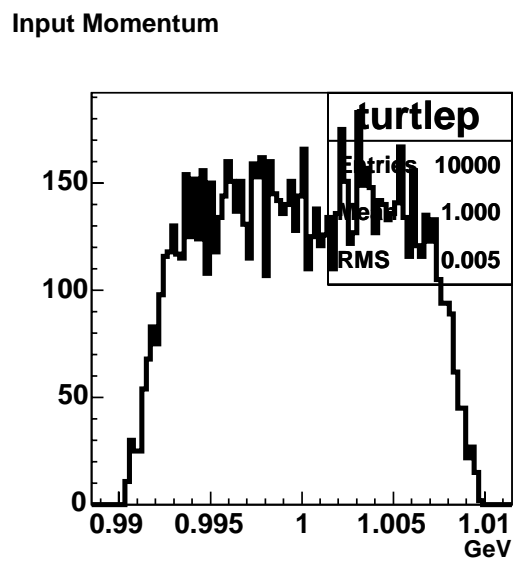

\section{Beam Spot, Pre-Trigger}

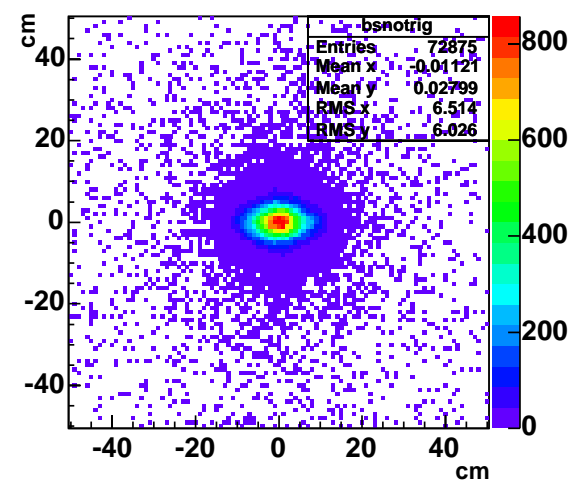

Vertex Position

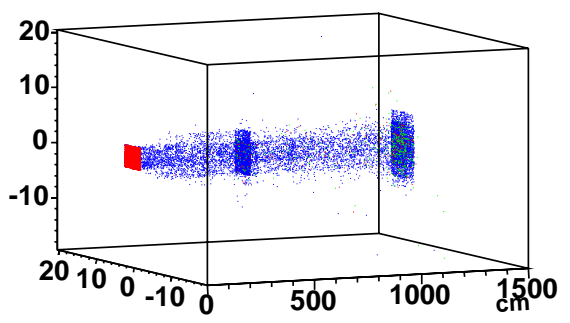

Beam Spot, Triggered Events

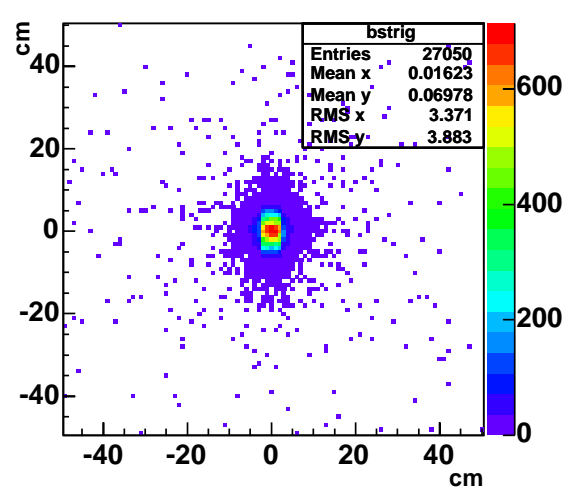

Figure 6.16: Top left plot shows the momentum distribution of particles produced by Decay Turtle, top right plot shows the vertex positions of the particles produced by a $1 \mathrm{GeV}$ positron in the T11, 2002 geometry for events that pass the trigger condition. Red indicates positrons, blue indicates photons, green indicates electrons. Bottom left shows the $\mathrm{x}$ and $\mathrm{y}$ position of all particles when incident on the first CalDet plane. Bottom right shows the same, but only for events which pass the trigger condition. 
Input Momentum

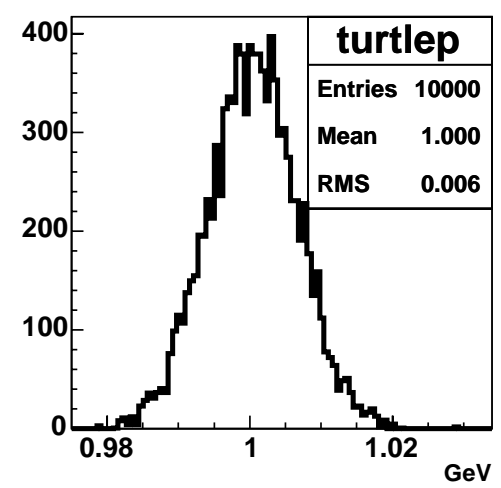

Beam Spot, Pre-Trigger

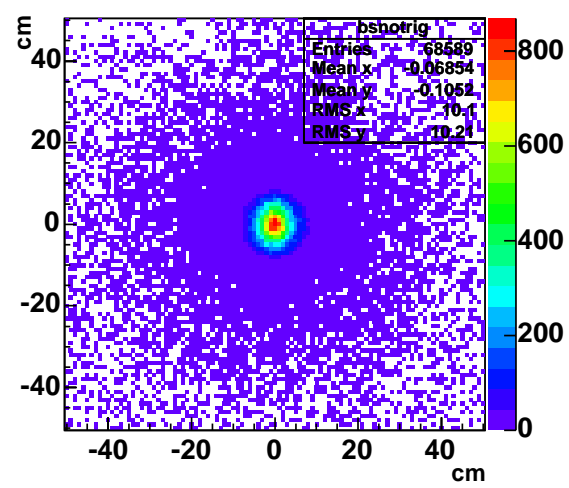

Vertex Position

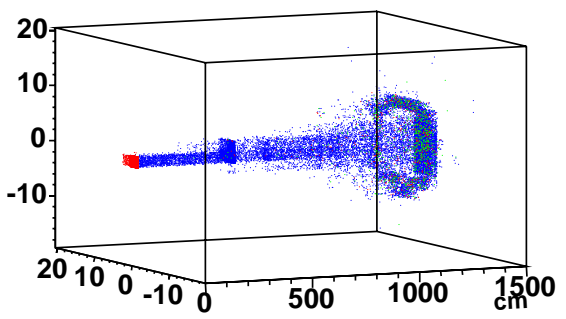

Beam Spot, Triggered Events

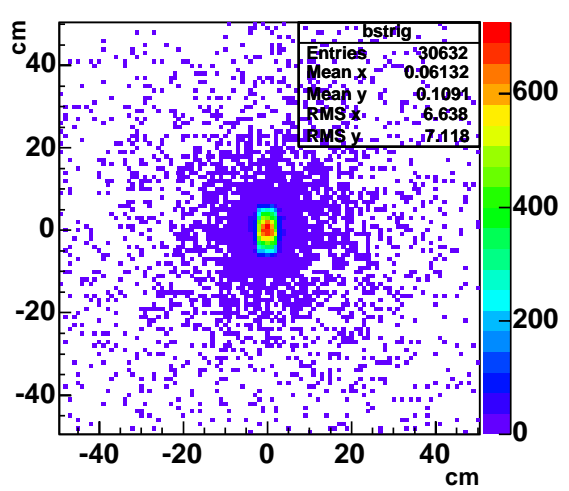

Figure 6.17: Top left plot shows the momentum distribution of particles produced by Decay Turtle, top right plot shows the vertex positions of the particles produced by a $1 \mathrm{GeV}$ positron in the T7, 2002 geometry for events that pass the trigger condition. Red indicates positrons, blue indicates photons, green indicates electrons. Bottom left shows the $\mathrm{x}$ and $\mathrm{y}$ position of all particles when incident on the first CalDet plane. Bottom right shows the same, but only for events which pass the trigger condition. 


\begin{tabular}{|l|l|l|}
\hline Configuration & Energy & Trigger Condition \\
\hline T11 2002 & $<1 \mathrm{GeV}$ & MID and DS TOF \\
T11 2002 & $\geq 1 \mathrm{GeV}$ & US and DS TOF \\
T7 2002 & All & US and DS TOF \\
T7 2003 & All & US,DS and Ext. DS TOF \\
T11 2003 & All & US,DS and Ext. DS TOF \\
\hline
\end{tabular}

Table 6.2: External enable trigger conditions used in data taking and in the beam line simulation.

simulation. Each plot shows the summed energy of particles reaching CalDet plane 0 , the energy of the particle with the maximum energy that reaches plane 0 , and the number of particles reaching plane 0 , for $1 \mathrm{GeV}$ positron events which pass the trigger requirement for each beam line. Appendix $\mathrm{C}$ shows the same distributions for other beam momenta. In general, electrons carry most of the energy of the initial particle; photons typically carry less than $10 \%$ of the initial energy. Most events are characterized by 2-3 particles interacting in the detector, usually one electron or positron and 1-2 photons. The absolute value of energy lost is roughly $20-30 \mathrm{MeV}$ for all momenta; however, the fractional difference between the nominal incident particle energy and the summed energy of the particles that reach the CalDet is greater for lower energies.

In 2003, different amounts of material were purposely introduced upstream of the detector in order to compare the results of the beam material simulation with the data. Such runs are described in Table 6.3. Figure 6.20 shows the response of the detector versus the simulated response of particles propagated through the beam line simulation. Adding extra material upstream of the detector changed the measured response by $8 \%$. The beam line simulation reproduces the shift, and the simulated response agrees with the data to within $1 \%$.

An independent indicator of the success of the beam line simulation is its ability to reproduce the low energy tail sometimes observed in the total MIP dis- 


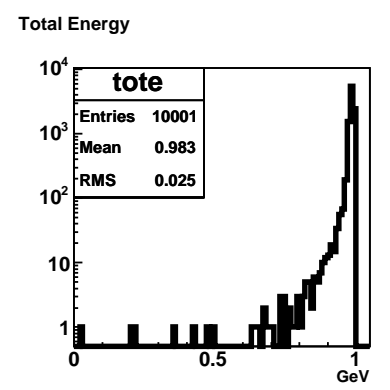

Max. Energy

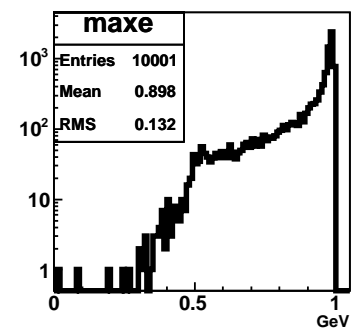

Number of Particles

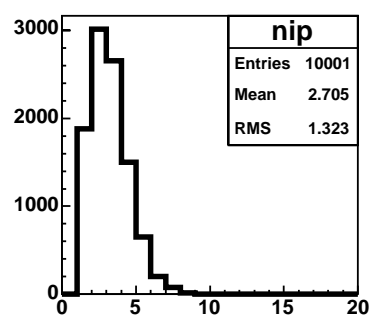

Total Energy, Electrons

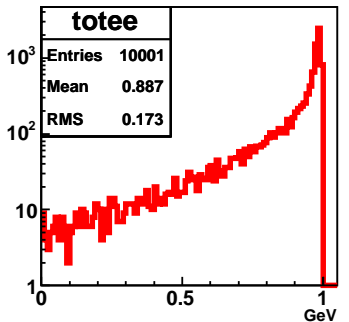

Max. Energy, Electrons

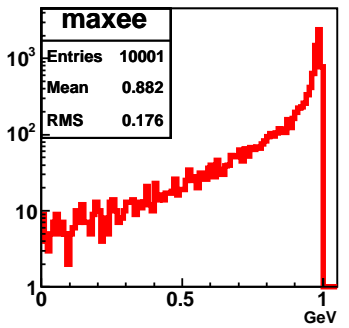

Number of Electrons

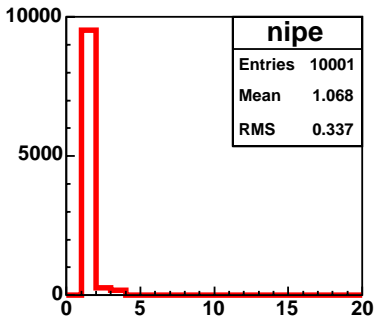

Total Energy, Gammas

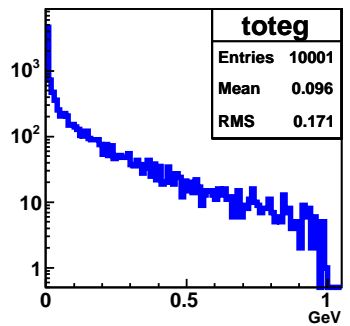

Max. Energy, Gammas

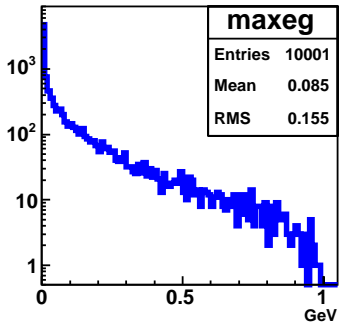

Number of Gammas

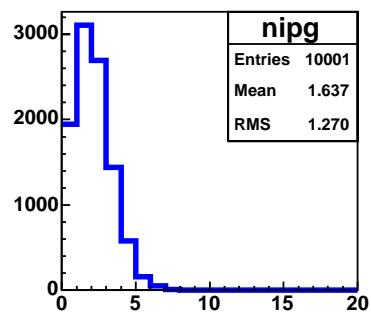

Figure 6.18: Characteristics of events produced in the T11, 2002 beam line simulation of $1 \mathrm{GeV}$ positrons that pass the trigger condition. Top row, the total energy of all the particles in black, the total energy of all electrons in red, and the total energy of all photons in blue. Middle row, the energy of the particle with the maximum energy, all particles in black, positrons in red, photons in blue. Bottom row, number of particles making up an event, all particles in black, positrons in red, photons in blue. 


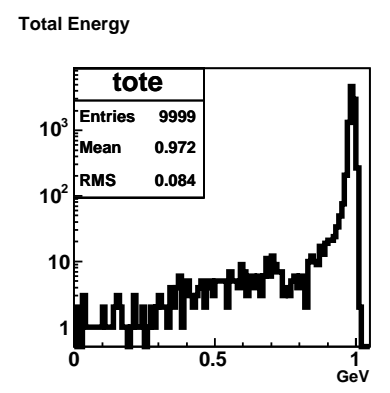

Max. Energy

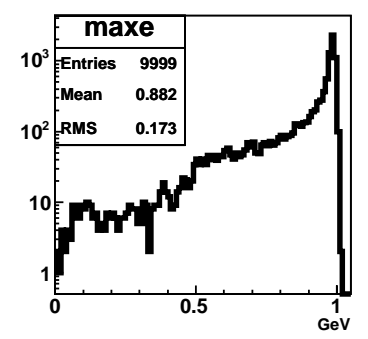

Number of Particles

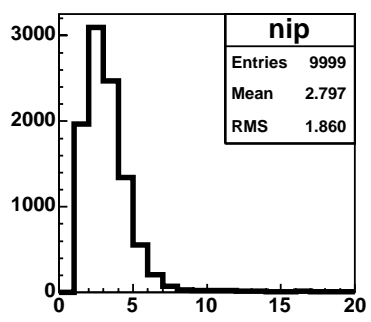

Total Energy, Electrons

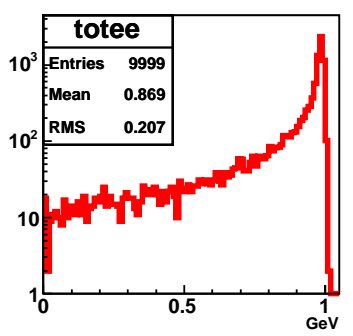

Max. Energy, Electrons

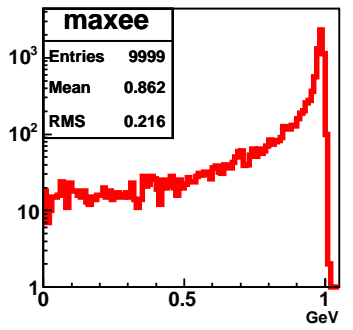

Number of Electrons

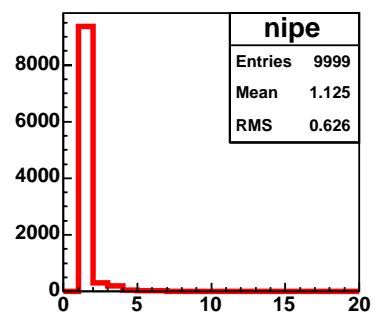

Total Energy, Gammas

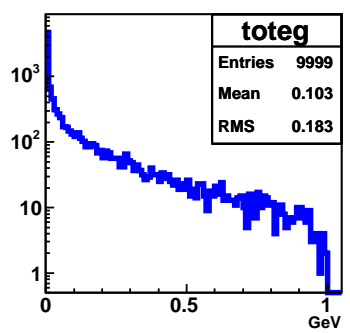

Max. Energy, Gammas

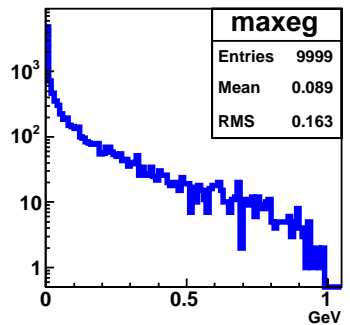

Number of Gammas

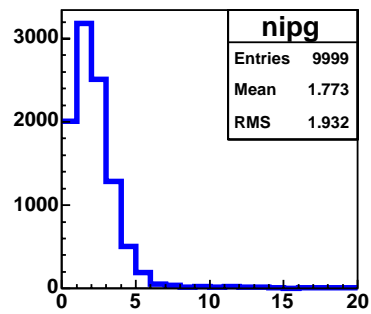

Figure 6.19: Characteristics of events produced in the T7, 2002 beam line simulation of $1 \mathrm{GeV}$ positrons that pass the trigger condition. Top row, the total energy of all the particles in an event in black, the total energy of all electrons in an event in red, and the total energy of all photons in blue. Middle row, the energy of the particle with the maximum energy, all particles in black, positrons in red, photons in blue. Bottom row, number of particles making up an event, all particles in black, positrons in red, photons in blue. 


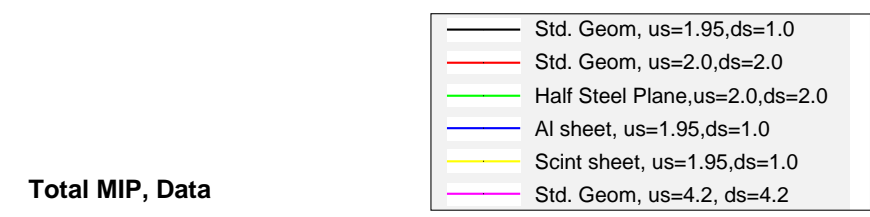

Total MIP, MC
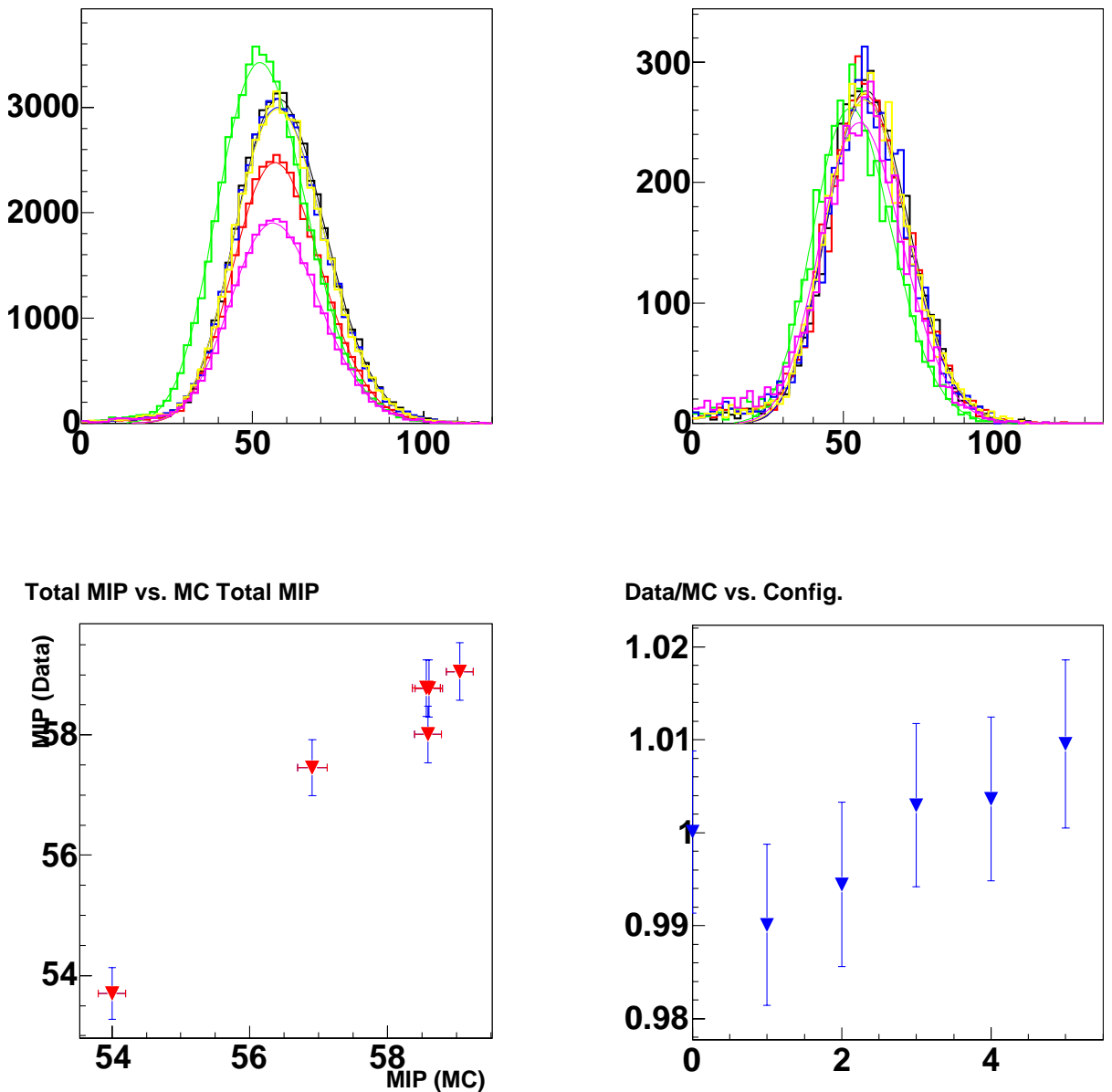

Figure 6.20: Top left plot shows the response distributions from several $1 \mathrm{GeV}$ runs taken with different materials in the beam line in front of the detector. Top right plot shows the simulated response distributions. Lower left plot shows the mean response from the data versus the mean response from the Monte Carlo. The lower right plot shows the ratio of the response from the data to that from the Monte Carlo versus run number. Adding extra material upstream of the detector induced a $8 \%$ shift in the detector response, a shift that was tracked within $1 \%$ in the Monte Carlo 


\begin{tabular}{|l|l|l|l|}
\hline Config. & $\begin{array}{l}\text { US CER } \\
\text { Pressure (ATM) }\end{array}$ & $\begin{array}{l}\text { DS CER } \\
\text { Pressure (ATM) }\end{array}$ & Run Numbers \\
\hline T7, 2 CER, 4 TOF & 1.95 & 1.00 & 70814 \\
(Std. Config.) & & & \\
T7, 2 CER, 4 TOF & 2.00 & 2.00 & 71343 \\
T7, 2 CER, 4 TOF & 4.20 & 4.20 & 71262 \\
T7, 2 CER, 4 TOF, & 2.00 & 2.00 & 71443,71447 \\
1/2" Steel Plane & & 1.00 & 70817 \\
T7, 2 CER, 4 TOF, & 1.95 & & \\
0.5 cm Al & & 1.00 & 70820 \\
T7, 2 CER, 4 TOF, & 1.95 & & \\
1 cm Scintillator & & & \\
\hline
\end{tabular}

Table 6.3: Special runs for validation of the beam line simulation

tribution. The events in the low energy tail arise from events in which the incident particle loses a large fraction of its energy upstream of the detector, typically inducing a shower before reaching the CalDet. While such events are present to varying degrees in all the data samples, they are most apparent in the 2003 data samples when two extra scintillator paddles were used to improve the resolution of the TOF system. Such events earned the moniker "splat events" because these events are typically characterized by an abnormally large number of hit strips in planes 0 and 1 , a wide transverse energy deposition, and a short depth. The Monte Carlo indicates that splat events can be identified using a number of topological cuts:

- Require the ratio of number of hit strips in planes 0 and 1 to the total number of hit strips in an event to be less than 0.5.

- Require the last plane (before a gap of more than 3 planes) divided by the MIP weighted radius (averaged over the strips within 6 strips of the event center and up to last plane) to be greater than 5 .

- Require the strip of maximum signal in plane 0 to be either strip 11 or 12 . 
- Require the ratio of number of strips hit in plane 0 to the total number of hit strips in an event to be less than 0.3 .

At a beam momentum of $1 \mathrm{GeV}$, these cuts remove $80 \%$ of splat events, with a loss of $3 \%$ of "good" events. Below $1 \mathrm{GeV}$ splat events are difficult to distinguish from "good" events. The distributions of the cut quantities for Monte Carlo events are shown in Figure 6.21, while the effect of the cuts on the response distribution is shown in Figure 6.22. The same distributions from a $1 \mathrm{GeV}$ data sample are shown in Figure 6.23 and the effect of these cuts on the response distribution is shown in Figure 6.24. Both the Monte Carlo and the data indicate that the 2002 data set is relatively free from "splat" events. This technique to remove splat events from the CalDet data samples is not used in the calibration of the detector response, but instead, is provided as reference for future studies into the topological properties of electron events. 

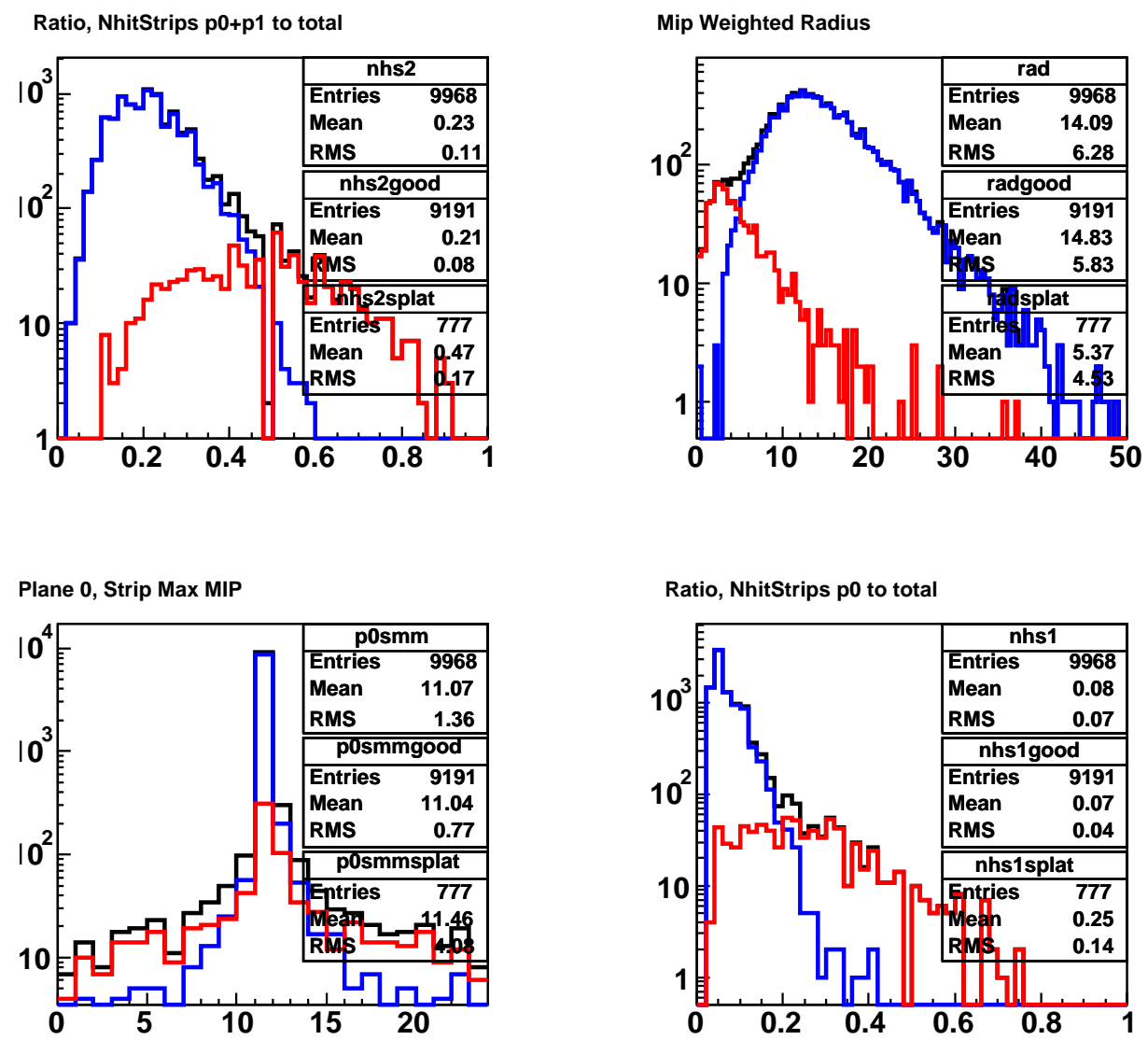

Figure 6.21: The cuts to identify "splat events" in the Monte Carlo. The top left plot shows the ratio of hit strips in plane 0 and 1 relative to the total. Black is for all events, blue is for events with total energy of all particles greater than $85 \%$ of incident energy as given by Monte Carlo truth, red is for events with less than $85 \%$ of incident energy ("splat events"). Top right shows end plane divided by MIP weighted radius. Bottom left plot shows strip of maximum MIP in plane 0, bottom right shows ratio of hit strips in plane zero to total number of hit strips. 


\section{Total MIPs}

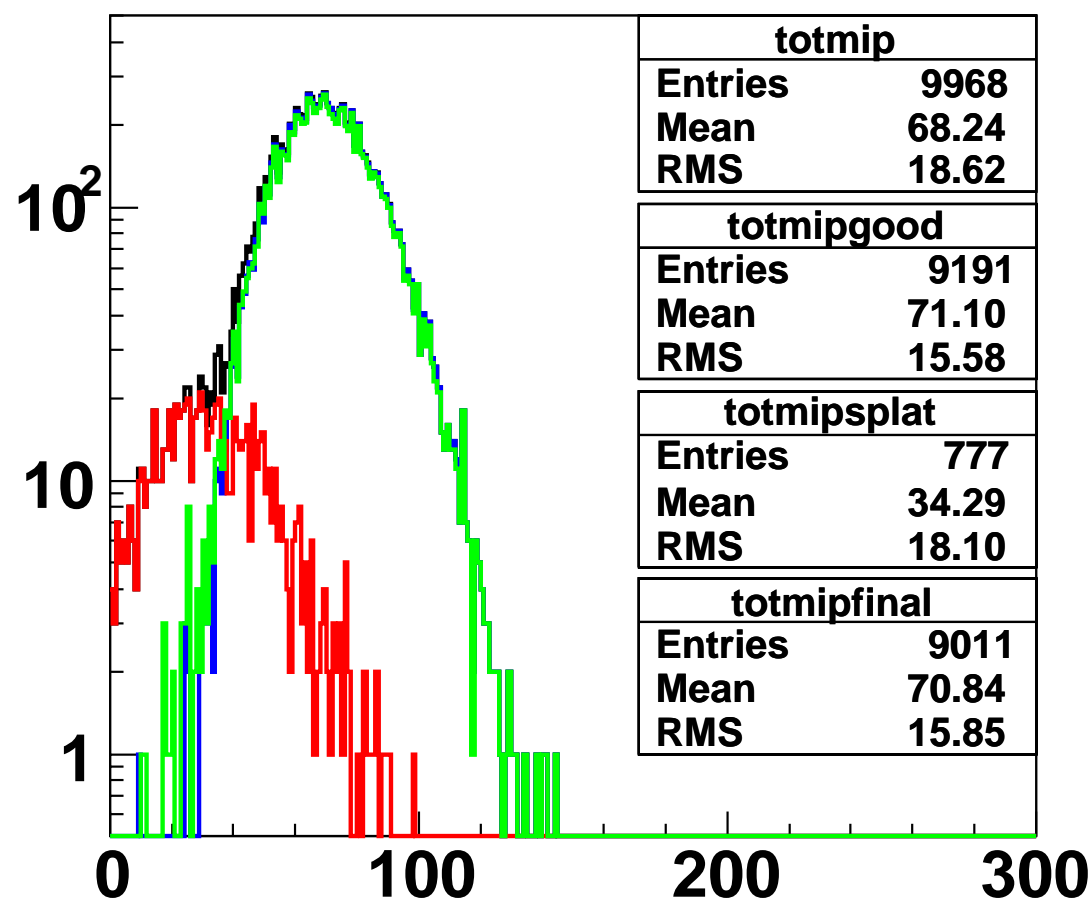

Figure 6.22: The detector response to $1 \mathrm{GeV}$ simulated electrons. Blue histogram shows events with greater than $85 \%$ of the incident energy, red shows events with less than $85 \%$ of incident energy and green is the result of the applying the cuts described in the text. 

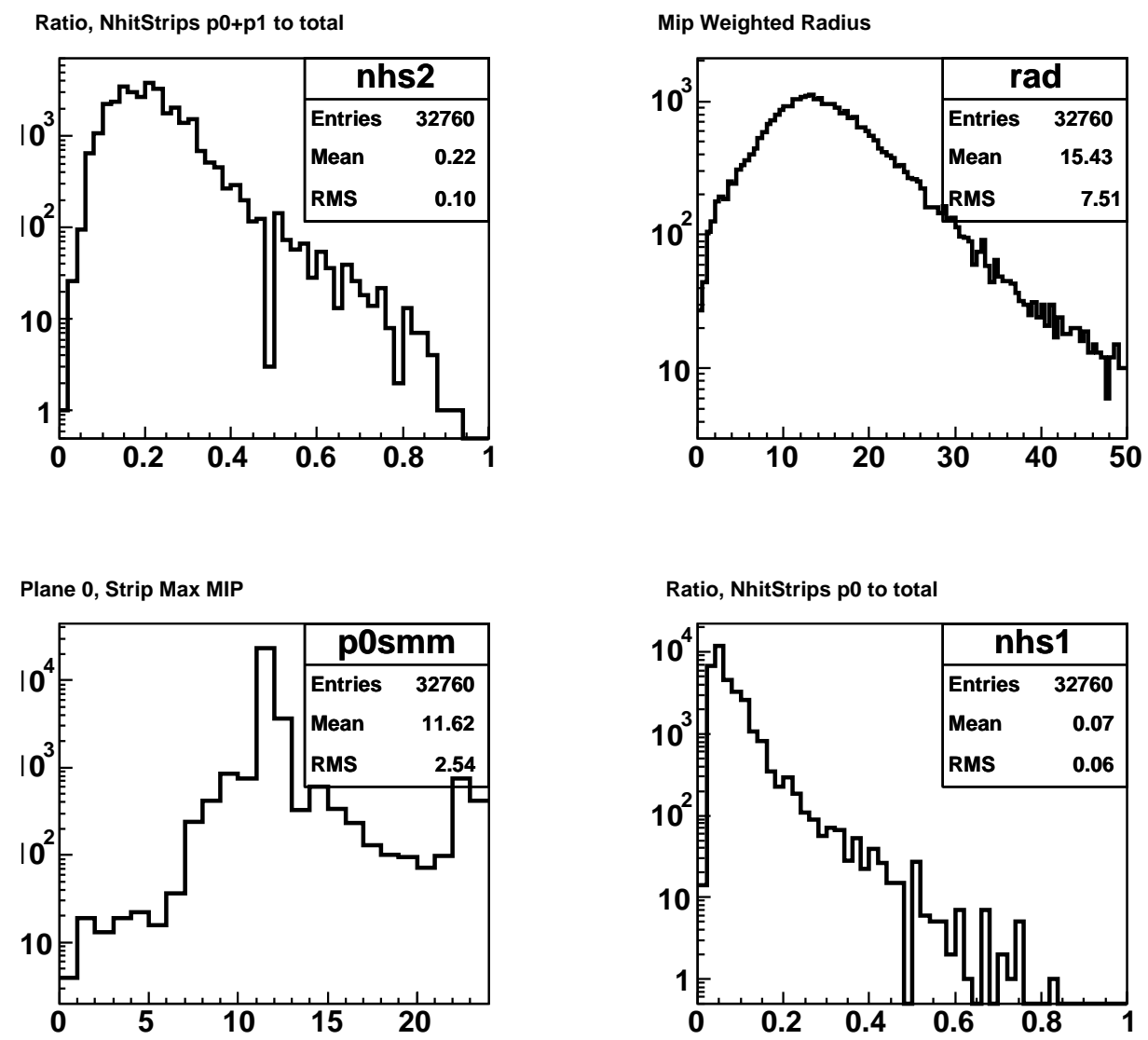

Figure 6.23: The cuts to identify "splat events" in the data. Top Left shows the ratio of hit strips in plane 0 and 1 . Top right shows end plane divided by MIP weighted radius. Bottom left plot shows strip of maximum MIP in plane 0 , bottom right shows ratio of hit strips in plane zero to total number of hit strips. 


\section{Total MIPs}

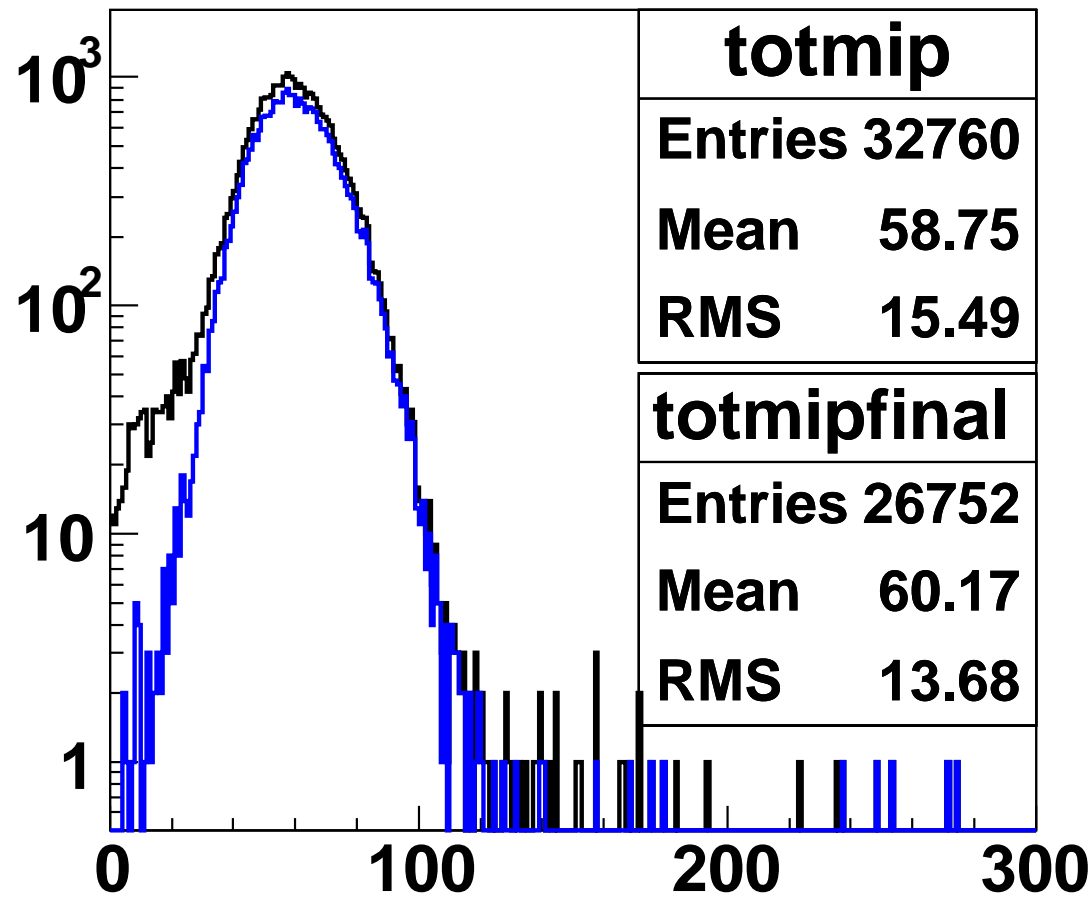

Figure 6.24: Response distribution for $1 \mathrm{GeV}$ electrons (data). Blue histogram is the result of the applying the cuts described in the text. 


\section{Chapter 7}

\section{Electron Characteristics}

In this chapter, the expected properties of electron interactions in a calorimeter are discussed, then the measured response and topological properties of electron showers are compared to the Monte Carlo expectation and contrasted against the properties of hadronic showers.

\subsection{Electromagnetic Interactions in Matter}

As an electron with energy above $1 \mathrm{GeV}$ travels through matter, it loses energy predominantly via bremsstrahlung [43]. The emitted photon in turn converts to produce an electron-positron pair, each of which can then undergo bremsstrahlung. In this way, an electron interacting in matter produces a cascade of particles, the number of particles in the shower approximately doubling with each series of interactions, and each new daughter particle carrying a smaller fraction of the total incident energy. Once the energy of the individual particles is below the pair production threshold, no new particles are created, and the rest of the energy of the shower particles is dissipated through ionization, Compton scattering, the photoelectric effect, and thermal excitation. 
Both the longitudinal and lateral development of electromagnetic showers can be parameterized in terms the radiation length $\left(X_{0}\right)$. The radiation length is the mean distance over which an electron loses all but $\frac{1}{e}$ of its energy due to bremsstrahlung, and the value of the radiation length is a function of the material being traversed. For a sampling calorimeter, the radiation length of the detector can be computed from the radiation lengths of its components using

$$
\frac{1}{X_{0}}=\sum_{j} \frac{w_{j}}{X_{0 j}}
$$

where $w_{j}$ is the fraction by weight of the $j^{\text {th }}$ detector layer and $X_{0 j}$ is the radiation length in the $j^{\text {th }}$ detector layer [43]. In CalDet, the radiation length is $4.1 \mathrm{~cm}$, or 0.69 planes, which is slightly longer than in the larger detectors due to the difference in the steel plane width.

In electron showers, the longitudinal distance from the beginning of the shower development at which the most daughter particles are present is given by

$$
z_{\max }=X_{0} \ln \frac{E_{0}}{E_{c}}-0.5
$$

where $E_{0}$ is the incident electron energy, and $E_{c}$ is the energy below which shower development stops and losses due to ionization dominate. In iron, $E_{c}$ is approximately $29 \mathrm{MeV}$. The transverse size of an electromagnetic shower is given by the Moliere radius:

$$
R_{M}=X_{0} \frac{21 \mathrm{MeV}}{E_{c}}
$$

In general, $90 \%$ of the energy deposited in an electromagnetic shower lies within a cylinder with radius $R_{M}$. In CalDet, the Moliere radius is on the order of $3 \mathrm{~cm}$, or less than one strip.

In the MINOS geometry, electromagnetic showers initiated by an electron or positron with energy in the $1-3 \mathrm{GeV}$ range, are expected to reach the maximum of shower development over $2-3$ planes, and the transverse size of the core is expected 
to be on the order of 1 strip. Compared to hadronic interactions, electromagnetic cascades are dense depositions of energy over a small volume.

\subsection{Comparison with Monte Carlo}

Electrons produced in $\nu_{e}$ charged current interactions will be identified using event topology. Monte Carlo is used to develop and study the performance of algorithms to identify the type of neutrino interactions. While neutrino events will induce multiple particle showers in the large MINOS detectors, it is essential to demonstrate that the Monte Carlo reproduces the characteristics of single particle interactions in the CalDet and that the basic event topologies are well modeled.

A number of steps are involved in simulating electrons interacting in CalDet. Prior to simulating the detector response, a flux file is generated using the beam line simulation detailed in Chapter 6. The events from the flux file are then input into the standard MINOS Monte Carlo chain, which consists of a GEANT3 based simulation, GMINOS, which models the interactions of particles in matter, and two MINOS offline packages, PhotonTransport and DetSim, which propagate photons produced in the scintillator and model the detector response [44], [45]. To adequately represent the data, some parameters involved in the simulation have been tuned away from the default values. Table 7.1 lists the non-default settings used in the simulation of CalDet data. The overall light level and the MIP scale are free parameters in the Monte Carlo. These values have been tuned so that the Monte Carlo response to positrons in each beam area matches the data at $1 \mathrm{GeV}$. Ideally, stopping muons would be used to tune these parameters, however an outstanding discrepancy in the range of stopping muons in the Monte Carlo makes this procedure undesirable [46].

Figures 7.1 through 7.7 show comparisons between data and Monte Carlo positrons. In each plot six momenta are shown; other momenta are shown in Appendix D. The black histogram shows the data, while the red histogram shows the 


\begin{tabular}{|l|l|}
\hline Package & Parameters Tuned \\
\hline GMINOS & $\begin{array}{l}\text { 1. Steel width: } 2.5 \mathrm{~cm} \\
\text { 2. Proper isotope ratio for CalDet steel } \\
\text { 3. All particle tracking cutoffs: } 10 \mathrm{keV} \text { (minimum) }\end{array}$ \\
& $\begin{array}{l}\text { 1. Birk's Constant: } 0.10 \mathrm{~m} / \mathrm{GeV} \\
\text { 2. DarkNoiseRate: } 100 \mathrm{~Hz}\end{array}$ \\
$\begin{array}{l}\text { 3. GreenNoiseRate: } 200 \mathrm{~Hz} \\
\text { 4. NoiseWindow: } 500 \mathrm{~ns}\end{array}$ \\
$\begin{array}{l}\text { 5. OverallLightOutput tuned to } 1 \mathrm{GeV} \text { electrons } \\
\text { 6. PE to MIP constant tuned to } 1 \mathrm{GeV} \text { electrons }\end{array}$ \\
$\begin{array}{l}\text { 1. PMT non-linearity is not simulated } \\
\text { 2. Dead VA chips are not simulated } \\
\text { 3. Sparsification threshold: } 8 \text { ADC } \\
\text { 4. Detector components have uniform response }\end{array}$ \\
\hline
\end{tabular}

Table 7.1: Non-default parameters set in simulating the CalDet

Monte Carlo. The value of $\chi^{2}$ given characterizes the differences between the Monte Carlo and the data. Figure 7.1 shows the distributions of the last plane in an event, where the last plane is defined to be the last plane with a hit of pulse height greater than 1.5 PE before a gap of four or more planes. The Monte Carlo mean last plane and shape of the distribution agree well with the data over all momenta. Figure 7.2 shows the number of strips hit in an event, while Figure 7.3 shows the number of strips with signal greater than 1.5 PE. The mean number of strips hit in the Monte Carlo is in general smaller than the mean number of hit strips in the data; however, when the number of strips with signal greater than 1.5 PE is compared, data and Monte Carlo agree favorably, indicating that the surplus of hit strips in the data are of low pulse height. Figure 7.4 shows the lateral extent of the events, defined as the response weighted distance of hits from the center of the event, summed over strips contained in a box 12 strips wide, and last planes long, centered around the event. At the lower beam momenta, the distributions do not differ dramatically, but 
at larger momenta, the Monte Carlo tends to overestimate the lateral extent of the shower. Figure 7.5 shows the plane of maximum energy deposition, and Figure 7.6 shows the fraction of the total response deposited in that plane. Both quantities show good agreement between data and Monte Carlo. Finally, Figure 7.7 shows the summed detector response in MIPs. Figure 7.8 shows the ratio of data to simulated detector response versus beam momentum. With the exception of the $3.6 \mathrm{GeV}$ point from T11, which is at the upper limit of the capabilities of the beam line, the Monte Carlo agrees with the measured response to within $2 \%$ across the entire momentum range.

\subsection{Contrasting Electrons and Hadrons}

Figures 7.9 through 7.15 show the same quantities listed above, but instead of comparing data and Monte Carlo, they contrast the properties of electrons and pions of the same momentum. Figure 7.9 shows the last plane in an event. At the lowest momenta, pions are shorter than electrons, but as the momentum increases, pions begin to penetrate deeper than electrons. Figures 7.10 and 7.11 show the number of hit strips in an event and the number of hit strips above a threshold of 1.5 PE. At the higher momenta, pions exhibit more activity than electrons. Figure 7.12 shows the transverse extent of pions and electrons. At lower momenta, electron and hadronic showers look similar, but at higher energies, hadrons tend to spread further laterally. Figure 7.13 shows the plane of maximum energy deposition, while Figure 7.14 shows the ratio of energy deposited in that plane relative to the total energy. Pions deposit more energy deeper in the detector, but also show wider fluctuations in the shower development than electrons. Figure 7.15 shows the detector response. At a given momentum, pions induce a smaller signal in the detector than electrons.

When looking at a neutrino induced event at the large MINOS detectors, the 
Last Plane, 1.5 PE cut, $0.2 \mathrm{GeV}$

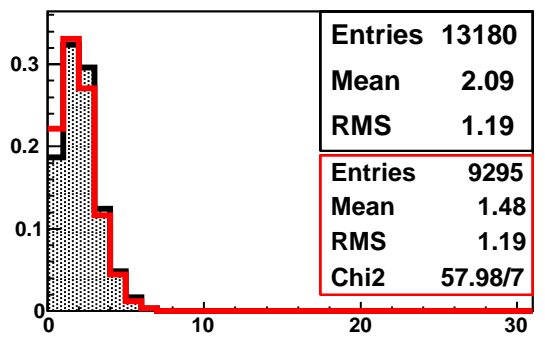

Last Plane, 1.5 PE cut, 2.0 GeV

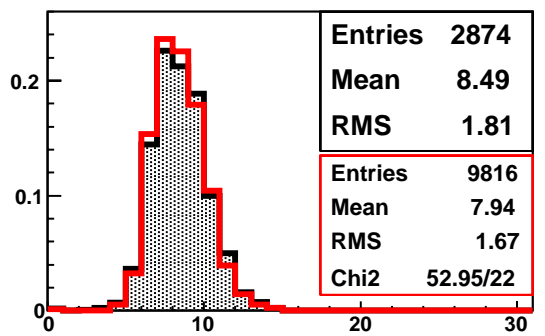

Last Plane, 1.5 PE cut, 5.0 GeV

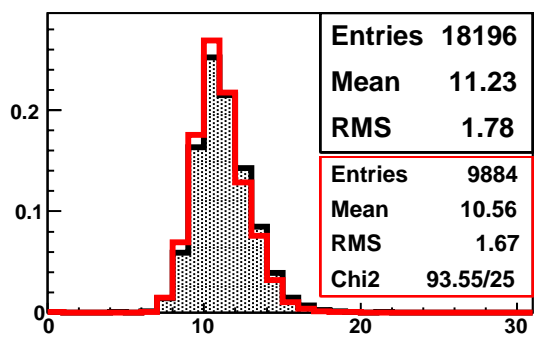

Last Plane, 1.5 PE cut, 1.0 GeV

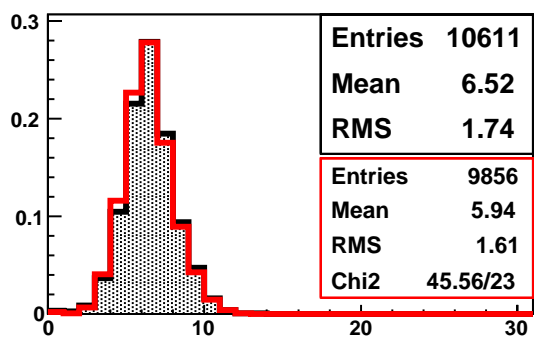

Last Plane, 1.5 PE cut, $3.2 \mathrm{GeV}$

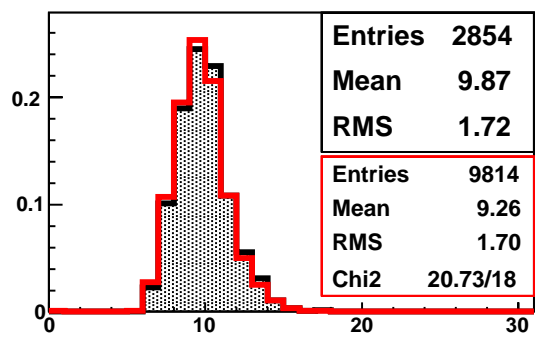

Last Plane, 1.5 PE cut, 7.0 GeV

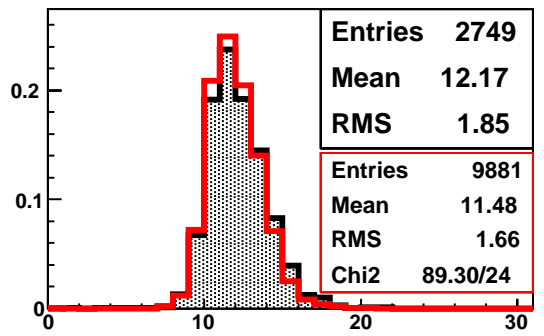

Figure 7.1: Comparison of last plane hit between data and Monte Carlo. Black distributions represent data, red distributions represent Monte Carlo. The momenta shown are $200 \mathrm{MeV}, 1 \mathrm{GeV}, 2 \mathrm{GeV}, 3.2 \mathrm{GeV}, 5 \mathrm{GeV}$, and $7 \mathrm{GeV}$. 


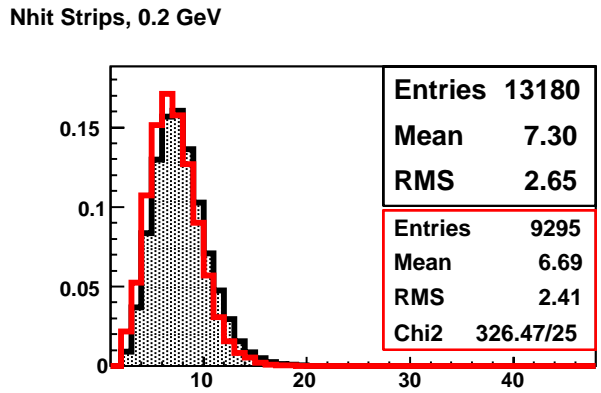

Nhit Strips, $2.0 \mathrm{GeV}$

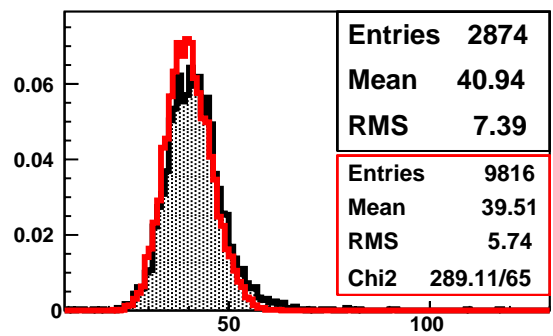

Nhit Strips, 5.0 GeV

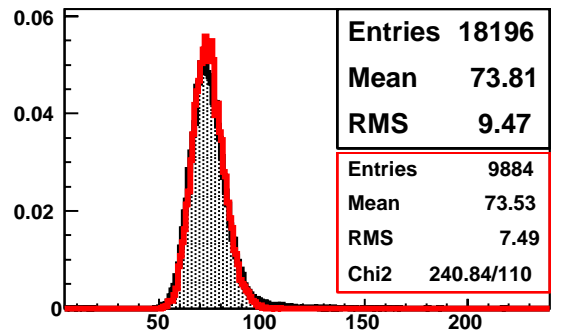

Nhit Strips, $1.0 \mathrm{GeV}$

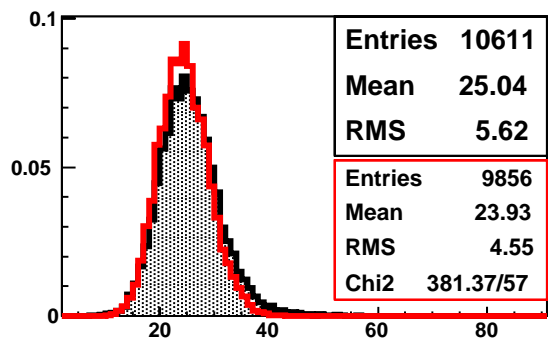

Nhit Strips, 3.2 GeV

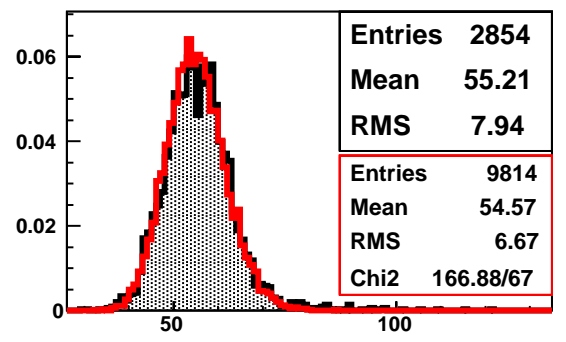

Nhit Strips, 7.0 GeV

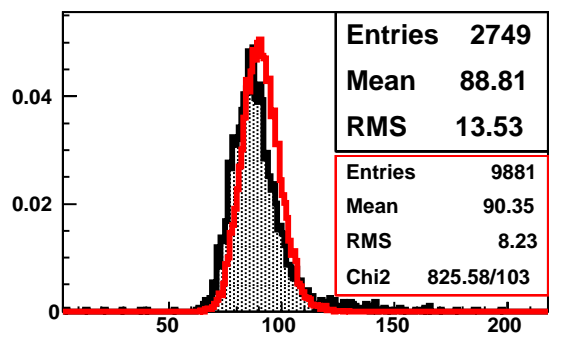

Figure 7.2: Comparison of the number of hit strips between data and Monte Carlo. Black distributions represent data, red distributions represent Monte Carlo. The momenta shown are $200 \mathrm{MeV}, 1 \mathrm{GeV}, 2 \mathrm{GeV}, 3.2 \mathrm{GeV}, 5 \mathrm{GeV}$, and $7 \mathrm{GeV}$. 
Nhit Strips, 1.5 PE Cut, 0.2 GeV

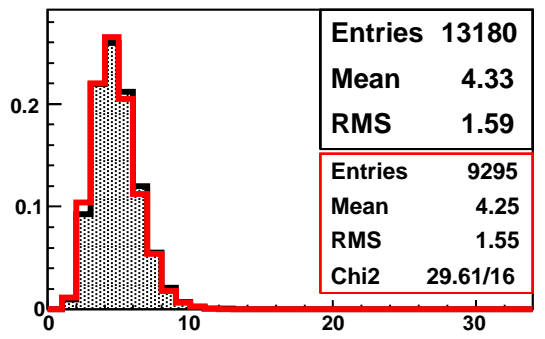

Nhit Strips, 1.5 PE Cut, 2.0 GeV

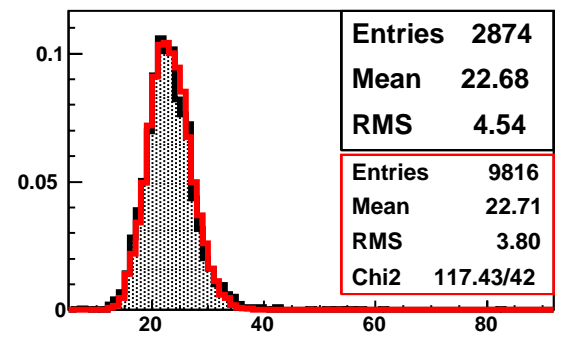

Nhit Strips, 1.5 PE Cut, 5.0 GeV

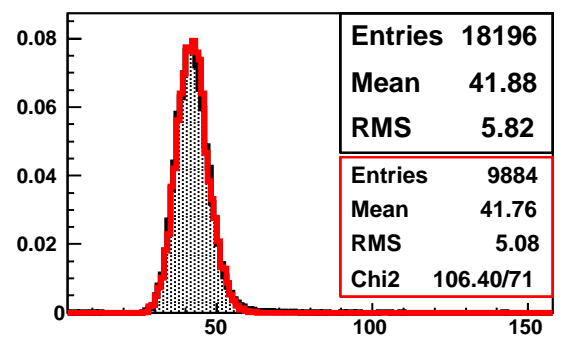

Nhit Strips, 1.5 PE Cut, 1.0 GeV

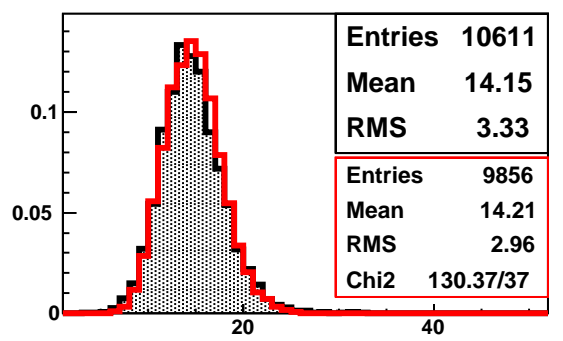

Nhit Strips, 1.5 PE Cut, 3.2 GeV

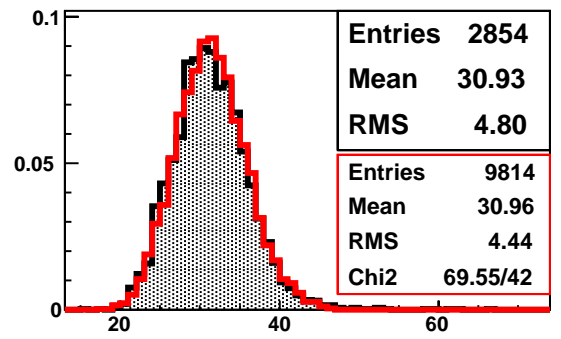

Nhit Strips, 1.5 PE Cut, 7.0 GeV

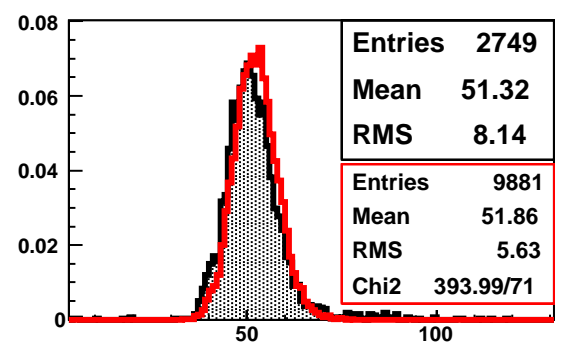

Figure 7.3: Comparison of number of strips hit above a 1.5 PE threshold between data and Monte Carlo. Black distributions represent data, red distributions represent Monte Carlo. The momenta shown are $200 \mathrm{MeV}, 1 \mathrm{GeV}, 2 \mathrm{GeV}, 3.2 \mathrm{GeV}$, $5 \mathrm{GeV}$, and $7 \mathrm{GeV}$. 


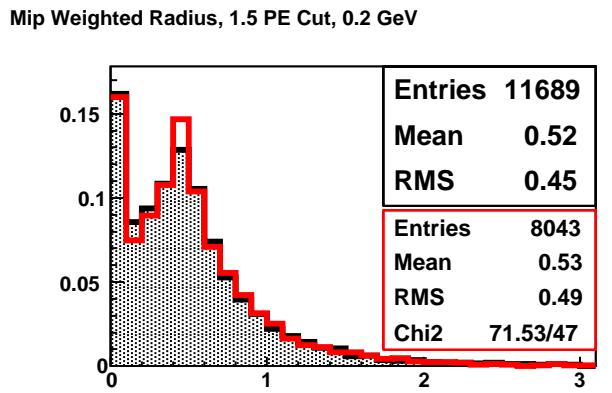

Mip Weighted Radius, 1.5 PE Cut, 2.0 GeV

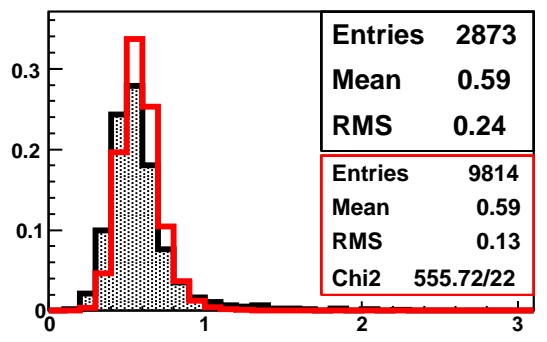

Mip Weighted Radius, 1.5 PE Cut, 5.0 GeV

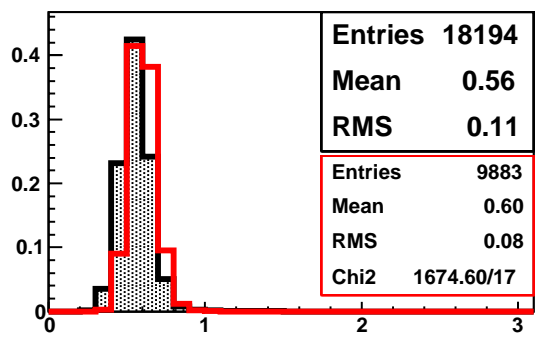

Mip Weighted Radius, 1.5 PE Cut, 1.0 GeV

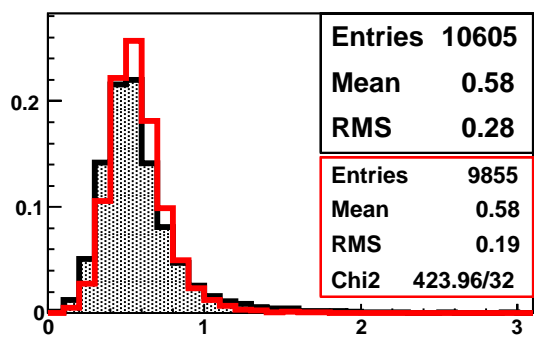

Mip Weighted Radius, 1.5 PE Cut, 3.2 GeV

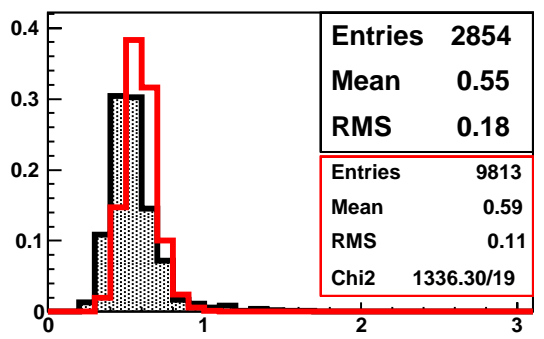

Mip Weighted Radius, 1.5 PE Cut, 7.0 GeV

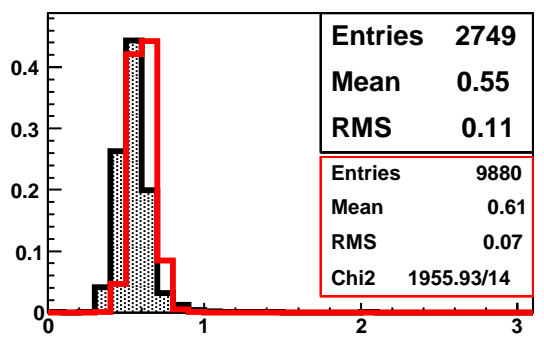

Figure 7.4: Comparison of the radius of electron events between data and Monte Carlo. Black distributions represent data, red distributions represent Monte Carlo. The momenta shown are $200 \mathrm{MeV}, 1 \mathrm{GeV}, 2 \mathrm{GeV}, 3.2 \mathrm{GeV}, 5 \mathrm{GeV}$, and $7 \mathrm{GeV}$. 
Plane of Shower Max, $0.2 \mathrm{GeV}$

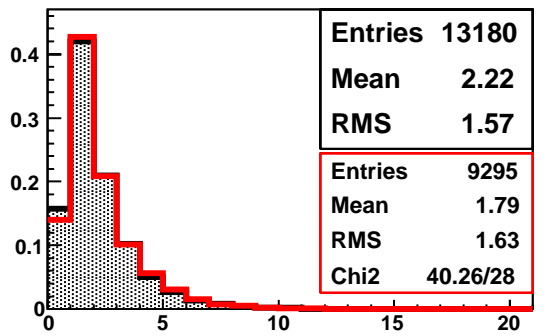

Plane of Shower Max, $2.0 \mathrm{GeV}$

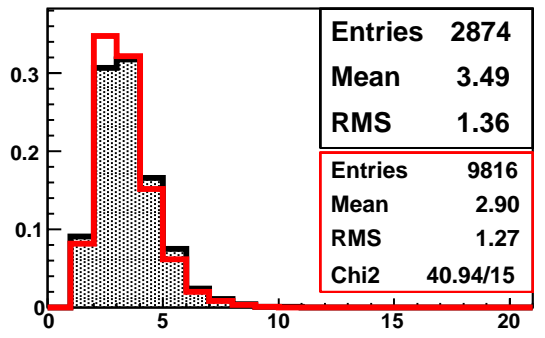

Plane of Shower Max, 5.0 GeV

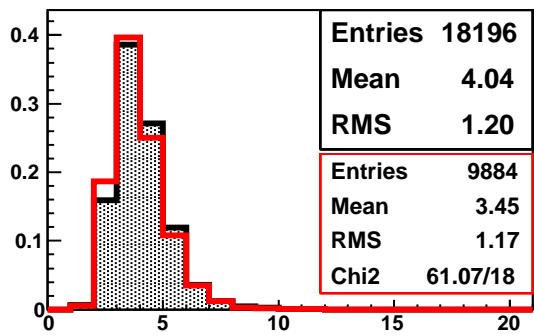

Plane of Shower Max, 1.0 GeV

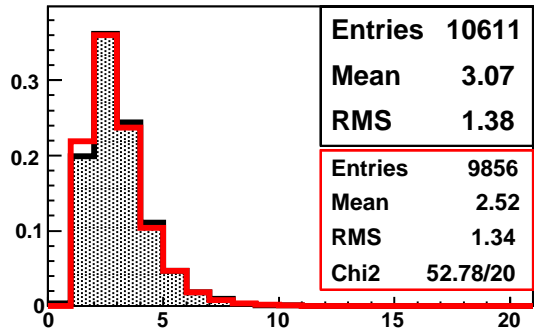

Plane of Shower Max, 3.2 GeV

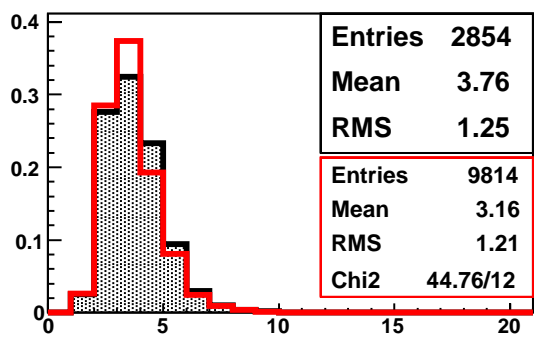

Plane of Shower Max, 7.0 GeV

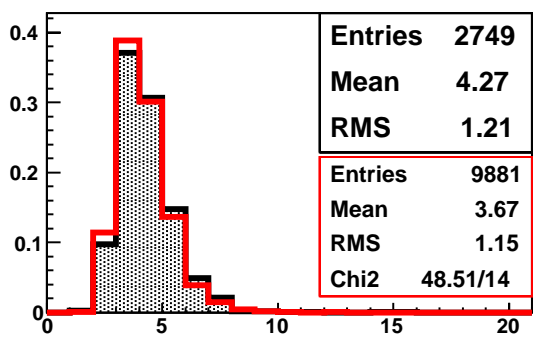

Figure 7.5: Comparison of the plane of shower max distributions between data and Monte Carlo. Black distributions represent data, red distributions represent Monte Carlo. The momenta shown are $200 \mathrm{MeV}, 1 \mathrm{GeV}, 2 \mathrm{GeV}, 3.2 \mathrm{GeV}, 5 \mathrm{GeV}$, and $7 \mathrm{GeV}$. 
MIPs in Plane of Shower Max, $0.2 \mathrm{GeV}$

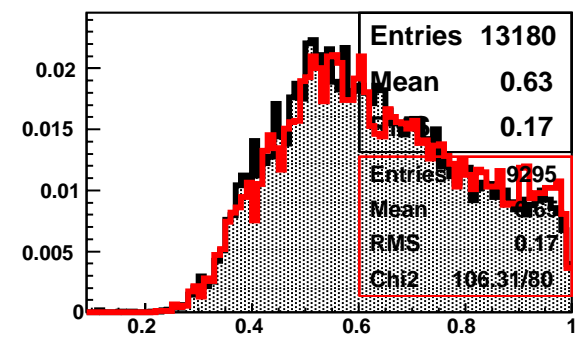

MIPs in Plane of Shower Max, $2.0 \mathrm{GeV}$

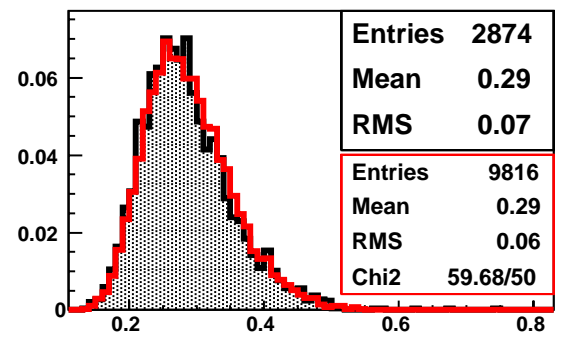

MIPs in Plane of Shower Max, 5.0 GeV

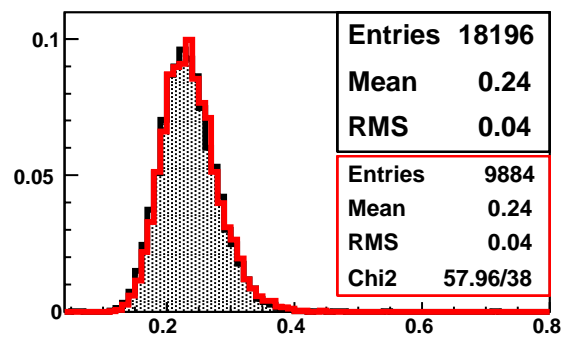

MIPs in Plane of Shower Max, $1.0 \mathrm{GeV}$

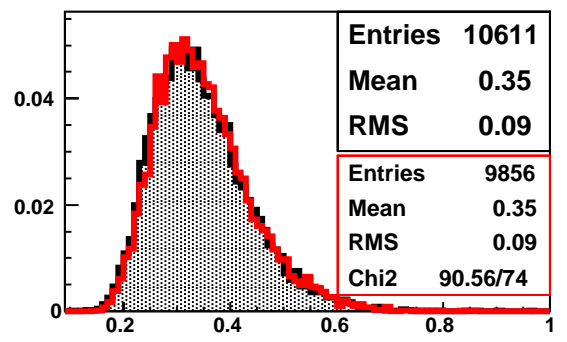

MIPs in Plane of Shower Max, 3.2 GeV

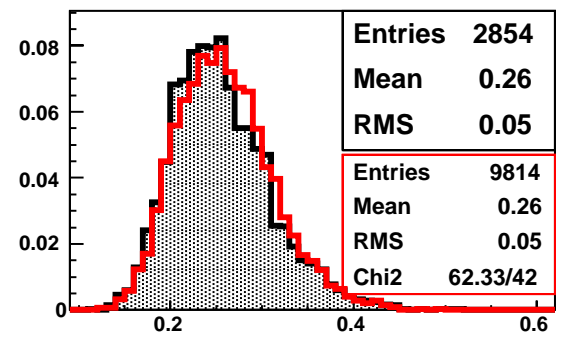

MIPs in Plane of Shower Max, 7.0 GeV

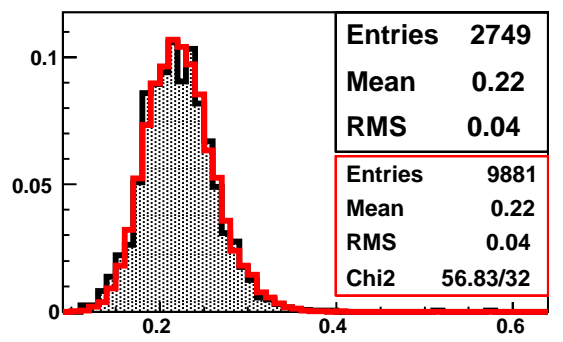

Figure 7.6: Comparison of the portion of energy deposited in the plane of shower max between data and Monte Carlo. Black distributions represent data, red distributions represent Monte Carlo. The momenta shown are $200 \mathrm{MeV}, 1 \mathrm{GeV}, 2 \mathrm{GeV}, 3.2 \mathrm{GeV}$, $5 \mathrm{GeV}$, and $7 \mathrm{GeV}$. 
Total MIP, $0.2 \mathrm{GeV}$

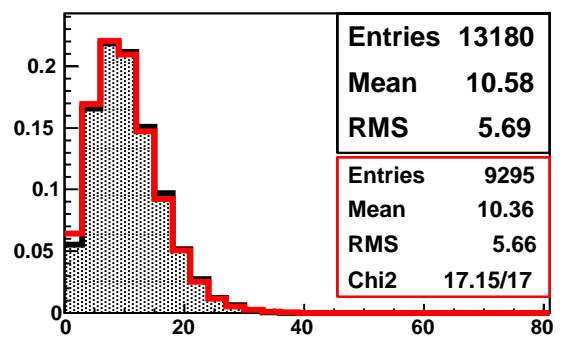

Total MIP, $2.0 \mathrm{GeV}$

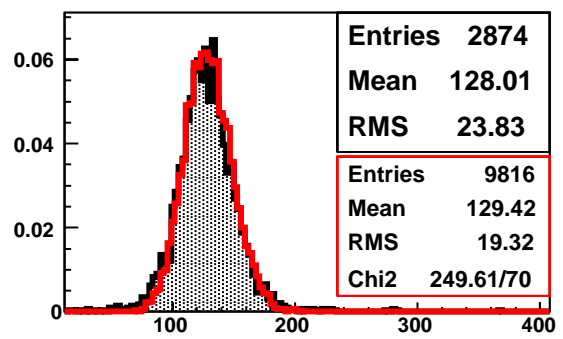

Total MIP, 5.0 GeV

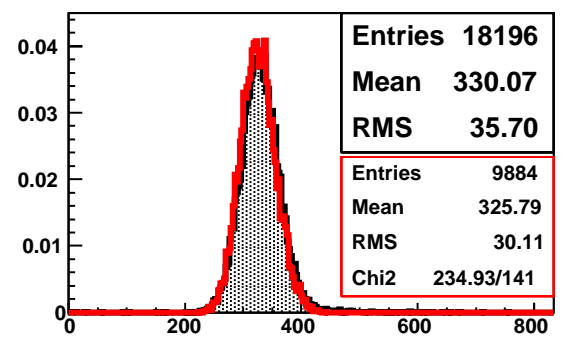

Total MIP, $1.0 \mathrm{GeV}$

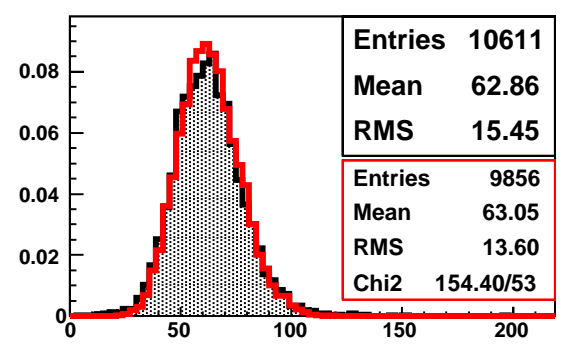

Total MIP, $3.2 \mathrm{GeV}$

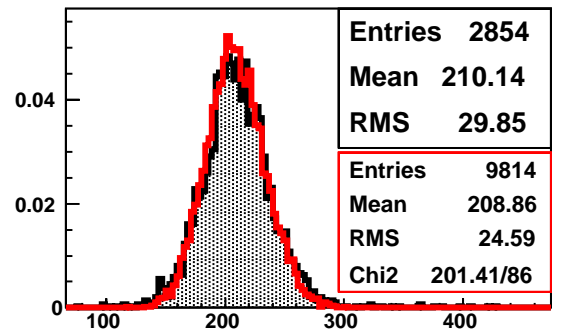

Total MIP, 7.0 GeV

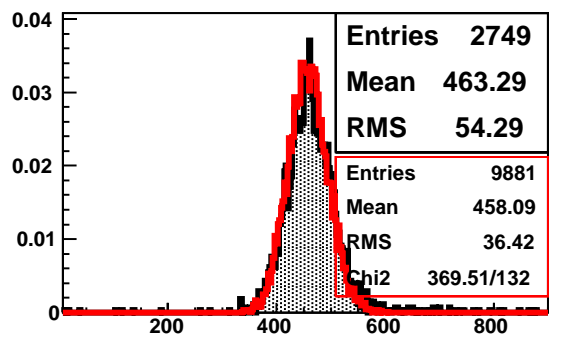

Figure 7.7: Comparison of the response between data and Monte Carlo. Black distributions represent data, red distributions represent Monte Carlo. The momenta shown are $200 \mathrm{MeV}, 1 \mathrm{GeV}, 2 \mathrm{GeV}, 3.2 \mathrm{GeV}, 5 \mathrm{GeV}$, and $7 \mathrm{GeV}$. 


\section{Ratio of Response, Data/MC}

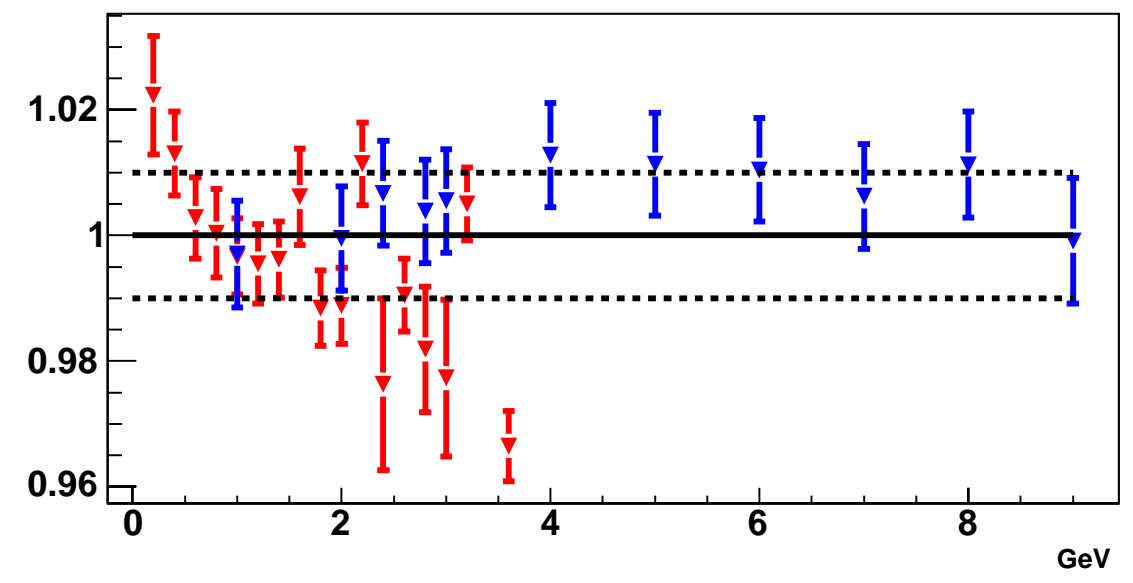

Figure 7.8: The ratio of response from positron data to that of Monte Carlo versus beam momentum. Runs taken in T11 are shown in red, runs from T7 are shown in blue.

momentum of the daughter particles is not known. Instead, the detector response is known, and event classification will be based on the comparison of particles that induce the same response. Figure 7.16 contrasts shower characteristics between $1 \mathrm{GeV}$ electron events and pion events over a range of momenta that exhibit a response consistent with that of $1 \mathrm{GeV}$ electrons. While no one quantity provides the power to distinguish hadronic showers from electron showers in the few $\mathrm{GeV}$ range, taken together, the topological properties of the shower will be a useful tool in distinguishing electromagnetic showers from hadronic showers. 
Last Plane, 1.5 PE cut, $0.2 \mathrm{GeV}$

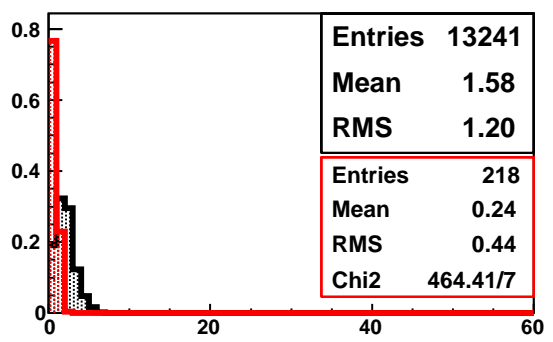

Last Plane, 1.5 PE cut, 2.0 GeV

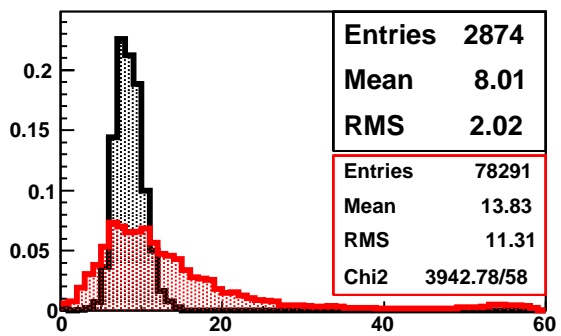

Last Plane, 1.5 PE cut, 5.0 GeV

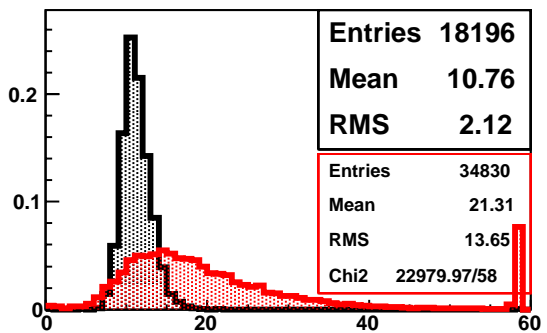

Last Plane, 1.5 PE cut, 1.0 GeV

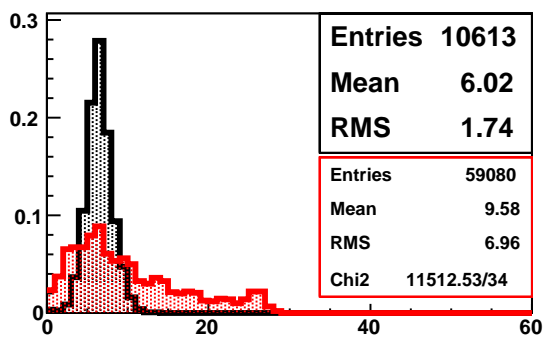

Last Plane, 1.5 PE cut, 3.2 GeV

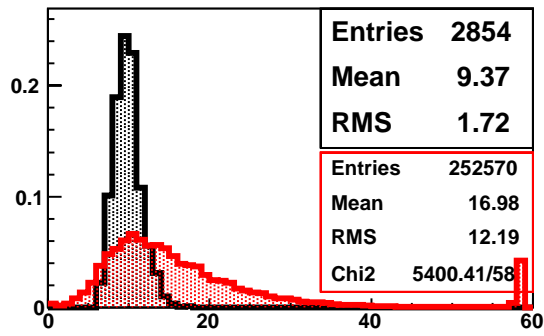

Last Plane, 1.5 PE cut, 7.0 GeV

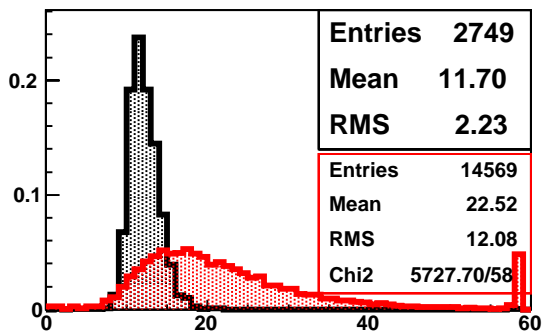

Figure 7.9: Comparison of the last plane in an event between electrons and and pions. Black distributions represent electrons, red distributions represent pions. The momenta shown are $200 \mathrm{MeV}, 1 \mathrm{GeV}, 2 \mathrm{GeV}, 3.2 \mathrm{GeV}, 5 \mathrm{GeV}$, and $7 \mathrm{GeV}$. 


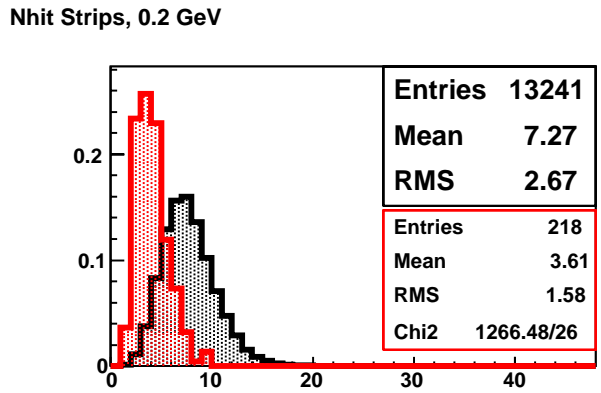

Nhit Strips, $2.0 \mathrm{GeV}$

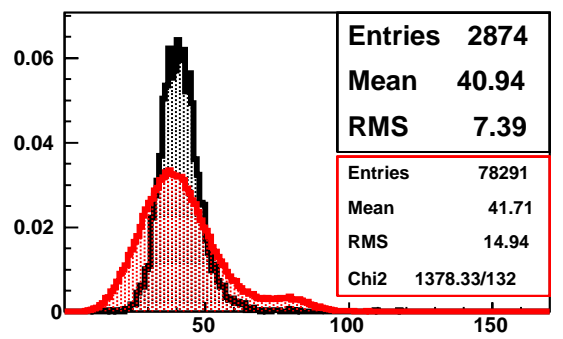

Nhit Strips, 5.0 GeV

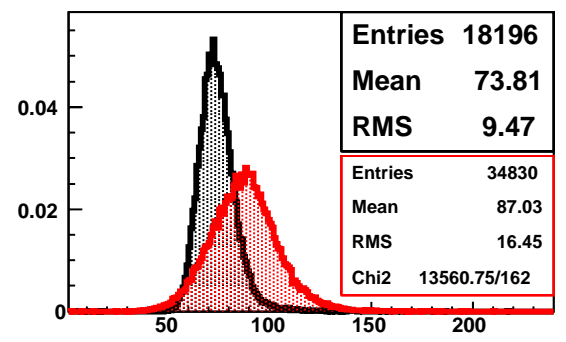

Nhit Strips, $1.0 \mathrm{GeV}$

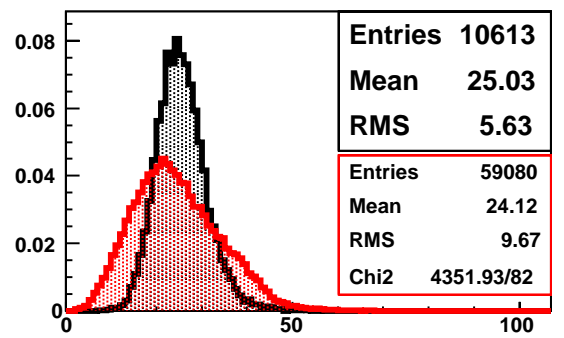

Nhit Strips, $3.2 \mathrm{GeV}$

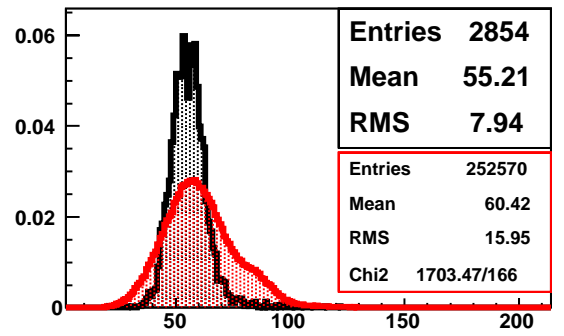

Nhit Strips, 7.0 GeV

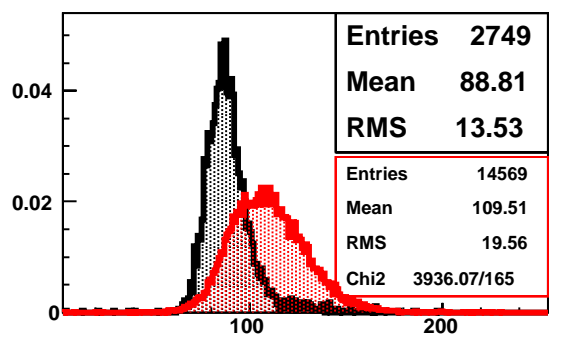

Figure 7.10: Comparison of number of hit strips in an event between electrons and and pions. Black distributions represent electrons, red distributions represent pions. The momenta shown are $200 \mathrm{MeV}, 1 \mathrm{GeV}, 2 \mathrm{GeV}, 3.2 \mathrm{GeV}, 5 \mathrm{GeV}$, and $7 \mathrm{GeV}$. 
Nhit Strips, 1.5 PE Cut, 0.2 GeV

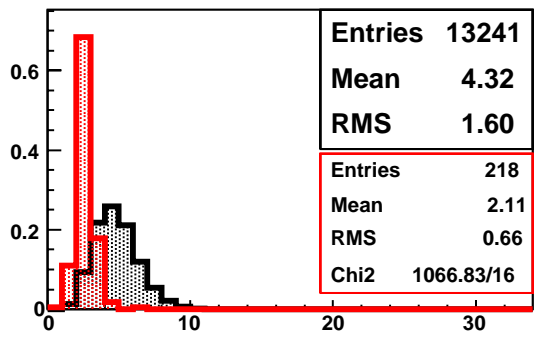

Nhit Strips, 1.5 PE Cut, 2.0 GeV

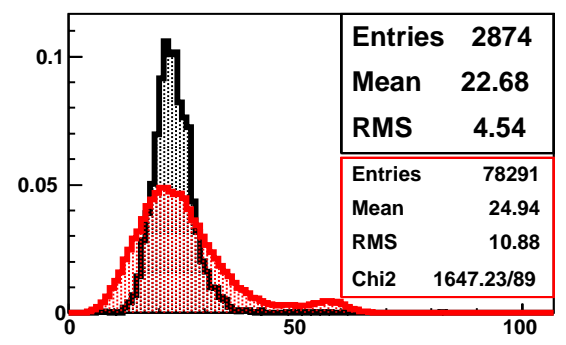

Nhit Strips, 1.5 PE Cut, 5.0 GeV

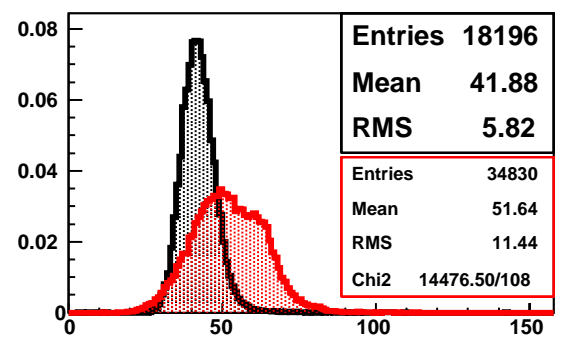

Nhit Strips, 1.5 PE Cut, 1.0 GeV

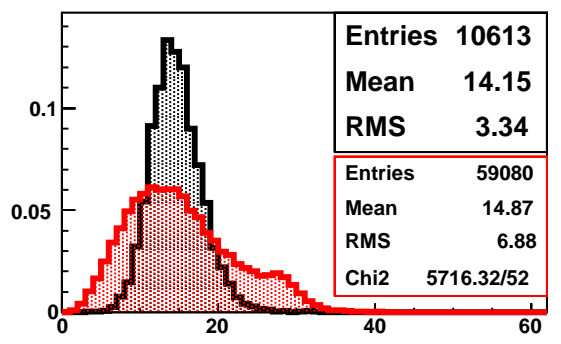

Nhit Strips, 1.5 PE Cut, 3.2 GeV

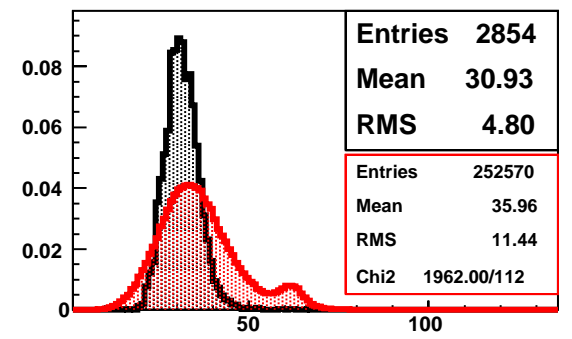

Nhit Strips, 1.5 PE Cut, 7.0 GeV

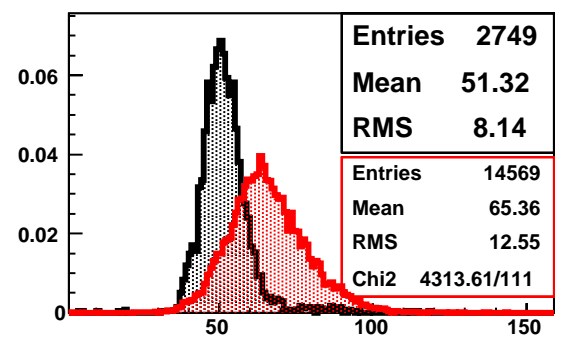

Figure 7.11: Comparison of number of hit strips above a 1.5 PE threshold in an event between electrons and and pions. Black distributions represent electrons, red distributions represent pions. The momenta shown are $200 \mathrm{MeV}, 1 \mathrm{GeV}, 2 \mathrm{GeV}$, $3.2 \mathrm{GeV}, 5 \mathrm{GeV}$, and $7 \mathrm{GeV}$. 
Mip Weighted Radius, 1.5 PE Cut, 0.2 GeV

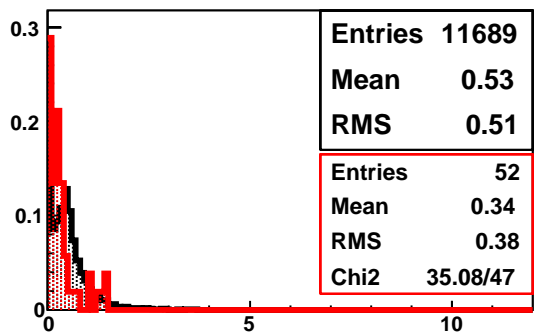

Mip Weighted Radius, 1.5 PE Cut, 2.0 GeV

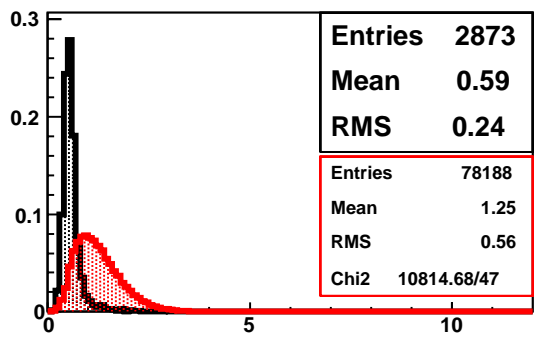

Mip Weighted Radius, 1.5 PE Cut, 5.0 GeV

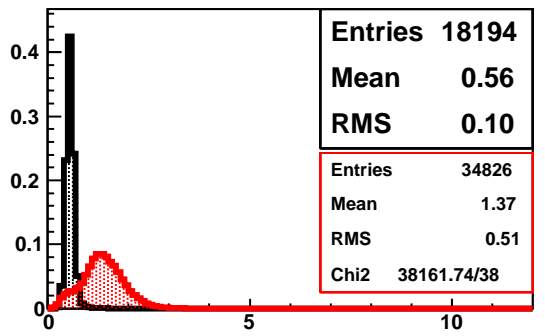

Mip Weighted Radius, 1.5 PE Cut, 1.0 GeV

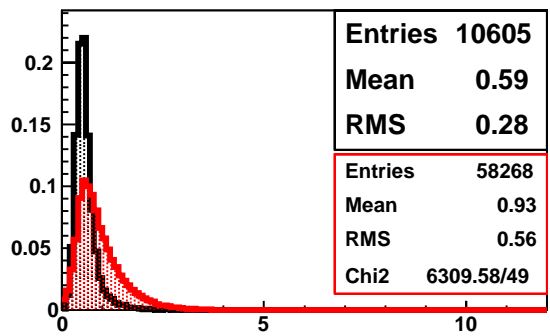

Mip Weighted Radius, 1.5 PE Cut, 3.2 GeV

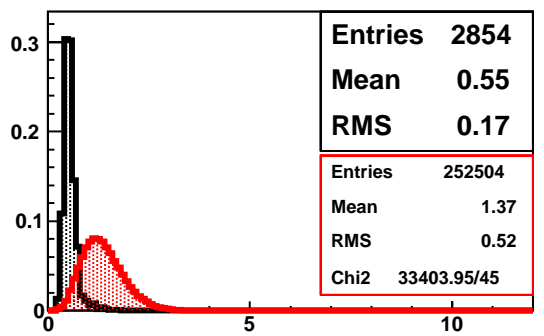

Mip Weighted Radius, 1.5 PE Cut, 7.0 GeV

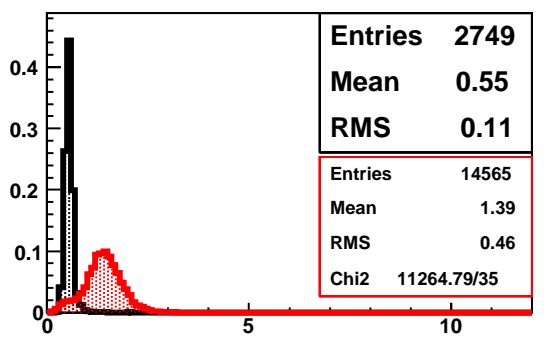

Figure 7.12: Comparison of the radius of an event between electrons and and pions. Black distributions represent electrons, red distributions represent pions. The momenta shown are $200 \mathrm{MeV}, 1 \mathrm{GeV}, 2 \mathrm{GeV}, 3.2 \mathrm{GeV}, 5 \mathrm{GeV}$, and $7 \mathrm{GeV}$. 
Plane of Shower Max, $0.2 \mathrm{GeV}$

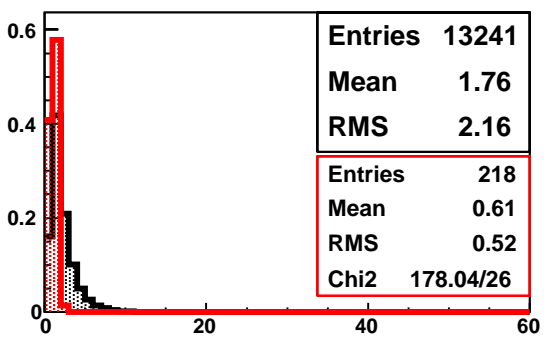

Plane of Shower Max, $2.0 \mathrm{GeV}$

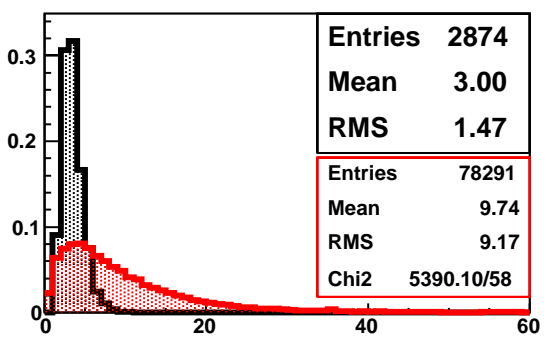

Plane of Shower Max, 5.0 GeV

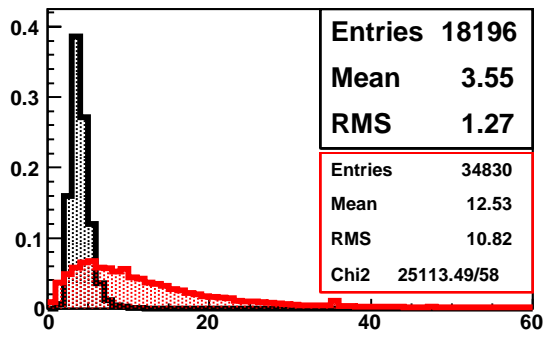

Plane of Shower Max, 1.0 GeV

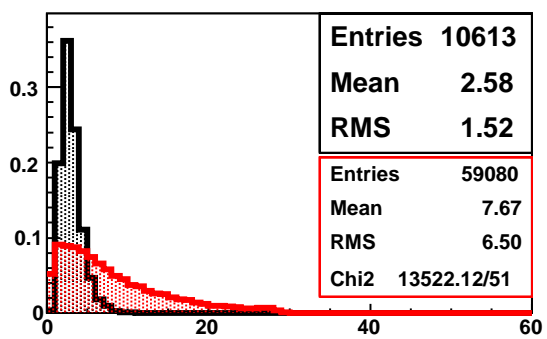

Plane of Shower Max, 3.2 GeV

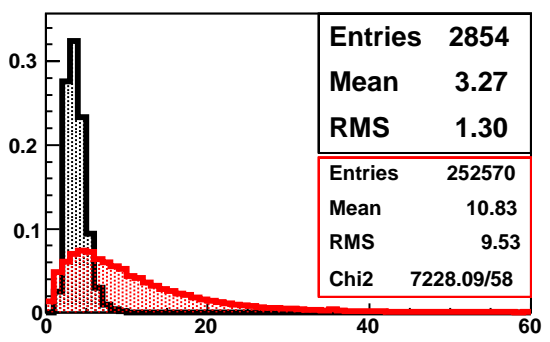

Plane of Shower Max, 7.0 GeV

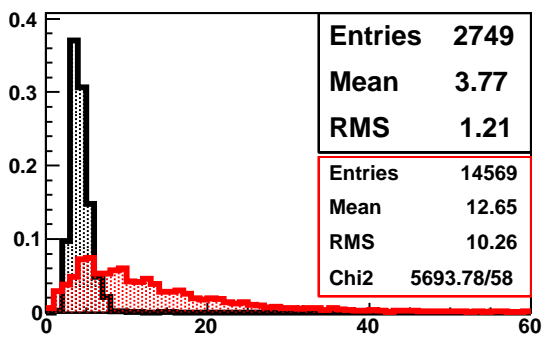

Figure 7.13: Comparison of the plane of shower maximum in an event between electrons and and pions. Black distributions represent electrons, red distributions represent pions. The momenta shown are $200 \mathrm{MeV}, 1 \mathrm{GeV}, 2 \mathrm{GeV}, 3.2 \mathrm{GeV}, 5 \mathrm{GeV}$, and $7 \mathrm{GeV}$. 
Signal Ratio in Plane of Shower Max, $0.2 \mathrm{GeV}$

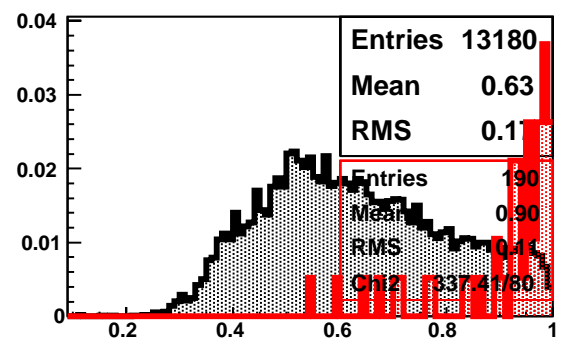

Signal Ratio in Plane of Shower Max, $2.0 \mathrm{GeV}$

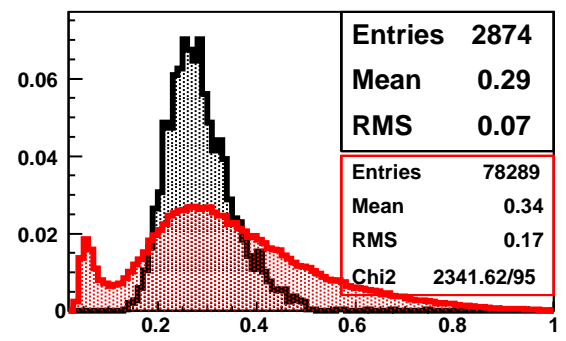

Signal Ratio in Plane of Shower Max, 5.0 GeV

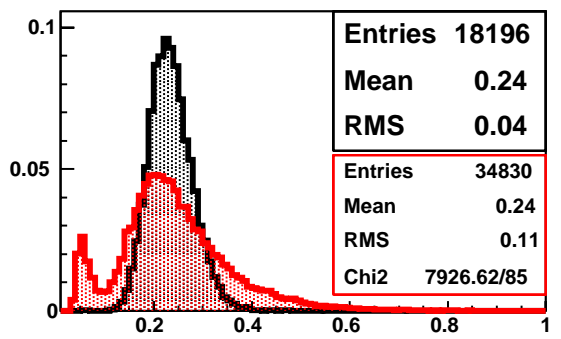

Signal Ratio in Plane of Shower Max, 1.0 GeV

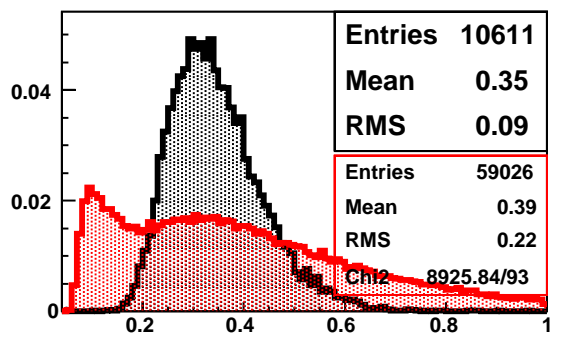

Signal Ratio in Plane of Shower Max, 3.2 GeV

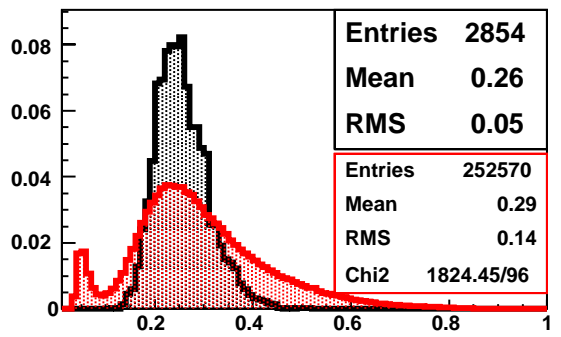

Signal Ratio in Plane of Shower Max, 7.0 GeV

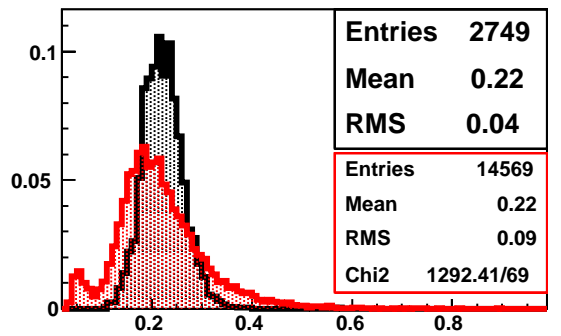

Figure 7.14: Comparison of the portion of energy deposited in plane of shower max in an event between electrons and and pions. Black distributions represent electrons, red distributions represent pions. The momenta shown are $200 \mathrm{MeV}, 1 \mathrm{GeV}, 2 \mathrm{GeV}$, $3.2 \mathrm{GeV}, 5 \mathrm{GeV}$, and $7 \mathrm{GeV}$. 


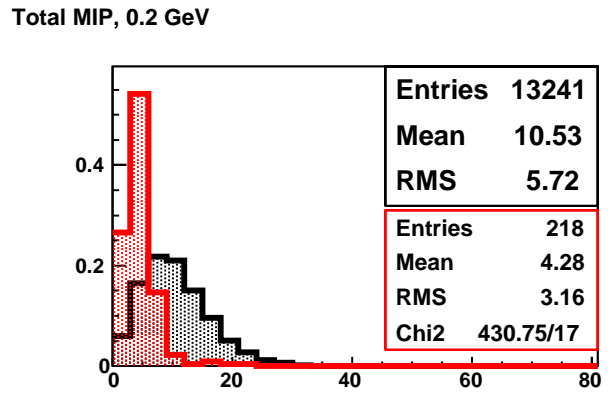

Total MIP, $2.0 \mathrm{GeV}$

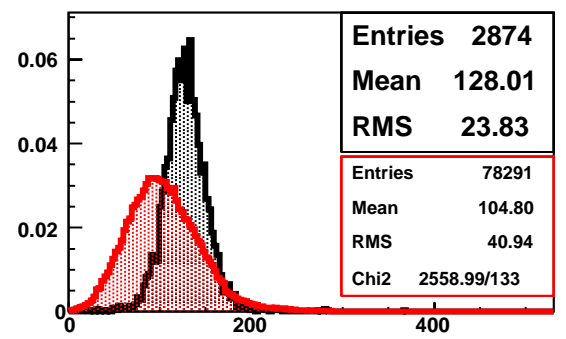

Total MIP, $5.0 \mathrm{GeV}$

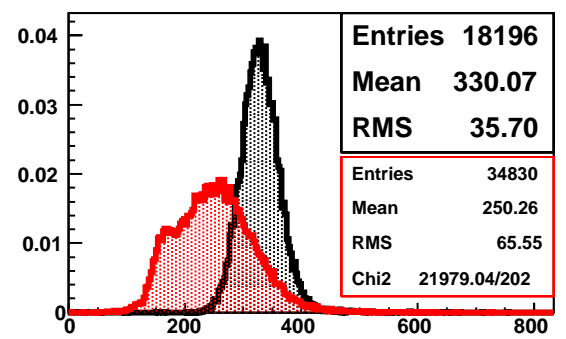

Total MIP, $1.0 \mathrm{GeV}$

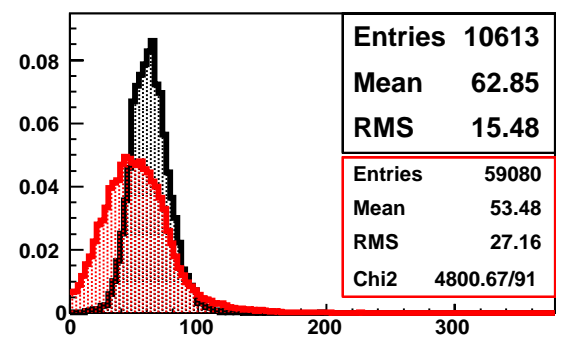

Total MIP, $3.2 \mathrm{GeV}$

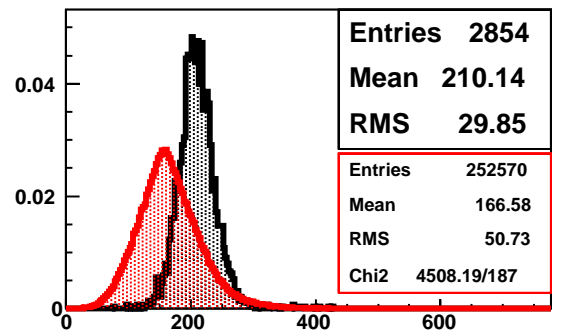

Total MIP, 7.0 GeV

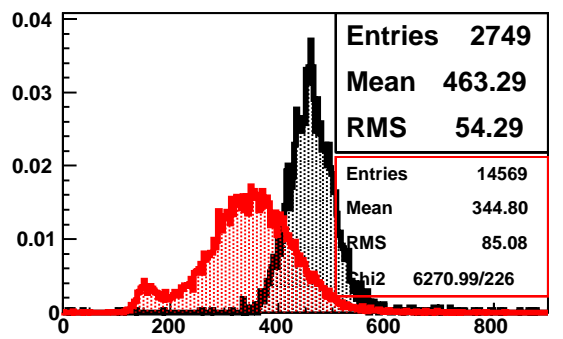

Figure 7.15: Comparison of response between electrons and and pions. Black distributions represent electrons, red distributions represent pions. The momenta shown are $200 \mathrm{MeV}, 1 \mathrm{GeV}, 2 \mathrm{GeV}, 3.2 \mathrm{GeV}, 5 \mathrm{GeV}$, and $7 \mathrm{GeV}$. 
Last Plane, 1.5 PE cut

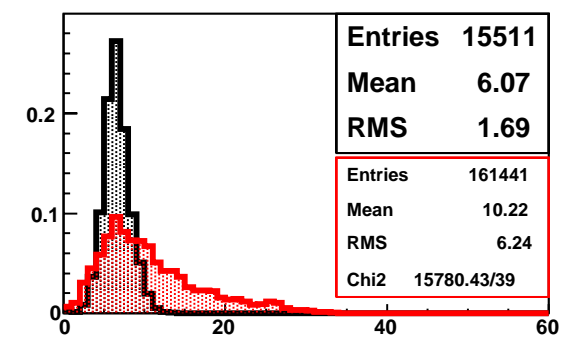

Nhit Strips, 1.5 PE Cut

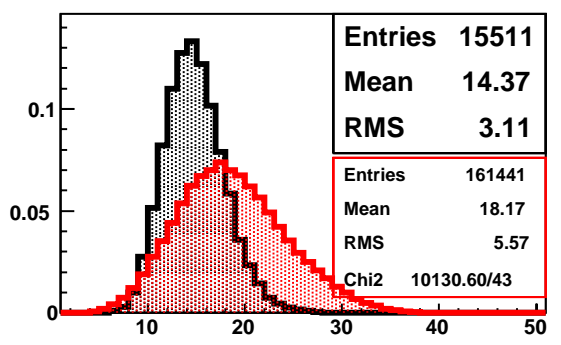

Plane of Shower Max

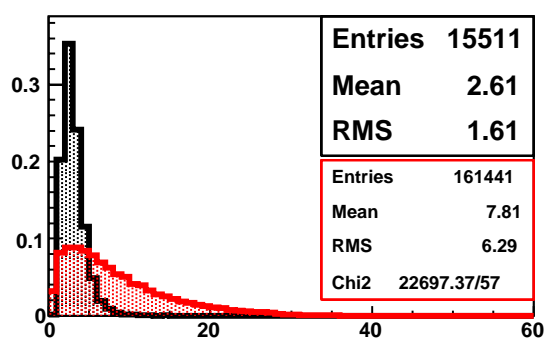

Nhit Strips

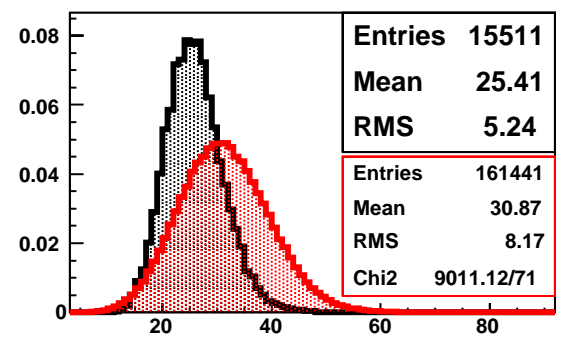

Mip Weighted Radius, 1.5 PE Cut

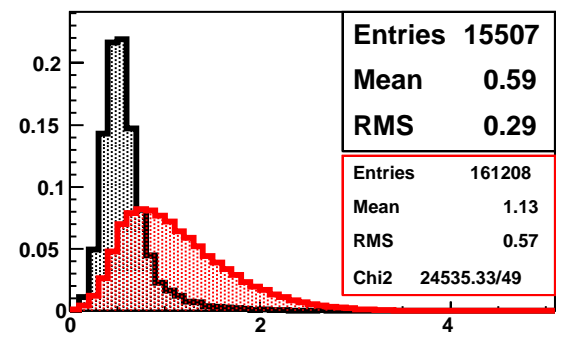

Signal Ratio in Plane of Shower Max

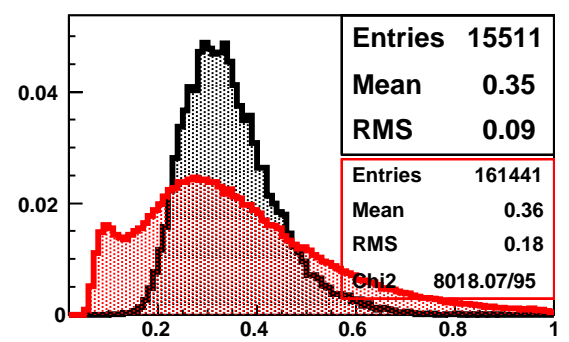

Figure 7.16: Comparison of topological properties between electrons and pions for events with response consistent with a $1 \mathrm{GeV}$ electron. Quantities shown are last plane, number of hit strips, number of hit strips above $1.5 \mathrm{PE}$, radius, plane of shower max, and portion of energy deposited in plane of shower max. 


\section{Chapter 8}

\section{Response and Resolution}

The ultimate goal of the calibration detector is to derive the detector response to showers in comparison to the response initiated by muons. In this chapter we discuss the procedure used to obtain the response at a given momentum, then parameterize the response of the detector to electrons and positrons as a function of the beam momentum.

\subsection{Deriving the Response}

In order to determine the detector response to electromagnetic interactions, electron events are selected using the criteria given in Chapter 5. The signal in each channel is converted to MIPs, and for each event, the signal in all channels from plane 1 through 60 is summed to obtain the total response. Plane 0 is used to cut on the position of the particle as it enters the detector, and, since plane 0 is not shielded in normal operation, the signal from this plane is not summed in the total response. The effect of ignoring the signal in plane 0 is explored further in Chapter 9.

To describe the response and resolution of the CalDet to electromagnetic interactions, a fit was performed on each of the total energy deposition distributions 
to extract a mean and RMS. A "continuous" Poisson distribution was used to fit the data [47]. The fitting function is given by

$$
\begin{aligned}
f\left(\frac{E}{S}, M\right) & =C \frac{M^{\frac{E}{S}} e^{-M}}{\Gamma\left(\frac{E}{S}+1\right)} \\
\mu & =S \times M \\
\sigma & =S \sqrt{M} \\
\text { Resolution } & =\frac{\sigma}{\mu}=\frac{1}{\sqrt{M}} .
\end{aligned}
$$

There are three fit parameters, $S, M$, and $C$. The mean, RMS, and resolution of the distribution can be expressed in terms of the fit parameters as given above. The fits were performed using the log likelihood method over a restricted range. The bounds of the range were set so that all but the $4 \%$ of entries in the tails of the distribution were included in the fit. Figures 8.1 through 8.4 show the CalDet response in MIPs for electrons and positrons. The top panel in each plot shows the response distributions for a selection of beam momenta. The bottom left panel shows the mean response divided by the beam momentum versus the beam momentum. The bottom right plot shows $\frac{\sigma}{\mu} \sqrt{P}$ versus $\sqrt{P}$, giving the electromagnetic energy resolution of the CalDet.

At first, a Gaussian fit was employed, however, the lower energy runs displayed an asymmetrical shape that can not be reproduced by a Gaussian. Figure 8.5 show examples of the Gaussian and Poisson fits to a $600 \mathrm{MeV}$ positron sample. The Poisson curve reproduces the shape of the data distribution nicely, giving a reduced $\chi^{2}$ of 2.0 compared to the reduced $\chi^{2}$ from the Gaussian fit of 8.9.

Figure 8.6 compares the Poisson fits to the Gaussian fits as a function of beam momentum. As shown in the top panel, the Poisson fit gives better $\chi^{2}$ values at lower beam momentum and equivalent values of $\chi^{2}$ at higher momenta. The second panel shows that the mean total energy deposited derived from the Poisson fit tends to be larger than the mean given by a Gaussian fit. The effect is on the 
Total MIP

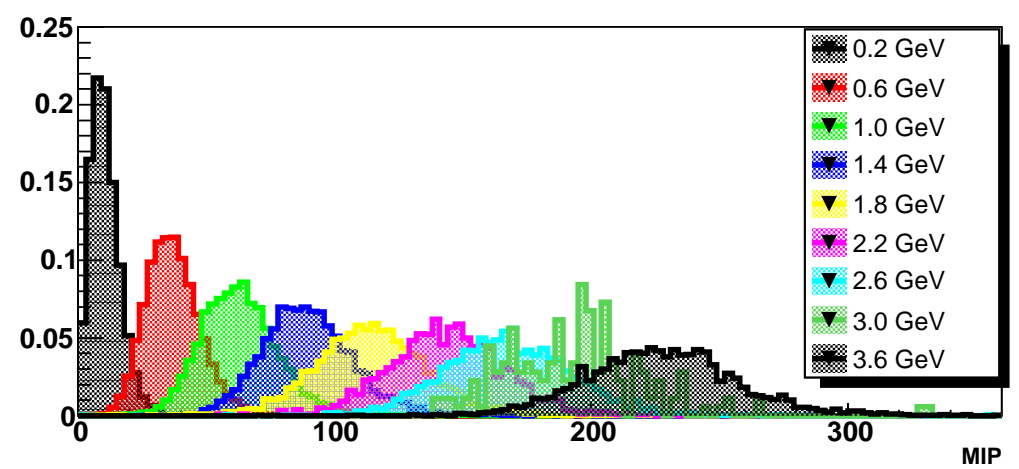

MIP/P vs. P

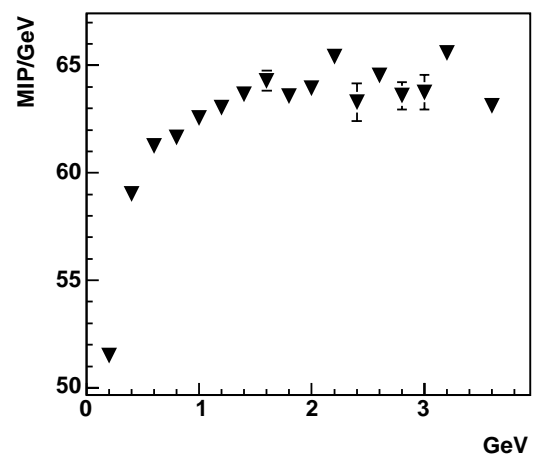

Resolution $\sqrt{\mathbf{P}}$ vs. $\sqrt{\mathbf{P}}$

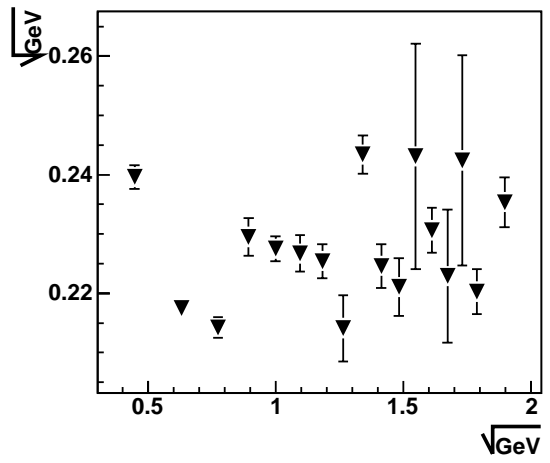

Figure 8.1: Total MIP distributions for T11, 2002 positive momenta runs. In the top plot each color represents a different beam momentum. The lower left plot shows the mean total MIP divided by the beam momentum versus the beam momentum. The lower right plot shows $\left(\frac{\sigma}{\mu}\right) \sqrt{P}$ versus $\sqrt{P}$. 
Total MIP

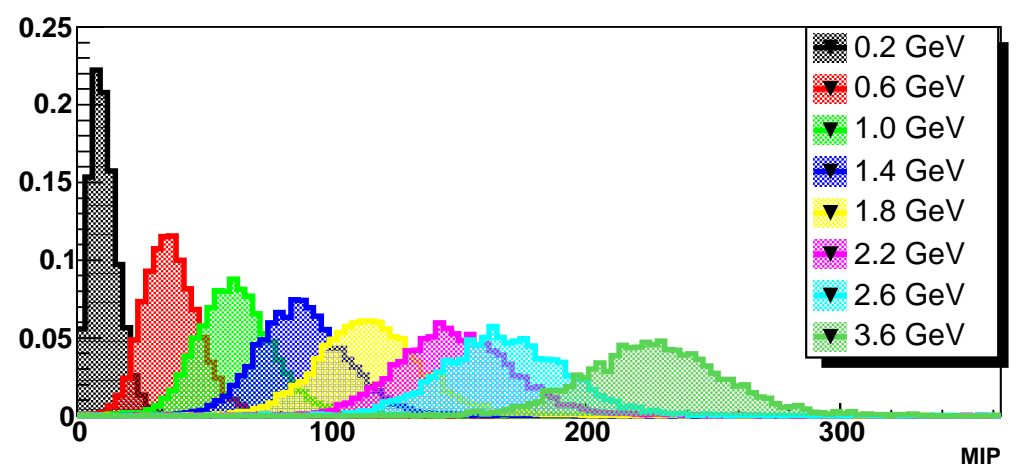

MIP/P vs. P

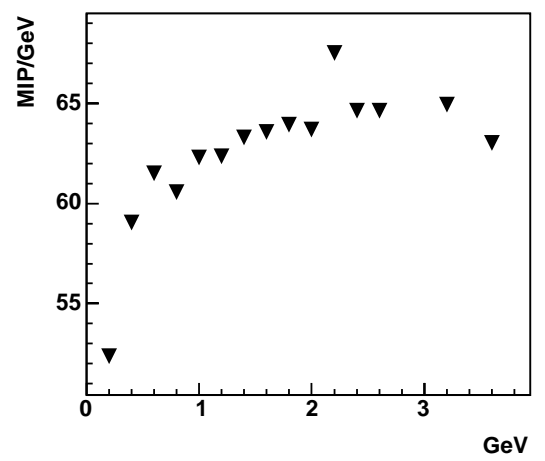

Resolution $\sqrt{\mathbf{P}}$ vs. $\sqrt{\mathbf{P}}$

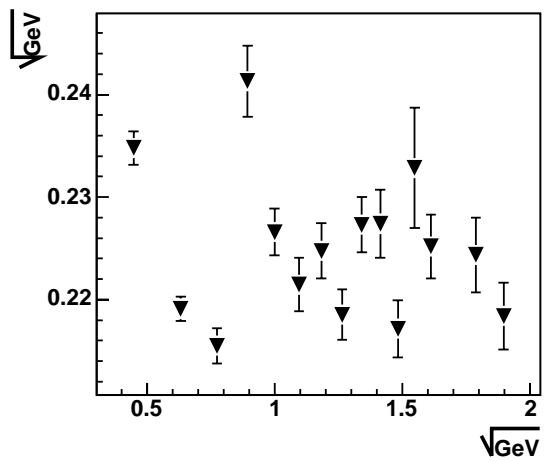

Figure 8.2: Total MIP distributions for T11, 2002 negative momenta runs. In the top plot each color represents a different beam momentum. The lower left plot shows the mean total MIP divided by the beam momentum versus the beam momentum. The lower right plot shows $\left(\frac{\sigma}{\mu}\right) \sqrt{P}$ versus $\sqrt{P}$. 
Total MIP

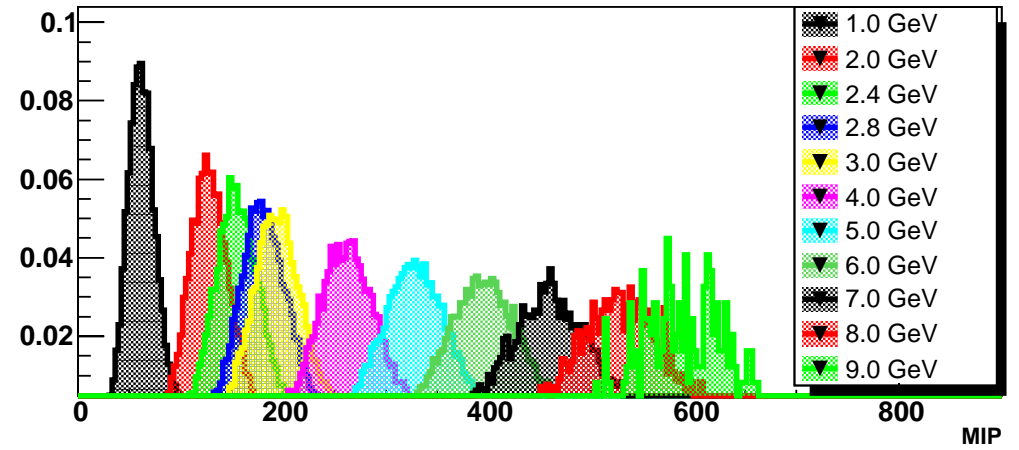

MIP/P vs. P

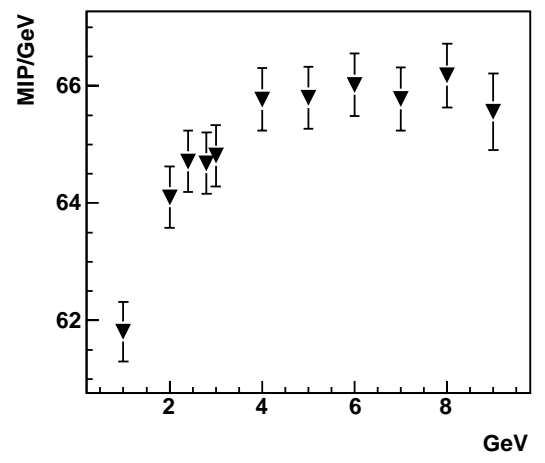

Resolution $\sqrt{\mathbf{P}}$ vs. $\sqrt{\mathbf{P}}$

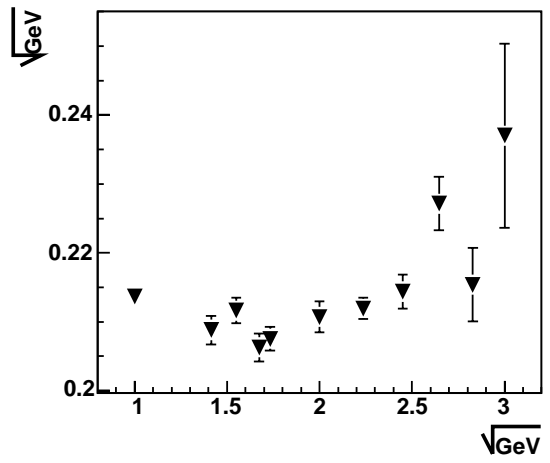

Figure 8.3: Total MIP distributions for T7, 2002 positive momenta runs. In the top plot, each color represents a different beam momentum. The lower left plot shows the mean total MIP divided by the beam momentum versus the beam momentum. The lower right plot shows $\left(\frac{\sigma}{\mu}\right) \sqrt{P}$ versus $\sqrt{P}$. 
Total MIP

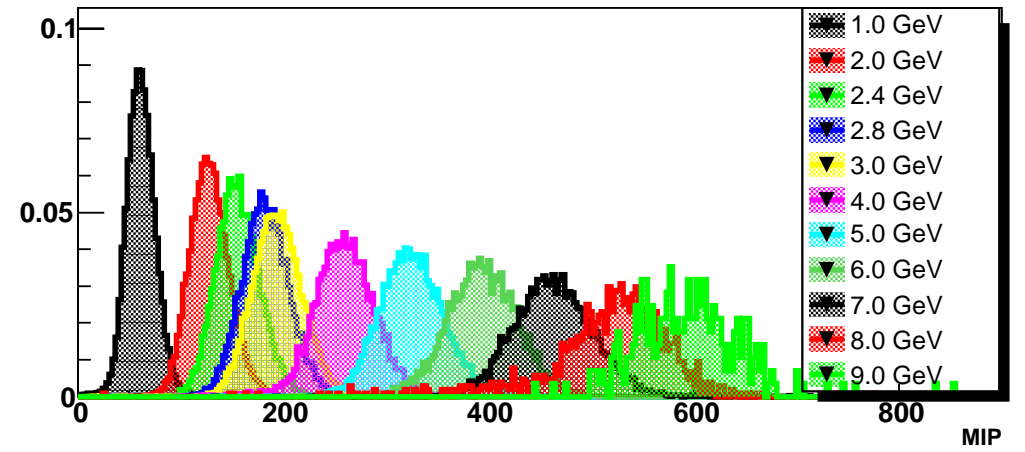

MIP/P vs. P

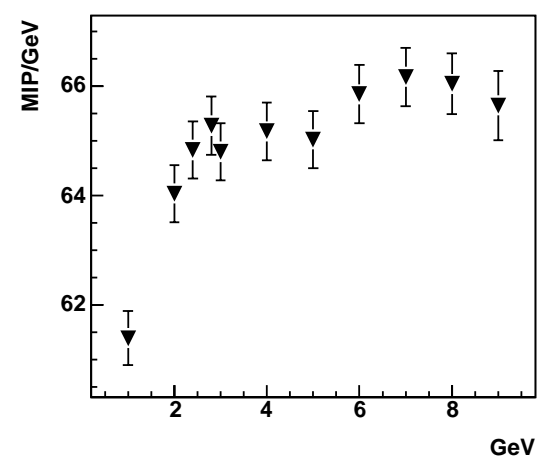

Resolution $\sqrt{\mathbf{P}}$ vs. $\sqrt{\mathbf{P}}$

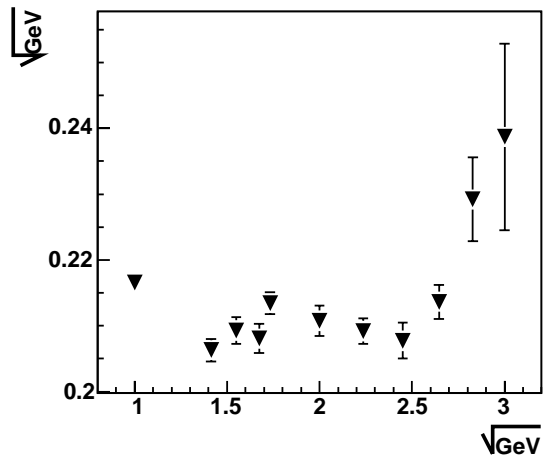

Figure 8.4: Total MIP distributions for T7, 2002 negative momenta runs. In the top plot, each color represents a different beam momentum. The lower left plot shows the mean total MIP divided by the beam momentum versus the beam momentum. The lower right plot shows $\left(\frac{\sigma}{\mu}\right) \sqrt{P}$ versus $\sqrt{P}$. 

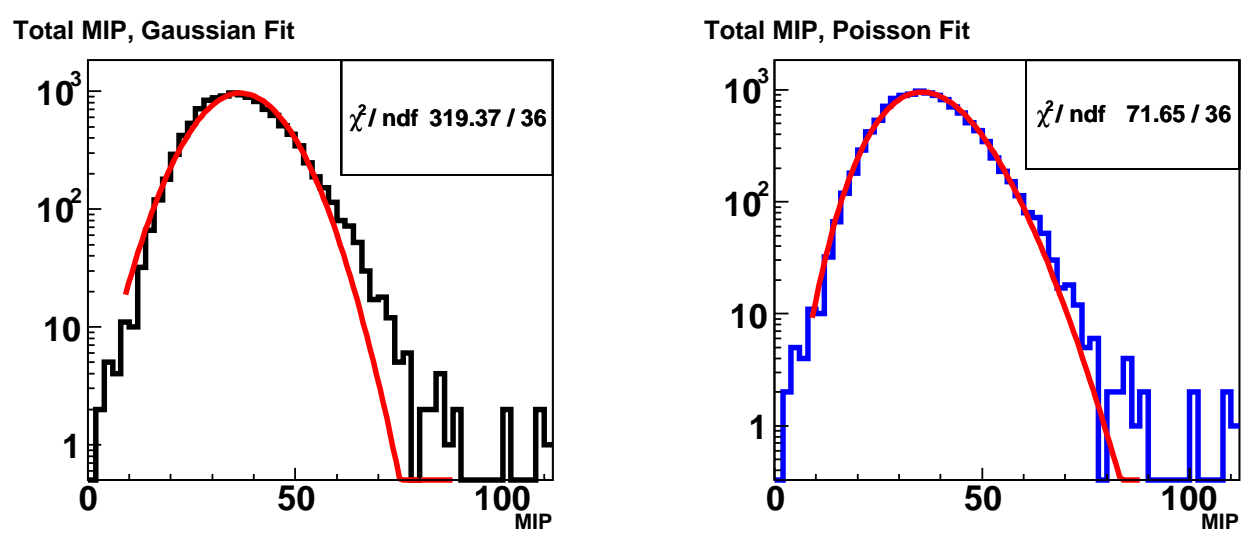

Figure 8.5: Total energy deposited in units of MIPs from a $600 \mathrm{MeV}$ positron run. To the left, the distribution is fitted with a Gaussian, producing a reduced $\chi^{2}$ of 8.9. To the right the distribution is fitted with a continuous Poisson function, giving a reduced $\chi^{2}$ of 2.0 .

order of a few percent at $200 \mathrm{MeV}$, but becomes negligible above 1-2 GeV. Finally, the last panel shows the difference in the resolution as obtained by the two different fits.

\subsection{Correcting for Upstream Energy Loss}

As discussed in Chapter 6, the energy lost by particles traveling through the material upstream of the detector must be taken into account when determining the detector response. In order to do so, the detector response was simulated with particles traveling through the upstream beam material and compared to the detector response due to particles created at the front face of CalDet. Figures 8.7 and 8.8 show this comparison for the T11 2002 and T7 2002 geometries respectively. Fitting the relation with a straight line gives a parameterized form of the correction for upstream energy loss. If $D$ is the mean response given by the Poisson fit to the data, $p_{1}$ the slope of the fitted correction, and $p_{0}$ is the y-intercept, then the corrected 

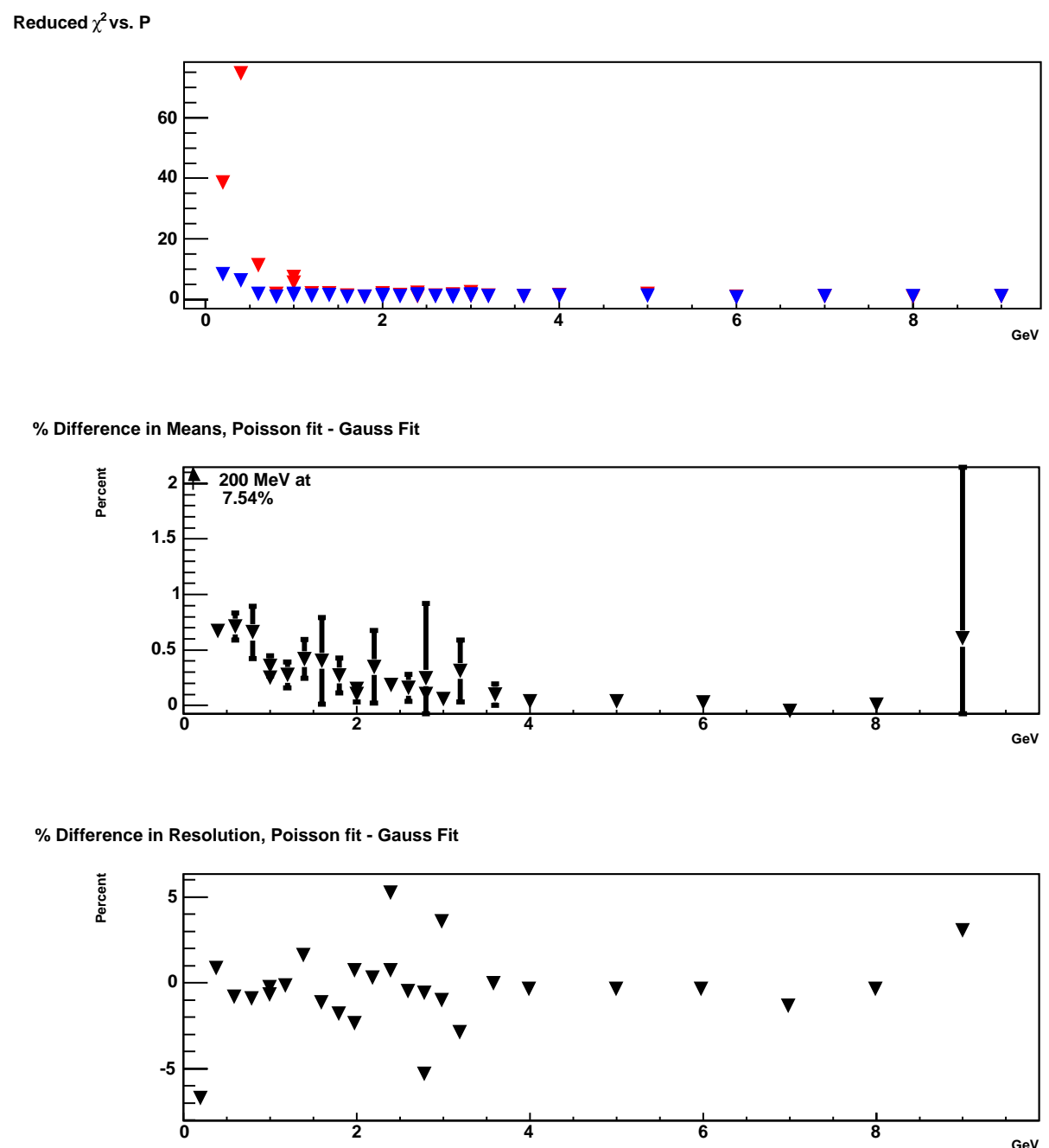

Figure 8.6: Top plot compares $\chi^{2}$ from Gaussian fit to total energy distribution (in red) to $\chi^{2}$ from Poisson fit (in blue) as a function of beam momentum. Middle plot shows the relative difference in the mean as given by the Poisson fit to the mean as given by the Gaussian fit as a function of beam momentum. Bottom plot shows the relative difference in the resolution as given by the Poisson fit minus the Gaussian fit versus the beam momentum. Above $1 \mathrm{GeV}$ the reduced $\chi^{2}$ averages about 1.4 for both fits. The average difference in the response is less than $0.1 \%$, while the average difference in resolution is $0.4 \%$. 
Simulated Response,with vs. w/o BeamSim

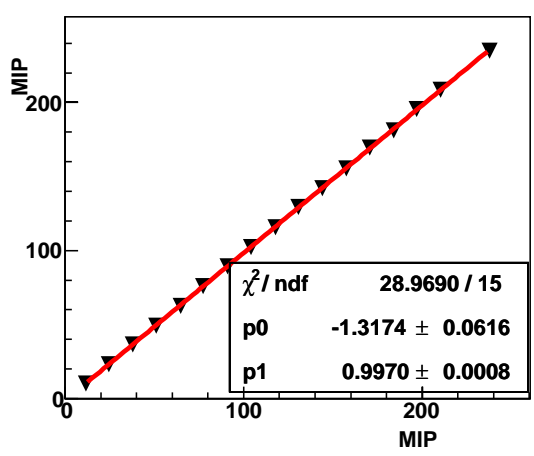

Response Ratio v. P, Fit

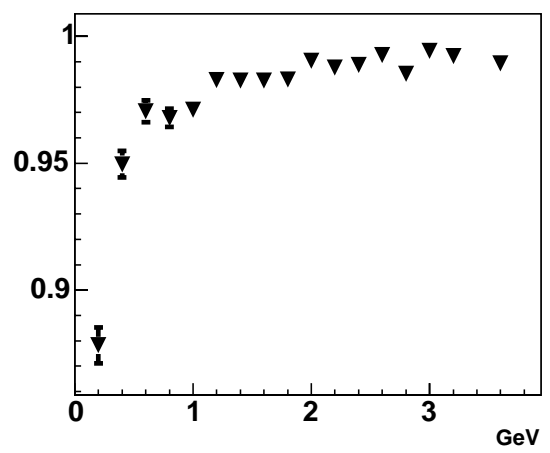

Figure 8.7: The upstream energy loss correction for T11, 2002. The left plot shows the simulated detector response including the energy loss determined using the beam line simulation versus the detector response with no energy loss. The fit parameters give the correction to be applied to the data. The right plot shows the ratio of the response with the energy losses included to the response without energy loss versus beam momentum.

response is given by

$$
D^{\prime}=\frac{D-p_{0}}{p_{1}}
$$

Figures 8.9 through 8.12 show the response before and after the correction for the upstream energy loss. The correction tends to increase the slope and y-intercept of the response parameterization and improves the $\chi^{2}$ for the straight line fit.

\subsection{Response and Resolution Versus Momentum}

Figure 8.13 shows the total MIP versus beam momentum for all 2002 runs. For a given beam momentum, the response from positive runs agrees within error with the response from negative runs. Moreover, the response measured in T11 agrees with the response measured in $\mathrm{T} 7$ at the same momentum. To extract the detector response as a function of beam momentum, a combined fit was performed over all the data from the 2002 beam runs, with the exception of the $\pm 3.6 \mathrm{GeV}$ and $\pm 2.2 \mathrm{GeV}$ 
Simulated Response, with vs. w/o BeamSim

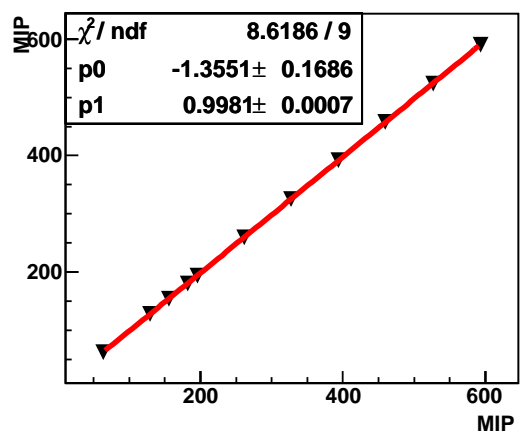

Response Ratio v. P, Fit

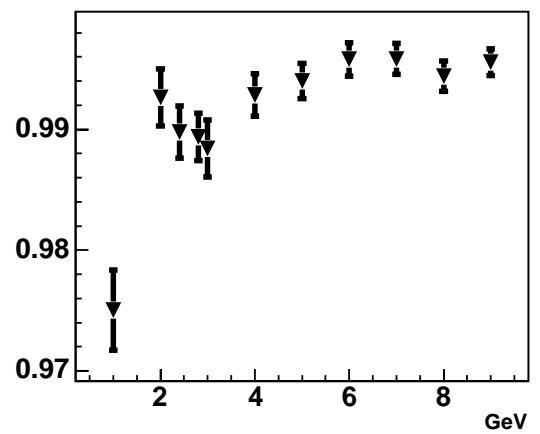

Figure 8.8: The upstream energy loss correction for T7, 2002. The left plot shows the simulated detector response including the energy loss determined using the beam line simulation versus the detector response with no energy loss. The fit parameters give the correction to be applied to the data. The right plot shows the ratio of the response with the energy losses included to the response without energy loss versus beam momentum

T11 points. The $\pm 3.6 \mathrm{GeV}$ points are at the edge of the capabilities of the T11 beam line. The $\pm 2.2 \mathrm{GeV}$ points are both deviate sufficiently from the rest of the data that it is suspected that one or more beam magnet or collimators settings were incorrect during these runs. From a combined fit over all the 2002 data samples, the detector response to electromagnetic interactions is

$$
(65.77 \pm 0.08) \frac{\mathrm{MIP}}{\mathrm{GeV}} \times \mathrm{P}-(1.46 \pm 0.06) \mathrm{MIP}
$$

Figure 8.14 shows the resolution of the electromagnetic response in each beam line. The plot shows $\frac{\sigma}{\mu}$ versus $\mathrm{P}$ for all runs taken in 2002. The resolution is parameterized using the function:

$$
\frac{\sigma}{\mu}=\frac{n}{P} \oplus \frac{m}{\sqrt{P}} \oplus k
$$

The fit curve corresponds to the best fit of the data to equation 8.7 where $k, m$, and $n$ are fit parameters. From the fit parameters, one sees that the electromagnetic 
Total MIP vs. $\mathbf{P}$

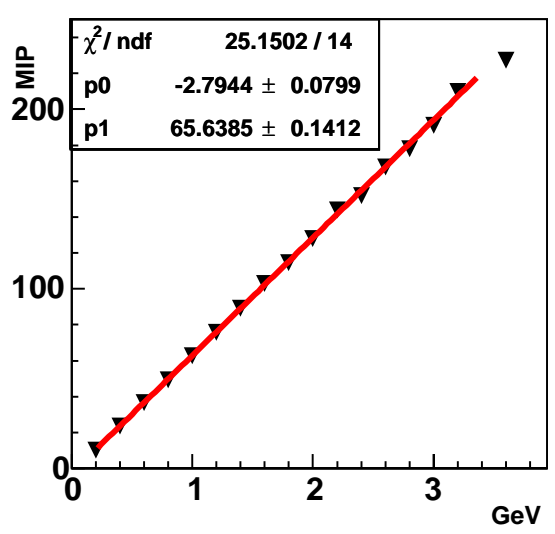

Total MIP (corrected) vs. P

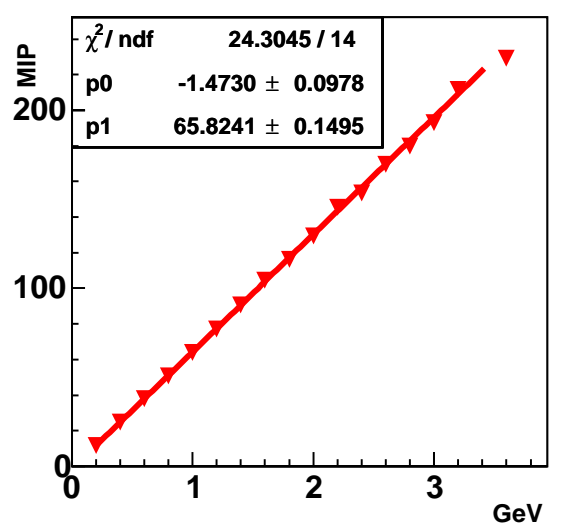

MIP/P vs. P

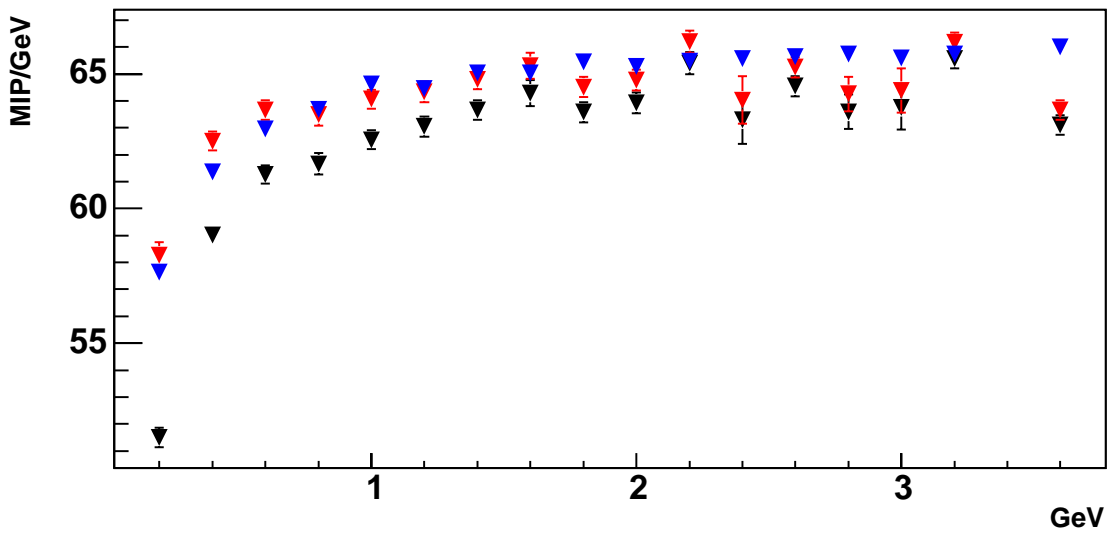

Figure 8.9: Top left shows the raw response versus momentum for T11, 2002 positrons. The top right plot shows the response after correcting for upstream energy loss. The bottom plot shows the response divided by momentum versus momentum, before the correction in black, after correction in red, and Monte Carlo without energy loss in blue. 
Total MIP vs. P

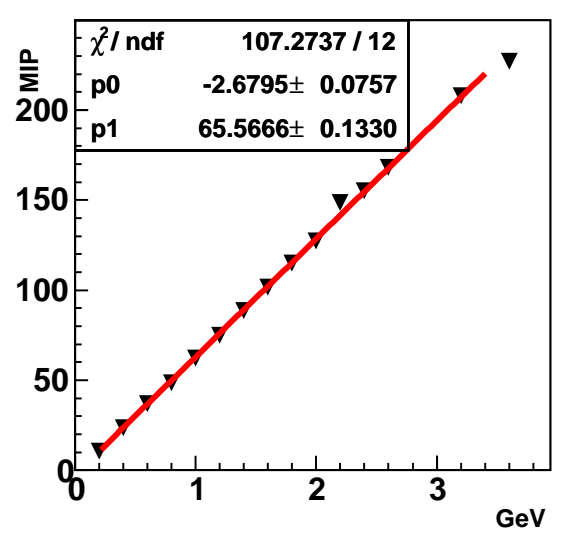

Total MIP (corrected) vs. P

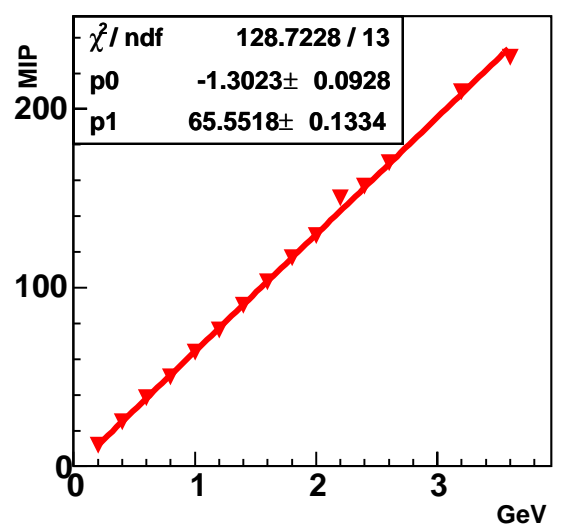

MIP/P vs. P

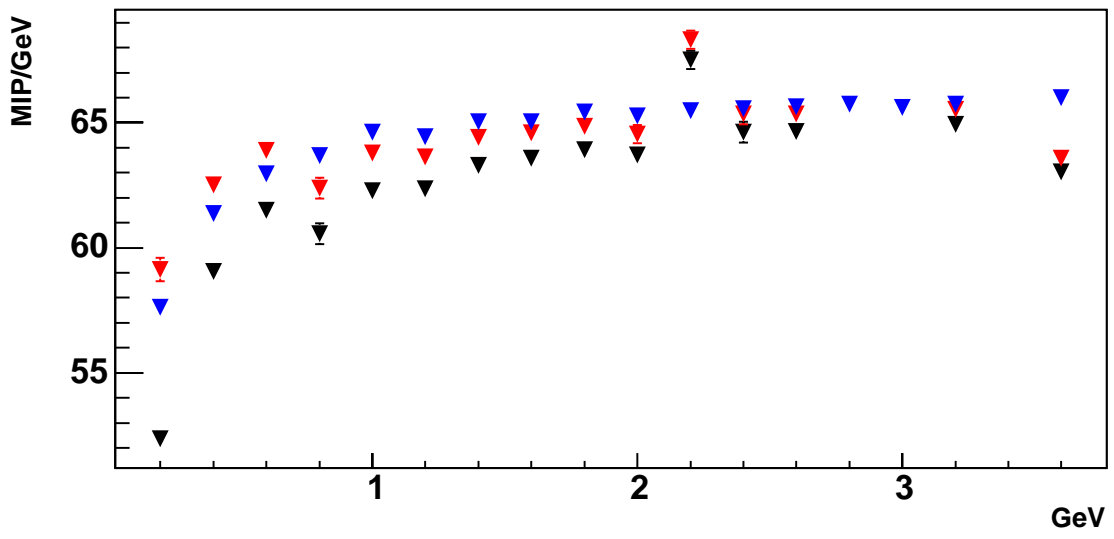

Figure 8.10: Top left shows the raw response versus momentum for T11, 2002 electrons. The top right plot shows the response after correcting for upstream energy loss. The bottom plot shows the response divided by momentum versus momentum, before the correction in black, after correction in red, and Monte Carlo without energy loss in blue. 
Total MIP vs. P

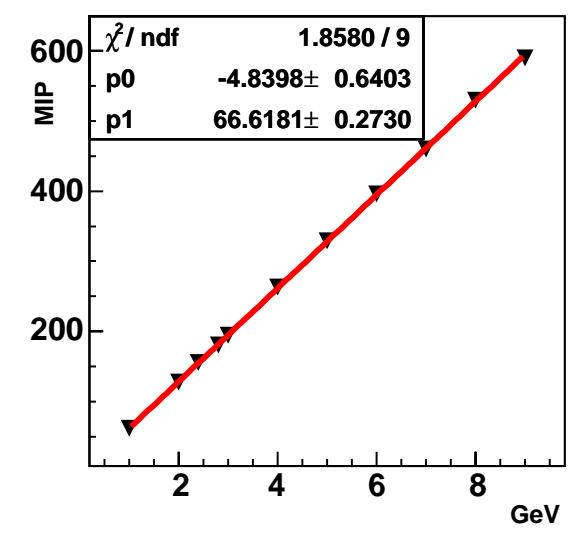

Total MIP (corrected) vs. P

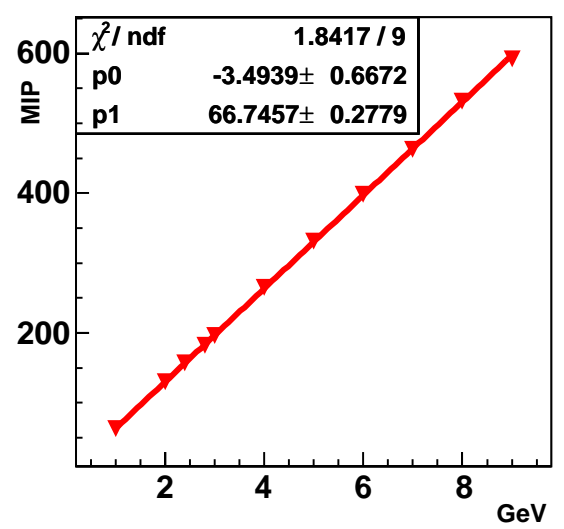

MIP/P vs. P

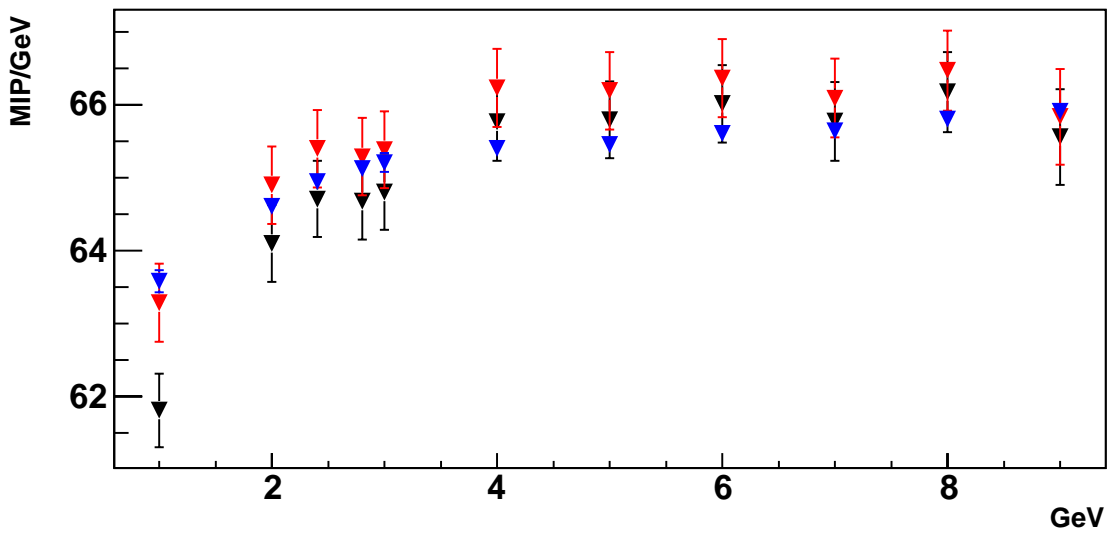

Figure 8.11: Top left shows the raw response versus momentum for T7, 2002 positrons. The top right plot shows the response after correcting for upstream energy loss. The bottom plot shows the response divided by momentum versus momentum, before the correction in black, after correction in red, and Monte Carlo without energy loss in blue. 
Total MIP vs. P

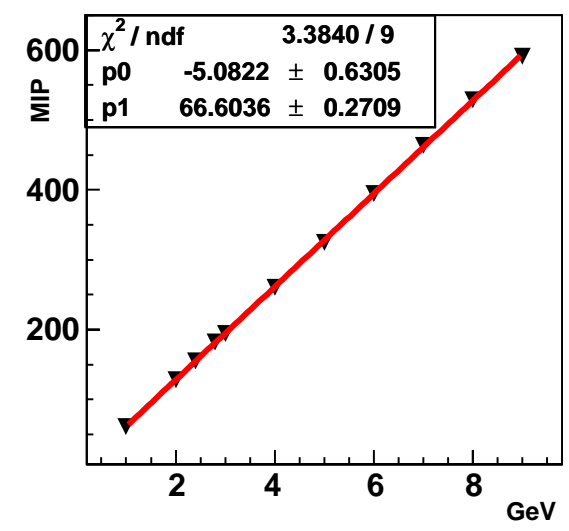

Total MIP (corrected) vs. P

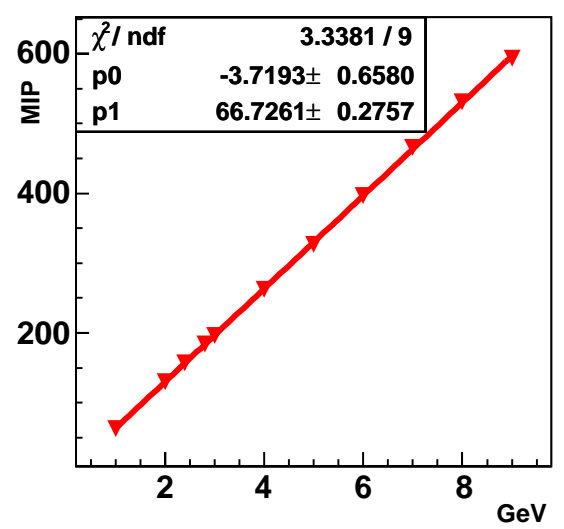

MIP/P vs. $\mathbf{P}$

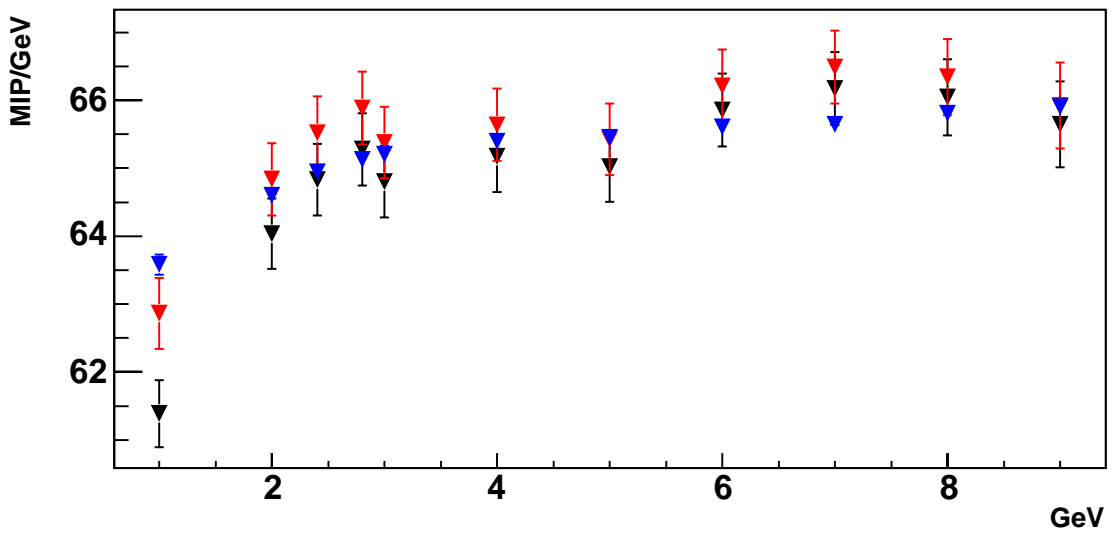

Figure 8.12: Top left shows the raw response versus momentum for T7, 2002 electrons. The top right plot shows the response after correcting for upstream energy loss. The bottom plot shows the response divided by momentum versus momentum, before the correction in black, after correction in red, and Monte Carlo without energy loss in blue. 


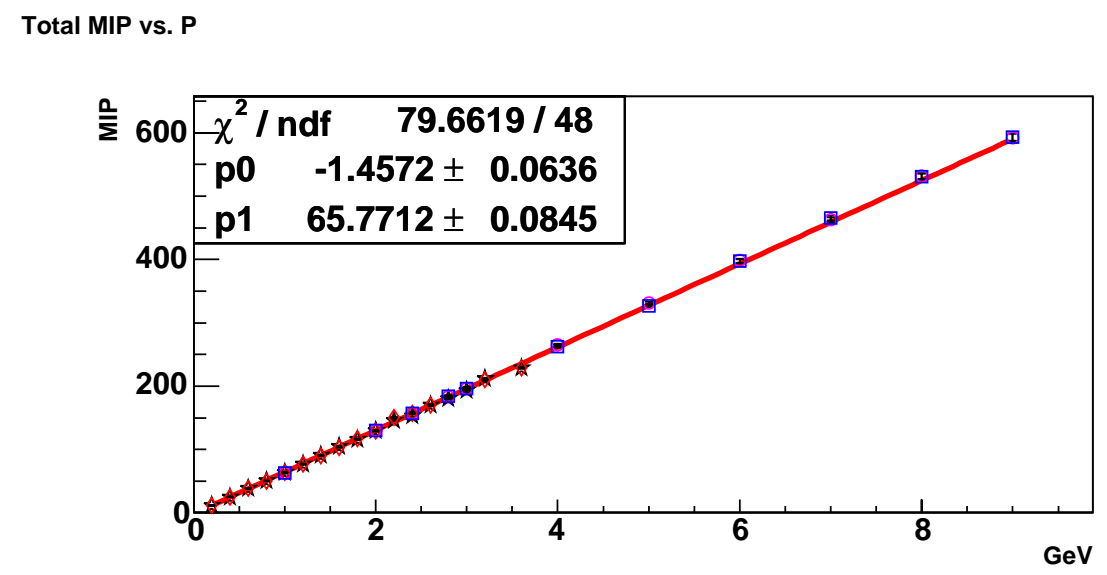

MIP/P vs. P

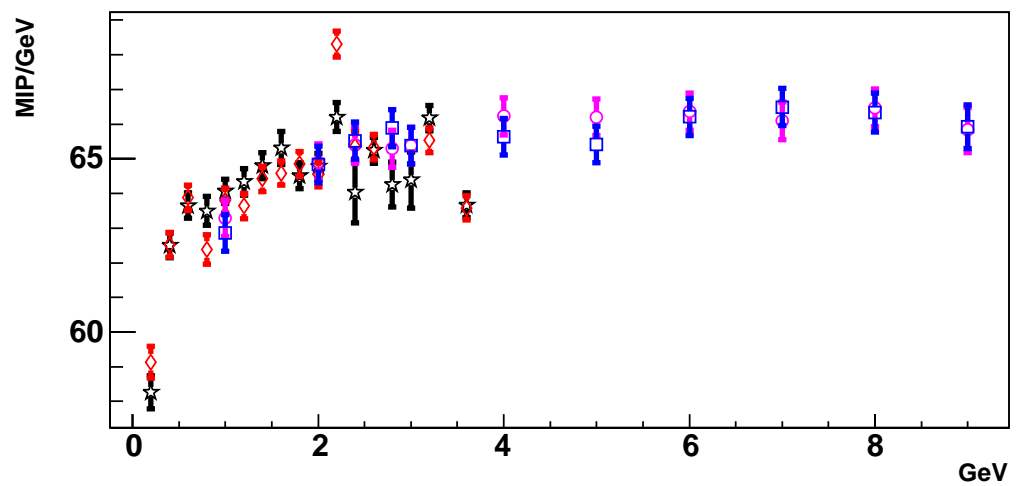

Figure 8.13: The top plot shows the detector response in MIPs versus the beam momentum. Black stars come from T11, 2002 positives, red diamonds from T11, 2002 negatives, blue squares from T7, 2002 positive runs and pink circles from T7, 2002 negative runs. All data points, except $\pm 3.6 \mathrm{GeV}$ and $\pm 2.2 \mathrm{GeV}$, are included in the straight line fit. The lower plot shows total MIP divided by beam momentum versus beam momentum. 
Resolution vs. $\mathbf{P}$

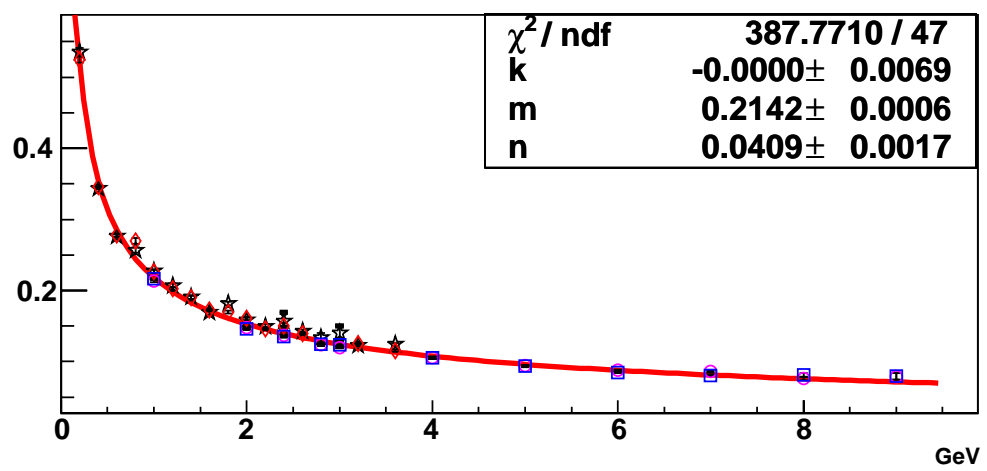

Figure 8.14: The electromagnetic energy resolution versus beam momentum. Black stars come from T11, 2002 positives, red diamonds from T11, 2002 negatives, blue squares from T7, 2002 positive runs and pink circles from T7, 2002 negative runs. All data points, except $\pm 3.6 \mathrm{GeV}$ and $\pm 2.2 \mathrm{GeV}$, are included in the fit which is given by a function of the form $\frac{\sigma}{\mu}=\frac{n}{P} \oplus \frac{m}{\sqrt{P}} \oplus k$.

energy resolution is

$$
\frac{\sigma}{\mu}=\frac{21.42 \pm 0.06 \%}{\sqrt{P}} \oplus \frac{4.1 \pm 0.2 \%}{P} .
$$

\subsection{Comparison of Near FEE Response}

At the time of writing, final drift point, linearity, and strip-to-strip calibration constants were not available for the 2003 CalDet test beam runs. The following plots are preliminary results from the 2003 data set that may change by a few percent once the final calibration constants are available.

Table 8.1 lists the subset of runs used to evaluate the 2003 detector response. Figures 8.15 through 8.17 show the total MIP distributions from selected beam momenta for the T7 2003, Near FEE with 3 m cables, the T7 2003, Near FEE with $1 \mathrm{~m}$ cables, and the T11 2003, Near FEE run configurations. Also shown is the 


\begin{tabular}{|l|l|l|l|l|}
\hline \multicolumn{3}{|c|}{ T7 } & \multicolumn{2}{c|}{ T11 } \\
\hline $\mathrm{P}(\mathrm{GeV})$ & \multicolumn{2}{|c|}{ Run Numbers } & $\mathrm{P}(\mathrm{GeV})$ & Run Numbers \\
\hline & $3 \mathrm{~m}$ Cables & $1 \mathrm{~m}$ Cables & & 1 m Cables \\
\hline 0.6 & 80300 & 90131 & 0.4 & 100247 \\
0.8 & 80304 & 90135 & 0.6 & 100253 \\
1.0 & 80007 & 90822 & 0.8 & 100257 \\
1.8 & 80277 & 90324 & 1.0 & 100203 \\
2.0 & 80284 & 90051 & 1.2 & 100171 \\
3.0 & 80013 & 90026 & 1.4 & 100144 \\
4.0 & 80045 & 90067 & 1.6 & 100261 \\
5.0 & 80180 & 90235 & 1.9 & 100306 \\
6.0 & 80212,80219 & 90167 & 2.0 & 100157 \\
7.0 & 80223,80227 & 90171 & 2.2 & 100333 \\
8.0 & 80231,80248 & 90175 & 2.8 & 100341,100342 \\
9.0 & 80254,80258 & 90183 & & \\
\hline
\end{tabular}

Table 8.1: Run numbers used for the 2003, Near FEE CalDet electron analysis

response versus beam momentum and the electromagnetic energy resolution versus beam momentum. The response measured with the Near FEE is approximately half that measured in 2002, as expected due to the single ended readout.

Figures 8.18 and 8.19 come from runs taken from the T7, 2003 Far/Near configuration. The response measured in the Far FEE is compared on an event by event basis with the response measured in the Near FEE. In Figure 8.18, the response measured in the Far Detector FEE is plotted versus the response recorded in the Near Detector FEE. Figure 8.19 shows the relative difference in total MIP versus total response. The mean difference between the two sets of electronics is $0.7 \%$ indicating that the two systems measure the same response when exposed to the same events. 
Total MIP

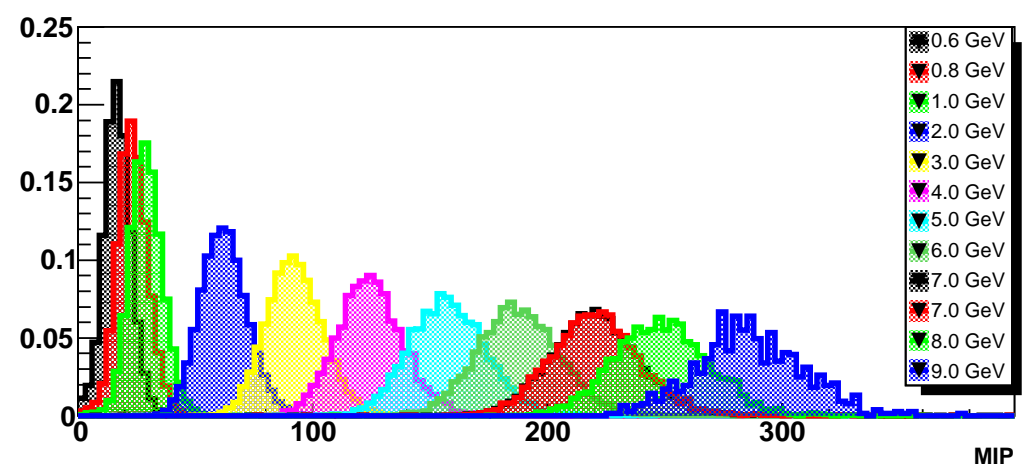

MIP/P vs. P

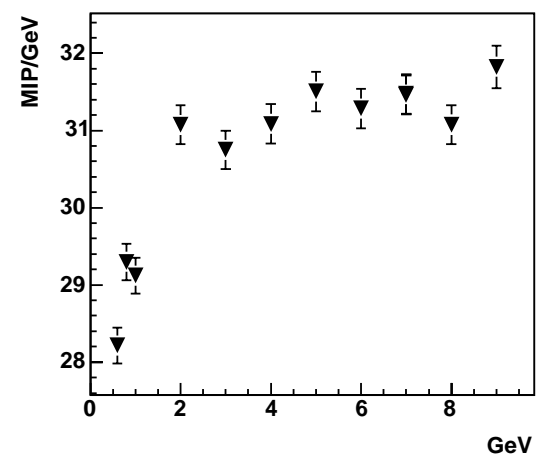

Resolution $\sqrt{\mathbf{P}}$ vs. $\sqrt{\mathbf{P}}$

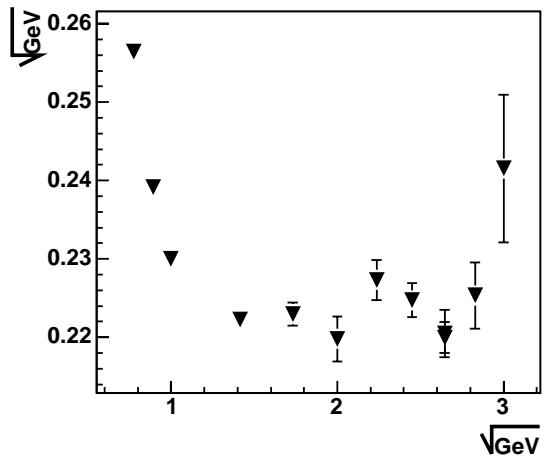

Figure 8.15: Total MIP distribution for T7, 2003, $3 \mathrm{~m}$ cables, positive momenta runs. In the top plot each color represents a different beam momentum. The lower left plot shows the total MIP divided by the beam momentum versus the beam momentum. The lower right plot shows $\left(\frac{\sigma}{\mu}\right) \sqrt{P}$ versus $\sqrt{P}$. 
Total MIP

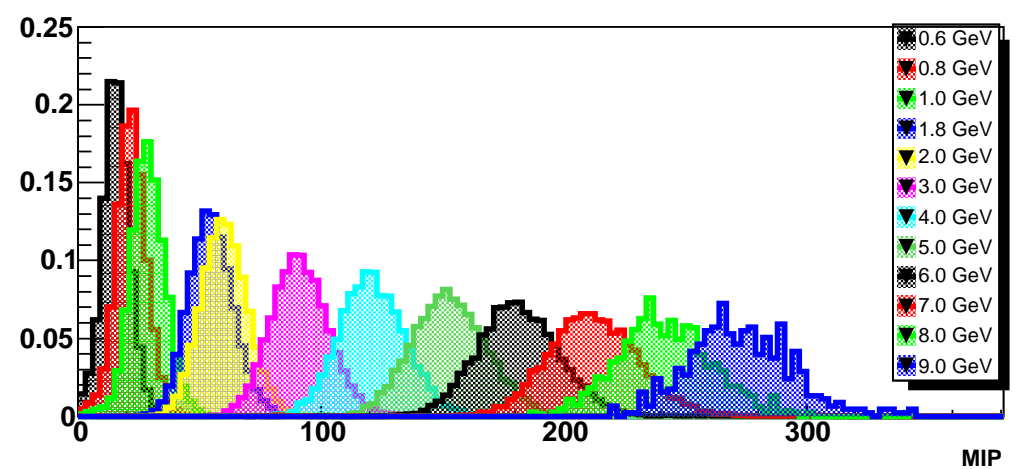

MIP/P vs. P

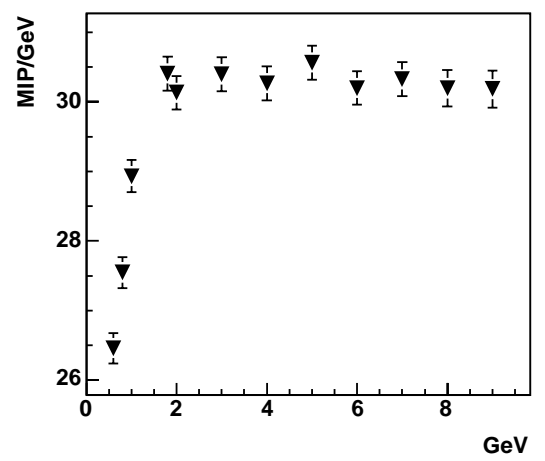

Resolution $\sqrt{\mathbf{P}}$ vs. $\sqrt{\mathbf{P}}$

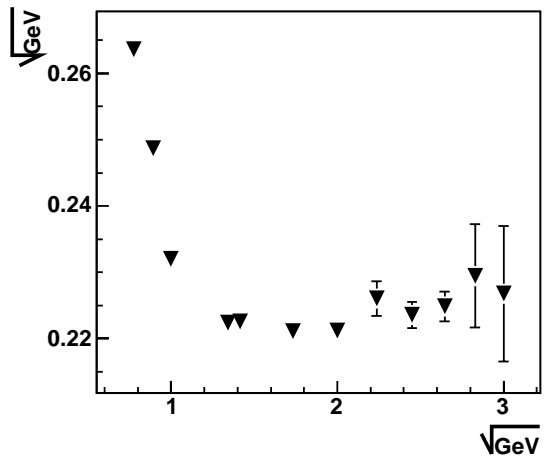

Figure 8.16: Total MIP distribution for T7, 2003, $1 \mathrm{~m}$ cables, positive momenta runs. In the top plot each color represents a different beam momentum. The lower left plot shows the total MIP divided by the beam momentum versus the beam momentum. The lower right plot shows $\left(\frac{\sigma}{\mu}\right) \sqrt{P}$ versus $\sqrt{P}$. 
Total MIP

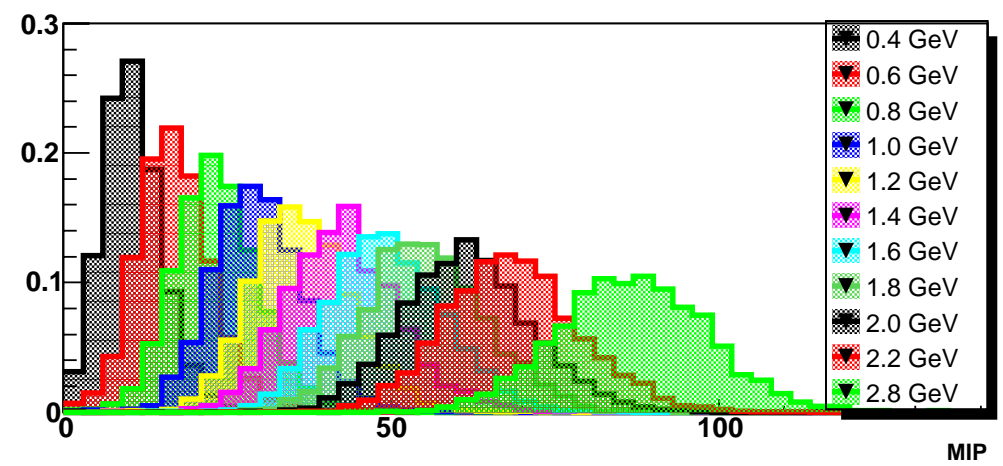

MIP/P vs. P

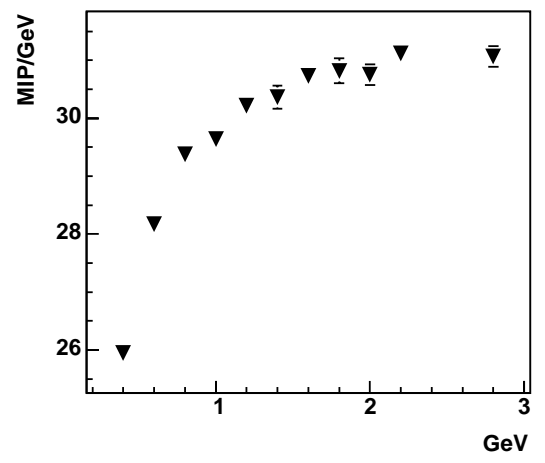

Resolution $\sqrt{\mathbf{P}}$ vs. $\sqrt{\mathbf{P}}$

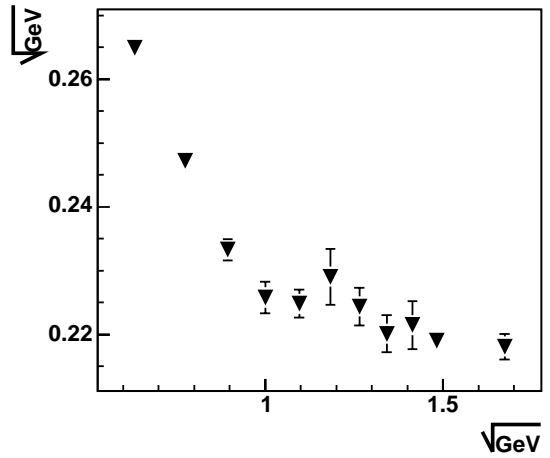

Figure 8.17: Total MIP distribution for T11, 2003 positive momenta runs. In the top plot each color represents a different beam momentum. The lower left plot shows the total MIP divided by the beam momentum versus the beam momentum. The lower right plot shows $\left(\frac{\sigma}{\mu}\right) \sqrt{P}$ versus $\sqrt{P}$. 
Far MIP vs. Near MIP

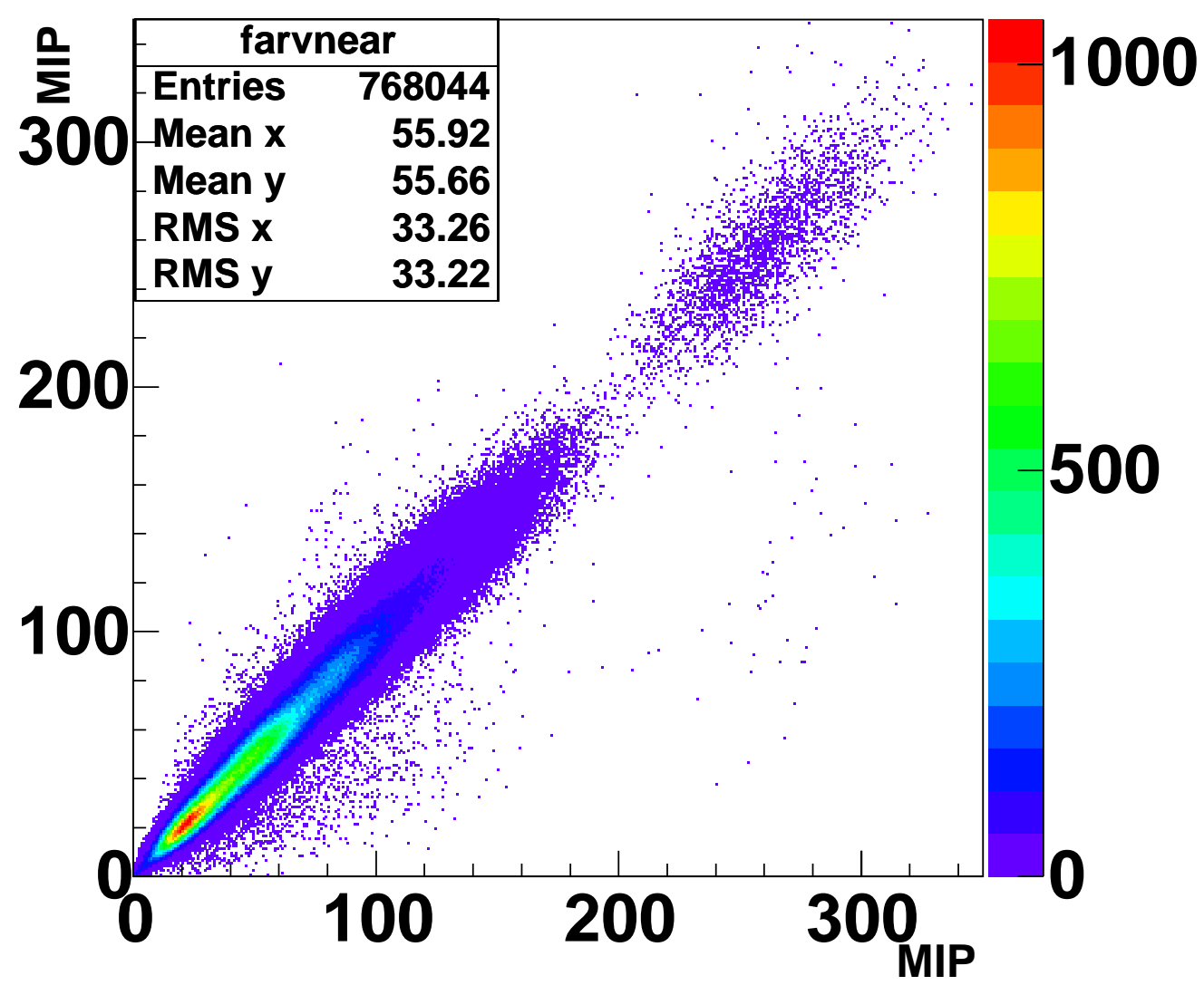

Figure 8.18: Total MIP from Far FEE versus total MIP from Near FEE. 

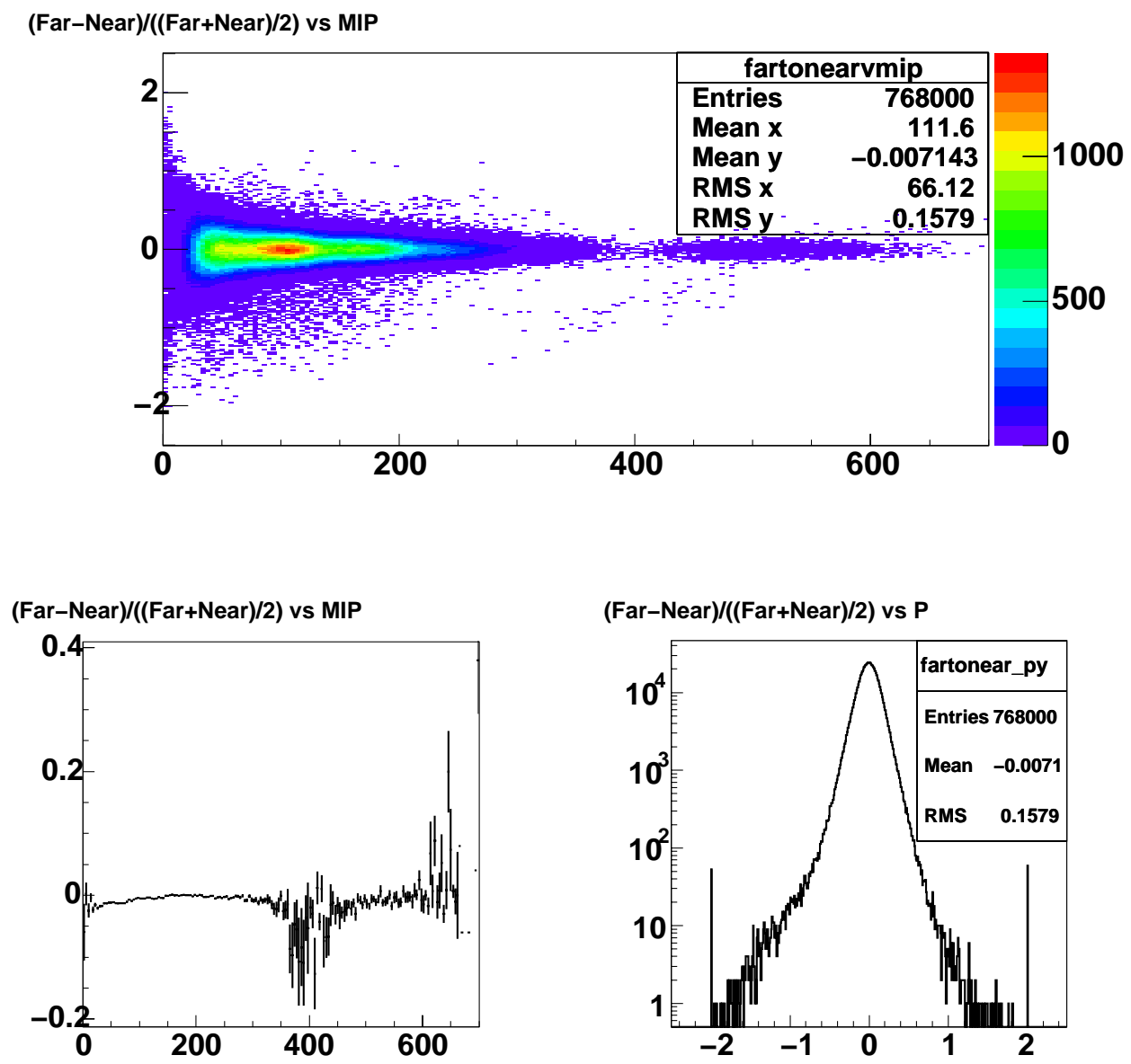

Figure 8.19: Top plot shows the relative difference in response between the Far FEE and Near FEE versus total response. Bottom left shows the profile histogram, while bottom right shows the y-projection. 


\section{Chapter 9}

\section{Looking Toward Neutrino}

\section{Interactions}

Studying the topological characteristics and response of electrons interacting in the MINOS geometry is fundamental to understanding the detectors. However, the MINOS oscillation measurements depend on the observation of secondary particles arising from the interactions of neutrinos. Electrons will be produced in the charged current interactions of electron neutrinos, but the final state produced will differ from that produced by single particle interactions in the CalDet. First, electrons produced in $\nu_{e}$ interactions will not all be produced normal to the detector planes, but instead at different angles. Second, electrons from $\nu_{e}$ events will not be produced at the front of a scintillator plane, rather, on average, in the middle of a steel plane. Finally, showers arising from $\nu_{e}$ interactions will be a superposition of hadronic and electromagnetic showers. 


\subsection{Electrons at Angled Incidence}

In the CalDet beam runs discussed thus far, the beam is normally incident to the CalDet planes; however, when an electron is produced from a neutrino interaction, it may be produced at an angle different than $90^{\circ}$ relative to the detector planes. To fully understand this effect, data were taken with the detector planes at the angles of $15^{\circ}, 30^{\circ}$, and $45^{\circ}$ relative to the beam direction. In this section we discuss the $30^{\circ}$ data taken in 2002. Figure 9.1 shows the CalDet in the T11 beam line in 2002 when the detector was angled. Due to time and space constraints, only the first 24 planes were instrumented. Figure 9.2 shows the strip versus plane distribution of hits in the angled detector. One can clearly see the angled incidence of particles in the odd planes. Figure 9.3 shows a single electron event at angled incidence. The angled incidence is evident even in the distribution of hits from a single event. By computing the response weighted center of the event in each plane, then performing a weighted least squares fit to the position of the center of the event versus plane number, the angle of the event can be be determined. Figure 9.4 shows the result of applying such a procedure to the data. When the beam is incident normal to the detector planes, the mean angle found in each view is $0.1^{\circ}$ with a width of approximately $9^{\circ}$. When the beam is incident at $30^{\circ}$, the mean angle found in the even planes is $0.1^{\circ}$ with a width of $11.0^{\circ}$, and the mean angle found in the odd planes is $28.4^{\circ}$ with a width of $10.4^{\circ}$.

An electron traveling through the detector at an angle effectively sees a factor

of $\frac{1}{\cos \theta}$ increase in plane width, where $\theta$ is the angle the electron trajectory makes with respect to the normal to the plane face. This effective increase in plane width could be expected to make a difference both in event topology and in response. To illustrate this, Figure 9.5 shows the theoretical $\frac{d E}{d x}$ given by

$$
\frac{d E}{d t}=\frac{E_{0}}{b} \frac{(b t)^{(a-1)} e^{-b t}}{\Gamma(a)}
$$




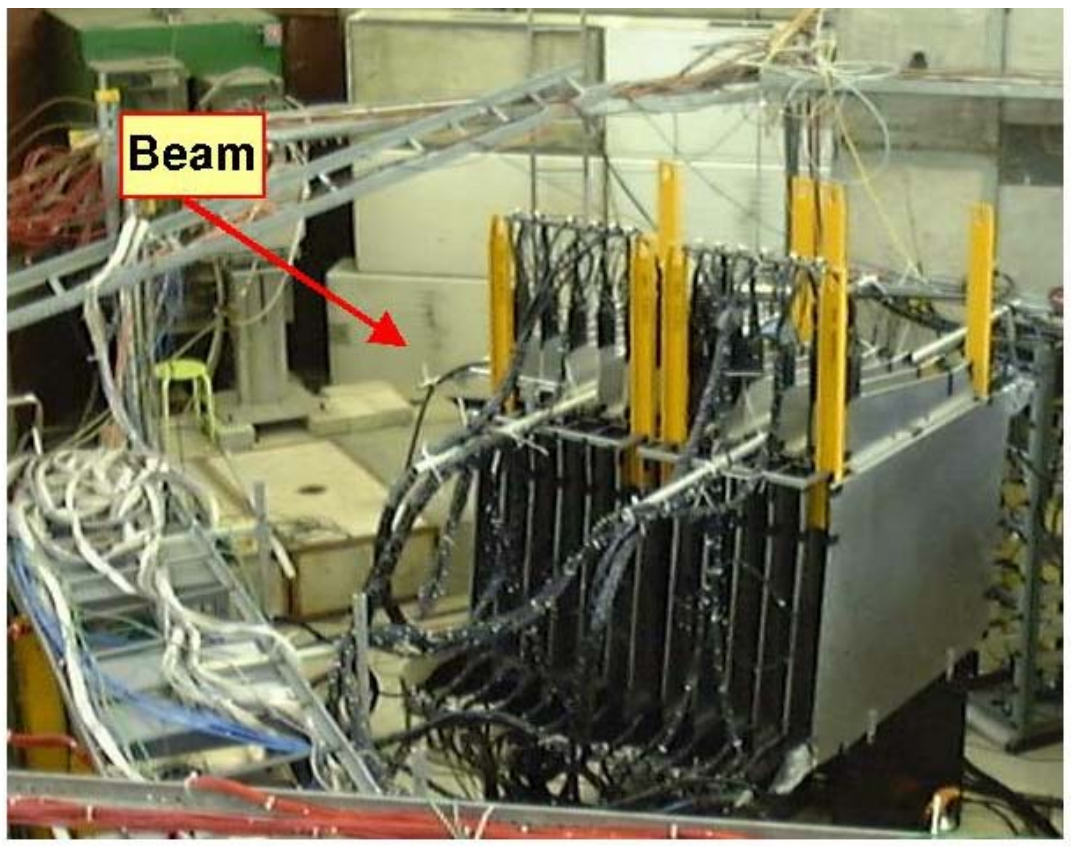

Figure 9.1: Photograph of the CalDet in the T11 beam line, angled $30^{\circ}$ with respect to the beam

$$
t=\frac{x}{X_{0}}
$$

where $E_{0}$ is the initial energy of the electron, $b$ and $a$ are energy dependent parameters, and $X_{0}$ is the radiation length [43]. The position and widths of the scintillator planes when the beam is incident normal to the detector are shown in red. The position and widths of the scintillator planes when the beam is incident at $30^{\circ}$ are shown in blue. When the detector is angled, the energy deposition curve is sampled at different positions, and the area over which it is sampled is different. Integrating the curve over the area bounded by the active planes gives the expected response. While the total response will depend on the detailed shape of the $\frac{d E}{d t}$ curve, Figure 9.5 suggests that differences in response between normal incidence and angled incidence on the order of $1 \%$ could be expected. 

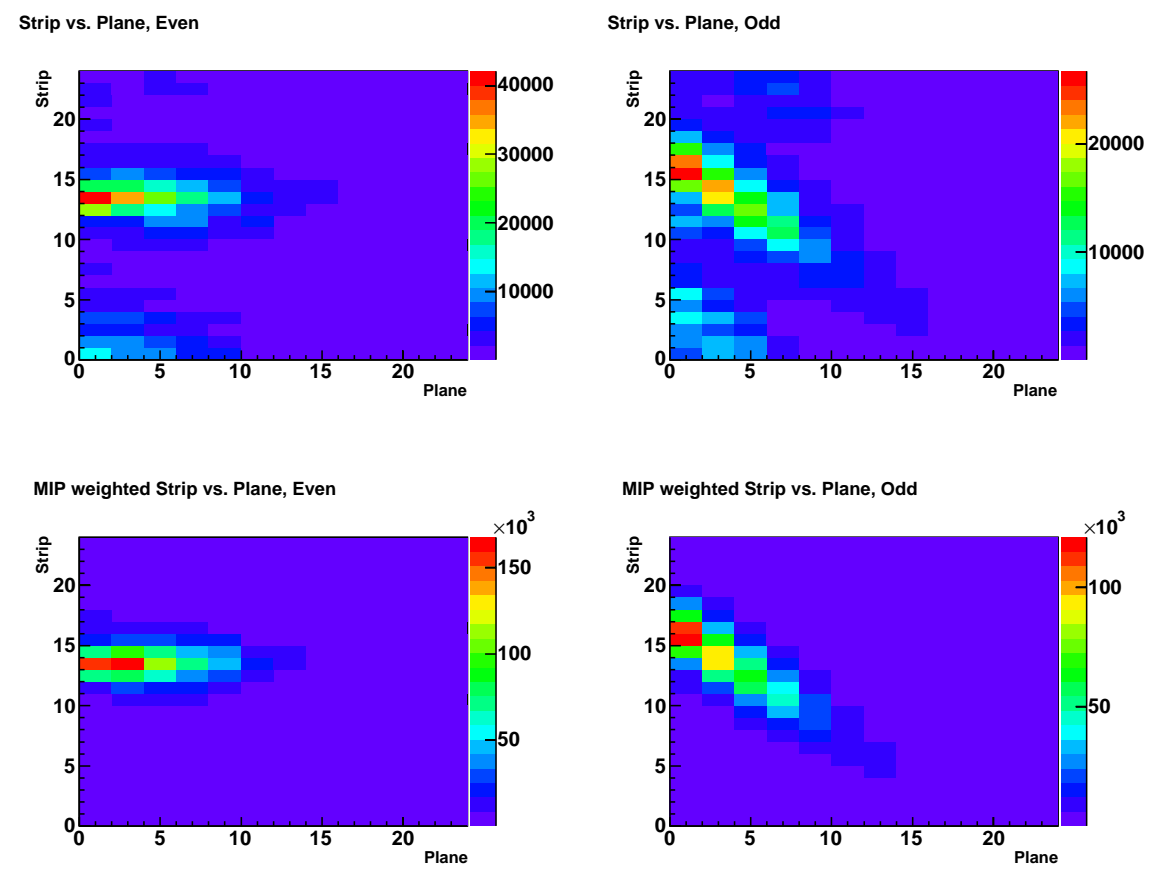

Figure 9.2: Top row of plots shows the strip versus plane distribution of hits for each of the two CalDet views. The bottom row of plots shows the same distribution weighted by energy deposited in the hit.

Figure 9.6 compares the last plane distributions between angle and normal incidence at different beam momenta. The angled electrons do not cross as many planes as the normal electrons, but the distance traveled given by dividing by $\cos 30^{\circ}$ is roughly the same. Figure 9.7 shows the number of hit strips above a $1.5 \mathrm{PE}$ threshold. The number of strips hit in an event does not change dramatically when the detector is angled. Figure 9.8 shows the transverse size of electron events at normal and angled incidence. Angled events appear fatter due to the fact that the transverse distance is computed relative to the detector planes, not relative to the beam direction. Figure 9.9 compares the plane of maximum energy deposition. Once again, the angled electrons reach shower max in fewer planes, but the actual distance traveled 


\begin{tabular}{c} 
Run: 41017 \\
Snarl: 101 \\
PID: Electron \\
Momentum: $1.2 \mathrm{GeV}$ \\
Total MIP: 83.84 , Even: 28.63 , Odd: 55.22 \\
\hline
\end{tabular}
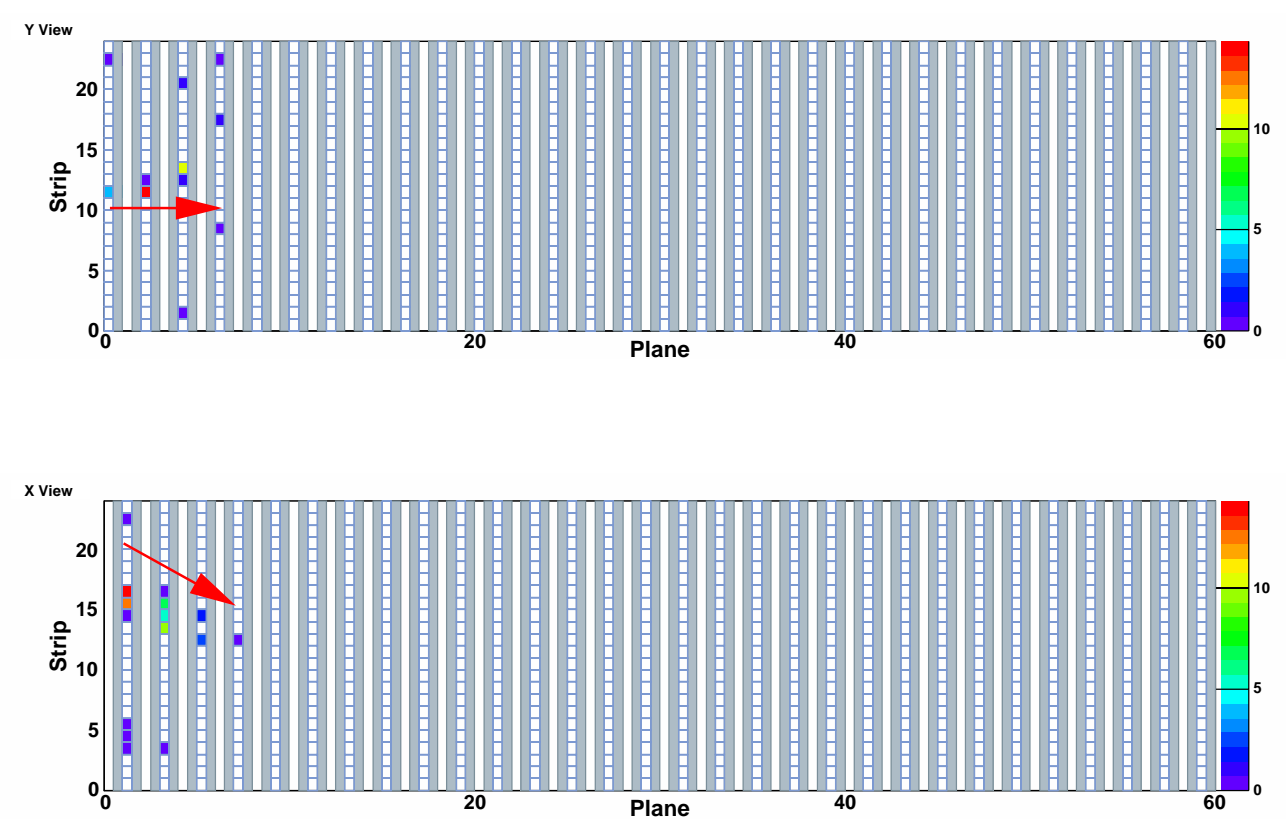

Figure 9.3: A $1.2 \mathrm{GeV}$ positron at angled incidence. 
Normal, Even View

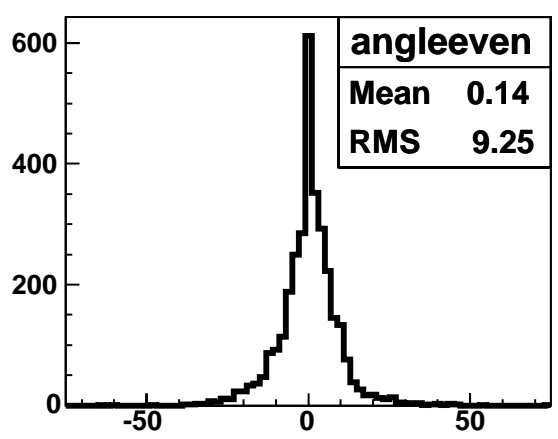

Angle, Even View

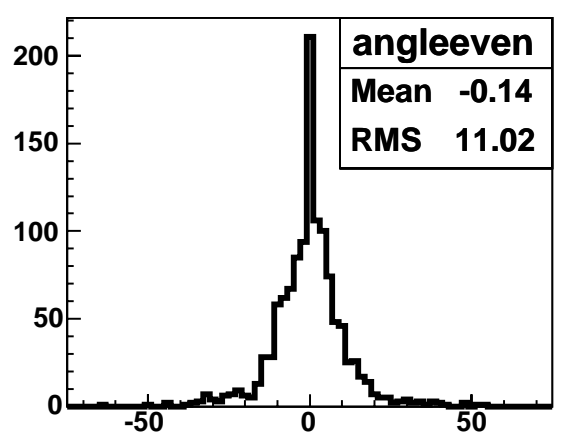

Normal, Odd View

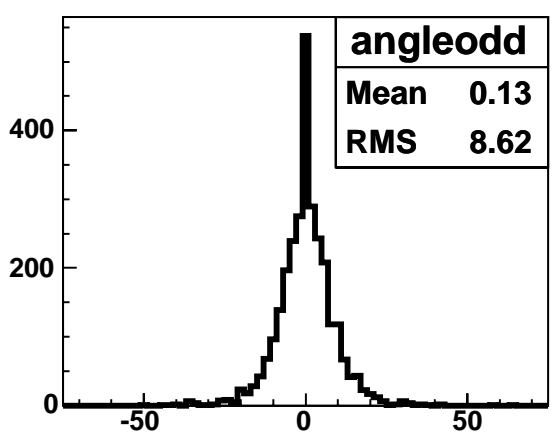

Angle, Odd View

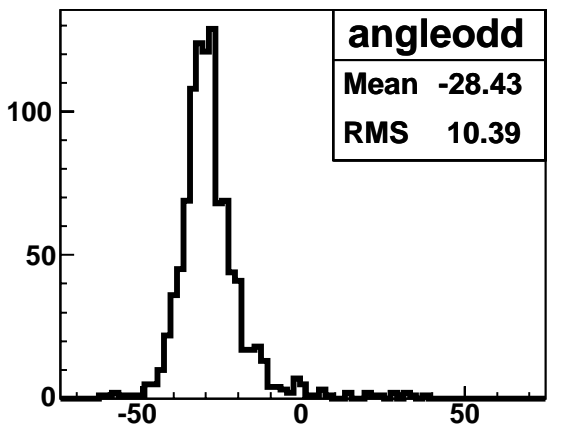

Figure 9.4: Left plots show the measured angle of events in the even planes, calculated on an event by event basis. Right plots show the measured angle of events in the odd planes. Top row of plots shows the measurement for the normally incident data, while the bottom row of plots shows the measurement for the angled data. 


\section{Energy Depositon Profile}

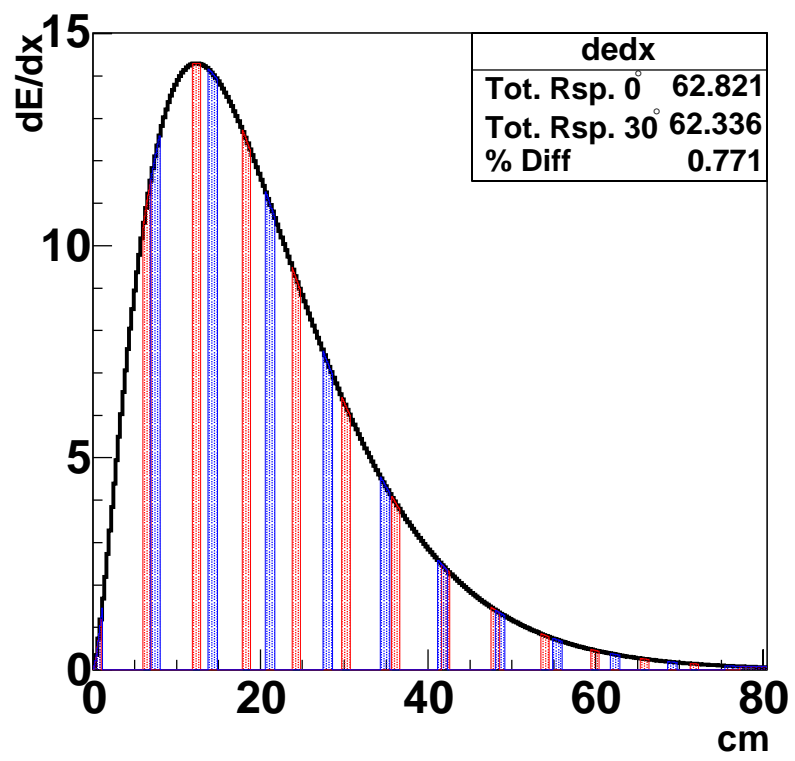

Figure 9.5: Parameterization of the energy deposited as a function of distance traveled for electron events. The position and widths of the scintillator planes when the beam is incident normal to the detector is shown in red. The position and widths of the scintillator planes when the beam is incident at $30^{\circ}$ is shown in blue. Integrating the curve over the areas corresponding to the active planes gives the total response. The relative difference in response between the normal and angled incidence is $0.8 \%$. 
is comparable to the normally incident electrons. Figure 9.10 compares the response between angle and normal data. Figure 9.11 shows the mean response over beam momentum versus beam momentum for positrons of angled incidence and normal incidence. The response measured from the angled electrons is about $2 \%$ smaller than that measured with the normally incident electrons. Some of this difference is due to the different amount of material traversed by an angled positron before reaching an active plane compared to that of a normally incident positron. Figure 9.12 compares the energy resolution between angled and normally incident positrons. The resolution increases by about $5 \%$ for the angled electrons. Unfortuanely, the LI system was not working properly during this run, so the response shown is not corrected for gain drifts over time. While the effect of the uncorrected gain drift is expected to be small, a precise statement regarding the change in response with angle will have to derived from the angle runs in the 2003 data set once the calibration constants have been finalized.

\subsection{First Plane Effects}

Electrons interacting in the CalDet pass through a leading plane of scintillator then a full steel plane before encountering an active detector plane. However, at the large MINOS detectors, a neutrino will, on average, interact in the center of a steel plane, thus a resulting electron will only travel through a half plane of steel before encountering an active detector plane. Figure 9.13 illustrates the difference between the two scenarios. To use the CalDet electromagnetic calibration at the large detectors, a correction must be applied to the CalDet response. To do this, the CalDet is simulated in the normal configuration, then further Monte Carlo is run in which an electron does not traverse the extra half steel and scintillator plane. The relation between the two different responses is fitted with a straight line, and the fit parameters give a correction to the CalDet response. Figure 9.14 shows the 
Last Plane, 1.5 PE cut, $-1.0 \mathrm{GeV}$

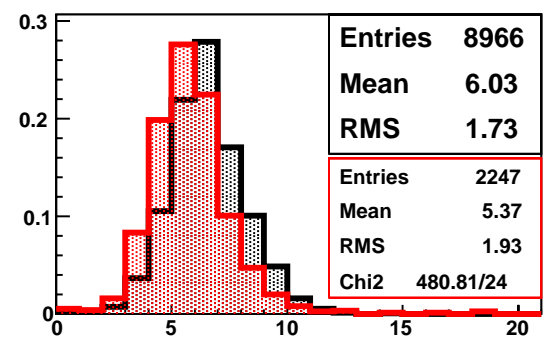

Last Plane, 1.5 PE cut, 0.8 GeV

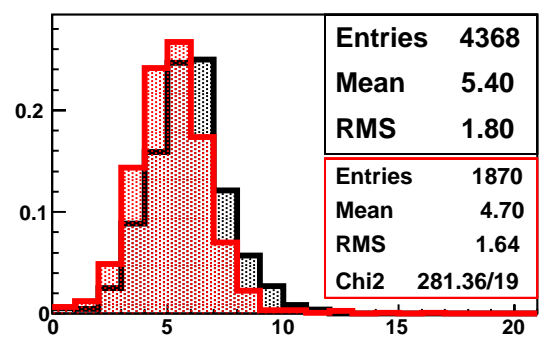

Last Plane, 1.5 PE cut, $-0.8 \mathrm{GeV}$

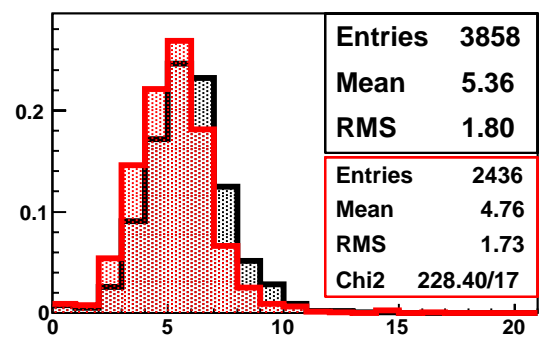

Last Plane, 1.5 PE cut, 1.0 GeV

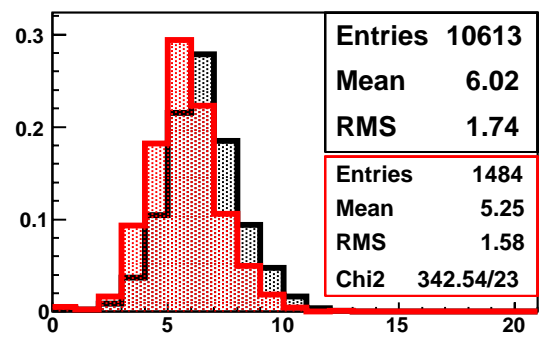

Last Plane, 1.5 PE cut, 1.2 GeV

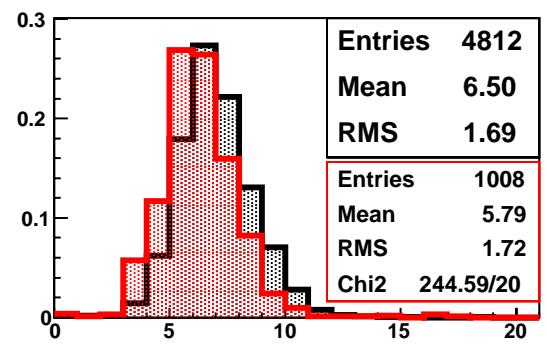

Figure 9.6: Comparison of number of hit planes between angled and normal incidence. Black histograms show normal incidence data, red histograms show angled data. Momenta shown are $-1 \mathrm{GeV},-0.8 \mathrm{GeV}, 0.8 \mathrm{GeV}, 1.0 \mathrm{GeV}$, and $1.2 \mathrm{GeV}$. 
Nhit Strips, 1.5 PE Cut, -1.0 GeV

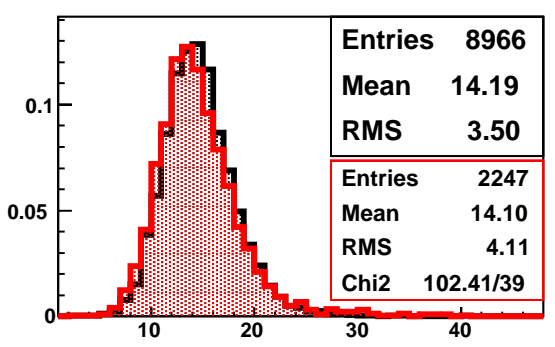

Nhit Strips, 1.5 PE Cut, $0.8 \mathrm{GeV}$

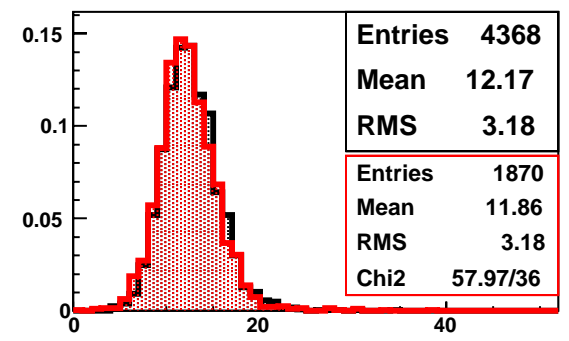

Nhit Strips, 1.5 PE Cut, -0.8 GeV

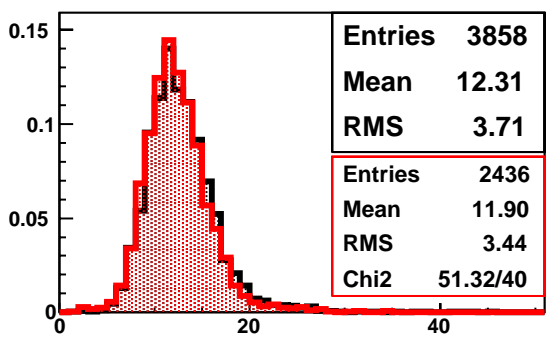

Nhit Strips, 1.5 PE Cut, 1.0 GeV

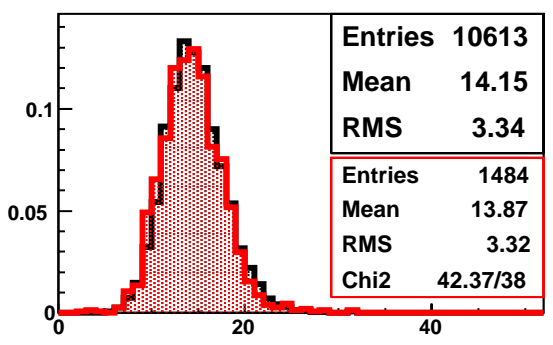

Nhit Strips, 1.5 PE Cut, 1.2 GeV

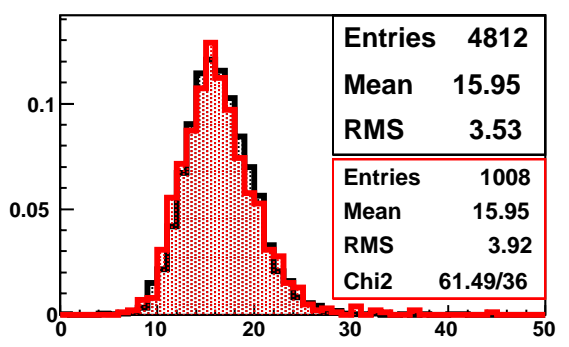

Figure 9.7: Comparison of the number of hit strips above 1.5 PE distributions for angled and normal incidence. Black histograms show normal incidence data, red histograms show angled data. Momenta shown are $-1 \mathrm{GeV},-0.8 \mathrm{GeV}, 0.8 \mathrm{GeV}$, $1.0 \mathrm{GeV}$, and $1.2 \mathrm{GeV}$. 
Mip Weighted Radius, 1.5 PE Cut, -1.0 GeV

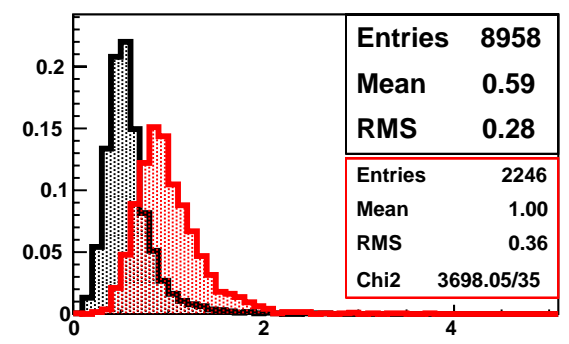

Mip Weighted Radius, 1.5 PE Cut, 0.8 GeV

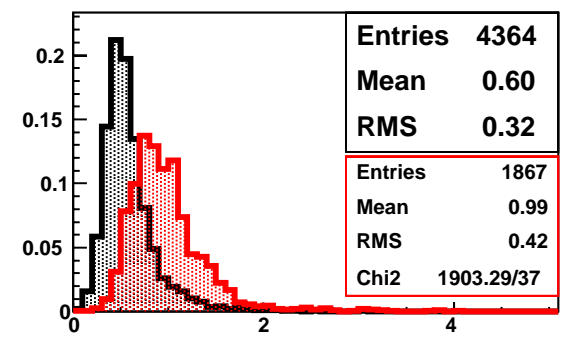

Mip Weighted Radius, 1.5 PE Cut, 1.2 GeV

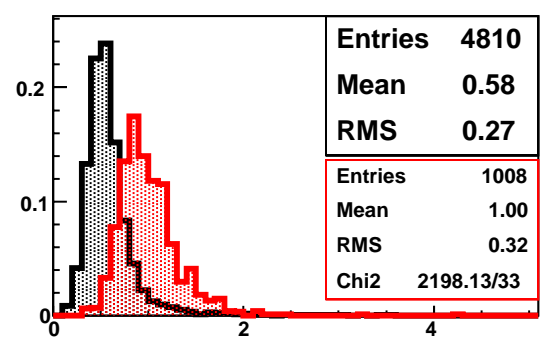

Mip Weighted Radius, 1.5 PE Cut, $-0.8 \mathrm{GeV}$

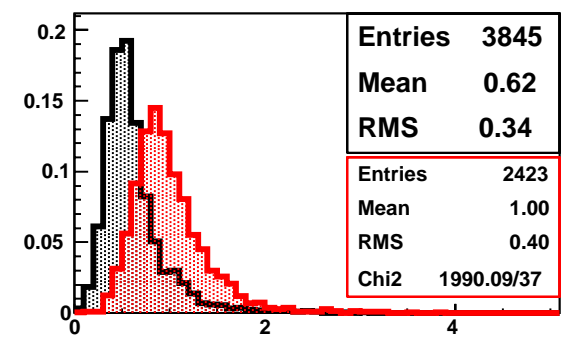

Mip Weighted Radius, 1.5 PE Cut, 1.0 GeV

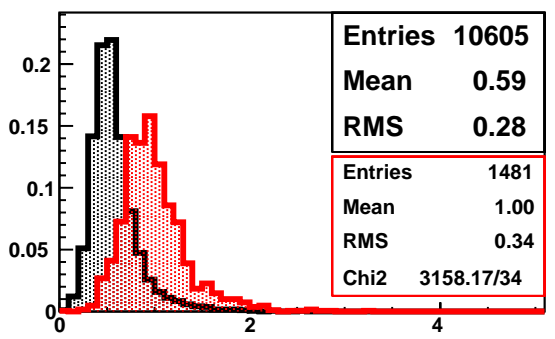

Figure 9.8: Comparison of the event radius for angled and normal incidence. Black histograms show normal incidence data, red histograms show angled data. Momenta shown are $-1 \mathrm{GeV},-0.8 \mathrm{GeV}, 0.8 \mathrm{GeV}, 1.0 \mathrm{GeV}$, and $1.2 \mathrm{GeV}$. 
Plane of Shower Max, $-1.0 \mathrm{GeV}$

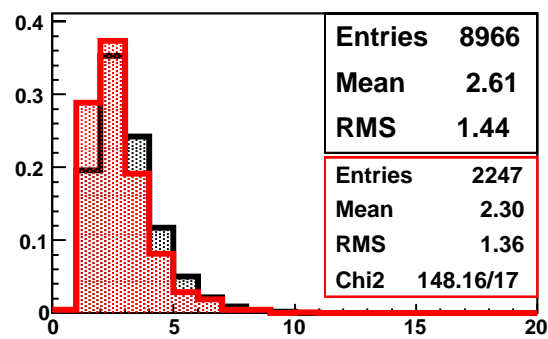

Plane of Shower Max, $0.8 \mathrm{GeV}$

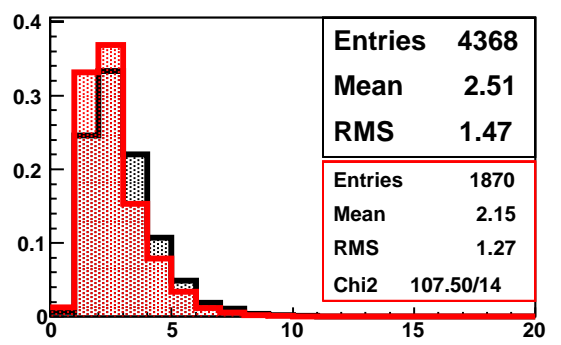

Plane of Shower Max, $-0.8 \mathrm{GeV}$

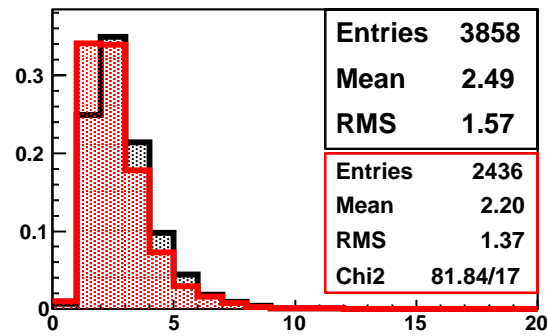

Plane of Shower Max, 1.0 GeV

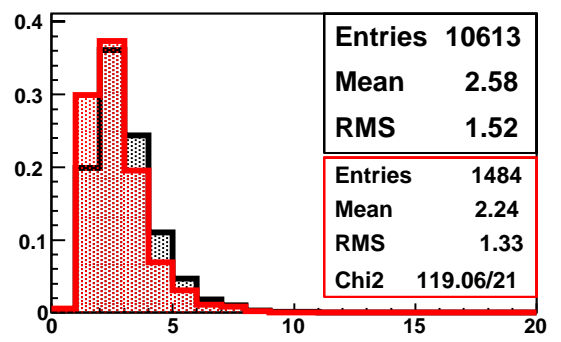

Plane of Shower Max, 1.2 GeV

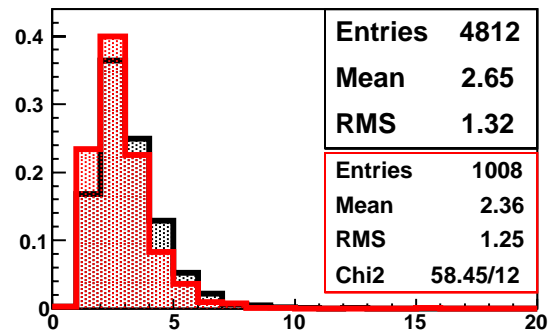

Figure 9.9: Comparison of plane of maximum energy deposition for angled and normal incidence. Black histograms show normal incidence data, red histograms show angled data. Momenta shown are $-1 \mathrm{GeV},-0.8 \mathrm{GeV}, 0.8 \mathrm{GeV}, 1.0 \mathrm{GeV}$, and $1.2 \mathrm{GeV}$. 
Total MIP, $-1.0 \mathrm{GeV}$

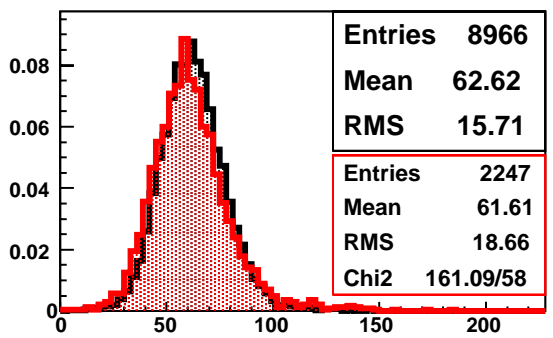

Total MIP, $0.8 \mathrm{GeV}$

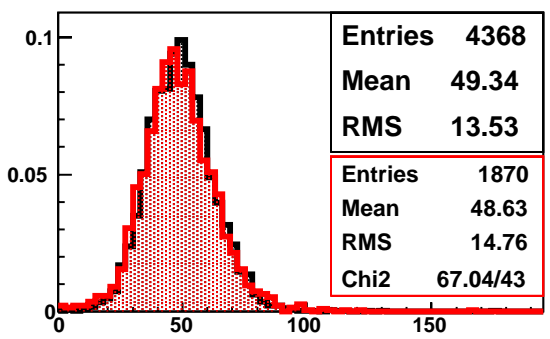

Total MIP, $-0.8 \mathrm{GeV}$

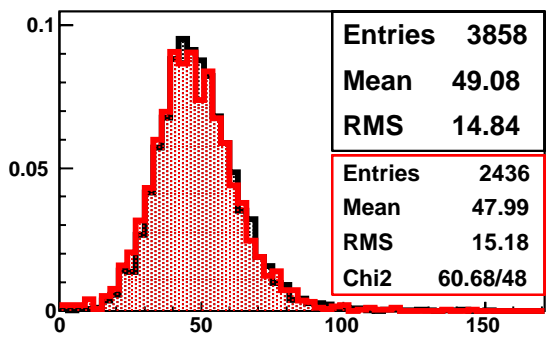

Total MIP, $1.0 \mathrm{GeV}$

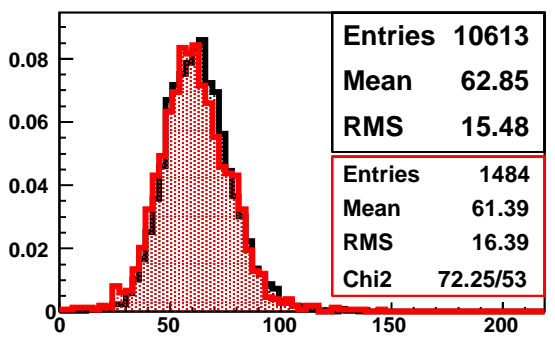

Total MIP, $1.2 \mathrm{GeV}$

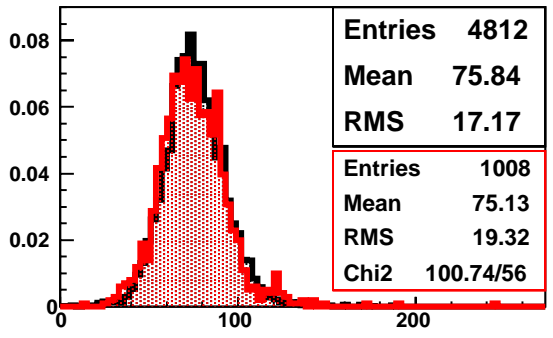

Figure 9.10: Comparison of total energy deposition for angled and normal incidence. Black histograms show normal incidence data, red histograms show angled data. Momenta shown are $-1 \mathrm{GeV},-0.8 \mathrm{GeV}, 0.8 \mathrm{GeV}, 1.0 \mathrm{GeV}$, and $1.2 \mathrm{GeV}$. 
MIP/P vs. P

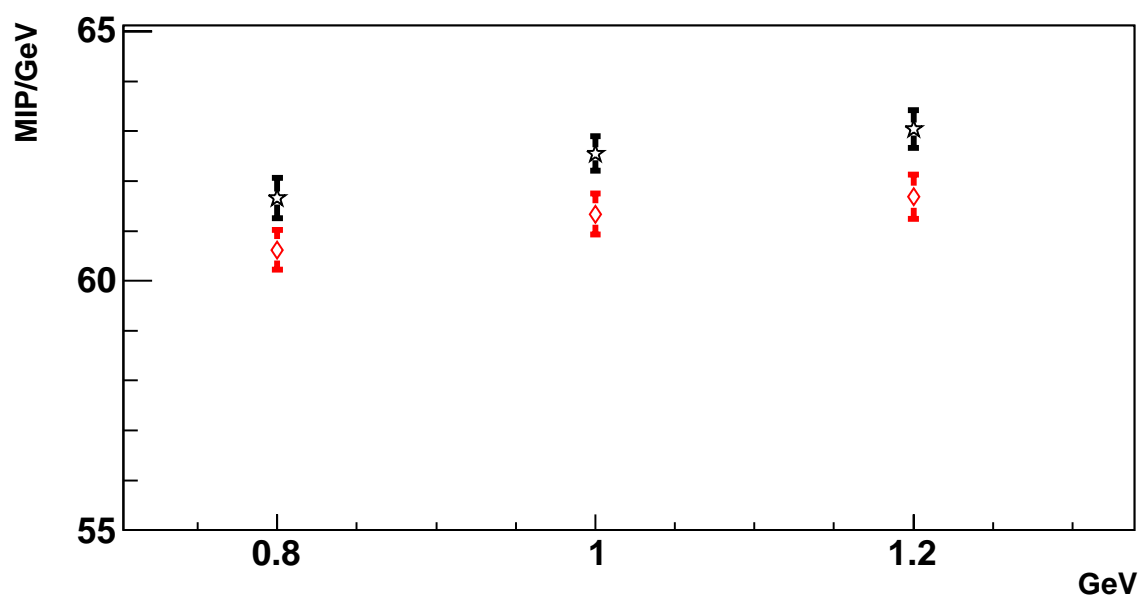

Figure 9.11: The mean response divided by beam momentum versus beam momentum. Normally incident positrons are shown with black stars, angled positrons are shown with red diamonds.

\section{Resolution $\sqrt{\mathrm{P}}$ vs. $\sqrt{\mathrm{P}}$}

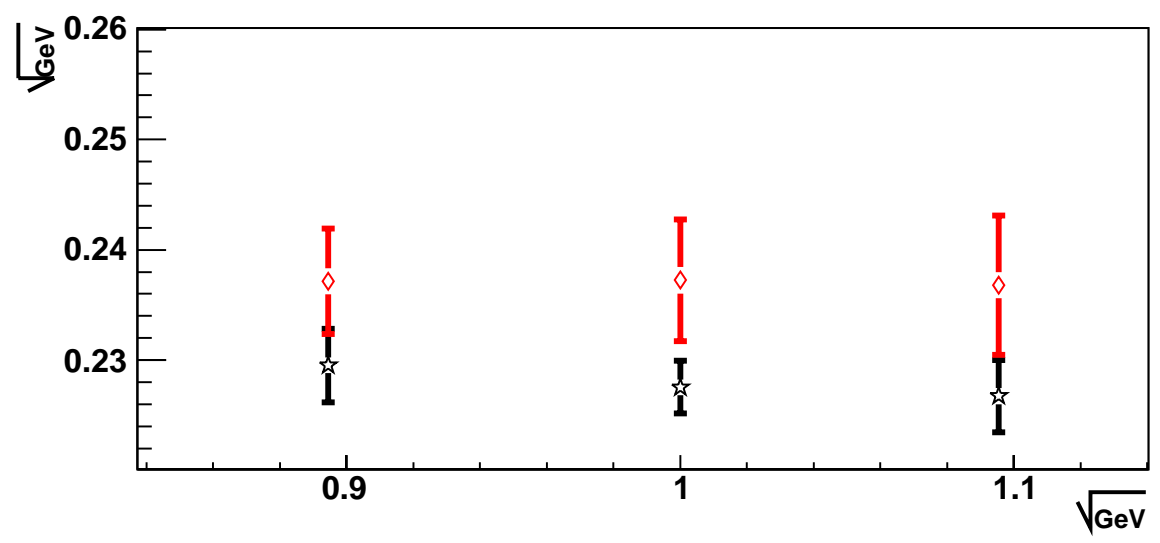

Figure 9.12: The energy resolution as characterized by $\frac{\sigma}{\mu} \sqrt{P}$ versus $\sqrt{P}$. Normally incident positrons are shown with black stars, angled positrons are shown with red diamonds. 
relation between the simulated detector response with and without the half plane. To verify the effect of this correction, data was taken in 2003 with a half steel plane in front of the detector. Figure 9.15 shows that after applying the correction to the data taken in the normal CalDet configuration, the response matches that from the data with the steel plane in front of the CalDet with the signal from plane 0 included in the response. Figure 9.16 shows the effect of the first plane correction on the data. The corrections counteract the fall-off of response at the lowest beam momenta. After the correction for the first plane, the response is

$$
(65.81 \pm 0.08) \frac{\mathrm{MIP}}{\mathrm{GeV}} \times \mathrm{P}-(0.23 \pm 0.07) \mathrm{MIP} .
$$

\subsection{Neutrinos in CalDet}

In a charged current, $\nu_{e}$ event, one expects not only a shower associated with the electron created, but also a hadronic shower. The extra hadronic activity changes the expected response and topology of the shower. Figures 9.17 through 9.22 show simulated charged current $\nu_{e}$ events in the CalDet at various energies. The following plots show the characteristics of showers generated by $\nu_{e}$, charged current interactions simulated in the CalDet. Also shown is a class of events with large components of electromagnetic activity as determined from Monte Carlo truth and the characteristics as measured at CalDet from single electron interactions. Figure 9.23 shows the length of the event, the number of strips hit in an event, the plane of maximum energy deposition, and the transverse extent of events, all versus response. Due to the hadronic component present in the average neutrino shower, the average $\nu_{e}$ shower penetrates further and induces more activity than single particle electron showers; however, the events with high electromagnetic content closely resemble CalDet electrons. Figure 9.24 shows the detector response versus incident particle 


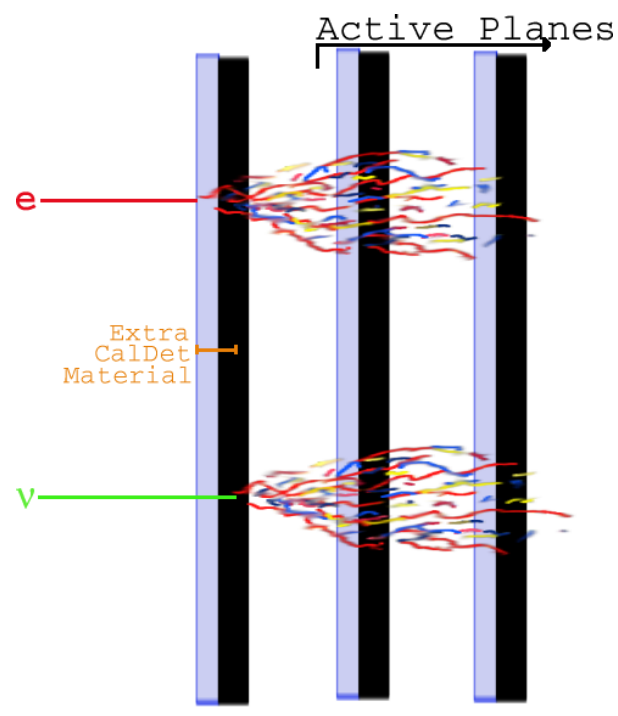

Figure 9.13: Illustration of the systematic difference between electron events measured at CalDet and electron events arising from neutrino interactions at the large MINOS detectors. At CalDet, electrons travel through a full scintillator plane and a full steel plane before encountering an active plane. At the large MINOS detectors, a neutrino will interact in the middle of a steel plane on average, and the resulting electron will travel only through a half plane of steel before encountering an active plane.

energy. Once again, the $\nu_{e}$ events with high electromagnetic content act much like single particle electrons.

Since the energy deposited in a neutrino interaction is deposited by a combination of electromagnetic interactions and hadronic interactions, to reconstruct the energy of the incident neutrino, one must not only know the detector's electromagnetic response, but also its response to hadronic activity. Figure 9.25 shows the ratio of electron response to pion response versus beam momentum for both particle polarities as measured in the CalDet. The superimposed curves are given by dividing the parameterized form of the electron response by the parameterized 

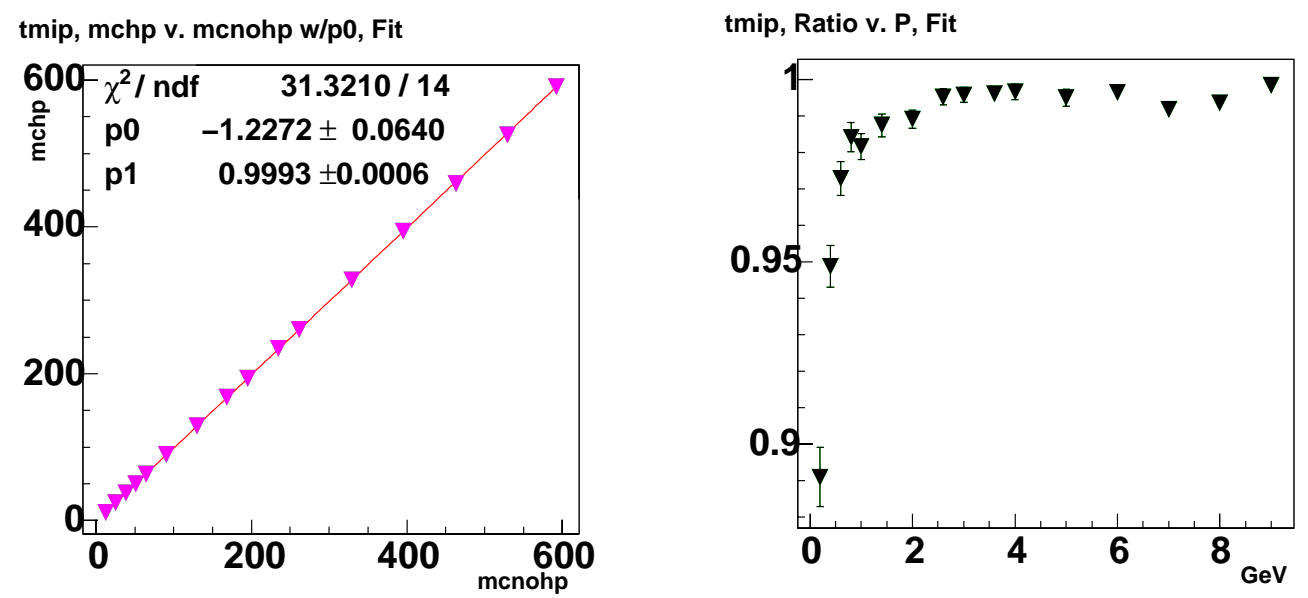

Figure 9.14: Left shows the simulated detector response in MIPs in the normal CalDet configuration versus the simulated response when particles only traverse half a steel plane before encountering an active plane. The fit parameters given provide a parameterized correction to the mean CalDet response. Right plot shows the ratio of normal configuration over half plane configuration versus beam momentum.

form of the pion response, $S$, given by [46]

$$
\begin{aligned}
& S=0.10+50.52 E_{\pi}+0.172 E_{\pi}^{2}\left(\pi^{+}\right) \\
& S=1.20+44.72 E_{\pi}+1.69 E_{\pi}^{2}-0.100 E_{\pi}^{3}\left(\pi^{-}\right) .
\end{aligned}
$$

The difference in the ratio between positives and negatives at low energies is due to differences in the detector response to $\pi^{-}$compared to $\pi^{+}$. As the energy increases, positives and negatives approach the same value of 1.27. Using the response versus momentum for both electrons and pions and the fraction of the shower induced by electromagnetic activity as obtained via Monte Carlo truth, the energy of the incident neutrino can be derived. The results of this procedure is shown in Figure 9.26. The reconstructed energy is on average about $2 \%$ higher than the true energy, with a width of $20 \%$ for all $\nu_{e}$ events. For $\nu_{e}$ showers with greater than $95 \%$ of the energy in 

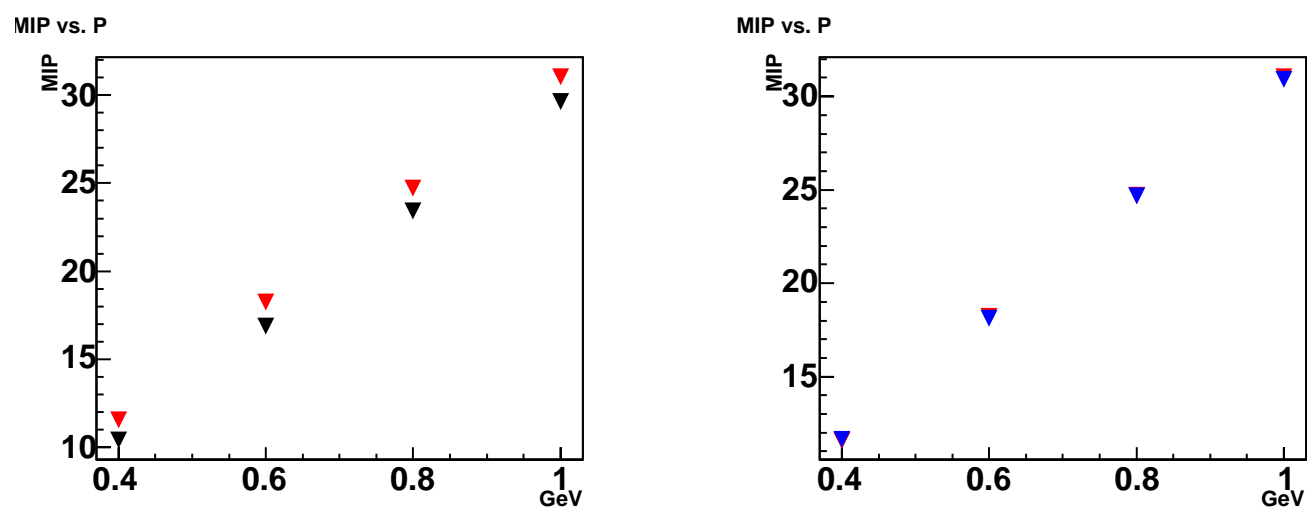

Response, Steel/No Steel vs. $\mathbf{P}$

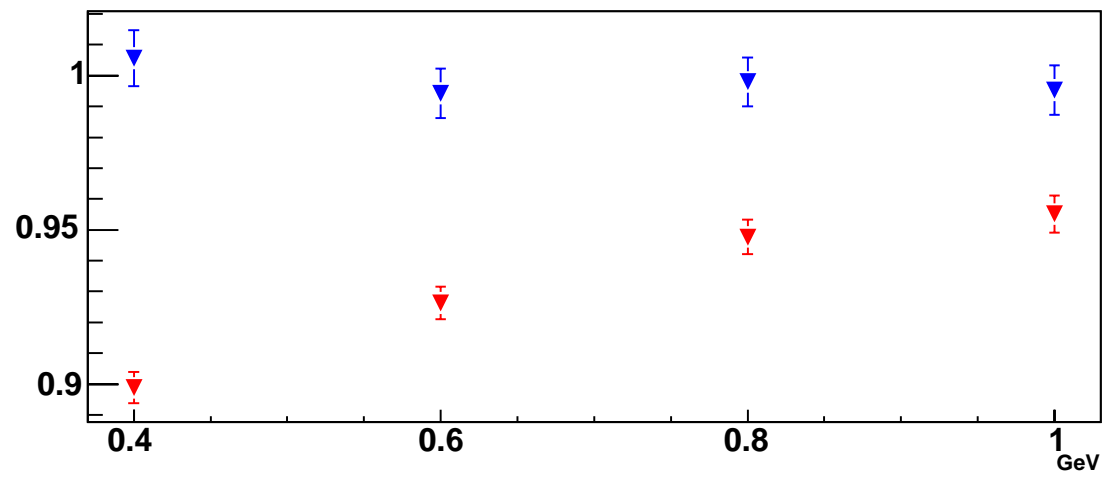

Figure 9.15: Top left shows the measured detector response versus beam momentum for the normal configuration in black and the half plane configuration in red. Top right shows the detector response versus beam momentum for the normal configuration after correcting for the effect of the extra CalDet planes in blue and for the half plane configuration in red (red points obscured). The lower plot shows the ratio of response, normal configuration over half plane configuration. Red points show the ratio before applying the correction. Blue points show the ratio after the correction. Applying the correction accounts for the difference between the two configurations. 
Resolution vs. $\mathbf{P}$

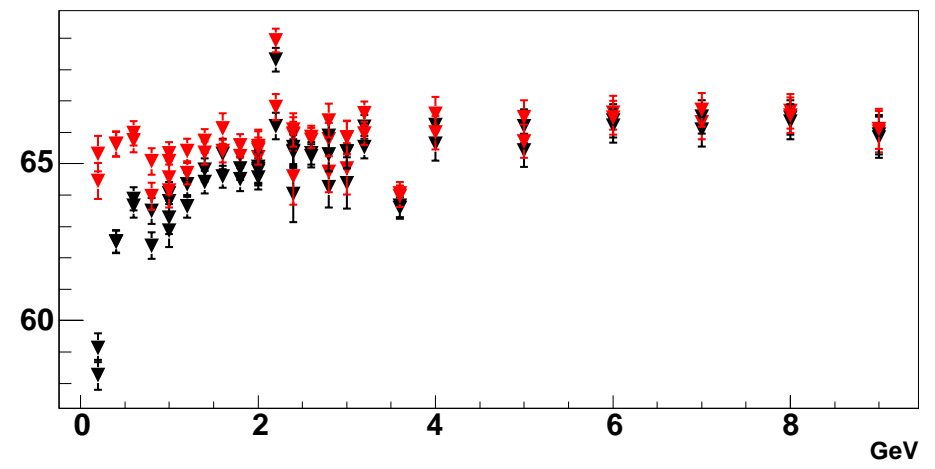

Total MIP vs. P

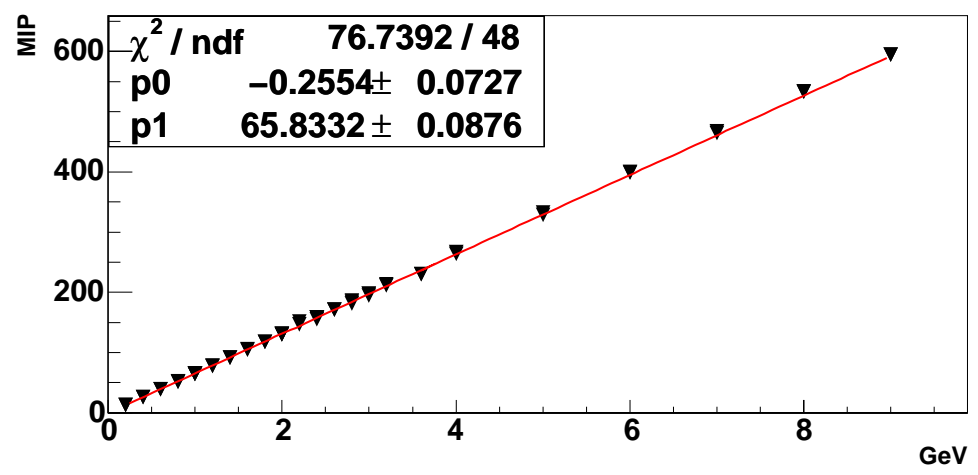

Figure 9.16: Top Plot shows the total MIP divided by beam momentum versus beam momentum. Black points show the response corrected for the energy loss, while red shows the result of the corrections for energy loss and for first plane effects. Bottom plot shows the total MIP versus beam momentum for the data corrected for first plane effects versus beam momentum. 

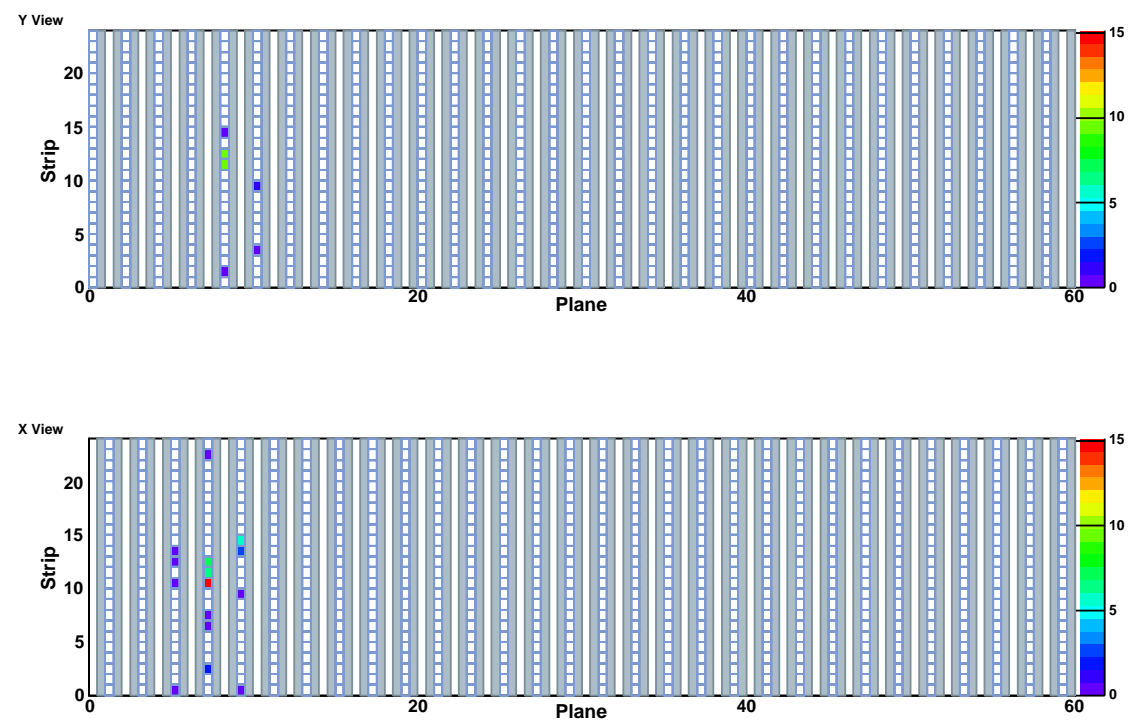

Figure 9.17: A $1.0 \mathrm{GeV}$, simulated $\nu_{e} \mathrm{CC}$ interaction
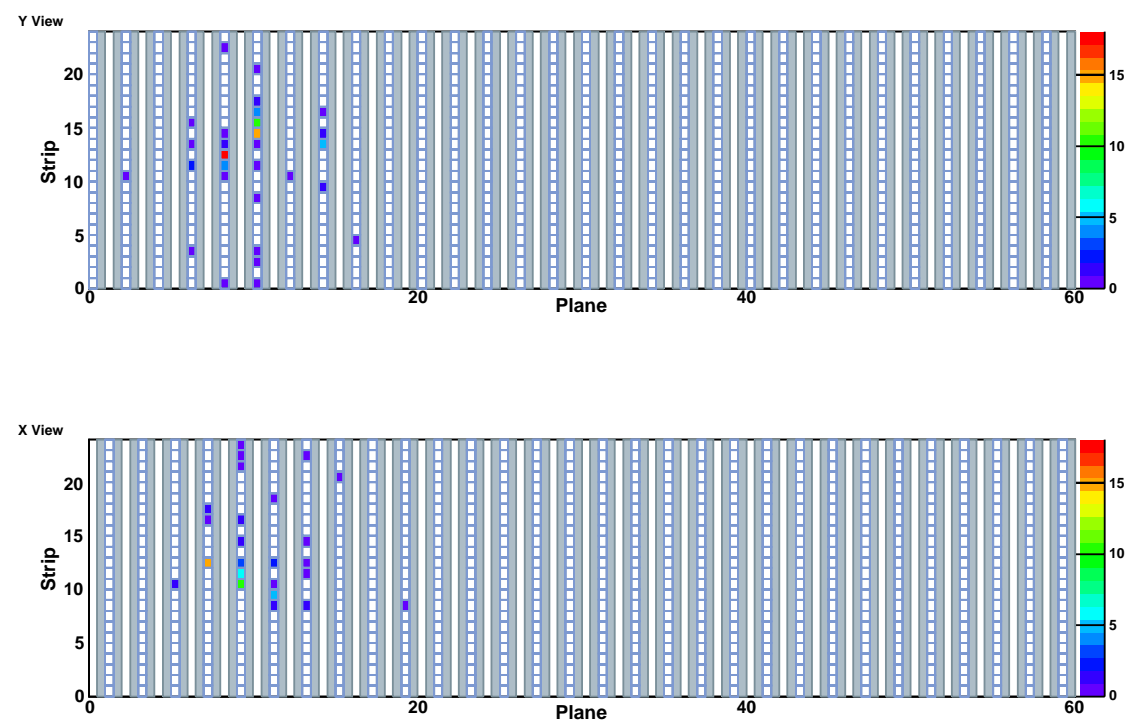

Figure 9.18: A $2.9 \mathrm{GeV}$, simulated $\nu_{e} \mathrm{CC}$ interaction 

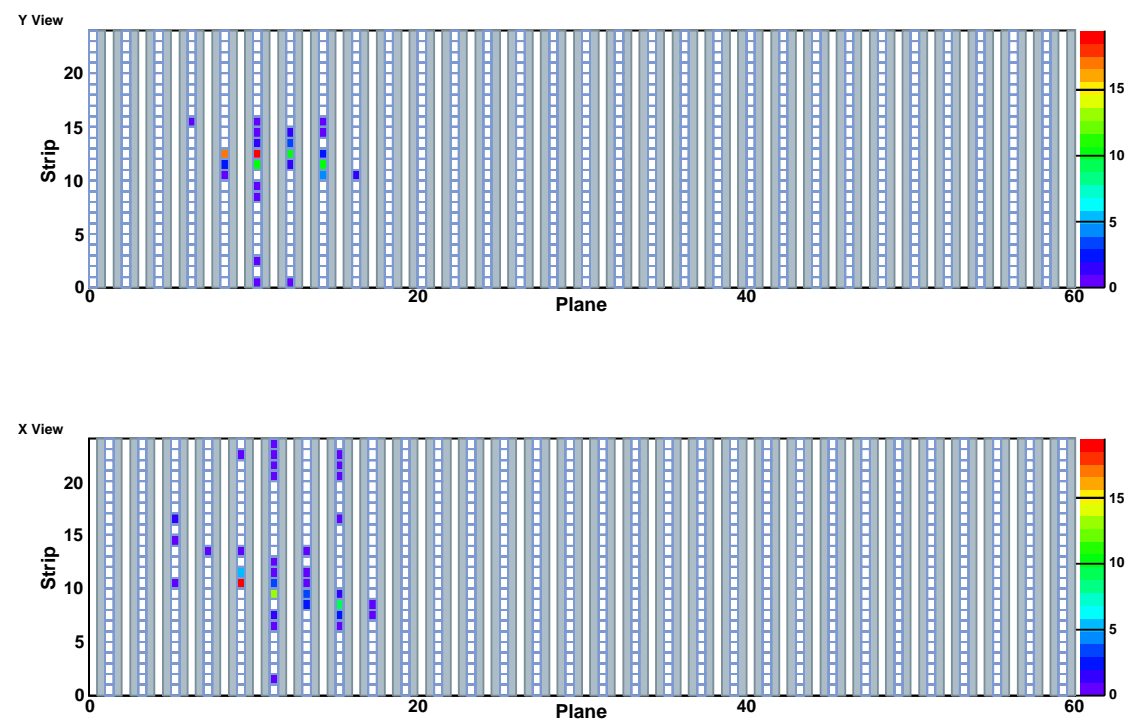

Figure 9.19: A $3.1 \mathrm{GeV}$, simulated $\nu_{e} \mathrm{CC}$ interaction
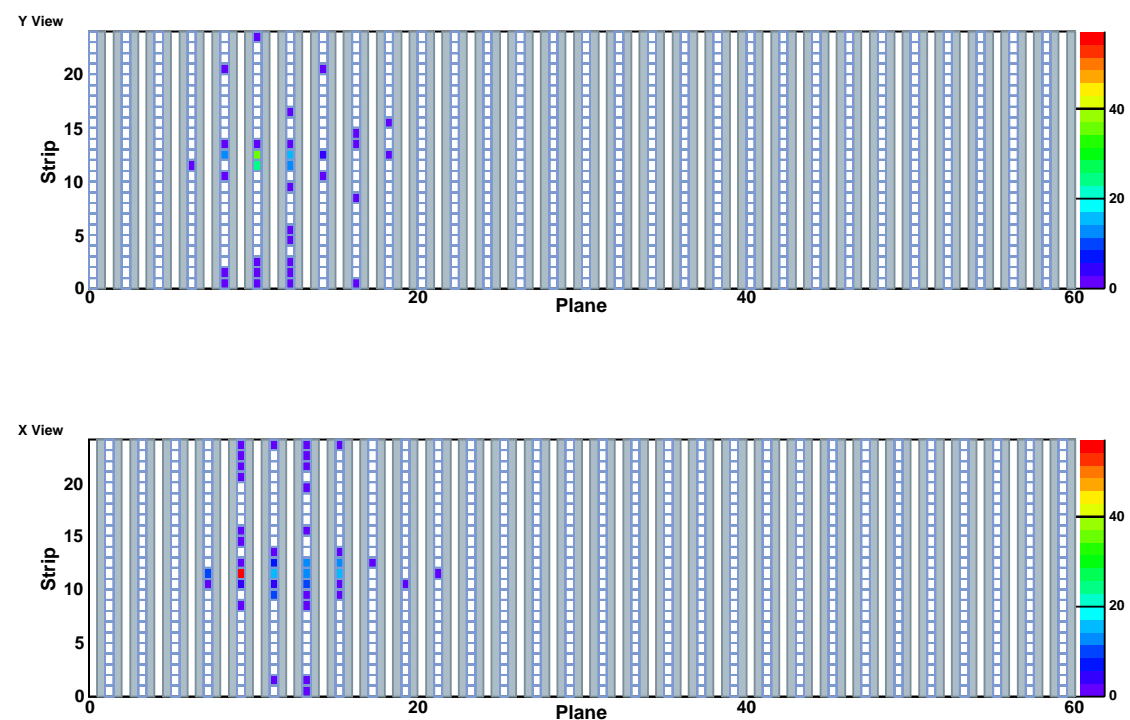

Figure 9.20: A $4.8 \mathrm{GeV}$, simulated $\nu_{e} \mathrm{CC}$ interaction 

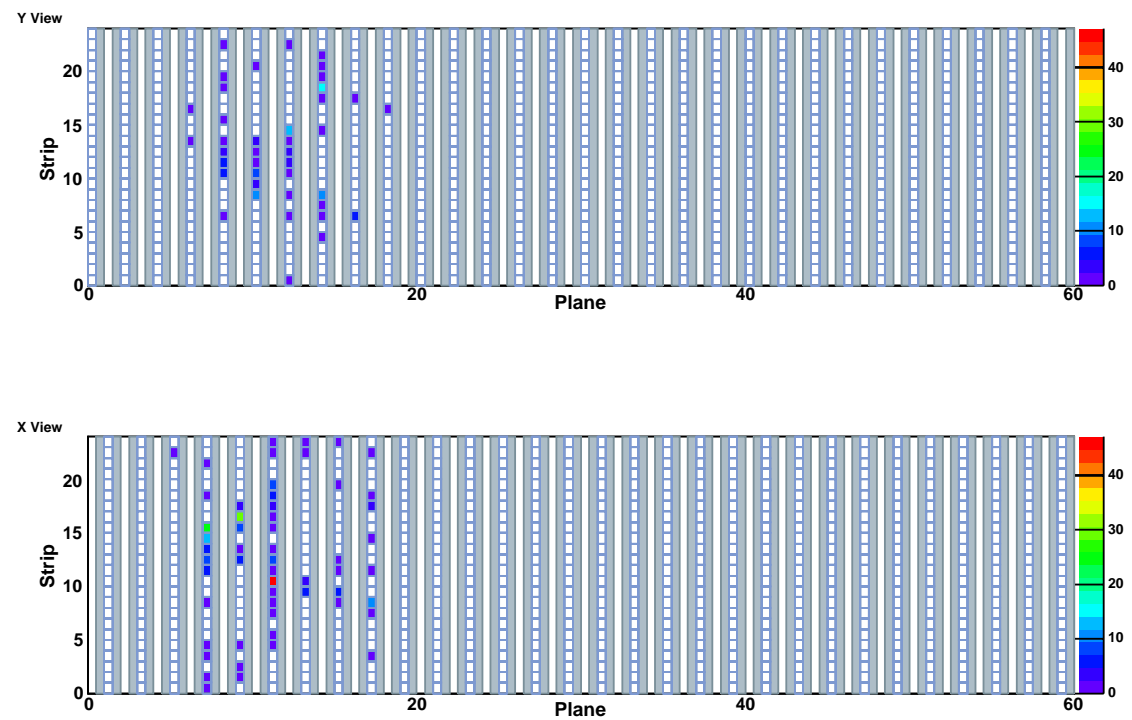

Figure 9.21: A $6.0 \mathrm{GeV}$, simulated $\nu_{e} \mathrm{CC}$ interaction
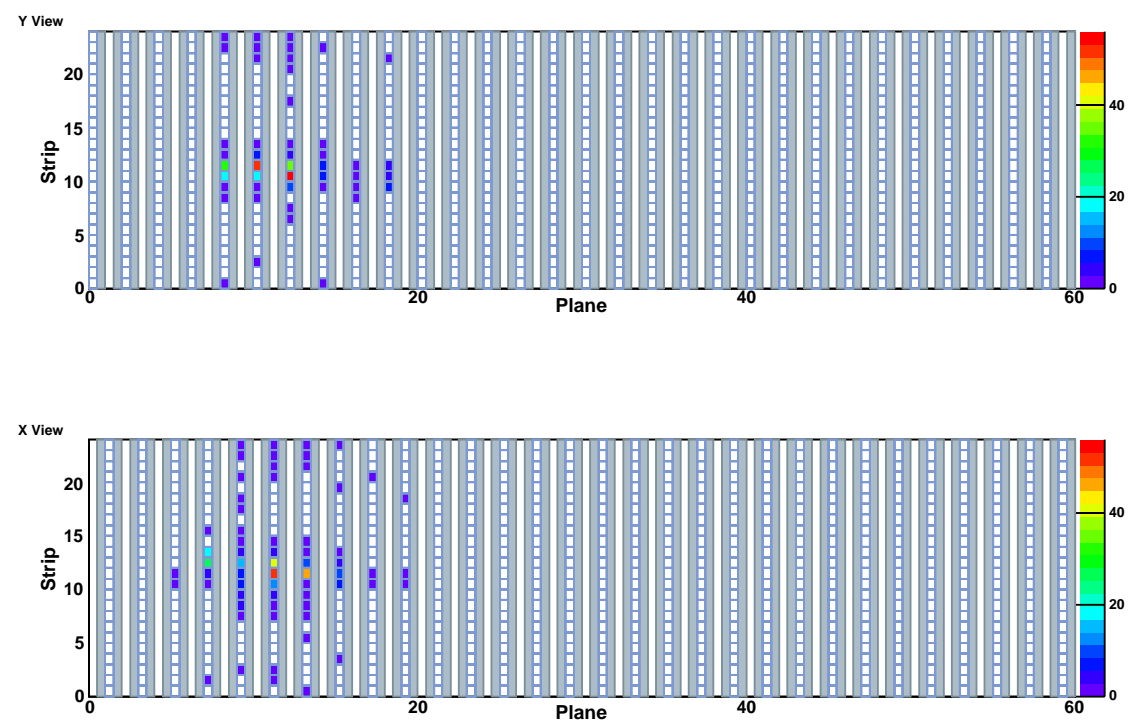

Figure 9.22: A $9.4 \mathrm{GeV}$, simulated $\nu_{e} \mathrm{CC}$ interaction 


\section{Last Plane vs. MIP}

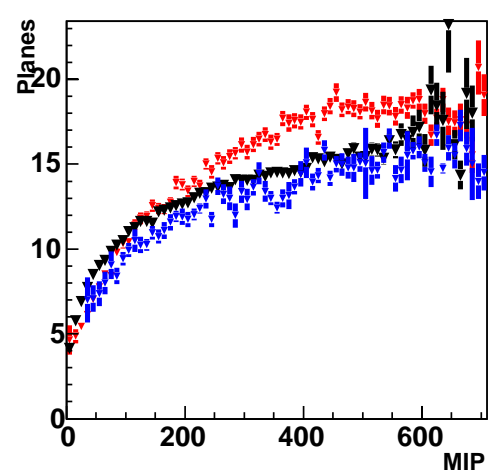

Plane of Shower Max vs. MIP

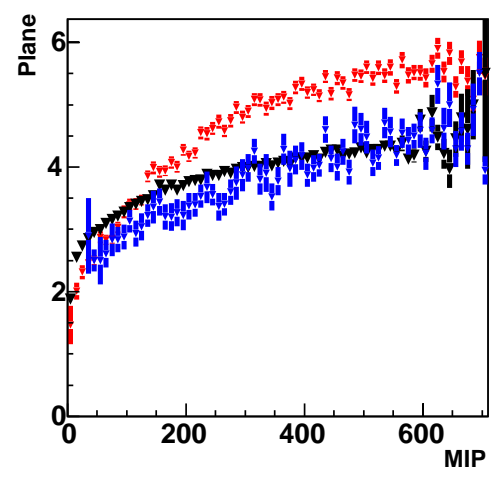

Number of Hit Strips vs. MIP, 1.5PE Cut

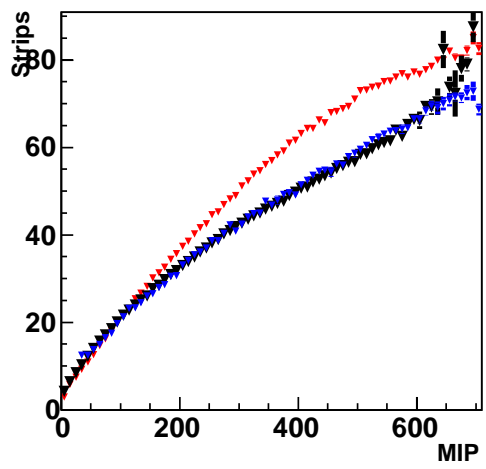

Radius vs. MIP

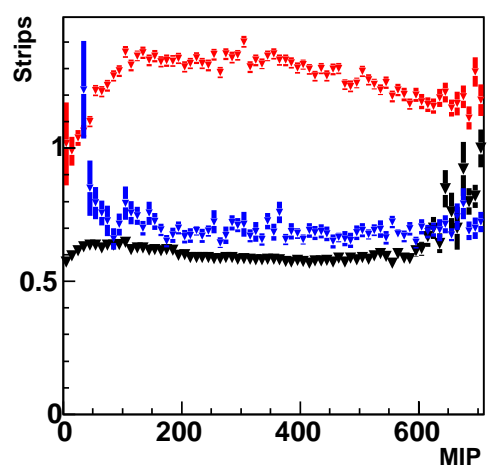

Figure 9.23: Top left shows the length of the event versus detector response. Top right shows the number of hit strips in an event above 1.5 PE. Bottom left shows the plane of maximum energy distribution, and bottom right shows the transverse extent of events. All $\nu_{e}$ charged current events are shown in red, $\nu_{e}$ charged current events with an electromagnetic component greater than $95 \%$ are shown in blue, and CalDet electrons are shown in black. 


\section{MIP vs $E_{\text {v }}$}

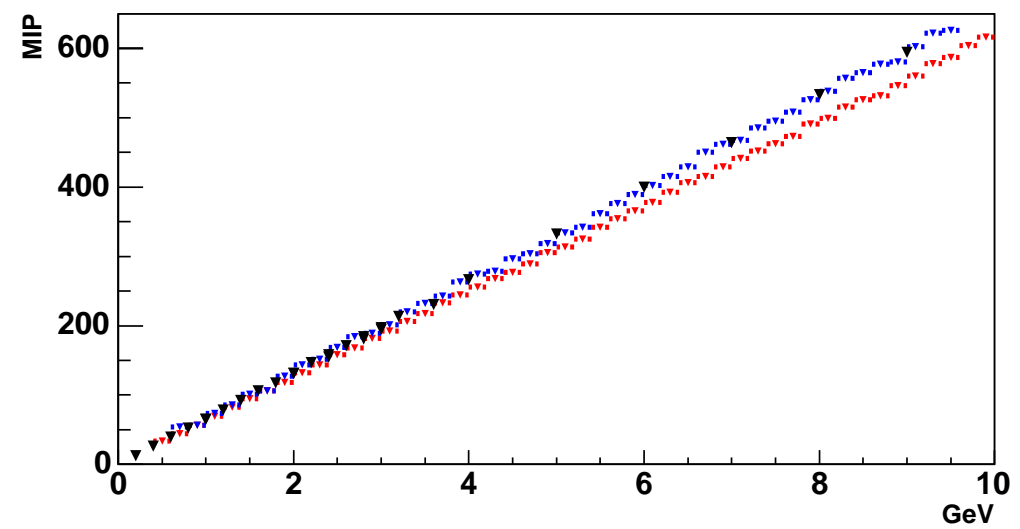

Figure 9.24: Total MIP versus incident energy. All $\nu_{e}$ charged current events are shown in red, $\nu_{e}$ charged current events with an electromagnetic component greater than $95 \%$ are shown in blue, while CalDet electrons are shown in black.

the electromagnetic portion of the shower, the difference between the reconstructed energy and the true energy is $1.2 \%$, and the spread is only $11.5 \%$. 
e/ $\pi$ vs. P

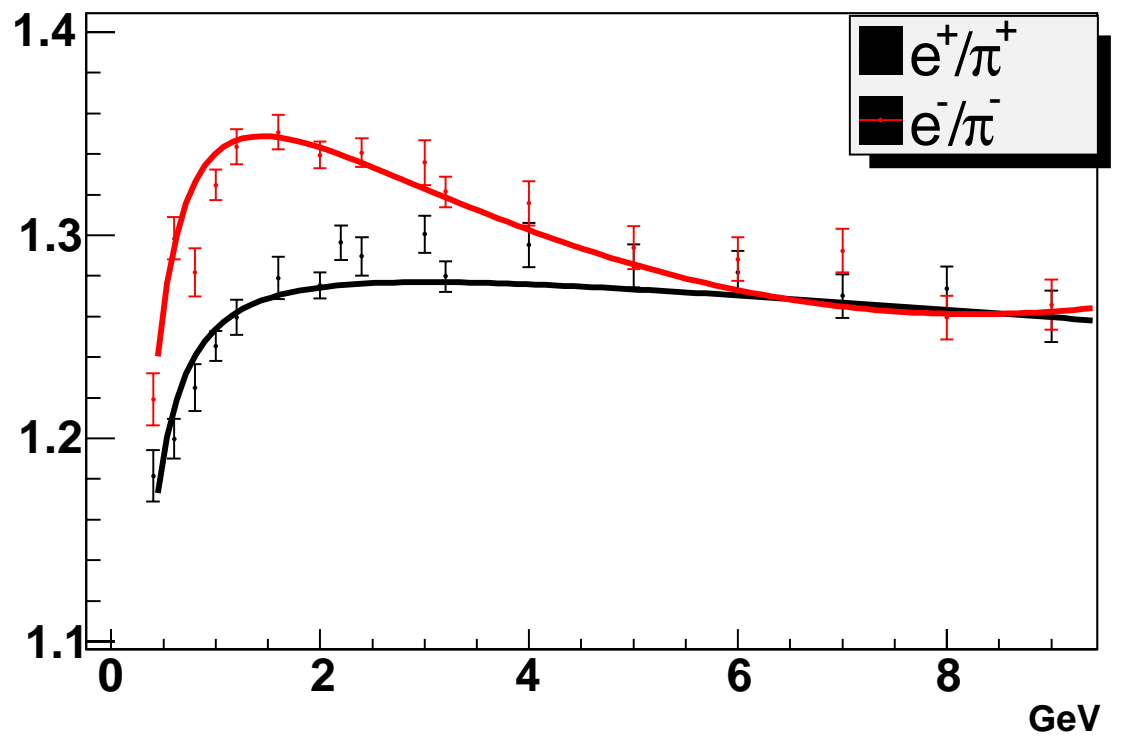

Figure 9.25: The ratio of electron response to pion response for both polarities. The curves are given by dividing the parameterized forms of the response for each of the particle species. 
(Reco-True)/True vs. True

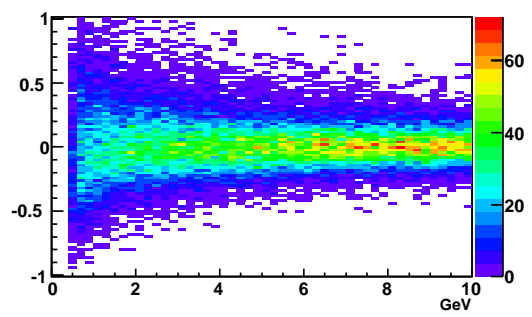

(Reco-True)/True vs. True, EMFrac $>0.85$

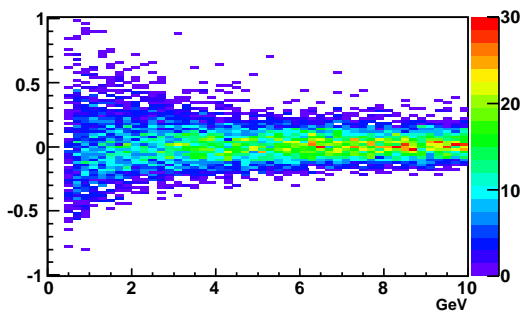

(Reco-True)/True vs. True, EMFrac $>0.95$

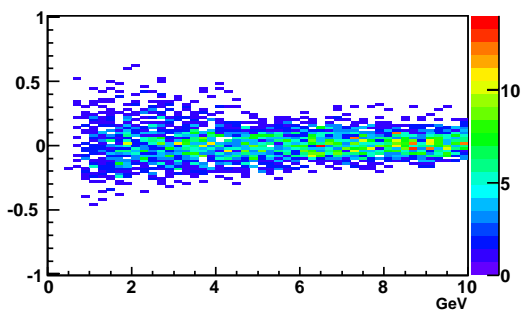

(Reco-True)/True

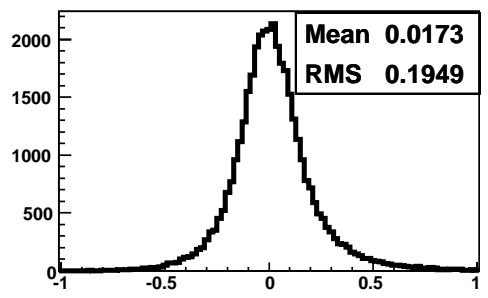

(Reco-True)/True, EMFrac $>0.85$

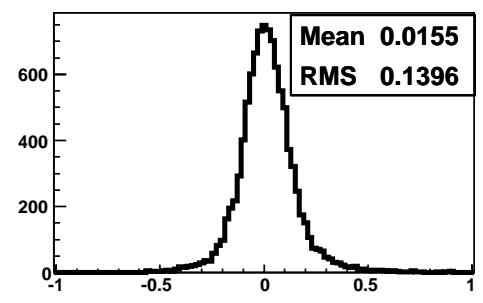

(Reco-True)/True, EMFrac $>0.95$

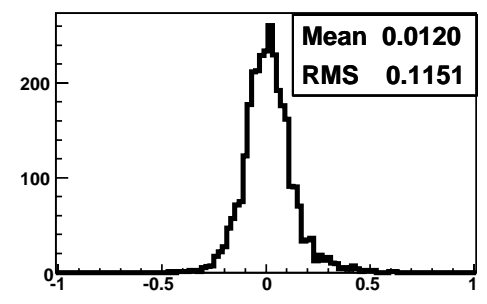

Figure 9.26: Top left shows the reconstructed energy minus true energy divided by true energy versus true energy for all $\nu_{e}$ charged current events. Top right shows the y projection. Middle left shows the reconstructed energy minus true energy divided by true energy versus true energy for $\nu_{e}$ charged current events with greater than $85 \%$ electromagnetic fraction. Middle right shows the y projection. Bottom left shows the reconstructed energy minus true energy divided by true energy versus true energy for $\nu_{e}$ charged current events with greater than $95 \%$ electromagnetic fraction. Bottom right shows the y projection. As the electromagnetic content of the shower increases, the reconstructed of the neutrino energy approaches the true value, and the width of the fractional deviation distribution decreases. 


\section{Chapter 10}

\section{Conclusions}

After ten years of development and construction, the MINOS long-baseline neutrino experiment is poised to make a precision measurement of the parameters governing neutrino oscillations. By comparing the $\nu_{\mu}$ charged current energy spectrum measured at the Far Detector with that extrapolated from the Near Detector spectrum, MINOS will be able to measure $\sin ^{2}(2 \theta)$ and $\Delta m^{2}$ to $10 \%$. While the two detector technique reduces the systematic uncertainties of the measurement, the absolute energy scale of particles interacting in each detector must be known with a precision of $5 \%$. To achieve this goal, a dedicated calibration detector, or CalDet, was exposed to particle beams in the CERN PS East Hall test beams. During the Fall months of 2001, 2002 and 2003, CalDet recorded the properties of over 30 million particle interactions of incident momenta spanning the range of interest to MINOS, namely the range from $200 \mathrm{MeV}$ to $10 \mathrm{GeV}$. These runs allowed for the study of the characteristics of electron, pion, muon, and proton interactions of both positive and negative charge.

This document describes the first global analysis of the electromagnetic interactions at CalDet. The conditions under which the data samples were collected has been documented. The steps necessary to calibrate the detector response have 
been discussed, and it was demonstrated that the calibration system is sufficient to correct $40 \%$ drifts in detector response to better than $0.5 \%$. The selection criteria necessary to select a clean sample of electrons were presented, and beam related effects that give rise to errors in the response measurement were explored. Furthermore, a simulation to calculate the energy lost in the upstream beam elements has been developed, and its effect was compared to specially collected data to prove its efficacy. The topological properties of electron events in the MINOS geometries were investigated and compared favorably to tuned Monte Carlo. Moreover, the properties of electromagetic showers were contrasted against those of hadronic showers. As expected, it was found that electron events are short, narrow dense depostions of energy, as opposed to pion events which are typically longer, wider and more diffuse. The CalDet response as a function of the incident momentum of the electron has been determined to be

$$
(65.77 \pm 0.08) \frac{\mathrm{MIP}}{\mathrm{GeV}} \times \mathrm{P}-(1.46 \pm 0.06) \mathrm{MIP}
$$

with an energy resolution of

$$
\frac{21.42 \pm 0.06 \%}{\sqrt{P}} \oplus \frac{4.1 \pm 0.2 \%}{P}
$$

A number of differences between the electrons produced in neutrino interactions and the electrons of CalDet have been explored. In particular, electrons incident at an angle to the detector planes were studied. While the characteristics of angled electrons do differ from the properties of normally incident electrons, the differences are understood and moreover, the angle of the event can be determined on an event by event basis. The effect of traveling through an extra scintillator plane and half a steel plane on the electromagnetic response was studied and special data samples were used to demonstrate that the effect can be corrected for using Monte Carlo. After correcting for the first plane effects, the detector response is found to 
be

$$
(65.81 \pm 0.08) \frac{\mathrm{MIP}}{\mathrm{GeV}} \times \mathrm{P}-(0.23 \pm 0.07) \mathrm{MIP} .
$$

In addition, although neutrino showers can be expected to be more complex than showers induced by single electrons, there is a class of $\nu_{e}$, charged current events with high electromagnetic content that closely resembles the electrons of the CalDet.

Its purpose fulfilled, the CalDet has been de-cabled for the last time. But even as the CalDet effort winds down, the MINOS collaboration looks forward to the first protons on target in December 2004, and the observation of the first beam neutrinos in both detectors shortly after. With the fundamentals of detector operation, single particle topology, and response established by CalDet, the real fun of studying neutrino oscillations begins. 


\section{Appendix A}

\section{Event Displays}

The following plots show electron, pion, proton and muon events at several beam momenta. The top plot in each panel shows the plane and strip position of each hit in the even planes while the bottom plot shows the position in the odd planes. The color scale gives the pulse height of the hit in MIPs. 

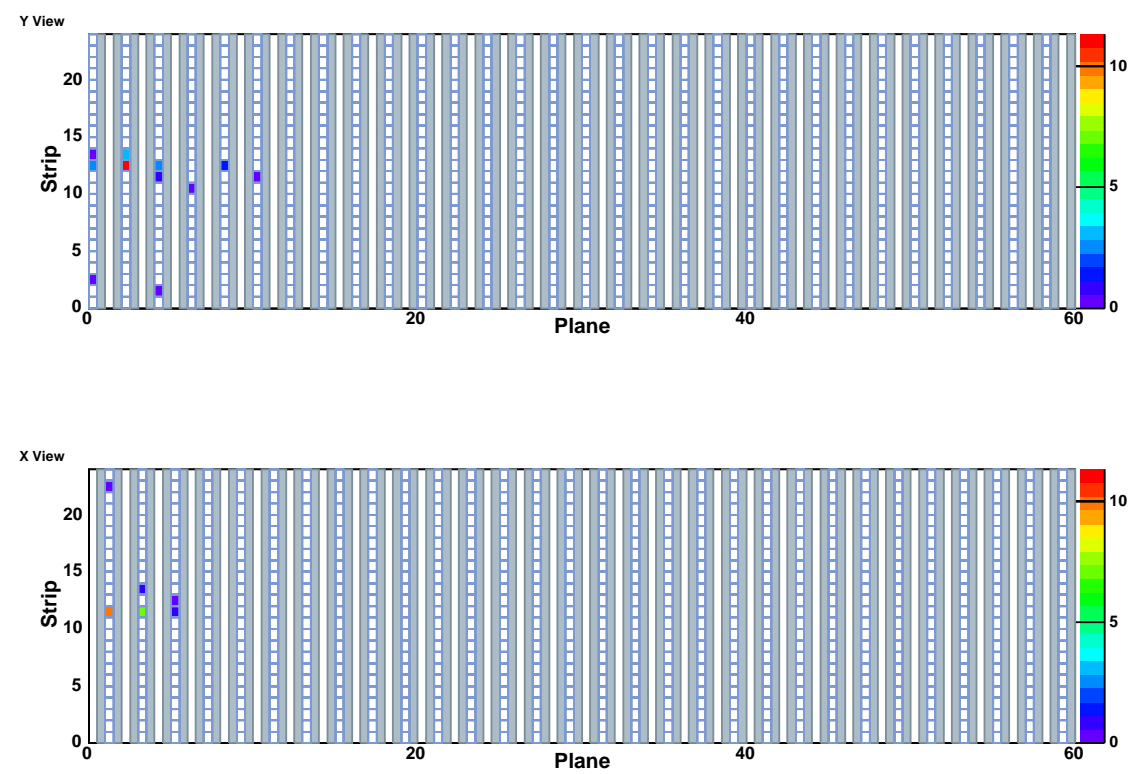

Figure A.1: A $0.6 \mathrm{GeV}$ electron
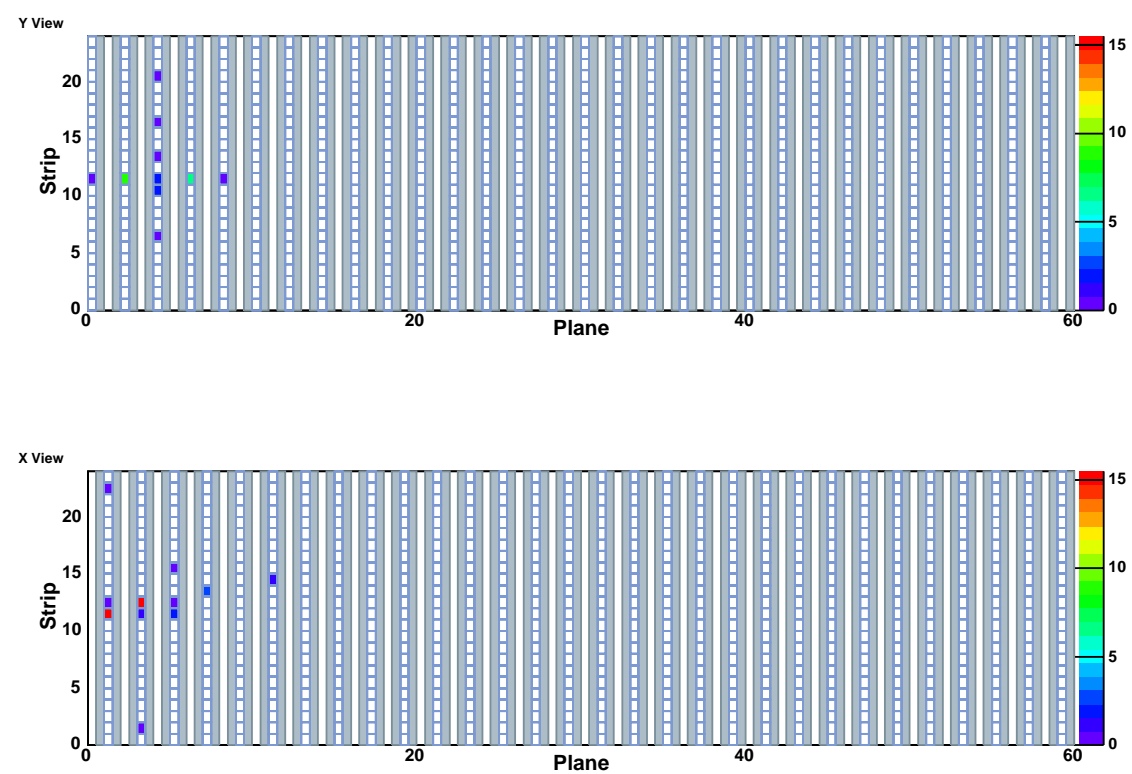

Figure A.2: A $1.0 \mathrm{GeV}$ electron 

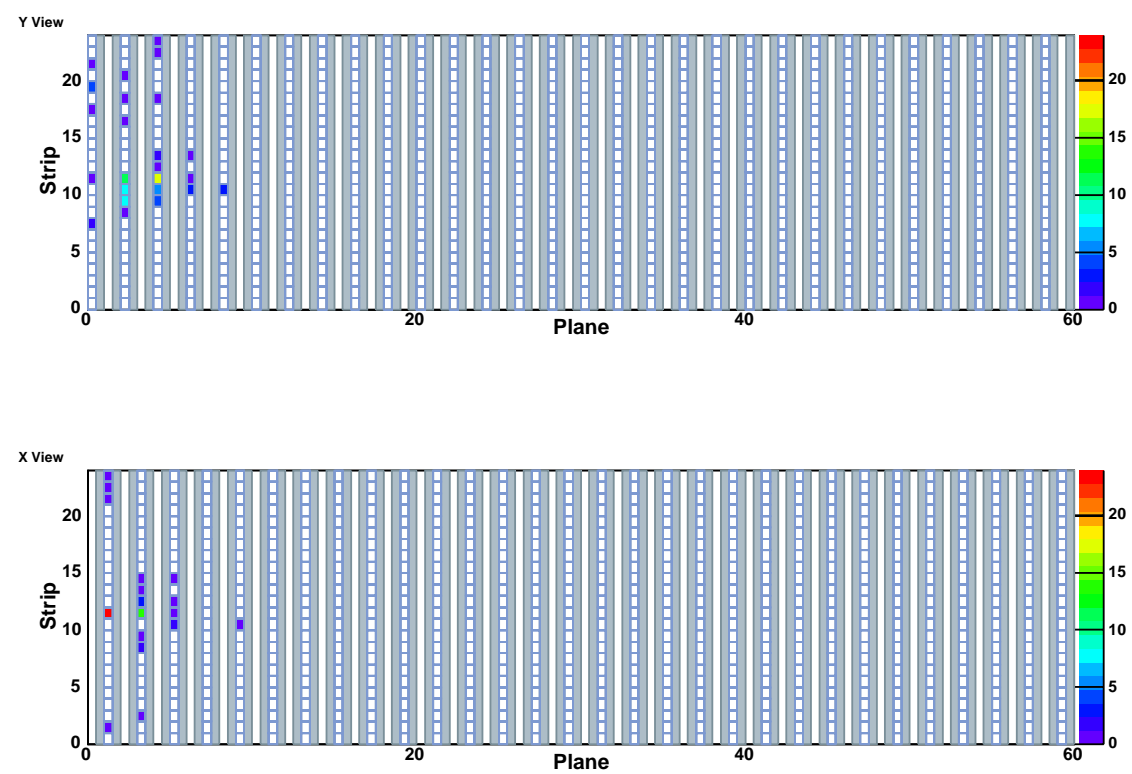

Figure A.3: A $2.0 \mathrm{GeV}$ electron
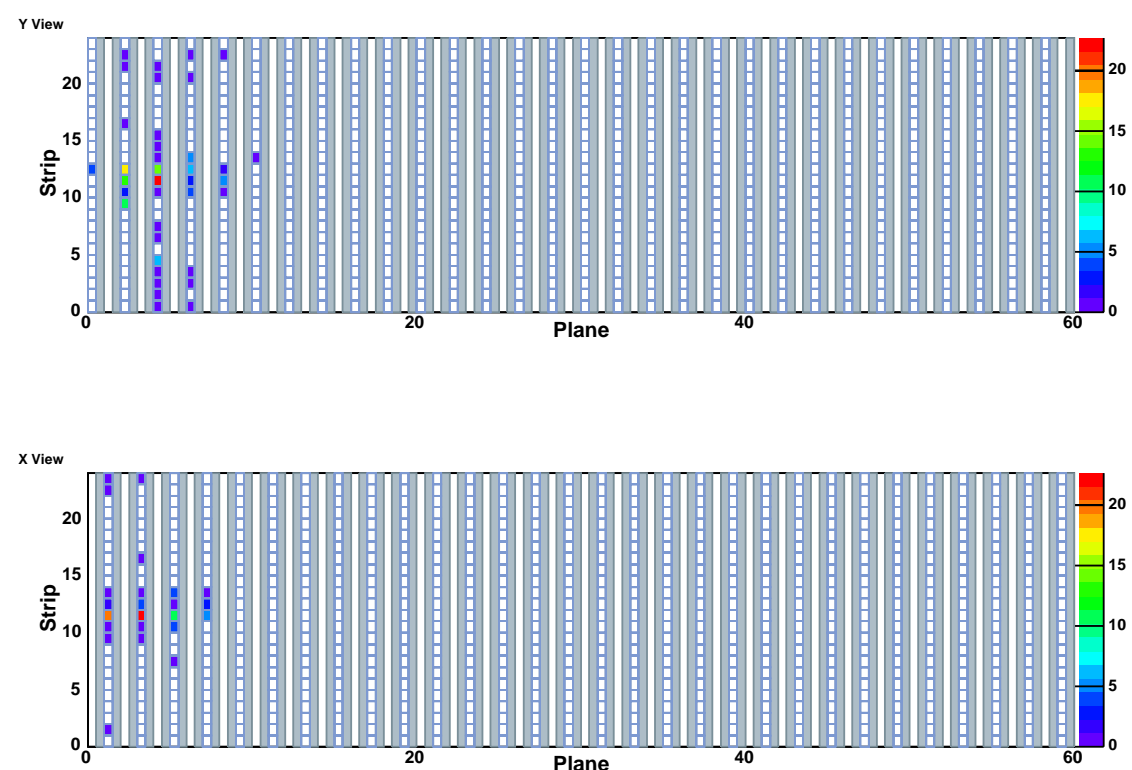

Figure A.4: A $3.0 \mathrm{GeV}$ electron 

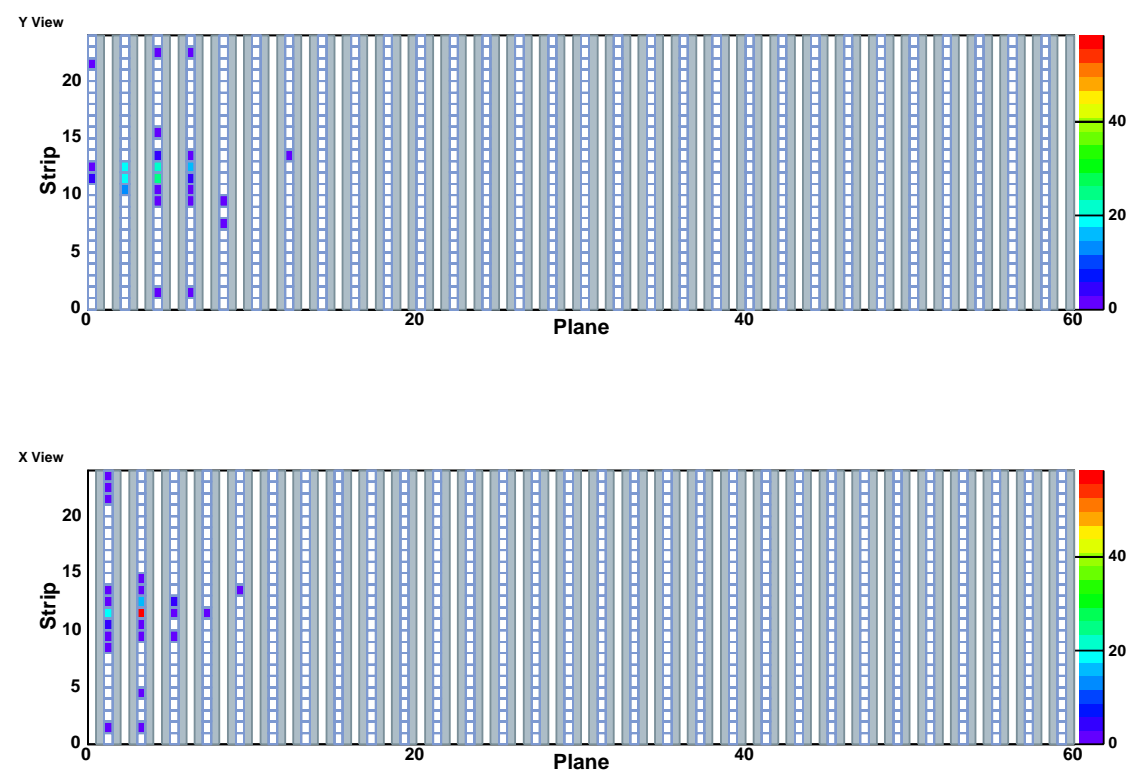

Figure A.5: A $4.0 \mathrm{GeV}$ electron
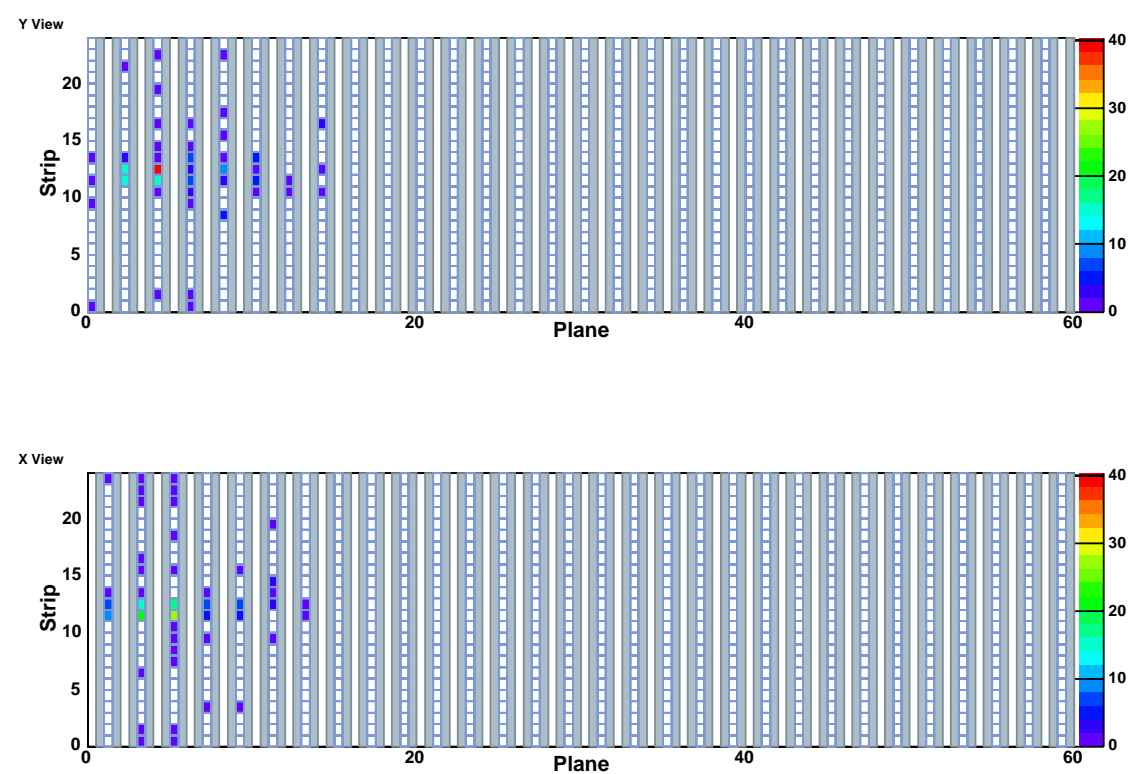

Figure A.6: A $5.0 \mathrm{GeV}$ electron 

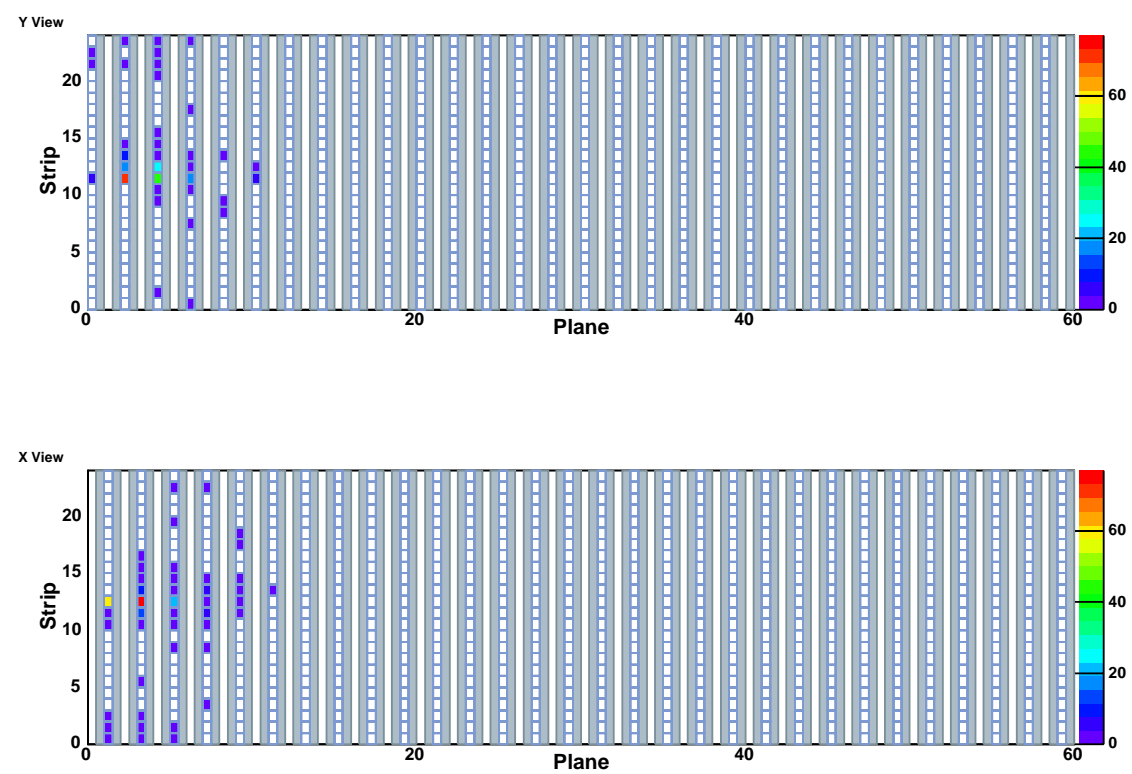

Figure A.7: A $6.0 \mathrm{GeV}$ electron
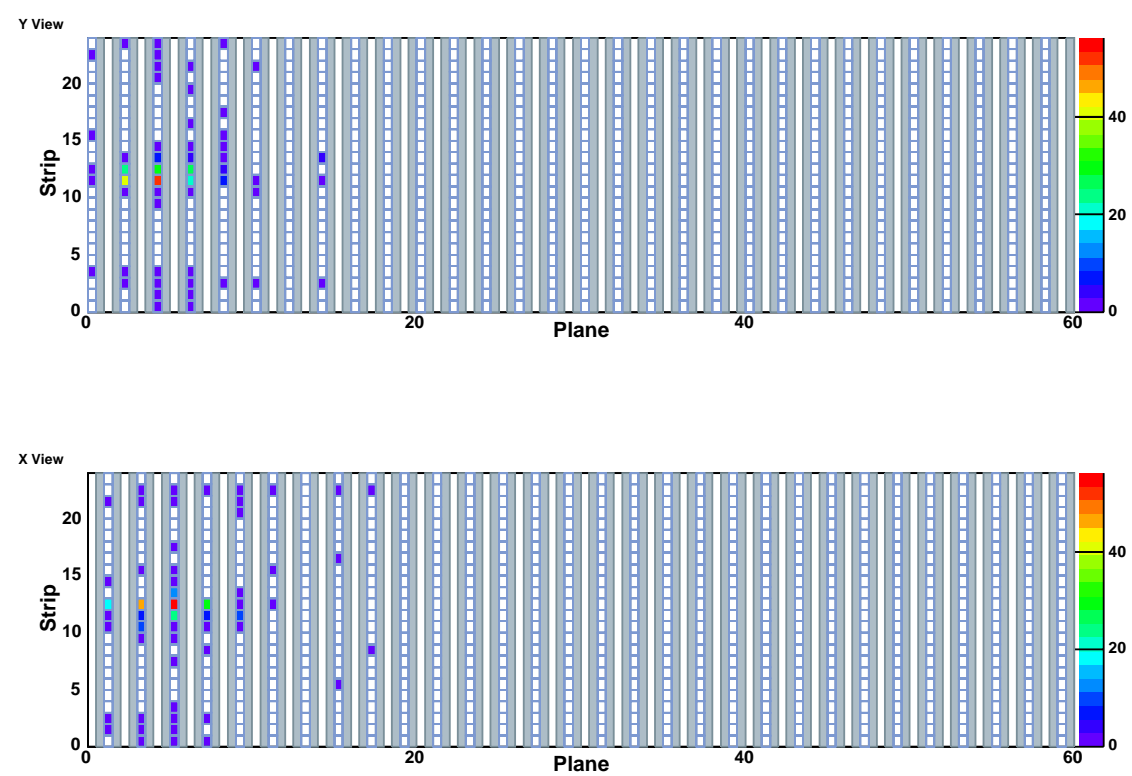

Figure A.8: A 7.0 GeV electron 

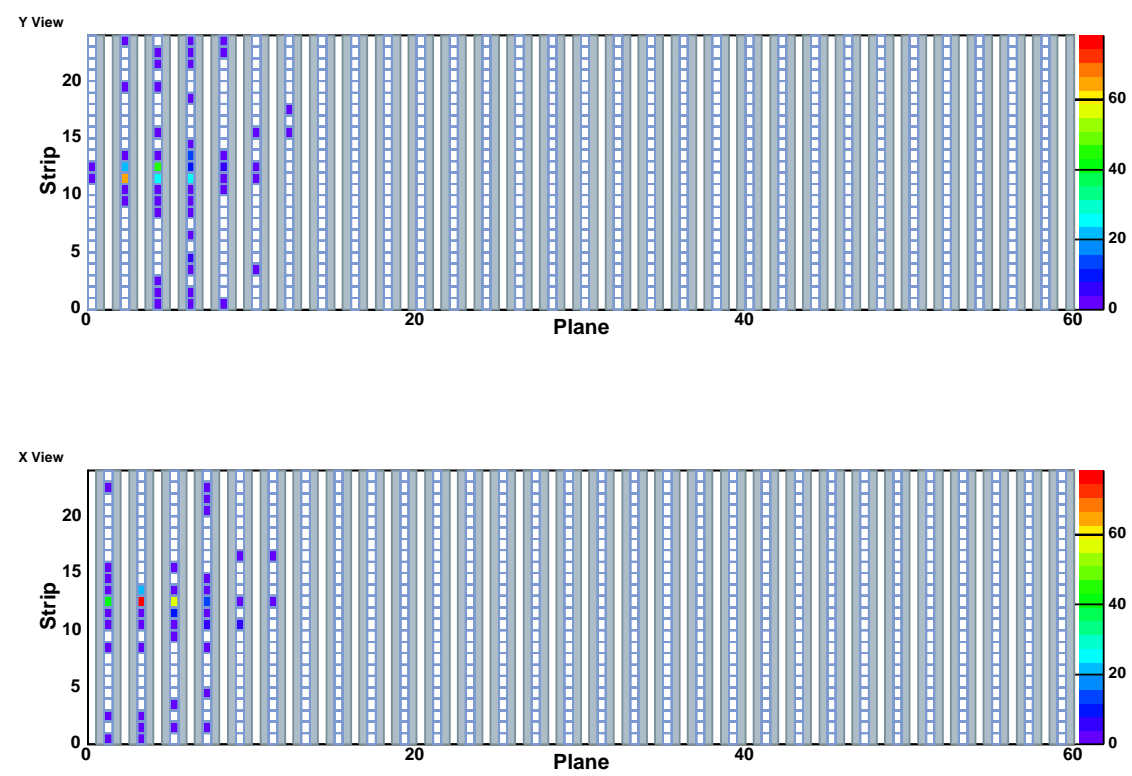

Figure A.9: A $8.0 \mathrm{GeV}$ electron
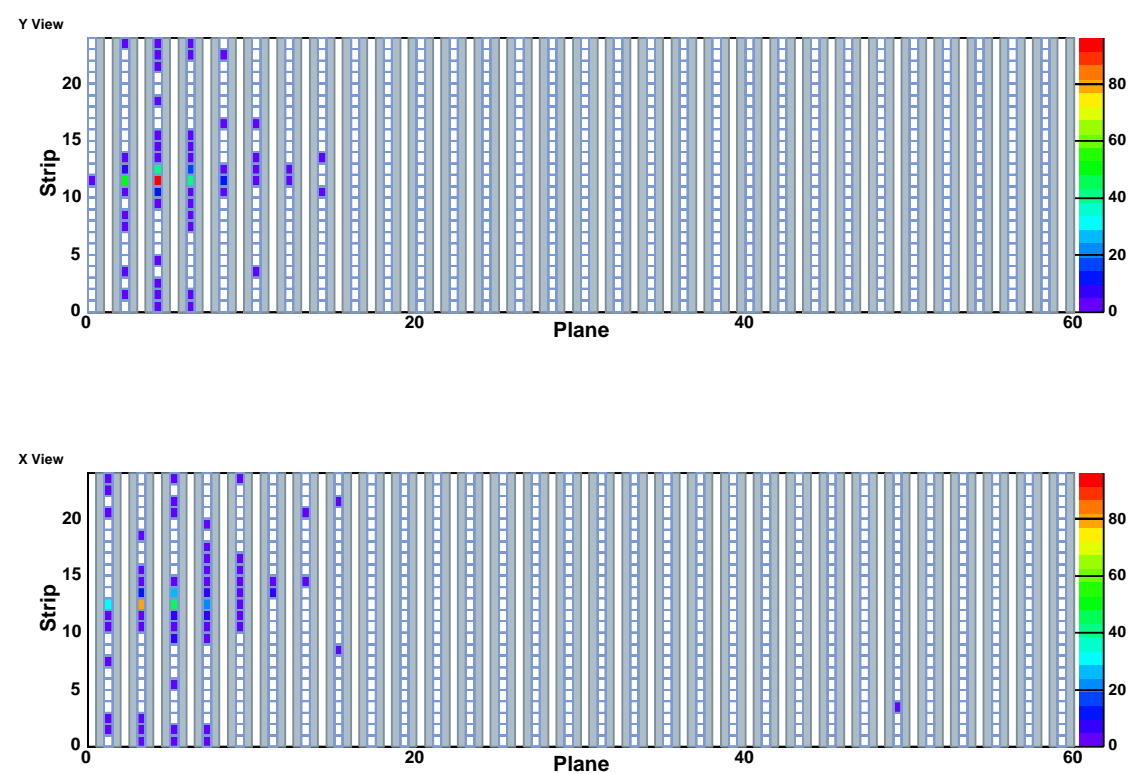

Figure A.10: A $9.0 \mathrm{GeV}$ electron 

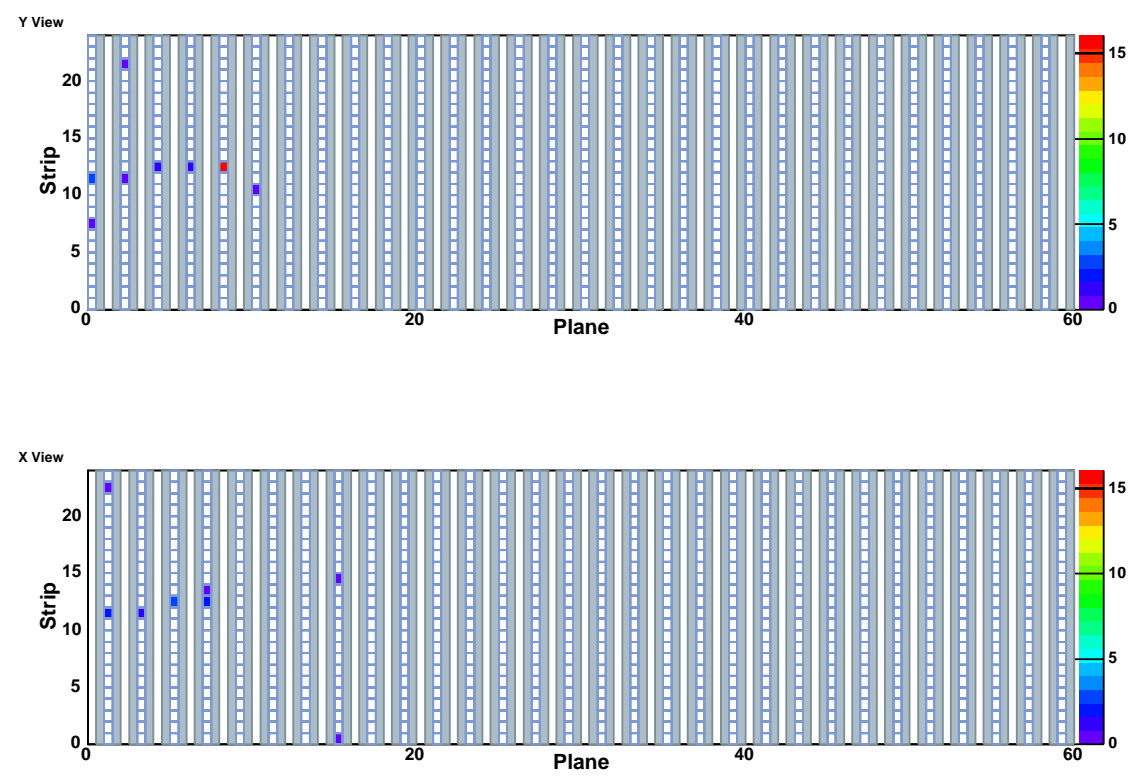

Figure A.11: A $0.6 \mathrm{GeV}$ pion
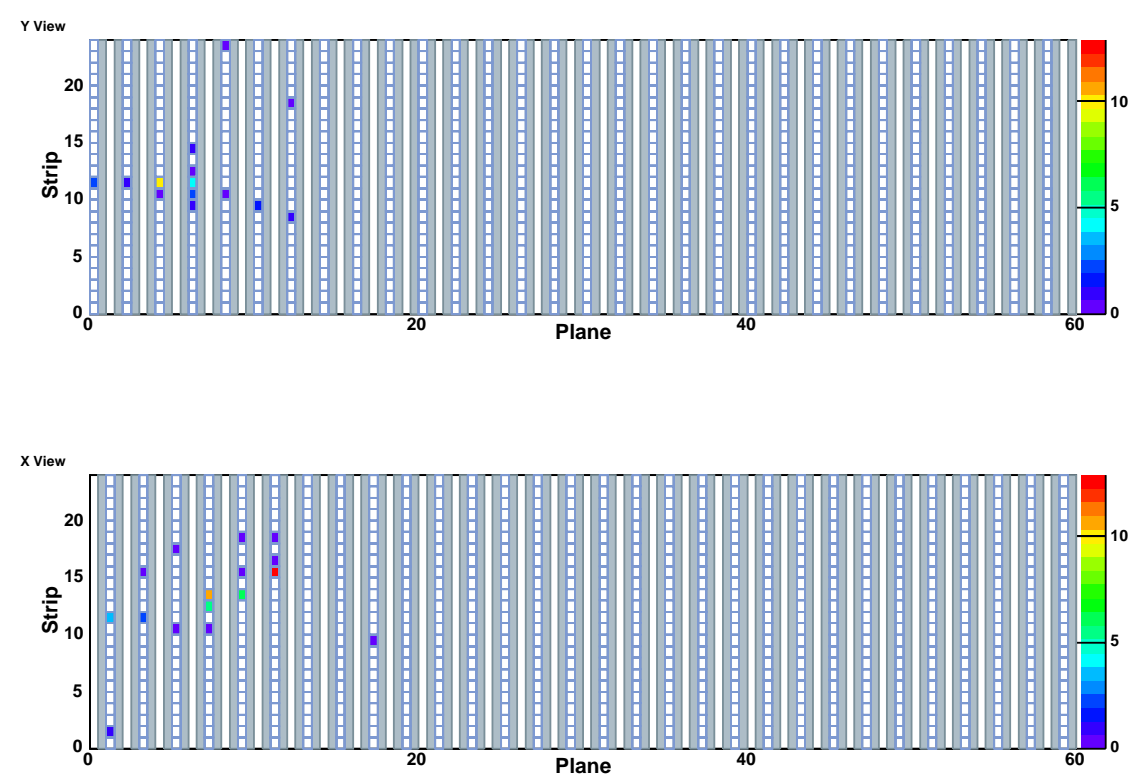

Figure A.12: A $1.0 \mathrm{GeV}$ pion 

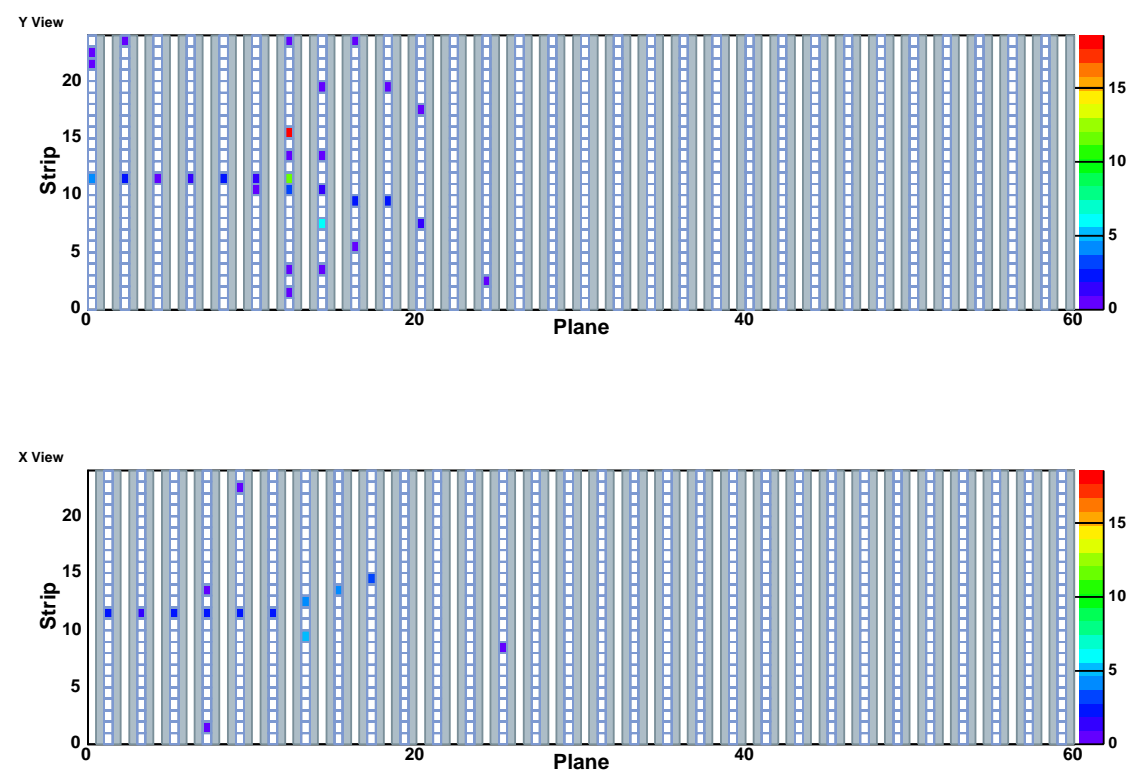

Figure A.13: A $2.0 \mathrm{GeV}$ pion
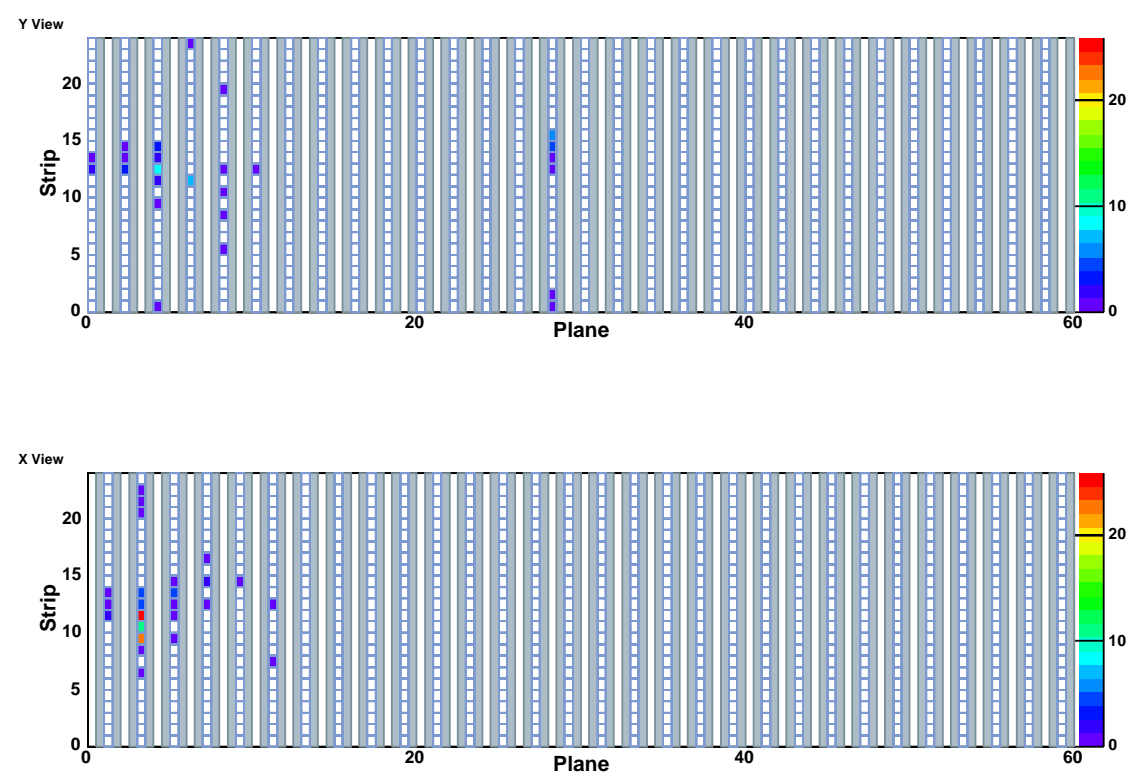

Figure A.14: A $3.0 \mathrm{GeV}$ pion 

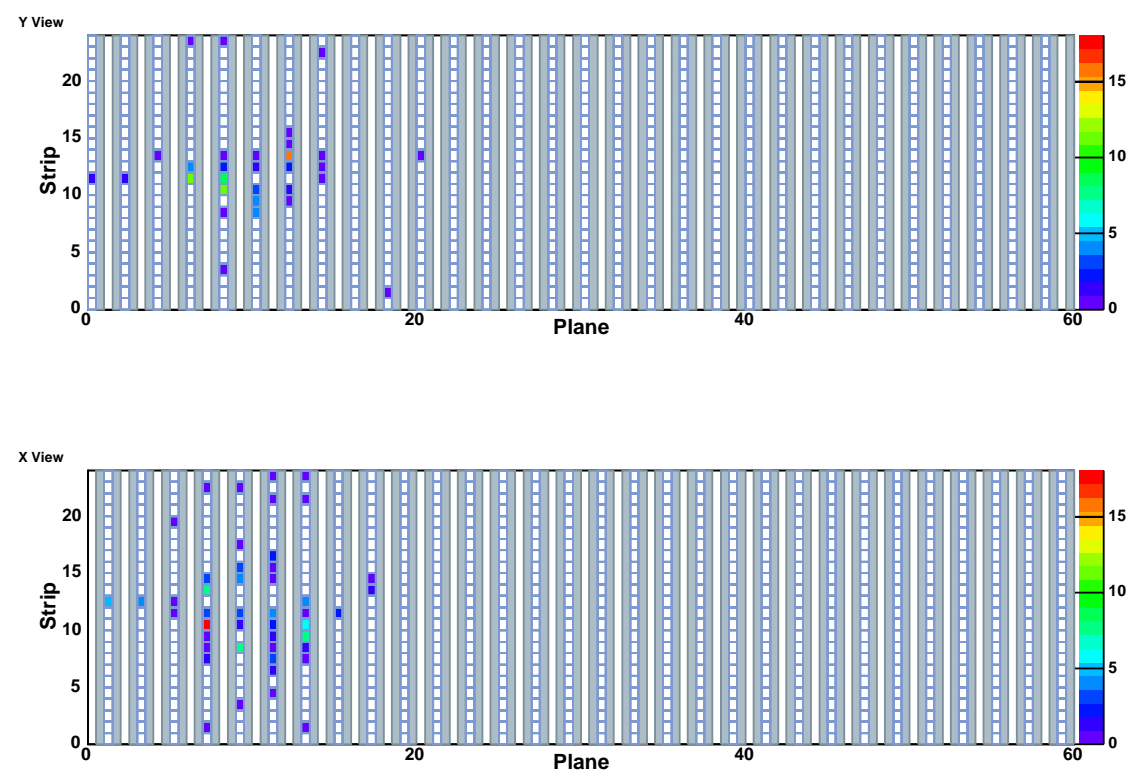

Figure A.15: A $4.0 \mathrm{GeV}$ pion
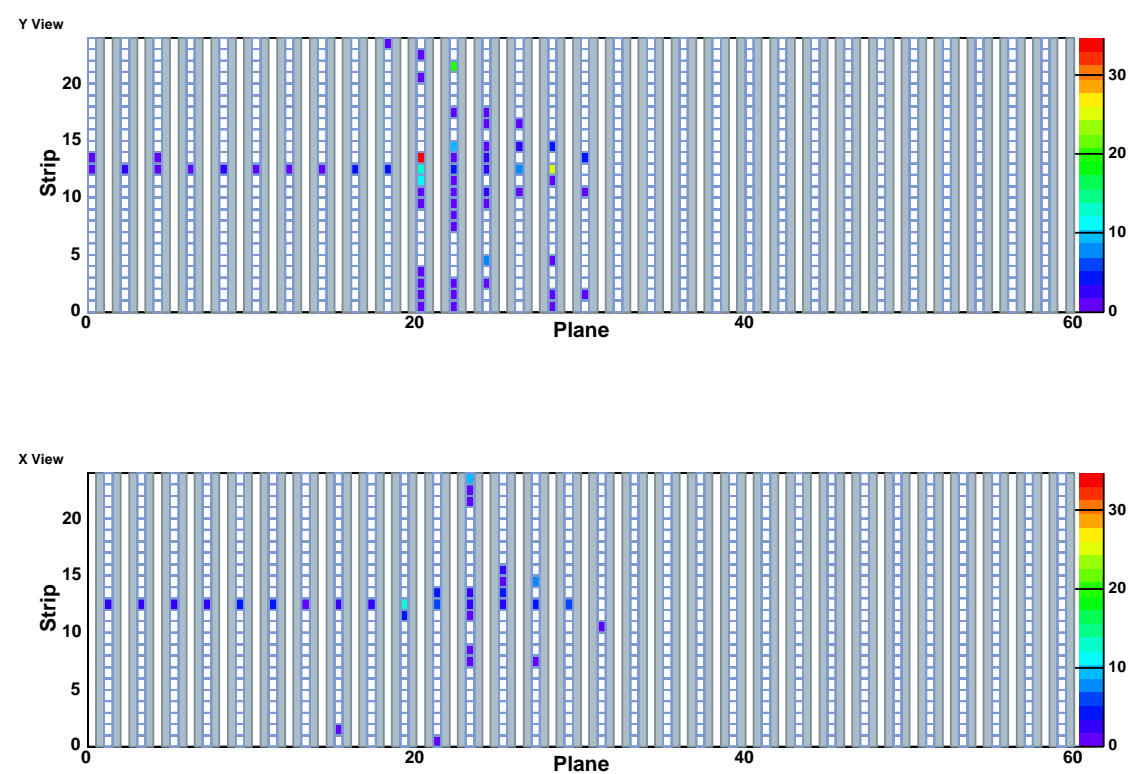

Figure A.16: A $5.0 \mathrm{GeV}$ pion 

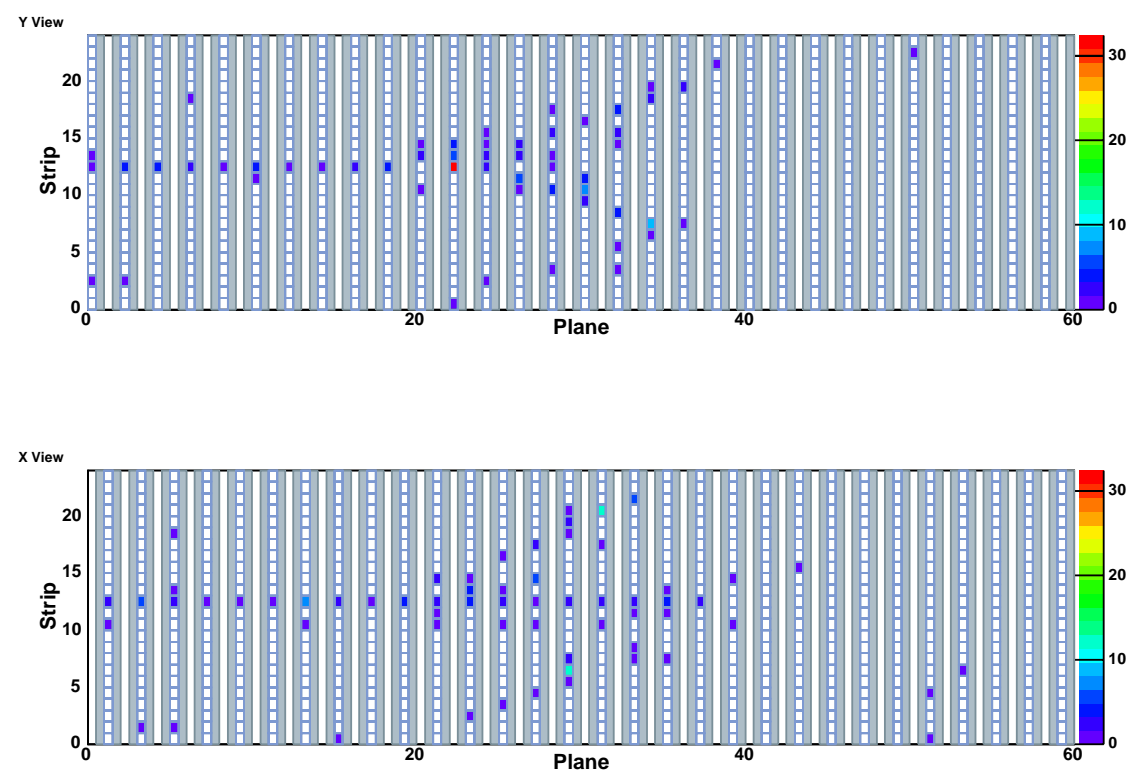

Figure A.17: A $6.0 \mathrm{GeV}$ pion
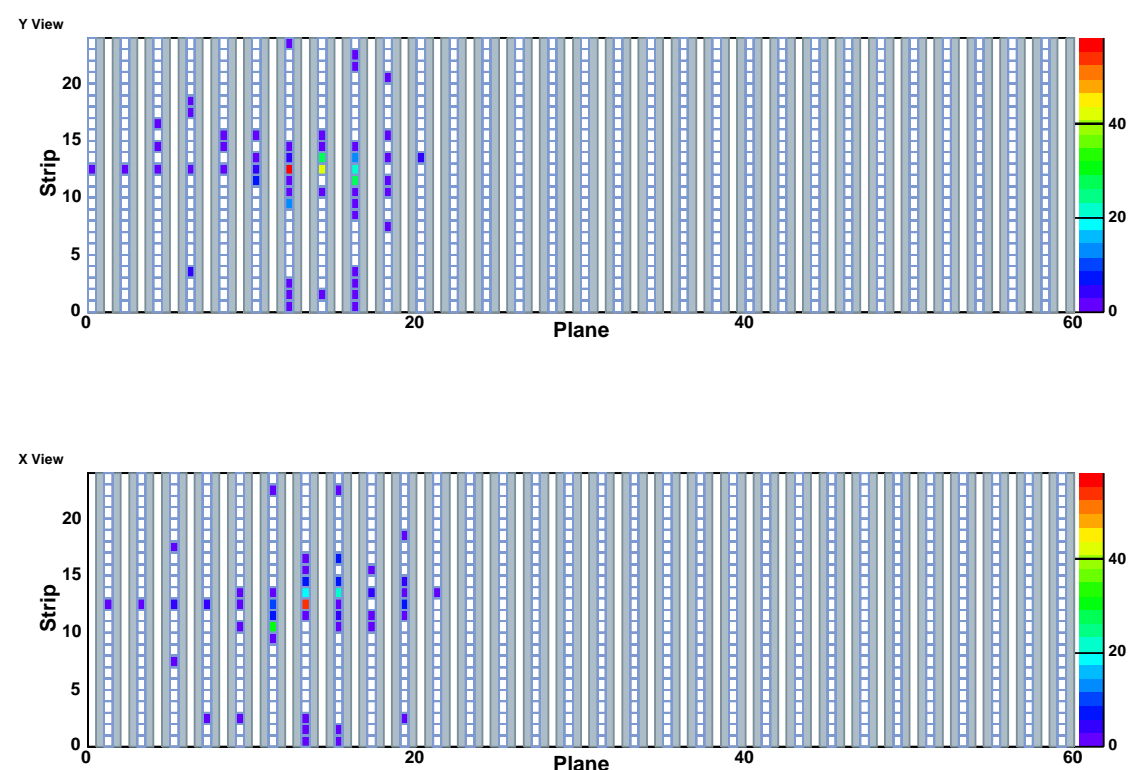

Figure A.18: A $7.0 \mathrm{GeV}$ pion 

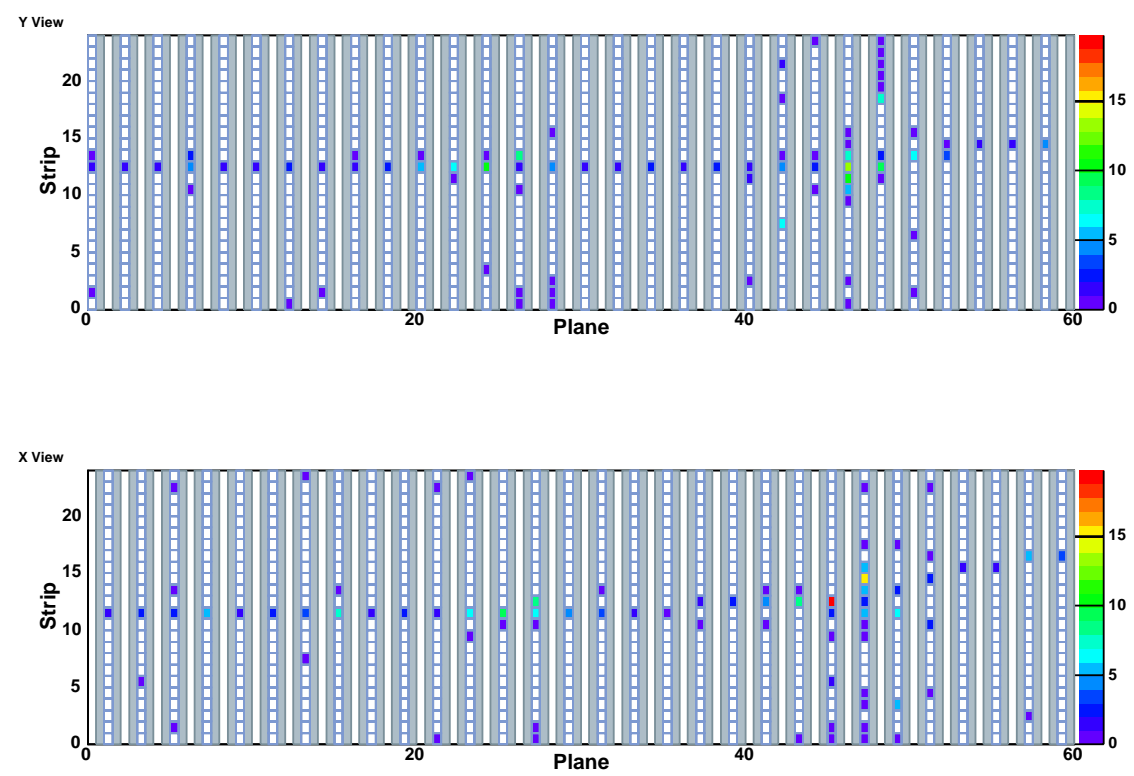

Figure A.19: A $8.0 \mathrm{GeV}$ pion
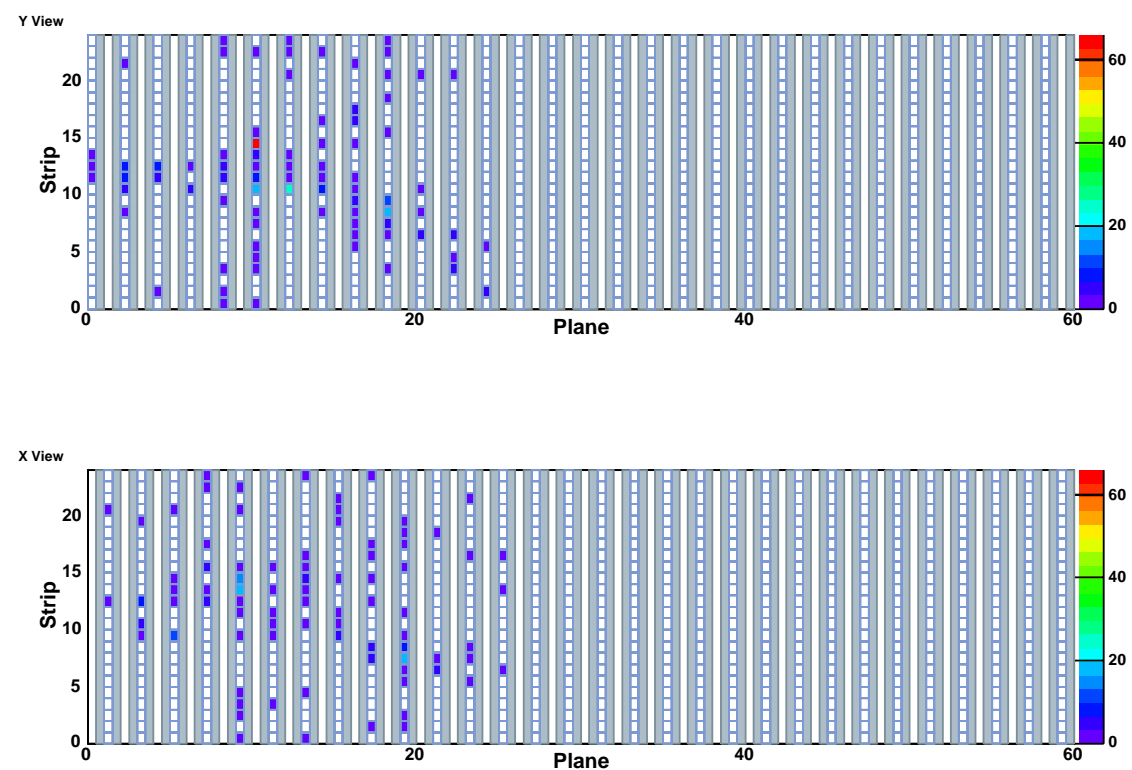

Figure A.20: A $9.0 \mathrm{GeV}$ pion 

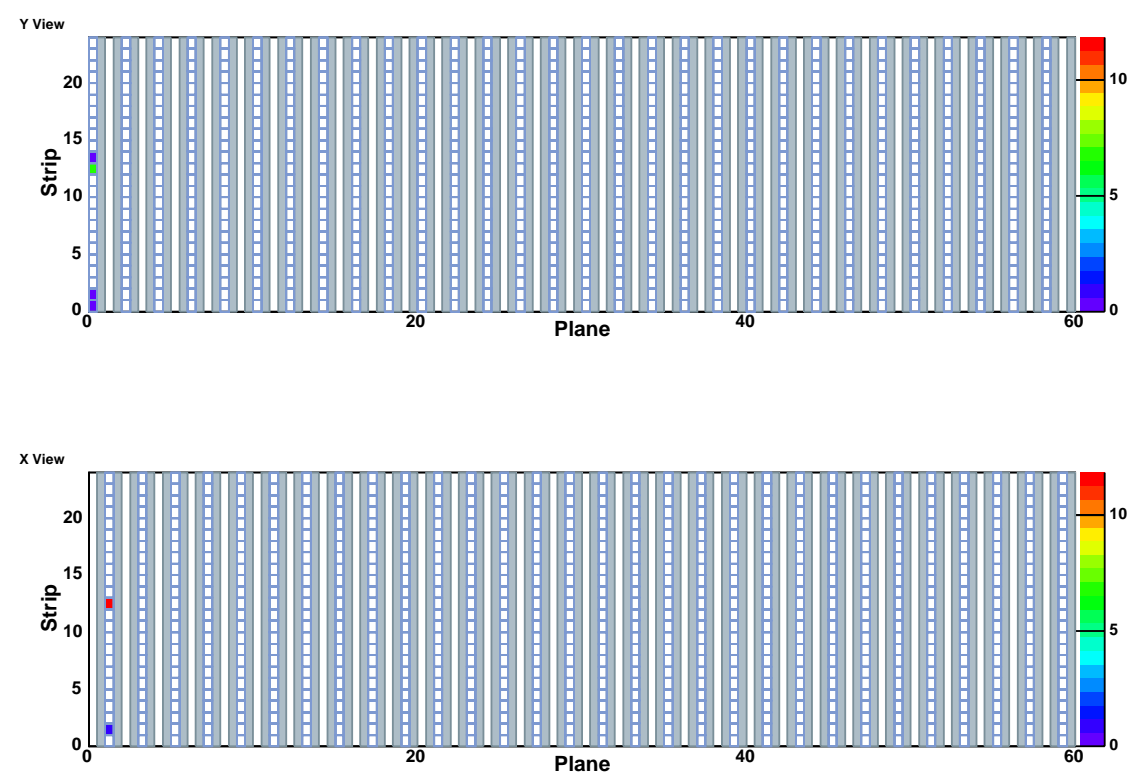

Figure A.21: A $0.6 \mathrm{GeV}$ proton
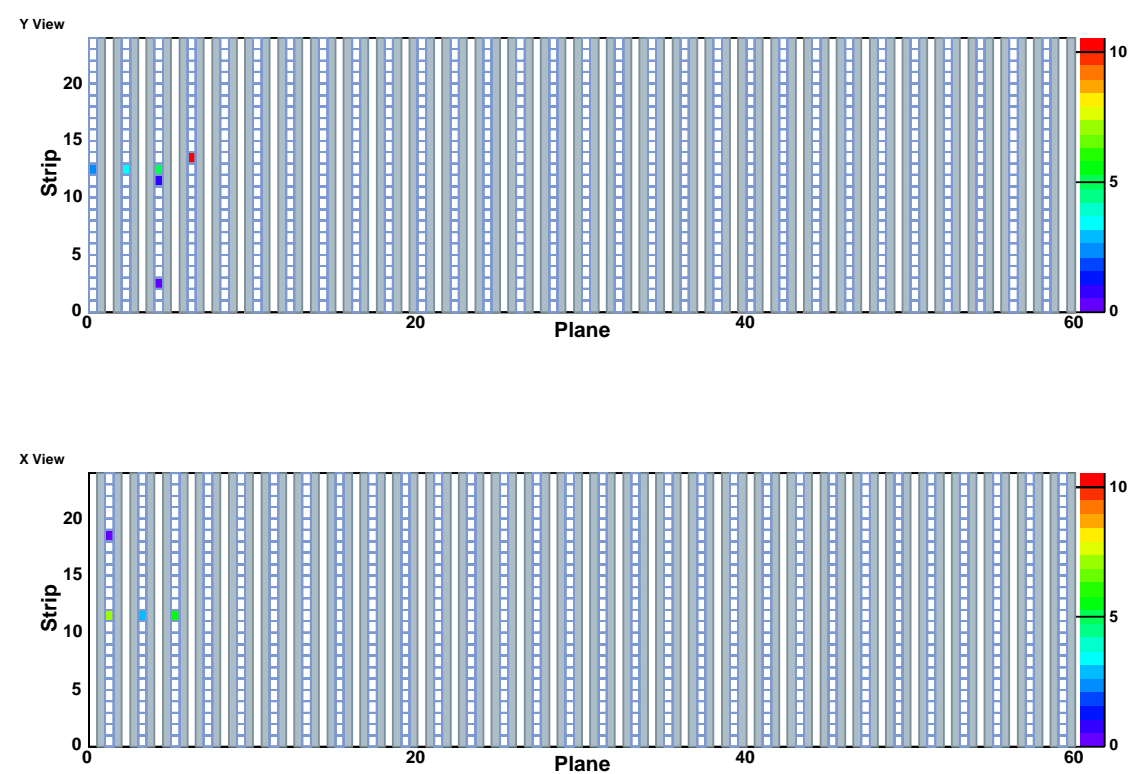

Figure A.22: A $1.0 \mathrm{GeV}$ proton 

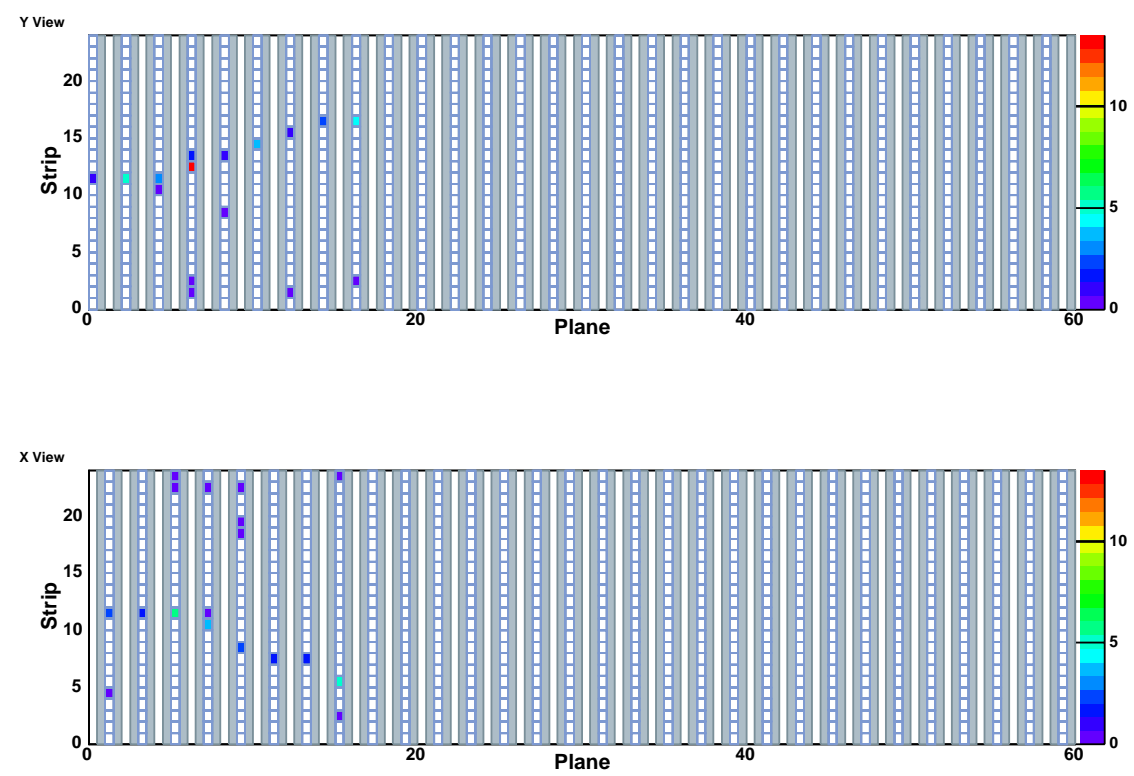

Figure A.23: A $2.0 \mathrm{GeV}$ proton
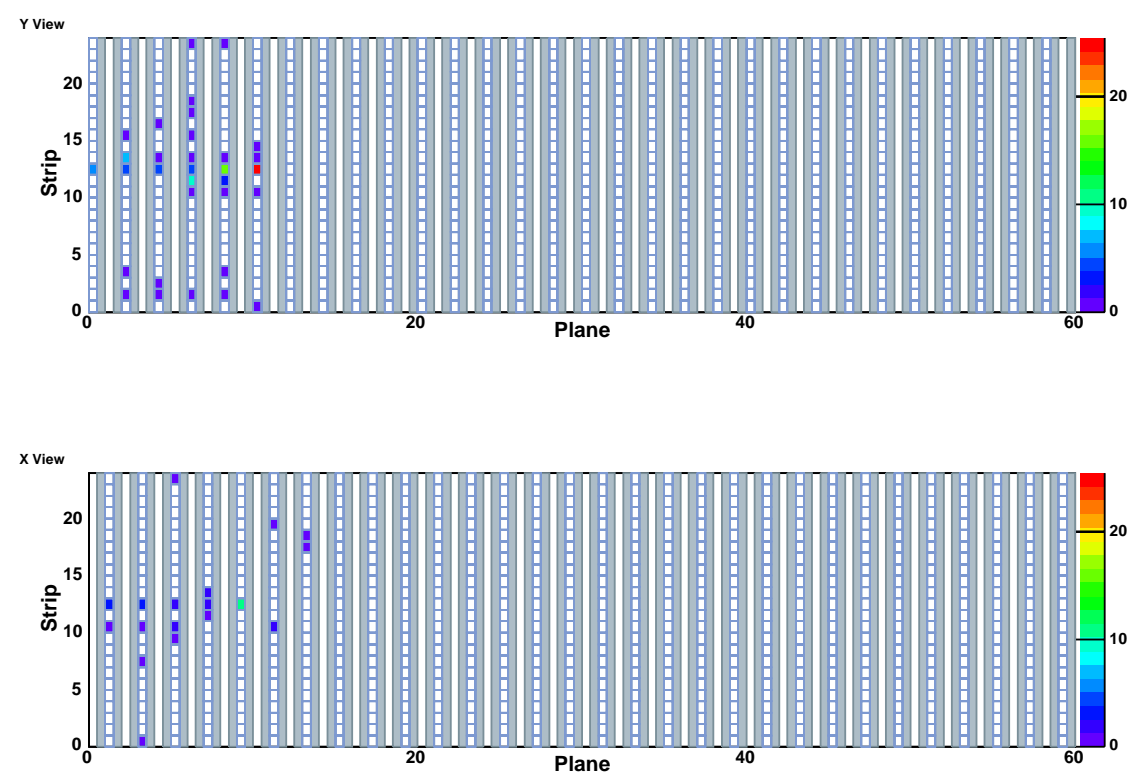

Figure A.24: A $3.0 \mathrm{GeV}$ proton 

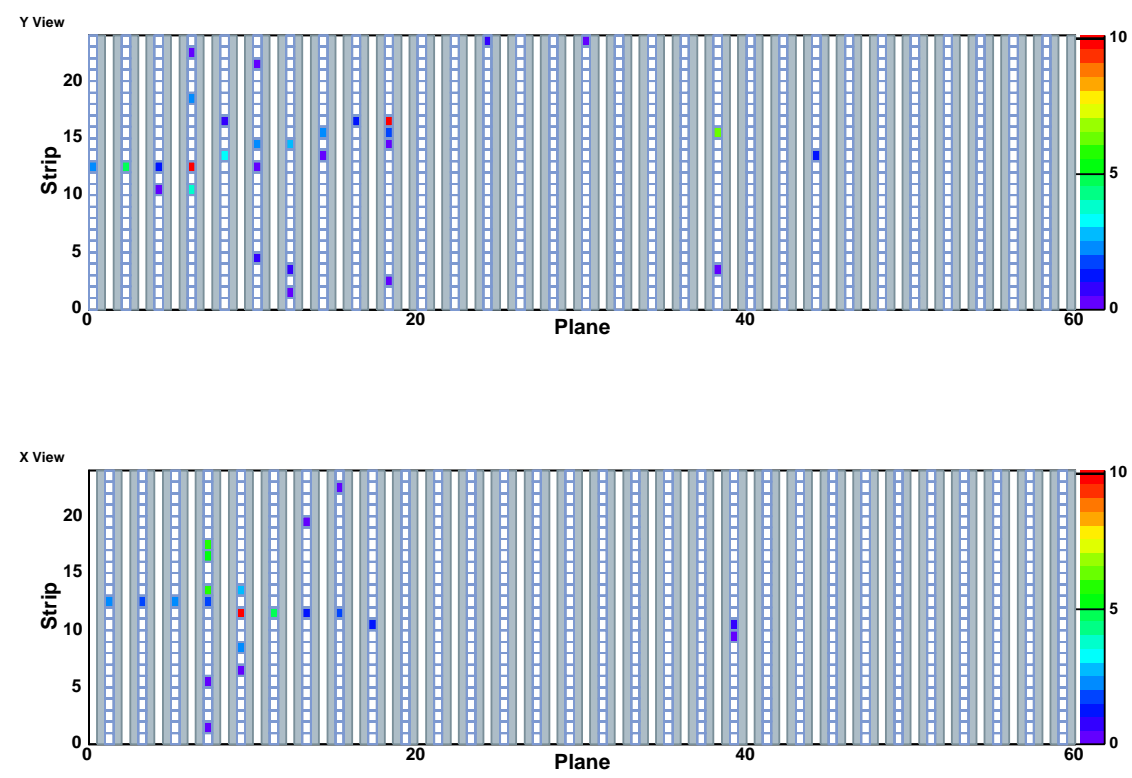

Figure A.25: A $4.0 \mathrm{GeV}$ proton
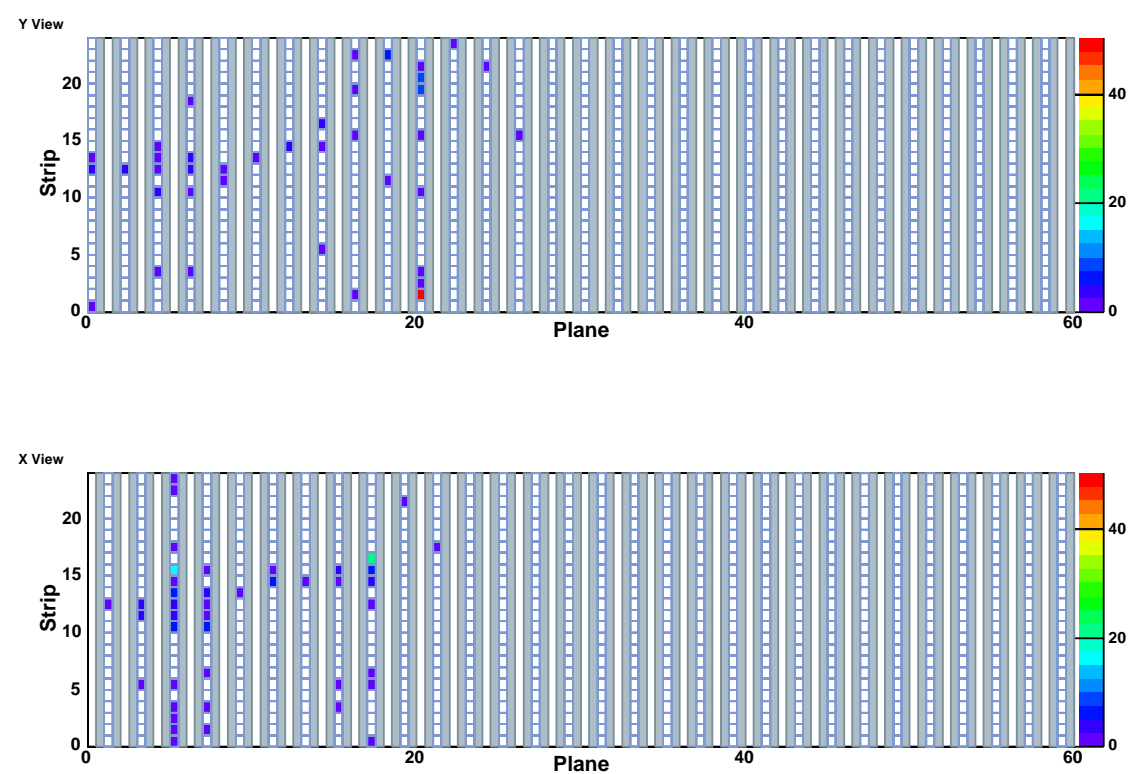

Figure A.26: A $5.0 \mathrm{GeV}$ proton 

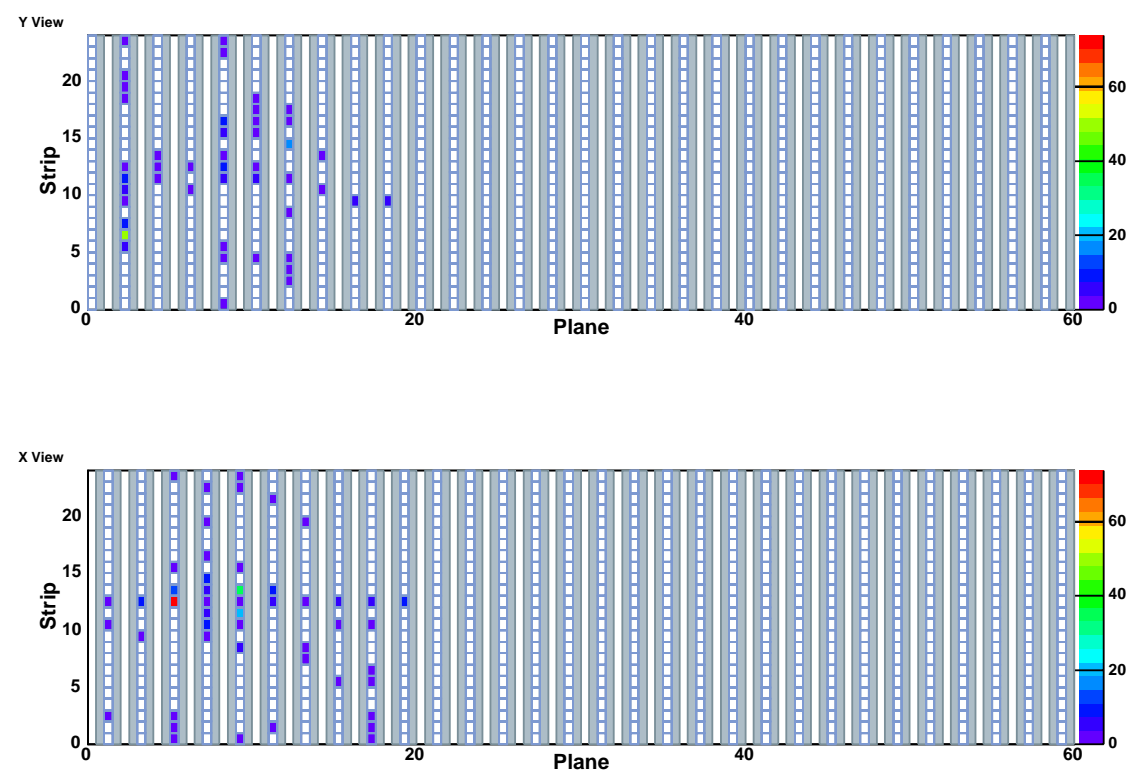

Figure A.27: A $6.0 \mathrm{GeV}$ proton
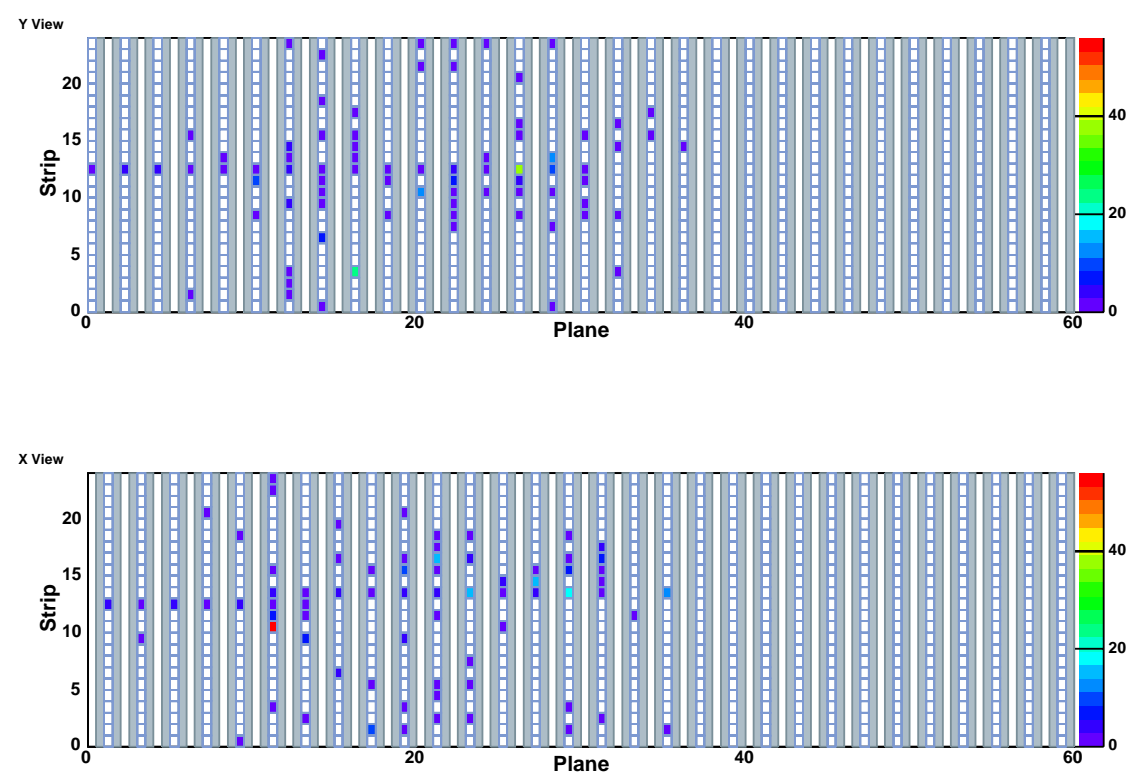

Figure A.28: A $7.0 \mathrm{GeV}$ proton 

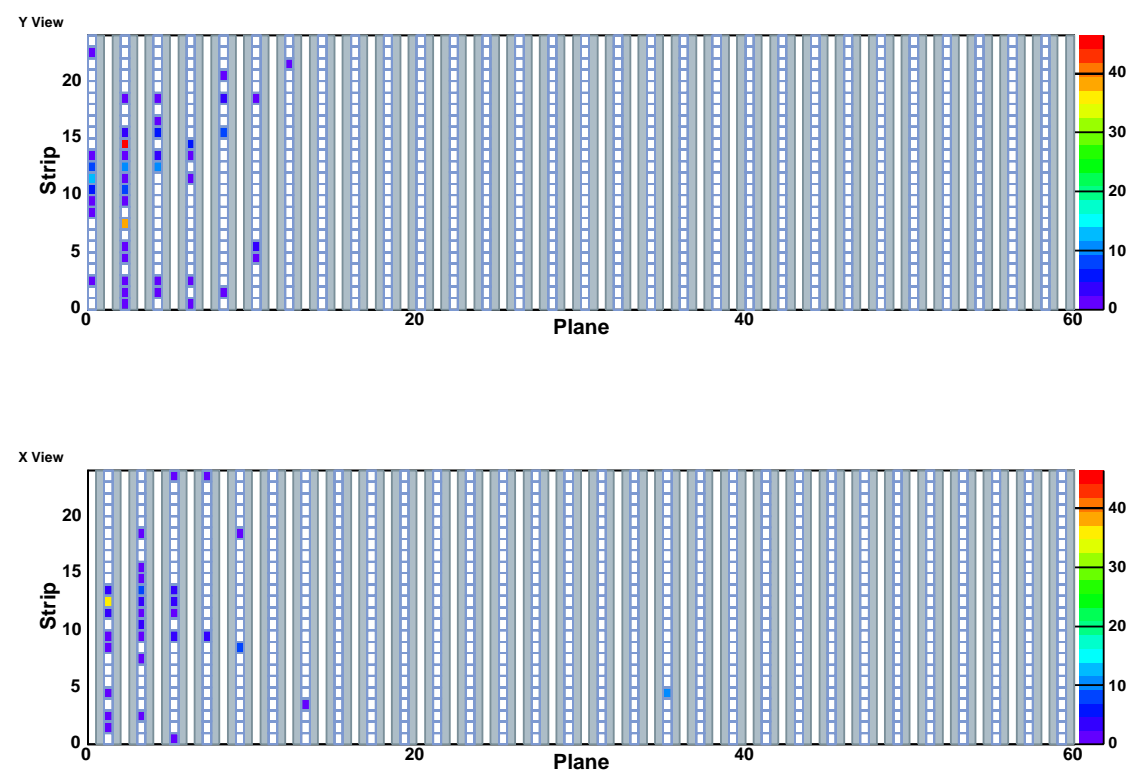

Figure A.29: A $8.0 \mathrm{GeV}$ proton
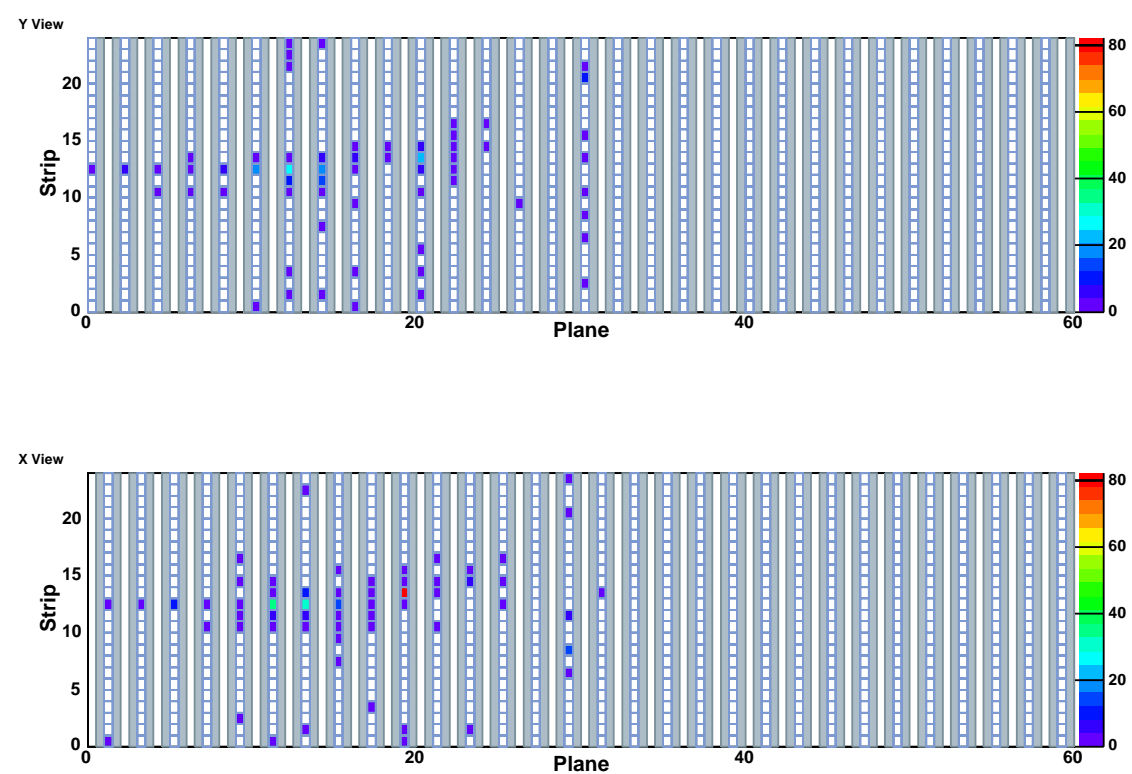

Figure A.30: A $9.0 \mathrm{GeV}$ proton 

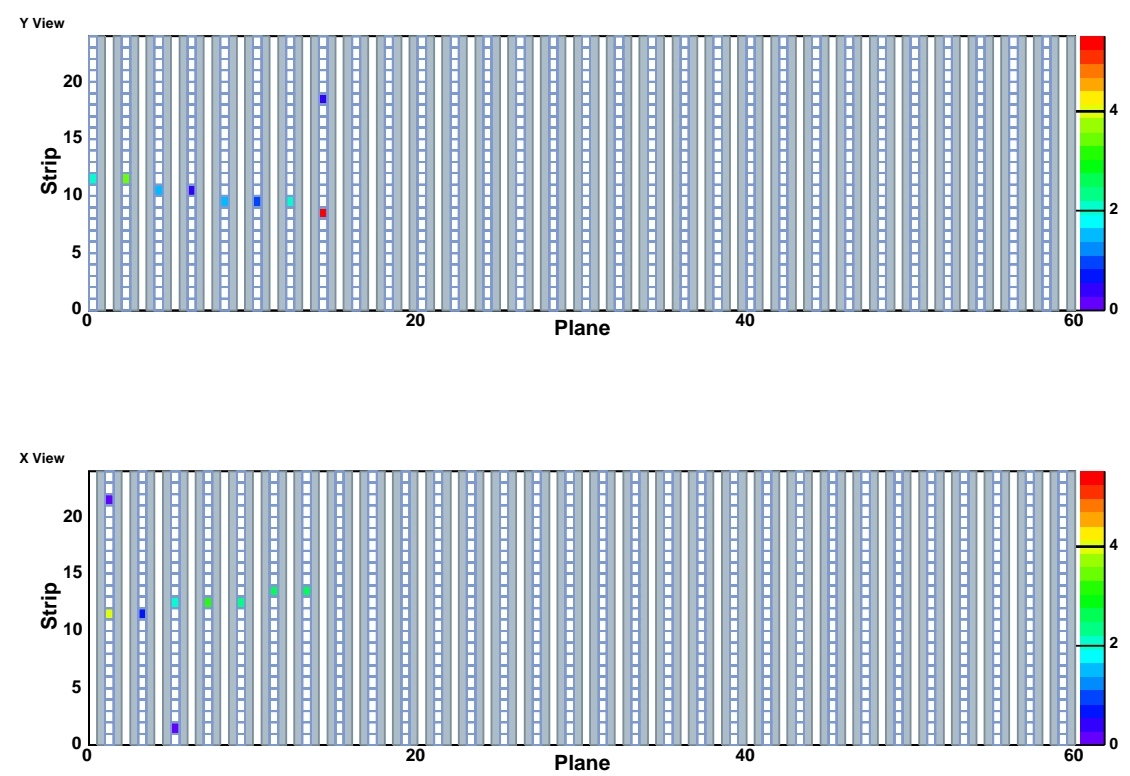

Figure A.31: A $0.6 \mathrm{GeV}$ muon
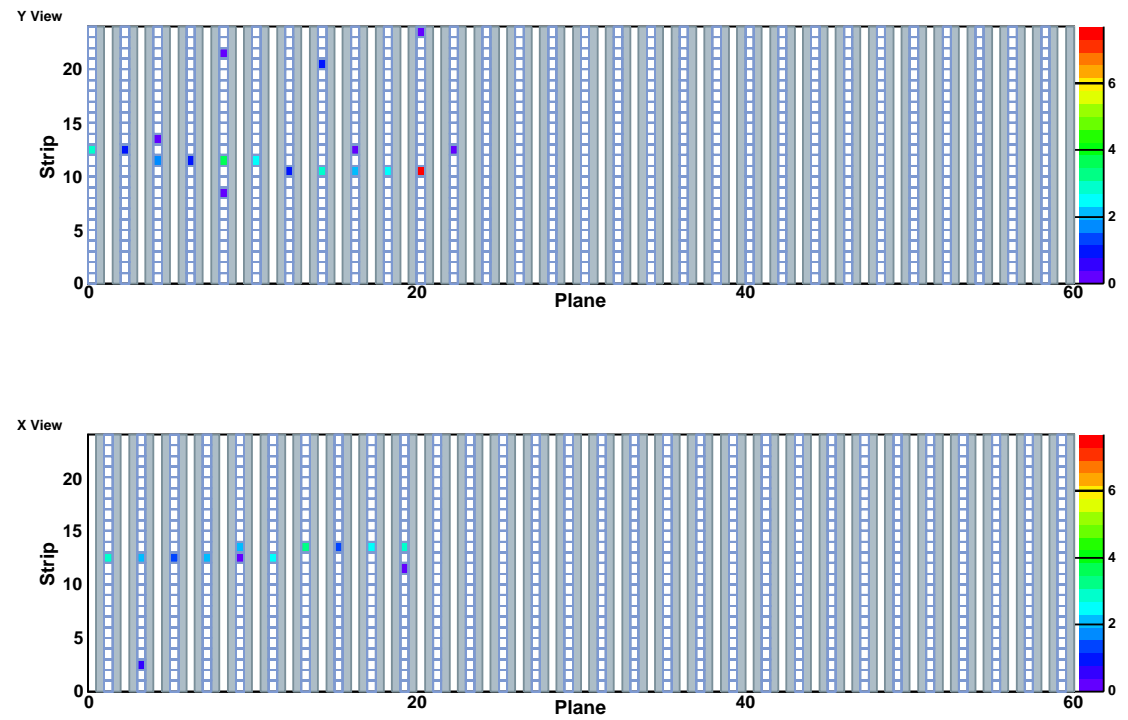

Figure A.32: A $0.8 \mathrm{GeV}$ muon 

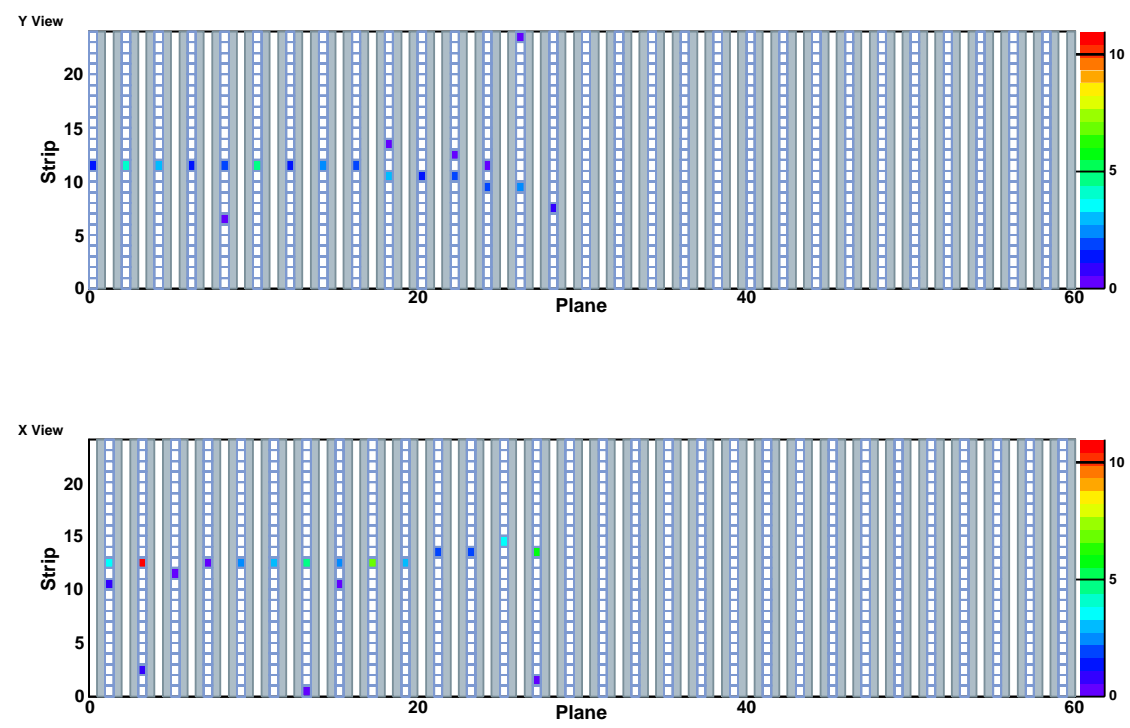

Figure A.33: A $1.0 \mathrm{GeV}$ muon
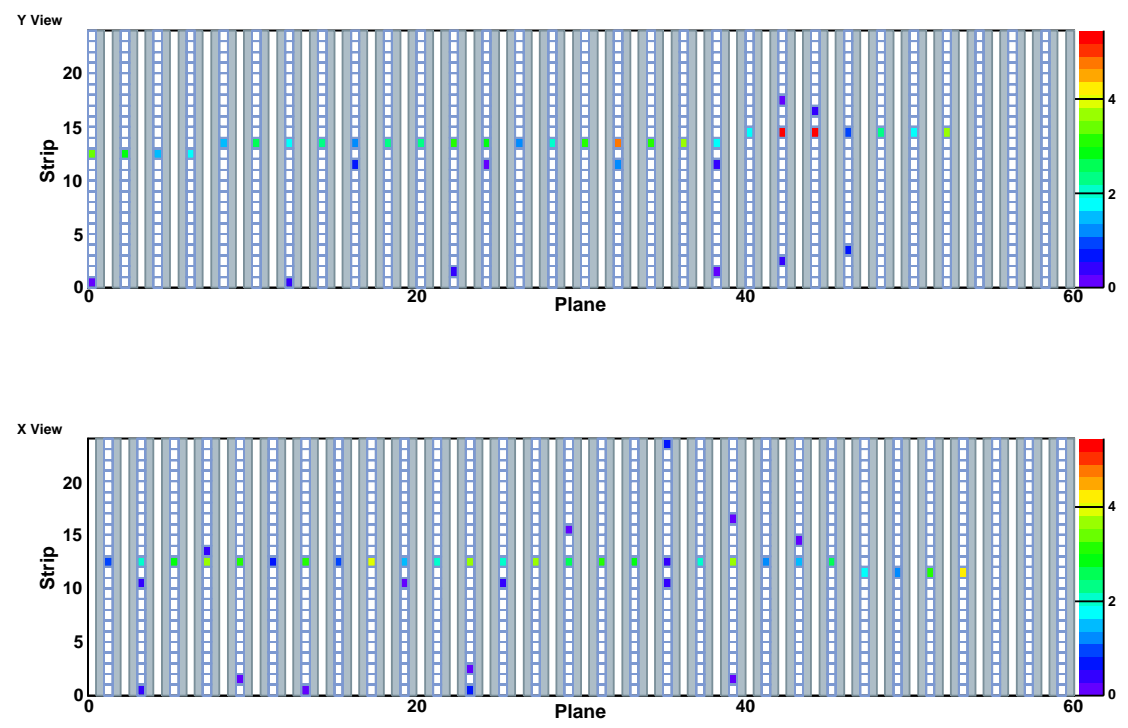

Figure A.34: A $1.8 \mathrm{GeV}$ muon 

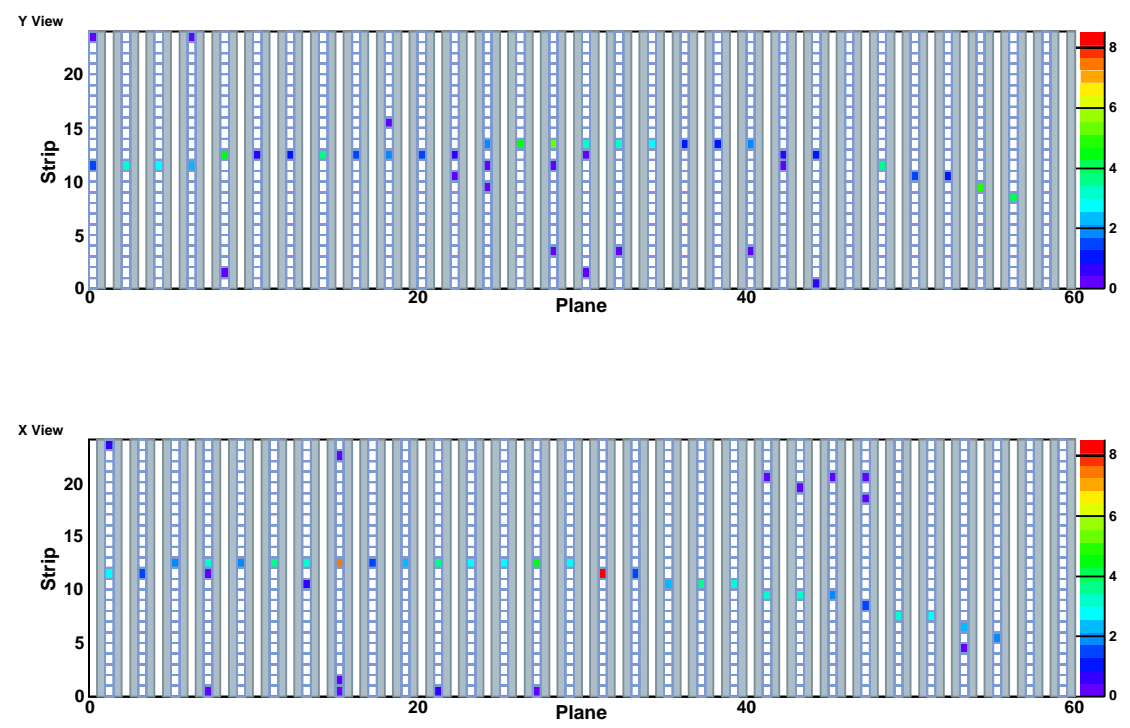

Figure A.35: A $2.0 \mathrm{GeV}$ muon
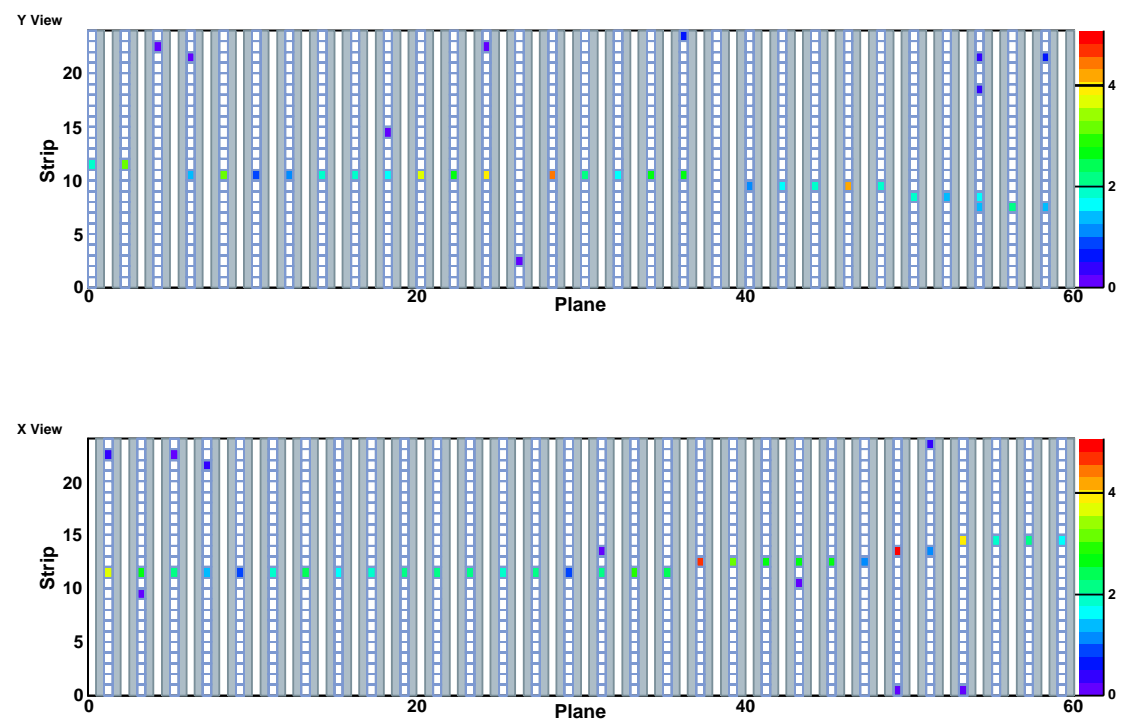

Figure A.36: A $3.0 \mathrm{GeV}$ muon 


\section{Appendix B}

\section{Electron Selection Cuts}

\section{B.1 The Cut Distributions}

The following plots show distributions of the electron selection cut quantities for several momenta in each beam line. For the set of plots for the T11 beam line, the top left plot shows the Cerenkov distribution, the top right plot shows the TOF distribution. The middle left shows the Čerenkov timing distribution, the middle right shows the overlapping event test statistic distribution. The bottom plot shows the distribution of strip of maximum MIP in plane 0 . For the set of plots for the T7 beam line, the top left plot shows the upstream Cerenkov distribution, the top right plot shows the downstream Čerenkov distribution. The second row, left shows the TOF distribution, the second row, right shows the upstream Cerenkov timing distribution. The third row, left shows the downstream Čerenkov distribution timing distribution. The third row right shows the overlapping event test statistic distribution. The bottom plot shows the distribution of strip of maximum MIP in plane 0. Black histograms show the sample after a requirement of a hit in a Čerenkov. Red histograms show the data sample after all cuts except for the one shown are made. Blue histograms show the data sample after all cuts are made. 

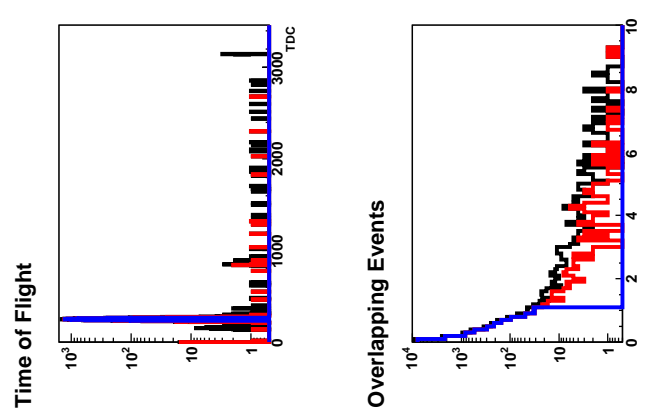

듬
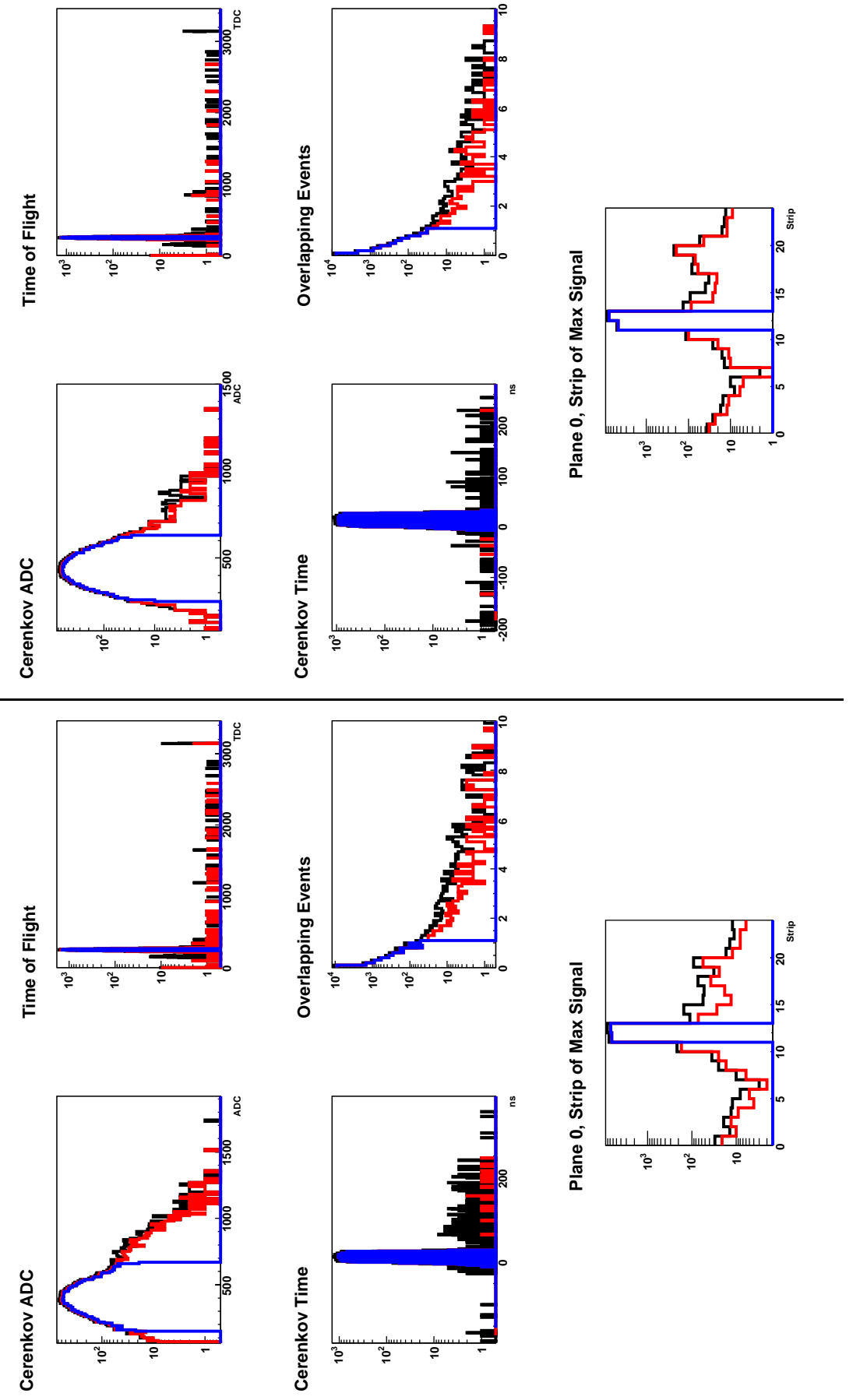

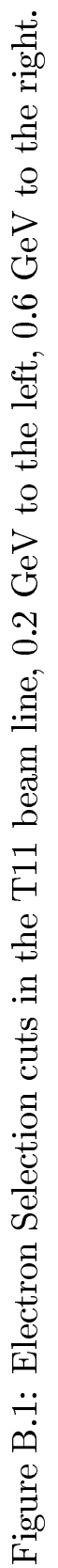



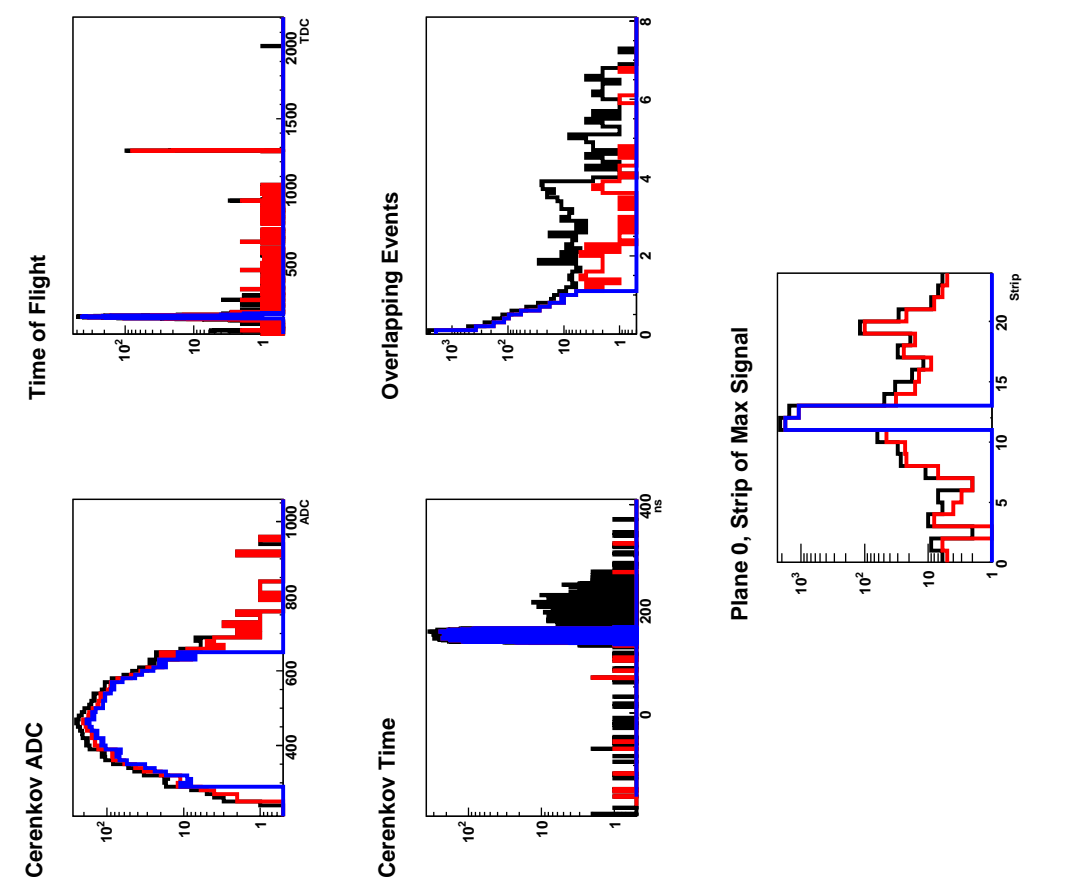

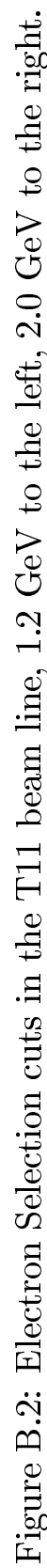
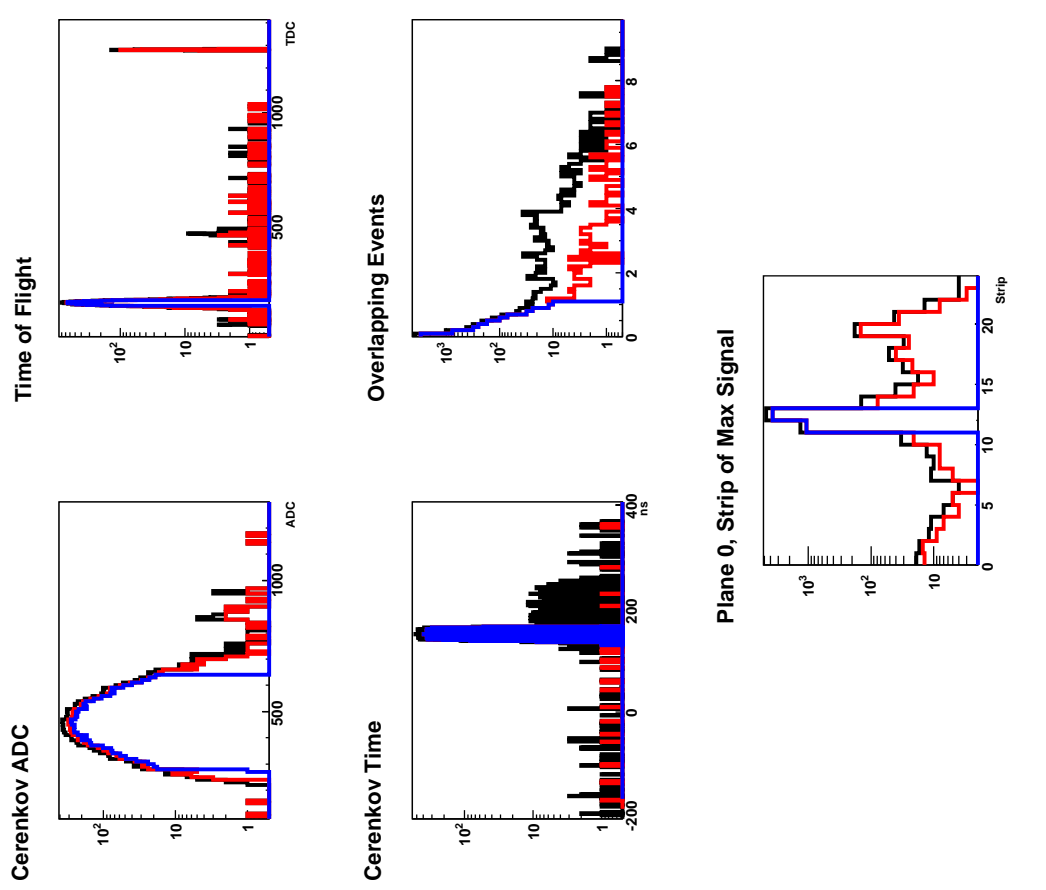

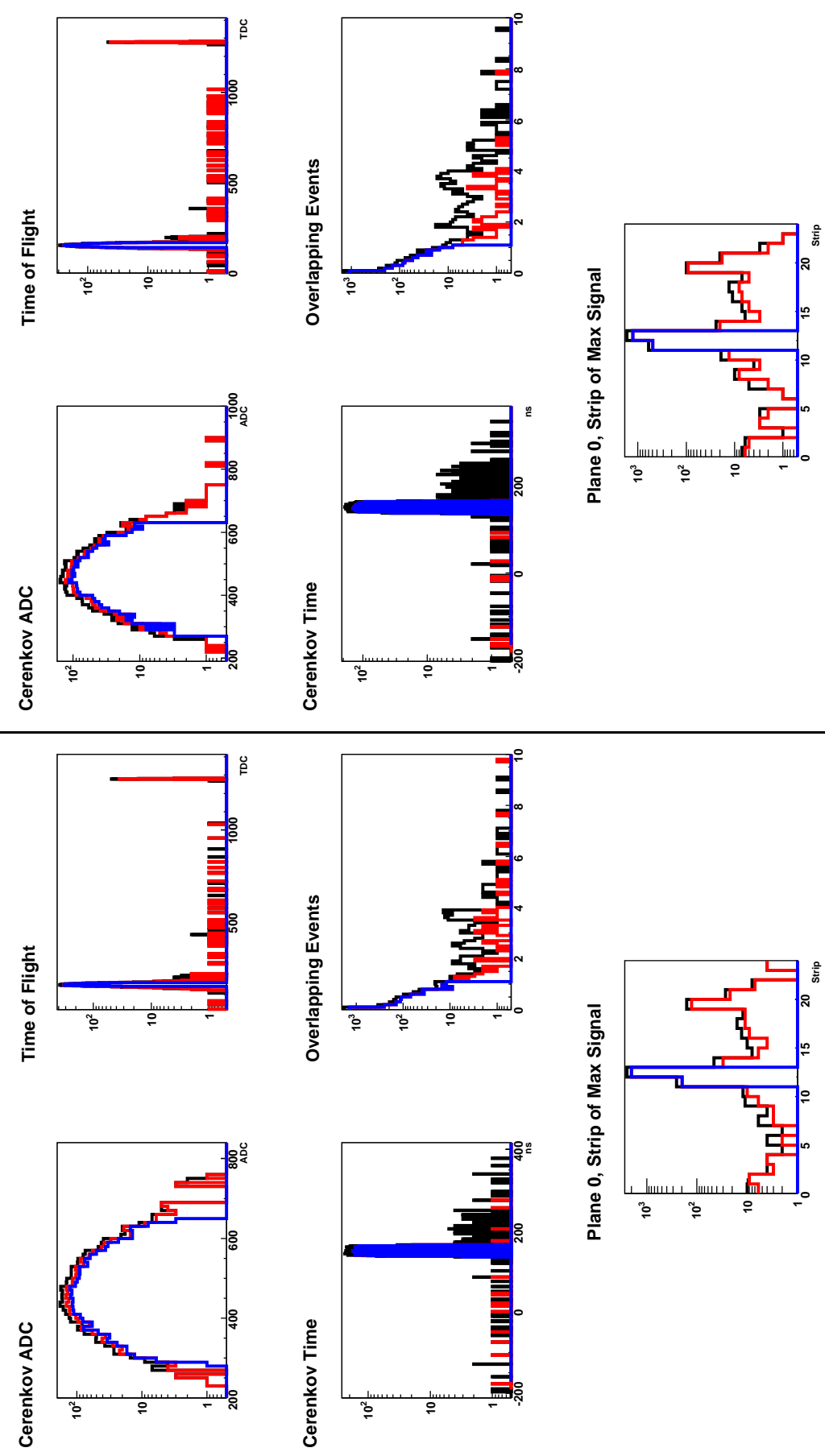

告0 

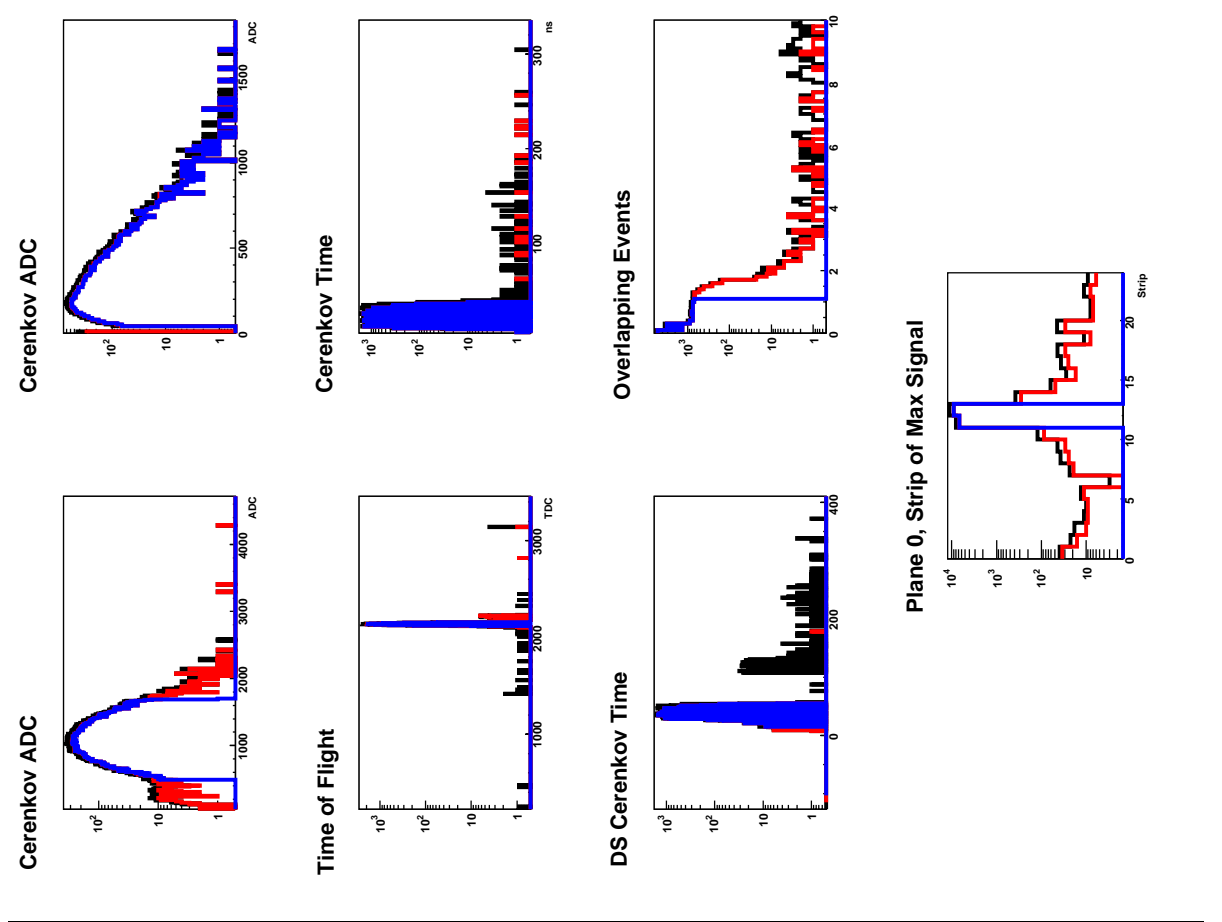

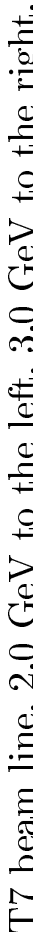
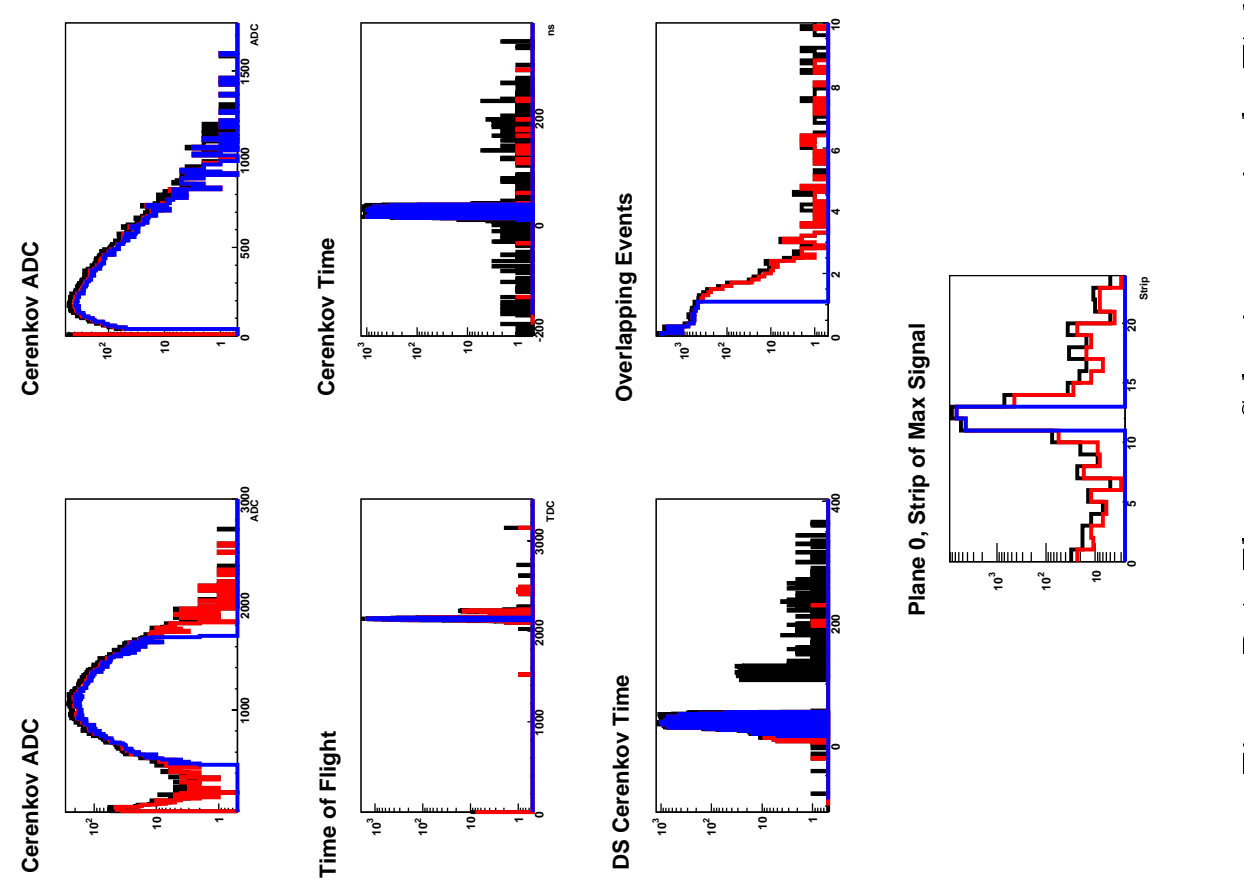

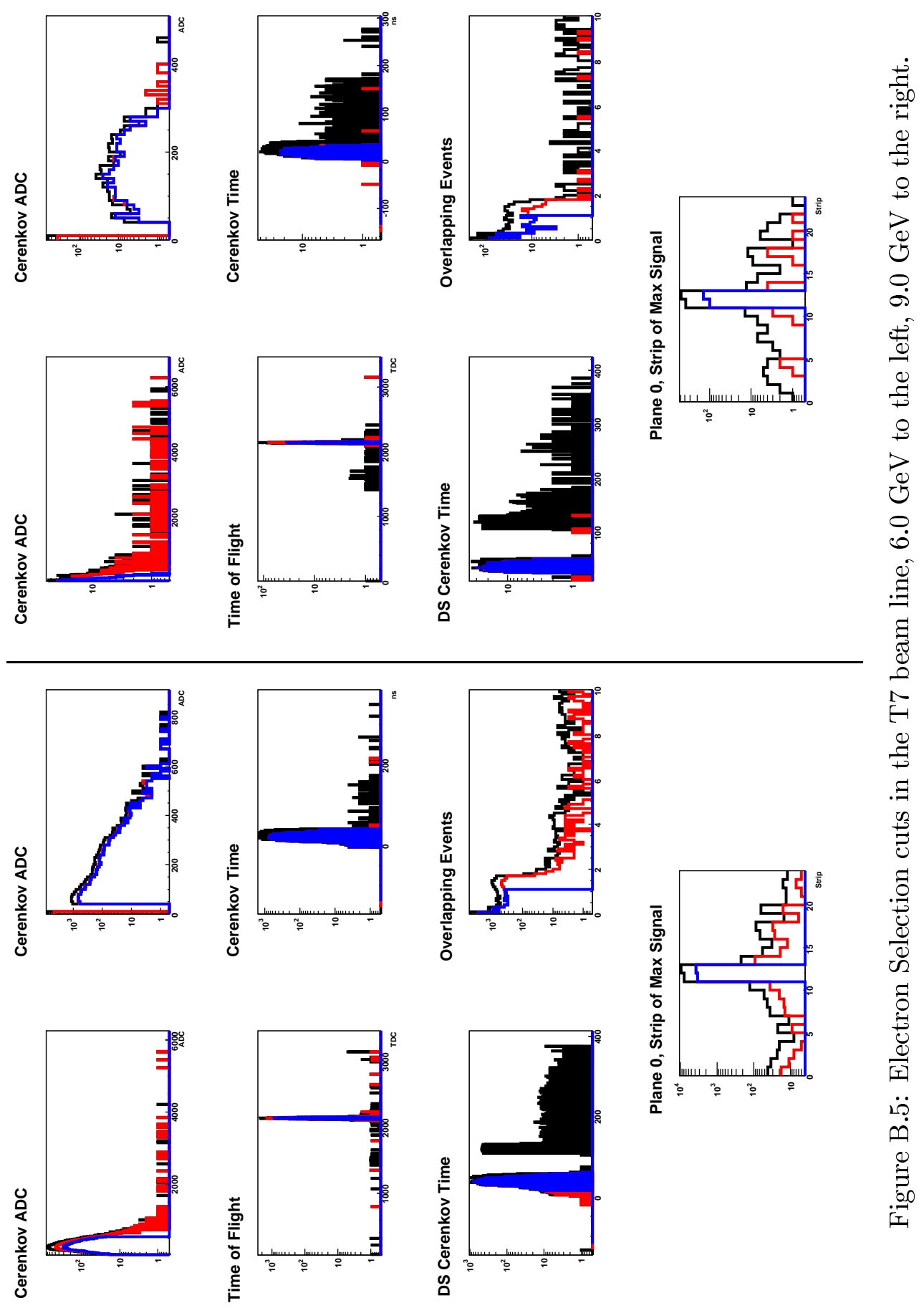


\section{B.2 The Number of Planes Hit Distributions}

The following plots show distributions of the number of hit planes for positron events selected by the criterion described in the text. Black shows the distribution for all events with a hit in the upstream Cerenkov. Red shows the number of hit planes for events that pass all cuts except the PID cut. Green shows the distribution for events that pass all cuts except the Cerenkov timing cut. Yellow shows the distribution for events that pass all cuts except the overlapping event cut. Purple shows the distribution for events that pass all cuts except the plane 0 strip max MIP cut.
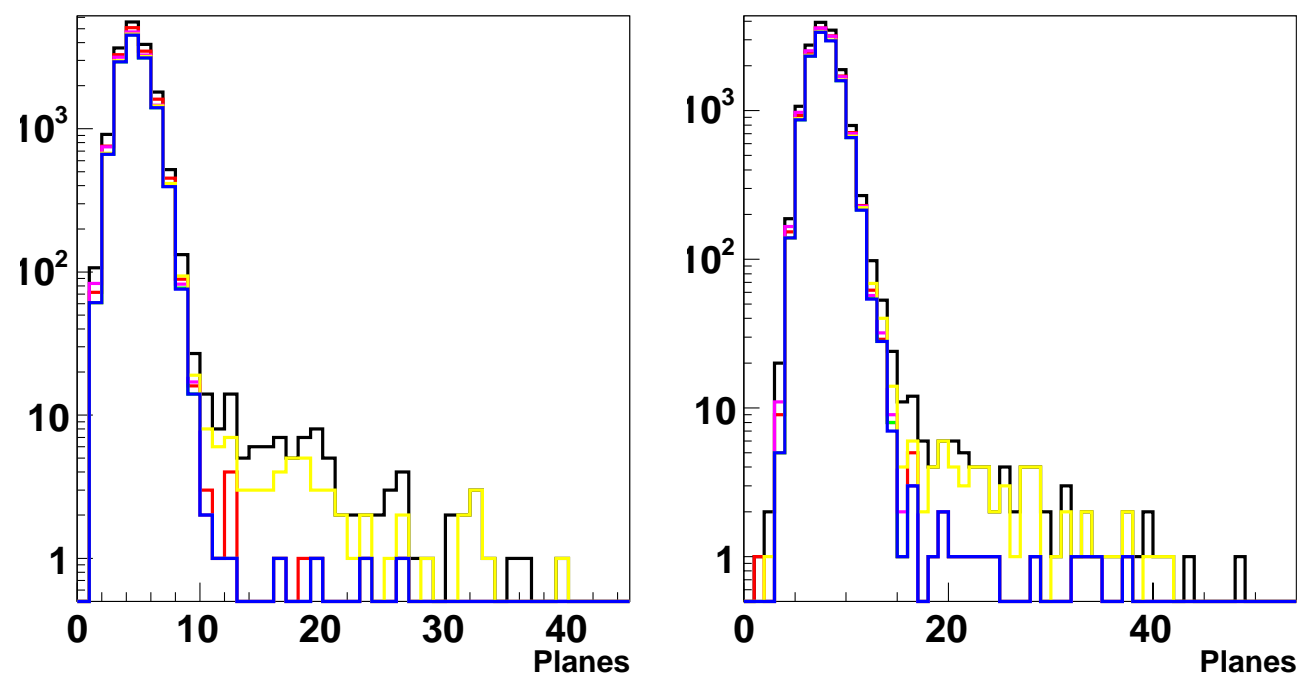

Figure B.6: Number of planes distributions from T11 2002, $0.2 \mathrm{GeV}$ to the left, $0.6 \mathrm{GeV}$ to the right. 

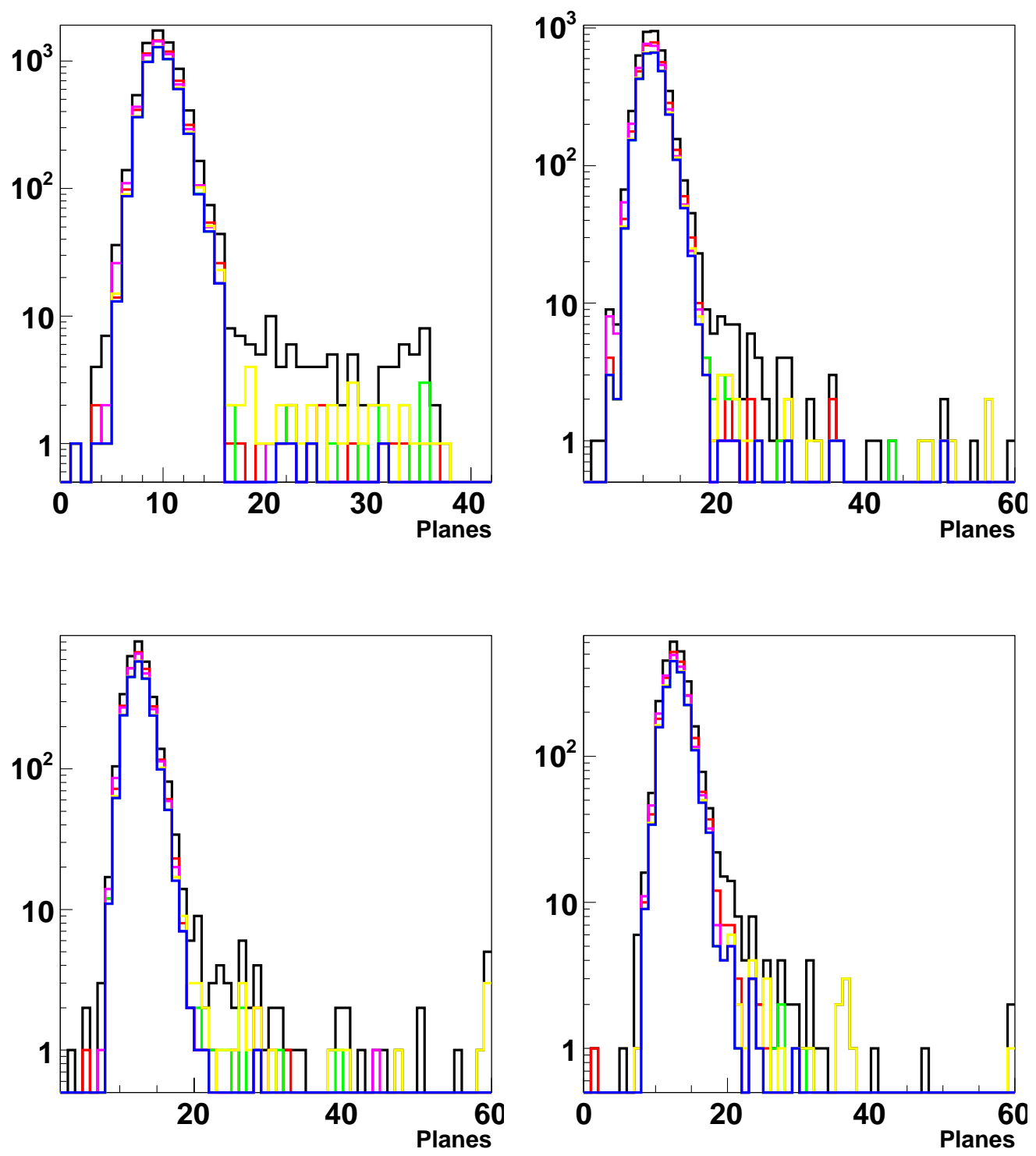

Figure B.7: Number of planes distributions from T11 2002, 1.2 GeV top left, 2.0 GeV top right, $3.2 \mathrm{GeV}$ bottom left, and $3.6 \mathrm{GeV}$ bottom right. 

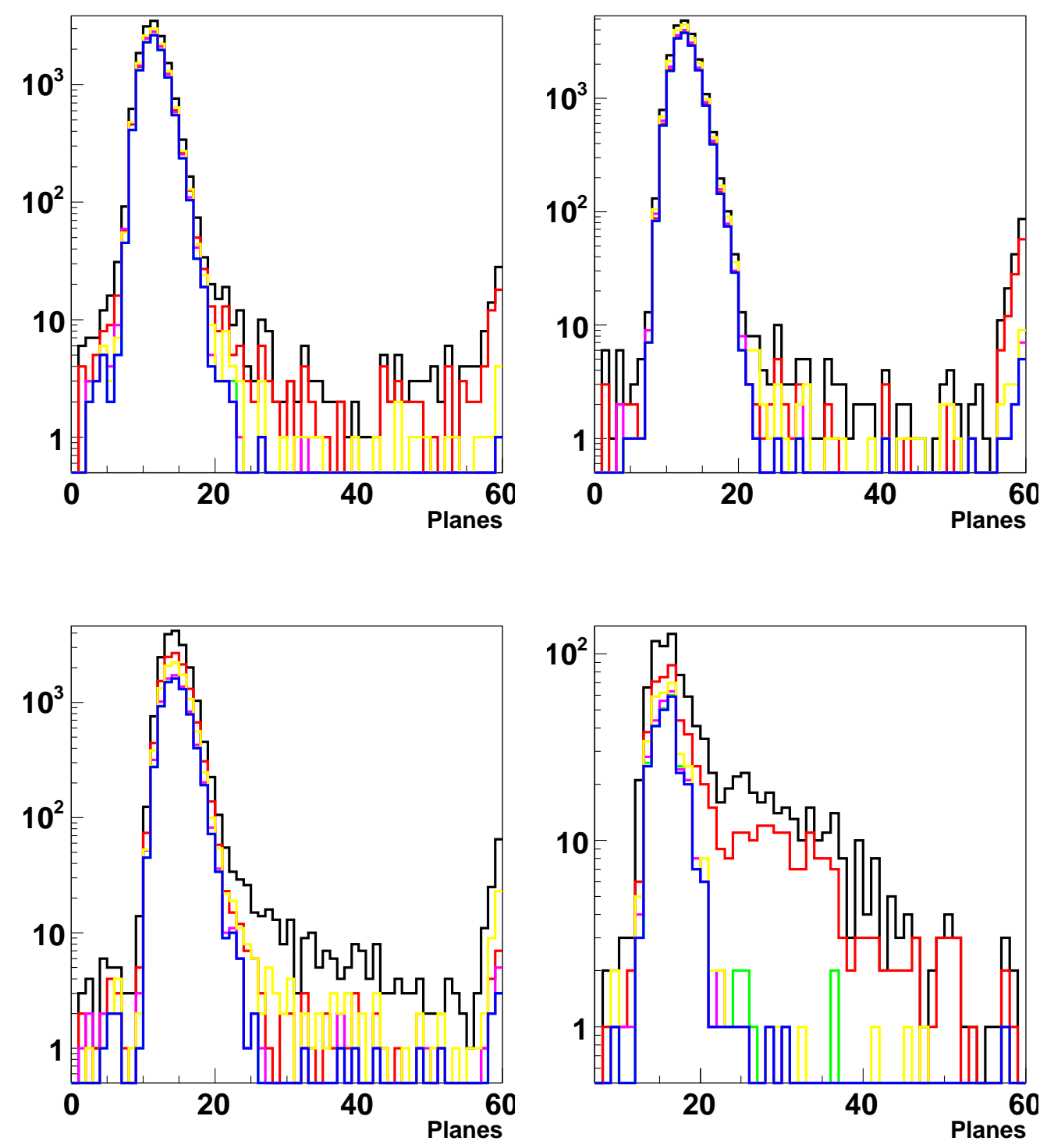

Figure B.8: Number of planes distributions from T7 2002, 2.0 GeV top left, $3.0 \mathrm{GeV}$ top right, $6.0 \mathrm{GeV}$ bottom left, and $9.0 \mathrm{GeV}$ bottom right. 


\section{Appendix C}

\section{Beam Line Simulation Plots}

\section{C.1 Momentum, Vertex Position, and Beam Spot}

The following plots show the momentum and spatial spread of the beam in the T11 and T7 beam lines as predicted by the beam line simulation. Each plot shows 4 figures. The top left plot shows the momentum spectrum predicted by the Turtle simulation, which is taken as the input to the GEANT3 beam line simulation. The top right plot shows the $(\mathrm{x}, \mathrm{y}, \mathrm{z})$ coordinates of the vertex position of each of the particles transported through the GEANT3 beam line simulation, red indicates positrons, blue indicates photons, while green indicates electrons. The lower left plot shows the position of all the particles when incident on the CalDet plane 0. The lower right plot shows the same, but for only those events that pass the simulated TOF trigger. 


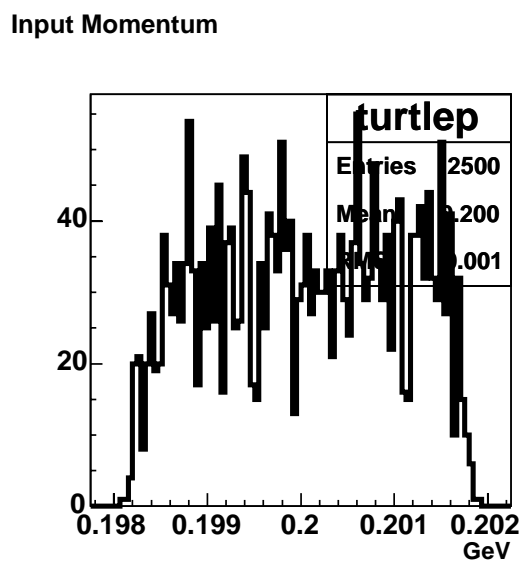

Beam Spot, Pre-Trigger

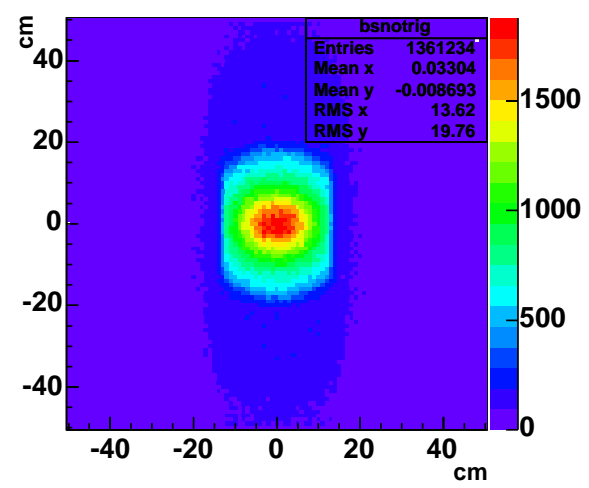

Vertex Position

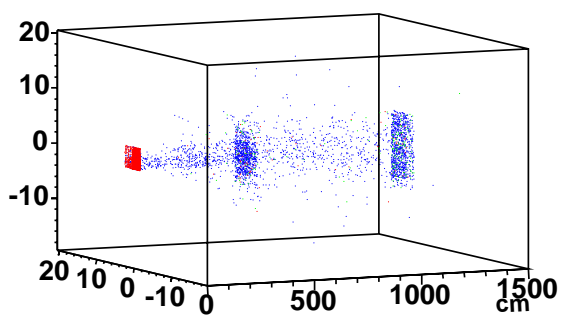

Beam Spot, Triggered Events

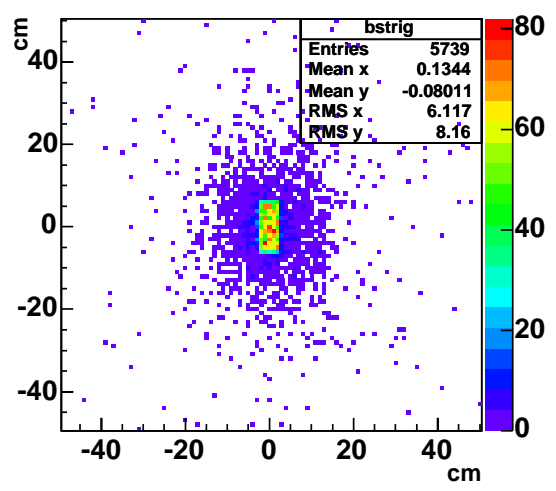

Figure C.1: Beam line simulation plots for $0.2 \mathrm{GeV}$ electrons in the T11 beam line. 

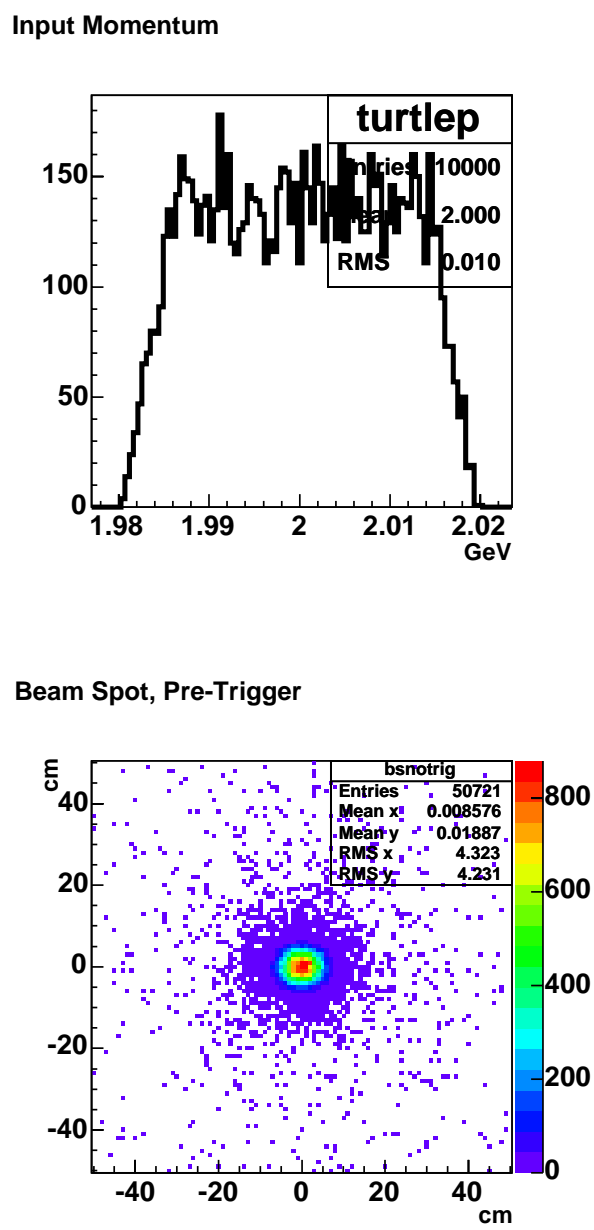

Vertex Position

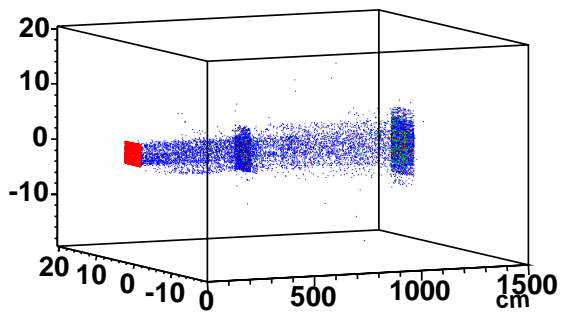

Beam Spot, Triggered Events

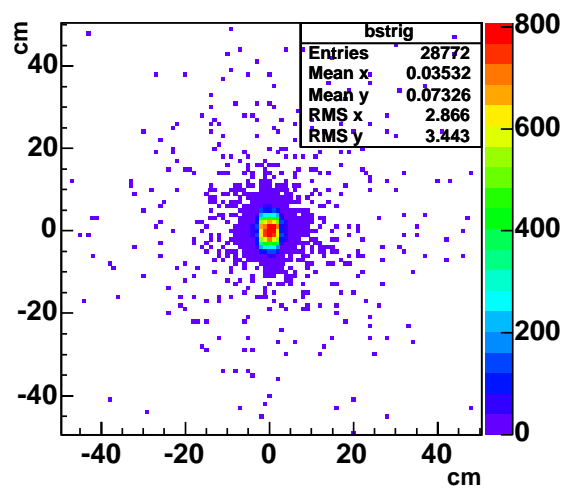

Figure C.2: Beam line simulation plots for $2.0 \mathrm{GeV}$ electrons in the $\mathrm{T} 11$ beam line. 
Input Momentum

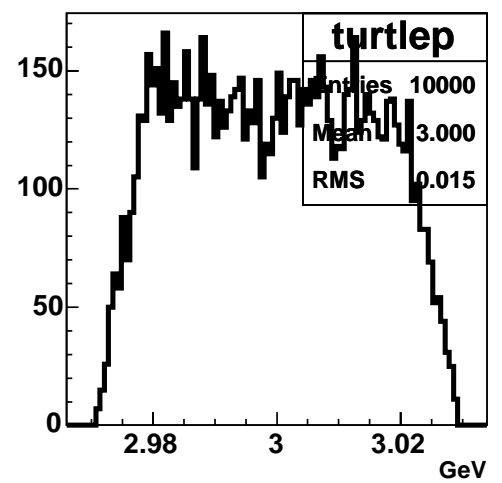

Beam Spot, Pre-Trigger

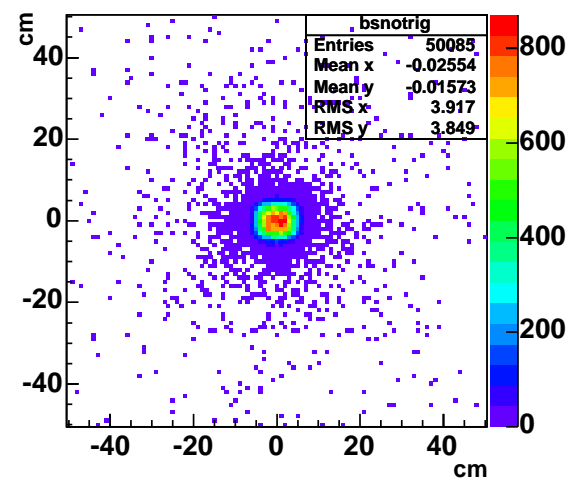

Vertex Position

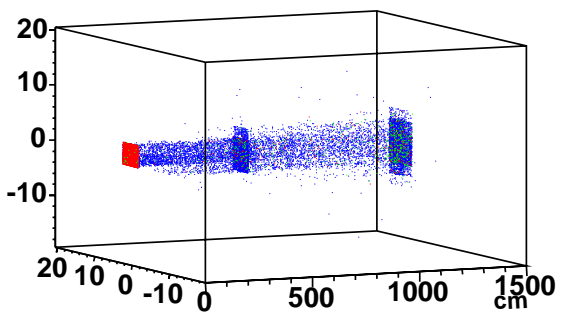

Beam Spot, Triggered Events

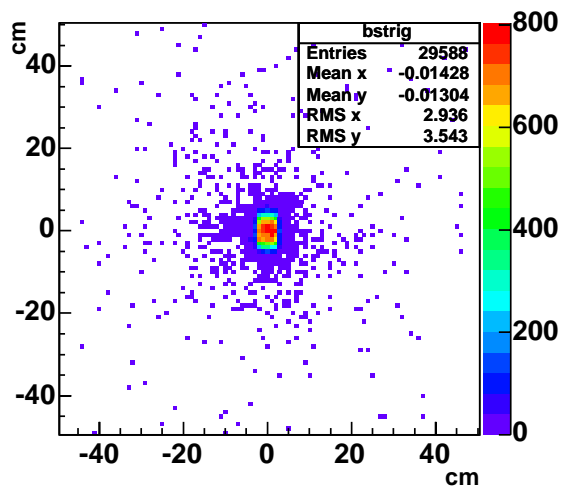

Figure C.3: Beam line simulation plots for $3.0 \mathrm{GeV}$ electrons in the T11 beam line. 

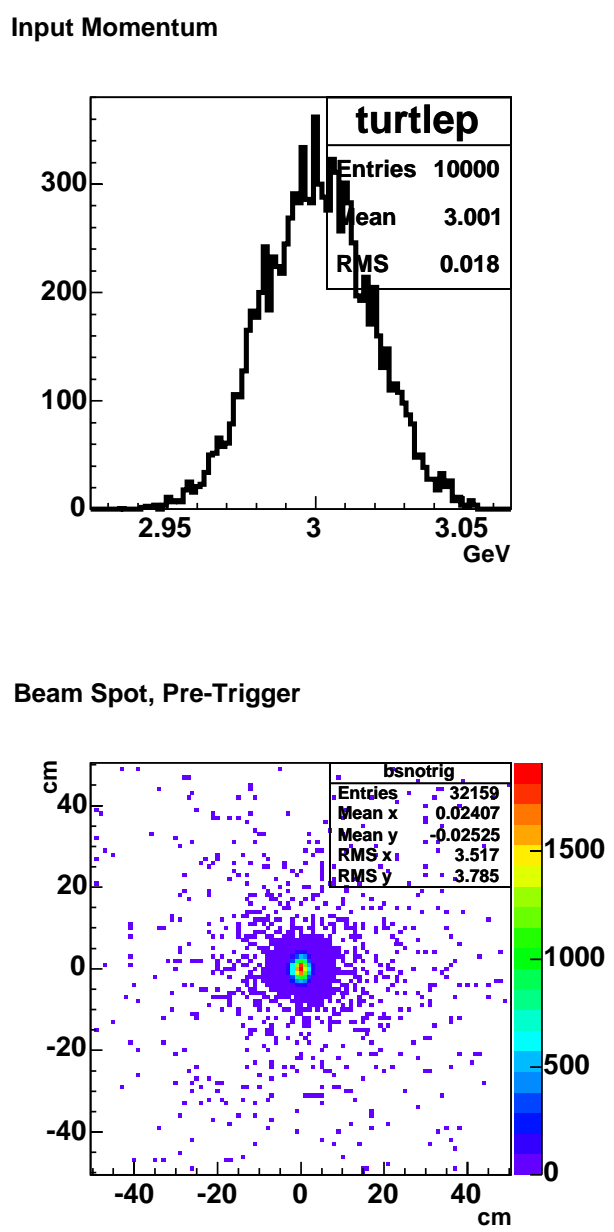

Vertex Position

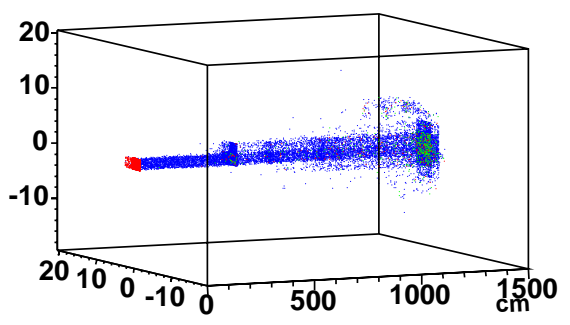

Beam Spot, Triggered Events

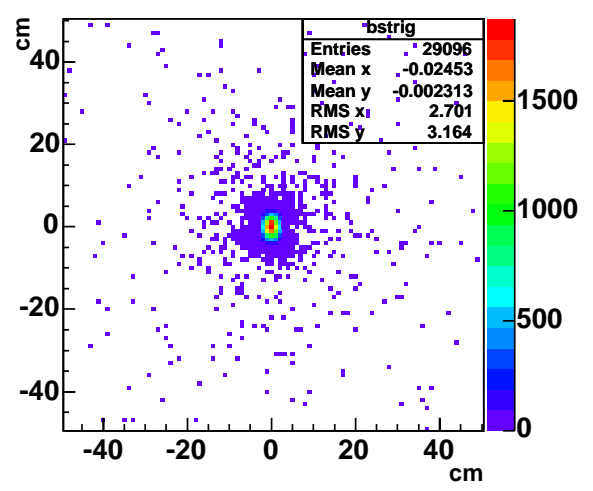

Figure C.4: Beam line simulation plots for $3.0 \mathrm{GeV}$ electrons in the $\mathrm{T} 7$ beam line. 


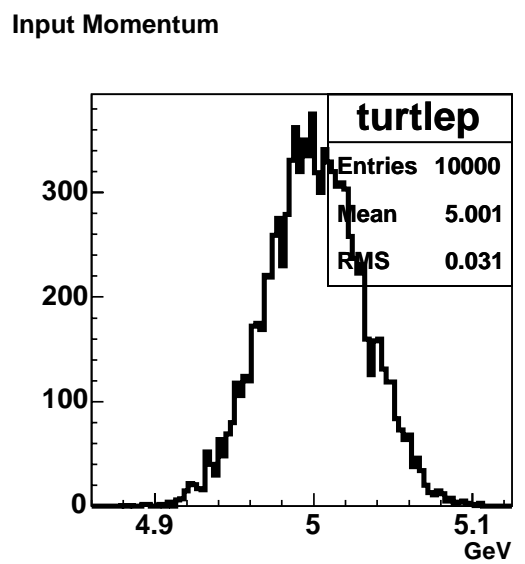

Beam Spot, Pre-Trigger

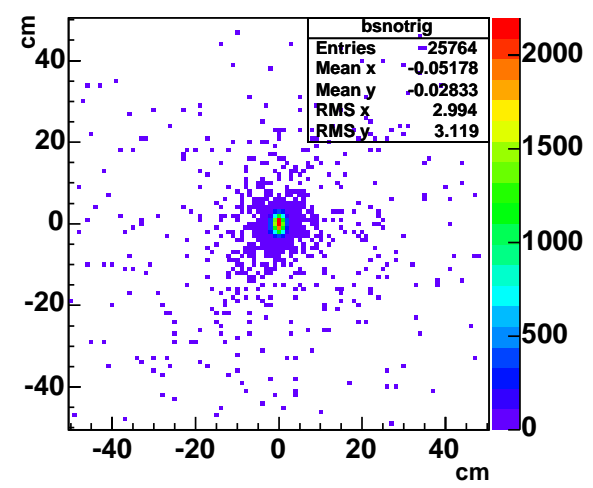

Vertex Position

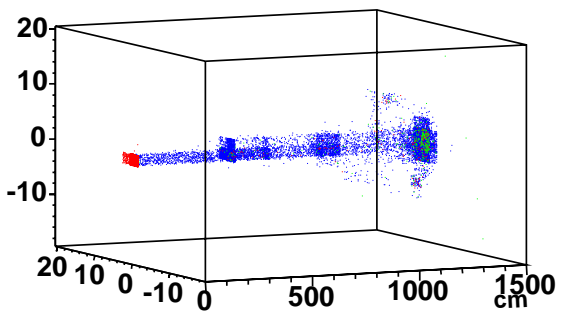

Beam Spot, Triggered Events

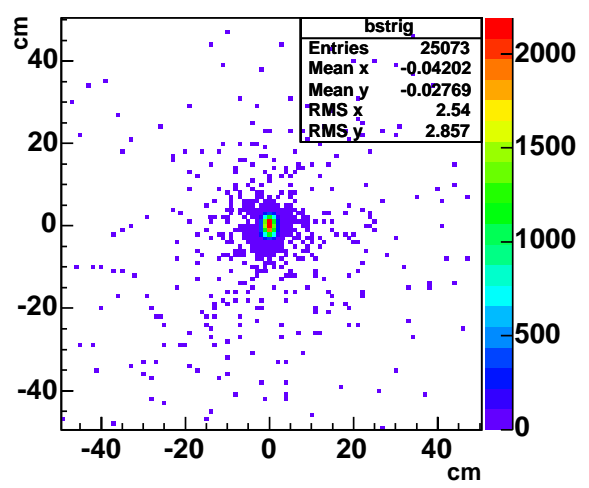

Figure C.5: Beam line simulation plots for $5.0 \mathrm{GeV}$ electrons in the $\mathrm{T} 7$ beam line. 
Input Momentum

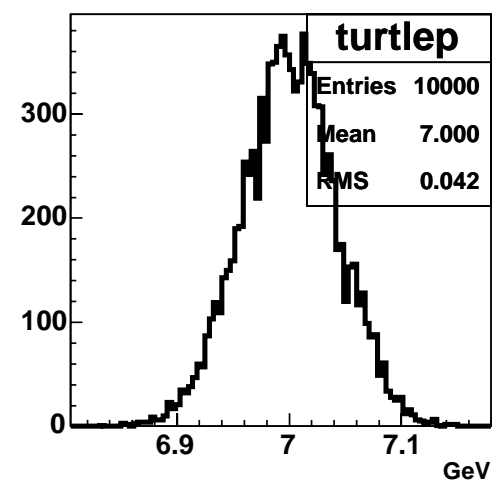

Vertex Position

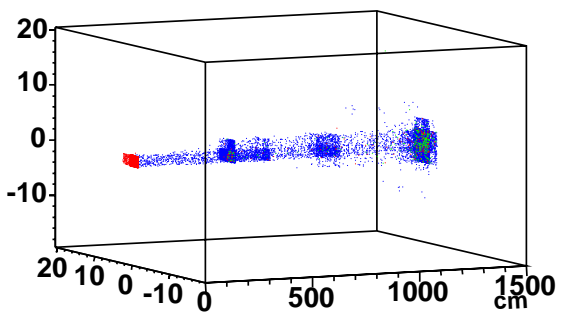

Beam Spot, Triggered Events

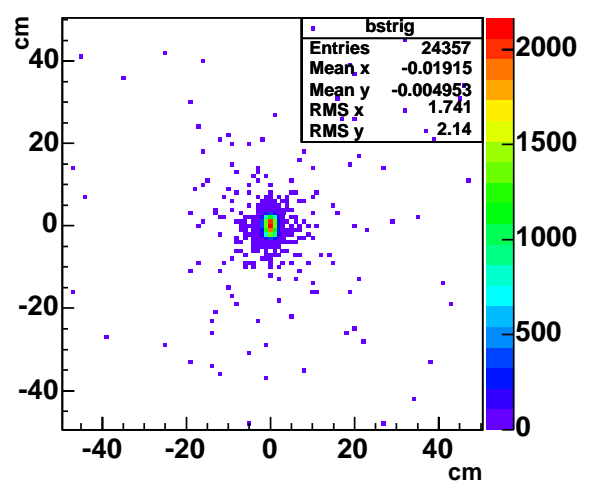

Figure C.6: Beam line simulation plots for $7.0 \mathrm{GeV}$ electrons in the $\mathrm{T} 7$ beam line. 


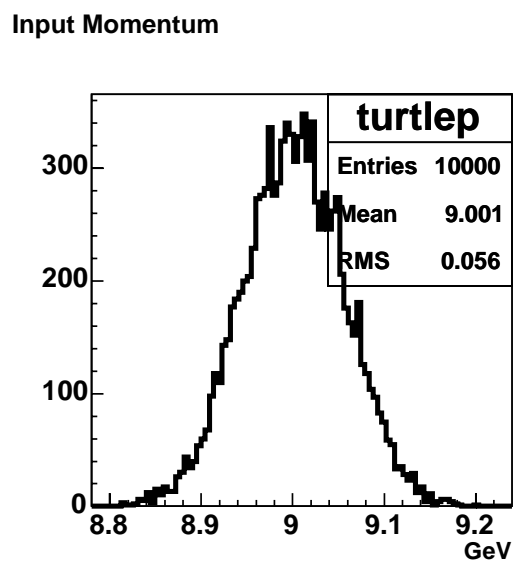

Beam Spot, Pre-Trigger

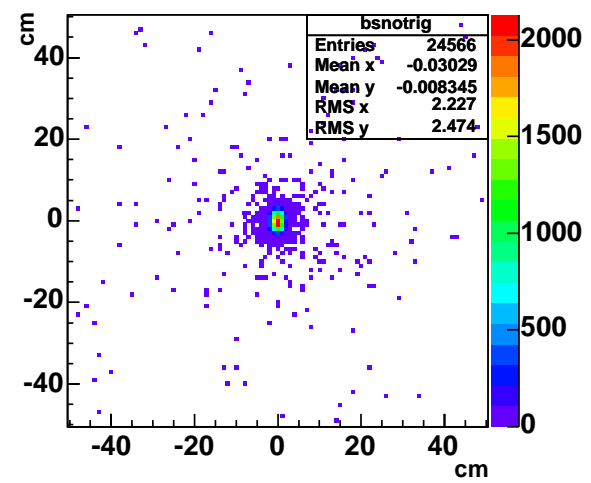

Vertex Position

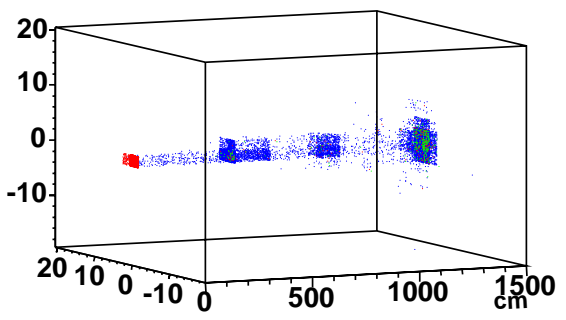

Beam Spot, Triggered Events

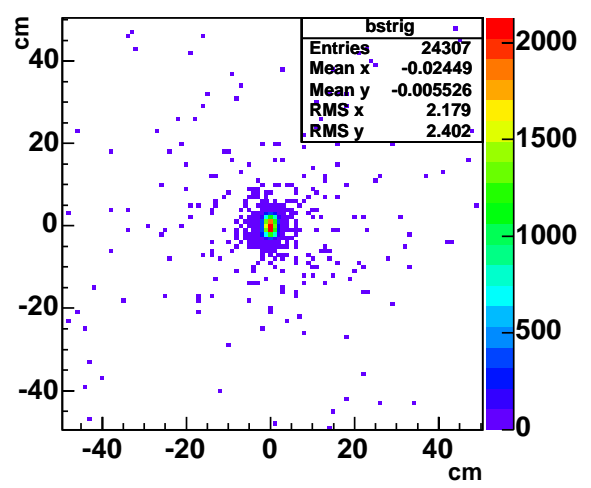

Figure C.7: Beam line simulation plots for $9.0 \mathrm{GeV}$ electrons in the $\mathrm{T} 7$ beam line. 


\section{C.2 Particle Multiplicities and Energy}

The following plots show the particle multiplicities and energy for events passing the TOF trigger in the GEANT3 beam line simulation. The top left plot shows the total energy in an event, summed over any daughter particles created while traversing the beam line material. The top middle plot shows the total energy, summed over electrons (and positrons) in the event, while the top right plot shows the total energy summed over photons. The middle row of plots shows the distribution of the maximum energy particle in an event, the left plot shows the maximum energy of any particle type, the middle the maximum electron (positron) energy, the right plot the maximum photon energy. The bottom row of plots shows the particle multiplicity in an event, the left plot shows all particles, the middle electrons (positrons) and the left photons. 

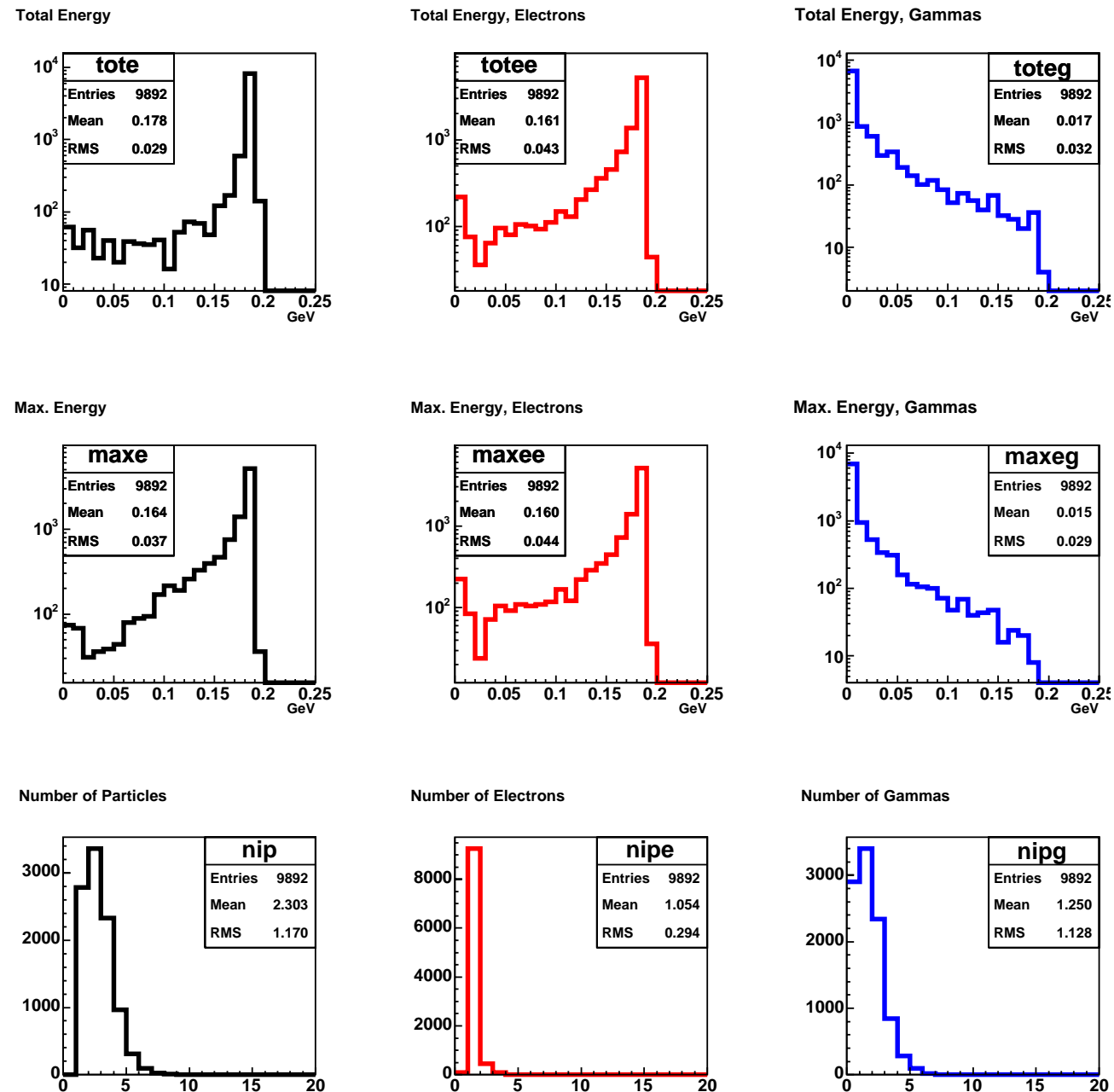

Figure C.8: Particle multiplicities and energy for particles created in the beam line simulation of $0.2 \mathrm{GeV}$ electrons in the $\mathrm{T} 11$ beam line. 

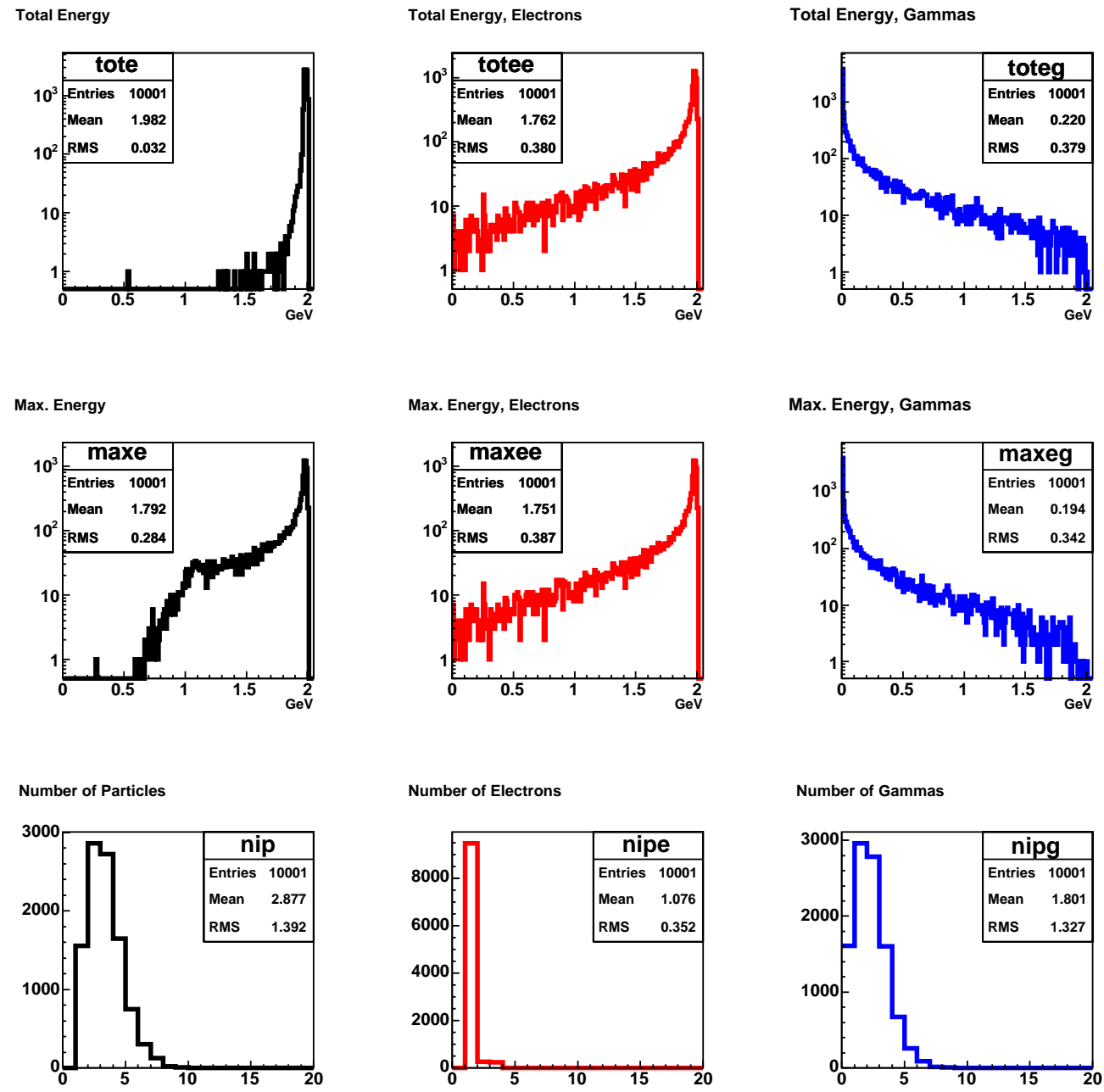

Figure C.9: Particle multiplicities and energy for particles created in the beam line simulation of $2.0 \mathrm{GeV}$ electrons in the $\mathrm{T} 11$ beam line. 

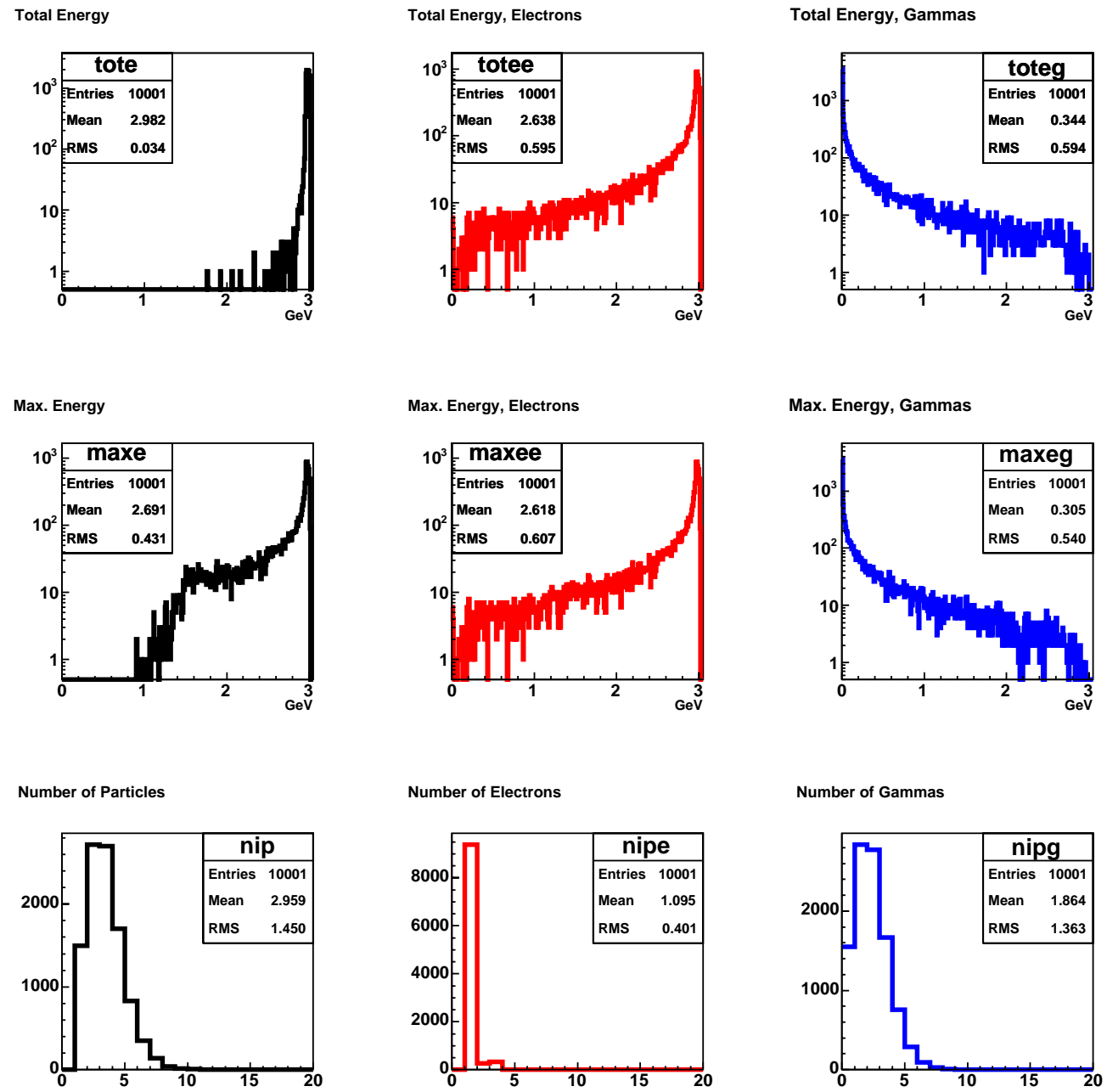

Figure C.10: Particle multiplicities and energy for particles created in the beam line simulation of $3.0 \mathrm{GeV}$ electrons in the $\mathrm{T} 11$ beam line. 

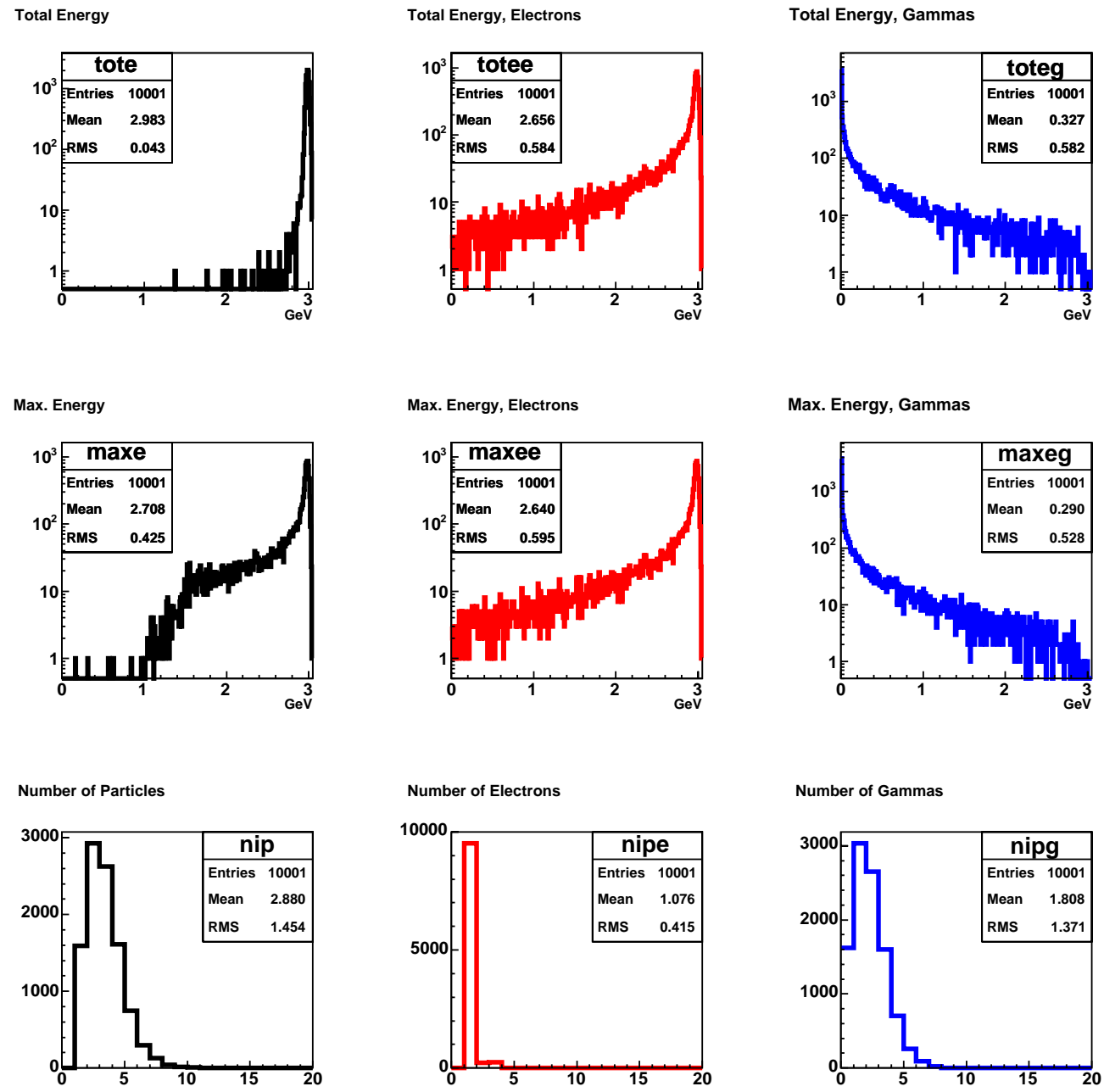

Figure C.11: Particle multiplicities and energy for particles created in the beam line simulation of $3.0 \mathrm{GeV}$ electrons in the $\mathrm{T} 7$ beam line. 


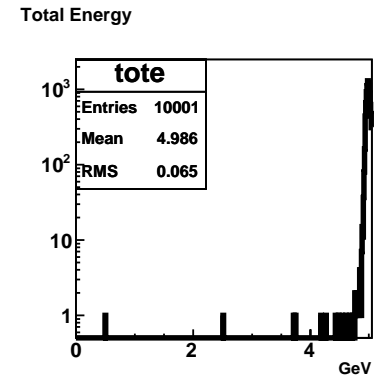

Max. Energy

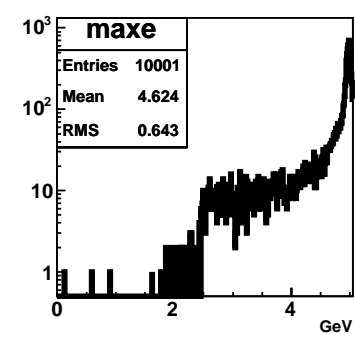

Number of Particles

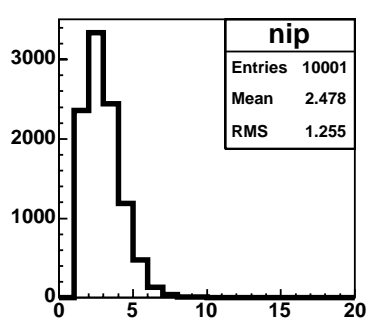

Total Energy, Electrons

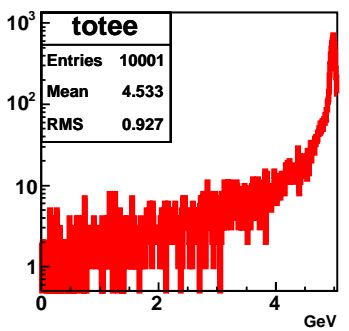

Max. Energy, Electrons

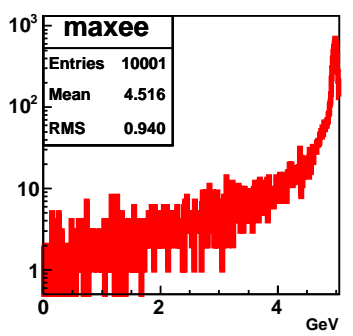

Number of Electrons

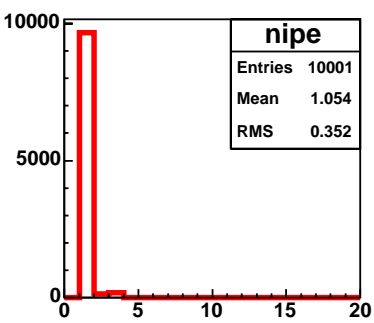

Total Energy, Gammas

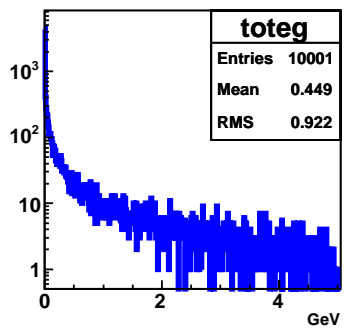

Max. Energy, Gammas

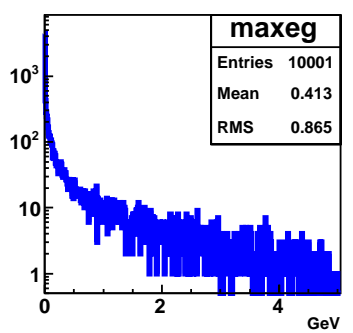

Number of Gammas

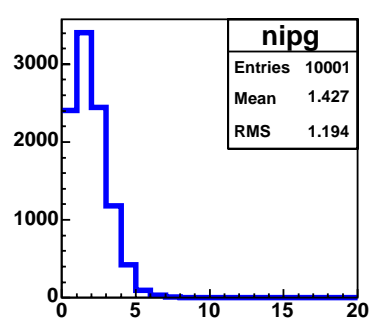

Figure C.12: Particle multiplicities and energy for particles created in the beam line simulation of $5.0 \mathrm{GeV}$ electrons in the $\mathrm{T} 7$ beam line. 


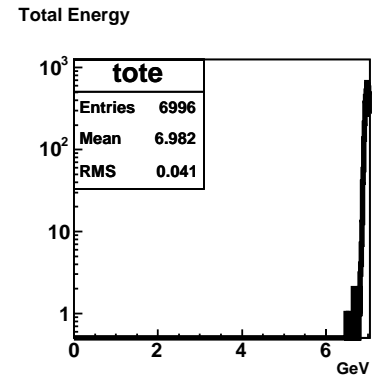

Max. Energy

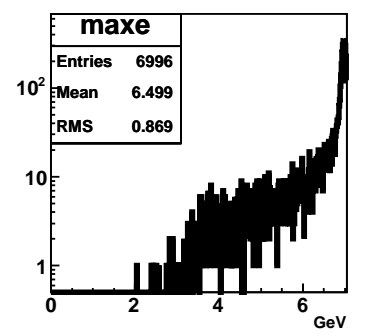

Number of Particles

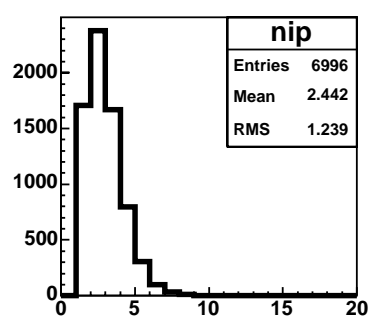

Total Energy, Electrons

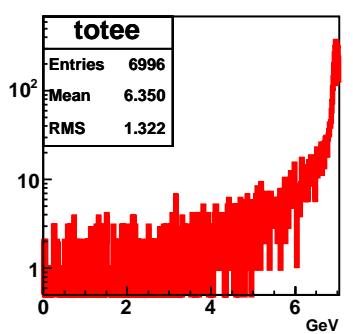

Max. Energy, Electrons

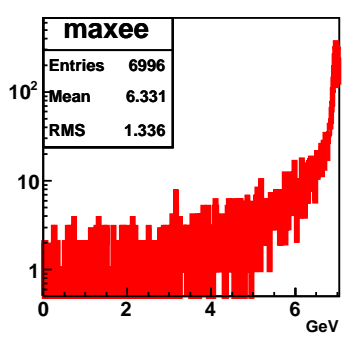

Number of Electrons

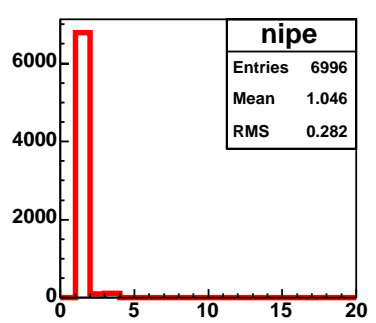

Total Energy, Gammas

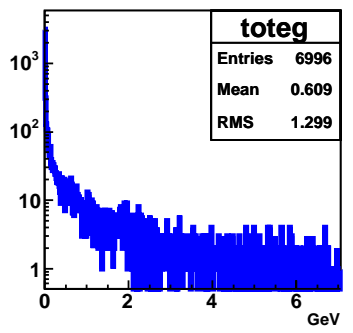

Max. Energy, Gammas

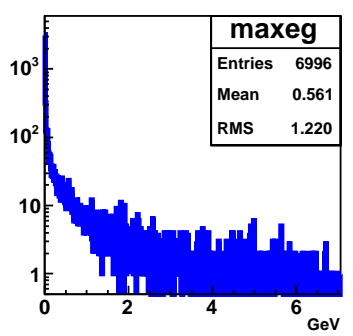

Number of Gammas

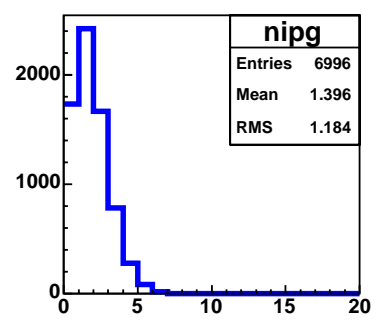

Figure C.13: Particle multiplicities and energy for particles created in the beam line simulation of $7.0 \mathrm{GeV}$ electrons in the $\mathrm{T} 7$ beam line. 


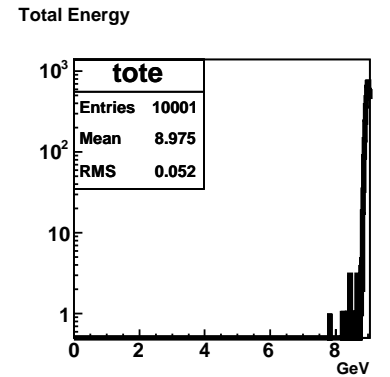

Max. Energy

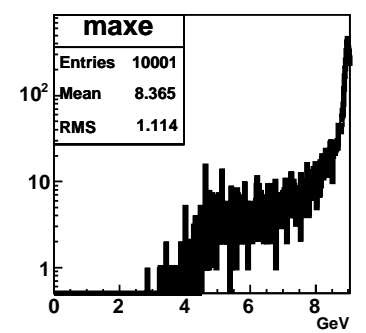

Number of Particles

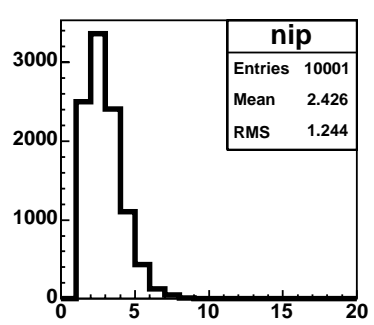

Total Energy, Electrons

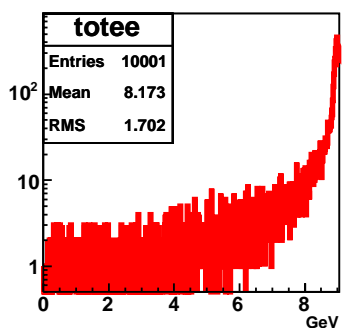

Max. Energy, Electrons

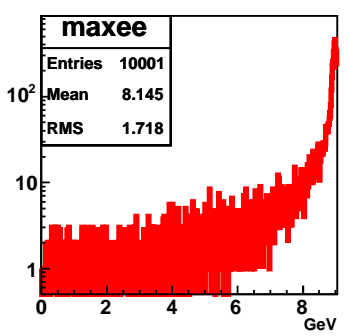

Number of Electrons

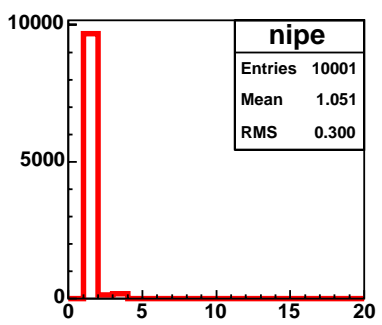

Total Energy, Gammas

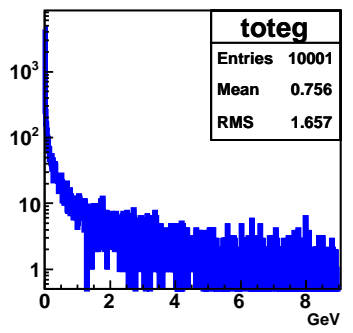

Max. Energy, Gammas

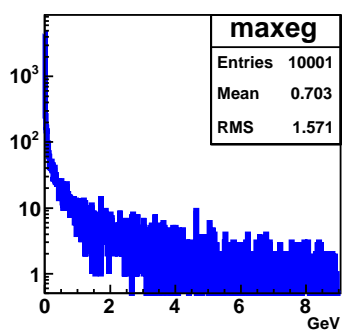

Number of Gammas

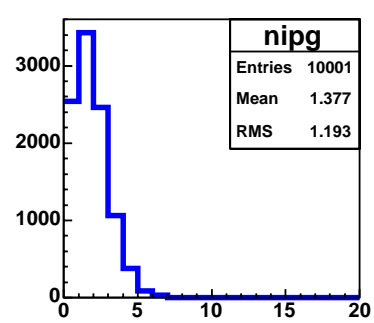

Figure C.14: Particle multiplicities and energy for particles created in the beam line simulation of $9.0 \mathrm{GeV}$ electrons in the $\mathrm{T} 7$ beam line. 


\section{Appendix D}

\section{Comparisons with Monte Carlo}

The plots in this appendix compare the properties of positrons as measured in the data to that simulated in Monte Carlo. Each page shows several momenta. The black histogram shows the data, while the red histogram shows the Monte Carlo. The value of $\chi^{2}$ given characterizes the differences between the Monte Carlo and the data. The first set of plots shows distributions of the last plane in an event. The next set of plots shows the number of strips hit in an event. The third set of plots show the number of strips with signal greater than 1.5 PE. The fourth set of plots show the lateral extent of the events. The fifth set of plots show the plane of maximum energy deposition, while the sixth set of plots show the fraction of the total response deposited in that plane. Finally, the seventh set of plots show the summed detector response in MIPs. 
Last Planecut, $0.2 \mathrm{GeV}$

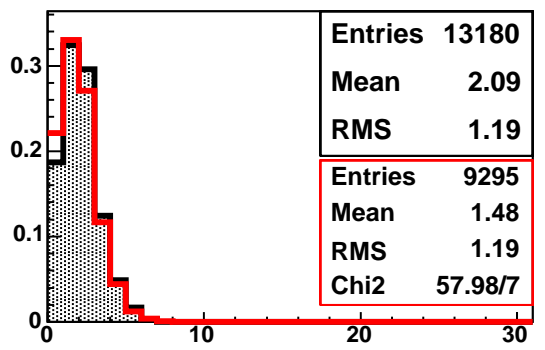

Last Planecut, $0.6 \mathrm{GeV}$

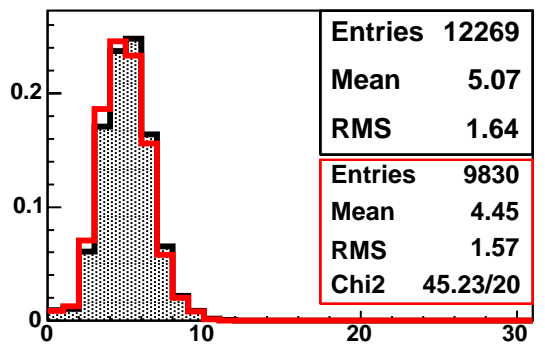

Last Planecut, $1.0 \mathrm{GeV}$

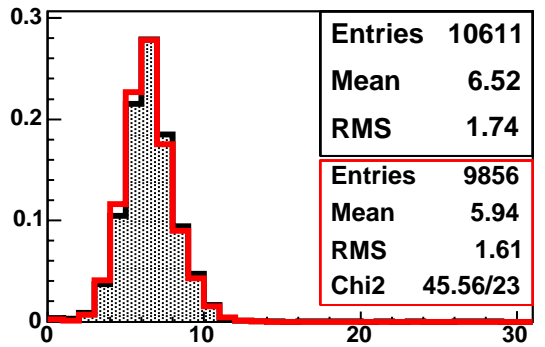

Last Planecut, $0.4 \mathrm{GeV}$

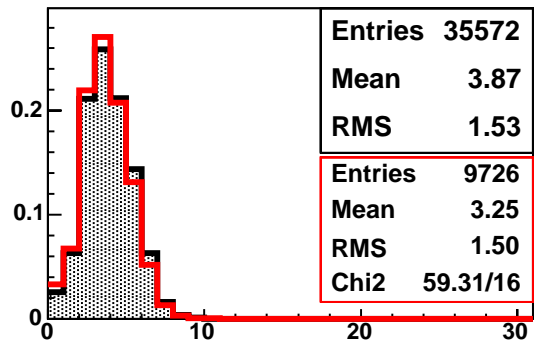

Last Planecut, $0.8 \mathrm{GeV}$

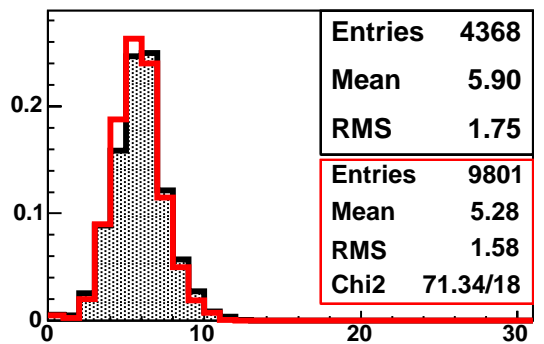

Last Planecut, 1.2 GeV

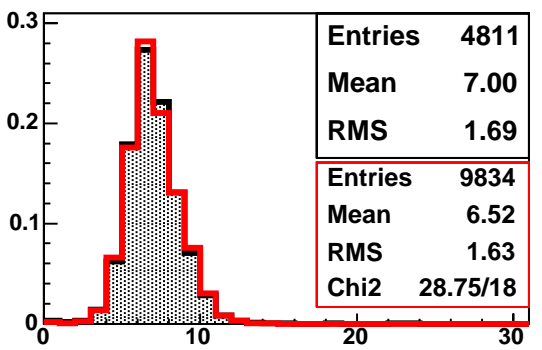

Figure D.1: Comparison of last plane distributions between data and Monte Carlo. 
Last Planecut, 1.4 GeV

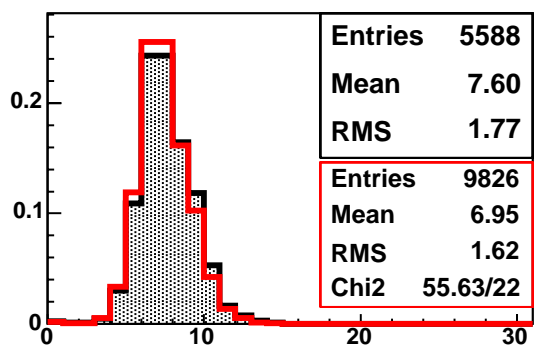

Last Planecut, $1.8 \mathrm{GeV}$

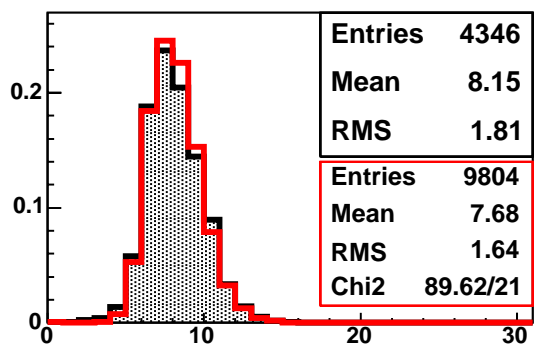

Last Planecut, 2.2 GeV

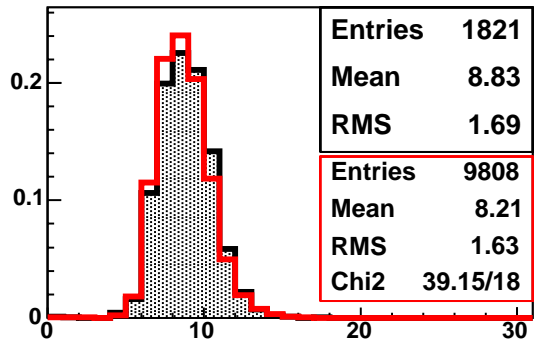

Last Planecut, $1.6 \mathrm{GeV}$

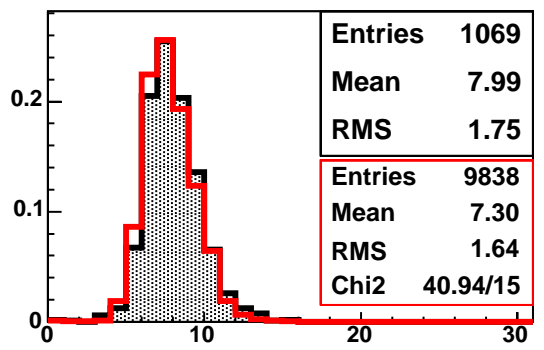

Last Planecut, $2.0 \mathrm{GeV}$

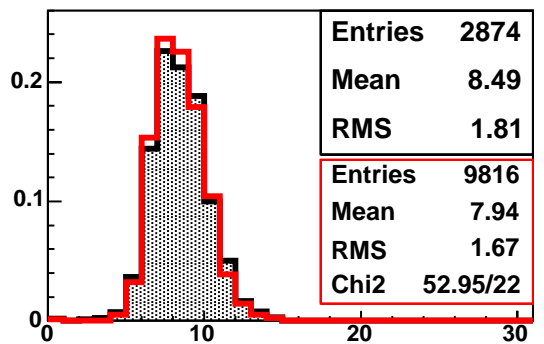

Last Planecut, $2.4 \mathrm{GeV}$

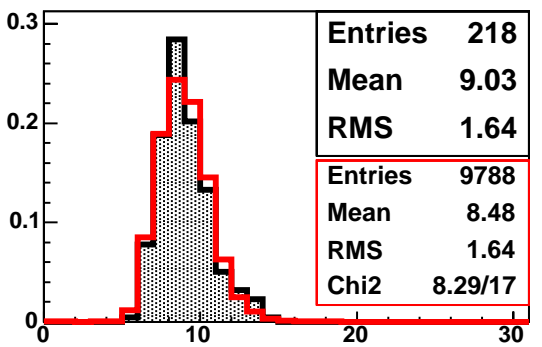

Figure D.2: Comparison of last plane distributions between data and Monte Carlo. 
Last Planecut, $2.6 \mathrm{GeV}$

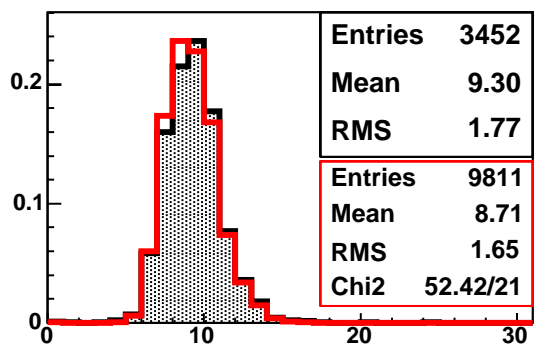

Last Planecut, $3.0 \mathrm{GeV}$

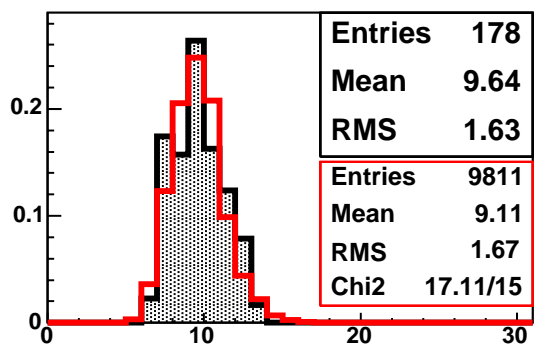

Last Planecut, $28 \mathrm{GeV}$

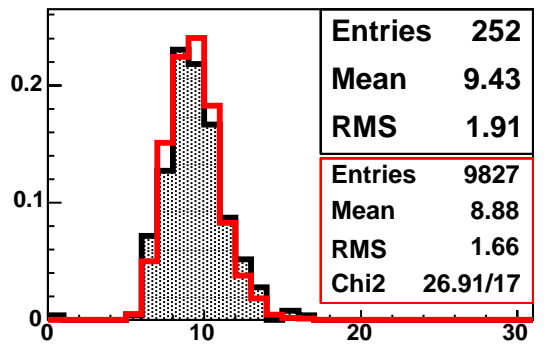

Last Planecut, $3.2 \mathrm{GeV}$

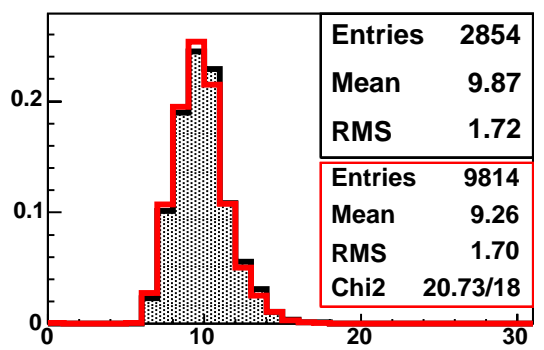

Last Planecut, 3.6 GeV

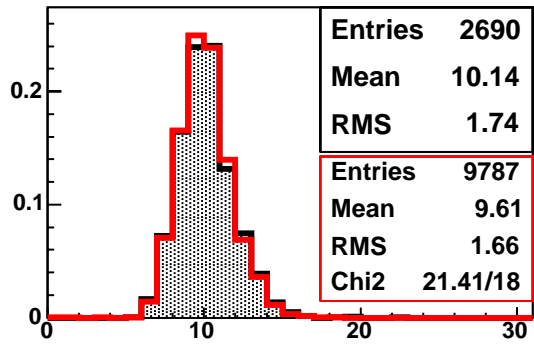

Figure D.3: Comparison of last plane distributions between data and Monte Carlo. 
Last Planecut, $1.0 \mathrm{GeV}$

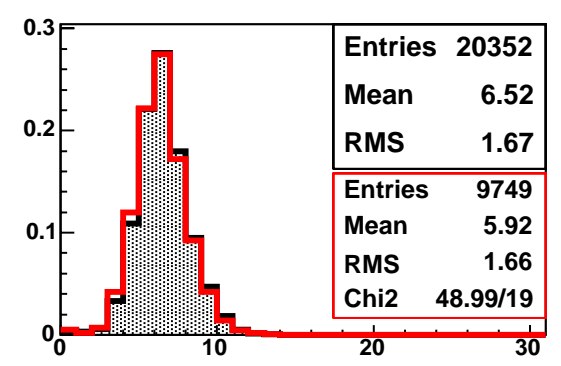

Last Planecut, $2.4 \mathrm{GeV}$

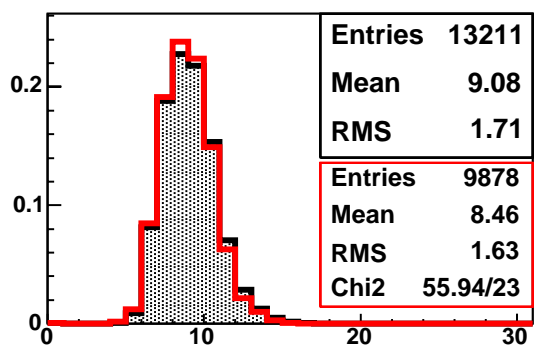

Last Planecut, $3.0 \mathrm{GeV}$

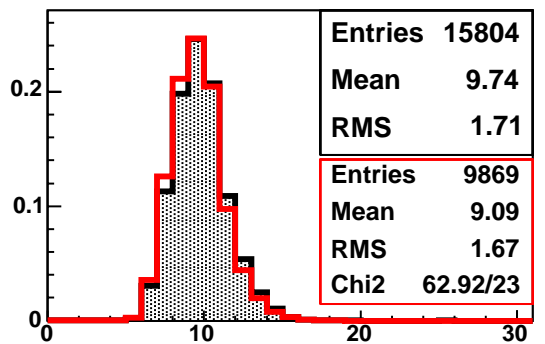

Last Planecut, $20 \mathrm{GeV}$

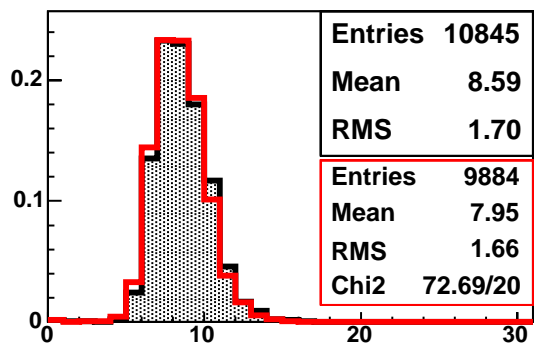

Last Planecut, $2.8 \mathrm{GeV}$

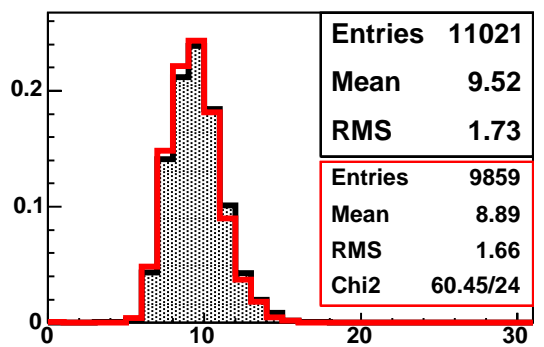

Last Planecut, $4.0 \mathrm{GeV}$

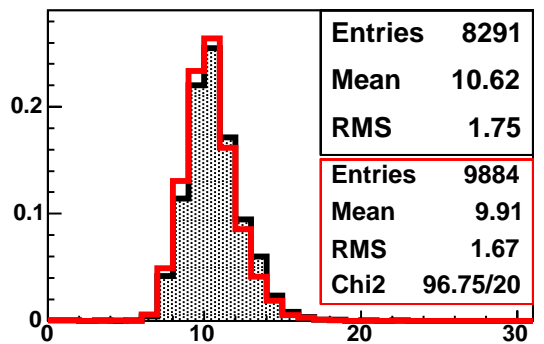

Figure D.4: Comparison of last plane distributions between data and Monte Carlo. 
Last Planecut, $5.0 \mathrm{GeV}$

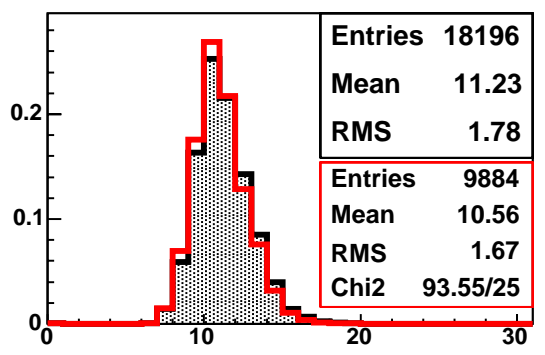

Last Planecut, $7.0 \mathrm{GeV}$

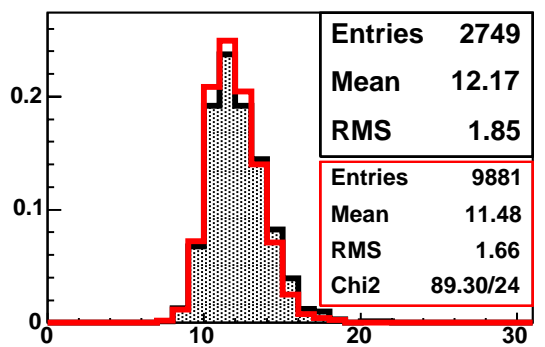

Last Planecut, $6.0 \mathrm{GeV}$

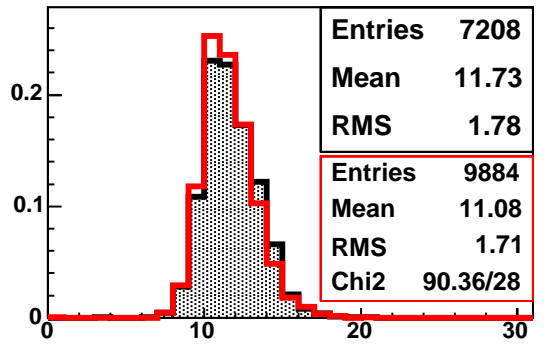

Last Planecut, $8.0 \mathrm{GeV}$

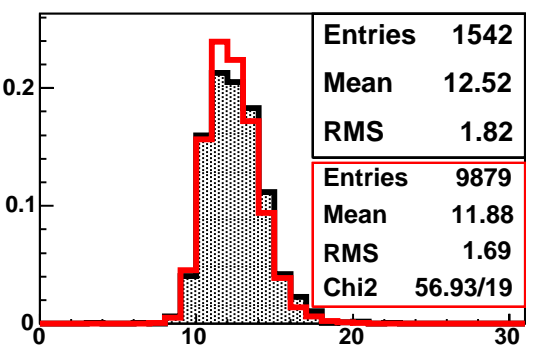

Last Planecut, $9.0 \mathrm{GeV}$

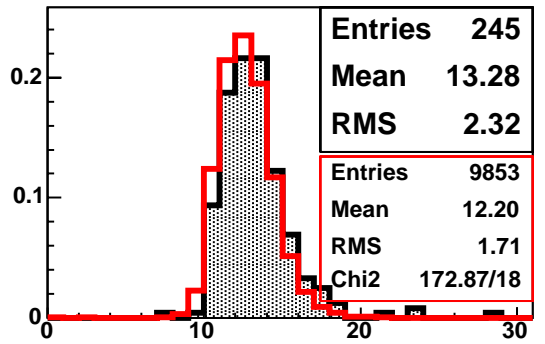

Figure D.5: Comparison of last plane distributions between data and Monte Carlo. 
Mip Weighted Radius, 1.5 PE Cut, 0.2 GeV

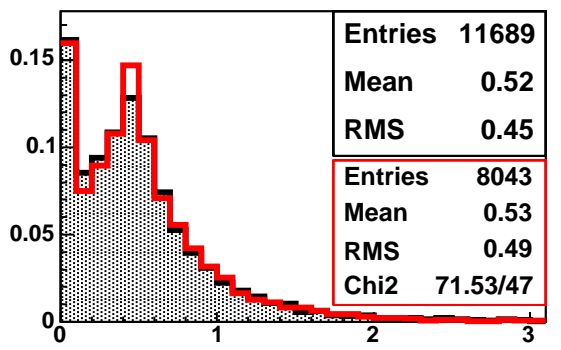

Mip Weighted Radius, 1.5 PE Cut, 0.6 GeV

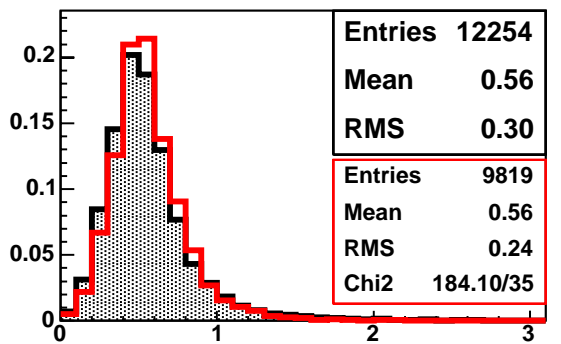

Mip Weighted Radius, 1.5 PE Cut, 1.0 GeV

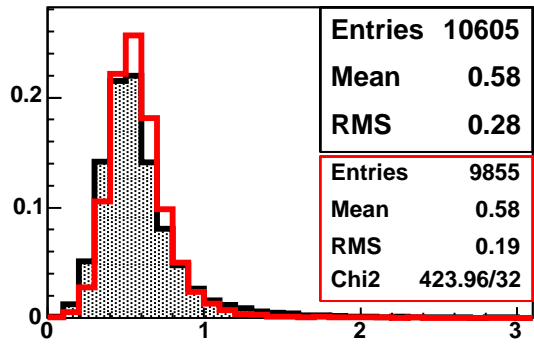

Mip Weighted Radius, 1.5 PE Cut, 0.4 GeV

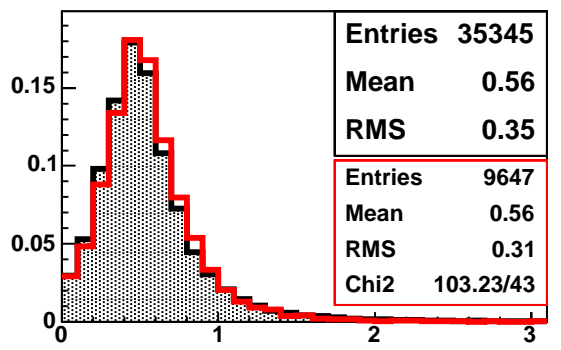

Mip Weighted Radius, 1.5 PE Cut, 0.8 GeV

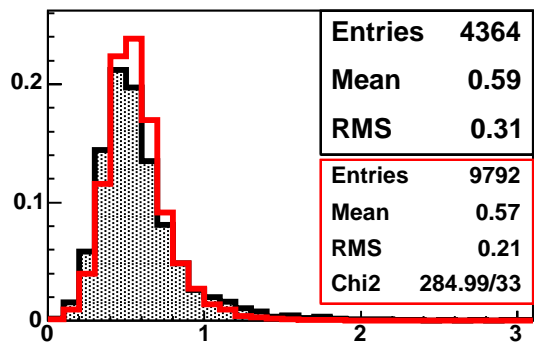

Mip Weighted Radius, 1.5 PE Cut, 1.2 GeV

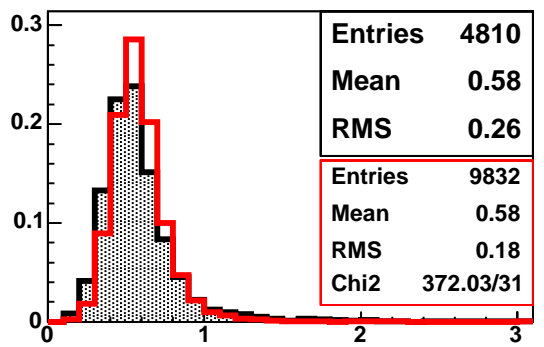

Figure D.6: Comparison of radial distributions between data and Monte Carlo. 
Mip Weighted Radius, 1.5 PE Cut, 1.4 GeV

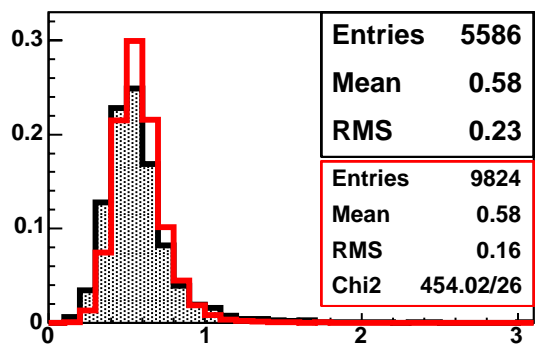

Mip Weighted Radius, 1.5 PE Cut, 1.8 GeV

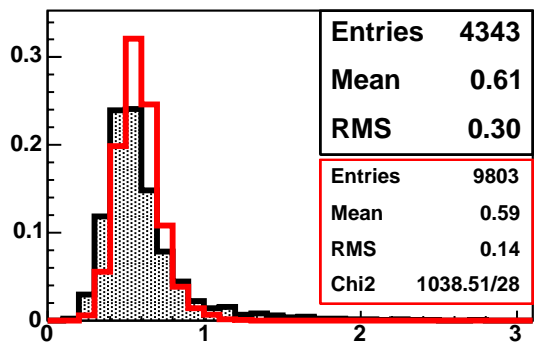

Mip Weighted Radius, 1.5 PE Cut, 2.2 GeV

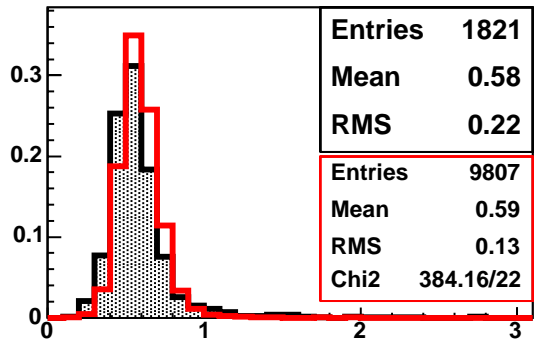

Mip Weighted Radius, 1.5 PE Cut, 1.6 GeV

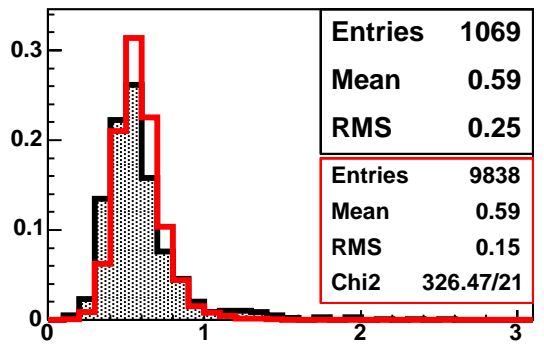

Mip Weighted Radius, 1.5 PE Cut, 2.0 GeV

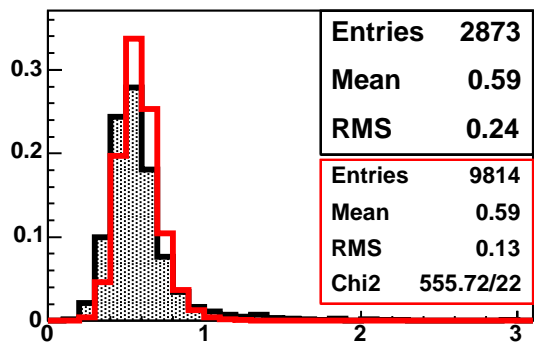

Mip Weighted Radius, 1.5 PE Cut, $2.4 \mathrm{GeV}$

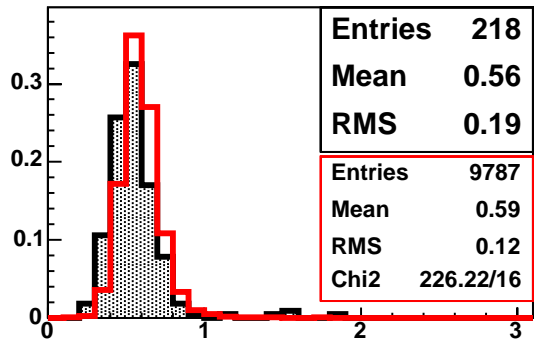

Figure D.7: Comparison of radial distributions between data and Monte Carlo. 
Mip Weighted Radius, 1.5 PE Cut, 2.6 GeV

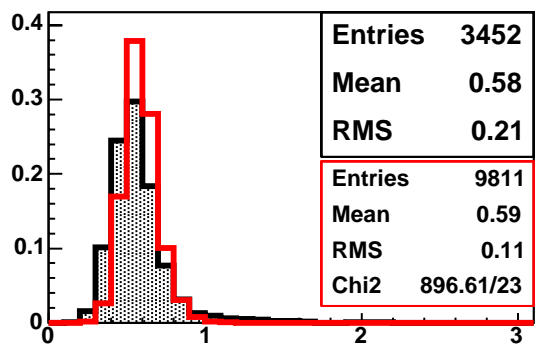

Mip Weighted Radius, 1.5 PE Cut, 3.0 GeV

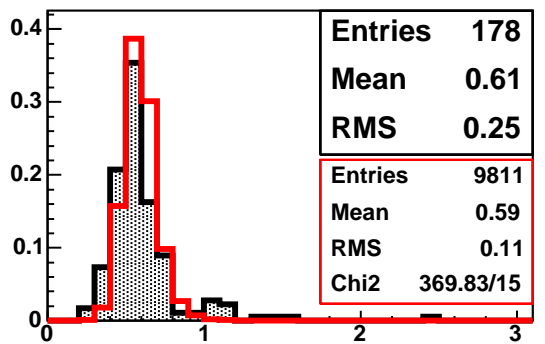

Mip Weighted Radius, 1.5 PE Cut, $2.8 \mathrm{GeV}$

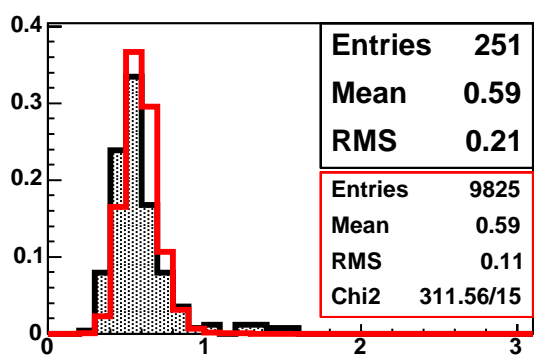

Mip Weighted Radius, 1.5 PE Cut, 3.2 GeV

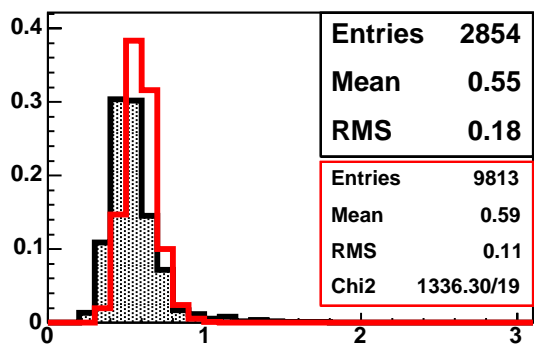

Mip Weighted Radius, 1.5 PE Cut, 3.6 GeV

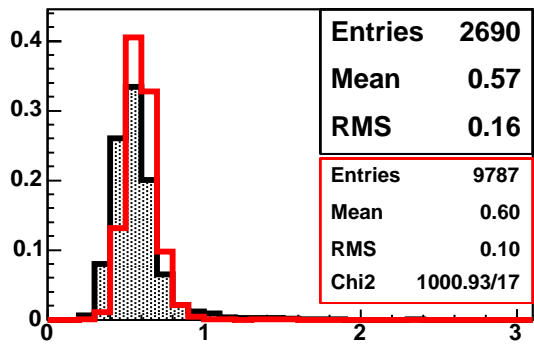

Figure D.8: Comparison of radial distributions between data and Monte Carlo. 
Mip Weighted Radius, 1.5 PE Cut, 1.0 GeV

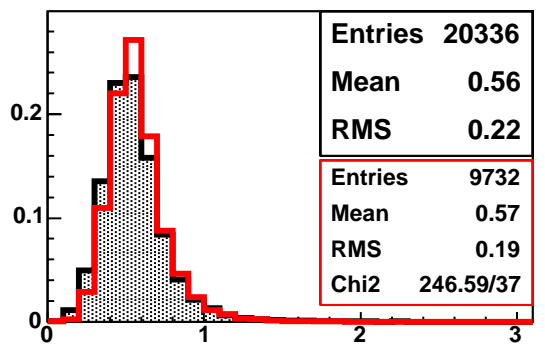

Mip Weighted Radius, 1.5 PE Cut, 2.4 GeV

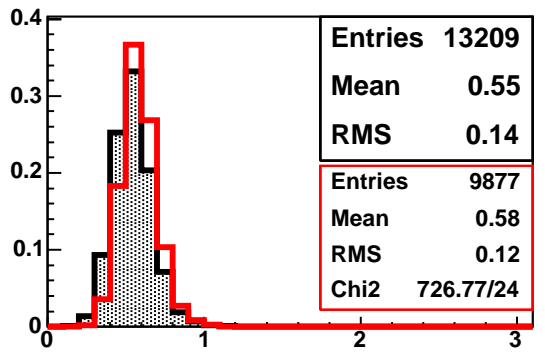

Mip Weighted Radius, 1.5 PE Cut, 3.0 GeV

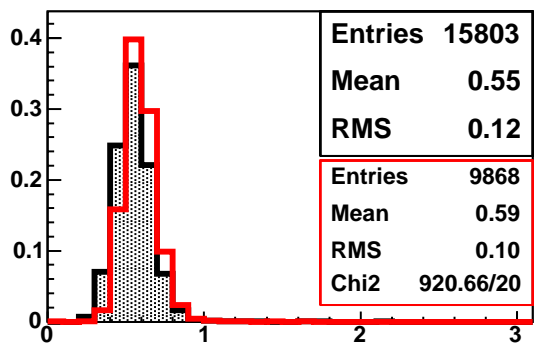

Mip Weighted Radius, 1.5 PE Cut, 2.0 GeV

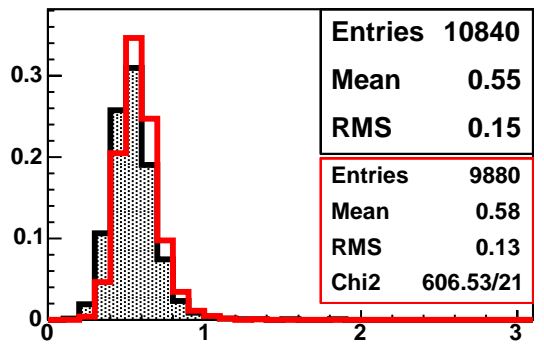

Mip Weighted Radius, 1.5 PE Cut, 2.8 GeV

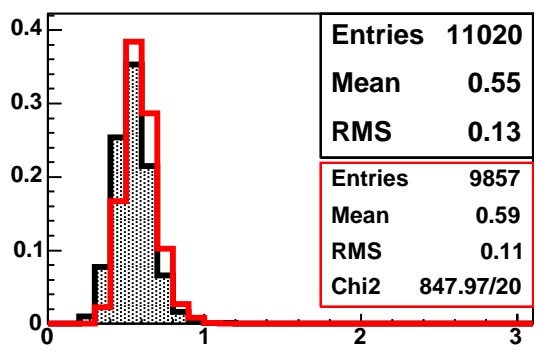

Mip Weighted Radius, 1.5 PE Cut, 4.0 GeV

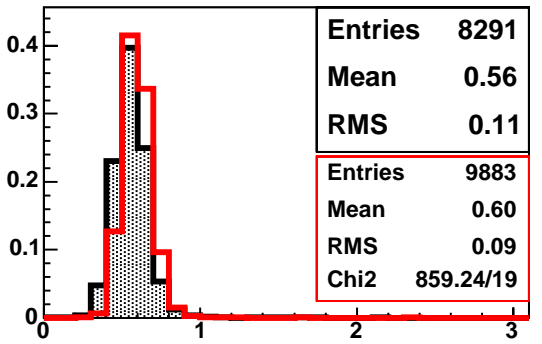

Figure D.9: Comparison of radial distributions between data and Monte Carlo. 
Mip Weighted Radius, 1.5 PE Cut, 5.0 GeV

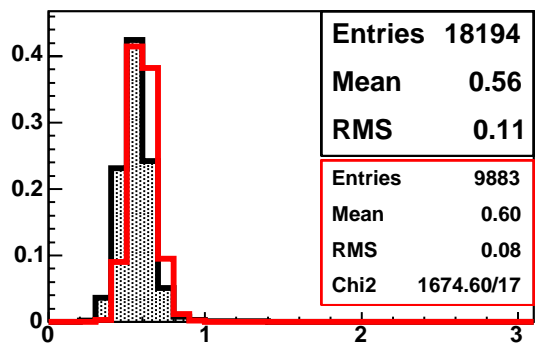

Mip Weighted Radius, 1.5 PE Cut, 7.0 GeV

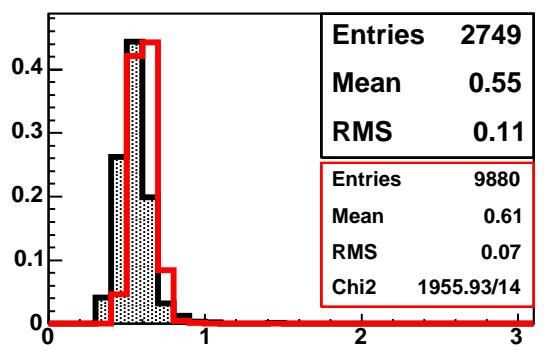

Mip Weighted Radius, 1.5 PE Cut, $6.0 \mathrm{GeV}$

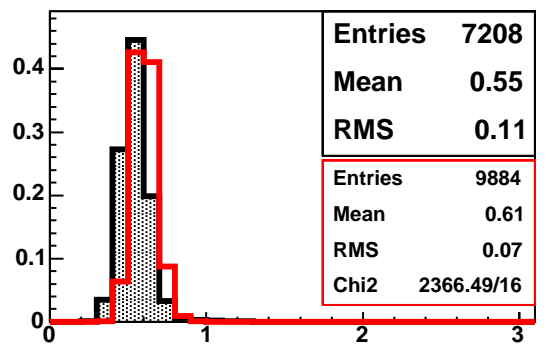

Mip Weighted Radius, 1.5 PE Cut, $8.0 \mathrm{GeV}$

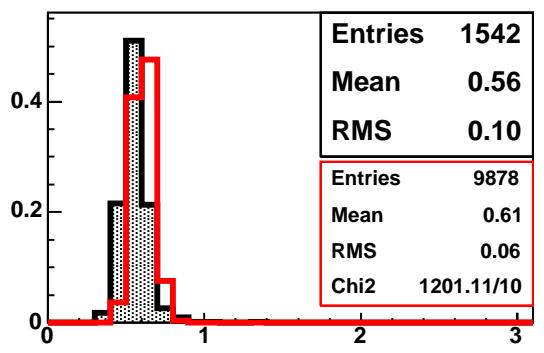

Mip Weighted Radius, 1.5 PE Cut, 9.0 GeV

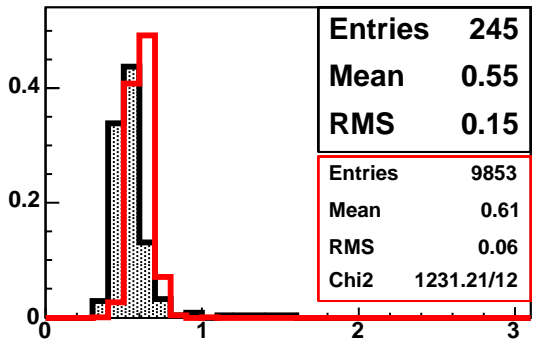

Figure D.10: Comparison of radial distributions between data and Monte Carlo. 
Nhit Strips, $0.2 \mathrm{GeV}$

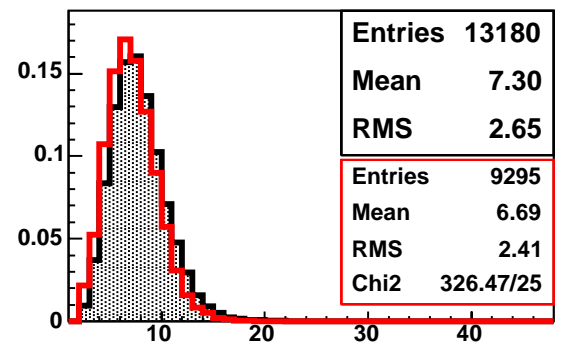

Nhit Strips, $0.6 \mathrm{GeV}$

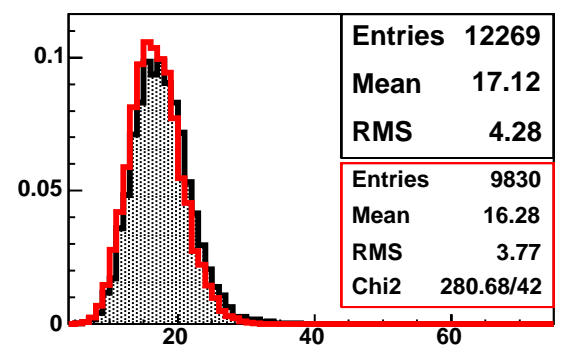

Nhit Strips, $1.0 \mathrm{GeV}$

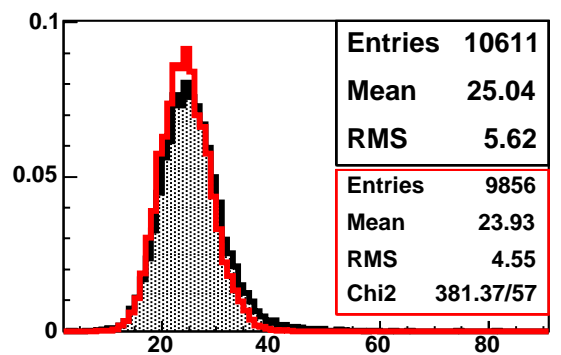

Nhit Strips, $0.4 \mathrm{GeV}$

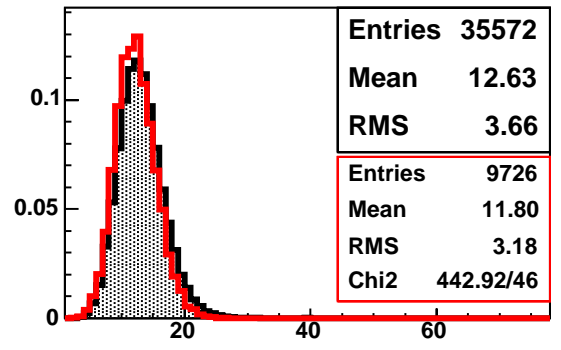

Nhit Strips, $0.8 \mathrm{GeV}$

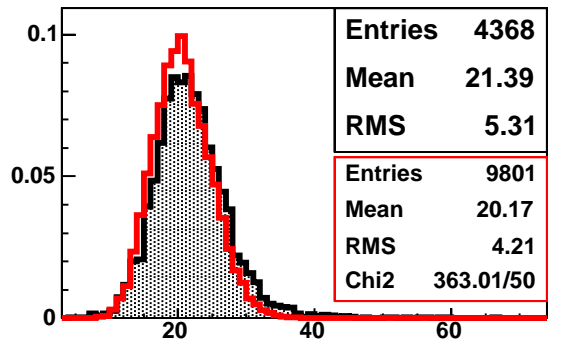

Nhit Strips, $1.2 \mathrm{GeV}$

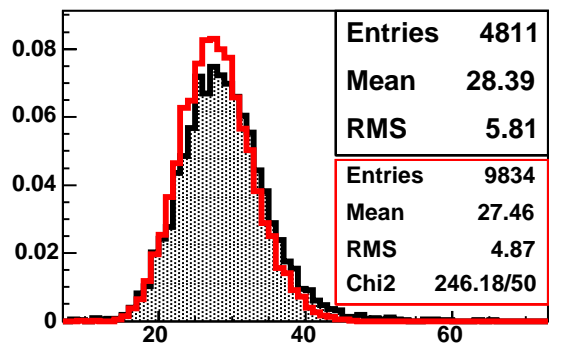

Figure D.11: Comparison of number of hit strips distributions between data and Monte Carlo. 
Nhit Strips, $1.4 \mathrm{GeV}$

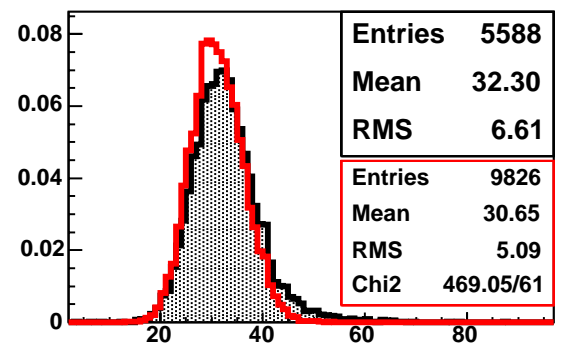

Nhit Strips, $1.8 \mathrm{GeV}$

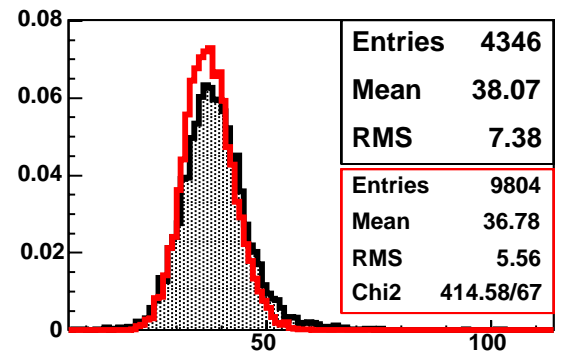

Nhit Strips, $2.2 \mathrm{GeV}$

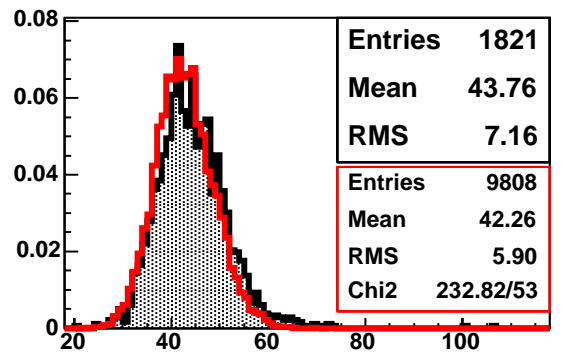

Nhit Strips, 1.6 GeV

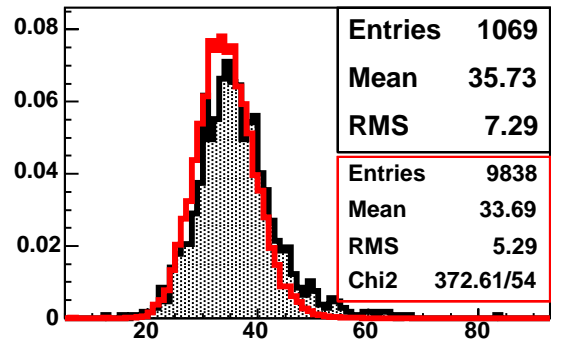

Nhit Strips, $2.0 \mathrm{GeV}$

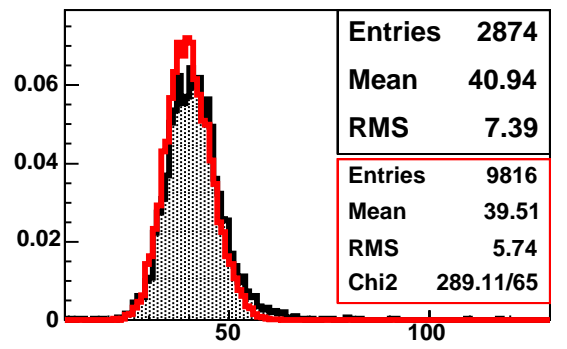

Nhit Strips, $2.4 \mathrm{GeV}$

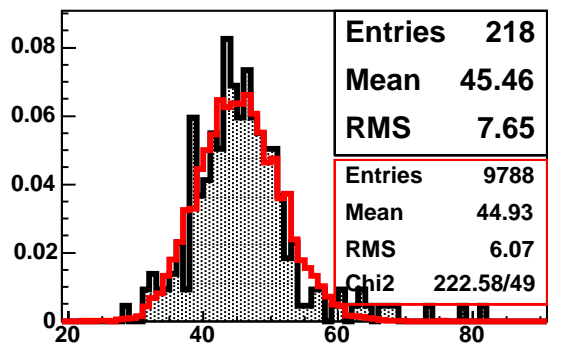

Figure D.12: Comparison of number of hit strips distributions between data and Monte Carlo. 
Nhit Strips, 2.6 GeV

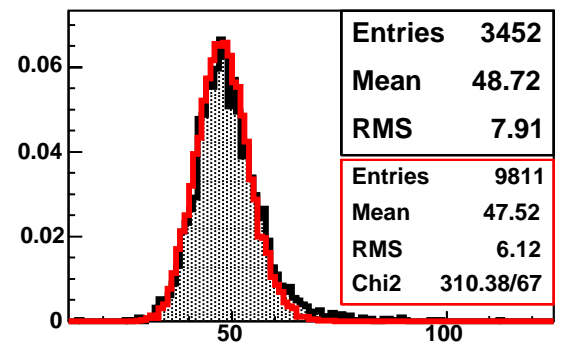

Nhit Strips, $3.0 \mathrm{GeV}$

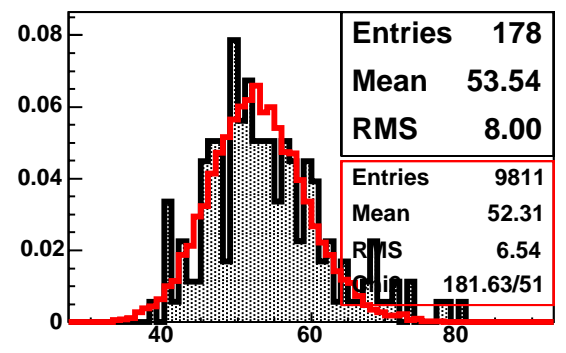

Nhit Strips, 3.6 GeV

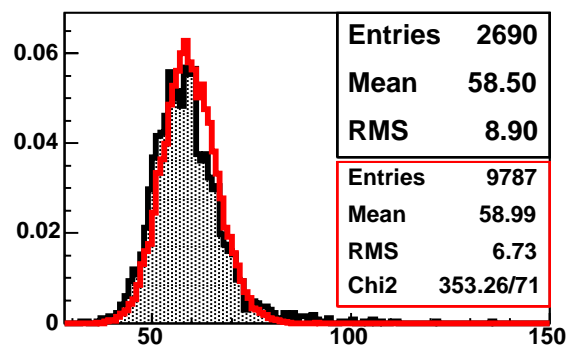

Nhit Strips, $2.8 \mathrm{GeV}$

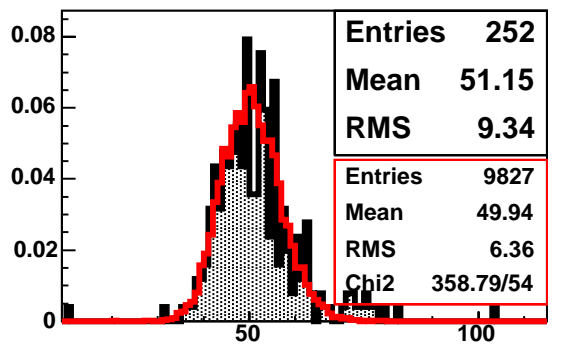

Nhit Strips, 3.2 GeV

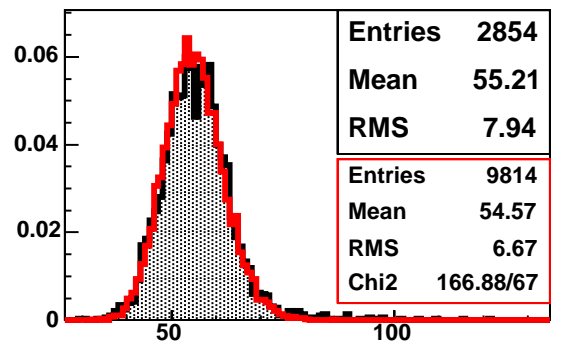

Figure D.13: Comparison of number of hit strips distributions between data and Monte Carlo. 
Nhit Strips, $1.0 \mathrm{GeV}$

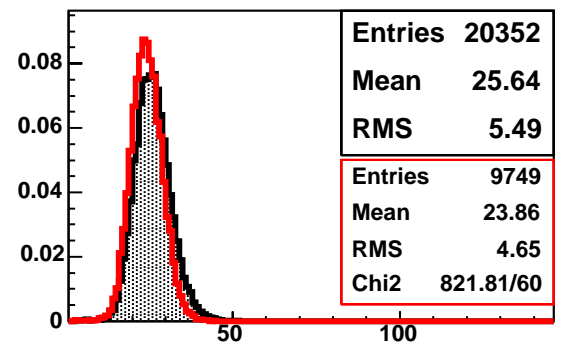

Nhit Strips, $2.4 \mathrm{GeV}$

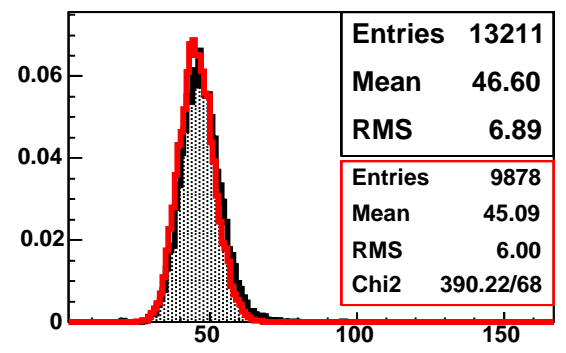

Nhit Strips, $3.0 \mathrm{GeV}$

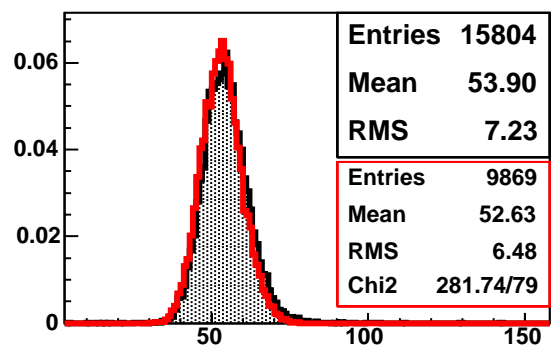

Nhit Strips, $2.0 \mathrm{GeV}$

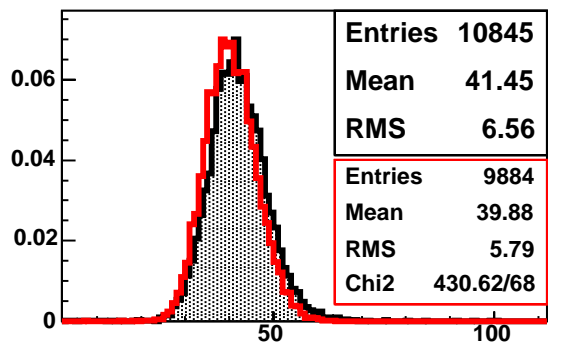

Nhit Strips, $2.8 \mathrm{GeV}$

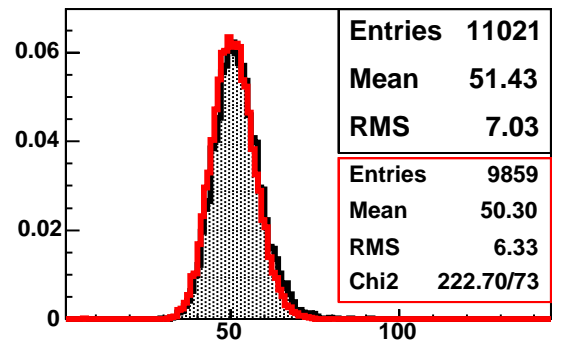

Nhit Strips, $4.0 \mathrm{GeV}$

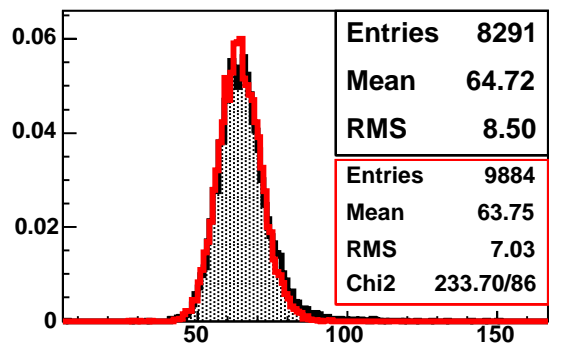

Figure D.14: Comparison of number of hit strips distributions between data and Monte Carlo. 
Nhit Strips, $5.0 \mathrm{GeV}$

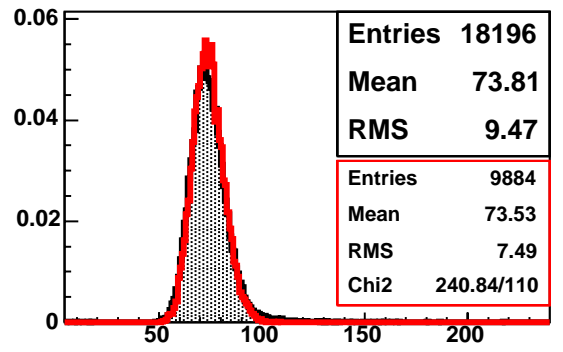

Nhit Strips, $7.0 \mathrm{GeV}$

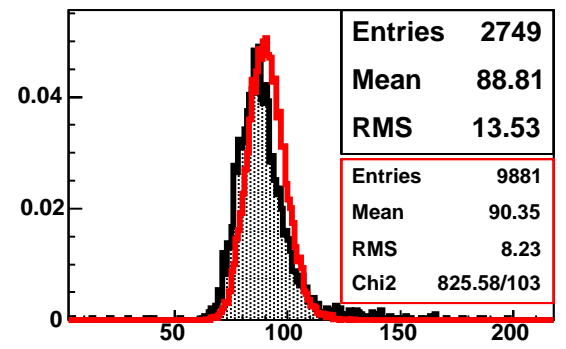

Nhit Strips, $6.0 \mathrm{GeV}$

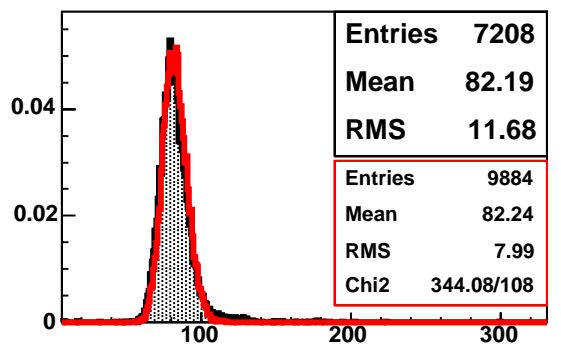

Nhit Strips, $8.0 \mathrm{GeV}$

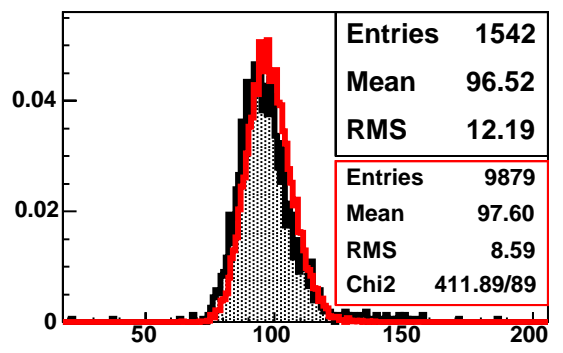

Nhit Strips, $9.0 \mathrm{GeV}$

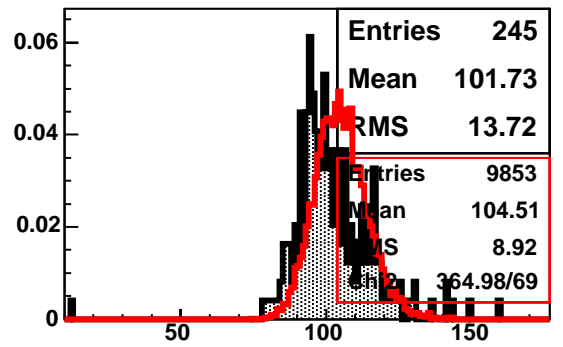

Figure D.15: Comparison of number of hit strips distributions between data and Monte Carlo. 
Nhit Strips, 1.5 PE Cut, 0.2 GeV

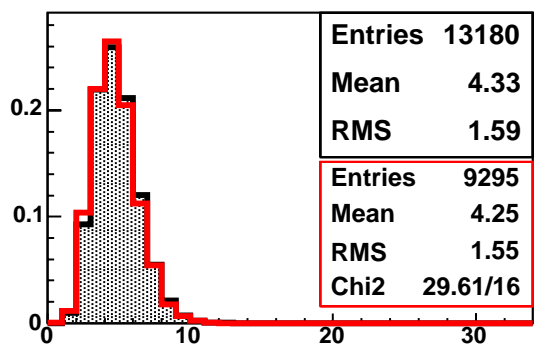

Nhit Strips, 1.5 PE Cut, 0.6 GeV

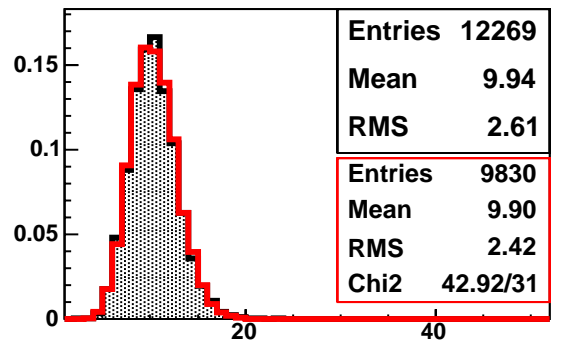

Nhit Strips, 1.5 PE Cut, 1.0 GeV

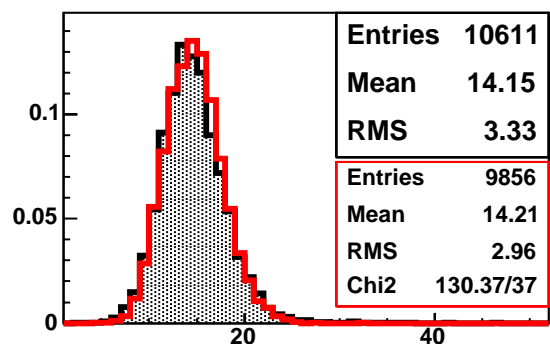

Nhit Strips, 1.5 PE Cut, 0.4 GeV

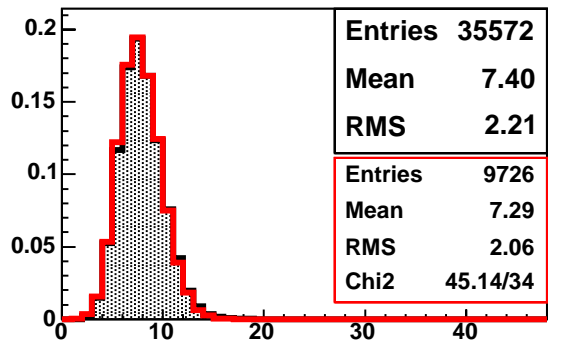

Nhit Strips, 1.5 PE Cut, $0.8 \mathrm{GeV}$

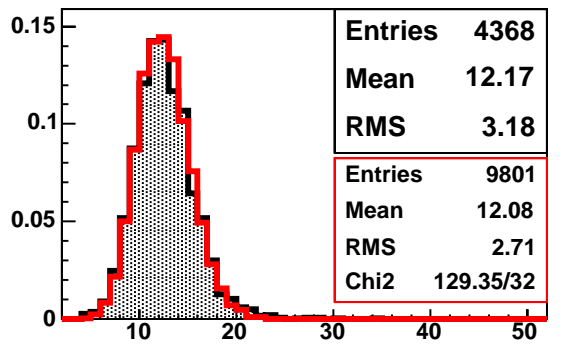

Nhit Strips, 1.5 PE Cut, 1.2 GeV

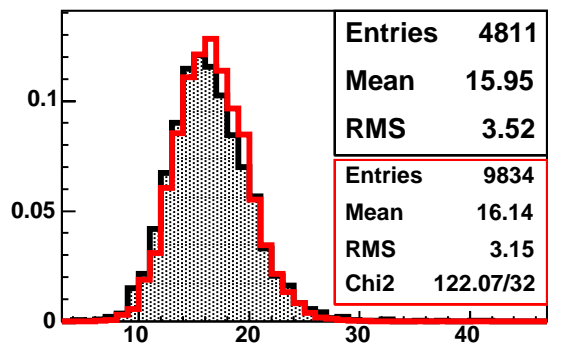

Figure D.16: Comparison of number of hit strips above 1.5 PE between data and Monte Carlo. 
Nhit Strips, 1.5 PE Cut, 1.4 GeV

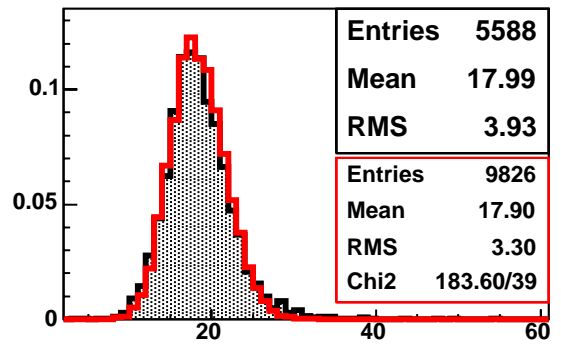

Nhit Strips, 1.5 PE Cut, 1.8 GeV

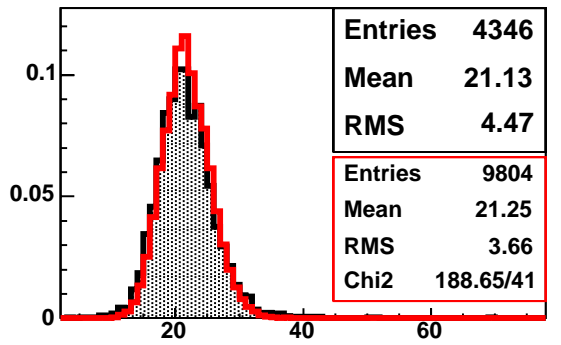

Nhit Strips, 1.5 PE Cut, 2.2 GeV

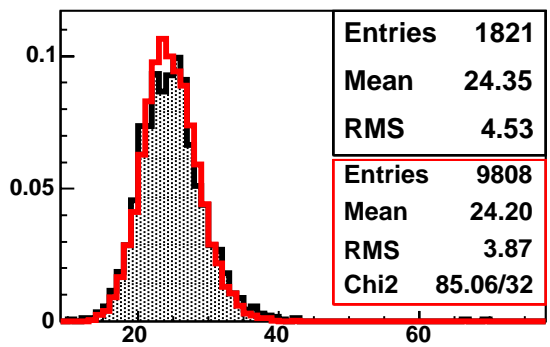

Nhit Strips, 1.5 PE Cut, 1.6 GeV

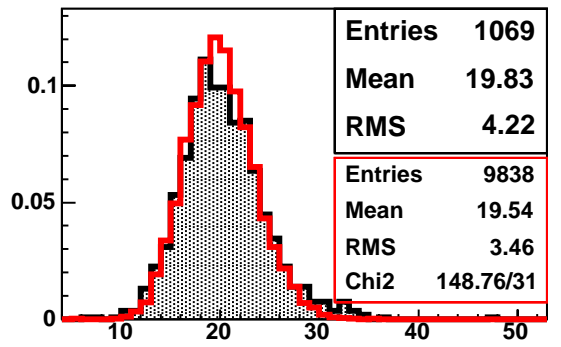

Nhit Strips, 1.5 PE Cut, 2.0 GeV

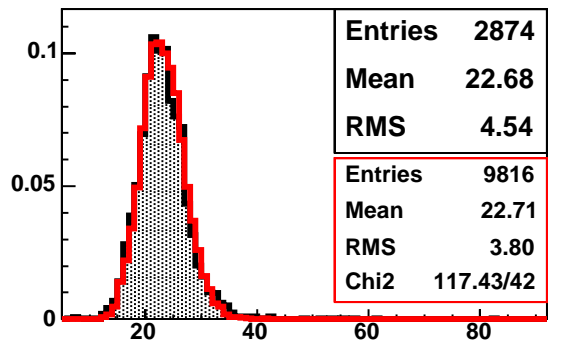

Nhit Strips, 1.5 PE Cut, 2.4 GeV

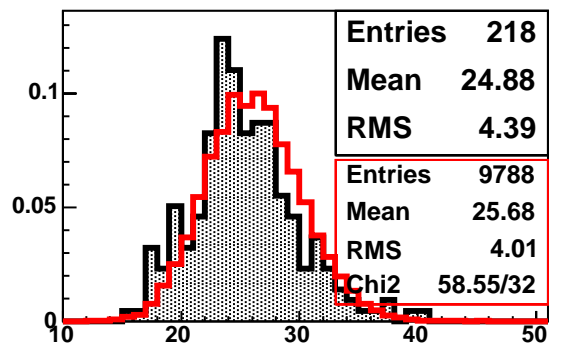

Figure D.17: Comparison of number of hit strips above 1.5 PE between data and Monte Carlo. 
Nhit Strips, 1.5 PE Cut, 2.6 GeV

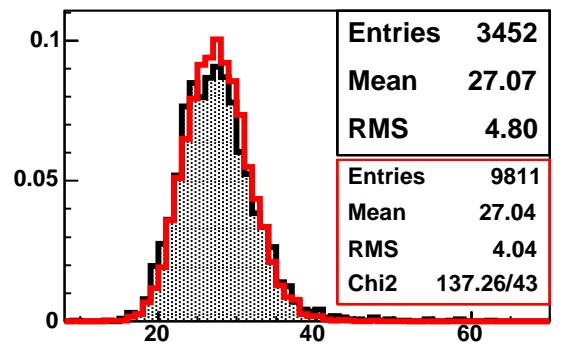

Nhit Strips, 1.5 PE Cut, 3.0 GeV

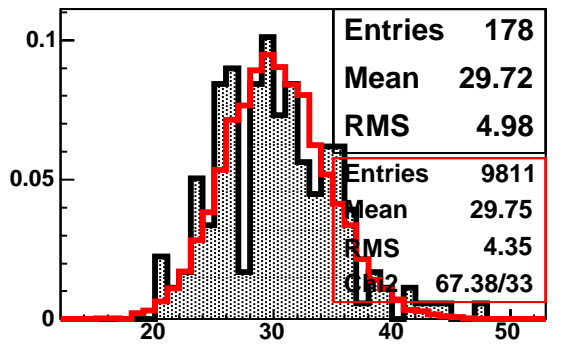

Nhit Strips, 1.5 PE Cut, 2.8 GeV

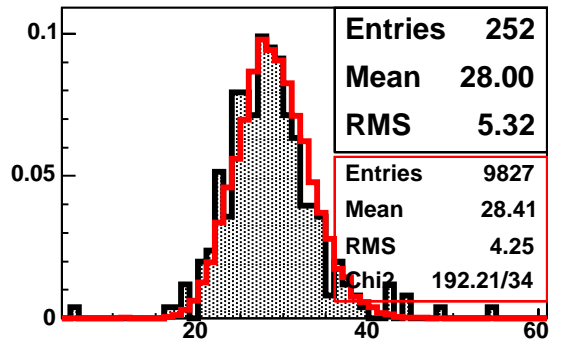

Nhit Strips, 1.5 PE Cut, 3.2 GeV

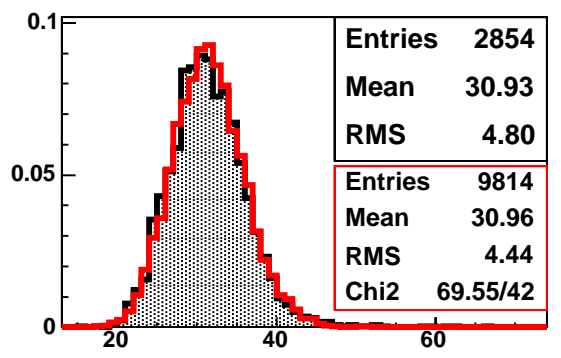

Nhit Strips, 1.5 PE Cut, 3.6 GeV

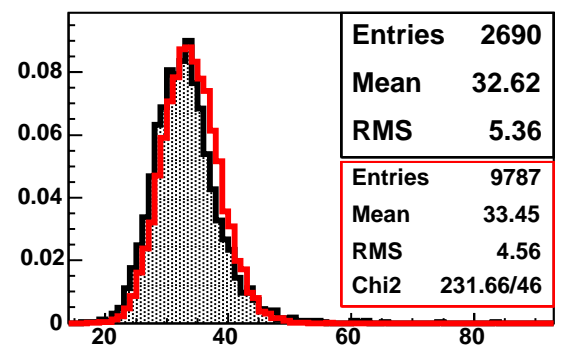

Figure D.18: Comparison of number of hit strips above 1.5 PE between data and Monte Carlo. 
Nhit Strips, 1.5 PE Cut, 1.0 GeV

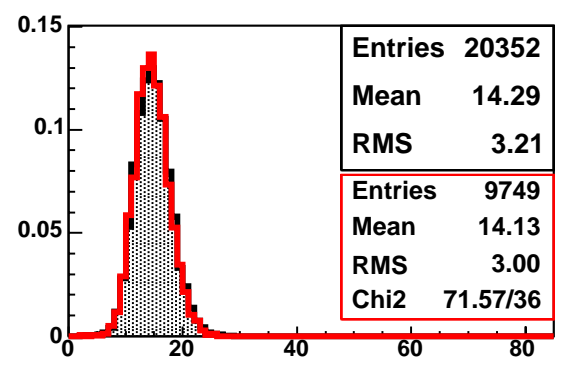

Nhit Strips, 1.5 PE Cut, 2.4 GeV

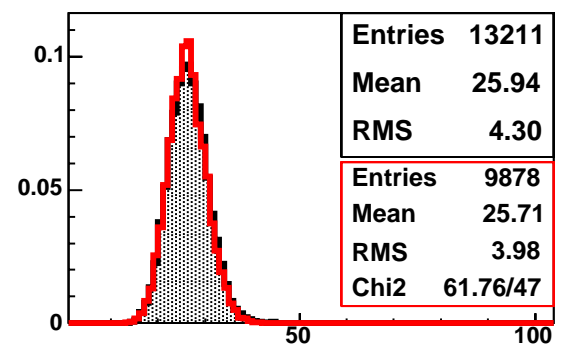

Nhit Strips, 1.5 PE Cut, 3.0 GeV

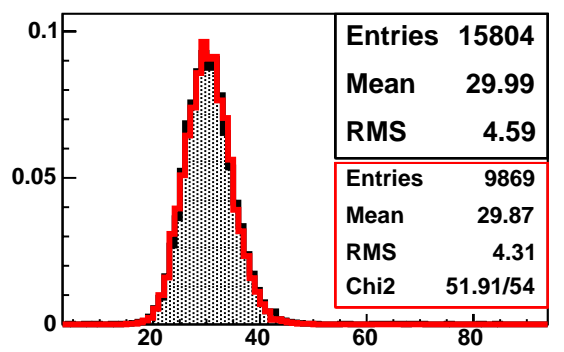

Nhit Strips, 1.5 PE Cut, 2.0 GeV

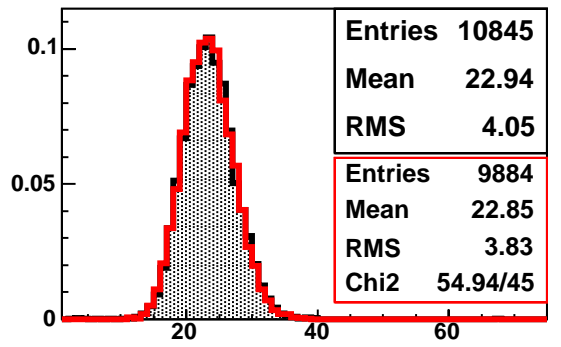

Nhit Strips, 1.5 PE Cut, 2.8 GeV

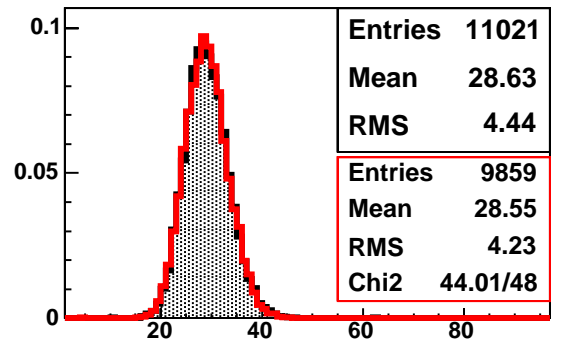

Nhit Strips, 1.5 PE Cut, 4.0 GeV

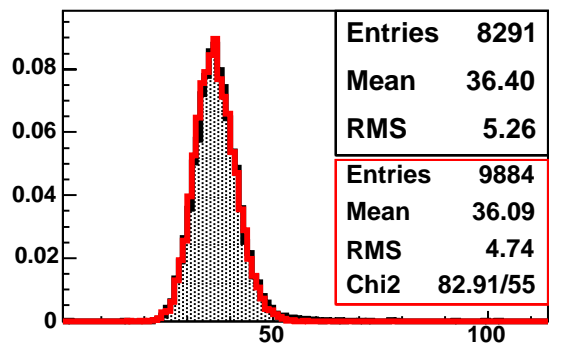

Figure D.19: Comparison of number of hit strips above 1.5 PE between data and Monte Carlo. 
Nhit Strips, 1.5 PE Cut, 5.0 GeV

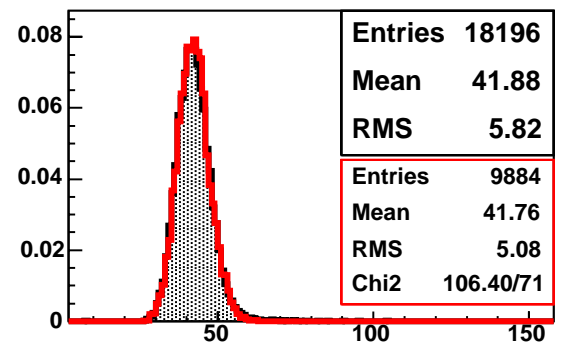

Nhit Strips, 1.5 PE Cut, 7.0 GeV

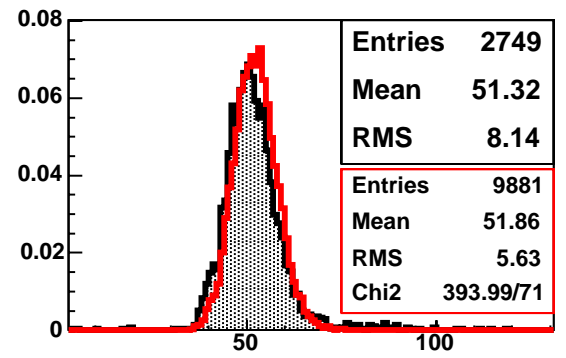

Nhit Strips, 1.5 PE Cut, 9.0 GeV

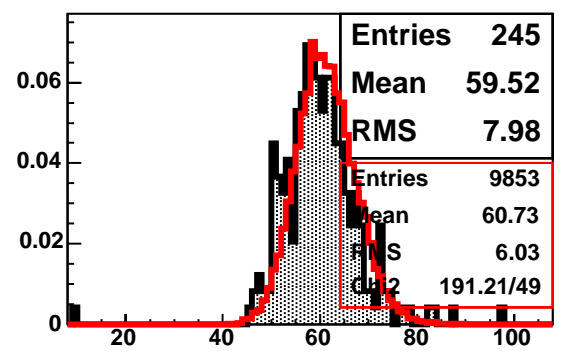

Nhit Strips, 1.5 PE Cut, $6.0 \mathrm{GeV}$

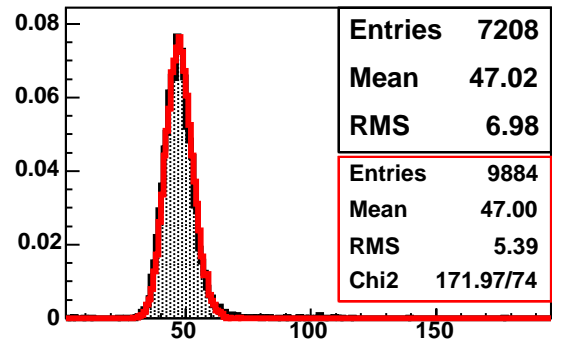

Nhit Strips, 1.5 PE Cut, 8.0 GeV

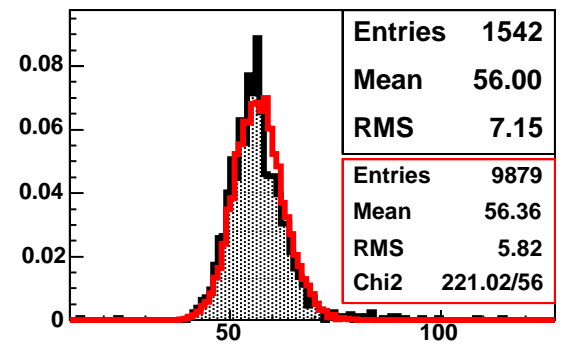

Figure D.20: Comparison of number of hit strips above 1.5 PE between data and Monte Carlo. 
Plane of Shower Max, $0.2 \mathrm{GeV}$

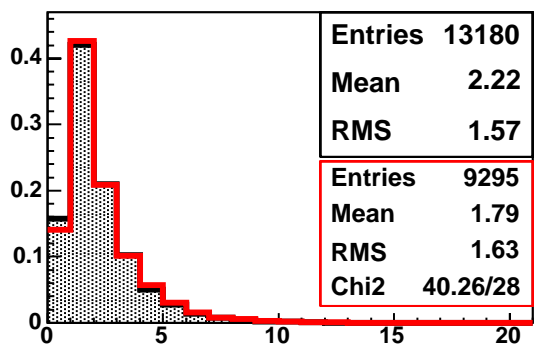

Plane of Shower Max, $0.6 \mathrm{GeV}$

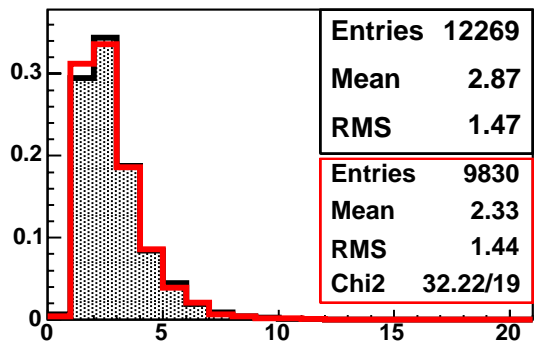

Plane of Shower Max, $1.0 \mathrm{GeV}$

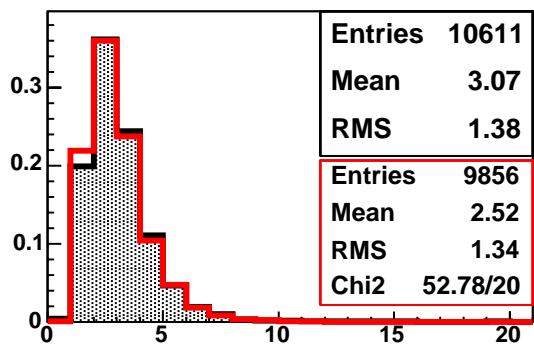

Plane of Shower Max, $0.4 \mathrm{GeV}$

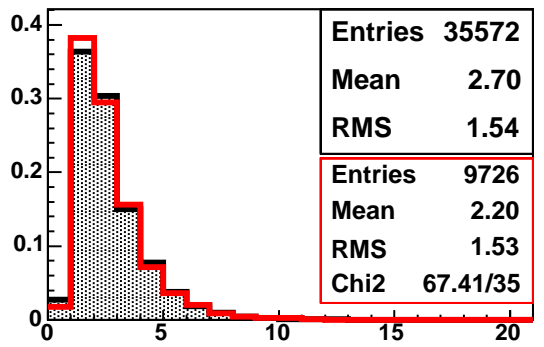

Plane of Shower Max, $0.8 \mathrm{GeV}$

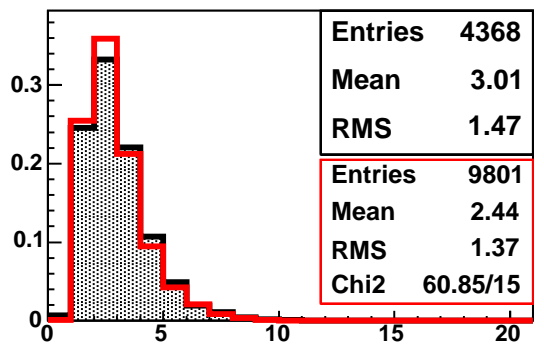

Plane of Shower Max, 1.2 GeV

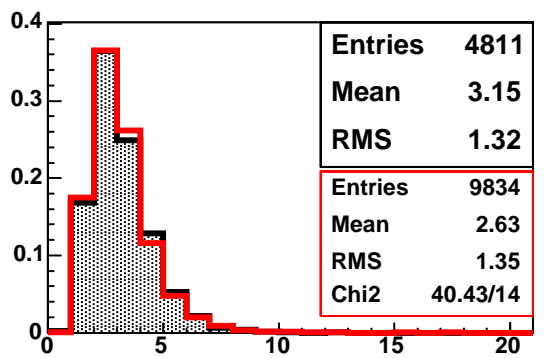

Figure D.21: Comparison of plane of shower max between data and Monte Carlo. 
Plane of Shower Max, $1.4 \mathrm{GeV}$

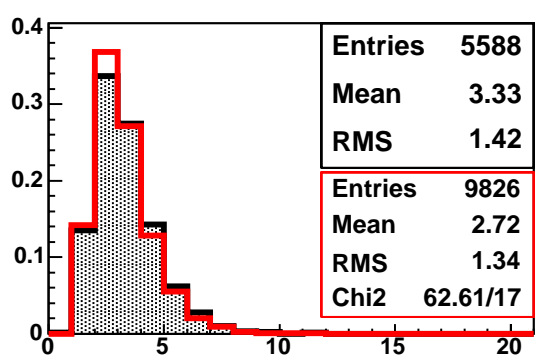

Plane of Shower Max, $1.8 \mathrm{GeV}$

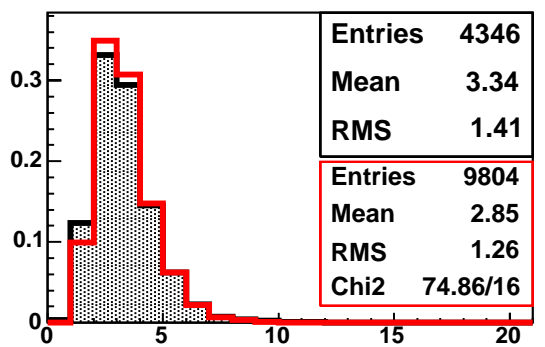

Plane of Shower Max, 2.2 GeV

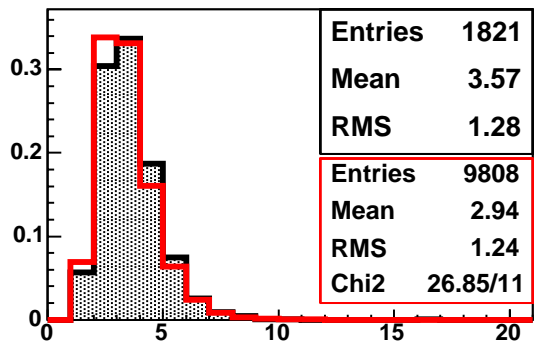

Plane of Shower Max, $1.6 \mathrm{GeV}$

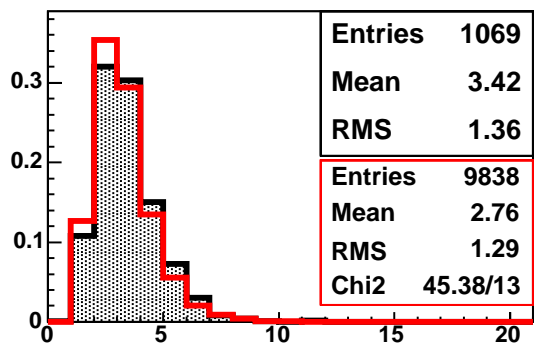

Plane of Shower Max, $2.0 \mathrm{GeV}$

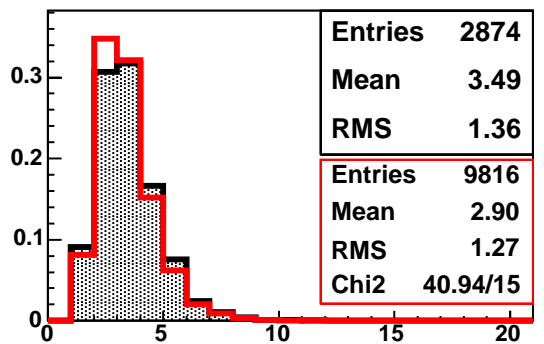

Plane of Shower Max, $2.4 \mathrm{GeV}$

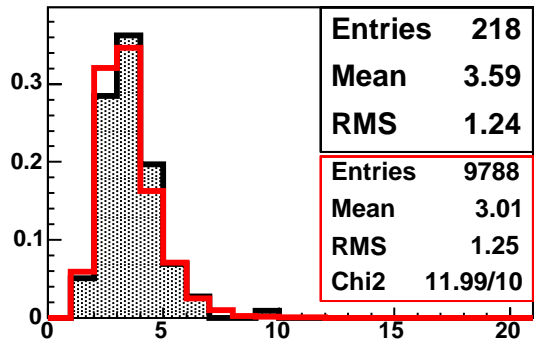

Figure D.22: Comparison of plane of shower max between data and Monte Carlo. 
Plane of Shower Max, $2.6 \mathrm{GeV}$

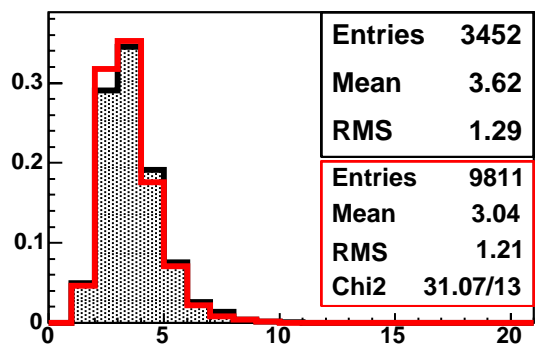

Plane of Shower Max, $3.0 \mathrm{GeV}$

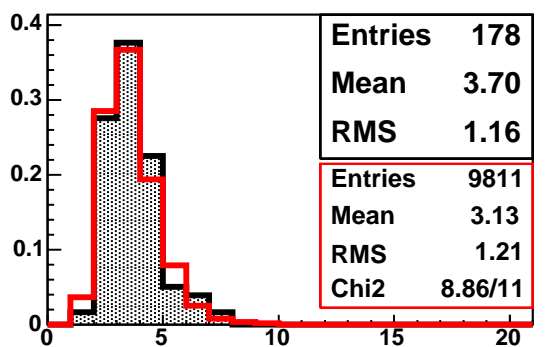

Plane of Shower Max, $28 \mathrm{GeV}$

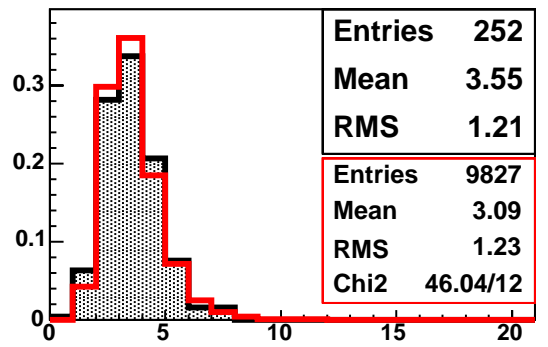

Plane of Shower Max, $3.2 \mathrm{GeV}$

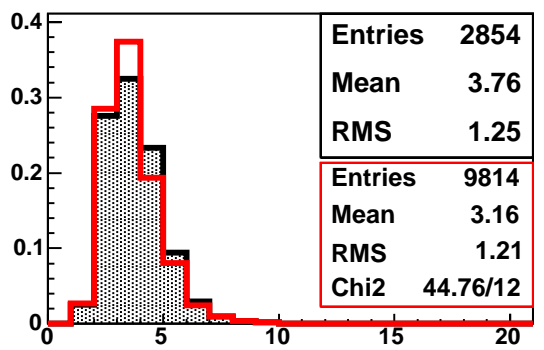

Plane of Shower Max, 3.6 GeV

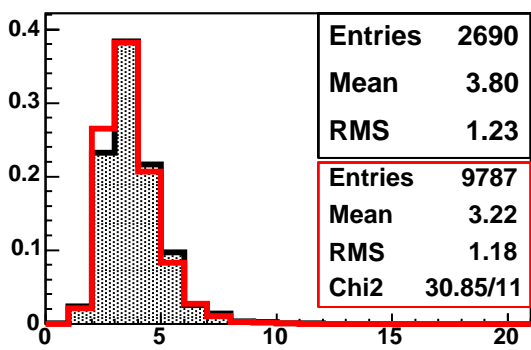

Figure D.23: Comparison of plane of shower max between data and Monte Carlo. 
Plane of Shower Max, $1.0 \mathrm{GeV}$

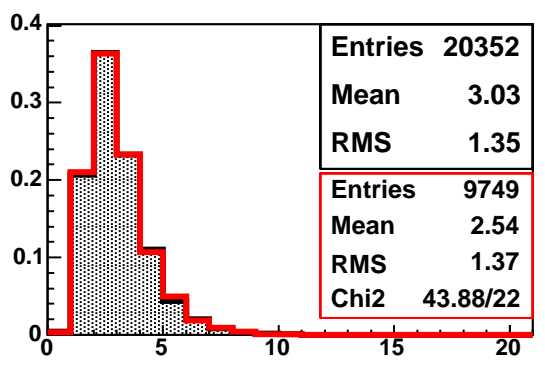

Plane of Shower Max, $2.4 \mathrm{GeV}$

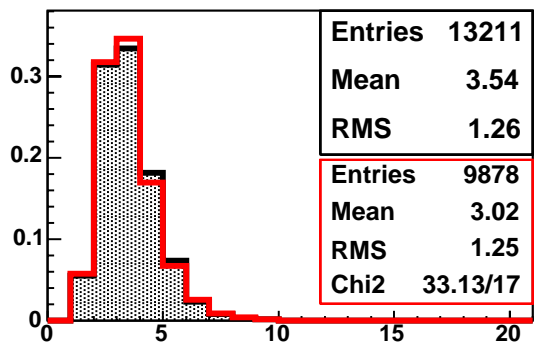

Plane of Shower Max, 3.0 GeV

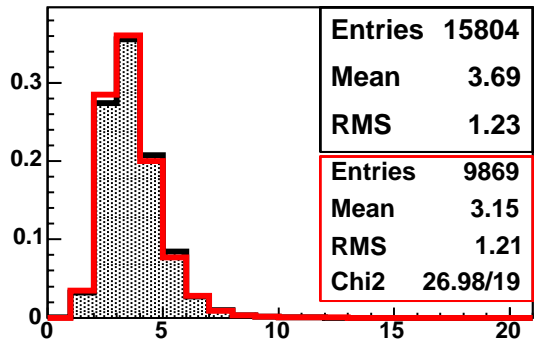

Plane of Shower Max, $20 \mathrm{GeV}$

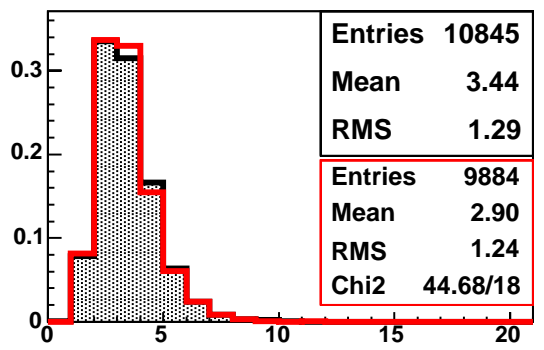

Plane of Shower Max, $2.8 \mathrm{GeV}$

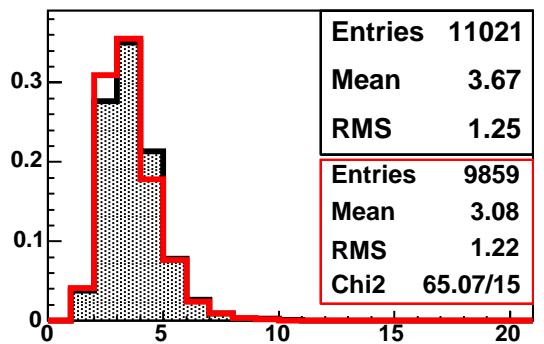

Plane of Shower Max, 4.0 GeV

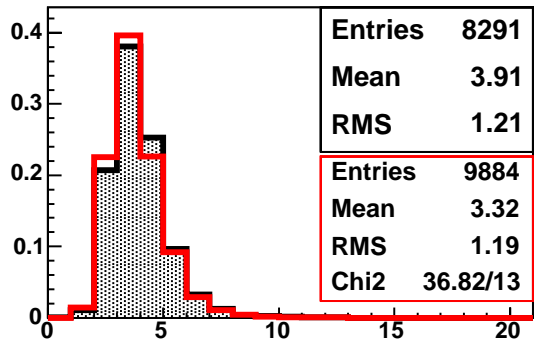

Figure D.24: Comparison of plane of shower max between data and Monte Carlo. 
Plane of Shower Max, 5.0 GeV

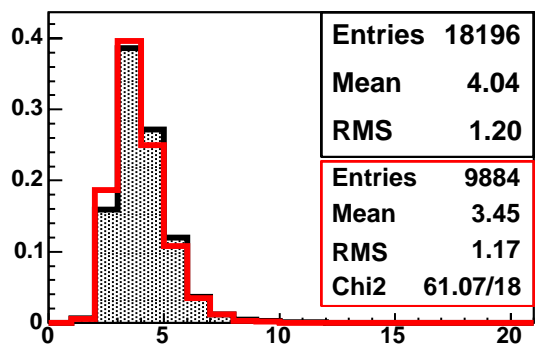

Plane of Shower Max, 7.0 GeV

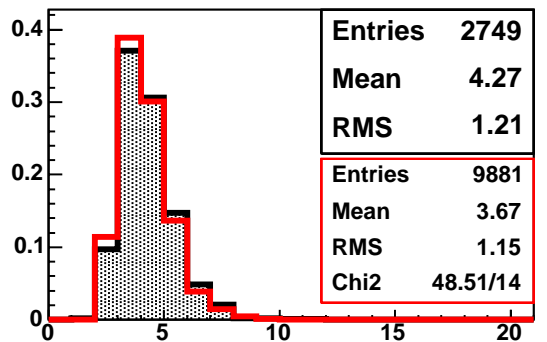

Plane of Shower Max, $6.0 \mathrm{GeV}$

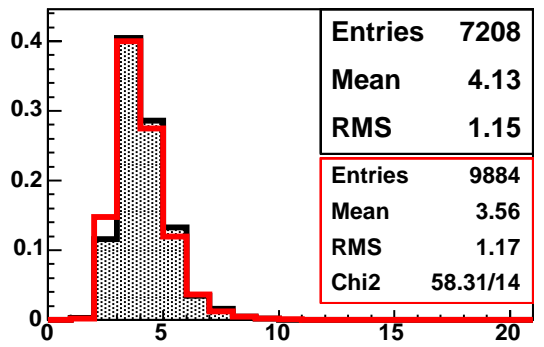

Plane of Shower Max, 8.0 GeV

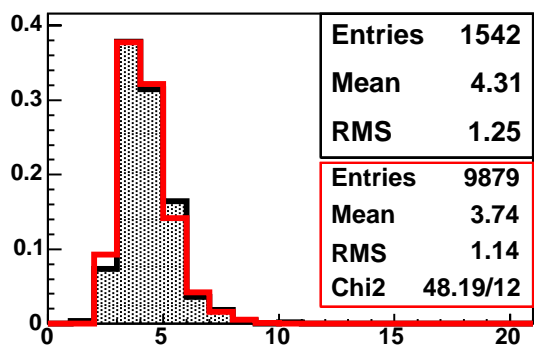

Plane of Shower Max, $9.0 \mathrm{GeV}$

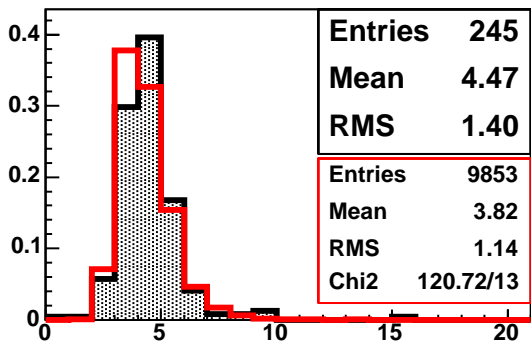

Figure D.25: Comparison of plane of shower max between data and Monte Carlo. 
MIPs in Plane of Shower Max, $0.2 \mathrm{GeV}$

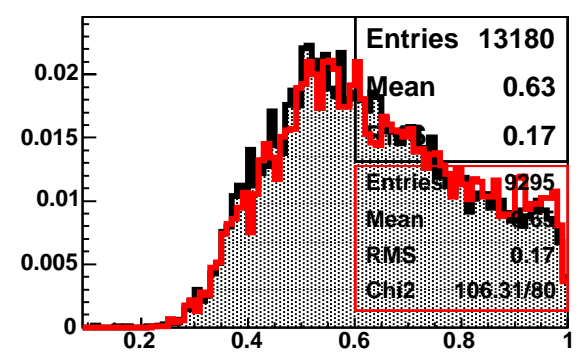

MIPs in Plane of Shower Max, $0.6 \mathrm{GeV}$

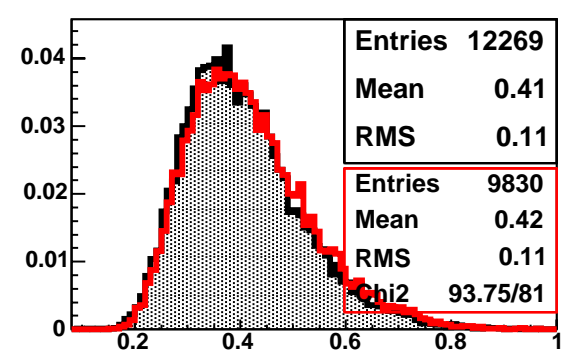

MIPs in Plane of Shower Max, 1.0 GeV

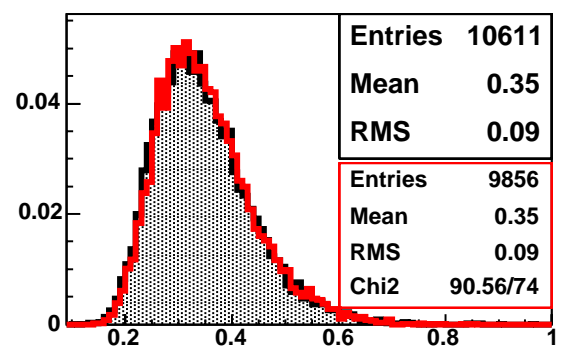

MIPs in Plane of Shower Max, $0.4 \mathrm{GeV}$

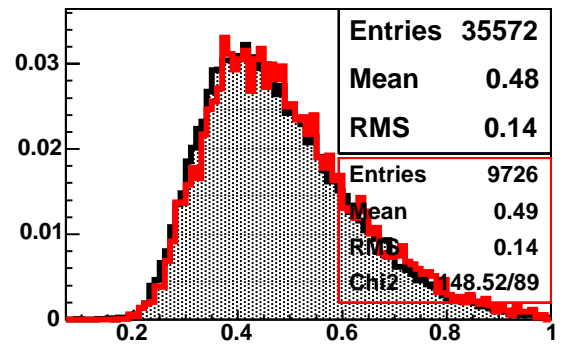

MIPs in Plane of Shower Max, $0.8 \mathrm{GeV}$

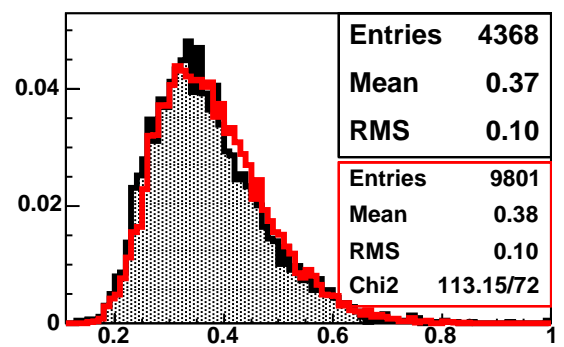

MIPs in Plane of Shower Max, $1.2 \mathrm{GeV}$

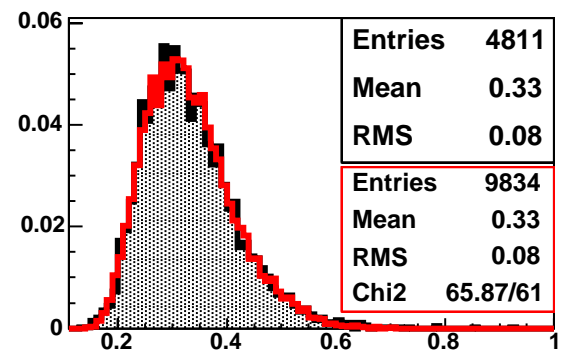

Figure D.26: Comparison of ratio of energy deposited in plane of shower max to total response between data and Monte Carlo. 
MIPs in Plane of Shower Max, $1.4 \mathrm{GeV}$

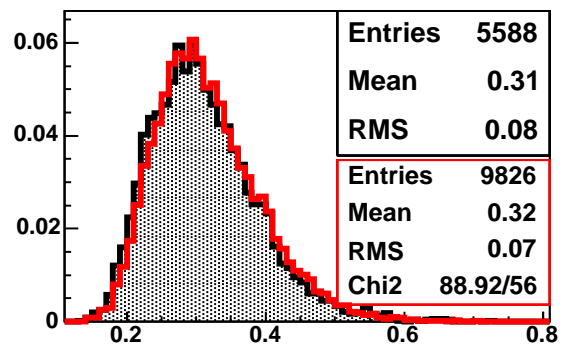

MIPs in Plane of Shower Max, 1.8 GeV

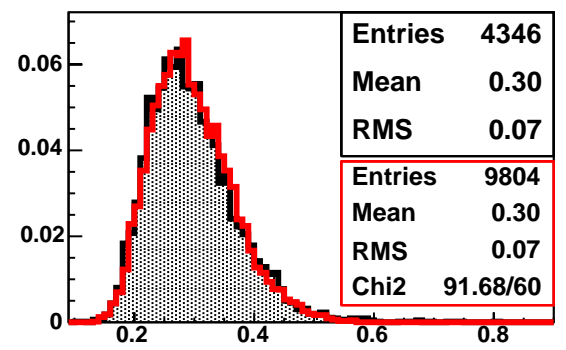

MIPs in Plane of Shower Max, $2.2 \mathrm{GeV}$

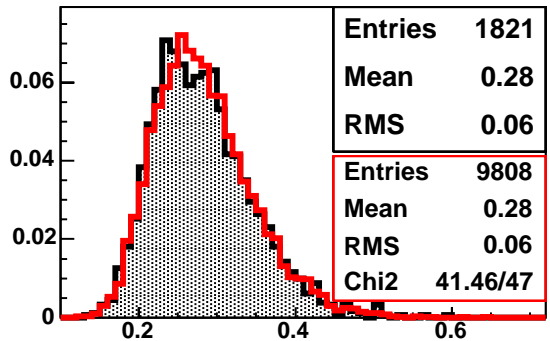

MIPs in Plane of Shower Max, 1.6 GeV

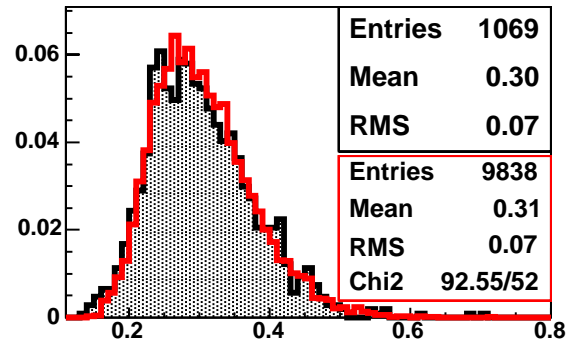

MIPs in Plane of Shower Max, $2.0 \mathrm{GeV}$

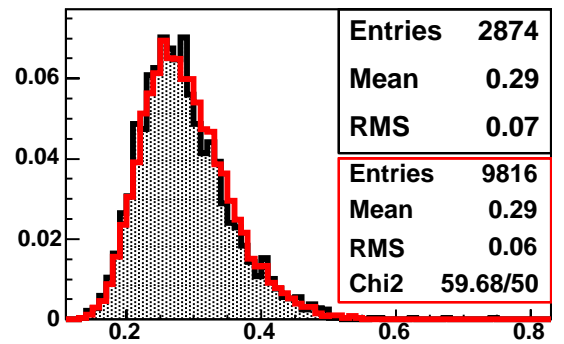

MIPs in Plane of Shower Max, $2.4 \mathrm{GeV}$

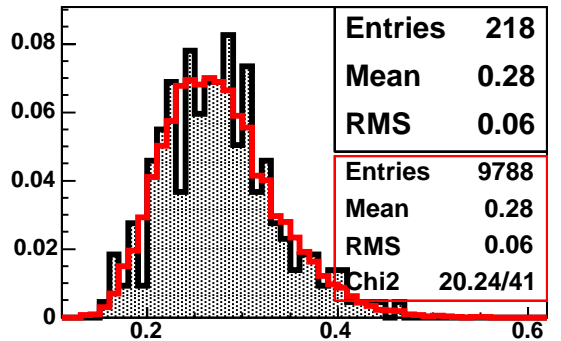

Figure D.27: Comparison of ratio of energy deposited in plane of shower max to total response between data and Monte Carlo. 
MIPs in Plane of Shower Max, $2.6 \mathrm{GeV}$

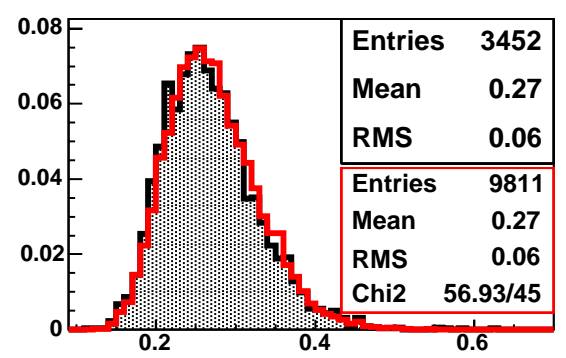

MIPs in Plane of Shower Max, $3.0 \mathrm{GeV}$

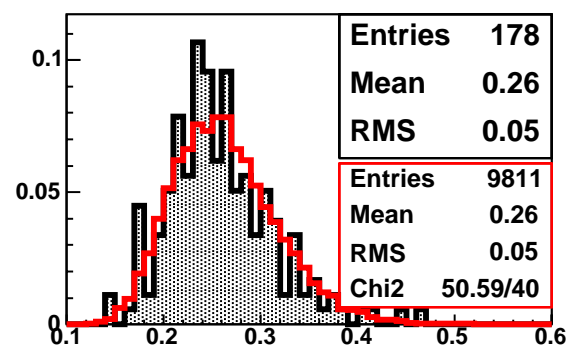

MIPs in Plane of Shower Max, $2.8 \mathrm{GeV}$

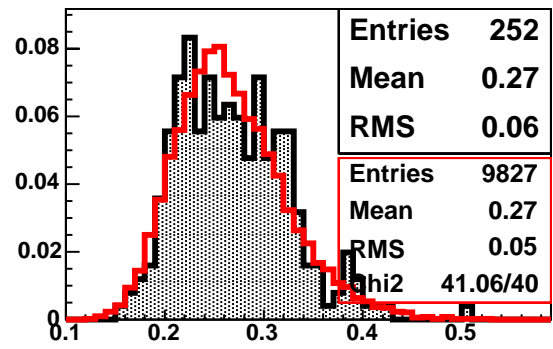

MIPs in Plane of Shower Max, 3.2 GeV

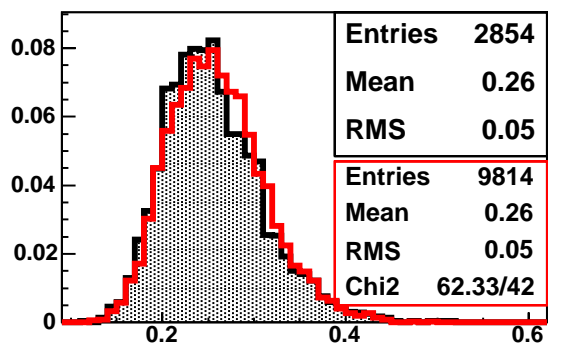

MIPs in Plane of Shower Max, $3.6 \mathrm{GeV}$

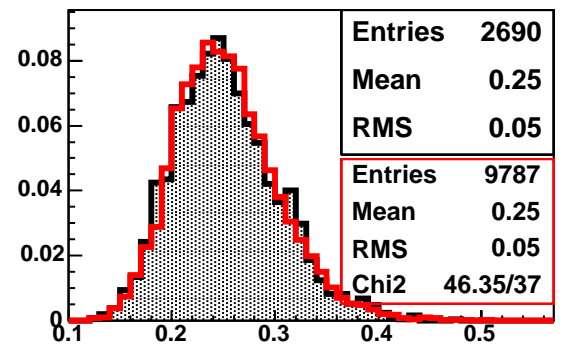

Figure D.28: Comparison of ratio of energy deposited in plane of shower max to total response between data and Monte Carlo. 
MIPs in Plane of Shower Max, $1.0 \mathrm{GeV}$

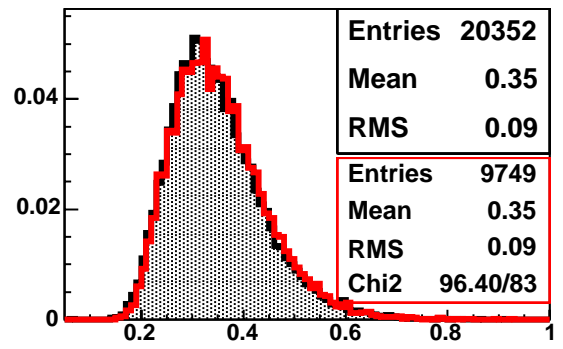

MIPs in Plane of Shower Max, $2.4 \mathrm{GeV}$

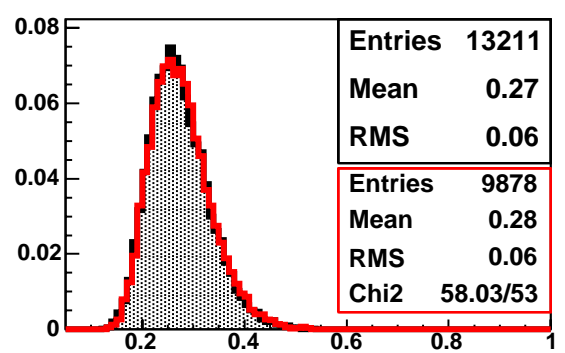

MIPs in Plane of Shower Max, 3.0 GeV

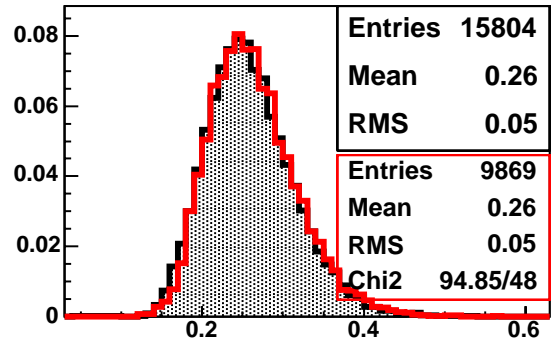

MIPs in Plane of Shower Max, $2.0 \mathrm{GeV}$

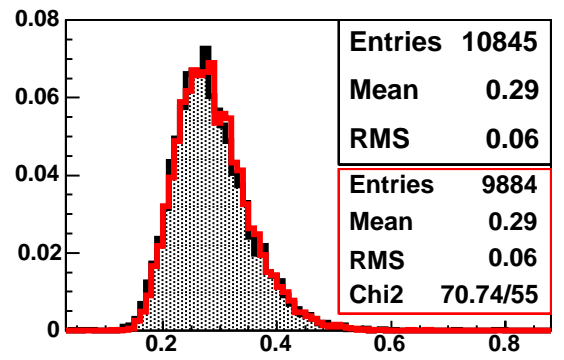

MIPs in Plane of Shower Max, $2.8 \mathrm{GeV}$

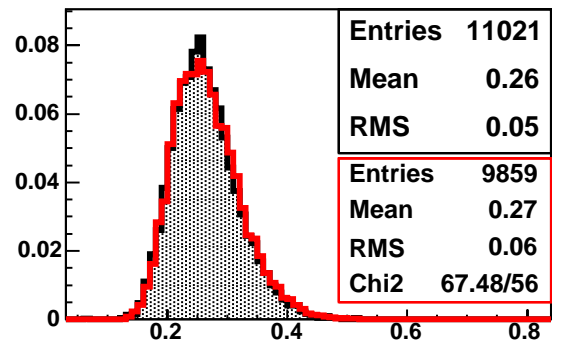

MIPs in Plane of Shower Max, 4.0 GeV

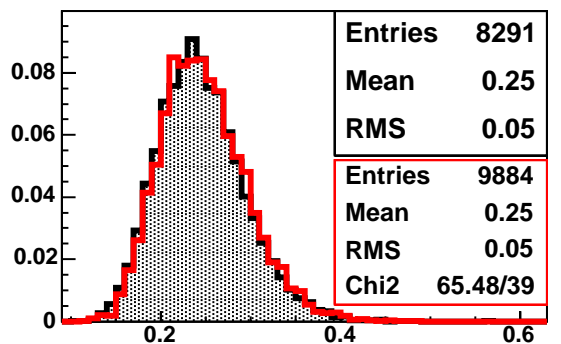

Figure D.29: Comparison of ratio of energy deposited in plane of shower max to total response between data and Monte Carlo. 
MIPs in Plane of Shower Max, $5.0 \mathrm{GeV}$

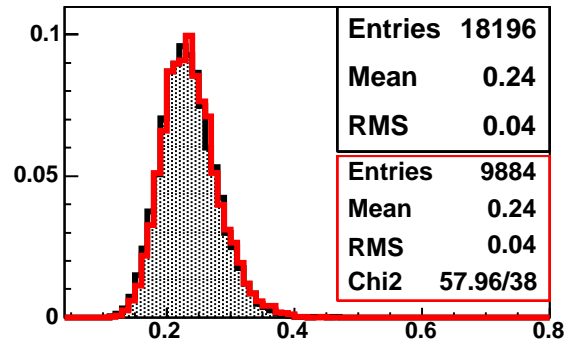

MIPs in Plane of Shower Max, $7.0 \mathrm{GeV}$

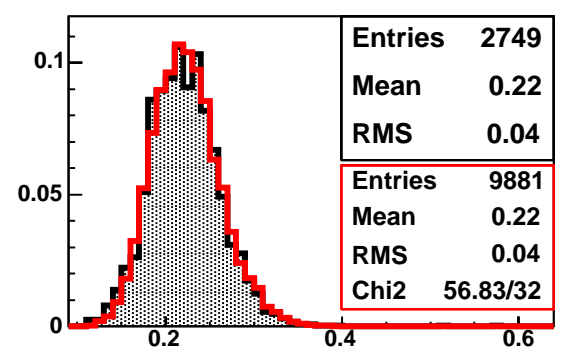

MIPs in Plane of Shower Max, $6.0 \mathrm{GeV}$

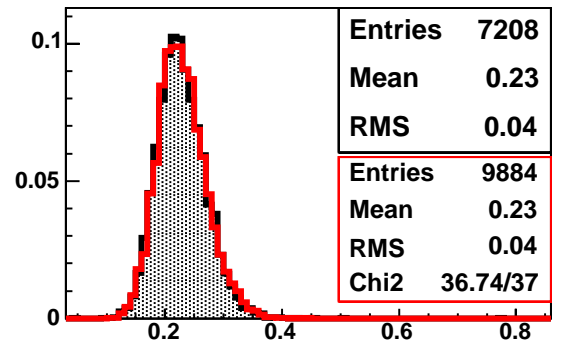

MIPs in Plane of Shower Max, 8.0 GeV

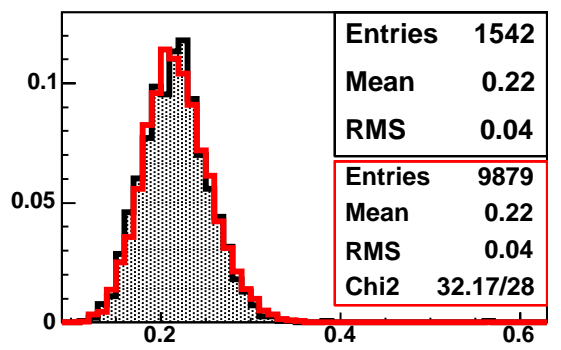

MIPs in Plane of Shower Max, $9.0 \mathrm{GeV}$

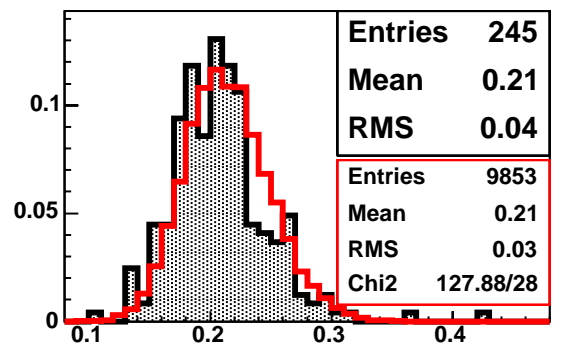

Figure D.30: Comparison of ratio of energy deposited in plane of shower max to total response between data and Monte Carlo. 
Total MIP, $0.2 \mathrm{GeV}$

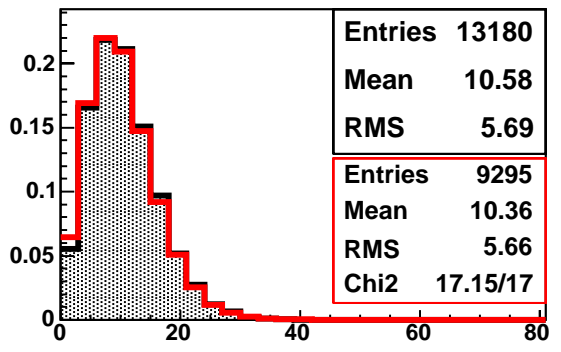

Total MIP, $0.6 \mathrm{GeV}$

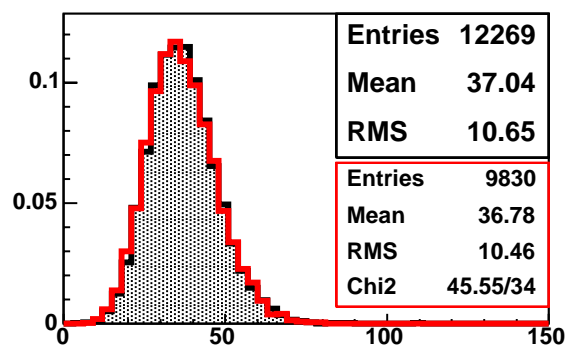

Total MIP, $1.0 \mathrm{GeV}$

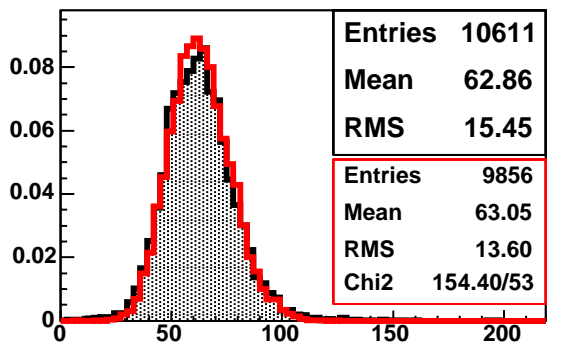

Total MIP, $0.4 \mathrm{GeV}$

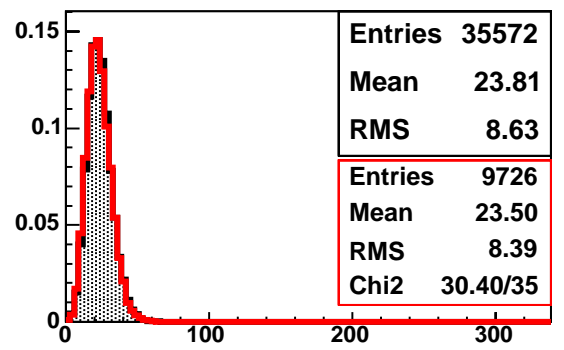

Total MIP, $0.8 \mathrm{GeV}$

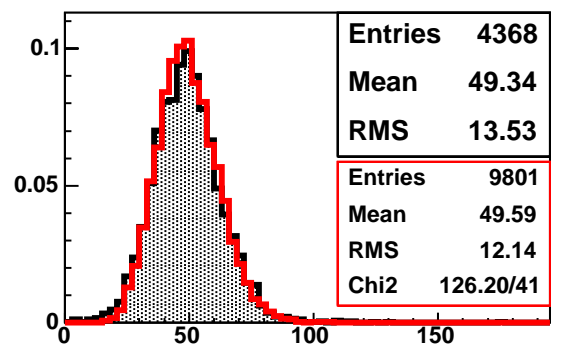

Total MIP, $1.2 \mathrm{GeV}$

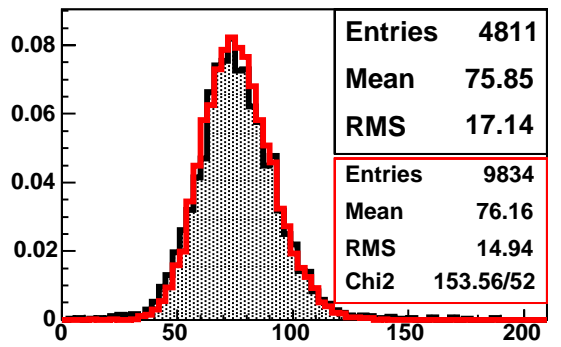

Figure D.31: Comparison of total MIP between data and Monte Carlo. 
Total MIP, $1.4 \mathrm{GeV}$

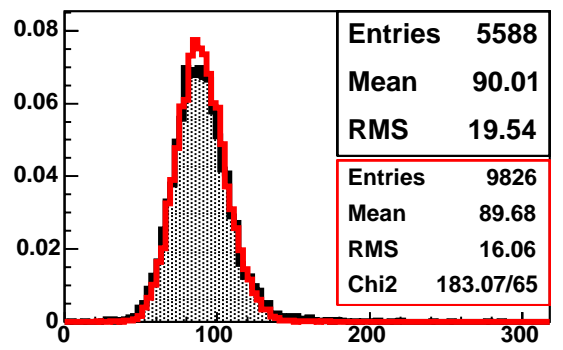

Total MIP, $1.8 \mathrm{GeV}$

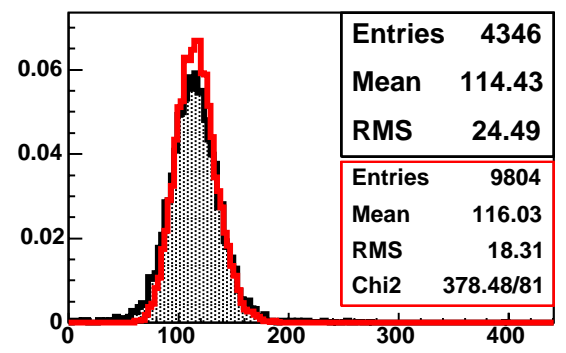

Total MIP, $2.2 \mathrm{GeV}$

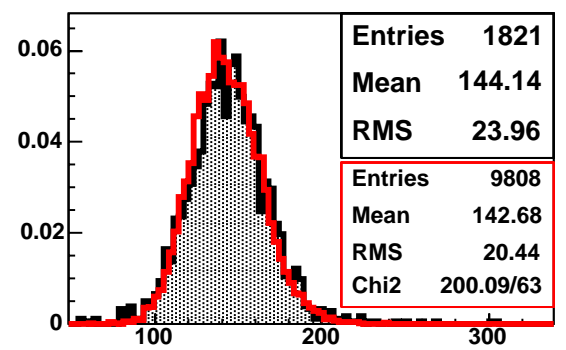

Total MIP, $1.6 \mathrm{GeV}$

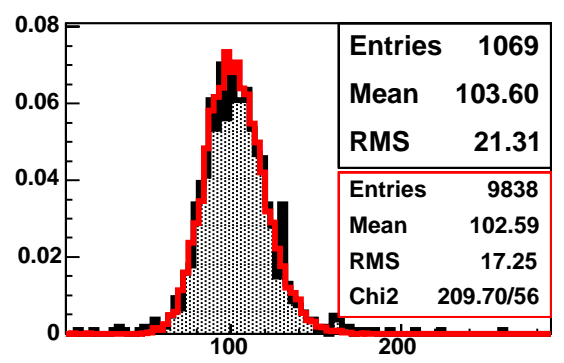

Total MIP, $2.0 \mathrm{GeV}$

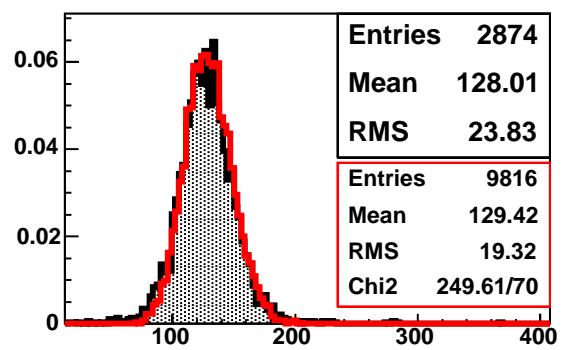

Total MIP, $2.4 \mathrm{GeV}$

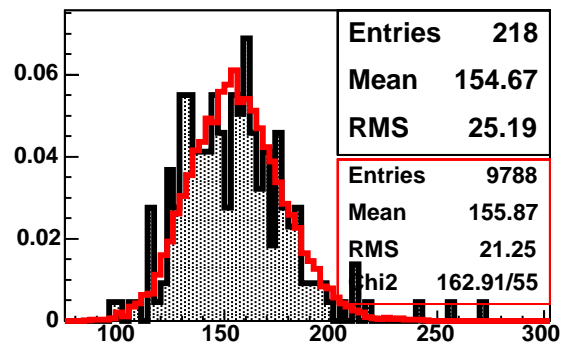

Figure D.32: Comparison of total MIP between data and Monte Carlo. 
Total MIP, $2.6 \mathrm{GeV}$

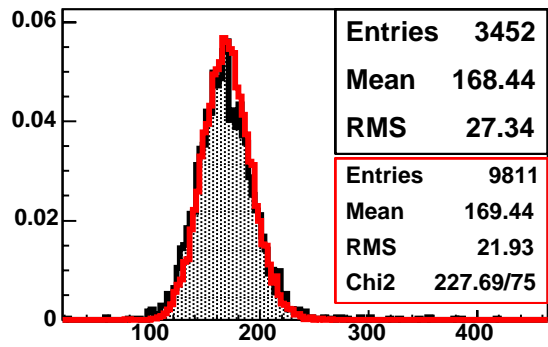

Total MIP, 3.0 GeV

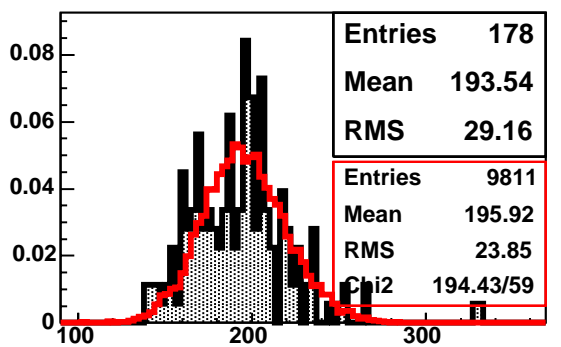

Total MIP, $3.6 \mathrm{GeV}$

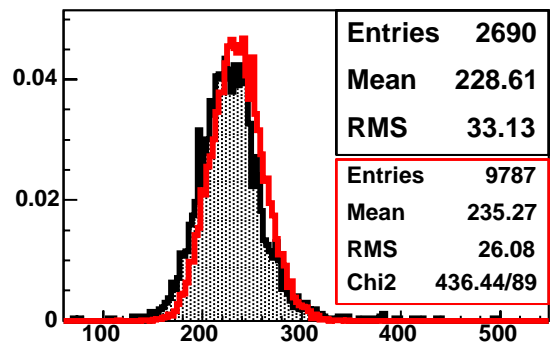

Total MIP $28 \mathrm{GeV}$

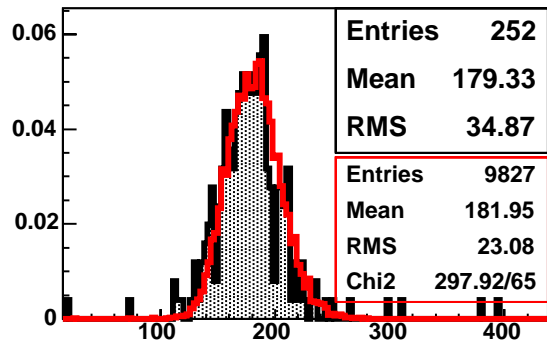

Total MIP, 3.2 GeV

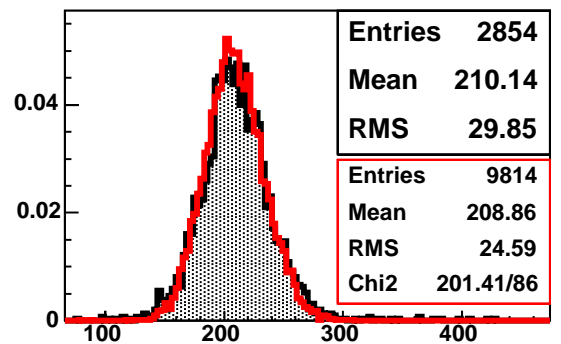

Figure D.33: Comparison of total MIP between data and Monte Carlo. 
Total MIP, $1.0 \mathrm{GeV}$

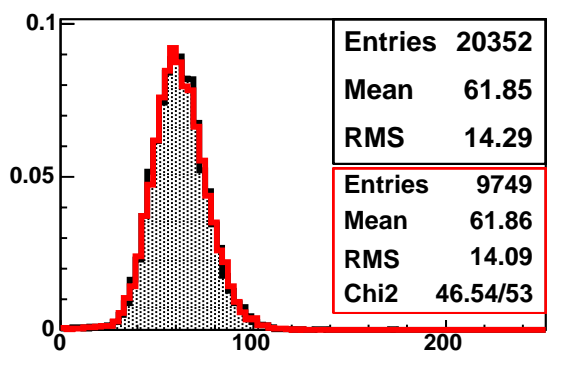

Total MIP, $2.4 \mathrm{GeV}$

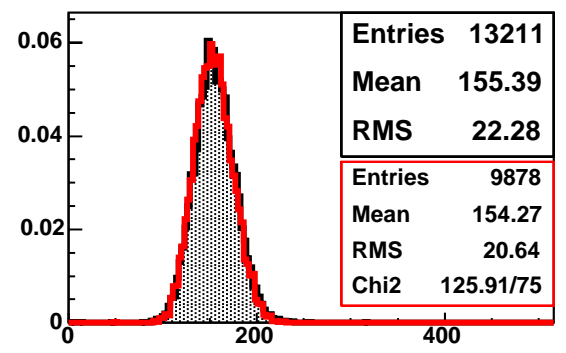

Total MIP, $3.0 \mathrm{GeV}$

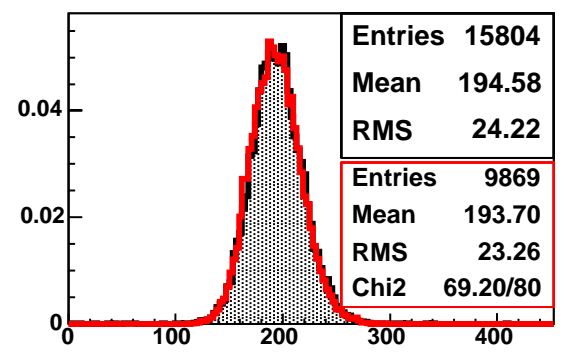

Total MIP, $2.0 \mathrm{GeV}$

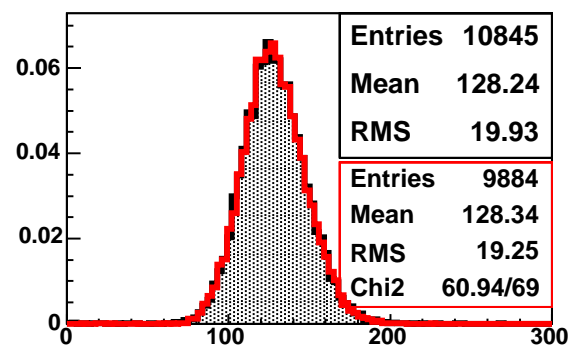

Total MIP, $2.8 \mathrm{GeV}$

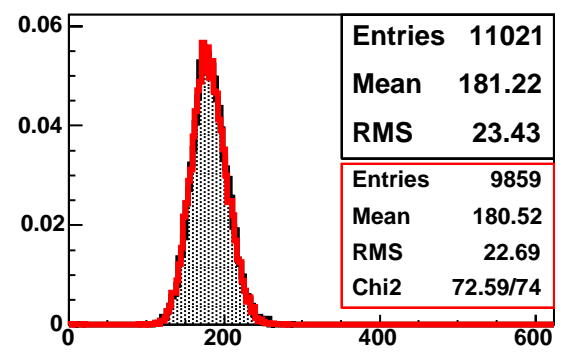

Total MIP, $4.0 \mathrm{GeV}$

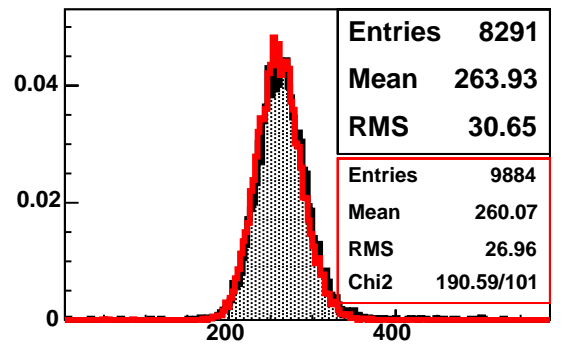

Figure D.34: Comparison of total MIP between data and Monte Carlo. 
Total MIP, $5.0 \mathrm{GeV}$

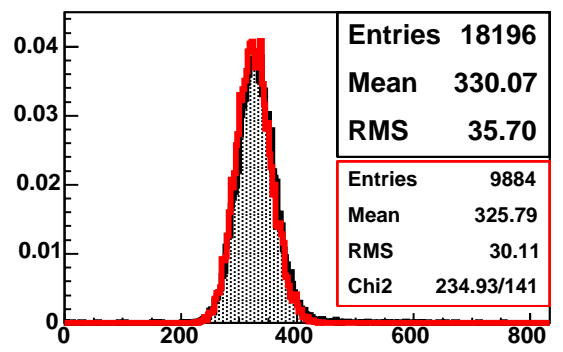

Total MIP, $7.0 \mathrm{GeV}$

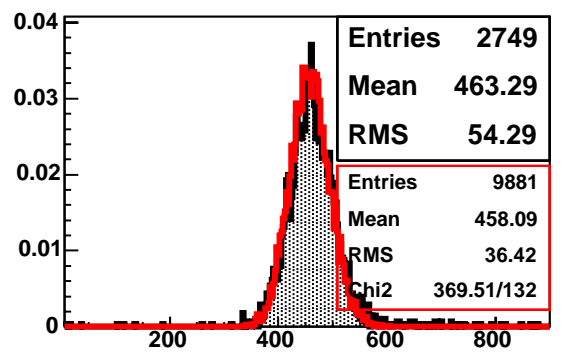

Total MIP, $9.0 \mathrm{GeV}$

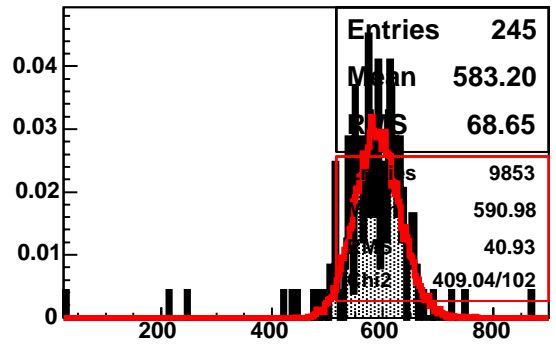

Total MIP $6.0 \mathrm{GeV}$

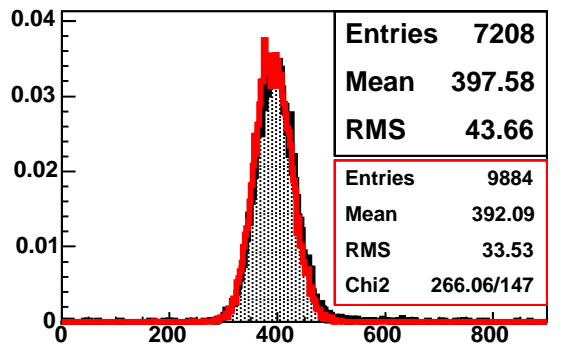

Total MIP, $8.0 \mathrm{GeV}$

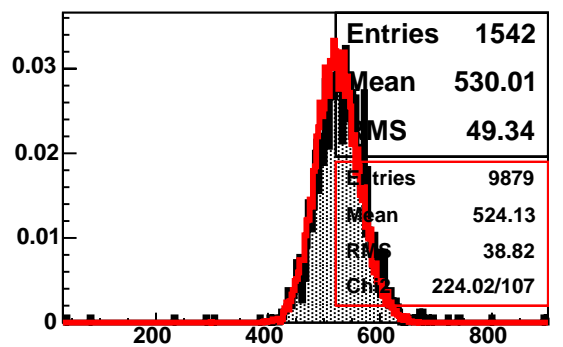

Figure D.35: Comparison of total MIP between data and Monte Carlo. 


\section{Appendix E}

\section{CalDetDST Offline Analysis Package}

\section{E.1 Introduction}

The study of the electromagnetic and hadronic interactions in the MINOS Calibration Detector was accomplished using a software suite composed of three packages designed for use in the MINOS offline software framework. The three packages, CalDetSI, CalDetPID and CalDetDST together apply the cuts needed to purify the CalDet data sample, interpret the particle identification information, and compute several derived quantities useful in analyzing the CalDet data. The functions defined in each of the packages are designed to work on CalDet data files that have been processed by the OfflineTrigger. Such files are produced offline from the raw data and have the filename extension .tdaq. Each package employs a class derived from the MINOS standard JobModule to accomplish the requisite analysis. The job modules are applied to the data in a sequence determined by a job module macro. An example of such a macro is provided via CVS in CalDetDST/macros/StdProduction/uberall.C. A slightly different macro is required for processing Monte Carlo events. An 
example of the Monte Carlo macro is provided in CalDetDST/macros/uber-mcall.C.

\section{E.2 CalDetSI}

The CalDetSI package is designed to extract CalDet specific information from the raw data. This package provides two main utilities, namely a candidate object to pass the CalDet specific information to other functions, and a set of cut utility functions that perform the cuts necessary to extract a clean CalDet data sample. The candidate package provides a handle to the CandCalDetSI object. Each method works on a single snarl at a time. The more useful data members contained in CandCalDetSI are listed in Table E.1.

The special electronics channels reserved for particle identification, trigger information, and timing differences between the Far Detector and the Near Detector are identified in the PLEXRAWCHANNELREADOUTTYPE offline database table. Each special channel has a readout type tag. Cerenkov1 in the CalDetSI context refers to the downstream Čerenkov, which has a readout type tag of "DSCER". Čerenkov2 refers to the middle Čerenkov, which has a readout type tag of "MIDCER". Cerenkov 3 refers to the upstream Cerenkov which has a readout type tag of "USCER". Channel 0 of the Oxford External Electronics box is identified by the readout type tag TADC0 but is not generally used. Channel 1 is identified by the readout type tag "TTAG" and is used as the TOF coincidence flag. Channel 2 is identified by the readout type tag "TimingFid" and is used to record the timing fiducial that tracks the drift of the Near Detector clock relative to the Far Detector clock when running in mixed electronics mode. Finally, the timestamp of one of the VARC input lemos is used as the time "toftime". It is identified in the PLEXRAWCHANNELREADOUTTYPE database table as "TTIME"

The second utility provided in the CalDetSI package is a JobModule called 


\begin{tabular}{|c|c|c|}
\hline Type & Fnct. Name & Description \\
\hline Bool_t & GetTriggerPMT() & $\begin{array}{l}\text { kTRUE if the trigger } \\
\text { PMT is digitized }\end{array}$ \\
\hline Bool_t & GetCosmicCnt () & $\begin{array}{l}\text { kTRUE if any } \\
\text { Cosmic Cntr is digitized }\end{array}$ \\
\hline Bool_t & GetFafErr() & $\begin{array}{l}\text { kTRUE if Faf } \\
\text { error encountered }\end{array}$ \\
\hline Bool_t & GetSparseErr() & $\begin{array}{l}\text { kTRUE if Sparsification } \\
\text { error encountered }\end{array}$ \\
\hline Int_t & GetTrigSource() & $\begin{array}{l}\text { bit field identifying } \\
\text { trigger source }\end{array}$ \\
\hline Int_t & GetKovADCn() & ADC of nth CER $(n=1,2,3)$ \\
\hline Int_t & GetKovTimeStampn() & $\begin{array}{l}\text { timestamp (FD ticks) } \\
\text { of hit in nth CER }\end{array}$ \\
\hline ULong_t & GetSnarlMinTimeStamp() & $\begin{array}{l}\text { timestamp (FD ticks) } \\
\text { of earliest snarl hit }\end{array}$ \\
\hline ULong_t & GetSnarlMaxTimeStamp () & $\begin{array}{l}\text { timestamp (FD ticks) } \\
\text { of latest snarl hit }\end{array}$ \\
\hline Int_t & GetTofTDC0() & $\begin{array}{l}\text { TDC from TDC Chan } 0 \\
\text { (DS/MID TOF paddle) }\end{array}$ \\
\hline Int_t & GetTofTDC1() & $\begin{array}{l}\text { TDC from TDC Chan } 1 \\
\text { (not used) }\end{array}$ \\
\hline Int_t & GetTofTDC2() & $\begin{array}{l}\text { TDC from TDC Chan } 2 \\
\text { (US TOF paddle) }\end{array}$ \\
\hline Int_t & GetTofADCn () & $\begin{array}{l}\mathrm{ADC} \text { from } \mathrm{nth}(\mathrm{n}=0,1,2 \\
) \text { input of } \mathrm{EVA}^{\dagger}\end{array}$ \\
\hline Int_t & GetTofADCTimeStampn() & $\begin{array}{l}\text { timestamp of hit } \\
\text { on nth input of EVA }\end{array}$ \\
\hline Int_t & GetTofTimeStamp () & $\begin{array}{l}\text { timestamp on VARC } \\
\text { lemo input }\end{array}$ \\
\hline Int_t & GetTickSinceLast() & $\begin{array}{l}\text { timestamps (FD ticks) } \\
\text { since last triggered event }\end{array}$ \\
\hline RawChanId & GetCerenkovChanneln() & $\begin{array}{l}\text { RawChannelId } \\
\text { of nth Čerenkov. }\end{array}$ \\
\hline vector\& & GetDeadChips () & dead chip RawChannelId's \\
\hline
\end{tabular}

Table E.1: Data members of CandCalDetSI, ${ }^{\dagger}$ External VA Electronics Box 
Cutter. Cutter consists of a number of functions defined to facilitate the pre-analysis cuts that are made on the CalDet data to obtain a clean sample. Table E.2 lists and describes the cuts that are defined in the Cutter package. Each cut can be toggled on or off in the job module macro by using the $\mathrm{Cmd}$ ("CutON(OFF) CntlString") method of the JobModule. The table lists the cuts in the order they are applied and also lists the default configuration of the cuts. Cutter writes out the results of cuts to a root file. The name of the root file may be specified by using the Config capability of JobModules to set the parameter "FileName".

\section{E.3 CalDetPID}

CalDetPID uses the read-out of the external particle identification detectors to decide what type of particle produced a given event. The nomenclature for the particle types is defined in CalDetParticleType and is also given in Table E.3. CalDetPID is also responsible for using the hit timing and the timing of the PID readout to determine whether or not the event was an "overlapping event". CalDetPID produces a candidate, which can then be passed to other job modules. There is also an associated class called NtpCalDetPID, which eventually gets written to the CalDetDST tree. The methods of CandCalDetPIDHandle are given in Table E.4. CalDetPID makes PID decisions based on values stored four database tables. Currently the data for these tables are stored in text files in CalDetPID/data and are loaded as temporary tables at run time. Eventually the PID database tables will be entered into the database permanently. Tables E.5, E.6, E.7, and E.8 list the requisite database tables and their fields. CalDetPID also provides another utility, that of comparing the hit times of an event to a template histogram in order to determine the likelihood of an event being an overlapper. This algorithm requires the existence of template histograms stored in root files within the CalDetPID/data directory. The particle ID package is run using the JobModule "RealCalDetPIDModule". The overlap- 


\begin{tabular}{|c|c|c|c|c|c|}
\hline & Fnct Prefix & Ctrl String & Hist Label & Pass Requirement & Default \\
\hline 1 & Trigger & TrigCut & Trig & $2 / 3$ plane trigger & $\mathrm{ON}$ \\
\hline 2 & TOFTrig & TOFTrig & TOFTrig & TOF coincidence & OFF \\
\hline 3 & Kov1Trig & Kov1TrigCut & Kov1Trig & non-zero CER1 & $\mathrm{OFF}$ \\
\hline 4 & Kov2Trig & Kov2TrigCut & Kov2Trig & non-zero CER2 & $\mathrm{OFF}$ \\
\hline 5 & Kov3Trig & Kov3TrigCut & Kov3Trig & non-zero CER3 & OFF \\
\hline 6 & KovORTrig & KovORTrigCut & KovORTrig & any non-zero CER & $\mathrm{OFF}$ \\
\hline 7 & AllKovTrig & AllKovTrigCut & AllKovTrig & all CER non-zero & $\mathrm{OFF}$ \\
\hline 8 & PIDOrTrig & PIDOrTrigCut & PIDTrig & Cut 2 or Cut 6 & OFF \\
\hline 9 & LITrig & LITrigCut & LITrig & event is not LI & $\mathrm{ON}$ \\
\hline 10 & DeadChip & DeadChipCut & $\mathrm{DC}$ & $\begin{array}{l}\text { No dead chips } \\
\text { below set plane }\end{array}$ & $\mathrm{ON}$ \\
\hline 11 & Kov & & Kov & $\begin{array}{l}\text { CER ADC } \\
\text { within set limit }\end{array}$ & OFF \\
\hline 12 & SparseError & SparseErrCut & $\mathrm{SE}$ & $\begin{array}{l}\text { No Sparsification } \\
\text { Errors }\end{array}$ & $\mathrm{ON}$ \\
\hline 13 & FafError & FafErrCut & Faf & No Faf Errors & $\mathrm{ON}$ \\
\hline 14 & OverShoot & & OS & $\begin{array}{l}\text { No chips in } \\
\text { overshoot period }^{\dagger}\end{array}$ & OFF \\
\hline 15 & TOF & TOFCut & TOF & $\begin{array}{l}\text { Any TDC } \\
\text { non-zero }^{\dagger}\end{array}$ & OFF \\
\hline 16 & $\begin{array}{l}\text { Cosmic- } \\
\text { Counter }\end{array}$ & CosmicCntCut & Cos & No Cosmic Cntr & $\mathrm{OFF}$ \\
\hline 17 & TrigPMT & TriggerPMTCut & PMT & No Trigger $\mathrm{PMT}^{\dagger}$ & $\mathrm{OFF}$ \\
\hline 18 & DeadKov & & DK & No dead CERs & $\mathrm{ON}$ \\
\hline 19 & NDError & NDErrorCut & $\mathrm{NDE}$ & No QIE errors & $\mathrm{ON}$ \\
\hline
\end{tabular}

Table E.2: Cut functions defined in Cutter. ${ }^{\dagger}$ Deprecated functions 


\begin{tabular}{|l|l|l|}
\hline Particle Type & Enum name & value \\
\hline Undetermined & kUnknown & 0x00 \\
$e$ & kElectron & 0x01 \\
$\mu$ & kMuon & 0x02 \\
$\pi$ & kPion & $0 \mathrm{x} 04$ \\
$K$ & kKaon & $0 \mathrm{x} 08$ \\
$p$ & kProton & $0 \mathrm{x} 16$ \\
\hline
\end{tabular}

Table E.3: Particle type nomenclature

\begin{tabular}{|c|c|c|}
\hline Method & Ntp variable & Description \\
\hline NoOverlap () & nov & Non-zero if all but \\
\hline InCerTime() & inct & $\begin{array}{l}\text { Non-zero if CER }(\mathrm{s}) \\
\text { signal time is in time }\end{array}$ \\
\hline GetPIDType() & cpid & Bit field describing particle type \\
\hline GetOLChi2() & olchi2 & $\begin{array}{l}\text { Chi2 from comparison of } \\
\text { event timing to template }\end{array}$ \\
\hline
\end{tabular}

Table E.4: Methods of CandCalDetPIDHandle

ping event identifier algorithms may be configured via RealCalDetPIDModule. The appropriate parameters are listed in Table E.9.

\section{E.4 CalDetDST}

The CalDetDST package was designed to create a TTree to hold many characteristics of CalDet beam events. There are three versions of the CalDetDST. The first, created by running the job module UberModule, contains derived event quantities, limited hit information, as well as the results of the Standard Reconstruction. The second, created by running the UberModuleLite job module was designed to provide quick feedback at the time of data taking. Trees created using UberModuleLite hold a subset of the data available in the UberModule tree variety. The final version, 


\begin{tabular}{|l|l|}
\hline CALDETTOFRANGE & \\
\hline PARTICLETYPE & Enum. particle type to which the row applies \\
TDC0LOW & Low end of acceptable TDC0 values \\
TDC0HIGH & High end of acceptable TDC0 values \\
TDC1LOW & Low end of acceptable TDC1 values \\
TDC1HIGH & High end of acceptable TDC1 values \\
TDC2LOW & Low end of acceptable TDC2 values \\
TDC2HIGH & High end of acceptable TDC2 values \\
TDC2MINUSTDC0LOW & Low end of acceptable TDC2-TDC0 values \\
TDC2MINUSTDC0HIGH & High end of acceptable TDC2-TDC0 values \\
TDC2MINUSTDC1LOW & Low end of acceptable TDC2-TDC1 values \\
TDC2MINUSTDC1HIGH & High end of acceptable TDC2-TDC1 values \\
\hline
\end{tabular}

Table E.5: TOFRange Database Table

\begin{tabular}{|l|l|}
\hline CALDETCERRANGE & \\
\hline PARTICLETYPE & Enum. particle type to which the row applies \\
CER0LOW & Low end of acceptable CER0 ADC values \\
CER0HIGH & High end of acceptable CER0 ADC values \\
CER1LOW & Low end of acceptable CER1 ADC values \\
CER1HIGH & High end of acceptable CER1 ADC values \\
CER2LOW & Low end of acceptable CER2 ADC values \\
CER2HIGH & High end of acceptable CER2 ADC values \\
\hline
\end{tabular}

Table E.6: CERRange Table

\begin{tabular}{|l|l|}
\hline CALDETOVERLAPWIN & \\
\hline PARTICLETYPE & Enum. particle type to which the row applies \\
WINLOW & Low end of acceptable hit time window \\
WINHIGH & High end of acceptable hit time window \\
\hline
\end{tabular}

Table E.7: OverLapWin Table 


\begin{tabular}{|l|l|}
\hline CALDETCERTIMEWIN & \\
\hline PARTICLETYPE & $\begin{array}{l}\text { Enum. particle type to which the row applies } \\
\text { WIN0LOW }\end{array}$ \\
WIN0HIGH & How end of acceptable CER0 time \\
WIN1LOW & Low end of acceptable CER0 time \\
WIN1HIGH & High end of acceptable CER1 time \\
WIN2LOW & Low end of acceptable CER2 time \\
WIN2HIGH & High end of acceptable CER2 time \\
\hline
\end{tabular}

Table E.8: CERRange Table

\begin{tabular}{|c|c|c|}
\hline Name & Description & Default \\
\hline NAllowedBefore & $\begin{array}{l}\text { Number of hits } \\
\text { allowed before WINLOW }\end{array}$ & 1 \\
\hline NAllowedAfter & $\begin{array}{l}\text { Number of hits } \\
\text { allowed after WINHIGH }\end{array}$ & 1 \\
\hline NAllowedOut & $\begin{array}{l}\text { Total number of hits } \\
\text { allowed out of WIN range }\end{array}$ & 2 \\
\hline NRequiredIn & $\begin{array}{l}\text { Number of hits } \\
\text { required to be in WIN range }\end{array}$ & 1 \\
\hline TimeFileNum & $\begin{array}{l}\text { Number of template } \\
\text { timing histogram file to use } \\
\text { (see example macro for details) }\end{array}$ & 0 \\
\hline
\end{tabular}

Table E.9: Configuration parameters for RealCalDetPIDModule 


\begin{tabular}{|l|l|l|}
\hline Return Type & Method & Description \\
\hline Int_t & GetRunNo & Returns the Run Number \\
Int_t & GetSubRunNo & Returns the Subrun Number \\
time_t & GetStartTime & $\begin{array}{l}\text { Returns the start } \\
\text { time of the sub run } \\
\text { Returns the temperature } \\
\text { Float_t }\end{array}$ \\
Float_t & GetTemperature & $\begin{array}{l}\text { at start of run(deprecated) } \\
\text { Returns the beam momentum }\end{array}$ \\
\hline
\end{tabular}

Table E.10: Methods of UberRecHeader

created by running the UberDST job module is no longer supported. The TTree that results from running UberModule is called "snarltree", and each row in snarltree contains a class called UberRecord. UberRecord derives from the standard offline ntuple class, and as such, each row in the tree is accompanied by a header called UberRecHeader. The methods that extract data from the header are listed in Table E.10. The beam momentum stored in the header class is read from the database table CALDETBEAMMOMENTUM, a supplemental database table that was created by hand; it is not extracted from the run comments. UberRecords hold another class called UberHit, which holds information specific to each of the hits in the event. The data stored in the UberHit are listed in table E.11. The data members contained in UberRecord are listed in Tables E.12 and E.13. 


\begin{tabular}{|l|l|l|}
\hline Type & Name & Description \\
\hline UShort_t & plane & plane number \\
UShort_t & strip & strip number \\
Int_t & padc & ADC on kPositive strip end \\
Int_t & nadc & ADC on kNegative strip end \\
Float_t & psiglin & linearized ADC on kPositive \\
Float_t & nsiglin & linearized ADC on kNegative \\
Float_t & pnpe & NPE on kPositive \\
Float_t & nnpe & NPE on kNegative \\
Float_t & pmip & MIP on kPositive \\
Float_t & nmip & MIP on kNegative \\
Float_t & ptime & time after first hit of event of hit on kPositive \\
Float_t & ntime & time after first hit of event of hit on kNegative \\
Int_t & pagg & aggregate plex number of kPositive end hit \\
Int_t & nagg & aggregate plex number of kNegative end hit \\
UInt_t & pbckt & number of buckets summed in positive side \\
UInt_t & nbckt & number of buckets summed in negative side \\
\hline
\end{tabular}

Table E.11: Data in UberHit 


\begin{tabular}{|c|c|c|}
\hline Type & Name & Description \\
\hline UInt_t & snarlno & snarl number \\
\hline Int_t & triggerword & offline trigger word \\
\hline Double_t & triggertime & Time (sec) of \\
\hline & & event WRT beginning of run \\
\hline UInt_t & nhits & number of hits in the event \\
\hline UShort_t & nhitplanes & number of planes hit \\
\hline UShort_t & nhitstrips & number of strips hit \\
\hline UShort_t & ndeadplanes & number of dead planes \\
\hline UShort_t & mindeadplaneno & smallest dead plane no \\
\hline & & (61-64, cosmic, 65-67 CER) \\
\hline Float_t & sigcorrconv & conversion from sigcorr to MIPs \\
\hline Float_t & totmip & total MIP in event (plane 0 not included) \\
\hline Int_t & maxadc & $\max$ hit $\mathrm{ADC}$ \\
\hline Float_t & maxnpe & $\max$ hit NPE \\
\hline Float_t & maxmip & $\max$ hit MIP \\
\hline Float_t & maxtime & time since beginning of event of last hit \\
\hline Float_t & p0totmip & tot MIPs deposited in plane 0 \\
\hline Float_t & p1totmip & tot MIPs deposited in plane 1 \\
\hline UShort_t & p0stripmaxmip & strip in plane 0 with max MIP(summed) \\
\hline Float_t & p0maxmiptstamp & time of hit in p0 with $\max$ MIP (one end) \\
\hline UShort_t & p1stripmaxmip & strip in plane 1 with max MIP(summed) \\
\hline Float_t & p1maxmiptstamp & time of hit in p1 with $\max$ MIP (one end) \\
\hline Float_t & mipweighaveplane & plane*MIP/totmip \\
\hline Float_t & mipweighcentereven & strip*MIP/totmip in even planes \\
\hline Float_t & mipweighcenterodd & strip*MIP/totmip in odd planes \\
\hline
\end{tabular}

Table E.12: Data in UberRecord. 


\begin{tabular}{|c|c|c|}
\hline Type & Name & Description \\
\hline Float_t & mipweighrad & $(\text { strip-center) })^{*} \mathrm{MIP} /$ totmip \\
\hline UShort_t & showermax & plane of maximum ADC \\
\hline Float_t & mipshowermax & MIP value in plane of shower max \\
\hline Float_t & totmipposeven & $\begin{array}{l}\text { MIPs summed over positive, } \\
\text { even strip ends (no plane 0) }\end{array}$ \\
\hline Float_t & totmipposodd & $\begin{array}{l}\text { MIPs summed over positive, } \\
\text { odd strip ends }\end{array}$ \\
\hline Float_t & totmipnegeven & $\begin{array}{l}\text { MIPs summed over negative, } \\
\text { even strip ends (no plane } 0 \text { ) }\end{array}$ \\
\hline Float_t & totmipnegodd & $\begin{array}{l}\text { MIPs summed over negative, } \\
\text { odd strip ends }\end{array}$ \\
\hline Int_t & ceradc $[3]$ & $\mathrm{ADC}$ from each $\mathrm{CER}^{\dagger}$ \\
\hline Float_t & certime $[3]$ & time-toftime of each CER \\
\hline Int_t & $\operatorname{toftdc}[3]$ & TDC from each TOF Paddle \\
\hline Int_t & tofadc $[3]$ & ADC from each of the EVA \\
\hline Float_t & tofhittime[3] & time-toftime of each EVA \\
\hline Float_t & toftime & time of external trigger \\
\hline NtpCalDetPID & cpid & PID Ntuple \\
\hline Float_t & summip $[60]$ & totmip summed over planes up to index \\
\hline UInt_t & nshw & Number of showers found by SR \\
\hline UInt_t & ntrk & number of tracks found by SR \\
\hline NtpSRShower & shw & shower ntuple ${ }^{\dagger \dagger}$ \\
\hline NtpSRTrack & & track ntuple ${ }^{\dagger \dagger}$ \\
\hline TClonesArray & *hitlist & Array of UberHits \\
\hline
\end{tabular}

Table E.13: Data in UberRecord. ${ }^{\dagger}$ cer[0] corresponds to the Čerenkov referenced by KovADC2 in CalDetSI, cer[1] corresponds to KovADC1, and cer[2] corresponds to KovADC3. ${ }^{\dagger \dagger}$ See Standard Reconstruction Documentation for description. 


\section{Appendix F}

\section{CalDetDBUtils Package}

The CalDetDBUtils package creates and reads many database tables specific to CalDet operations. The following sections detail the use of each of the classes provided.

\section{F.1 DCSEnvCal}

The class DCSEnvCal facilitates reading the database table DCS_ENV_CAL, which stores data from the environmental monitoring devices employed in the 2003 beam runs. Table F.1 describes the data stored in the database. The CAL_CERENKOV fields store the raw voltages output by the pressure transducers. These voltages are converted to absolute pressure using the function GetCerPres(int num).

\section{F.2 DCSMagCal}

The class DCSMagCal facilitates reading the database table DCS_MAG_CAL, which stores the values of the beam line magnet currents for the 2003 beam runs. Table F.2 describes the fields associated with DCS_MAG_CAL. 


\begin{tabular}{|l|l|}
\hline DCS_ENV_CAL & \\
\hline CAL_TEMPERATURE & Temperature at Field Point unit \\
CAL_HUMIDITY & Humidity \\
CAL_PRESSURE & Atmospheric Pressure \\
CAL_ISTRIG & Triggered DCS readout? \\
CAL_TRIGGERRATE & Rate of DCS Readouts \\
CAL_THERM1-32 & Thermocouple voltages around detector \\
CAL_CERENKOV1 & US Čerenkov pressure transducer voltage \\
CAL_CERENKOV2 & DS Čerenkov pressure transducer voltage \\
CAL_CERENKOV3 & US Čerenkov pressure transducer voltage \\
CAL_CERENKOV4 & DS Čerenkov pressure transducer voltage \\
CAL_CERENKOV5 & atmospheric pressure transducer voltage \\
CAL_COUNT1 & Spill Counter \\
CAL_COUNT2 & Number of US TOF hits \\
CAL_COUNT3 & Number of Gated TOF Coinc. \\
CAL_COUNT4 & Number of DS TOF hits \\
CAL_COUNT5 & Number of Ungated TOF Coinc. \\
CAL_COUNT6 & Number of DS Čerenkov hits \\
CAL_COUNT7 & Number of US Čerenkov hits \\
CAL_COUNT8 & Spare \\
\hline
\end{tabular}

Table F.1: DCS_ENV_CAL database table description 


\begin{tabular}{|l|l|}
\hline DCS_MAG_CAL & \\
\hline CAL_IST7 & Is data for T7? \\
CAL_ZT7_BHZ01S7 & Current in first T7 bending mag. \\
CAL_ZT7_BHZ01SN7 & Current in duplicate T7 bending mag. \\
CAL_ZT7_BHZ02 & Current in second T7 bending mag. \\
CAL_ZT7_QFO01 & Current in T7 quad. \\
CAL_ZT7_QDE02 & Current in T7 quad. \\
CAL_ZT7_QFO03 & Current in T7 quad. \\
CAL_ZT7_QDE04 & Current in T7 quad. \\
CAL_ZT7_QFO05 & Current in T7 quad. \\
CAL_ZT11_QDE01 & Current in T11 quad. \\
CAL_ZT11_QFO02 & Current in T11 quad. \\
CAL_ZT11_BHZ01 & Current in first T11 bending mag. \\
CAL_ZT11_QFO03 & Current in T11 quad. \\
CAL_ZT11_BHZ02 & Current in second T11 bending mag. \\
CAL_ZT11_QFO04 & Current in T11 quad. \\
CAL_ZT11_QDE05 & Current in T11 quad. \\
CAL_ZT11_BVT01 & Current in T11 pitching mag. \\
\hline
\end{tabular}

Table F.2: DCS_MAG_CAL database table description 


\section{F.3 CalDetSuperCycle}

The class CalDetSuperCycle creates and reads a database table that stores the PS super cycle period for each of the beam running periods. The table created, called CALDETSUPERCYCLE, is currently loaded at the beginning of each job to the temp database using the function LoadSuperCycle. The data written to the table is stored in the text file CalDetDBUtils/data/SuperCyclePeriod.txt. The database table holds has one row named SCPERIOD, which holds the super cycle period associated with the given validity range.

\section{F.4 SpillErrors}

The class CalDetSpillErrors creates and reads a database table that stores the errors associated with the differences among spills. The CALDETSPILLERRORS table is loaded into the temp database at the beginning of each job using the function LoadBeamErrors. The data written to the table is stored in CalDetDBUtils/data/SpillErrors.txt. Table F.3 describes the data stored in the database table.

\section{F.5 MomSetErrors}

The class CalDetMomSetErrors creates and reads a database table designed to store the systematic errors on the response measurement. The CALDETMOMSETER-

RORS table is loaded into the temp database at the beginning of each job using the function LoadBeamErrors. The data written to the table is stored in CalDetDBUtils/data/MomSetErrors.txt. The database table CALDETMOMSETERRORS has one row named BEAMSYSERR which holds the value of the systematic error of the response measurement associated with a given validity range. 


\begin{tabular}{|l|l|}
\hline CALDETSPILLERRORS & \\
\hline TMIPMAXSPILL & Response from events in spill with most events \\
ERRTMIPMAXSPILL & Error in response from spill with most events \\
NEVTMAXSPILL & Number of events in spill with most events \\
TMIPALLSPILL & Response from events in all spills \\
ERRTMIPALLSPILL & Error in response from all spills \\
NEVTALLSPILL & Number of events in all spills \\
TMIPMINSPILL & Response from events in spill with fewest events \\
ERRTMIPMINSPILL & Error in response from spill with fewest events \\
NEVTMINSPILL & Number of events in spill with fewest events \\
SPILLDEV & Relative difference in response between \\
& spill with most events and all spills \\
ERRSPILLDEV & Error in the relative difference \\
\hline
\end{tabular}

Table F.3: CALDETSPILLERRORS database table description

\section{F.6 BeamLoss}

The class CalDetBeamLoss creates and reads a database table designed to store the parameters to correct for upstream energy loss as derived using the beam line simulation. The CALDETBEAMLOSS table is loaded into the temp database at the beginning of each job using the function LoadBeamErrors. The data written to the table is stored in CalDetDBUtils/data/BeamLoss.txt. The details of the CALDETBEAMLOSS database table are given in Table F.4. An accessory class called CalDetBeamLossCalib provides functions that apply the corrections. The function ApplyJustBeamMatCorr(int, float, float, float\&) takes the particle ID, the raw response and the error in the raw response and returns a response corrected for the upstream energy loss. The error in the corrected response is assigned to the last parameter. The function ApplyJustHalfPlaneCorr(int, float, float, float\&) takes the particle ID, the raw response and the error in the raw response and returns a response corrected for first plane effects. The error in the corrected response is assigned to the last parameter. The function ApplyBeamLossCorr(int, float, float, 


\begin{tabular}{|l|l|}
\hline CALDETBEAMLOSS & \\
\hline PID & Particle ID for which the correction applies \\
BLSLOPE & Slope of the correction for the energy loss \\
BLSLOPEERR & Error in the slope of the energy loss correction \\
BLINTERCEPT & Y-Intercept of the correction for the energy loss \\
BLINTERCEPTERR & Error in the Y-Intercept of the energy loss correction \\
HFPLSLOPE & Slope of the correction for the first plane \\
HFPLSLOPEERR & Error in the slope of the first plane correction \\
HFPLINTERCEPT & Y-Intercept of the correction for the first plane \\
HFPLINTERCEPTERR & Error in the Y-Intercept of the first plane correction \\
\hline
\end{tabular}

Table F.4: CALDETBEAMLOSS database table description

float\&) takes the particle ID, the raw response and the error in the raw response and returns a response corrected for both energy loss and first plane effects. The error in the corrected response is assigned to the last parameter. 


\section{Bibliography}

[1] Pauli's now famous letter was not originally published. The idea was proposed in a letter dated December 4, 1930 and was addressed to the attendees of a conference in Tubingen. The letter has since been reproduced in a number of places.

[2] F. Reines and C. L. Cowan, "Detection of the Free Neutrino," Phys. Rev. 92 (1953) 830-831.

[3] G. Danby et al., "Observation of high-energy neutrino reactions and the existence of two kinds of neutrinos," Phys. Rev. Lett. 9 (1962) 36-44.

[4] DONUT Collaboration, K. Kodama et al., "Observation of tau-neutrino interactions," Phys. Lett. B504 (2001) 218-224.

[5] LEP Collaboration, "Electroweak parameters of the Z0 resonance and the Standard Model: the LEP Collaborations," Phys. Lett. B276 (1992) 247-253.

[6] Gargamelle Neutrino Collaboration, F. J. Hasert et al., "Observation of neutrino-like interactions without muon or electron in the Gargamelle neutrino experiment," Phys. Lett. B46 (1973) 138-140.

[7] B. T. Cleveland et al., "Measurement of the Solar Electron Neutrino Flux with the Homestake Chlorine Detector," Astrophys. J. 496 (1998) 505-526. 
[8] Super-Kamiokande Collaboration, Y. Fukuda et al., "Evidence for oscillation of atmospheric neutrinos," Phys. Rev. Lett. 81 (1998) 1562-1567.

[9] Super-Kamiokande Collaboration, T. Toshito, "Super-Kamiokande Atmospheric Neutrino Results," hep-ex/0105023.

[10] K2K Collaboration, M. H. Ahn et al., "Indications of Neutrino Oscillation in a 250-km Long-Baseline Experiment," Phys. Rev. Lett. 90 (2003) 041801.

[11] SNO Collaboration, Q. R. Ahmad et al., "Direct Evidence for Neutrino Flavor Transformation from Neutral-Current Interactions in the Sudbury Neutrino Observatory," Phys. Rev. Lett. 89 (2002) 011301.

[12] KamLAND Collaboration, "Measurement of Neutrino Oscillation with KamLAND: Evidence of Spectral Distortion." June 13, 2004.

[13] LSND Collaboration, C. Athanassopoulos et al., "Evidence for $\bar{\nu}_{\mu} \rightarrow \bar{\nu}_{e}$ Oscillation from the LSND Experiment at the Los Alamos Meson Physics Facility," Phys. Rev. Lett. 77 (1996) 3082-3085.

[14] A. Strumia, "Interpreting the LSND anomaly: Sterile neutrinos or CPTviolation or...?," Phys. Lett. B539 (2002) 91-101.

[15] MiniBooNE Collaboration, I. Stancu et al., "The MiniBooNE detector technical design report,". FERMILAB-TM-2207.

[16] NOvA Collaboration, "NOvA: Proposal to Build an Off-Axis Detector to Study muon- neutrino $\rightarrow$ electron-neutrino Oscillations in the NuMI Beamline,". FERMILAB-PROPOSAL-0929.

[17] Y. Itow et al., "The JHF-Kamioka neutrino project," hep-ex/0106019.

[18] V. D. Barger et al., "Neutrino decay and atmospheric neutrinos," Phys. Lett. B462 (1999) 109-114. 
[19] E. Lisi, A. Marrone, and D. Montanino, "Probing possible decoherence effects in atmospheric neutrino oscillations," Phys. Rev. Lett. 85 (2000) 1166-1169.

[20] MINOS Collaboration, "Proposal for a Five Year Run Plan for MINOS," 2003. MINOS/NuMI internal note, NuMI-930.

[21] D. A. Petyt, " $\nu_{\mu} \rightarrow \nu_{s}$ in MINOS," 2000. MINOS/NuMI internal note, NuMI-L-691.

[22] CHOOZ Collaboration, M. Apollonio et al., "Initial results from the CHOOZ long baseline reactor neutrino oscillation experiment," Phys. Lett. B420 (1998) 397-404.

[23] MINOS Collaboration, "The NuMI Technical Design Report," 2002.

[24] D. Indurthy et al., "Ion Chambers for Monitoring the NuMI Neutrino Beam at Fermilab," 2004. MINOS/NuMI internal note, NuMI-CONF-Beam-1028.

[25] MINOS Collaboration, "The MINOS Detectors Technical Design Report," 1998. MINOS/NuMI internal note, NuMI-L-337.

[26] MINOS Collaboration, "The MINOS Detector Design Parameter Book, Version 1.3," 2001.

[27] B. Rebel, Private Correspondence, 2004.

[28] K. Lang et al., "Characterization of 1,600 of 16-Anode PMTs for the MINOS Far Detector,". To be submitted.

[29] N. Felt et al., "Far Detector Front End Electronics," 2001. MINOS/NuMI internal note, NuMI-901.

[30] R. Lee, "Event Reconstruction in the Near Detector," 2000. MINOS/NuMI internal note, NuMI-NOTE-COMP-917. 
[31] N. Tagg et al., "Performance of Hamamatsu 64-Anode Photomultiplier Tubes for Use with Wavelength-shifting Fibre-optic Readout of Scintillator,". To be submitted.

[32] G. Drake et al., "Overview of the Front End Electronics for the Near Detector," 1999. MINOS/NuMI internal note, NuMI-L-628.

[33] "CERN PS East Area Hall Beam Line Documentation." http://psdata.web.cern.ch/psdata/www/eastareaop/index .html.

[34] MINOS Collaboration, P. Adamson et al., "The MINOS light injection calibration system," Nucl. Instrum. Meth. A492 (2002) 325-343.

[35] R. Nichol, "Light Injection Calibration at the Calibration Detector," 2003. MINOS/NuMI internal note, NuMI-974.

[36] MINOS Collaboration, P. Adamson et al., "On the linearity of the MINOS light-injection calibration system," Nucl. Instrum. Meth. A521 (2004) $361-366$.

[37] C. Smith, Calibration of the MINOS Detectors and Extraction of Neutrino Oscillation Parameters. PhD thesis, University College London, 2002.

[38] R. Nichol and C. Smith, "Strip-to-Strip Calibration at the Calibration Detector," 2003. MINOS/NuMI internal note, NuMI-972.

[39] J. Hartnell. Private Correspondence, 2004. To be explored further in his Doctoral Thesis, Oxford University.

[40] P. Adamson and P. Vahle, "The Caldet Trigger," 2003. MINOS/NuMI internal note, NuMI-NOTE-COMP-978. 
[41] U. Rohrer, "PSI Graphic Turtle Framework by U. Rohrer based on a CERN-SLAC-FERMILAB version by K.L. Brown et al." http://people.web.psi.ch/rohrer_u/turtle.htm, 2003.

[42] R. Brun et al., "GEANT Detector Description and Simulation Tool," 1994. CERN Program Library Long Writeup W5013.

[43] Particle Data Group Collaboration, K. Hagiwara et al., "Review of particle physics," Phys. Rev. D66 (2002) 010001.

[44] R. Hatcher, "Active Detector (FLS) Simulation in gminos and reco.minos," 1999. MINOS/NuMI internal note, NuMI-L-479.

[45] N. Tagg, "DetSim and PhotonTransport: MINOS Detector and Front-End Simulation." http://www-numi.fnal.gov/offline_software/srt_public_ context/WebDocs/detsim/DetSim_Documentation.html.

[46] M. Kordosky, Hadronic Interactions in the MINOS Detectors. PhD thesis, University of Texas at Austin, 2004.

[47] W. K. Sakumoto et al., "Calibration of the CCFR Target Calorimeter," Nucl. Instrum. Meth. A294 (1990) 179-192. 


\section{Vita}

Patricia LaVern Vahle was born in Omaha, Nebraska on February 6, 1975, the first daughter of Michael and Tamara Vahle. After graduating as valedictorian of Eldorado High School in Albuquerque, New Mexico in 1993, she attended New Mexico State University in Las Cruces, New Mexico. Her summers during college were spent working as an Undergraduate Research Assistant at Argonne National Laboratory. During the school year, Patricia was employed as a tutor at the Mathematics Learning Center at NMSU. In December, 1997 she received the degree of Bachelor of Science in Physics and Mathematics, graduating with High Honors. In August of 1998, Patricia entered the Graduate School of the University of Texas. She began working on the MINOS experiment in 2000.

Permanent Address: 1613 Pablo Ct. NE

Albuquerque, NM 87112

This dissertation was typeset with $\operatorname{LATE}_{\mathrm{E}} 2_{\varepsilon}{ }^{1}$ by the author.

\footnotetext{
${ }^{1} \mathrm{LTT}_{\mathrm{E}} \mathrm{X} 2 \varepsilon$ is an extension of $\mathrm{LTT}_{\mathrm{E}} \mathrm{X}$. ${ }^{\mathrm{A}} \mathrm{T}_{\mathrm{E}} \mathrm{X}$ is a collection of macros for $\mathrm{T}_{\mathrm{E}} \mathrm{X}$. $\mathrm{T}_{\mathrm{E}} \mathrm{X}$ is a trademark of the American Mathematical Society. The macros used in formatting this dissertation were written by Dinesh Das, Department of Computer Sciences, The University of Texas at Austin, and extended by Bert Kay and James A. Bednar.
} 